Bond University

Research Repository

\title{
Modified shuttle test-paeds: a valid cardiorespiratory fitness measure for children
}

Milne, Nikki; Simmonds, Michael; Hing, Wayne A

Licence:

Free to read

Link to output in Bond University research repository.

Recommended citation(APA):

Milne, N., Simmonds, M., \& Hing, W. A. (2015). Modified shuttle test-paeds: a valid cardiorespiratory fitness measure for children. 104. Abstract from CONNECT 2015 Physiotherapy Conference, Gold Coast, Queensland, Australia.

\section{General rights}

Copyright and moral rights for the publications made accessible in the public portal are retained by the authors and/or other copyright owners and it is a condition of accessing publications that users recognise and abide by the legal requirements associated with these rights.

For more information, or if you believe that this document breaches copyright, please contact the Bond University research repository coordinator. 

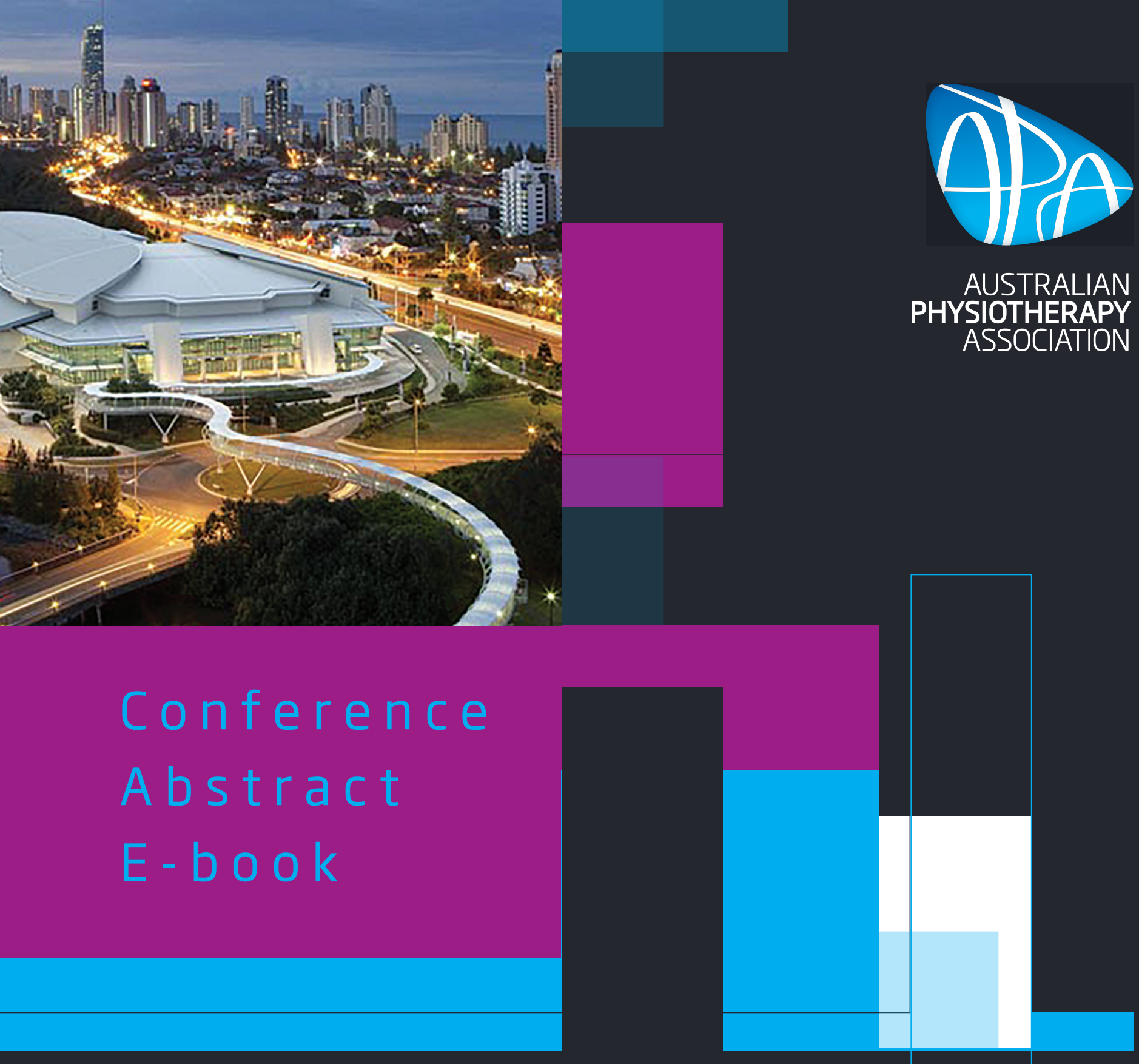

www.physiotherapy.asn.au/Conference 2015
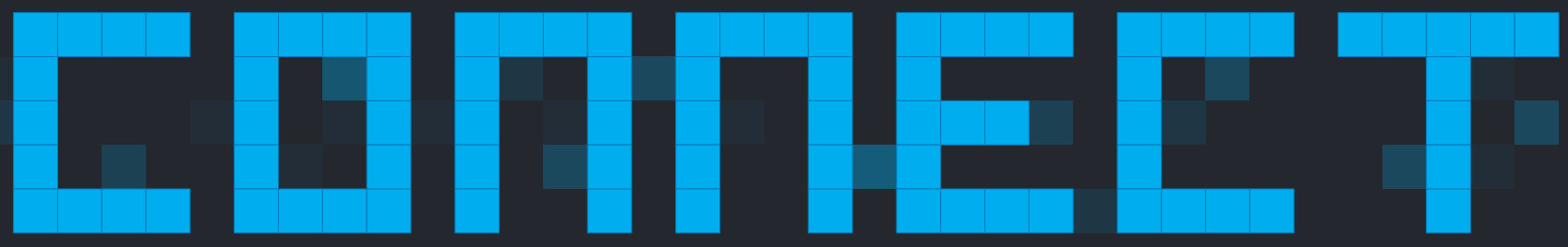

Physiotherapy Conference 2015

Gold Coast Convention and Exhibition Centre 3 - 6 october 
HOW TO GET PUBLISHED IN A PEERREVIEWED JOURNAL

\section{Abbott J $\mathrm{H}^{1}$}

Journal of Orthopaedic \& Sports Physical Therapy

In this, the third presentation in the session "How to get started in sports research, utilise your existing populations and get publishing", Dr Abbott, Editor-in-Chief-Elect for the Journal of Orthopaedic \& Sports Physical Therapy (JOSPT), will discuss: how to get started with writing and publishing: what a peer-reviewed journal looks for in a submission; how to prepare an article for submission; how to choose a journal to submit to; and what happens after you've submitted it. He will discuss the 'rules' of authorship, and offer some manuscript writing tips.

\section{Key Practice Points}

- How to get started with writing and publishing

- What a peer-reviewed journal looks for in a submission

- How to prepare an article for submission

- How to choose a journal to submit to

- What happens after you've submitted it

- The 'rules' of authorship

- Some manuscript writing tips

\section{THE SUBSTANTIAL PERSONAL BURDEN OF HIP AND KNEE OSTEOARTHRITIS AMONG YOUNGER PEOPLE}

Ackerman IN ${ }^{1,2}$, Bucknill $A^{2}$, Page RS ${ }^{3}$, Broughton $N^{4}$, Roberts $C^{2}$, Cavka B², Schoch $\mathrm{P}^{5}$, Brand CA²

${ }^{1}$ The University of Melbourne, Melbourne

2Melbourne Health, Melbourne

${ }^{3}$ Barwon Health and Deakin University, Geelong

${ }^{4}$ Peninsula Health, Frankston

${ }^{5}$ Barwon Health

Question: What is the Health-Related Quality of Life (HRQLL) and level of psychological distress experienced by younger people with hip or knee osteoarthritis, compared with population norms?

Design: Multi-centre cross-sectional study.

Participants: People aged 20-55 years with hip or knee osteoarthritis $(n=147)$ were recruited from three major Victorian public hospitals following screening of orthopaedic clinic lists and referrals, and through community-based advertisements.

Outcome Measures: HRQoL was assessed using the Assessment of Quality of Life (AQoL) instrument (range -0.04-1.00; scored worst to best; minimal important difference 0.06 units). Psychological distress was assessed using the K10 instrument (range 10-50; 22-29=high distress; 30 $50=$ very high distress). Data were compared to age- and gender-matched Australian norms.

Results: Comparison with population data revealed a very large reduction in HRQoL (mean reduction - $0.35 \mathrm{AQoL}$ units, $95 \% \mathrm{Cl}-0.40$ to -0.31 ). The prevalence of high psychological distress among the sample was substantially greater than for the population (31\% vs 8\%), as was the prevalence of very high distress (16\% vs $4 \%$ ). Participants were $>4$ times more likely to report high/very high psychological distress, compared with the population (relative risk 4.19,95\% Cl 3.53-4.98).

Conclusion: This study highlights the substantial personal burden of hip and knee osteoarthritis among younger people, and provides evidence to support the provision of targeted osteoarthritis services for people of working age.

Key Practice Points:

- Osteoarthritis has a major psychosocial impact

- Holistic management approaches should address physical and psychological wellbeing

- Osteoarthritis models of care for younger patients should incorporate screening for psychological distress and appropriate referral/ support mechanisms
MIXED METHODS EVALUATION OF A COMPREHENSIVE OSTEOARTHRITIS HIP AND KNEE SERVICE; PATIENT, CLINICIAN AND ADMINISTRATIVE PERSPECTIVES

Cavka B ${ }^{1}$, Ackerman I², Tacey M², Wicks I $^{3}$, Bucknill A ${ }^{4}$, Brand CA²

IPhysiotherapy Department, Royal Melbourne Hospital, Melbourne

2Melbourne Epicentre, Royal Melbourne Hospital, Melbourne

${ }^{3}$ Rheumatology Unit, Royal Melbourne Hospital, Melbourne

${ }^{4}$ Orthopaedic Unit, Royal Melbourne Hospital, Melbourne

Question: What is the impact of a targeted Osteoarthritis Hip and Knee Service (OAHKS) on efficiency outcomes and how is the new service perceived by patients and clinicians?

Design: Mixed methods design.

Participants: Wait times were evaluated for 250 random patients referred from general practice (GP) in 2003-2005 (pre-OAHKS implementation) and 2007-2012 (post-implementation). Interviews and focus groups were conducted with patients $(n=15)$, clinicians $(n=16$, advanced practice physiotherapists, orthopaedic surgeons, rheumatologists) and members of the original project team $(n=6)$.

Outcome Measures: Key wait times (GP referral to initial appointment initial appointment to consent for surgery, consent for surgery to procedure). Interviews and focus groups explored enablers and barriers to implementation, service satisfaction and patient experience.

Results: Median wait time from GP referral to initial appointment decreased significantly from 105 days pre-OAKHS implementation to 78 days post-implementation $(\pi=0.005)$. OAHKS patients waited a shorter time for their initial appointment compared to patients triaged to general orthopaedic clinics (median 63 versus 93 days, $\pi=0.002$ ). OAHKS patients waited a shorter time for their procedure (median 141 versus 216 days, $\pi=0.006$ ) compared to orthopaedic clinic patients. Qualitative data indicate patients, clinicians and project team members are highly satisfied with and value the OAHKS.

Conclusion: Implementation of the OAHKS at RMH has improved access to care for patients with hip and knee osteoarthritis through reduction of key wait times. The service was implemented as initially planned.

Key Practice Points:

- OAHKS has reduced key patient wait times

- The service is valued by patients, clinicians and stakeholders

\section{WWW.HEARTONLINE.ORG.AU: WHAT'S NEW?}

Adsett $]^{1}$, Hickey $\mathrm{A}^{2}$

${ }^{1}$ Royal Brisbane and Women's Hospital, Brisbane

2The Prince Charles Hospital, Brisbane

Background: Accessing evidence based tools to support the management of patients with cardiovascular disease is frequently difficult due to time and resource constraints and relevant information is of ten dispersed across multiple sources.

Purpose: Heart Education Assessment Rehabilitation Toolkit (HEART) Online provides clinical tools for Physiotherapists and other members of the multidisciplinary team to enable the assessment, planning, implementation and evaluation of care for patients with coronary artery disease and heart failure.

Methods: HEART Online was launched in May 2013 and has since been continuously modified based upon periodic targeted surveys and expert reviewer feedback. Ongoing evaluation is also provided through Google Analytics and the "contact us" pages. The site is currently undergoing a major revision which amongst other things includes a new web build, enhanced navigation and capability for access on mobile platforms. It is anticipated the enhanced site will be completed by July 2015 .

Results: HEART Online has an average of 3,100 visits to the site per month. The site is widely used in every state and territory of Australia and international visitors are growing. During 2014, visitors were mainly from English speaking countries: Australia (68\%) followed by USA (12\%), UK (6.5\%) then Canada, India and New Zealand. Feedback has resulted in: new pages on medications for acute coronary syndromes; sleep and heart failure; implantable devices; and revised sections on sexual activity and smoking. The 33 downloadable resources (such as clinical forms and letters, clinical management algorithms and tables, and patients handouts) have been revised to improve presentation and the ease of reading.

Conclusion: HEART Online has been developed by lead clinicians and academics from around Australia and has the potential to improve standards of evidence-based practice in cardiovascular care. The popularity of the site suggests that it is meeting an unmet need. Updates demonstrate the benefits of the web in being responsive to current and future needs of clinical practice. 
AOUATIC EXERCISE TRAINING AND STABLE HEART FAILURE: CURRENT RECOMMENDATIONS FROM A SYSTEMATIC REVIEW AND META-ANALYSIS

Adsett $]^{1}$, Mudge $\mathrm{A}^{2}$, Morris $\mathrm{N}^{3}$, Kuys $\mathrm{S}^{4}$, Paratz ${ }^{3,5}$ ${ }^{1}$ Heart Failure Service, Royal Brisbane and Women's Hospital, Australia 'Dept Internal Medicine and Aged Care, Royal Brisbane and Women's Hospital, Australia

${ }^{3}$ School of Allied Health Sciences, Griffith University, Gold Coast, Australia ${ }^{4}$ School of Physiotherapy, Australian Catholic University, Australia 5Physiotherapy Dept, Royal Brisbane and Women's Hospital, Australia

Background: Exercise training is a recommended component of the comprehensive approach to heart failure management: however traditional training programmes may not be suitable for all patients. Those with significant co-morbid disease, orthopaedic issues or balance disturbances for example, often find participation in these programmes difficult. Aquatic exercise (exercise conducted in thermoneutral water) may provide a suitable alternative, however progress has been hampered by historical concerns regarding safety.

Purpose: A meta-analysis and review of the evidence was conducted to determine the efficacy of aquatic exercise training for individuals with heart failure compared to traditional land-based exercise programmes.

Methods: A systematic search was conducted for studies published prior to March 2014, using MEDLINE, PUBMED, Cochrane Library, CINAHL and PEDro databases. Key words and common synonyms relating to aquatic exercise and heart failure comprised the search strategy. Interventions included aquatic exercise or a combination of aquatic plus land-based training, whilst comparator protocols included usual care or land-based training alone. The primary outcome was exercise performance. Studies reporting on muscle strength, quality of life and a range of haemodynamic and physiological parameters were also reviewed.

Results: Eight studies met criteria, accounting for 156 participants. Meta-analysis identified studies including aquatic exercise to be superior to comparator protocols for 6 minute walk test $(p<0.004)$ and peak powe $(p<0.044)$. Compared to traditional land-based training programmes, aquatic exercise training provided similar benefits for VO2 peak, muscle strength and quality of life, though was not superior. Cardiac dimensions, left ventricular ejection fraction, cardiac output and BNP were not influenced by aquatic exercise training. No serious adverse events were reported for any of the 156 participants.

Conclusions: For those with stable heart failure, aquatic exercise training improves exercise capacity, muscle strength and quality of life similar to land-based training programmes. This form of exercise may provide a safe and effective alternative for those unable to participate in traditional exercise programmes.

\section{CLINICAL GEMS FOR TREATMENT OF HIP PAIN}

\section{Agolley D ${ }^{1}$, Grimaldi A2 , Sims $\mathrm{K}^{3}$, Wisbey-Roth $\mathrm{T}^{4}$}

IJohn Flynn Private Hospital, Gold Coast Surgical Hospital \& Tweed Heads Hospital

2Physiotec Physiotherapy, Brisbane \& The University of Queensland ${ }^{3}$ Cricket Australia \& The University of Queensland

${ }^{4}$ Bounce Back Active Rehabilitation Systems \& Wisbey-Roth Consulting This panel will bring together leading Australian physiotherapists and orthopaedic surgeons to discuss the latest evidence pertaining to the clinical management of hip pain and pathology in adults. The panel comprises experts who are both researchers and clinicians, and so will bring a strong evidence-based approach to the discussion. Each presenter will discuss a case commonly seen in their clinical practice, followed by questions from the other panel members and the audience. Cases presented will include femoro-acetabular impingement, gluteal tendinopathy and early hip osteoarthritis. The session will be interactive, with audience members encouraged to provide questions and alternative perspectives to the panel.

\section{Key Practice Points:}

- An understanding of current clinical management of common presentations of hip pain in adults

- An understanding of current evidence pertaining to clinical management of common presentations of hip pain in adults

- An opportunity to actively discuss these outcomes with experts in the field of hip pain in adults
PAIN AND SELF-REPORTED CENTRAL SENSITISATION SYMPTOMS ARE ASSOCIATED WITH BRAIN GAMMA-AMINOBUTYRIC LEVELS IN MIGRAINE: INSIGHTS FOR PHYSIOTHERAPY ASSESSMENT

Aguila MER ${ }^{1,2}$, Rebbeck $\mathrm{T}^{1}$, Leaver $\mathrm{AM}^{1}{ }^{1}$, Lagopoulos $]^{3}$, Brennan $\mathrm{PC}^{1}$, Hübscher $\mathrm{M}^{1,4}$, Refshauge KM ${ }^{1}$

${ }^{1}$ The University of Sydney Faculty of Health Sciences, Lidcombe ¿University of the Philippines College of Allied Medical Professions, Manila, Philippines

${ }^{3}$ Brain and Mind Research Institute, Sydney Medical School, Camperdown ${ }^{4}$ Neuroscience Research Australia and The University of New South Wales, Randwick

Question: Is there a relationship between migraine clinical characteristics and brain gamma-aminobutyric acid (GABA) levels?

Design: Case-control study

Participants: Twenty adults fulfilling the International Classification of Headache Disorders-II criteria for migraine and 20 age- and gendermatched controls.

Outcome Measures: Pain, central sensitisation symptoms, negative emotional state symptoms, and perceived disability were measured using Short-Form McGill Pain Questionnaire-2 (maximum score 10), Central Sensitization Inventory (maximum score 100), Depression Anxiety Stress Scales-21 (maximum score 42), and Headache Impact Test-6 (maximum score 78), respectively. Scores were compared between migraine and control groups using Wilcoxon signed ranks test. Secondary analysis of brain GABA levels of the same cohort measured using proton magnetic resonance spectroscopy was conducted. Relationships between clinical characteristics and GABA levels were examined using correlation analyses.

Results: The migraine group had significantly higher scores on pain [median (interquartile range) 2.3 (0.8 to 3.1)], central sensitisation [32 (30 to 42.5)] and disability [63.0 (57.2 to 65.5)] than the control group ( $p<$ $0.05)$. There was fair positive association between GABA levels and pain score $(\rho=.47, p=.036)$ and central sensitisation score $(\rho=.48, p=.034)$. GABA levels did not correlate with disability.

Conclusion: Increased GABA levels are associated with higher pain and central sensitisation scores. This relationship corroborates the potential of GABA as a migraine biomarker and contributor to migraine mechanisms.

\section{Key Practice Points:}

- GABA is a potential diagnostic biomarker for migraine

- Self-report questionnaires may be useful in migraine assessment

- Measuring clinical characteristics in migraine

\section{HOW EFFECTIVE ARE F-MARC INJURY PREVENTION PROGRAMS FOR SOCCER PLAYERS? A SYSTEMATIC REVIEW AND META-ANALYSIS}

Al Attar WS ${ }^{1}$, Soomro N ${ }^{1}$, Sinclair PJ ${ }^{1}$, Pappas E ${ }^{1}$, Sanders RH ${ }^{1}$ ${ }^{1}$ Faculty of Health Sciences, the University of Sydney

Question: How Effective are F-MARC Injury Prevention Programs for Soccer Players?

Design: Systematic review with meta-analysis

Participants: Soccer players.

Intervention: F-MARC injury prevention programs for soccer players. These F-MARC programs included the FIFA 11+, the FIFA 11, and the FIFA Bricks.

Outcome Measures: Presence or absence of lower limb and overal injuries: Injury rate in the control group versus the intervention group.

Results: The pooled results based on total injuries per 1000 hours of exposure showed that the F-MARC programs had a statistically significant reduction in the overall injury risk ratio to $0.771(95 \% \mathrm{Cl}: 0.647$ to $0.918, \mathrm{p}=0.003)$ and lower extremity injury risk ratio of 0.762 ( $95 \%$ Cl: 0.621 to $0.935, p=0.009$ ) Moreover, F-MARC '11+' had a statistically significant reduction in the overal injury risk ratio of 0.654 ( $95 \%$ Cl: 0.537 to $0.798, p<0.001)$ and lower extremity injury risk ratio of 0.612 ( $95 \%$ Cl: 0.475 to $0.788, p<0.001$ ). However, F-MARC ' 11 ' did not reach significance for overall and lower extremity injury reduction.

Conclusions: This systematic review and meta-analysis indicated that use of the F-MARC injury prevention programs, particularly the '11+' program, decreases the risk of injuries among soccer players.

\section{Key Practice Points:}

- There is good evidence that F-MARC injury prevention programs can reduce injury between $20 \%$ and $50 \%$ in the long term compared to the teams that do not engage in the F-MARC programs

- The ' $11+$ ' in particular was shown to be highly effective in reducing overall and lower extremity injury 
A PILOT QUASI-RANDOMIZED CONTROL TRIAL ON NON-INVASIVE NERVE STIMULATION IN THE MANAGEMENT OF PAEDIATRIC CHRONIC PAIN

McCormick $\mathrm{M}^{1}$, Bott $\mathrm{A}^{1}$, Aldridge $\mathrm{V}^{2}$, Lang $\mathrm{T}^{1}$, Pacey $\mathrm{V}^{2}$

${ }^{1}$ Department of Pain and Palliative Care, Sydney Children's Hospital

2Department of Health Professions, Macquarie University

Question: Will paediatric chronic pain patients who receive non-invasive nerve stimulation display greater improvement in functioning and pain levels than those not receiving any of this treatment?

Design: Quasi-randomized controlled trial

Participants: 17 paediatric chronic pain patients (mean age 12.9yrs, 14 females) were recruited from a tertiary paediatric pain clinic.

Intervention: Non-invasive nerve stimulation device or waitlist contro for 6 weeks. Participants were instructed to use the device whenever they experienced pain or felt the device may be beneficial.

Outcome Measures: Primary outcome is child-reported pain intensity measured by the Faces Pain Scale. Secondary outcome is child-reported pain interference with daily life measured by the Pain Interference Scale. Measures were taken at baseline and immediately post-intervention.

Results: Most participants' primary site of chronic pain was their abdomen or lower limb. After 6 weeks of intervention the non-invasive nerve stimulation group ( $n=7$ ) displayed a mean decrease in child-reported pain intensity of 2.57/10 (SD=1.51, $p=0.004)$. The control group $(n=4)$ displayed a mean difference of no change $($ mean $=0.000, S D=2.83, p=1.00)$. There was no significant difference in the change in child-reported pain intensity between the groups (mean $=1.27, S D=1.8, p=0.076$ )

Conclusion: These preliminary results suggest non-invasive nerve stimulation may be useful in reducing pain intensity. More participants are required to provide a better understanding of the influence of these devices.

\section{Key Practice Points:}

- Non-invasive nerve stimulation may reduce pain intensity experienced by paediatric chronic pain patients

- Non-invasive nerve stimulation can be used in addition to exercise to manage paediatric chronic pain

\section{BRAIN AND SPINAL CORD STIMULATION FOR THE TREATMENT OF CHRONIC HEADACHE}

Alhassani $\mathrm{G}^{1}$, Schabrun $\mathrm{SM}^{1}$

${ }^{1}$ University of Western Sydney, Brain Rehabilitation and Neuroplasticity Unit Campbelltown Campus

Question: What is the effect of a combined brain and spinal cord direct current stimulation treatment on chronic headache frequency, intensity, duration and pain sensitivity?

Design: An ABA design that involved three stages (baseline, intervention and post-intervention)

Participants: Five individuals, three that suffered from chronic tension type headache and two individuals with chronic migraine.

Intervention: Five consecutive daily sessions of a 20 minute brain stimulation treatment delivered to the primary motor cortex, followed by a 20 minute spinal cord stimulation treatment delivered over the 10 th thoracic vertebrae at $1 \mathrm{~mA}$ intensity.

Outcome Measures: Pain sensitivity was recorded immediately before and after treatment using a pressure algometer to record pressure pain threshold. Headache symptoms of frequency, intensity and duration were recorded via a headache diary four weeks before and after treatment.

Results: Headache frequency reduced in all participants (mean number

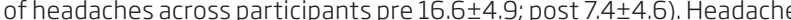
duration and intensity, and pressure-pain sensitivity, were unaltered in all participants.

Conclusion: These preliminary data indicate that a combined brain and spinal cord stimulation may reduce headache frequency in headache sufferers, but when a headache is triggered, duration and intensity are unaltered.

\section{Key Practice Points:}

- A combined brain and spinal cord stimulation may reduce headache frequency.

- When a headache is triggered, pain duration and intensity are unaltered.

- A reduction in headache frequency with combined brain and spinal cord stimulation does not appear to be related to reduced pain sensitivity.
IMPROVING ACCESS TO PULMONARY REHABILITATION - NEW DEVELOPMENTS

\section{Alison JA $\mathrm{A}^{1,2,3}$}

${ }^{1}$ Discipline of Physiotherapy, Faculty of Health Science University of Sydney ${ }^{2}$ Allied Health Professorial Unit, Sydney Local Health District ${ }^{3}$ Department of Physiotherapy, Royal Prince Alfred Hospital Pulmonary rehabilitation has high level evidence of efficacy in the management of people with chronic lung disease, however access to pulmonary rehabilitation in Australia is limited. To enable the translation of the evidence for pulmonary rehabilitation into clinical practice, the reasons for limited access need to be determined and strategies to improve access to pulmonary rehabilitation are needed. The presentation will provide the most recent evidence for the benefits of pulmonary rehabilitation and discuss reasons for limited access and strategies to improve access. Such strategies include: alternative modes of delivery of pulmonary rehabilitation; educational programs available to health professionals to upskill in pulmonary rehabilitation; support networks and resources; and new and proposed funding models.

\section{Key Practice Points:}

- This session will enable participants to know the most recent evidence for pulmonary rehabilitation as an intervention in the management of chronic lung disease and strategies that can improve access to pulmonary rehabilitation.

\section{YEAR IN REVIEW- PULMONARY REHABILITATION AND EXERCISE PRESCRIPTION}

\section{Alison JA 1,2,3}

${ }^{1}$ Discipline of Physiotherapy, Faculty of Health Sciences, University of Sydney 2Sydney Local Health District, Sydney

${ }^{3}$ Department of Physiotherapy, Royal Prince Alfred Hospital, Sydney

Recent literature in the area of exercise prescription, pulmonary

rehabilitation and physical activity in people with chronic lung diseases will be reviewed.

\section{Key Practice Points:}

- This session will provide participants with an update of current research in exercise prescription, pulmonary rehabilitation and physical activity which may aid evidence-based practice.

\section{CONNECTING WITH MEN: GIVING THE EVIDENCE A CHANCE}

\section{Allingham $\mathrm{C}^{1}$ \\ 'Strong Men's Business}

Men's health is far more than prostate cancer, incontinence and erectile dysfunction. The health of men encompasses not only these biological issues (and many more) but also their psychosocial aspects of health attitudes and behaviours, and those of the health professionals they consult. Men have many strengths, but preserving their own health is not prominent amongst them. In fact, the very essence of masculinity is constructed on the four pillars of risk taking, stoicism, excess and lack of concern for self. It is arguable whether a male patient and their health professional are even speaking the same language when it comes to health, which makes communication challenging at best. Men tend to focus on what is working well (or well enough) whereas we health professionals are single minded in our focus of what is not working well, asking about signs and symptoms that have not yet reached the conscious recognition for the man concerned. While the evidence base for our interventions grows, it is of little practical use if the client is not playing his role as an active participant in best practice therapy. The goal of this presentation is to acknowledge the differences in how men perceive and report their health and to offer practical suggestions for health professionals to improve their connection with their male clients to facilitate a more powerful outcome for both the therapist and the patient in the interests of the men, their families and the community.

\section{Key Practice Points:}

- This presentation will facilitate more effective understanding and communication with male clients in the therapeutic exchange, enabling improved patient compliance and an enhanced opportunity for evidence based treatments to exert their effects. 


\section{REDUCED MUSCLE STRENGTH, POWER, MUSCLE MASS AND ACTIVATION AS RISK FACTORS FOR POOR OUTCOMES AFTER TOTAL KNEE ARTHROPLASTY: A SYSTEMATIC REVIEW}

\section{Alnagmoosh $A^{1,2}$, Harmer $A^{1}$, Simic $M^{1}$, Fransen $M^{1}$}

${ }^{1}$ The University of Sydney, Sydney

2The University of Dammam, Dammam, Saudi Arabia

Questions: Do low muscle strength, power, muscle mass and activation predict ongoing pain and activity limitations after total knee arthroplasty (TKA)?

Design: Systematic review of randomized controlled trials, longitudinal and cross sectional studies.

Participants: People with unilateral or bilateral TKA.

Intervention: OVID, EMBASE and CINAHL databases were searched from their inception to May 2014. Two reviewers (AA and ARH) independently determined study eligibility. Two reviewers (AA and MS) extracted the data and assessed study risk of bias.

Outcome Measures: Eligible studies required measures of muscle strength, power, muscle mass, or activation of the knee extensors or knee flexors prior to, or very soon after, TKA and self-reported pain or activity limitations at a later period (at least three months following TKA).

Results: Among the 786 search identified records, only 13 longitudinal and eight cross-sectional studies had the required data. Only 12 longitudinal studies were considered of high methodological quality, with only four studies able to provide longitudinal associations between a muscle performance measure and self-reported pain or activity limitations. No study provided a full prediction model.

Conclusion: There is an absolute lack of studies evaluating the risk of pain and activity limitations after TKA associated with poor knee extensor or flexor muscle function.

Key Practice Points:

- Reducing pain and activity limitations is an integral part of rehabilitation post TKA

- There is still little evidence for the role of poor knee extensor or knee flexor muscle function for self-reported outcomes after TKA.

\section{IS PAIN REALLY THE PROBLEM? - A CASE REPORT}

\section{Althorpe T}

\section{Curtin University, WA}

Wanneroo Physiotherapy

The purpose of this case report is to discuss and compare patient centred outcomes with the application of evidence-based practice in the management of a lower limb injury. A 13-year old female presented with lateral leg and foot pain that was preventing her continued participation in her sport of netball. Assessment revealed multiple domains of impairment. including sub optimal jumping and landing kinematics that placed the client in a high-risk category for lower limb injury. Management focused on a multi modal approach to address intrinsic and extrinsic risk factors and patient centred outcomes. This case report discusses factors affecting the resolution of presenting symptoms, return to sport, patient's desired outcome and the optimal management of this patient.

\section{Key Practice Points:}

- Identification of jumping and landing kinematics that may be a risk factor forknee pathology and strategies to improve this

- Consideration of adolescent specific development characteristics that may impact on patient presentation and rehabilitation

- Clinical consideration of patient centred outcomes and whether this provides optimal outcomes for the patient.

\section{INTEGRATING MOBILE HEALTH AND PHYSICAL ACTIVITY TO REDUCE THE BURDEN OF CHRONIC LOW BACK PAIN TRIAL (IMPACT): A STUDY PROTOCOL}

Amorim $\mathrm{AB}^{1}$, Pappas $\mathrm{E}^{1}$, Simic $\mathrm{M}^{1}$, Ferreira $\mathrm{ML}^{2,3}$, Tiedemann $\mathrm{A}^{2}$, Jennings $\mathrm{M}^{4}$, Ferreira $\mathrm{P}^{1}$

${ }^{1}$ The University of Sydney, Sydney, Australia

${ }^{2}$ The George Institute for Global Health, Sydney Medical School, The University of Sydney, Sydney, Australia

IInstitute of Bone and Joint Research, The Kolling Institute, The University of Sydney, Sydney, Australia

${ }^{4}$ Physiotherapy Department, Liverpool Hospital, South Western Sydney Local Health District, NSW, Australia

Question: What is the effect of a mobile health-supported physical activity intervention on rates of pain recurrence and care seeking in people with chronic low back pain after discharge from treatment?

Design: Single blinded randomised controlled trial with concealed allocation and intention-to-treat analysis.

Participants: Sixty-eight patients with chronic low back pain persisting for over 12 weeks but without serious spine pathology and who have been discharged from a physiotherapy program. Eligible participants will be regular users of an internet-connected mobile device and fluent in English.

Intervention: The experimental group will receive an advice booklet in addition to a 6-month, tailored physical activity plan with health coaching sessions delivered fortnightly by a specifically designed WebApp and Fitbit pedometer. The control group will receive the advice booklet only.

Outcome Measures: Primary outcomes will be health care seeking associated with low back pain, pain and disability. Secondary outcomes will be physical activity engagement, goal attainment, quality of life, sleep quality, depression, fear avoidance, cognitive function and lower extremity function.

Results: Recruitment will start in June 2015

Conclusion: This model aims to prevent back pain recurrence, reduce care seeking and also empower patients to self-manage their low back pain by offering them a contemporary patient-centred physical activity program with the support of mobile health technology.

Trial registration: ACTRN12615000189527.

\section{Key Practice Points:}

- Pain recurrence is common in low back pain patients.

- This new approach of care has the potential to reduce recurrence and care seeking in low back pain patients.

\section{DOES SEDENTARISM INCREASE THE RISK OF LOW BACK PAIN: A PROSPECTIVE CO- TWIN STUDY OF SPANISH TWINS}

Amorim $\mathrm{AB}^{1}$, Pappas $\mathrm{E}^{1}$, Simic $\mathrm{M}^{1}$, Ferreira $\mathrm{ML}^{2}$, 3 , Ordoñana JR $\mathrm{R}^{4}$, Ferreira $\mathrm{P}^{1}$

${ }^{1}$ Faculty of Health Sciences, The University of Sydney, Sydney, NSW, Australia ${ }^{2}$ The George Institute for Global Health, Sydney Medical School, The University of Sydney, Sydney, NSW, Australia

Institute of Bone and Joint Research, The Kolling Institute, The University of Sydney, Sydney, Australia

${ }^{4}$ Murcia Twin Registry, Department of Human Anatomy and Psychobiology. University of Murcia, and IMIB-Arrixaca, Spain

Question: Is sedentary behaviour a risk factor for low back pain?

Design: Longitudinal observational study with a within-pair twin casecontrol design.

Participants: 1454 adult twins registered in the Murcia Twin Registry Spain, free of low back pain at baseline were followed for 2-4 years.

Outcome Measures: The main outcomes of this study were measures related to incidental chronic low back pain, care-seeking and activity limitation associated with low back pain. Sedentary behaviour was the explanatory variable.

Results: Data on LBP at follow-up was available for 1095 twins, In the total sample analysis, sedentary behaviour did not increase the incidence of chronic low back pain (OR:1.3, 95\% Cl: 0.93 to $1.70, p=1.13$ ). The within-pair twin case-control analyses for monozygotic twins only (when genetics is controlled for) showed a tendency for an increase in risk of low back pain in sedentary twins although (OR:4.0, 95\% Cl: 0.84 to $18.83, p=0.08)$. Similar results were found for the remaining outcomes.

Conclusion: Having a sedentary lifestyle is likely to increase the risk of future development of low back pain. Results of this study highlight the importance of controlling for genetics when investigating risks for low back pain.

\section{Key Practice Points:}

- Sedentary behaviour might increase the risk of chronic low back pain in adults.

- Preventative strategies to reduce low back pain incidence should focus on decreasing sedentary behavior. 
EIGHT MINUTES OF TREADMILL RUNNING FAMILIARISATION IS REQUIRED TO ACHIEVE CONSISTENT BIOMECHANICS IN BAREFOOT AND SHOD CONDITIONS

Arnold BJW $\mathbf{W}^{1,2}$, Horan $\mathrm{SA}^{1,2}$, Weeks $\mathrm{BK}^{1,2}$

${ }^{1}$ School of Allied Health Sciences, Griffith University, Gold Coast

2Centre for Musculoskeletal Research, Menzies Health Institute Queensland, Gold Coast

Question: How long does it take to achieve consistent biomechanical parameters (i.e. familiarise) when treadmill running in barefoot and shod conditions?

Design: Observational laboratory-based design with participants randomly allocated to run either barefoot $(n=13)$ or shod $(n=13)$ for 20 -minutes on an instrumented treadmill at a self-selected comfortable pace.

Participants: Twenty-six healthy men $(22.2 \pm 2.8$ years $)$ inexperienced in treadmill running.

Outcome Measures: Three-dimensional lower-limb sagittal-plane kinematics, ground reaction force and spatio-temporal data were collected at two-minute intervals and examined using one-way repeated measures ANOVA and ICCS.

Results: Temporal differences were observed for peak hip flexion during swing for the barefoot condition ( $F=3.448, p=0.02$ ) until 8 min. For the shod condition, temporal differences were observed until 8 min for peak vertical ground reaction force ( $F=4.245, p<0.001$, while for peak dorsiflexion during stance temporal differences were observed only after 10 min $(F=3.18, p=0.02)$. Reliability analysis revealed high levels of consistency (ICC > 0.90) across all consecutive time-points for al dependent variables for both conditions after 8 min of treadmill running.

Conclusion: Variation exists in biomechanical parameters for up to $8 \mathrm{~min}$ when running on a treadmill under barefoot and shod conditions.

\section{Key Practice Points}

- Biomechanics take time to adjust to treadmill running

- Whether shod or barefoot, 8 min of familiarisation is necessary to achieve consistent biomechanics

- Treadmill running studies should incorporate a familiarisation period of adequate length to enable valid conclusions to be drawn from biomechanical data

\section{A GRADED COLD EXPOSURE INTERVENTION CAN IMPROVE WIDESPREAD COLD HYPERSENSITIVITY. A CASE STUDY USING A NOVEL INTERVENTION}

\section{Azoory R}

University of Sydney, Sydney

Cold hypersensitivity is an established feature of chronic whiplash associated disorders and an indicator of poor prognosis. There is paucity of literature that investigates treatments to reduce sensitivity to cold stimulus. This single-subject case study aimed to treat cold hypersensitivity, provocative of neck pain, in a patient who had otherwise recovered from initial pain and disability following whiplash injury. A novel eight-week graded activity-modelled approach cold exposure program was designed according to previous protocols for graded activity programs that use principles of cognitive behavioural therapy. Psychological and physical measures including the Neck Disability Index, Numerical Rating Scales for pain, and cold pain thresholds (using Quantitative Sensory Testing), as well as a novel functional measure of cold exposure tolerance were recorded weekly. A modified version of the Tampa Scale for Kinesiophobia was created to reflect cold-related fear, and taken pre and post intervention. Results found improved reported cold exposure tolerance from five minutes until onset of neck pain pre-intervention, to 120 minutes post. The modified Tampa Scale score improved by 27 points (minimally clinically important change is four points). Cold pain thresholds, Neck Disability Index scores and Numerical Rating Scale scores for pain remained unchanged. The results of this case study suggest that individually tailored, graded cold exposure programs may be efficacious in treating reduced tolerance for cold stimulus. Further research in this area will assist in understanding the potential of such programs and possible mechanisms such as reducing cold-related fear that may have influenced findings.
HARNESSING EMPLOYEE ENGAGEMENT IN A PHYSIOTHERAPY DEPARTMENT: MOVING FROM A CULTURE OF BLAME TO A CULTURE OF SUCCESS

\section{Bach A $^{1}$}

${ }^{1}$ Epworth Healthcare, Rehabilitation Division, Melbourne, Victoria

Question: Can a targeted action plan shift employee engagement levels from blame to success in a hospital physiotherapy department in less than 18 months?

Design: Pre-post cohort survey study.

Participants: The physiotherapy department of an inner Melbourne private hospital, with 25 respondents to the survey (60\% response rate).

Intervention: A departmental action plan was implemented based on Best Practice Australia's six pillars of cultural change: engagement; leadership values \& behaviours; quality \& innovation; safety; and consumer outcomes.

Outcome Measures: Employee engagement levels were measured via a benchmarked electronic survey (administered through Best Practice Australia) pre and post action plan implementation. This measured the proportion of staff currently in an engagement cycle, versus swinging voters and those in a disengagement cycle.

Results: Employee engagement improved from 24\% to 76\% between October 2013 and March 2015. The overall rating improved from a classification of a culture of "blame" (20-30\% engagement level) to a culture of "success" (>60\% engagement level). $93 \%$ of itemised questions improved from pre to post survey results.

Conclusion: The results of this study indicate that a well-established action plan can shift the culture of a large physiotherapy department from blame to success in less than 18 months.

\section{Key Practice Points:}

- Employee engagement is a crucial precursor to the provision of a highperforming physiotherapy service.

- A department-wide action plan can transform a workplace culture from blame to success in less than 18 months.

- A strongly positive culture within a workplace drives strategy and outcomes.

\section{HOW CAN WE FUTURE-PROOF \\ THE PROFESSION: FACTORS INFLUENCING THE JOB SATISFACTION OF PHYSIOTHERAPISTS}

\section{Bacopanos E, Edgar S}

Questions: What is the job satisfaction of graduate physiotherapists from The University of Notre Dame Australia? What is the relationship between job satisfaction and demographic factors? What is the attrition rate of graduate physiotherapists and the factors influencing attrition?

Design: Descriptive survey design.

Participants: One hundred and fifty seven graduates (2006-2012) completed the online survey.

Outcome Measures: The online survey incorporated the Job Satisfaction Rating Scale, developed specifically for physiotherapists. Comparison between job satisfaction scores and demographics was undertaken to determine any statistically significant relationships.

Results: The overall job satisfaction of Notre Dame graduates was high, however the majority did not predict a long-term career in physiotherapy. Lowered job satisfaction was related to working in the cardiorespiratory area of physiotherapy and working in multiple jobs since graduation. Lack of challenge and workplace flexibility influenced job satisfaction ratings. The study also identified reasons for attrition from the profession highlighting lack of autonomy and career progression.

Conclusion: The study highlighted differences in job satisfaction for physiotherapists working in different clinical streams. Lack of career progression and limited scope of practice were influential in early career attrition. Extended scope roles currently being considered by the profession, may influence the future job satisfaction and attrition rates of physiotherapists

\section{Key Practice Points:}

- Job satisfaction is influenced by area of practice and number of jobs held since graduation

- Lack of career progression and autonomy can be linked to attrition from the profession

- Extended scope roles may influence the longevity of physiotherapists in the workforce 
STERNAL MICROMOTION FOLLOWING CARDIAC SURGERY: A 3-MONTH PROSPECTIVE, OBSERVATIONAL STUDY

Balachandran $\mathrm{S}^{1,2}$, Denehy $L^{2}$, Lee $A^{2}$, Royse $A^{2,3}$, El-Ansary $D^{2}$

${ }^{1}$ Eastern Health, Melbourne

ZUniversity of Melbourne, Melbourne

3Melbourne Health, Melbourne

Question: How much sternal micromotion occurs during dynamic upper limb and trunk tasks in patients following cardiac surgery?

Design: Prospective, observational study.

Participants: Seventy-five patients following cardiac surgery via a median sternotomy.

Outcome Measures: Sternal micromotion (vertical and horizontal direction) was measured using ultrasound at rest and during five dynamic tasks (deep inspiration, coughing, unilateral and bilateral upper limb elevation, and sit to stand), at three postoperative time points (T1:3-5 days, T2:6 weeks and T3:3 months). Sternal pain and function were also assessed.

Results: There was a significant decrease in the mean separation (T1-T3:0.11 mm) and overlap (T1-T3:0.08 mm) of the sternal edges in the vertical and horizontal directions, respectively, over time. This correlated with a significant decrease in sternal pain and increase in function over time. Coughing produced the greatest mean increase in sternal micromotion (0.14-0.16 mm) and sternal pain. Five participants demonstrated sternal union on ultrasound at T3.

Conclusion: The magnitude of sternal micromotion decreased over time and correlated with a decrease in sternal pain and increase in function. Coughing produced the greatest magnitude of sternal micromotion and pain. Further investigation of the factors that affect sternal micromotion and healing is warranted.

\section{Key Practice Points:}

- Current sternal precautions may be overly restrictive and not warranted in all patients.

- The results support caution with coughing as a routine intervention.

- Patient-specific sternal care that is informed by patient comfort and the assessment of risk for sternal complications may be more appropriate to facilitate recovery.

\section{HOW TO USE A CLINICAL PATHWAY IN THE MANAGEMENT OF MUSCULOSKELETAL CONDITIONS}

Bandong $\mathrm{AN}^{1}$, Leaver $\mathrm{A}^{1}$, Rebbeck $\mathrm{T}^{1}$

${ }^{1}$ University of Sydney Faculty of Health Sciences, Sydney

Background: A clinical pathway of care is a structured multidisciplinary plan that details steps in the care of patients with a specific clinical problem (Rotter et al., 2010). Clinical pathways, which provide a mechanism for tracking outcomes and describing variances from optimal outcomes, have not been used as extensively in musculoskeletal healthcare as in other fields. Clinical pathways link evidence to practice, taking into consideration local context, healthcare systems, and time frames to optimize outcomes and maximize clinical efficiency (Rotter et al., 2010). In the absence of wellestablished pathways for musculoskeletal conditions, clinicians can use the principles of clinical pathways to systematically examine current practice and communicate details of management to external stakeholders

Aims / objectives: Participants will understand some of the principles underpinning the use of clinical pathways- triage assessment, evidencebased management, and regular review of outcomes; and incorporate these into management of chosen musculoskeletal conditions.

\section{Approach:}

1. Brief presentation on the use and components of clinical pathways in health care based on the presenters' current research.

2. Interactive case-based workshop that develops consensus-based pathways for two selected musculoskeletal conditions.

3. Discussion about using the pathways in explaining and justifying physiotherapy management plans to external stakeholders.

\section{Conclusion / Key Practice Points:}

- A clinical pathway of care provides a streamlined approach to addressing patient needs.

- Using the principles of clinical pathways, practitioners might better justify and communicate details of a management plan to external stakeholders.

- Clinical pathways can potentially be used as a quality assurance tool to improve outcomes.
WHAT IS THE EFFECT OF ADDITIONAL PHYSIOTHERAPY ON SITTING BALANCE FOLLOWING STROKE COMPARED TO STANDARD PHYSIOTHERAPY TREATMENT: A SYSTEMATIC REVIEW

Bank $J^{1}$, Charles $\mathrm{K}^{1}$, Morgan $\mathrm{P}^{1}$

${ }^{1}$ Monash University, Frankston

Question: What is the efficacy of additional physiotherapy treatment to address sitting balance dysfunction following stroke? What outcome measures are used? Does practice intensity or intervention target affect outcomes?

Design: Systematic review with meta-analysis of randomised controlled trials

Participants: Acute and chronic patients following stroke with impaired sitting balance

Intervention: Studies that evaluated the impact of an additional physiotherapy treatment in addition to standard physiotherapy were included. The interventions were defined as targeting impairments, activity limitations, or both.

Outcome Measures: Outcome measures that evaluated aspects of sitting balance were included: Trunk Impairment Scale (TIS), Trunk Control Test (TCT), Motor Assessment Scale (Item 3), Postural Assessment Scale for Stroke, or customized measures of sitting balance performance.

Results: Eleven studies met inclusion criteria. Nine targeted the ICF (International Classification of Function, Disability and Health) domain of Activity. The TCT was used as a primary outcome measure in five studies, and the TIS was used in four. There was a significant effect (SMD =1.67, $95 \% \mathrm{Cl}=0.54$ to 2.80 ) favoring intervention as measured by the TIS. No evidence supported the effect of additional treatment on sitting balance as measured by the TCT (SMD $=-1.53,95 \% \mathrm{Cl}=-9.37$ to 6.32$)$.

Conclusion: The current evidence supports strategies that target deficits at the activity level and increase total treatment time. The TIS is most responsive as a measure of treatment efficacy. Further research is required using recommended outcome measures to facilitate generation of a minimum data set and data pooling.

\section{Key Practice Points:}

- The Trunk Impairment Scale is recommended as a measure for sitting balance performance

- Physiotherapy interventions for sitting balance after stroke should be targeted at the activity leve

- More favourable outcomes may be achieved by increasing total treatment time 


\section{SEVERITY AND DISTRIBUTION OF SPASTICITY DOES NOT LIMIT MOBILITY OR INFLUENCE COMPENSATORY STRATEGIES FOLLOWING TRAUMATIC BRAIN INJURY}

Banky $\mathbf{M}^{1}$, Williams $\mathrm{G}^{1,2,3}$, Oliver $]^{4,5,6}$

IPhysiotherapy Department, Epworth Healthcare

2Physiotherapy Department, The University of Melbourne

${ }^{3}$ Physiotherapy Department, Latrobe University

${ }^{4}$ Rehabilitation Medicine, Epworth HealthCare

${ }^{5}$ Rehabilitation Medicine, Monash University

E Epworth Monash Rehabilitation Unit (EMReM)

Questions: Does the severity of lower limb spasticity effect mobility outcomes following traumatic brain injury? Does the distribution of spasticity influence proximal compensation strategies following traumatic brain injury?

Design: Cross sectional cohort study.

Participants: Ninety-three people attending physiotherapy for mobility limitations following a traumatic brain injury.

Outcome Measures: The High-Level Mobility Assessment Tool (HiMAT) gait velocity, Modified Tardieu scale and ankle and hip power generation.

Results: No significant relationship was found between the severity of lower limb spasticity and self-selected walking speed $(p=.07)$ or HiMAT scores $(p=.22)$. There was a strong positive relationship between ankle power generation and both self-selected walking speed $(p<.001)$ and HiMAT scores $(p<.001)$. Proximal compensation strategies did not vary significantly between groups with different distributions of lower limb spasticity $(p=.22)$.

Conclusion: The ability to generate ankle power has a large impact on mobility outcomes following traumatic brain injury. Although spasticity was prevalent, the severity did not impact mobility outcomes. Proximal compensation strategies were not influenced by the presence or distribution of lower limb spasticity following traumatic brain injury.

\section{Key Practice Points:}

- The distribution and severity of lower limb spasticity did not impact mobility outcomes or proximal compensation strategies following traumatic brain injury.

- The ability to generate ankle and hip power has greater association with mobility outcomes than the distribution and severity of spasticity.

- Clinical measures of lower limb spasticity may not be valid.

Ethics approval: Epworth HealthCare and the University of Melbourne.

\section{ANKLE PLANTARFLEXOR SPASTICITY DOES NOT RESTRICT THE RECOVERY OF ANKLE PLANTARFLEXOR STRENGTH OR ANKLE POWER GENERATION FOLLOWING TRAUMATIC BRAIN INJURY}

Banky $\mathbf{M}^{1}$, Williams $\mathrm{G}^{1,2,3}$, Oliver $]^{4,5,6}$

${ }^{1}$ Physiotherapy Department, Epworth Healthcare

2Physiotherapy Department, The University of Melbourne

3Physiotherapy Department, Latrobe University

${ }^{4}$ Rehabilitation Medicine, Epworth HealthCare

${ }^{5}$ Rehabilitation Medicine, Monash University

${ }^{6}$ Epworth Monash Rehabilitation Unit (EMReM)

Questions: Does the presence of plantarflexor spasticity affect muscle strength or ankle power generation during walking?

Design: Cross sectional cohort study.

Participants: Seventy-five ambulant people who were attending physiotherapy for mobility limitations as a result of a traumatic brain injury. Outcome Measures: Gait velocity, Modified Tardieu scale, ankle power generation using three dimensional gait analysis and plantarflexor strength using a hand held dynamometer.

Results: Participants with ankle plantarflexor spasticity had significantly lower initial self-selected walking speeds than those without plantarflexo spasticity $(p=.03)$, however there was no significant difference in ankle plantarflexor strength or ankle power generation. Participants with ankle plantarflexor spasticity were not restricted in the recovery of self-selected walking speed $(p=.39)$, ankle plantarflexor strength $(p=.91)$, or ankle power generation $(p=.22)$ at the six month follow up when compared to those without spasticity.

Conclusion: People with ankle plantarflexor spasticity have significantly greater initial mobility limitations than those without spasticity, yet retain the capacity for recovery of self-selected walking speed.

\section{Key Practice Points:}

- The presence of ankle plantarflexor spasticity did not compromise plantarflexor strength or ankle power generation during walking.

- The primary benefit of injecting Botulinum Toxin-A into the calf may be to provide a window of opportunity to strengthen the muscle as opposed to solely reducing spasticity.

- These findings may not be applicable to other muscle groups due to the unique role of the calf in gait.

Ethics approval: Epworth HealthCare and the University of Melbourne

\section{DISTRIBUTION OF LOWER LIMB SPASTICITY DOES NOT INFLUENCE MOBILITY OUTCOME FOLLOWING TRAUMATIC BRAIN INJURY: AN OBSERVATIONAL STUDY}

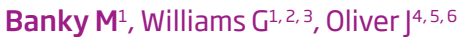

${ }^{1}$ Physiotherapy Department, Epworth Healthcare

2Physiotherapy Department, The University of Melbourne

${ }^{3}$ Physiotherapy Department, Latrobe University

${ }^{4}$ Rehabilitation Medicine, Epworth HealthCare

${ }^{5}$ Rehabilitation Medicine, Monash University

${ }^{6}$ Epworth Monash Rehabilitation Unit (EMReM)

Question: Does the presence and distribution of lower limb spasticity impact mobility outcome following traumatic brain injury?

Design: Cross sectional cohort study.

Participants: Ninety-three ambulant people attending physiotherapy for mobility limitations following a traumatic brain injury.

Outcome Measures: The High-Level Mobility Assessment Tool, gait velocity and the Modified Tardieu scale.

Results: Lower limb spasticity was common (63.4\%), with a dista distribution being the most prevalent (44.1\%). Participants with spasticity had greater initial mobility limitations compared to participants without spasticity $(p=0.03)$.The distribution of lower limb spasticity had no impact on mobility outcomes ( $p=0.31$ ). There was no difference in mobility outcomes between those with a uni-vs bilateral distribution of spasticity $(p=0.64)$. There was no difference in mobility at the six month follow up when comparing people with and without spasticity $(p=0.48)$

Conclusion: Individuals with lower limb spasticity have greater initial mobility limitations compared to those without spasticity following traumatic brain injury. Participants demonstrated equivalent ability to improve their mobility over six months despite the presence of spasticity. The distribution of spasticity did not impact mobility outcomes.

\section{Key Practice Points:}

- There is long term potential to improve mobility despite the presence of lower limb spasticity following traumatic brain injury.

- The distribution of lower limb spasticity did not impact mobility following traumatic brain injury.

- It may be possible that other impairments, such as weakness, may have a greater impact on mobility outcomes than spasticity.

Ethics approval: Epworth HealthCare and the University of Melbourne. 


\section{ARE PHYSICAL ACTIVITY LEVELS RELATED TO A CHILD'S FUNCTIONAL CAPACITY OR DEVELOPMENT?}

Banney $\mathrm{PJ}^{1}$, Danks $\mathrm{M}^{1}$, Low Choy $\mathrm{N}^{1,2}$

${ }^{1}$ Australian Catholic University, Brisbane

2The Prince Charles Hospital \& Metro North, Brisbane

Questions: Is there association between child report, parent report and accelerometer Measures of physical activity in typically developing children? Does level of physical activity influence functional capacity or maturity of gait in children?

Design: Prospective observational study

Participants: Twenty-six typically developing children (mean age 10.7 y \pm 1.49; $46 \%$ female) and their parents.

Outcome Measures: Physical activity level was measured by accelerometer (worn for one week) and by parent and child report using the Children's Leisure Activity Study Survey. Functional capacity was assessed using the Six Minute Walk Test. Step width, a measure of maturity of gait was recorded using the Gaitrite instrumented walkway.

Results: Accelerometer measures identified only $19 \%$ of the children as undertaking one hour of moderate to vigorous physical activity per day. However, 92\% of the typically developing children met this recommended guideline for physical activity according to parent or self-report. Child report of activity levels was moderately associated with maturity of gait measure $(r=-0.434, p=0.03)$. There was no significant relationship between measures of physical activity and functional capacity.

Conclusion: Parent and child report overestimated levels of moderate to vigorous activity compared to accelerometer measures. While a child's perception of their level of activity was associated with the gait maturity neither time in moderate to vigorous activity or sedentary behaviour in these typically developing children influenced functional capacity measure. Key Practice Points:

- As current recommended levels of physical activity are designed to be monitored by parents, calibration of equivalent accelerometer measures is required.

\section{GENERALIZED OUTPATIENT}

\section{REHABILITATION PROGRAM COMPARED TO USUAL CARE IN PEOPLE WITH MULTIMORBIDITY: FEASIBILITY OUTCOMES FROM A PILOT RANDOMIZED CONTROLLED TRIAL}

Barker $\mathrm{K}^{1}$, Ritchie $\mathrm{K}^{1}$, Lee $\mathrm{AL}^{2}$, Holland $\mathrm{AE}^{3-4}$, Haines $\mathrm{TP}^{5}$,

Turczyniak $\mathrm{M}^{1}$, Thomas $\mathrm{L}^{1}$, Boote $\mathrm{C}^{1}$, Saliba $\rfloor^{1}$, Lowe $\mathrm{S}^{1}$, Pazsa F ${ }^{1}$,

Skinner $\mathrm{EH}^{1,5-6}$

${ }^{1}$ Western Health, Melbourne

2West Park Healthcare Centre, Toronto, Canada

La Trobe University, Melbourne

${ }^{4}$ Alfred Health, Melbourne

5 Monash University, Melbourne

The University of Melbourne, Melbourne

Questions: What is the effect of a generalized outpatient rehabilitation program compared to usual care on functional exercise tolerance in people with multimorbidity?

Design: Pilot randomized controlled trial (concealed allocation, assessor blinding and intention-to-treat analysis).

Participants: Sixteen individuals with multiple chronic conditions, not usually eligible for disease-specific rehabilitation programs (e.g. pulmonary, cardiac, heart failure rehabilitation). Exclusion criteria included medical instability, inability to walk 50 metres.

Interventions: Intervention group attended a generic chronic disease outpatient exercise rehabilitation program. Participants attended twice weekly exercise and weekly education for eight weeks. Exercise consisted of aerobic, upper and lower limb resistance exercises. Control group participated in usual care (not including structured exercise rehabilitation).

Outcome Measures: Feasibility measures included numbers screened, recruited and completed. The primary outcome was change in functional exercise capacity (six-minute walk test (6MWT)). Secondary outcomes included quality of life.

Results: One hundred and two patients were screened to recruit sixteen participants (eight intervention, eight control). Participants were mostly female (69\%) with mean (SD) age 65 (12) years, BMI 33 (8) and functiona comorbidity index 6 (2). Presenting conditions included hypertension $(63 \%)$, diabetes $(50 \%)$, cancer (44\%), Intervention patients completed median (IQR) 14 (6 - 16) sessions. One participant (6\%) withdrew after commencing rehabilitation; two (12\%) were lost to follow-up. Intervention group 6MWT distance increased by mean (SD) 0.7 (73.3) metres compared to 22.6 (29.5) metres (control); mean difference (95\% Cl) -21.9 (-88.1, 44.2), $p>0.05$.
Conclusion: This trial was feasible and informs the development of a large randomized controlled trial powered for superiority.

Trial registration: ACTRN12614001187639.

\section{GENERALIZED COMPARED TO DISEASE- SPECIFIC OUTPATIENT REHABILITATION IN PEOPLE WITH MULTIMORBIDITY: FEASIBILITY OUTCOMES FROM A PILOT RANDOMIZED CONTROLLED TRIAL}

Barker $K^{1}$, Ritchie $K^{1}$, Lee $A^{2}$, Holland $A E^{3-4}$, Haines TP Turczyniak $\mathrm{M}^{1}$, Thomas $\mathrm{L}^{1}$, Boote $\mathrm{C}^{1}$, Saliba $]^{1}$, Lowe ${ }^{1}$, Pazsa F ${ }^{1}$, Skinner $\mathrm{EH}^{1,5-6}$

${ }^{1}$ Western Health, Melbourne

2West Park Healthcare Centre, Toronto, Canada

${ }^{3}$ La Trobe University, Melbourne

${ }^{4}$ Alfred Health, Melbourne

5 Monash University, Melbourne

${ }^{6}$ The University of Melbourne, Melbourne

Questions: What is the feasibility of generalized compared to diseasespecific outpatient rehabilitation in people with multimorbidity?

Design: Pilot feasibility randomized controlled trial (concealed allocation, assessor blinding, intention-to-treat analysis).

Participants: Seventeen individuals with a chronic disease eligible for disease-specific rehabilitation (e.g. pulmonary, cardiac, heart failure rehabilitation) and at least one other chronic condition. Exclusion criteria included medical instability, inability to walk 50 metres.

Interventions: Intervention group attended generic chronic disease outpatient exercise rehabilitation. Control group attended usual diseasespecific outpatient exercise rehabilitation. Participants attended twice weekly exercise and weekly education for eight weeks. Exercise consisted of aerobic, upper and lower limb resistance exercises.

Outcome Measures: Feasibility measures included numbers screened, recruited and completed. The primary outcome was change in functional exercise capacity (six-minute walk test (6MWT)). Secondary outcomes included quality of life.

Results: Sixty-one patients were screened to recruit seventeen participants (nine intervention, eight control); one withdrew prior to rehabilitation. Participants were mostly male (59\%) with mean (SD) age 70 (9) years, BMI 29 (7), functional comorbidity index 5 (3). Presenting conditions included heart disease (53\%), chronic obstructive pulmonary disease/asthma (35\%). Intervention and control patients completed median (IQR) 16 (8 - 15); 12 (9 - 14) sessions respectively. One participant (6\%) withdrew after commencing; two (12\%) were lost to follow-up. Intervention group 6MWT distance increased by mean (SD) 22.1 (45.1) metres compared to $22.3(57.4)$ metres (control); mean difference $(95 \% \mathrm{Cl})$ $-0.125(-67.1,66.9), p>0.05$

Conclusion: This trial was feasible and informs the development of a large non-inferiority randomized controlled trial.

Trial registration: ACTRN12614001186640

\section{COMMUNITY REHAB NO: 'FROM LITTLE THINGS, BIG THINGS GROW'}

\section{Barker R}

Community Rehab nQ, Northern Australia Primary Health James Cook University, Darwin

Community Rehabilitation northern Queensland (Community Rehab NQ) is a novel person-centred, student-assisted service for people with neurological conditions who reside in regional, rural and remote northern Queensland. Arising from a collaborative venture between James Cook University and Northern Queensland Primary Health, Community Rehab NQ was designed to address unmet need for rehabilitation services while at the same time, build workforce capacity for northern Queensland. This presentation outlines the development of Community Rehab nQ from its small beginnings in 2007 to the comprehensive service it is today. Starting with a series of pilot trials tailored to the needs of people from northern Queensland, recommendations for a sustainable rehabilitation service were generated. Subsequently, a comprehensive proposal for Community Rehab nQ was developed using an iterative process of planning, consultation with stakeholders, data gathering and compilation of a service model. Support for this proposal from local, state and national governments led to allocation of $\$ 16$ million to establish the service over a three-year period. Now in 2015, Community Rehab n0 provides a service to over 400 people per year, clinical placements for over 60 health professional students per year, and has a workforce that includes 18 health professionals across 11 professions. 


\section{AUSTRALIAN CHILDREN NEED BASIC MOVEMENT SKILL COMPETENCE FOR THEIR HEALTH}

\section{Barnett L}

Deakin University

Many Australian children are not meeting physical activity guidelines, are not cardiovascularly fit and are overweight or obese. Furthermore, around half of Australian children are not competent in basic movement skills; the ability to catch, run and jump. Even in adolescence, when we would expect these skills to be mastered, movement skill competency is low. Therefore acting preventatively to improve motor competence is crucial. This presentation will use a lifespan approach to discuss the importance of children's movement competence to future health. Dr Barnett will present a range of her studies covering the age period from pre-school to upper high school, and the key work of others in the field, to demonstrate the current state of evidence in this area and the remaining research questions to be answered. Importantly, there is now compelling evidence demonstrating that poor childhood movement skill competence and poor perceptions of competence, links to subsequent reduced physical activity and healthrelated physical fitness, and a higher chance of becoming overweight/ obese. Finally Dr Barnett will present evidence on whether movement skill competency gained through an intervention can be sustained.

\section{Key Practice Points:}

- Physiotherapists have a crucial role to play - they are primary contact practitioners and have the opportunity to screen and promote movement skill competency in children and youth.

- This presentation will give practitioners a fresh perspective on the importance of movement skill development for all children.

\section{HOW DO ENTRY-LEVEL STUDENTS EXPERIENCE WAYS OF THINKING AND PRACTISING IN PHYSIOTHERAPY}

\section{Barradell S 1,2}

${ }^{1}$ School of Allied Health, La Trobe University

2Faculty of Education, University of Sydney

Question: How do physiotherapy students experience physiotherapy through entry-level education?

Design: Semi-structured interviews. This presentation reports on an aspect of a larger phenomenologically oriented inquiry.

Participants: Current entry-level physiotherapy students / recent graduates of an Australian entry-level physiotherapy program Objectives: There is an increasing expectation that universities produce work ready graduates; individuals who not only think and practise in the ways of their chosen discipline but who also have the capability to develop beyond those defined abilities. Institutions often have particular ideas about what disciplinary practices entail and what their importance is for developing student learning. These ideas and practices become re-contextualised and translated into curriculum. Yet little is known about students' experiences of ways of thinking and practising - particularly in physiotherapy.

Results: The student experiences of ways of thinking and practising in physiotherapy will be presented as specific themes. Metaphors will be used to describe the different dimensions of each theme; the different ways that themes were experienced.

Conclusion: The study is unique in that it begins with a focus on physiotherapy students' experiences of disciplinary learning in order to inform subsequent curriculum renewal with academics and clinical / professional partners.

\section{Key Practice Points:}

- It is critical to have an understanding of the nature of practice in order to educate for practice.

- Ways of thinking and practising help to consider particular learning outcomes and have the potential to shift persistent disciplinary ideas.

- Inclusion of students in educational research brings students into the community of practice in its fullest potential.
SENSORY NERVES CONTAINING CALCITONIN GENE-RELATED PEPTIDE IN STRUCTURES COMMONLY ASSOCIATED WITH CHRONIC AND RECURRENT PAIN

Barry CM, Kestell GR, Haberberger RV, Gibbins IL

Anatomy and Histology, and Centre for Neuroscience, School of Medicine, Flinders University, Adelaide

Question: How are sensory nerve subpopulations distributed in gastrocnemius, erector spinae, latissimus dorsi, thoracolumbar fascia and cranial dura?

Design: Basic science, descriptive and quantitative study (C57BI/6 mice, $\mathrm{n}$ $=4$ to 6 per group).

Outcome Measures: Neuron soma size, neurochemical content, location and frequency (retrograde axonal tracing, multiple labelling immunofluorescence, quantification).

Results: Most (75\%) dorsal root ganglion neurons traced from muscle contained calcitonin gene-related peptide (CGRP) and 70\% of these did not contain substance P. In muscles and fascia, most nerve fibres contained CGRP, a third of which lacked substance P. In dura, a higher proportion of CGRP fibres lacked substance P. Neurons containing CGRP alone had larger mean soma size $(p<0.001)$ and their fibres less frequently accompanied blood vessels. Innervation density was three times higher in thoracolumbar fascia compared to back muscles $(p<0.05)$

Conclusion: All muscles and connective tissue structures investigated were predominantly innervated by sensory neurons containing CGRP, an important modulator of pain signal transmission. There are two distinct populations of these neurons and their fibers are more frequent in thoracolumbar fascia compared to back muscles.

\section{Key Practice Points:}

- It is uncertain how subpopulations of sensory fibres may provide distinct contributions to pain associated with muscle, fascia and dura.

- CGRP released by primary afferent neurons at their peripheral or central projections may contribute to altered neuronal sensitivity.

- Following muscle injury, primary afferent neurons containing CGRP demonstrate plastic changes that may augment and prolong pain.

\section{PERFORMANCE IN PHYSIOTHERAPY CLINICAL EXIT EXAMINATIONS: THE RELATIONSHIP TO ACADEMIC AND CLINICAL PLACEMENT ASSESSMENT}

Barry RK ${ }^{1}$, Newstead C] ${ }^{1}$, Osmotherly PG ${ }^{1}$, Johnston $\mathrm{CL}^{1}$ ${ }^{1}$ The University of Newcastle, Australia

Question: Is there a relationship between performance in clinical exit examinations and entry-level training course assessments (academic and clinical) in physiotherapy students?

Design: Retrospective cohort study

Participants: Undergraduate students $(n=219)$ enrolled in the Bachelor of Physiotherapy program at The University of Newcastle between 2005 and 2009 who completed a clinical exit examination.

Outcome Measures: Measurements included clinical exit examination score and entry-level training course assessment scores (academic and clinical) in three core content areas: musculoskeletal, cardiopulmonary and neurological physiotherapy. Relationships were determined using correlation and linear regression.

Results: There was little to low correlation between exit examination and entry-level training course assessment scores in core content areas. Only two academic courses showed predictive value for exit examination performance; musculoskeletal physiotherapy 1 and cardiopulmonary academic average. These courses accounted for $7.7 \%$ and $9.4 \%$ respectively of exit examination variance. Performance in clinical placements had no predictive value for exit examination performance in core content areas.

Conclusion: Performance in entry-level training course assessment (academic and clinical) was not well correlated with exit examination performance. The value of clinical exit examinations and the aspect of clinical practice measured by these remain unclear.

\section{Key Practice Points:}

- Clinical exit examinations do not appear to measure the same domains as clinical placement or academic assessments in physiotherapy students.

- The domain of practice measured by clinical exit examinations is unknown and should be the focus of future research.

- The value and role of exit examinations in physiotherapy programs remains unclear 


\section{THE IMPACT OF PHYSICAL \\ ACTIVITY LEVELS IN THE ICU ON \\ FUNCTIONAL PATIENT OUTCOMES: AN OBSERVATIONAL STUDY}

Beach $\mathrm{LJ}^{1}$, Fetterplace $\mathrm{K}^{2}$, Edbrooke $\mathrm{L}^{3}$, Parry SM ${ }^{3}$, Curtis R ${ }^{1}$, Rechnitzer $\mathrm{T}^{4}$, Berney $\mathrm{S}^{5}$, Denehy $\mathrm{L}^{3}$

${ }^{1}$ Department of Physiotherapy, Royal Melbourne Hospital, Melbourne ${ }^{2}$ Department of Clinical Nutrition, Royal Melbourne Hospital, Melbourne ${ }^{3}$ Department of Physiotherapy, The University of Melbourne, Melbourne ${ }^{4}$ Department of Intensive Care, Royal Melbourne Hospital, Melbourne ${ }^{5}$ Department of Physiotherapy, Austin Health, Melbourne

Questions: What level of physical activity is performed in the ICU and what are the barriers? What is the relationship between muscle strength and physical function? What are the predictors of length of stay and discharge destination?

Design: prospective observational study

Participants: Sixty participants in a tertiary ICU; mechanically ventilated for at least 48 hours and requiring five or more days of ICU care.

Outcome Measures: Physical activity duration and steps (accelerometry), barriers to physical activity, muscle strength (Medical Research Council scale), physical function (Physical Function in Intensive care Test-scored), and delirium (Confusion Assessment Method ICU).

Results: Physical activity duration (minutes); median (IQR) 16.8[0.60 152.4] and steps 4[1.2-7.0]. The main barrier to physical activity (day five) was sedation; $n(\%) 19(46)$. There was a strong correlation between strength and function at ICU discharge $(r=0.79, p<0.001)$. Days of delirium (beta $0.73, p<0.001$ ) explained $53 \%$ of the variation in ICU length of stay Participants discharged home demonstrated higher strength $(p=0.04)$ and function (mean difference 1.40 (95\% Cl 0.07 to 2.74), $p=0.04$ ) scores at ICU discharge, compared to those discharged elsewhere

Conclusion: Participants in this study demonstrated low levels of physical activity and the main barrier was sedation.

\section{Key Practice Points:}

- Culture change to increase physical activity levels, in addition to rehabilitation, should be considered

- Delirium duration impacts length of stay. Investigating the impact of physical activity levels on delirium is required.

- Improving strength and function in ICU may impact discharge destination

\section{UNDERSTANDING THE CONCEPT OF PAIN SENSITIVITY IN CLINICAL PRACTICE}

\section{Beales D}

${ }^{1}$ School of Physiotherapy and Exercise Science, Curtin University

Clinically, level of pain sensitivity is a recognisable feature of any patients presentation. However, there is contrasting evidence as to how much increased pain sensitivity relates to the experience of pain itself and associated disability. The contrasting evidence is reflected in confusion amongst health care practitioners in relation to the clinical significance of increased pain sensitivity, and how to consider increased pain sensitivity within the development of individualised management programs. This presentation aims to provide guidance for clinicians in integrating the concept of pain sensitivity into clinical practice including:

- Clinical meaning of pain sensitivity research performed at Curtin University at the population level will be highlighted. We have completed pain sensitivity profiling in 23 year olds of the Western Australian Pregnancy Cohort 'Raine' Study. Specific investigations include HPA-axis functional as a predictor of pain sensitivity/musculoskeletal pain, and pain sensitivity in menstrual pain.

- Clinical meaning of pain sensitivity research performed at Curtin University in specific clinical cohorts will be highlighted. This will include discussion of clusters of pain sensitivity in chronic low back pain, and pain sensitivity in pregnancy related lumbopelvic pain.

- A biopsychosocial framework for assessing pain sensitivity with be discussed.

- Potential strategies for management of pain sensitivity will be presented, along with strategies that are not useful

\section{Key Practice Points:}

- Participants will have enhanced understanding of potential causes of increased pain sensitivity from a biopsychosocial perspective.

- Participants will have increased understanding of specific assessment for increased pain sensitivity in the clinic.

- Participant will understand what aspects of clinical practice may be unuseful in the management of increased pain sensitivity

- Participants will enhance their clinical reasoning process for managing increased pain sensitivity

\section{UNDERSTANDING THE CONCEPT OF BELIEFS IN CLINICAL PRACTICE}

\section{Beales $D^{1}$}

1School of Physiotherapy and Exercise Science, Curtin University

In life, beliefs underlie our identities as individuals, and our interactions with others. The same holds true in health and medicine. Beliefs have wide influence in determining the effect of pain at an individual level. Beliefs underpin the communication between health care practitioners and other stakeholders in healthcare.

This presentation aims to provide guidance for clinicians in implicitly integrating the concept of beliefs into clinical practice including:

- Clinical meaning of beliefs research performed at the population level will be highlighted. We have completed beliefs profiling in 23 year olds of the Western Australian Pregnancy Cohort 'Raine' Study and the Busselton Healthy Aging Study. A focus has been on the future course and inevitability of back pain (the Back Beliefs Questionnaire)

- Research performed looking at mismatched beliefs amongst different stakeholders in the workers' compensation settings will be presented.

- Research attempting to influence beliefs for behavioural change will be discussed.

- Strategies for managing beliefs at a individual level and systems based level will be introduce.

\section{Key Practice Points:}

- Participants will have enhanced understanding of potential role of beliefs at an individual level.

- Participants will have enhanced understanding of potential role of beliefs at a practitioner level, specifically related to intra-health care practitioner beliefs.

- Participants will have increased understanding of specific assessment of beliefs in the clinic.

- Participants will understand strategies for integration of beliefs assessment at a systems level

- Participants will enhance their clinical reasoning process for managing beliefs.

\section{ADVANCED MUSCULOSKELETAL PRACTICE PHYSIOTHERAPY: CLINICAL DIAGNOSIS AND RADIOLOGICAL IMAGING}

Bennett $L^{1}$, Haley $C^{1}$, Harding $\mathrm{P}^{1}$, Holland $\mathrm{A}^{1,2}$

${ }^{1}$ Department of Physiotherapy, The Alfred, Melbourne

${ }^{2}$ Alfred Health Physiotherapy Clinical School, La Trobe University, Melbourne

Question: To review the clinical presentations of patients seen by an advanced musculoskeletal practice physiotherapist in an orthopaedic physiotherapy screening clinic and to determine if clinical diagnosis by the physiotherapist correlated with reported MRI findings.

Design: Retrospective audit of patients seen in The Alfred orthopaedic outpatient physiotherapy screening clinic between July and December 2010

Participants: One hundred and eighty nine patients [97 males, (51\%)] were triaged for assessment by an advanced musculoskeletal practice physiotherapist. The mean age was 54 years (range 17-99 years).

Outcome Measures: Data describing physiotherapists' clinical diagnoses, physiotherapist initiated imaging and surgical waitlisting was collated. The correlation of pre- imaging clinical diagnosis to radiological imaging findings was performed by an independent assessor.

Results: Forty patients (21\%) had physiotherapy-initiated MRI Musculoskeletal diagnosis by the physiotherapist was consistent with MRI findings in 38 patients (95\%). Seventy five patients (40\%) had knee complaints with 18 (24\%) of these undergoing arthroscopy. Fourteen (78\%) had an MRI prior to this surgery.

Conclusion: Physiotherapists' diagnoses of musculoskeletal conditions correlate well with MRI findings. Knee conditions were the most common presentations in our screening clinic with physiotherapists' clinical findings usually confirmed with MRI prior to knee arthroscopy. Some knee arthroscopies were performed without MRI in our institution.

\section{Key Practice Points:}

- Gain knowledge about the range of conditions seen by advanced musculoskeletal practice physiotherapists in an orthopaedic screening clinic

- Provide evidence supporting the clinical diagnostic expertise of advanced musculoskeletal practice physiotherapists

- Identify physiotherapy initiated imaging referral patterns 
AVERT: AN INTERNATIONAL CLINICAL

TRIAL TESTING THE EFFICACY AND

SAFETY OF EARLY MOBILISATION

WITHIN 24 HOURS OF STROKE ONSET IMPLICATIONS FOR CLINICAL PRACTICE

\section{Bernhardt J}

The Florey Institute of Neuroscience and Mental Health and La Trobe University, Melbourne, Australia

The conduct and completion of the AVERT Phase III trial represents a significant milestone in stroke rehabilitation research. Including over 2000 patients, 56 recruiting sites, 5 countries and involving over 900 clinical and research staff, AVERT is the largest randomised controlled trial of a complex intervention ever undertaken. The trial was primarily led by physiotherapists at each of the sites. The intervention focused on early mobility training, delivered by both physiotherapists and nurses and the primary research question was "Does starting mobility training within 24 hours of stroke and continuing frequently thereafter improve functional outcome at 3 months more than current usual care?" The trial took 8 years to complete and first results were reported in The Lancet in April 2015. We found that counter to findings from our Phase II trial, higher dose, very early intervention led to fewer stroke patients achieving no or little disability (mRS 0-2) at 3 months when compared to lower dose usual care, which also started on average within 24 hours of stroke onset. So what does this mean for our clinical practice? In this talk, Julie will expand on these early results with a focus on providing some take home messages for clinicians.

\section{THE FUTURE OF STROKE REHABILITATION RESEARCH: WHERE TO FROM HERE?}

\section{Bernhardt J}

The Florey Institute of Neuroscience and Mental Health and La Trobe University, Melbourne, Australia

Effective rehabilitation, delivered at the right time, in the right place, can significantly reduce the burden of stroke. There is strong evidence demonstrating that the brain has remarkable capacity for plasticity and reorganisation, however applying this knowledge clinically is in its infancy Rehabilitation research is too often characterised by small, underpowered studies on highly selected populations that contribute little or no evidence. Building the evidence base of interventions to help recovery is a global research priority. At present the research effort in Australia is fragmented with pockets of activity but no strong collaborative efforts. Interventions are rarely underpinned by sound neuroscience and we lack capacity. In this presentation, Julie will outline current national and international efforts to improve the science of rehabilitation research including advancing understanding of the neurobiology of stroke recovery and key impediments to recovery; accelerating development of novel treatments underpinned by neuroscience and more carefully target treatments to individuals; developing world class trial platforms and training; improving translation pathways and systems and developing a'stroke rehabilitation research community'

\section{CAN PEOPLE WITH A WEAK ARM POST STROKE USE A DYNAMIC ORTHOSIS TO COMPLETE A HOME BASED INTENSIVE TRAINING PROGRAM?}

Bew P, Carter M

Brighton Health Campus, Brighton

Question: In an outpatient setting, is it feasible for people with a weak upper limb after stroke to use a dynamic orthosis?

Design: Prospective, observational study

Participants: 4 stroke survivors ( 3 males and 1 female) average 50 years of age (38 - 62 years) living in the community. Average time post-stroke was 24 months (11 - 39 months).

Intervention: Each person was fitted with a customized dynamic orthosis (Saebo ${ }^{\circledR}$ ) and prescribed an individualised exercise program completed at home for a minimum 90 minutes daily. No other specific arm exercises were done. Each person was reassessed after one month.

Outcome Measures: Participants recorded how many repetitions were completed each session. Grip strength was recorded monthly. Ease of donning and doffing the splints and any adverse incidents were also recorded by each person.

Results: On average each person completed 5557 repetitions in the first month at an average of 106 repetitions per day. Grip strength improved an average of $3.5 \mathrm{~kg}(1.3-4 \mathrm{~kg})$. All participants achieved independence in donning / doffing the orthosis. No adverse issues were reported.

Conclusion: These preliminary findings suggest that use of a dynamic upper limb orthosis is feasible as is person's participation in a high dose home based training program. Such involvement may facilitate a person's functional recovery and further study is indicated.

\section{Key Practice Points:}

- It is feasible for a dynamic orthosis to be used by people in an outpatient setting.

- Such a device may assist participation in a home based program.

- Further studies need to be done.

\section{CROSS CULTURAL COMPETENCY, COMMUNICATION AND CLINICAL REASONING}

\section{Bialocerkowski A ${ }^{1}$, Edwards I $^{2}$, Jackson $\mathrm{H}^{3}$}

${ }^{1}$ Head of School of Allied Health, Griffith University ¿School of Health Sciences, University of South Australia ${ }^{3}$ Department of Linguistics, Macquarie University

Australia is home to people of many nationalities, cultures and beliefs; one in four Australians were born outside this nation and nearly one in five of us speak a language other than English. Together we can communicate in over 260 languages (Department of Social Services). Being aware of how our own cultures and belief systems differ from those we are seeking to influence is key to being an effective clinician or educator. This is the basis of cross cultural competence. Through the medium of an engaging and interactive Q\&A style forum, we will explore what it is to be cross culturally competent in physiotherapy practice. Our invited speakers include a linguistics expert working in the organisational and health sectors, an academic clinician who has practised in the Middle Eastern and South East Asia, and an academic leader who developed strategies for teaching culturally sensitive students, and for enhancing cultural competency in clinical practice. Guided by their experiences and expertise, we will discuss how we can develop cultural competence, with particular reference to communication and clinical reasoning skills, in ourselves, our staff and our students.

\section{Key Practice Points:}

- Better patient and student outcomes and greater job satisfaction can be gained through enhanced cultural competence. 


\section{PHYSICAL ACTIVITY AND EXERCISE INFORMATION FOR PEOPLE WITH PARKINSON'S DISEASE: EXPRESSED PREFERENCE FOR SOURCE AND FORMAT}

Bilney BE ${ }^{1}$, McGinley JL' ${ }^{1}$ Danoudis $\mathrm{M}^{1,2}$, Morris $\mathrm{ME}^{3}$, Higgins RO ${ }^{1,4,5,6}$ ${ }^{1}$ Department of Physiotherapy, School of Health Science, University of Melbourne, Melbourne

2Physiotherapy, Kingston Centre, Monash Health, Melbourne

${ }^{3}$ School of Allied Health, College of Science, Health and Engineering, La Trobe University Melbourne

${ }^{4}$ Heart Research Centre, Royal Melbourne Hospital, Melbourne

${ }^{5}$ Cabrini Health, Melbourne

${ }^{6}$ Psychology, Faculty of Health, Deakin University, Melbourne

Question: What are the current and preferred source and format of physical activity and exercise information for people with Parkinson's disease (PD)?

Design: Cross sectional, self-administered online or paper-based questionnaire.

Participants: Respondents were diagnosed with PD and living in the community $(n=357)$. They had a mean age of 68.6 years (SD 8.9, range 36 - 90) and disease symptoms ranged from mild to severe (median Hoehn Yahr 2). The majority were male (60\%), and educated to Year 12 or higher (72\%).

Outcome Measures: Source and format of received physical activity and exercise information. Nominated preferred source and format of activity and exercise information.

Results: The majority of respondents reported they had received activity or exercise information (89.4\%), most commonly from a physiotherapist (54\%) or neurologist (53.5\%). Other sources included general practitioners $(44.2 \%)$, books (42\%), internet (35.5\%), family members $(24.4 \%)$ and others with PD (23.8\%). Information was most frequently delivered verbally, with physiotherapists providing written advice more often than other health professionals (46.9\%). Respondents identified a preference for information from physiotherapists (25.4\%) or specialist physiotherapists (7.4\%) and PD Associations (15.2\%). Respondents preferred paper based (50.8\%) to verbal information (23.6\%).

Conclusion: Physical activity and exercise information should match the preferences for delivery source and format of people with PD.

\section{Key Practice Points:}

- Information preferences should guide source and format of activity and exercise advice

- Advice from professionals with perceived specialist knowledge of PD is preferred

- Written resources for activity and exercise advice should be developed for people with PD

\section{AN ACCURATE, RELIABLE AND VALID ELECTRONIC APP TO MEASURE PAIN}

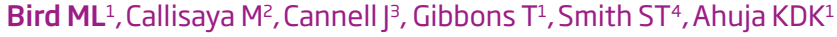
${ }^{1}$ School of Health Sciences, University of Tasmania, Launceston, TAS Australia 2Menzies Institute for Medical Research, University of Tasmania, Hobart, TAS Australia

${ }^{3}$ The Tasmanian Health Organisation, Launceston, TAS, Australia

${ }^{4}$ The University of the Sunshine Coast, QLD, Australia

Question: Is an application developed for the iPad to measure pain using a Visual Analogue Scale (VAS) accurate, reliable and valid?

Design: Accuracy was tested using an observational study design. Reliability and validity were tested using a crossover RCT design.

Participants: One researcher collected accuracy data (101 data points). Twenty two community dwelling older adults were then recruited to test reliability and validity.

Intervention: Each participant completed eight VAS (four iPad and four paper) in a randomised order.

Outcome Measures: Accuracy was determined by physically measuring iPad scale on screen and comparing it to the results from the programme. Reliability was tested using intraclass coefficients (ICC) $(95 \% \mathrm{CI})$, and validity tested using Bland-Altman graphs (measuring bias and limits of agreement) and correlations to describe the relationships.

Results: Accuracy was high resulting in means \pm SD 48.4 \pm 27.3 and 48.8 \pm 27.5 for paper and iPad respectively. Reliability for both tools was excellent with iPadVAS (ICC, 95\%CI) (0.90 0.82 to 0.95) and paperVAS within individual (0.96.92 to 98). Linear regression demonstrated a strong relationship $\left(R^{2}=0.904\right)$. There was a small negative bias towards the iPad $(-2.6 \pm 5.0)$ with limits of agreement $(95 \% \mathrm{Cl})$ from -12.4 to 7.1 .

Conclusion: The iPadVAS for Pain provides an efficient way of collecting data and is accurate and reliable in healthy older adults.

\section{Key Practice Points:}

- Data collected using iPads is accurate, reliable and valid

- They may streamline data collection and reduce transcription errors

- Secure recording and storage of data is possible

- Participatory health care in monitoring chronic pain conditions may be enhanced

\section{IMPROVING ENGAGEMENT WITH EXERCISE AND PHYSICAL ACTIVITY USING TECHNOLOGY}

\section{Bird ML}

The School of Health Sciences, The Faculty of Health, University of Tasmania

Question: Can technology provide an engaging intervention and streamline monitoring of client progress?

Design: Motion Capture Interactive Technology from two intervention studies

Participants: Study 1. Outpatients attending a University exercise clinic

Study 2. Inpatient stroke clients undergoing rehabilitation

Intervention: Intervention 1. (Oupatient) A Balance exergame in a circuit class

Intervention 2. (Inpatient) RCT exergame using stroke specific software or usual rehabilitation

Outcome Measures: Client's perceived benefit and enjoyment. Clinician's perception regarding their workload and benefit for clients.

Results: Outpatient clients reported significant changes to perception of exergames for multiple health benefits after exposure. Inpatient clients and clinicians indicated that different program levels are required for therapist assisted and independent practice. Clinicians indicated that remote monitoring of client performance may have positive workload implications. Inbuilt assessment tools need to be tested for validity and reliability.

Conclusion: Motion capture sof tware has potential benefits for engaging older adults across different settings for a variety of health goals and changing therapy delivery.

\section{Key Practice Points:}

- Older adults have limited exposure to exergames. Outpatient participation in an exergame increased their perceived benefit of this type of activity in improving their health

- Client engagement can be enhanced with motion capture software to improve activity and meet a variety of health goals in rehabilitation as well as preventative health contexts

- Stroke specific motion capture software can be adjusted for independent practice or more challenging therapist assisted programs

- Software that records outcome measures may provide a valuable tool for long term data storage or remote monitoring of performance. 


\section{CLINICAL MEASUREMENT OF SITTING BALANCE AFTER STROKE: A SYSTEMATIC REVIEW}

Birnbaum MA ${ }^{1,2}$, Hill K², Kinsella R ${ }^{1}$, Black S ${ }^{1}$, Clark R ${ }^{3}$, Brock K ${ }^{1}$

${ }^{1}$ St. Vincent's Hospital, Melbourne

${ }^{2}$ Curtin University, Perth

${ }^{3}$ Australian Catholic University, Melbourne

Question: What published clinical sitting measurement scales containing dynamic tasks exist for use with stroke survivors, and what psychometric properties have been evaluated to support their use?

Design: Systematic Review with data from quantitative studies.

Participants: Individuals following stroke.

Outcome Measures: Databases, including CINAHL, MEDLINE, EMBASE, Cochrane, Pubmed and AMED were searched from inception to Octobe 2014. The search strategy included terms relating to sitting, balance, and postural control. Motor or functional measures were included when separate data existed for the sitting balance component of the measure. Two reviewers independently selected and extracted data from the identified articles and assessed the methodological quality of the papers using the Consensus-based Standards for selection of health status Measurement Instruments (COSMIN) checklist.

Results: Fourteen clinical sitting measurement scales (38 papers) met the inclusion criteria and various measurement properties were evaluated. The methodological quality of the majority of the included studies was rated as poor to fair using the COSMIN checklist, with common limitations including small sample size and inappropriate use of statistical methods.

Conclusion: There is a need for higher quality studies exploring measurement properties of current sitting balance measures for stroke survivors.

\section{Key Practice Points:}

- There is currently no 'gold standard' clinical measure for assessing sitting balance for stroke survivors.

- The methodological quality of many previous studies investigating the psychometric properties of sitting balance measures is poor to fair.

- There is a need for higher quality studies exploring measurement properties of current sitting balance measures for individuals following stroke.

\section{MEASURING LATEROPULSION}

\section{FOLLOWING STROKE IN THE CLINICAL} SETTING: A FEASIBILITY STUDY USING WII TECHNOLOGIES

Birnbaum MA ${ }^{1,2}$, Brock K ${ }^{1}$, Clark R $^{3}$, Hill K

${ }^{1}$ St. Vincent's Hospital, Melbourne

${ }^{2}$ Curtin University, Perth

${ }^{3}$ Australian Catholic University, Melbourne

Question: Is it feasible to use Wii Balance Board derived centre of pressure data as measures of balance in people with lateropulsion following stroke? Design: Prospective repeated measures study.

Participants: Ten individuals with lateropulsion, between one and twelve weeks post stroke participated in this study Participants were recruited from admissions to the Stroke Unit and Rehabilitation Units of a tertiary hospital between April and November 2014.

Outcome Measures: Participants were assessed on four occasions over a two-week period, performing a number of tasks in sitting and standing on the Wii Balance Board, in addition to clinical measures. Feasibility was determined by participant retention and compliance with assessment procedures.

Results: Participant retention was 100\%. Compliance with assessment procedures was 30\% predominantly due to participant fatigue. Wii Balance Board-derived centre of pressure data appeared to capture useful information in sitting and standing about individuals with different levels of lateropulsion severity and displayed change over time.

Conclusion: The use of Wii Balance Board technology as a measure for balance in individuals with lateropulsion appears feasible using a reduced number of tasks. Results from this pilot study have informed a larger longitudinal measurement study, currently underway.

\section{Key Practice Points:}

- The use of Wii Balance Board technology to assess sitting and standing balance with individuals with lateropulsion appears feasible.

- Use of Wii Balance Boards as a research tool may capture useful information about balance in individuals with lateropulsion.

- For individuals with lateropulsion early following stroke, fatigue should be taken into consideration when designing research protocols.

\section{LATERAL ELBOW PAIN: CLINICAL PATHWAYS AND THE ROLE OF THE MULTIDISCIPLINARY TEAM}

Bisset $\mathrm{L}^{\mathrm{I}}$

${ }^{1}$ Menzies Health Institute Queensland, Griffith University

The clinical characteristics of patients presenting with lateral elbow pain can vary from localised elbow pain to widespread sensory and motor changes. Physiotherapy is known to be effective in many of these patients, yet for those who do not respond to conservative treatment, we often see them 'hand-balled' from one health care practitioner to another, receiving injection therapies, medicines and surgery with variable outcomes. Physiotherapists are often the first point of contact for people with lateral elbow pain, and are therefore in a position to advise on the best clinical pathway for these patients. This presentation will provide an evidence-based clinical pathway for lateral elbow pain, and discuss treatment options based on clinical presentation, prognostic indicators and underlying pathophysiology.

\section{THE IMPORTANCE OF UNDERSTANDING ENERGY BALANCE}

\section{Blair SN}

Arnold School of Public Health, University of South Carolina, USA

It is indisputable that obesity rates have been increasing in most countries around the world over the past few decades. The causes of this epidemic are complex and are not well understood. It is clear that persistent weight gain over time is caused by individuals being in positive caloric balance, consuming more calories than they burn on too many days. Therefore the obesity epidemic is due to too many people being in positive caloric balance, where their energy intake exceeds their energy expenditure. Although positive energy balance can be caused by increases in intake, decreases in expenditure, or a combination of the two; most of the attention in the scientific and lay press focuses on the intake side of the equation. This imbalance in attention to the energy expenditure side of energy balance and a major focus on the intake side is unlikely to produce policies, strategies, and tactics that will be effective in reducing the obesity epidemic. This lecture will include information on both sides of the energy balance equation and I will discuss current flawed strategies and make suggestions for new directions.

\section{PHYSICAL ACTIVITY: THE BIGGEST PUBLIC HEALTH PROBLEM OF THE 21ST CENTURY}

\section{Blair SN}

Arnold School of Public Health, University of South Carolina, USA

Sedentary habits are highly prevalent in most countries of the world. In the U.S. approximately $25-35 \%$ of adults are inactive, meaning that they have sedentary jobs, no regular physical activity program, and are generally sedentary around the house and yard. Given that sedentary and unfit individuals are at approximately two-fold higher risk for many health conditions than those who are moderately active and fit, the population attributable risk (par) of inactivity is high. In the aerobics center longitudinal study (acls) the par for low fitness in more than 50,00 women and men followed for many years is $16-17 \%$ of deaths. This is far higher than other putative risk factors for mortality. For example, obesity accounts for $2-3 \%$ of deaths in this cohort. Another example from the acls is that in 3,293 obese men (bmi ${ }^{3} 30.0$ ), 27\% of the deaths might have been avoided if none of the men had prevalent cardiovascular disease at baseline whereas $44 \%$ of the deaths might have been avoided if none of the men had been unfit. The independent relative risks for death are comparable for prevalent cardiovascular disease $(r r=2.4)$ and for low fitness ( $r r=2.3)$. Over the past few decades we have largely engineered the need for physical activity at home, on the job, and during leisure-time out of the daily lives of most people in industrialized societies. To address the major public health problem of physical inactivity we will need to consider and evaluate societal, environmental, and individual approaches to making physical activity more common for more people more of the time. 


\section{FROM CLINICAL PHYSIOTHERAPIST TO CLASSROOM EDUCATOR: A PILOT PROJECT}

Barradell $\mathrm{S}^{1}$, Mastwyk $\mathrm{S}^{1}$, McConville J1, Tang $\mathrm{CY}^{1}$, Leo $]^{1,2}$, Blackstock FB ${ }^{1}$

${ }^{1}$ Physiotherapy, School of Allied Health, College of Science Health and Engineering La Trobe University, Melbourne

¿Education Team, College of Science Health and Engineering, La Trobe University, Melbourne

Question: What are the experiences of physiotherapists who teach a theory-based subject in an alternative teaching and learning space? Design: Qualitative study using a phenomenological approach to explore the lived experience of physiotherapists teaching in a new environment. Participants: Purposeful sampling of physiotherapy staff within a metropolitan healthcare organisation. Participants were eligible if they had recent experience supervising students and agreed to teach into a theory subject.

Intervention: Participants completed a blended educational program designed by university-based academics, prior to commencement of the 4-week teaching period. Participants taught dedicated classes within new teaching and learning spaces attached to this healthcare organisation. The curricula were provided by the university so as to ensure that the learning outcomes were identical to the academic led classes conducted in a university-based classroom. Two semi-structured interviews were conducted with each participant at the beginning and end of the teaching period respectively.

Outcome Measures: Information from the semi-structured interviews were coded and analysed for common themes regarding the experiences of physiotherapists.

Results: Themes that capture the essence of the experience for all six participants were generated.

Conclusion: This pilot provides a deeper understanding of the experiences of physiotherapists in new, and often unfamiliar teaching and learning roles, encouraging consideration of the implications of inter-institution partnerships for future physiotherapy education.

\section{Key Practice Points:}

- The physiotherapist's self-perceived identity was broadened and there was a shift in their conceptions of teaching and learning.

- Clinical educators involved in teaching a theory-based subject may find it challenging to balance multiple roles.

\section{THE EFFECT OF FEMORAL NERVE CATHETERS ON EARLY MOBILISATION AND HOSPITAL LENGTH OF STAY FOLLOWING ELECTIVE PRIMARY KNEE REPLACEMENT SURGERY}

\section{Blake EL}

Physiotherapy Department, Sir Charles Gairdner Hospital, Perth

Question: Does the use of femoral nerve catheters affect the patient's ability to mobilise on day 1 post knee replacement surgery and does early mobilisation impact hospital length of stay (LOS)?

Design: Prospective observational cohort study.

Outcome Measures: presence of motor block on day 1, achievement of mobilisation on day 1 , reasons for not mobilising on day 1 , average hospital LOS.

Participants: 123 patients who had an elective primary knee replacement with a femoral nerve catheter during 2014

Results: From the 123 patients, 82 (67\%) did not mobilise on day 1 . Of these 82 patients, 66 (80\%) did not mobilise due to the effects of motor block. Other reasons included pain and dizziness. Patients who mobilised on day 1 had an average LOS of 4.1 days compared to 4.9 days for those that did not mobilise on day 1 .

Conclusion: Motor block was the primary reason for patients not mobilising on day 1 which was directly related to the presence of a femoral nerve catheter. Patients who mobilised on day 1 were, on average, discharged earlier than those that did not mobilise on day 1 . This resulted in a decreased LOS and hospital costs.

\section{Key Practice Points:}

- Presence of a femoral nerve catheter is highly likely to affect the patient's ability to achieve early mobilisation.

- There is a correlation between mobilisation on day 1 in patients post knee replacement surgery and hospital length of stay.

- Anaesthetists should investigate other quadriceps sparing techniques that aim to eliminate motor block.

\section{HAS THE ATHLETE TRAINED ENOUGH TO RETURN TO PLAY?}

\section{Blanch P}

Griffith University, Brisbane, Queensland

Swimming Australia

With history of past injury commonly reported as a predictor of subsequent injury the decisions around return to play are increasingly important. Much has been written on the politics of who should make the decision and within those arguments a comprehensive check list of considerations has been developed. One of the core metrics suggested is around functional tests. Functional tests come in a myriad of forms and for the lower limb injuries may include timed runs, vertical jump, hop tests and the star excursion balance test. While it may be argued that the functional test has some clinical utility in evaluating the rehabilitation progress of an athlete, recent investigations have demonstrated that these tests offer very little predictive value for injury. Functional tests provide little insight as to how the athlete will withstand the rigours of full training and competition. There appears to be a missing component in the return to play literature. Recently we have demonstrated that 'spikes' in an athlete's training and playing load is a significant injury risk factor. This is often perceived as something extraordinary in a current week. However, due to circumstances where chronic training load has ebbed over the previous month for different reasons of which injury may be one, a relatively 'normal' training and playing week may be enough to exceed the athlete's load capabilities. "Has the athlete prepared enough for what we are asking them to do?" should be a question in the return to play decision.

\section{HOSPITAL COSTS OF RESPIRATORY COMPLICATIONS FOLLOWING ABDOMINAL SURGERY: IMPLICATIONS FOR SERVICE PROVISION AND INTERPRETATION OF CLINICAL TRIALS}

Boden I', 2, Skinner $\mathrm{EH}^{2,3}$, Browning $\mathrm{L}^{2,4}$, Denehy $\mathrm{L}^{2}$

${ }^{1}$ Physiotherapy Department, Launceston General Hospital, Launceston Department of Physiotherapy, The University of Melbourne, Melbourne ${ }^{3}$ Department of Physiotherapy, Western Health, Melbourne

${ }^{4}$ Divison of Allied Health, Western Health, Melbourne

Question: What are the hospital costs and length of stay consequences of respiratory complications following abdominal surgery?

Design: Prospective multi-centre observational study

Participants: Patients ( $n=321$ ) having elective major abdominal surgery at three Australian and New Zealand hospitals. Patients received standardised post-operative physiotherapy.

Outcome Measures: Costs and length of stay were collected from hospital clinical activity and coding data for each participant. Respiratory complications were measured daily using standardised diagnostic criteria.

Results: Length of stay was longer (mean difference 5.4 days, 95\% CI 3.8 to 7.0 days), and hospital costs were greater in participants with respiratory complications compared to those without following both upper abdominal (mean difference $\$ 18,409,95 \%$ CI $\$ 11,732$ to $\$ 25,086$ ) and prolonged lower abdominal/laparoscopic surgery (mean difference $\$ 13,639,95 \%$ Cl \$6,319 to $\$ 20,959)$

Conclusion: Post-operative respiratory complications are costly. A 5\% reduction (equivalent to a number needed to treat of 20 ) would save \$AUD 100,000 and 30 bed days per 100 patients. Some trials conclude that respiratory physiotherapy is ineffective when performed in addition to early ambulation. Sample sizes were calculated to measure large reductions $(>10 \%)$ in respiratory complications. Our study suggests that measuring smaller reductions in complications could be worthwhile. Larger clinical trials measuring smaller effect sizes with cost-benefit analysis are needed.

\section{Key Practice Points:}

- Post-operative respiratory complications significantly increase length of stay and are very costly.

- Even small reductions in complication rates would have high fiscal and bed stay benefits.

- Preventative therapies, including physiotherapy, with moderate numbers needed to treat could be cost effective. 


\section{THE MELBOURNE RISK PREDICTION TOOL ACCURATELY PREDICTS PATIENTS UNLIKELY TO GET A RESPIRATORY COMPLICATION FOLLOWING MAJOR OPEN UPPER ABDOMINAL SURGERY}

Boden I', ${ }^{1,}$, Browning $L^{2,3}$, Skinner $E H^{2,4}$, Denehy $L^{2}$

1Physiotherapy Department, Launceston General Hospital, Launceston 'Department of Physiotherapy, The University of Melbourne, Melbourne ${ }^{3}$ Divison of Allied Health, Western Health, Melbourne

${ }^{4}$ Department of Physiotherapy, Western Health, Melbourne

Question: Can the Melbourne risk prediction tool predict the development of a respiratory complication following major upper abdominal surgery?

Design: Prospective multi-centre assessor-blinded observational study

Participants: Two hundred and thirty nine patients having elective upper abdominal surgery at three Australian and New Zealand hospitals.

Intervention: Pre-operatively, participants were assessed for risk of developing a post-operative pulmonary complication using the five-factor Melbourne risk prediction tool (operation type, respiratory comorbidity, smoking status, operation duration, self-rated physical activity,). All participants received a standardised early ambulation program.

Outcome Measures: Post-operative respiratory complications were assessed daily with standardised diagnostic criteria by assessors blind to the participant's risk rating.

Results: The Melbourne risk prediction tool predicted 55\% of participants as high risk and $45 \%$ as low risk. Fifty-two participants (22\%, 95\% Cl 17\% to $28 \%$ ) developed a respiratory complication. Sensitivity of the tool was $80 \%$ and specificity $52 \%$. The negative predictive value was $90 \%$ and positive predictive value was $33 \%$

Conclusion: The Melbourne risk prediction tool has excellent accuracy in predicting patients unlikely to develop a respiratory complication following upper abdominal surgery. This study contributes to external validation and clinical utility testing of this tool.

Trial registration: ANZTRN 12613000664741

\section{Key Practice Points:}

- The Melbourne risk prediction tool accurately predicts low risk of respiratory complications following elective open upper abdominal surgery.

- Physiotherapists can be confident that patients calculated as low risk are unlikely to develop a respiratory complication.

- This tool assists in deciding who does not require respiratory physiotherapy services in addition to an early ambulation program.

\section{LUNG INFECTION PREVENTION POST- \\ SURGERY MAJOR ABDOMINAL WITH \\ PRE-OPERATIVE PHYSIOTHERAPY \\ (LIPPSMACK POP) TRIAL: AN \\ INTERNATIONAL MULTI-CENTRE RANDOMISED CONTROLLED TRIAL}

Boden $\mathrm{I}^{1,2}$, Browning $\mathrm{L}^{2,3}$, Skinner $\mathrm{EH}^{2,4}$, Reeve ${ }^{5,6}$, Anderson $\mathrm{L}^{6}$, Hill C', Robertson IK ${ }^{8}$, Denehy $L^{2}$

IPhysiotherapy Department, Launceston General Hospital, Launceston 2Department of Physiotherapy, The University of Melbourne, Melbourne ${ }^{3}$ Divison of Allied Health, Western Health, Melbourne

${ }^{4}$ Department of Physiotherapy, Western Health, Melbourne

${ }^{5}$ Department of Physiotherapy, Auckland University of Technology, Auckland, New Zealand

${ }^{6}$ Physiotherapy Department, North Shore Hospital, Auckland, New Zealand 7Physiotherapy Department, North West Regional Hospital, Burnie ¿School of Health Sciences, University of Tasmania, Launceston

Question: Does pre-operative physiotherapy education prevent post-operative pulmonary complications (PPC) following upper abdominal surgery?

Design: Multi-centre, double-blinded, randomised controlled trial with concealed allocation and intention-to-treat analysis.

Participants: Four-hundred and forty-one patients undergoing elective upper abdominal surgery at three Australian and New Zealand hospitals. Intervention: All participants received a physiotherapy assessment and information booklet within six weeks of surgery. The intervention group received an additional physiotherapy education and training session. Post-operatively, early ambulation was standardised for all patients and no additional respiratory exercises were provided.
Outcome measures: Daily assessment of PPC using standardised diagnostic criteria. Secondary outcomes included pneumonia, intensive care readmission, hours from operation to mobilise $>1$ minute, discharge day from physiotherapy, and length of stay (LOS).

Results: A total of 434 participants completed the trial. The intervention significantly reduced PPC incidence to $10.5 \%$ (95\%CI 7 to $15 \%$ : $p<0.001)$ compared to $27 \%$ (95\% $\mathrm{Cl} 21$ to $33 \%$ ) in the control group (ARR $16.5 \%$ (95\% Cl 9 to $24 \%$ ), NNT 6 (95\% Cl 4 to 11$)$ ). Pneumonia (20\% v $8 \%$ $\mathrm{p}<0.001), \operatorname{LOS}(10.35$ days $\vee 9.15$ days, $\mathrm{p}=0.046)$ and ICU readmissions $(10 \%$ $v 6 \%, p=0.06$ ) were also less in the intervention group.

Conclusion: A single pre-operative physiotherapy education and training session prevents PPC following major abdominal surgery.

Trial registration: ANZTRN 12613000664741

Key Practice Points:

- LIPPSMAck POP is the largest double-blinded controlled trial investigating physiotherapy to prevent PPC following abdominal surgery.

- PEDro score 9/10

- This study provides conclusive evidence that pre-operative physiotherapy education services should be provided to all patients prior to major abdominal surgery.

\section{ALLIED HEALTH ASSISTANTS CAN SAFELY AND EFFECTIVELY PROVIDE EARLY AMBULATION FOLLOWING MAJOR UPPER ABDOMINAL SURGERY}

Boden I, 2, Browning $L^{2,3}$, Skinner $E H^{2,4}$, Denehy $L^{2}$

${ }^{1}$ Physiotherapy Department, Launceston General Hospital, Launceston Department of Physiotherapy, The University of Melbourne, Melbourne ${ }^{3}$ Divison of Allied Health, Western Health, Melbourne ${ }^{4}$ Department of Physiotherapy, Western Health, Melbourne

Question: Can Allied Health Assistants safely provide early ambulation above a minimum intensity level and duration following major upper abdominal surgery?

Design: Prospective multi-centre observational study

Participants: One-hundred and fifty-eight patients after elective open upper abdominal surgery at three Australian and New Zealand hospitals.

Intervention: Patients received a daily standardised post-operative early ambulation program. Physiotherapists assisted patients until they were ambulant longer than one minute. Thereafter, Allied Health Assistants assisted patient ambulation until discharge from physiotherapy services according to a threshold scoring tool.

Outcome Measures: Intensity (0-10 visual analogue scale) and duration (minutes) of ambulation episodes, location of treatment, adverse events, physiotherapy referral requests, surgical attachments, analgesia, and oxygen requirements were recorded each session.

Results: On the first post-operative day $77 \%$ patients ambulated $>1$ minute and were then transferred to ambulation services by Allied Health Assistants. Two-hundred-and-sixty Allied Health Assistant sessions were provided (mean sessions per patient 2, range 0 to 8 ), averaging >10 minutes at a mean intensity of $3 / 10.10 \%$ of patients had an epidural and $16 \%$ were located in intensive care. There were no adverse events.

Conclusion: Allied Health Assistants can safely and effectively provide a progressive, moderate intensity early ambulation program under the direction of a physiotherapist following upper abdominal surgery.

Trial registration: ANZTRN 12613000664741.

\section{Key Practice Points:}

- Allied Health Assistants, supervised by physiotherapists, can safely and effectively provide early ambulation programs following upper abdominal surgery.

- Allied Health Assistants are a low-cost option to provide early ambulation programs.

- Physiotherapists could redirect resources to higher risk patients. 


\section{GAIT RETRAINING FOR THE MANAGEMENT OF PATELLOFEMORAL PAIN IN RUNNERS: A CASE SERIES}

Bonacci J ${ }^{1}$, Saunders $\mathrm{N}^{1}$, Vicenzino $\mathrm{B}^{2}$

${ }^{1}$ School of Exercise \& Nutrition Sciences, Deakin University, Geelong ¿School of Health \& Rehabilitation Sciences: Physiotherapy, The University of Queensland, Brisbane

Question: Is gait retraining effective at reducing symptoms and patellofemoral joint kinetics in individuals with patellofemoral pain.

Design: Case series.

Participants: Four participants with patellofemoral pain

Intervention: Participants underwent a 6 -week gait retraining program that incorporated a minimalist shoe and a $10 \%$ increase in running cadence. Cadence was controlled using a metronome with a faded feedback protocol used over the 6 -weeks. Three-dimensional kinematics and kinetics were used to assess participants running gait prior to, immediately after, and 6 -weeks following the intervention.

Outcome Measures: Global rating of change $(-7$ to +7$)$ and patellofemoral joint reaction force.

Results: All participants maintained the $10 \%$ increase in running cadence at 6 and 12 weeks. Patellofemoral joint reaction force during running was reduced by $28 \%$ (95\% Cl 16\% to 39\%, $p=0.005)$ and $23 \%(95 \% \mathrm{Cl} 3 \%$ to $43 \%, p=0.04$ ) at 6 and 12 weeks following the intervention, respectively. All participants reported an improvement in their symptoms at 6 and 12 weeks. Three of the four participants reported a global rating of change of $\geq 6$ out of 7 , indicating a marked improvement in their condition.

Conclusion: These results suggest that gait retraining incorporating an alteration in running cadence and use of a minimalist shoe may be effective in the management of patellofemoral pain.

\section{Key Practice Points}

- Gait retraining improves symptoms in individuals with patellofemoral pain

- The reduction in patellofemoral joint kinetics may account for the improvement in symptoms

- Assessment of running gait may be warranted in individuals with patellofemoral pain

\section{EFFECTS OF SHOE TYPE AND CADENCE ON PATELLOFEMORAL JOINT KINETICS IN RUNNERS WITH PATELLOFEMORAL PAIN}

Bonacci ] ${ }^{1}$, Saunders $N^{1}$, Vicenzino $\mathrm{B}^{2}$

${ }^{1}$ School of Exercise \& Nutrition Sciences, Deakin University, Geelong 2School of Health \& Rehabilitation Sciences: Physiotherapy, The University of Queensland, Brisbane

Question: What influence does cadence and shoe type have on patellofemoral joint kinetics in individuals with patellofemoral pain?

Design: Randomised, within-participant experimental study.

Participants: Ten participants with patellofemoral pain

Intervention: Participants performed running trials on an instrumented treadmill in a neutral cushioned shoe and a minimalist shoe under two conditions (freely chosen running cadence and a 10\% increase in cadence). Three-dimensional kinematics were collected and marker trajectory and kinetic data input into a biomechanical model of the patellofemoral joint.

Outcome Measures: Patellofemoral joint reaction force and stress

Results: There were significant main effects for both shoe type ( $p=$ 0.001 ) and cadence ( $p=0.019)$. Running in the minimalist shoe reduced patellofemoral joint reaction force and stress by $14.3 \%$ (95\% CI 8.4\% to $20.3 \%$ ) and $18 \%$ (95\% Cl $7.5 \%$ to $28 \%$ ), respectively. Running with a $10 \%$ increase in cadence reduced patellofemoral joint reaction force and stress by $16.5 \%$ (95\% Cl 6.9\% to 26\%) and 20\% (95\% Cl 6.1\% to 34\%), respectively. There was no significant interaction effect between shoe type and cadence.

Conclusion: Utilising a minimalist shoe or adopting a running pattern with a higher cadence are both effective at reducing patellofemoral joint kinetics in individuals with patellofemoral pain.

\section{Key Practice Points:}

- Cadence and shoe type both influence patellofemoral joint kinetics

- Running with an increased cadence or in a minimalist shoe result in similar reductions in patellofemoral joint kinetics

- Assessment of gait and shoe type may be warranted in runners with patellofemoral pain

\section{CURRENT TELEMEDICINE-BASED INTERVENTIONS ARE NOT EFFECTIVE IN MANAGING CHRONIC LOW BACK PAIN: SYSTEMATIC REVIEW WITH META-ANALYSIS}

Borges Dario $A^{1}$, Moreti $A^{2}$, Almeida $L^{3}$, Simic $M^{1}$, Papas $E^{1}$, Ferreira $\mathrm{M}^{4}$, Refshauge $\mathrm{K}^{1}$, Ferreira $\mathrm{PH}^{1}$

${ }^{1}$ Discipline of Physiotherapy, Faculty of Health Sciences, The University of Sydney, Sydney, Australia

2Discipline of Physiotherapy, Center of Biological Sciences and Health, Federal University of São Carlos, São Carlos, Brazil

${ }^{3}$ Discipline of Physiotherapy, Institute of Health Sciences, Federal University of Bahia, Salvador, Brazil

${ }^{4}$ The George Institute for Global Health, Sydney Medical School, The University of Sydney, Sydney, Australia

Question: Is telemedicine effective in reducing pain and disability in people with chronic low back pain (LBP)?

Design: Systematic review with meta-analysis of randomized clinical trials. Participants: Individuals with nonspecific chronic LBP.

Intervention: Any type of telemedicine interventions (i.e. e-mail discussion, online chat-) used to reduce pain and disability.

Outcome Measures: Pain measured with visual analogue scale. Disability measured with Roland-Morris Disability Questionnaire or Oswestry Disability Index. For the meta-analyses, outcomes were converted to 0-100 scale and effect sizes were described as weighted mean differences (WMD) with $95 \%$ confidence intervals (CI).

Results: 4 trials were identified and included in a meta-analysis Telemedicine interventions had no effect on pain (4 trials short-term follow-up; WMD -2.61 points; $95 \% \mathrm{Cl}-5.23$ to $0.01 ; \mathrm{I}^{2}=0 \%$ / 2 trials longterm follow-up; WMD: -0.94 points; $95 \%$ Cl -6.71 to 4.84; $\left.\right|^{2}=40 \%$ ) or disability (4 trials short-term follow-up; WMD: -1.85 points; $95 \% \mathrm{Cl}-4.32$ to $0.62 ; 1^{2}=0 \% / 2$ trials long-term follow-up; WMD: 0.13 points; $95 \% \mathrm{Cl}-4.1$ to $4.4 ; 1^{2}=0 \%$ ) when compared to minimal interventions.

Conclusion: There is 'moderate quality evidence' that current telemedicine interventions do not reduce pain and disability in chronic LBP in the short or long-term. Although it is still premature to dismiss telemedicine-based strategies to manage chronic LBP it is clear that current interventions need to be re-designed.

\section{Key Practice Points:}

- Telemedicine interventions only do not reduce pain and disability in chronic LBP.

- Further research is needed to investigate the potential of telemedicine to benefit LBP patients. 
OBESITY DOES NOT INCREASE THE

RISK OF CHRONIC LOW BACK PAIN. A PROSPECTIVE STUDY OF SPANISH ADULT TWINS

Borges Dario A ${ }^{1}$, Ferreira $\mathrm{ML}^{2}$, Refshauge $\mathrm{K}^{1}$, Ordoñana JR $\mathrm{R}^{3,4}$, Ferreira $\mathrm{PH}^{1}$

${ }^{1}$ Discipline of Physiotherapy, Faculty of Health Sciences, The University of Sydney, Sydney, Australia

The George Institute for Global Health, Sydney Medical School, The

University of Sydney, Sydney, Australia

3Murcia Twin Registry, Department of Human Anatomy and Psychobiology,

University of Murcia, Spain

4IMIB-Arrixaca, Murcia, Spain

Questions: Do obesity related measures (body mass index; \% fat mass; waist circumference; waist-hip ratio) increase the risk of chronic low back pain (LBP) when genetics and early shared environment are considered?

Design: Prospective observational study.

Participants: 1454 adult twins (759 males) free of LBP at baseline were followed for 2-4 years.

Outcome Measures: Chronic LBP was assessed with the question in the follow-up "Have you ever suffered from chronic LBP?" Chronic LBP was defined as LBP > 6 months. To assess chronic disabling LBP, those participants identified with chronic LBP were asked "Was this pain bad enough to limit your usual activities or change your daily routine for more than one day?"

Results: Data on LBP at follow-up was available for 1098 twins (76\% of the baseline sample). Obesity related measures (body mass index; \% fat mass; waist circumference; waist-hip ratio) did not increase the risk of developing chronic LBP or chronic disabling LBP ( $p>0.05)$. Similar results were found for both outcomes when early environment and genetic factors shared by twins were adjusted in the analyses.

Conclusion: Obesity related measures do not increase the risk of chronic LBP or chronic disabling LBP in adults. Our findings do not support a causa direct relationship between obesity and chronic LBP.

Key Practice Points:

- Obesity does not increase the risk of chronic LBP in adults.

- Our findings suggest that preventative strategies to reduce LBP incidence should focus on other modifiable risk factors.

\section{CENTRE OF GRAVITY: CAN START LOCATION BE USED TO REFLECT MEAN LOCATION IN THE ANTERO- POSTERIOR PLANE?}

Boughen $\mathrm{J}^{1}$, Johnston $\mathrm{V}^{1}$, Khan $\mathrm{A}^{1}$, Nitz $]^{2}$

${ }^{1}$ The School of Health and Rehabilitation Sciences, The University of Queensland, Brisbane

2Formerly, the School of Health and Rehabilitation Sciences, The University of Queensland, Brisbane

Question: Does start location of centre of gravity in three test trials fall in the same forward or backward sector as mean location in the same trials in stance on a firm surface with eyes open? Is this so for all age decades?

Design: Cross-sectional study.

Participants: Sixty men and women (40-80 years), independently mobile within the community, were tested standing with eyes open on a Kistle force-plate (firm surface) on just one occasion.

Outcome Measures: Start and mean locations of centre of gravity for each of $3 \times 10$ sec trials were categorized to forwards or backwards of the medial malleoli if at least two of the three locations were in the same sector.

Results: Analysis is in progress. Delays in gaining access to some data and statistical software have prevented preliminary results from being available in time for abstract submission.

Conclusion: This study contributes to knowledge of how centre of gravity measures from force-plate data might be interpreted in the clinical setting. It builds on previous work for tests with altered sensory inputs on one specific commercially available force-plate system.

\section{Key Practice Points}

- Contributes to understanding of centre of gravity location in the anteroposterior plane in standing balance.

- Enhances understanding of the behaviour of centre of gravity under different surface and vision conditions.

- Explores how data from a force-plate system currently used in some rehabilitation centres might be used to monitor centre of gravity location in assessment and treatment.

\section{POSTING AND HARDNESS OF FOOT ORTHOSES MODIFY PLANTAR PRESSURE AND COMFORT IN CYCLING}

Bousie JA ${ }^{1}$, Vicenzino $B^{2}$, Blanch $P$

${ }^{1}$ CQUniversity

2The University of Queensland

Question: Does hardness and posting of foot orthoses impact on plantar profile and perceived comfort and support during cycling?

Design: Randomised, within-participant experimental study.

Participants: 23 healthy cyclists.

Intervention: Participants performed steady-state seated cycling. Eight orthosis conditions, varying in hardness and posting, were evaluated.

Outcome Measures: Plantar contact area (CA), mean pressure (MP) and peak pressure (PP) were measured and perceived comfort and support of the cycling shoe were rated.

Results: Softer orthoses significantly increased CA\% $(p=0.014)$ across the midfoot and heel and decreased it at the hallux, toes and mid and lateral forefoot. MP\% $(p=0.034)$ and PP\% $(p=0.012)$ were significantly increased at the mid and lateral forefoot with a reduction in $\mathrm{MP} \%$ at the midfoot and in PP\% at the hallux and toes. Forefoot and rearfoot posting significantly decreased CA\% $(p=0.018)$ at the midfoot and heel and increased it at the toes and forefoot. Posting significantly altered PP\% ( $p=$ 0.013 ) with results varying with posting position. A medial rearfoot posting significantly altered comfort $(p=0.036)$ and support $(p=0.018)$ at the arch and heel.

Conclusion: Plantar pressure and contact area are modulated by orthosis hardness and posting. A medial forefoot posting impacts on perceived comfort and support at the arch and heel.

Key Practice Points:

- Plantar profile is modulated by orthosis hardness and posting.

- Medial forefoot posting influences perceived comfort and support.

- Further research is required to investigate the impact of orthoses on symptomatic cyclists.

\section{MYOFASCIAL TRIGGER POINTS IN EOUINE PECTORAL MUSCLES AND THEIR ASSOCIATION WITH GIRTHY BEHAVIOUR}

\section{Bowen $\mathrm{A}^{1}$}

\section{${ }^{1} A B$ Physiotherapy Services}

Question: Are myofascial trigger points (MTrPs) in equine pectoral muscles associated with girthy behaviour?

Design: Cross-sectional Clinical Study

Participants: 38 low level performance horses in Western Australia.

Outcome Measures: Owners were questioned on girthy behaviours and their frequency; this was transformed into a girthiness score. Horses received routine assessment and treatment with palpation of their pectoral muscles recorded in detail onto a reference map. For each taut band identified direct pressure was applied and the behavioural response scored 0-3 to estimate severity. Mean MTrP severity was calculated for each horse and for each cell. This data was mapped and analysed using Chi square for trend.

Results: MTrPs were present in the pectoral muscles of all horses in this study. The most common locations were the axillary portion of posterior pectoral, to the right of the sternum in the girth region and towards the edges of muscles. Girthy horses had higher scores for severity than notgirthy horses $(p=0.008)$

Conclusion: MTrPs are common in the pectoral muscles and girthy horses are more severely affected. Knowledge of where MTrPs typically occur could lead to the development of efficient treatments and preventions, to improve comfort for horses, optimise performance and reduce dangerous girthy behaviour.

\section{Key Practice Points:}

- The most common sites for MTrPs in equine pectoral muscles.

- The most severe MTrP sites in equine pectoral muscles.

- Awareness of association between MTrPs in the pectoral muscles and girthy behaviour. 


\section{TRANSITION OF ADOLESCENTS WITH ON-GOING UROLOGICAL CARE NEEDS FROM PAEDIATRIC TO ADULT SERVICES}

\section{Bower W}

Sub-Acute Services - Continence and Department of Rehabilitation, The Royal Melbourne Hospital, Melbourne Health

Children with urinary tract disorders are managed by teams or individual pediatricians, urologists, nephrologists, gastroenterologists, neurologists and nurses. At some point adolescents or young adults with lifelong urological care need to move from a child-centered to an adult-centered health system. The actual physical change is referred to as the transfer whilst the process preceding this move constitutes the transition of care. Urological patients, with congenital or other lifelong care needs, are now entering adulthood in larger numbers than previously, necessitating new planning processes for tailored transfer of management. Adult teams must become familiar with new clinical problems in multiple organ systems and anticipate issues provoked by adolescence and physical growth. During this period of transitional care the paediatric clinician or team assists the young patient to build attitudes, skills and understanding of processes needed to maximize the function of their urinary tract - thus taking responsibility for their own health needs. Tools such as the Readiness Scale can be incorporated into standard clinical care. Preparation must also address negotiating the adult health care system, psychosocial, educational or vocational issues and mental wellbeing. This presentation will identify management and health-service problems related to children with congenital or acquired urological conditions who advance into adulthood and the clinical implications this has for long-term health and specialist care. Specific issues face both pediatric and adult -care clinicians, necessitating a systems-approach model.

\section{THE ROLE OF EXERCISE AND PHYSICAL ACTIVITY IN CANCER CARE AND LYMPHOEDEMA MANAGEMENT}

\section{Box RC ${ }^{1}$}

${ }^{1}$ QLD Lymphoedema and Breast Oncology Physiotherapy, Grange, QLD 4051 AUSTRALIA

Cancer treatments can result in a number of long-term problems that impact on quality of life. A prospective surveillance model of care incorporating a rehabilitation framework, should be integral to Physiotherapy services provided. Principles of chronic condition management are essential. The benefits of exercise and physical activity for general health and well-being are established. The body of evidence for its use with the cancer population has grown exponentially in the last decade.Promoting change in a person is essential to enable the levels of intensity required to achieve benefits during cancer treatment, minimise the impact of ongoing sequelae, reduce cancer recurrence, and maintain functional capacity for optimal quality of life to be met. The presence of, or risk of developing, Lymphoedema is not a reason for lower levels of physical activity or avoidance of certain types of exercise. Translating research into daily clinical practice, within a patient-focussed model, is fundamental in the approach used to optimise outcomes following a cancer diagnosis for each individual patient. Aquatic physiotherapy, is a component of the 'MOVES for Life $\odot$ programme' to enable individuals to achieve recommended levels of intensity.

\section{Key Practice Points:}

- Both specific therapeutic exercise and physical activity have a role in the management of sequelae from cancer treatments.

- Mode of exercise and physical activity should be individualised to maximise participation to achieve benefits.

- Prescription should be graduated and progressive, monitoring for outcomes to ensure maximal participation without adverse events.

Further research is needed to establish the additional benefits from aquatic physiotherapy

\section{VERY EARLY BRAIN STRUCTURE AND NEUROLOGICAL FUNCTION DETECTS BRAIN INJURY IN PRETERM INFANTS AT 30 WEEKS AND 40 WEEKS POSTMENSTRUAL AGE}

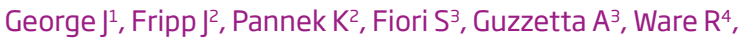
Rose $\mathrm{S}^{3}$, Colditz $\mathrm{P}^{5}$, Boyd $\mathrm{R}^{1}$

${ }^{1}$ Queensland Cerebral Palsy and Rehabilitation Research Centre, School of Medicine, The University of Queensland, Brisbane, Australia

2The Australia E-Health Research Centre, Brisbane, Australia

${ }^{3}$ Stella Maris Scientific Institute, Department of Developmental Neuroscience, Pisa, Italy

${ }^{4}$ Children's Health Research Centre and School of Population Health, The University of Queensland, Brisbane, Australia

${ }^{5}$ Centre for Clinical Research, The University of Queensland,

Brisbane, Australia

Question: Examining the relationship between neurological function and structural MRI at 30 and 40 weeks postmenstrual-age in very preterm infants.

Design: Prospective cohort study

Participants: At 30weeks: 67 infants born<31weeks, 39 male, age at birth 28.3wks(1.6), birthweight 1113g(283g), age at MRI 32.3wks(1.4) neurological score 15.7(3.63). At 40weeks: 56 re-assessed: age at MRI 40.9wks(1.4), neurological score 23.9(3.5)

Outcome Measures: Infants assessed at 30-32weeks and 40-42weeks postmenstrual-age with 3T MRI and the Hammersmith Neonatal Neurological Examination. Kidokoro scoring of structural MRI's generated white matter, grey matter and global injury scores. Multivariable logistic regression, adjusted for GA at birth, sex, birthweight and age at MR $(p<0.05)$.

Results: At 30wks, neurological scores significantly related to white matter $M(S D)=3.10(2.23)$, regression coefficient $(B)=-0.16,95 \% \mathrm{Cl}=-0.32$ to $0, p=0.05$; grey matter $=2.36(1.62), \mathrm{B}=-0.10,95 \% \mathrm{Cl}=-0.19$ to -0.01 , $p=0.04$; and global scores $=5.46(3.29), B=-0.26,95 \% \mathrm{Cl}=-0.48$ to -0.04 , $p=0.02$. At 40 weeks, neurological scores were significantly related to grey matter $=0.86(1.02), \mathrm{B}=-0.1,95 \% \mathrm{Cl}=-0.19$ to $0.02, p=0.02$; with a trend to global scores $=3.66(2.89), \mathrm{B}=-0.24,95 \% \mathrm{Cl}=-0.49$ to $0.01, p=0.06$; and $\mathrm{did}$ not relate to white matter $=2.8(2.4), \mathrm{B}=-0.13,95 \% \mathrm{Cl}=-0.34$ to $-0.07, p=0.2$.

Conclusion: A significant relationship exists between MRI brain structure and neurological function in preterm born infants at 30 weeks postmenstrual age and is reconfirmed at 40 weeks.

\section{Key Practice Points:}

- Early MRI brain structure and neurological function have an important role in the very early detection of brain injury in infants at risk of CP.

- Early MRI is feasible in this population.

- Neurological assessment identifies those infants with lesions evident on brain MRI at 30 weeks. 
RELATIONSHIP BETWEEN EARLY BRAIN STRUCTURE ON MRI, WHITE MATTER INTEGRITY (DIFFUSION MRI) AND NEUROLOGICAL FUNCTION AT 30 WEEKS POSTMENSTRUAL AGE IN INFANTS BORN VERY PRETERM

George $J^{1}$, Fripp $]^{2}$, Shen $\mathrm{K}^{2}$, Pannek K², Fiori ${ }^{3}$, Chan $\mathrm{A}^{2}$, Ware R ${ }^{4}$, Rose $\mathrm{S}^{3}$, Colditz $\mathrm{P}^{5}$, Boyd $\mathrm{R}^{1}$

${ }^{1}$ Queensland Cerebral Palsy and Rehabilitation Research Centre, School of Medicine, The University of Queensland, Brisbane, Australia

2 The Australia E-Health Research Centre, Brisbane, Australia

${ }^{3}$ Children's Health Research Centre and School of Population Health, The

University of Queensland, Brisbane, Australia

${ }^{4}$ Centre for Clinical Research, The University of Queensland

Brisbane, Australia

Question: To examine the relationship between very early brain structure (structural MRI), white matter integrity (diffusion MRI) and neurological function at 30 weeks postmenstrual-age in very preterm infants.

Design: Prospective cohort study

Participants: At 30wks PMA: 57 infants, born <31weeks, 24 male, age at birth 28.31weeks(1.62), birthweight 1099g(269g), age at MRI 32.19weeks(1.47), neurological scores 15.53(4.37).

Outcome Measures: Infants assessed at 30 -32weeks postmenstrual-age with $3 T$ MRI and the Hammersmith Neonatal Neurological Examination.

Structural MRI segmented in grey matter, white matter and corticospinal fluid, and labelled using Albert Atlas (MilxView). A population specific fractional anisotropy atlas created aligned to JHU neonatal Atlas. Relationship between regional volumetric or diffusion scores (Frontal, Parietal, Temporal, Occipital, Thalamus, Ventricle) and neurological measures assessed using multivariate regression, adjusted for gestational age at birth, sex, birthweight, brain size and age at MRI, statistical significance set at $5 \%(R)$

Results: The neurological scores, brain size and age at MRI were associated with volumetric measures. In particular, grey matter fraction ( $R^{2}$ adj=0.3; $F=7.0 ; p<.05$ ), occipital ( $R^{2}$ adj0.33; $F=7.4 ; p<.02$ ), and temporal ( $R^{2}$ adj 0.09:F=5.0,p<.04; grey matter superior posterior). Neurological scores were associated with fractional anisotropy, in particular, corpus callosum ( $\left.R^{2} a d j=0.1 ; F=2.7 ; p<.05\right) \&$ fornix $\left(R^{2} a d j=0.09 ; F=2.7 ; p<.05\right)$.

Adjustment for age at birth and MRI was important.

Conclusion: A modest relationship was determined between neurological assessment and structural or diffusion MRI at 30 weeks postmenstrual-age.

\section{Key Practice Points}

- Advanced imaging plays an important role in earlier detection of infants at risk of Cerebral Palsy.

- Bedside clinical tools feasible at this age and provide insight on brain integrity

- MRI feasible at 30-32 weeks.

\section{ESTABLISHMENT OF A PHYSIOTHERAPY BRONCHOSCOPY SERVICE INICU}

\section{Bradley $\mathbf{S}^{1}$}

IPhysiotherapy Department and Intensive Care Unit, The Alfred, Melbourne, Victoria. Australia

The Alfred, under a workforce innovation grant provided by the Victorian Department of Health and Human Services, is establishing an Advanced Respiratory Physiotherapy in ICU (ARP-ICU) program. This program, led by senior physiotherapy and medical staff in ICU, is applying the same principles and methods used in establishing The Alfred's Advanced Musculoskeletal Physiotherapy services. The program is currently focussed on developing and implementing an independent practice physiotherapy bronchoscopy service in ICU. Over 800 bronchoscopies are performed per annum in The Alfred's ICU to assist with sputum clearance or to obtain diagnostic lavage samples for microbiology. It has been recognised that the physiotherapy staff in ICU could be trained to perform many of these bronchoscopies in intubated / ventilated ICU patients, as an extension of their current role in airway and secretion management. This presentation will discuss how this advanced practice role has become feasible through the support of the ICU medical team, as well as the recognition of senior physiotherapy staff to apply their extensive respiratory care skills to the advanced practice of bronchoscopy. Scope of practice, key clinical governance issues and some of the perceived barriers that needed to be overcome will also be discussed. In addition the framework for selection, education, training, supervision and assessment of each physiotherapist, and some of the proposed methods to evaluate the effectiveness, safety and efficacy of the physiotherapy bronchoscopy service in ICU as a whole will also be raised.

\section{Key Practice Points:}

- Implementation of advanced practice in critical care

- Competency and credentialing frameworks

- Physiotherapy performed bronchoscopy
SUPRASCAPULAR NERVE STIMULATION MODULATES MOTOR CORTEX

EXCITABILITY IN HEALTHY ADULTS BUT NOT IN CHRONIC SHOULDER PAIN; IMPLICATIONS FOR TREATMENT OF SHOULDER PAIN USING ACUPUNCTURE

\section{Bradnam LV ${ }^{1,2}$}

${ }^{1}$ Discipline of Physiotherapy, School of Health Sciences, Faculty of Medicine, Nursing and Health Sciences, Flinders University, South Australia 2Discipline of Physiotherapy, Graduate School of Health, University of Technology Sydney, Sydney, Australia

Question: To compare integration of sensory inputs in the primary motor cortex between healthy adults and people experiencing chronic shoulder pain.

Design: Comparative and non-blinded interventional study Participants: 18 healthy adults and eight patients with chronic shoulder pain were recruited.

Intervention: Sensory nerve stimulation

Outcome Measures: Transcranial magnetic stimulation (TMS) to assess cortical neurophysiology. Pain intensity in patients was assessed by a visual analogue scale.

Results: Low intensity, sensory nerve stimulation reduced the size of the TMS induced motor-evoked potential (afferent inhibition) in the shoulder motor cortex in controls (all p < 0.03). Inhibition in the motor cortex was assessed using the cortical silent period and inhibition was reduced by sensory nerve stimulation (all $p<0.0001$ ). However, shoulder pain patients demonstrated higher active motor threshold $(p=0.046)$, less afferent inhibition ( $p=0.044)$, longer cortical silent periods $(p=0.048$ ) and less reduction of the cortical silent period to sensory nerve stimulation ( $p=$ 0.045 ). Higher motor thresholds were related to higher pain scores ( $p=$ 0.009). The finding that low intensity sensory nerve stimulation does not modify cortical inhibition in people with chronic shoulder pain has implications for the use of acupuncture as an intervention in chronic pain disorders.

Conclusion: Sensory stimulation modifies shoulder motor cortex neurophysiology in healthy adults by not in those with chronic pain. Acupuncture interventions that reduce cortical inhibition might improve function in chronic shoulder pain patients. Studies are needed to determine the best parameters of stimulation to effectively improve the integration of sensory inputs by the motor cortex in chronic pain.

\section{Key Practice Points:}

- Shoulder pain alters inhibitory function in shoulder motor cortex, which may impact on motor learning and therefore rehabilitation

- Low intensity sensory input does not modify cortical inhibition in chronic shoulder pain patients

- Acupuncture interventions that reduce cortical inhibition may improve outcomes in chronic shoulder pain 


\section{MODULATION OF THE CEREBELLUM USING THETA-BURST STIMULATION IN PEOPLE WITH CERVICAL DYSTONIA}

Bradnam LV ${ }^{1,2}$, McDonnell MN³ , Ridding MC4

${ }^{1}$ Graduate School of Health, University of Technology Sydney, Sydney, Australia

2Discipline of Physiotherapy, Flinders University, Adelaide, Australia

${ }^{3}$ Sansom Institute for Healthcare Research, University of South Australia, Adelaide, Australia

${ }^{4}$ Robinson Research Institute, School of Paediatrics and Reproductive Health, University of Adelaide, Adelaide, Australia

Question: Non-invasive stimulation brain stimulation may improve Cervica Dystonia. The objective was to assess effects of intermittent theta-burst stimulation of the cerebellum on dystonia symptoms, quality of life and cortical neurophysiology using Transcranial Magnetic Stimulation (TMS)

Design: A randomised, double-blind, sham controlled trial

Participants: Sixteen participants were randomised into real $(n=8)$ or sham $(n=8)$ groups

Intervention: Ten sessions of stimulation were delivered by an independent therapist followed by neck exercises guided by a video.

Outcome Measures: TMS assessed motor-evoked potentials and cortical silent periods from the upper trapezius muscles. Dystonia severity and quality of life were assessed by the TWSTRS and the CDQ24.

Results: TWSTRS was significantly reduced by real stimulation $(\mathrm{X} 2(2)=$ 8.86, $p=0.012)$, but not sham $(X 2(2)=0.67, p=0.72)$, with no difference between groups (all $p>0.66$ ). TWSTRS pain subsection also reduced for real stimulation $(X 2(2)=9.74, p=0.008)$, with a significant difference between groups $(p=0.040)$. For CDQ-24, there was a reduction for real (X2 $(4)=11.14, p=0.025)$, but not sham $(X 2(4)=4.69, p=0.32)$ stimulation, with a group difference 12 weeks later $(p=0.04)$. Cortical neurophysiology did not change (all $p>0.21$ ). Performance of the grooved pegboard task improved for real stimulation ( $p=0.03$ ).

Conclusion: Intermittent theta-burst to the cerebellum may provide a novel treatment intervention for people with cervical dystonia, but a larger study is needed

\section{Key Practice Points:}

- The cerebellum may play a role in the pathophysiology of cervical dystonia

- Interventions that target cerebellar function may be a therapeutic option - Non-invasive stimulation of the cerebellum appears to improve quality of life, this may be driven by reduction in pain

\section{HOW TO MANAGE CERVICAL DYSTONIA}

\section{Bradnam $\mathrm{L}^{1}$}

${ }^{1}$ University of Technology, Sydney, Sydney, Australia

Background: Cervical Dystonia is a recalcitrant movement disorder with sensory, motor, perceptual and cognitive deficits. Standard medical care is treatment by botulinum toxin injections. Patients are rarely referred to physiotherapists for rehabilitation. This session will provide a concise evidence-based overview of the assessment and management of people with cervical dystonia by physiotherapists

Aims / objectives: The aim of this session is to enhance physiotherapy management of cervical dystonia.

The objectives are to:

1. Understand the evidence-base for physiotherapy in treatment of cervical dystonia

2. Highlight common signs and symptoms to enhance clinical reasoning

3. Identify treatments that target the deficits associated with cervical dystonia

At the end of this session, participant will have achieved the following learning outcomes:

1. Better understand the pathophysiology, aietology and incidence of cervical dystonia

2. Enhanced clinical reasoning of presenting signs and symptoms across sensory, motor, perceptual and cognitive domains

3. Cognisant of the current evidence-base for physiotherapy rehabilitation

4. Knowledge of interventions that address the common deficits of cervical dystonia

Approach: Introductory overview lecture (5 minutes), then participants will work in small groups to identify the main problems from a video recording of a person with cervical dystonia. Appropriate treatment to address sensory, motor, perceptual and cognitive issues will be debated within groups using a list of evidence-based interventions. In the session closing segment, participants will have the opportunity to suggest additional interventions for particular impairments and function based on their clinical experience.

\section{Conclusion / Key Practice Points:}

- Improve knowledge of the movement disorder, cervical dystonia

- Identify main deficits and signs and symptoms experienced by patients

- Enhance clinical reasoning and knowledge of evidence underpinning physiotherapy for cervical dystonia

- Apply a range of interventions that target key deficits to improve patient outcomes

TESTING A SHAM DRY NEEDLE FOR THE CERVICAL SPINE IN HEALTHY ADULTS: A PILOT STUDY AND TWO RANDOMISED CONTROLLED TRIALS

Braithwaite $\mathrm{FA}^{1,2}$, Walters $]^{1,2}$, McEvoy $\mathrm{M}^{1,2}$

${ }^{1}$ School of Health Sciences, University of South Australia

${ }^{2}$ The International Centre for Allied Health Evidence (iCAHE)

Question: Can healthy adults distinguish between a true and nonpenetrating sham dry needle in the cervical area?

Design: Pilot and two double-blinded randomised controlled trials. Participants: Healthy adults ( $n=116$ ); pilot $n=16$, part one (no previous needling: 'naïve') $n=50$, part two (non-naïve) $n=50$

Intervention: The study tested the blinding success of a sham needle (toothpick in a plastic guide-tube), by application of a true and sham needle in the cervical area of blinded participants.

Outcome Measures: Primary outcome: participant perception of group allocation. Secondary outcomes: responses to two open-ended questions about the procedure: 'Why did you think you had the needle/sham?', and, 'Can you briefly describe what you felt?'. All outcomes were assessed immediately after the procedure.

Results: Participants were unable to distinguish whether the true or sham dry needle had been applied (naïve $p=0.32$; non-naïve $p=0.78$ ) Qualitative data revealed the sham felt similar to a true needle.

Conclusion: This inexpensive and easily replicable non-penetrating sham needle is likely to blind naïve and non-naive healthy adults to whether the true or sham dry needle has been applied.

\section{Key Practice Points:}

- There is no consensus on a credible sham for needling studies, limiting confidence in testing the effectiveness of dry needling interventions.

- Participants reported basing their judgment of group allocation on sensation and could not distinguish between the sham and true needle.

- Following further validation in participants with cervical pathology, this sham may be used in future trials to separate therapeutic and placebo effects.

Trail registration: Australian New Zealand Clinical Trails Registry (ANZCTR) ACTRN12614000285651

Ethics approval: Obtained from the Health Sciences Divisional Human Research Ethics Committee, University of South Australia. 


\section{SMART ARM WITH OR WITHOUT ELECTRICAL STIMULATION COMPARED TO USUAL CARE IN STROKE INPATIENTS WITH SEVERE DISABILITY: RANDOMISED CONTROLLED TRIAL}

Brauer SG ${ }^{1}$, Hayward KS ${ }^{1,2}$, Carson RG ${ }^{3}$, Lloyd D ${ }^{4}$, Cresswell AG $^{5}$, Barker RN ${ }^{2}$

${ }^{1}$ School of Health and Rehabilitation Sciences, The University of Queensland, Brisbane, Australia

${ }^{2}$ College of Health Sciences, James Cook University, Townsville, Australia

${ }^{3}$ Trinity College institute of Neuroscience \& School of Psychology, Trinity College Dublin, Ireland

${ }^{4}$ Queensland Brain Institute, The University of Queensland

Brisbane, Australia

${ }^{5}$ School of Human Movement and Nutrition Sciences, The University of Queensland, Brisbane, Australia

Question: What is the effect of SMART Arm training with or without outcome-triggered electrical stimulation on function compared with usual care in stroke inpatients?

Design: Multicentre randomised controlled trial, concealed allocation assessor blinding and intention-to-treat

Participants: Fifty stroke inpatients with severe arm disability.

Intervention: Either (1) SMART Arm training with outcome-triggered stimulation (SMART Arm-stim) and usual therapy, (2) SMART Arm-alone and usual therapy, or (3) usual therapy (control) for 20, 1-hour sessions over 4-weeks.

Outcome Measures: Participants were assessed at baseline, postintervention; 26- and 52-weeks follow up. The primary outcome measure was Motor Assessment Scale item 6 (MAS-6) at post-intervention, with a score of >1 point classified as a clinically meaningful improvement in arm function.

Results: Post-intervention, 65\% of SMART Arm-stim, 53\% of SMART Arm-alone and $47 \%$ of controls improved in arm function. At 26-weeks, $69 \%$ of SMART Arm-stim, $88 \%$ of SMART Arm-alone and $59 \%$ of controls improved $(p=0.098)$. Both SMART Arm groups had greater odds (1.241.31) of a higher MAS-6 score than controls at this timepoint. There was no difference between groups at post or 52-weeks ( $p>0.543$ ).

Conclusion: SMART Arm with or without stimulation was as effective as usual care to improve arm function in stroke survivors with severe arm disability

Trial registration: ACTRN12608000457347

Key Practice Points:

- Stroke survivors with severe arm disability can improve function with SMART Arm or usual care

- Improvements continued at 26-weeks but reduced at 52-weeks.

- Pragmatic approach to SMART Arm training duration and use of electrical stimulation warrants investigation

\section{MEASUREMENT OF WRIST JOINT \\ MOTION: DOES THE HALO DEVICE AGREE WITH THE UNIVERSAL GONIOMETER?}

\section{Breckenridge $\mathrm{J}^{1,2}$, Hirschhorn ${ }^{1,3}$, James $\mathrm{B}^{4}$}

${ }^{1}$ The Clinical Research Institute

¿2entral West Orthopaedic \& Sports Physiotherapy

${ }^{3}$ Department of Physiotherapy, Westmead Hospital

${ }^{4}$ The University of Kentucky

Question: Measurement of wrist joint motion: Does the HALO digital laser goniometer device agree with the universal goniometer?

Design: Cross sectional experimental study.

Participants: Adults without upper limb pathology ( $n=27$, age 32 \pm 11years).

Procedure: Participants sat comfortably with their forearm resting on a table. Left and right wrist joints were respectively positioned in a series of four randomly determined flexion and extension angles, using a universal goniometer. A researcher, blinded to the HALO device's LCD (output) screen, then performed repeated wrist joint flexion/extension measures, using the HALO device.

Outcome Measures: i) Pearson's correlation coefficients between goniometer-set and HALO measured wrist joint flexion/extension angles; ii) Bland-Altman limits of agreement between goniometer-set and HALOmeasured wrist joint flexion/extension angles.

Results: There were significant and strong correlations between goniometer-set and HALO-measured wrist joint angles for both flexion $(R=0.985, p<0.001)$, and extension ( $R=0.894, p<0.001)$. Mean difference between the HALO measurement and goniometer-set for flexion was $2^{\circ}$, (limits of agreement $7^{\circ}$ to $-3^{\circ}$ ). Extension mean difference was $4^{\circ}$, (limits of agreement $13^{\circ}$ to $-4^{\circ}$
Conclusion: Measures of wrist joint range of motion obtained with the HALO device correlate strongly with those set using a universal goniometer. Limits of agreement between the two methods of measurement are acceptable and consistent across a wide range of wrist joint positions. Our results suggest that the HALO device may be considered a valid alternative to the traditionally used universal goniometer for the measurement of wrist joint range of motion.

\section{Key Practice Points:}

- The HALO device is a new method of joint range measurement and is a valid alternative to the universal goniometer for the measurement of wrist joint flexion/extension range of motion.

- Physiotherapists considering the use of novel technologies should test their validity before clinical application

\section{THE ROLE OF SHORT-TERM GOAL ACHIEVEMENT IN PREDICTING DISCHARGE DESTINATION IN PEOPLE ADMITTED TO NEUROLOGICAL REHABILITATION WITH SEVERE FUNCTIONAL DEFICITS}

Brimblecombe $\mathrm{J}^{1}$, Black S1, Brock K ${ }^{1}$

${ }^{1}$ St Vincent's Hospital Melbourne

Question: Which measure of progress in early phase neurological rehabilitation best predicts discharge destination and motor improvement at discharge?

Design: Prospective observational cohort study.

Participants: 53 consecutive patients with a neurological diagnosis admitted to rehabilitation with an admission score below 47 on the motor subset of the Functional Independence Measure (FIM motor).

Outcome Measures: Two measures evaluated at team meetings in the first two weeks after admission were utilised; FIM motor change and short-term goal achievement in the domains of the FIM motor. Discharge destination and FIM motor change at discharge were collected.

Results: Logistic regression was conducted with the outcome variable discharge destination (home or semi-independent living versus high dependency care) and the predictors being median goal achievement and change in FIM motor over the first two weeks. Of the variables, goal achievement was the only significant predictor in the model $(p=.026)$. For FIM motor change by discharge, linear regression analysis indicated that both variables contributed significantly to the model; FIM motor change ( $p$ $=.003)$ and goal achievement $(p=.016)$.

Conclusion: This study reaffirms the importance of individualised goal setting as a key practice feature for rehabilitation patients with severe neurological impairment.

\section{Key Practice Points:}

- Short-term goal achievement in the early phase post admission is an indicator of the person's potential to return home or to semiindependent living.

- Individualised short-term goal achievement may be more useful in predicting discharge destination than a standardised measure such as the FIM motor. 


\section{IMPLEMENTATION OF THE CHRONIC STROKE REVIEW TOOL AND INTERVENTION GUIDE (REV-TIG)}

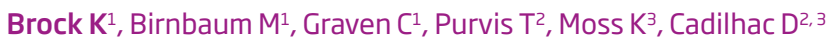

${ }^{1}$ St Vincent's Hospital Melbourne

${ }^{2}$ Translational Public Health and Evaluation Division, Stroke and

Ageing Research Centre, School of Clinical Sciences at Monash Health

Monash University

${ }^{3}$ The Florey Institute of Neuroscience and Mental Health

Question: What are consumer and clinician perceptions of the Chronic Stroke Rev-TIG, a new resource for assessment and management of people with stroke in community rehabilitation settings?

Design: Mixed methods: post pilot program implementation questionnaires and focus group.

Participants: 37 stroke survivors (median age 60 years, 63\% male, median time since stroke 12 months) and 22 clinicians from 8 community rehabilitation settings.

Intervention: Comprehensive assessment utilising the Rev-TIG.

Outcome Measures: Survivors of stroke participated in Rev-TIG assessments, completed questionnaires about their experiences and participated in a focus group. Clinicians completed questionnaires regarding the content and utility of the Rev-TIG and it's application for individual client management.

Results: Clinicians believed the Rev-TIG was useful and focused on areas of importance to survivors of stroke; $92 \%$ would recommend it to other clinicians. Both clinicians and clients found the comprehensive review assessment worthwhile. Clinicians reported increased confidence in exploring alterations in mood and participation in valued activities with clients. Clients felt the assessment covered domains that were important to them. Areas identified for further development of the Rev-TIG included a greater focus on emotional changes after stroke and adjustment to major life changes; community access; and sexuality and intimacy.

Conclusion: The Rev-TIG is a promising tool for enhancing communitybased rehabilitation practice for survivors of stroke.

\section{Key Practice Points:}

- Comprehensive assessment of people with chronic stroke is considered worthwhile by stroke survivors and clinicians

- The Chronic Stroke Rev-TIG improved confidence of clinicians in exploring alterations of mood and participation in valued activities

\section{SPORTING PAEDIATRIC HIP}

\section{Brooksbank J2, Vladusic S ${ }^{1}$, Grimaldi A ${ }^{3}$, Mosler A}

${ }^{1}$ Senior Clinical Physiotherapist - Royal Children's Hospital Melbourne ¿Sports physiotherapist, Movement Solutions Brisbane and Gold Coast \& Associate lecturer University of Queensland

${ }^{3}$ Physiotec Physiotherapy, Brisbane \& The University of Queensland, Brisbane ${ }^{4}$ Sports Physiotherapist Aspetar Qatar Orthopaedic and Sports Medicine Hospital, Doha

The NPG and SPA present this combined group session that brings together clinical experts to share their experiences regarding the "Paediatric Sporting Hip". More attention is being focused that suggests that later adult hip pain presentations may be related to previous hip pathology and thus a complete lifespan approach to understanding early hip development and pathology is relevant to all physiotherapists. Physiotherapists working predominantly with children will also benefit from the session as it aims to address the specific needs of young dancers and athletes and the impact of hip pathology on sporting performance.

\section{Key Practice Points:}

- Neonatal Hip - overview of congenital hip dysplasia screening and treatment

- Paediatric Hip - discussion of the limping child with reference to common pathological presentations and red flags for physiotherapists

- Adolescent Hip - overview of growth related changes and common

pathological presentations in teenagers

- Hip Presentations in young Dancers and Athletes

- Impact of paediatric and adolescent Pathology in sport
WATER IMMERSION FOR ELITE ATHLETES - PRACTICAL TIPS AND A LOOK TOWARDS THE FUTURE

\section{Brophy-Williams $\mathbf{N}^{1}$ \\ ${ }^{1}$ New South Wales Institute of Sport, Sydney, Australia}

The use of hydrotherapy dates back hundreds of years, yet in today's world of sports science, there is still a great deal of research in the area as we aim to continually refine implementation of hydrotherapy protocols.This presentation will outline the underlying mechanisms of water immersion (hot, cold and contrast) and how these impact the physiology of exercise recovery. These mechanisms will be linked to the more conventional use of hydrotherapy in the sporting arena. The hot topic of periodising recovery will also be discussed. Much like the way training and nutrition can be periodised, debate has raged recently over the ideal application of water immersion. Can ice baths actually hinder our performance? When not to use water immersion, and how it may be a hindrance, will also be addressed, as well as how we can incorporate these theories into a daily training environment.

The presentation will include take home tips on how, when and why water immersion can be used effectively for your clients.

\section{Key Practice Points:}

- Improve your understanding of recovery practices of elite athletes

- Apply these learnings to the general sporting arena

- Reconsider or possibly update current hydrotherapy practices for exercise recovery

\section{A PROGRESSIVE EXERCISE PROGRAM FOLLOWING A DISTAL RADIAL FRACTURE - A RANDOMISED CONTROLLED TRIAL}

Bruder $\mathbf{A}^{1}$, Shields $\mathrm{N}^{1,2}$, Dodd $\mathrm{KJ}^{3}$, Hau R ${ }^{4}$, Taylor $\mathrm{NF}^{1,5}$

${ }^{1}$ School of Allied Health, La Trobe University, Melbourne 2Department of Allied Health, Northern Health, Melbourne ${ }^{3}$ La Trobe University, Melbourne

${ }^{4}$ Department of Orthopaedic Surgery, Northern Health, Melbourne ${ }^{5}$ Allied Health Clinical Research Office, Eastern Health, Melbourne

Question: Does a program of exercise and advice improve activity compared to advice alone following distal radial fracture?

Design: A phase II randomised controlled trial with concealed allocation, assessor blinding and intention-to-treat analysis.

Participants: Thirty-three adults (25 female, mean age 54 years) following distal radial fracture managed in a cast.

Intervention: A six-week program of progressive exercise (including home exercises) and structured advice implemented over three consultations by a physiotherapist compared to a program of structured advice only delivered by a physiotherapist over three consultations.

Outcome Measures: The primary outcome was upper limb activity limitations assessed by the Patient Rated Wrist Evaluation and QuickDASH. Secondary outcomes were wrist range of movement, grip strength and pain. Outcomes were measured at baseline, after the program (week 7) and at 3 month follow-up (week 24)

Results: There were no significant between-group differences or effects favouring the intervention group for any outcome at any time-point. Significant within-group effects that exceeded the minimal clinically important difference were found in both groups for the primary outcome of upper limb activity at both time-points.

Conclusion: The prescription of exercise in addition to a structured advice program during rehabilitation may convey no extra benefit following distal radial fracture managed in a cast.

Trial registration: ACTRN12612000118808

\section{Key Practice Points:}

- The prescription of exercise in addition to a structured advice program over three physiotherapy consultations may convey no extra benefit following distal radial fracture managed in a cast.

- Advice may be a useful intervention to restore upper limb activity after distal radius fracture. 
HOSPITAL INPATIENT VERSUS HOME-

BASED REHABILITATION AFTER

KNEE ARTHROPLASTY (THE HIHO

STUDY): PRELIMINARY RESULTS OF A

RANDOMISED CONTROLLED TRIAL

Buhagiar $M A^{1,2}$, Naylor JM $M^{2,3,4}$, Harris $I A^{2,3,4}$, Xuan $W^{2,3}$, Kohler $F^{1,2}$, Wright RJ ${ }^{5}$, Fortunato $\mathrm{R}^{6}$

${ }^{1}$ HammondCare, Sydney

2South West Sydney Clinical School, UNSW, Sydney

3Ingham Institute of Applied Medical Research, Sydney

${ }^{4}$ Whitlam Orthopaedic Research Centre, Liverpool Hospital, Sydney

${ }^{5}$ Fairfield Hospital, Sydney

${ }^{6}$ Campbelltown Hospital, Sydney

Question: Does inpatient rehabilitation followed by a hybrid home program provide superior recovery of functional mobility compared to a hybrid home program alone following total knee arthroplasty?

Design: Two-arm parallel randomised controlled trial.

Participants: 165 consenting participants who had undergone a primary total knee arthroplasty across two centres.

Intervention: Once cleared for discharge from acute care, participants were randomly allocated to either 10 days of inpatient rehabilitation followed by usual care (a 6-week hybrid home program) or usual care.

Outcome Measures: Primary outcome - 6 -minute walk test at 26 weeks. Secondary outcomes - 15-metre walk test, Oxford Knee Score and EQ-5D.

Results: Preliminary results were analysed at 10 and 26 weeks after surgery for 150 participants. No significant differences between the groups were shown, even when allowing for covariates. Between groups, the mean difference (with a 95\% confidence interval) for the measured outcomes were: 6 -minute walk test: $0.4 \mathrm{~m}$ (-34 to 35); 15 -metre walk test: $0.3 \mathrm{sec}$ (-1.1 to 1.7); Oxford Knee Score: 2.2 (-0.7 to 5.1); and EQ-5D visual analogue scale: 2.1 (-8.2 to 4.0).

Conclusion: Inpatient rehabilitation does not provide participants with a superior level of recovery across a range of outcomes following total knee arthroplasty when compared to a hybrid home program.

Trial registration: NCT01583153

\section{Key Practice Points:}

- Inpatient rehabilitation doesn't provide improved outcomes for patients following total knee arthroplasty.

- Other benefits, unrelated to these outcomes, may exist and require further investigation.

- Current results apply only to those who, prior to surgery, were deemed unlikely to require inpatient rehabilitation.
"GAINING CONTROL" A PROSPECTIVE MIXED-METHODS STUDY EXPLORING THE FACTORS ASSOCIATED WITH IMPROVEMENTS IN FEAR IN PEOPLE WITH CHRONIC BACK PAIN

Bunzli S' ${ }^{1}$, Smith $A^{1}$, Schütze R², O'Sullivan $\mathrm{P}^{1}$

${ }^{1}$ School of Physiotherapy and Exercise Science, Curtin University, Perth ¿School of Psychology and Speech Pathology, Curtin University, Perth

Question: Fear-reduction interventions targeting pain-catastrophising have reported modest effects. To better understand the mechanisms involved in fear-reduction, we asked: What factors are associated with improvements in fear in people with chronic back pain?

Design: A prospective mixed-methods study

Intervention: Non-interventional

Participants: 31 individuals with chronic back pain and high fear (>40/68 Tampa), recruited from musculoskeletal clinics.

Outcome Measures: Semi-structured interviews and self-report questionnaires at baseline and four months. At baseline, participants reported two main beliefs underlying fear. At follow-up, change in fear and factors associated with improvement in fear were identified.

Results: At follow-up, 18 (58\%) participants described improvements in fear. Regardless of beliefs at baseline, the overarching theme amongst improvers was 'gaining control' over the pain experience, achieved through improvements in pain predictability, controllability, intensity, and reduced goal conflict. For improvers with damage beliefs at baseline, gaining control was also achieved through a new conceptual understanding of pain coupled with targeted pain-control. Triangulation of qualitative with quantitative data confirmed statistically significant differences on changes in pain control $(p<0.001)$, self-efficacy $(p=0.029)$, intensity $(p=0.03)$ and back beliefs ( $p=0.004)$ between improvers and non-improvers.

Conclusion: This study suggests different pathways to fear-reduction that may be facilitated by clinicians considering the beliefs underlying fear and providing individualised management to improve pain control.

\section{Key Practice Points:}

- Pain-related fear is modifiable over a four-month period

- Individuals who gain control over the pain experience may experience fear-reduction.

- Improving pain predictability, controllability, intensity and back beliefs may assist in gaining control over the pain experience.

\section{HOW TO UNDERSTAND AND TREAT PAIN-RELATED FEAR USING A ‘COMMON- SENSE' FRAMEWORK}

Bunzli S ${ }^{1}$, Smith $A^{1}$, Schütze $R^{2}$, O'Sullivan $P^{1}$

${ }^{1}$ School of Physiotherapy and Exercise Science, Curtin University, Perth 2School of Psychology and Speech Pathology, Curtin University, Perth

Background: Interventions based on the Fear Avoidance Model that target catastrophising as a catalyst of the fear-avoidance-disability cycle have yielded modest effects. Our qualitative research into chronic back pain and high fear highlights limitations in this model and the need to account for heterogeneous beliefs underlying fear; contextual factors that influence beliefs; and the multiple pathways to fear-reduction.

objectives: To present an alternative framework to understand and treat fear: the Common-Sense Model.

Approach: Three case studies drawn from our research illustrate how the process underlying the development, maintenance and reduction in fear will be similar for all people despite variance in individual experience. Individuals make sense of pain by forming a'representation' based on their beliefs about the label, causes, consequences, time-line and controllability of symptoms at any given time. The representation guides problem-solving behaviour. Fear may be a 'common-sense' response to a threatening representation. If pain continues to be experienced as unpredictable, uncontrollable and intense despite problem-solving behaviour, the representation will no longer be useful in guiding sensible decisions. This inability to make sense of pain may perpetuate fear. Reconceptualising the meaning of pain and developing effective pain control strategies may assist individuals to make sense of pain and facilitate fear-reduction.

\section{Key Practice Points:}

Participants will learn:

- The influence clinicians can have in the formation of threatening pain representations.

- How to reconceptualise pain so it makes sense to individuals

- How to equip individuals with pain control strategies by improving pain predictability, controllability and intensity 


\section{THE IMPACT OF CLASSIFICATION OF PHYSICAL ACTIVITY IN CHRONIC OBSTRUCTIVE PULMONARY DISEASE ON RESULTS FROM PHYSICAL ACTIVITY MONITORING}

Burge $\mathrm{AT}^{1,2}$, Hill C] ${ }^{3,4}$, Lee $\mathrm{AL}^{1,3}$, Moore $\mathrm{R}^{4}$, Nicolson $\mathrm{C}^{2}$, Cox NS , Lahham $A^{2}$, Ndongo $R^{4}$, McDonald $C^{3}$, Mahal $A^{5}$, Holland $A E^{1,2,3}$

${ }^{1}$ Physiotherapy, The Alfred, Prahran

2Physiotherapy, La Trobe University, Bundoora

IInstitute for Breathing and Sleep, Austin Health, Heidelberg

${ }^{4}$ Physiotherapy, Austin Health, Heidelberg

${ }^{5}$ Department of Epidemiology and Preventative Medicine, Monash University, Prahran

Question: What is the effect of different physical activity classifications in people with chronic obstructive pulmonary disease on time spent in physical activity, according to public health recommendations?

Design: Prospective observational study.

Participants: Sixty six people with chronic obstructive pulmonary disease. Intervention: Physical activity monitoring using Sensewear armband.

Outcome Measures: Participants were assessed for disease severity, dyspnoea and obesity. Results were analysed using steps, physical activity level, time (total/day, accumulated in bouts $\geq 10 \mathrm{~min}$ ) spent in physical activity according to intensity classified as $\geq 1.5 \mathrm{METs}, \geq 3 \mathrm{METs}$ or relative to age (45-64 years $\geq 4$ METs, 65-79 years $\geq 3.2 \mathrm{METs}, \geq 80$ years $\geq 2$ METs). Results: Decreased steps ( $p<0.001$ ) and physical activity time (bouts) $\geq 3$ METs ( $p=0.025$ ) were seen with increasing dyspnoea. Decreased time was observed with increasing obesity using classifications of age $(p<0.001)$ and $\geq 3$ METs ( $p=0.009$ ). The proportion of participants who achieved recommended physical activity levels varied according to the classification system used from 20\% (age) to 90\% (21.5 METs).

Conclusion: In people with COPD, a relationship between dyspnoea and obesity with physical activity was observed, but not disease severity. Physical activity monitoring results are influenced by the method of analysis.

\section{Key Practice Points:}

- There are a wide range of methods used for defining physical activity.

- Dyspnoea and obesity had an inverse relationship with physical activity, but this was not observed with disease severity.

- The classification method has a profound impact on the proportion of people who are considered physically active.

\section{GENERAL PRACTITIONERS' VIEWS ABOUT AN ORTHOPAEDIC CLINICAL ASSESSMENT SERVICE}

\section{Burn DE ${ }^{1}$, May $S^{2}$, Edwards L ${ }^{3}$}

${ }^{1}$ Walsall Healthcare NHS Trust, Walsall Manor Hospital, Trauma Orthopaedic and Musculoskeletal Services, Moat Road, Walsall, United Kingdom, WS29PS ${ }^{2}$ Faculty of Health and Wellbeing, Sheffield Hallam University, Sheffield, UK ${ }^{3}$ ATOS Healthcare, Nottingham, UK

Questions: What are general practitioners' views about an orthopaedic clinical assessment service run by extended scope physiotherapists? How do they believe the service could be improved? How do they feel about services providing care for patients suffering from orthopaedic conditions?

Design: Prospective cohort study with purposive sampling

Participants: Fifteen consenting general practitioners volunteered from across Walsall. Fourteen separate GP practices were represented.

Intervention: General practitioners were interviewed. The interviews were recorded, transcribed and analysed for emergent themes. Interviews were analysed sequentially by date performed until saturation. Themes were discussed, and disagreements evaluated until agreement was found between the two main authors. The third author then analysed randomly selected interview transcripts.

Outcome Measures: Emergent themes were identified through analysis of the interview transcripts.

Results: The study found general practitioners refer to specialist services, including assessment services, because of wait times, locality, patient experience, general practitioner experience and knowledge of available services.

Conclusion: General practitioners saw the orthopaedic clinical assessment service as another referral choice for patients suffering orthopaedic pathologies. They identified some difficulty in understanding the different services including the various professional roles involved. To assist with their understanding, they described requesting advertising for the different services and the clinicians involved or streamlining of services by provider services. Further detailed research addressing the limitations in this research design is indicated to investigate general practitioners' thoughts and behaviours in relation to referral patterns as general practitioners took on commissioning for services from April 2013.

\section{Key Practice Points:}

- General practitioners identified difficulty understanding different services and various professional roles involved

- General practitioners requested advertising of services and the clinicians involved or streamlining of services by providers

- Further studies are required addressing the limitations of this study. These studies should investigate thoughts and behaviours of general practitioners in relation to decision making processes for referrals with patients suffering orthopaedic conditions

\section{ORTHOPAEDIC TRIAGE: COST EFFECTIVENESS, DIAGNOSTIC/SURGICAL AND MANAGEMENT RATES}

Burn DE¹, Beeson E

Walsall Healthcare NHS Trust, Walsall Manor Hospital, Trauma Orthopaedic and Musculoskeletal Services, Moat Road, Walsall, United Kingdom, WS29PS

Questions: Does triage of orthopaedic referrals by extended scope practitioners cost less? How many patients following triage assessment required diagnostics? Of those patients referred to orthopaedics following triage assessment, what percentage were appropriate and required surgical intervention?

Design: Prospective cohort study with purposive sampling

Participants: 273 patients from 1395 referred by general practitioners on paper to or thopaedics were triaged for assessment. The remaining patients continued the normal pathway.

Intervention: The experimental group consisted of patients triaged for assessment by extended scope practitioner (triage assessment). Patients were assessed and results collected. The remaining patients formed the control group and continued normal route for assessment by orthopaedics.

Outcome Measures: Costing was calculated for the total of the patient pathway, utilising unit cost per appointment and then compared. Outcomes of paper triage and triage assessment were collected. Outcomes of assessment were also collected for patients referred to orthopaedics following triage assessment.

Results: The new pathway demonstrated a $27.3 \%$ cost saving versus normal route. Following triage assessment, 13.9\% patients required an orthopaedic assessment. Of those patients requiring an orthopaedic assessment, $75 \%$ required surgery (compared to $57 \%$ for normal route) and $80.5 \%$ of referrals were appropriate.

Conclusion: This is the first known study investigating cost effectiveness across a patient pathway of orthopaedic triage. The results showed improved cost-effectiveness and improved surgical percentage rates in comparison to the normal pathway. Larger studies are required to determine cost and clinical effectiveness of utilising extended scope practitioners for orthopaedic triage.

\section{Key Practice Points:}

- Orthopaedic triage by an extended scope practitioner is cost effective

- Orthopaedic triage increases the percentage of patients requiring surgical intervention

- Further high quality studies with larger numbers of patients and clinicians is indicated to investigate this emerging area of physiotherapy practice 


\section{THE EXPERIENCE OF LIVING WITH CHRONIC NEUROPATHIC PAIN IN SPINAL CORD INJURY}

Buscemi V ${ }^{1}$, Cassidy $\mathrm{E}^{2}$

IUniversity of Western Sydney, School of Science and Health, Locked bag 1797 Penrith, NSW 2751

Brunel University London, Kingston Lane, Uxbridge, Middlesex UB8 3PH, United Kingdom

Question: How do people with chronic neuropathic pain after spinal cord injury $(\mathrm{SCl})$ view and manage their pain and what do they need to better cope with it?

Design: Focus groups and individual interviews for participants uncomfortable in group setting.

Participants: Twenty-five participants were contacted from an Italian Spinal Cord Unit. Nine outpatients agreed, five declined and one person was excluded as their chronic neuropathic pain was not due to SCl.

Outcome Measures: A topic guide was characterized by four open-ended questions exploring the following questions: how does chronic neuropathic pain influence life? What are an individual's beliefs about pain? How do people manage their pain and what do they need to better cope with it?

Results: Two themes emerged. The first theme described how chronic neuropathic pain negatively impacted on an individual's physical and psychological health and how this in turn, negatively influenced the intensity of pain. The second theme summarized how individuals developed coping strategies and knowledge into their condition independently, and through an unsystematic process of trial and error.

Conclusion: The findings of this study increase awareness of the chronic neuropathic pain experience after $\mathrm{SCl}$ and should help to stimulate local health communities to improve practice.

\section{Key Practice Points:}

- Pain intensity, physical and psychological factors are different faces of the same coin.

- There is a need to provide individuals with appropriate resources to develop knowledge regarding their condition.

- Future studies should seek to identify the most effective method to disseminate information to this population.

\section{STUDENT SELF-EVALUATION OF CLINICAL REASONING AND DECISION-MAKING SKILLS WITHIN A PHYSIOTHERAPY CASE-BASED LEARNING UNIT}

Butler $]^{1}$, Dwyer $\mathrm{G}^{2}$, Hough J ${ }^{1}$, Danks $\mathrm{M}^{1}$

${ }^{1}$ Australian Catholic University, Sydney

2The University of Western Sydney

Question: What are the perceptions of undergraduate students about their paediatric physiotherapy clinical reasoning and decision-making skills during completion of a case-based unit?

Design: Prospective cohort survey. Participants completed a questionnaire at four time periods (commencement of the unit and after each of three cases). Participants rated their abilities on nine areas associated with clinical reasoning and decision-making using a 5-point Likert scale.

Participants: One hundred and twenty seven students enrolled in the unit (92\% response rate).

Results: Initially, percent agreement in students' capacity ranged from $21.9 \%$ (ability to critique and synthesise new information) to $53.6 \%$ (ability to recognise key information). Subsequently there was significant improvement in rating of skills. Rating increased between $53.5 \%$ to $89.6 \%$ (Case Study 1), 67.1\% to 89.6\% (Case Study 2) and $71.2 \%$ to $84.8 \%$ (Case Study 3). Improvement in clinical reasoning rating was greatest between the commencement of the unit and completion of the first case study ( $p<0.0001)$. There was no further significant change in most areas except in ability to identify learning needs $(p=0.038)$, identify and utilise effective learning strategies ( $p=0.038)$, and to critique and synthesise new information ( $p=0.016)$.

Conclusion: Learning in this format was successful in developing students clinical reasoning and decision-making capacities. Change was most significant after their first experience of a case. The process areas of learning and decision-making took longer for students to feel they were achieving desired outcomes.

\section{Key Practice Points:}

- Students take longer to be confident in the process of knowledge acquisition/information gathering compared with learning knowledge content.

\section{PHYSICAL ACTIVITY AND CHILDHOOD ACADEMIC ACHIEVEMENT: A CRITICAL REVIEW}

Cacciotti $\mathrm{K}^{1}$, Milne $\mathrm{N}^{1}$, Orr $\mathrm{R}^{1}$

${ }^{1}$ Faculty of Health Sciences and Medicine, Institute of Health and Sport, Bond University, Gold Coast, Australia

Question: What effect does physical activity have on academic achievement?

Design: Critical review of the literature on physical activity and childhood academic achievement from 1990 to September 2014.

Participants: School aged children between 6-12 years of age.

Intervention: Physical activity was defined as any bodily movement produced by skeletal muscles that require energy expenditure with exercise considered as a subset of physical activity.

Outcome Measures: Academic outcome measures included standardized tests (e.g. Wechsler Individual Achievement Test, The Standard Progressive Matrices, The Palmetto Achievement Challenge Tests,), grades from academic classes (e.g. mathematics, science, English/language) and psychometric cognitive tests.

Results: Of 170 potential articles, 10 studies with generally high methodological quality (mean Downs and Black score of 74\%: range 64\% to $82 \%$ : Krippendorff's alpha=0.86) were evaluated. All 10 studies utilised a diverse variety of physical interventions, with each modality resulting in positive impacts towards children's academic achievements.

Conclusion: Physical activity, delivered in different physical modalities, both during and out of school hours had a favourable effect on academic achievement and cognitive functioning and performance.

\section{Key Practice Points:}

- Increasing physical activity in the school environment can provide positive effects on academic achievement and cognitive functioning in school-aged children.

- Investigating the links between physical activity and academic achievement may help provide evidence for inclusion of increased physical activity time in schools.

- Further exploration on dose and specificity of physical activity to provide optimal cognitive and academic achievement outcomes for school-aged children is warranted.

\section{TRAUMA-FOCUSED COGNITIVE BEHAVIOURAL THERAPY (CBT) AND PHYSIOTHERAPY FOR CHRONIC WHIPLASH: STUDY PROTOCOL FOR A RANDOMISED CONTROLLED TRIAL}

Sterling $\mathrm{M}^{1}$, McGregor $\mathrm{L}^{1}$, Campbell L $\mathrm{L}^{1}$, Kenardy ${ }^{2}$, Andersen $\mathrm{T}^{3}$ ${ }^{1}$ CRE Road Traffic Injury, CONROD, Menzies Health Institute Queensland, Griffith University

${ }^{2}$ CONROD Research Injury Centre, The University of Qld

3University of Southern Denmark, Odense, Denmark

Question: Is trauma-focused CBT (delivered by a clinical psychologist) and physiotherapy effective for people with chronic whiplash associated disorders (WAD).

Design: Randomised controlled trial with concealed allocation, assessor blinding and intention-to-treat analysis

Participants: 110 participants (18 and 70 years) with chronic WAD (> 3 months < 5 years duration), reporting at least moderate pain and disability and a diagnosis of posttraumatic stress disorder (PTSD).

Intervention: Trauma-focussed CBT (delivered by a clinical psychologist) delivered once a week over 10 weeks followed by clinical guideline adherent physiotherapy delivered over a 6 week period. The control intervention will supportive therapy (delivered by a clinical psychologist) followed by the same 6 week physiotherapy program.

Outcome Measures: The primary outcome measure is the Neck Disability Index with secondary outcomes of pain intensity, presence of PTSD, perceived recovery, pain specific self-efficacy and catastrophizing. Outcomes will be measured at 10 and 16 weeks, 6 and 12 months. Analysis will be blinded and by intention to treat.

Conclusion: This study will provide a definitive evaluation of the effects of adding trauma-focused CBT to physiotherapy for individuals with chronic WAD and will have immediate clinical applicability. Trial Registration: The trial has been submitted to ANZCTR for prospective registration. Funding: NHMRC Project Grant 1059310

\section{Key Practice Points:}

- Participants will be made aware of new developments in the management of chronic whiplash that are currently undergoing evaluation.

- Participants will learn of the complexities of chronic whiplash and how inter-disciplinary treatment approaches may be required. 
COGNITIVE FUNCTIONAL THERAPY PROMOTES CHANGE IN HIGHLY FEARFUL PATIENT WITH PERSISTENT LOW BACK PAIN

Caneiro JP1, 2, Smith $\mathrm{A}^{1}$, Rabey $\mathrm{M}^{1}$, O'Sullivan $\mathrm{P}^{1,2}$

${ }^{1}$ School of Physiotherapy and Curtin Health Innovation Research Institute, Curtin University, Perth

${ }^{2}$ Body Logic Physiotherapy, Perth

Questions: Is a Cognitive Functional Therapy intervention able to promote change in a patient with persistent low back pain (PLBP) and high painrelated fear? What are the qualitative factors underlying this process of change?

Design: a case report. Intervention was delivered over six sessions in a three-month period. 6 months follow up.

Participant: male with a twenty-five years history of LBP with a belief that his spine is degenerating and pain signifies damage and further suffering.

Intervention: Cognitive Functional Therapy targeting maladaptive cognitive, functional and lifestyle behaviours considered to promote pain and disability, delivered in an integrated manner.

Outcome Measures: Self-report appraisals of bending in relation to fear, pain expectancy and experience during bending scored on a Numeric Rating Scale (NRS), Orebro Musculoskeletal Screening (OMPSQ), TAMPA Kinesiophobia Scale. A six-month follow up interview was conducted to identify the patients' individual experience during the intervention, and perception of barriers and facilitators for change.

Results: reduction in self report appraisals of fear, pain expectancy and pain during bending, culiminating in increased activity participation and restoration of daily activities. A six months follow up demonstrated substantial changes in pain-related fear (TSK: pre - 47, post - 31) and multidimensional risk profile (OMPQ: pre - 61, post - 46). The interview revealed the patient has accepted a biopsychosocial understanding of pain and acquired self-management skills. The interview also revealled that the facilitators for change were: a trusting relationship with the threapist, dethreatening of radiology and positive experiences in a new way of moving.

Conclusion: This report outlines the journey of a patient and his process to change and achievement of independence. The CFT intervention was successful in reducing pain-related fear and disability. Therapeutic alliance, education delivered in relation to the person's story, and development of pain control via new movement strategies were facilitators of this process.

\section{ACTIVITY BASED WORK - IS THIS A SUSTAINABLE ERGONOMIC SOLUTION TO OFFICE WORK?}

\section{Caple $\mathrm{D}^{1}$, McAtamney $\mathrm{L}^{2}$}

${ }^{1}$ David Caple \& Associates Pty Ltd, Melbourne

${ }^{2}$ Atune Health Centres Pty Ltd, Newcastle

Activity Based Work (ABW) has been introduced across Australian corporate, and government offices to improve the efficiency of nett rentable floor space, and to provide a more dynamic work environment. It moves away from an entitlement based space allocation with fixed workstations to a building based approach offering a variety of working environments to suit the range of activities required. Regardless of the architectural design and layout of the work areas, the building still provides a work place for the managers and staff to achieve their respective work outcomes. The challenge in a sustainable ergonomics solution, is to ensure that the physical, cognitive and psychosocial needs of the occupants of this building can be optimised. Through the input into the design process, and subsequent evaluation of the ABW work areas we are now in a better position to understand the holistic nature of this changing work environment and the impact that it has on ergonomics risk factors. The key requirements to enable the ABW to be introduced include suitable mobile technology; Wi-Fi reliability; digitisation of records; flexible telecommunication systems; and organisational structures that enable this system to be successful. It is evident that the most of the major office projects across Australia are now using the ABW approach. The OHS professionals and researchers need to assess the impact of this system of work to ensure the needs of the staff health and safety are maintained.
DOES HYDROTHERAPY IMPROVE

A PATIENT'S SENSE OF MENTAL

WELLBEING TO A GREATER EXTENT

THAN PHYSIOTHERAPY PRESCRIBED

HOME EXERCISE PROGRAMS ALONE? A PILOT STUDY

Carere $\mathrm{A}^{1}$, Warburton $\mathrm{M}^{2}$, Orr RM ${ }^{1}$

${ }^{1}$ Bond University, Gold Coast

2Gateway Physiotherapy, Brisbane

Question: Does hydrotherapy improve the mental wellbeing of individuals with chronic conditions to a greater extent than a physiotherapy prescribed home exercise program alone?

Design: Randomised crossover pilot study

Intervention: During the intervention period hydrotherapy sessions were conducted for 45 minutes, twice weekly for four weeks (mean water temperature $\left.32.7 \pm 1.1^{\circ} \mathrm{C}\right)$.

Outcome Measures: Warwick-Edinburgh Well-being Scale (WEWBS) and Short Form 36 (SF36) self-administered questionnaires.

Results: Ten participants (female $n=9$ : male $n=1$ : mean age $=70 \pm 8.85$ years) were included in this study. Following a paired t-test used to assess the relative intervention effect, significant improvements $(p=0.034)$ in mental wellbeing, as measured by the WEWBS, were found following the hydrotherapy intervention period. SF36-Physical Capacity Scores approached, but did not achieve significance $(p=0.061)$ while the SF36 Mental Capacity Scores did not reach statistical significance $(p=0.538)$ following the hydrotherapy intervention. No significant difference in change in outcome measures was detected when sequencing effects were examined using independent t-tests following the cross over $(p=0.153$ to 0.793$)$.

Conclusion: Hydrotherapy may enhance mental wellbeing and health as well as physical health to a greater extent than a physiotherapy prescribed home exercise program alone for individuals with a chronic condition. However this was not consistent among mental wellbeing and health scales.

\section{Key Practice Points:}

- Hydrotherapy is a viable treatment to improve a sense of wellbeing in patients with chronic conditions.

- Objective outcome results may vary depending on the tool used.

- Hydrotherapy may also provide ancillary improvements in physical capacity as measure by the SF 36 in these patients.

\section{HOW TO ADAPT PHYSIOTHERAPY PRACTICE TO OPTIMISE CULTURALLY SENSITIVE, PATIENT-CENTRED CARE}

\section{Carroll HG', Wall KA², Pritchard SA ${ }^{3}$}

${ }^{1}$ Alice Springs Hospital, Northern Territory Government, Department of Health ${ }^{2}$ Alice Springs Hospital, Northern Territory Government, Department of Health 3Monash University, Melbourne

Background: Central Australia is a region diverse by language and cultural traditions. Building upon evidence-based foundations, physiotherapy practice requires creativity and flexibility to connect with culture and facilitate consumer engagement in health services.

Aims/Objectives: To develop an understanding of the relationships between culture and health with consideration of equitable access to best practice service provision, and to explore approaches to physiotherapy that are culturally sensitive and patient centred.

Approach: The workshop will facilitate discussion of the flexibility and creativity required in practice, to support clients to actively engage through individualised plans for health and wellbeing. Experiences of physiotherapists working in Central Australia will be shared and discussed with recognition of local Aboriginal culture, exploring the need for health professionals to connect with cultural diversity in practice. A reflection task will encourage participants to consider their own background, traditions and priorities, and how these factors contribute to perception of health and engagement in services. Participants will be presented with approaches to culturally sensitive physiotherapy and guided through the continuum from critical care to community practice, with learning materials provided to address clinical examples.

\section{Conclusion/Key Practice Points:}

Participants will be provided with a greater understanding of culturally sensitive and patient centred practice, with skills transferrable to their own clinical environments. The session will aim to optimise engagement and provision of services to patients of diverse backgrounds, specifically in:

- Culturally sensitive assessment and treatment;

- Individualised goal setting;

- Shared education between the physiotherapist and client; and,

- Responsive discharge planning. 


\section{THE PRAGMATIC IMPLEMENTATION OF A 6-DAY PHYSIOTHERAPY SERVICE IN REHABILITATION}

Caruana $\mathrm{EL}^{1,2}$, Kuys $^{3}$, Clarke ${ }^{3}$, Brauer SG ${ }^{1}$

${ }^{1}$ The University of Queensland, School of Health and Rehabilitation Sciences, Brisbane, Australia

2St Andrew's War Memorial Hospital, Brisbane, Australia

${ }^{3}$ Australian Catholic University, Brisbane, Australia

Question: Is a 6-day rehabilitation physiotherapy service effective in reducing length of stay and improving outcomes?

Design: Prospective pragmatic cohort study with historical comparison

Participants: 536 patients admitted to a rehabilitation unit over a twoyear period: 2011 (intervention) and 2010 (historical control)

Intervention: A four-hour Saturday physiotherapy service was provided to intervention participants meeting eligibility criteria of: admitted Thursday/ Friday; making daily progress or likely to deteriorate if not seen; admitted for $\leq$ one week; requiring a maximum of one-assist to mobilise. Other patients received a 5-day physiotherapy service.

Outcome Measures: The primary outcome measure was rehabilitation length of stay. Secondary measures included clinical gait and balance measures.

Results: Patients in the two cohorts did not differ in age and gender. In 2011,162 participants received 6 -day physiotherapy (60\%), attending on average 1.67 (SD1.56) extra physiotherapy sessions during their admission. There was a trend for patients in 2011 to have a shorter length of stay (1.7 days, $95 \% \mathrm{Cl}-0.532$ to 3.920 ) compared to 2010. Patients in 2011 had better gait and balance on discharge compared to the 2010 cohort (Timed Up and Go mean difference 3.34s, p=0.036, 95\% 0.77 to 5.89; static stance time mean difference $1.83 \mathrm{~s}, \mathrm{p}=0.004,95 \% \mathrm{Cl}-3.93$ to 0.27 ).

Conclusion: Provision of a 6 -day physiotherapy service in a realworld rehabilitation setting has resulted in reduced length of stay and improvements in discharge gait and balance. This could lead to cost-savings for hospitals and improve flow through of patients.

\section{Key Practice Points:}

- Introduction of a 6-day physiotherapy service in a rehabilitation facility for patients meeting pragmatic eligibility criteria has resulted in reduced length of stay, and improvements in gait and balance measures on discharge.

- Implementation of this half-day service yields similar results to those in previous studies of a full-day service.

- These results provide evidence that may facilitate others to implement a 6-day rehabilitation service.

\section{LOWER LIMB REHABILITATION IN TEAM ATHLETES}

\section{Charlton P}

Australian Institute of Sport

This session will discuss high level lower limb rehabilitation with strength and conditioning principles using physiotherapy clinical reasoning. Specifically, the session will review the principles of strength and power program design with respect to athlete rehabilitation and will discuss bridging the gap between the clinic and successful return to play. The session will also encompass minimising risk of re-injury once an athlete has returned to training and competition and the importance of maintaining lower limb load during injury rehabilitation.

\section{Key Practice Points:}

- Parameters of rehabilitation program design

- Clinical reasoning regarding exercise prescription for returning to contact/collision and impact sports

- Matching exercise prescription with demands of the sport

- Progression of exercise and rehabilitation beyond theraband

\section{THE USE OF INERTIAL SENSORS TO MANAGE LOAD}

\section{Charlton P}

Australian Institute of Sport

This session will introduce commercially available inertial sensors and discuss a cost/benefit analysis of various systems and their use in a variety of sports.

\section{Key Practice Points:}

- Implementation of inertial sensors in elite spor

- Integration of systems into athlete training environments

- Data collection using inertial systems

- Interpretation of results with respect to evidence

- Towards best practice planning and periodization using inertial systems

\section{RELIABILITY AND VALIDITY OF A \\ SMARTPHONE, BUBBLE INCLINOMETER \\ AND MOTION ANALYSIS SYSTEM FOR MEASUREMENT OF HIP JOINT RANGE OF MOTION}

Charlton PC ${ }^{1}$, Mentiplay BF², Pua Y-H ${ }^{3}$, Clark RA²

${ }^{1}$ Australian Institute of Sport, Canberra

2Australian Catholic University, Melbourne

${ }^{3}$ Singapore General Hospital, Singapore

Question: Is a customised Smartphone application a reliable and valid tool for measuring hip joint range of motion?

Design: Intra-tester reliability with concurrent validity

Participants: 20 young, healthy males (mean \pm SD age: $23 \pm 4.6$ years, height: $179.0 \pm 7.4 \mathrm{~cm}$, mass: $78.9 \pm 11.4 \mathrm{~kg}$ )

Outcome Measures: Passive hip joint range of motion was recorded for seven movement directions on two occasions. Data from a Smartphone, inclinometer and a three dimensional motion analysis system (degrees) were collected simultaneously. Intraclass correlation coefficients, coefficients of variation and standard error of measurement were used to assess reliability. To assess validity, fixed and proportional biases were additionally used.

Results: The smartphone demonstrated good to excellent reliability (ICCS >0.75) for four out of the seven movements and moderate to good reliability (ICC $=0.63-0.68$ ) for the remaining three. The Smartphone application demonstrated excellent validity when compared to the three dimensional motion analysis system for all movements (ICCS >0.88) except one, which displayed moderate to good validity $(\mathrm{ICC}=0.71)$.

Conclusion: Smartphones are portable and widely available tools that are mostly reliable and valid for assessing passive hip joint range of motion with potential for large-scale use when a bubble inclinometer is not available. However, caution must be taken in its implementation as some movement axes demonstrated only moderate reliability.

\section{Key Practice Points:}

- Smartphones are portable and widely available and may be used to reliably measure and monitor some aspects of hip joint range of motion.

- A Smartphone application has potential for large-scale screening in clinical and sporting settings. 


\section{WORKPLACE-BASED INTERVENTIONS FOR NECK PAIN IN OFFICE WORKERS: A SYSTEMATIC REVIEW AND META-ANALYSIS OF RANDOMISED CONTROLLED TRIALS}

Chen $\mathrm{X}^{1}$, Coombes BK ${ }^{2}$, Sjøgaard $\mathrm{G}^{3}$, Jun $\mathrm{D}^{1}$, O'Leary $\mathrm{S}^{1,4}$, Johnston $\mathrm{V}^{1}$ 'School of Health and Rehabilitation Sciences, The University of Queensland, Brisbane

¿School of Biomedical Sciences, The University of Queensland, Brisbane ${ }^{3}$ Department of Sports Science and Clinical Biomechanics, University of Southern Denmark, Denmark

${ }^{4}$ Physiotherapy Department, Royal Brisbane and Women's Hospital, Brisbane Question: What is the effectiveness of workplace-based interventions on the prevention and reduction of neck pain in office workers?

Design: Systematic review with meta-analysis of randomised controlled trials (RCTs)

Participants: Computer-based office workers

Intervention: Workplace-based interventions

Outcome Measures: Intensity or incidence of neck pain

Results: Twenty-six RCTs (5016 participants) of high methodological quality were included. Meta-analysis found strong evidence for a minimum of ten weeks neck/shoulder strengthening exercises in office workers with neck pain (cases) (SMD 0.59, 95\% Cl 0.29 to 0.89), and strong evidence for lack of effect in the general office worker population (general population) The greatest benefits were found for studies with the highest adherence to exercise. There was also strong evidence for general fitness training in cases (SMD 0.43,95\% Cl 0.08 to 0.78). There was strong evidence for the lack of effect of multi-component ergonomic interventions in the general population, but limited evidence of benefit in cases. Moderate to strong evidence was found for a lack of effectiveness of group education and myofeedback and limited evidence to support work-break interventions.

Conclusion: Workplace-based exercise interventions were effective in reducing neck pain in office workers. Benefits were limited to cases and were larger when interventions were targeted to the neck/shoulder. Adherence was an important factor influencing the effectiveness of exercise.

\section{Key Practice Points}

- There is strong evidence for workplace-based neck/shoulder strengthening and general fitness exercises for reducing neck pain in office workers

- There is strong evidence for the lack of effect of ergonomic interventions in the general office worker population and limited evidence in neck cases.

\section{PRACTICE BEHAVIORS, BUT NOT PATIENT OUTCOMES, CHANGE AFTER A LONGITUDINAL PROFESSIONAL DEVELOPMENT ACTIVITY}

Chipchase $\mathrm{L}^{1,2}$, Cavaleri $\mathrm{R}^{1}$, Jull $\mathrm{G}^{2}$

'School of Science and Health, University of Western Sydney, Sydney 2Division of Physiotherapy, School of Health and Rehabilitation Sciences, The University of Queensland, Brisbane

Question: Does a traditional workshop with a follow-up educational meeting change practice behaviour and patient outcomes compared to a traditional workshop alone?

Design: A single blind pre-test post-test randomised controlled trial.

Participants: Twenty-three physiotherapists with a musculoskeletal caseload.

Intervention: All participants undertook the two-day workshop with the intervention group completing an additional five-hour follow-up session a month later.

Outcome Measures: Practice behaviour was measured with a semistructured questionnaire. Clinical outcomes were assessed with the Neck Disability Index (NDI). Participants administered the NDI to 10 successive neck pain patients on the first and at fourth treatment occasion before and after the educational intervention/s.

Results: All participants reported improved confidence in a range of examination and management techniques $(p<0.05)$ but only motor relearning for the cervical flexors and exercises for cervical kinaesthetic sense were used more often after the workshop by the intervention group $(p<0.05)$. Patients of both groups demonstrated a reduction in NDI scores (seven points on 50 point scale) after three treatments, however there was no difference between groups in patients' NDI scores before and after the educational interventions ( $p=0.11$ ).

Conclusion: A weekend workshop with a five-hour follow-up offering an evidence-based approach to the diagnosis and management of neck disorders changed practitioner behavior but did not impact on clinical outcomes.

\section{Key Practice Points:}

- Longitudinal professional development results in changes to practice behavior but not clinical outcomes.

- Further research is needed to evaluate whether additional educational strategies can enhance patient outcomes to ensure they are clinically meaningful.

\section{CULTURAL RESPONSIVENESS: STUDENT PHYSIOTHERAPISTS' PERCEPTIONS ON THEIR PREPAREDNESS AND EDUCATION}

Pourkazemi $\mathrm{F}^{1}$, Chipchase $\mathrm{L}^{1}$

ISchool of Science and Health, The University of Western Sydney, Sydney

Question: What are the perceptions of third year physiotherapy students of their preparedness for culturally diverse practice and the educational activities to enhance this attribute?

Design: An observational study.

Participants: Forty-four third year physiotherapy students.

Outcome Measures: Students were asked to complete questionnaires that sought their perceptions of preparedness for culturally diverse practice, the value of various educational activities and their open mindedness (Rokeach Dogmatism Scale)

Results: Forty-four students completed the questionnaires ( $F: M=18: 22$ age 22.3 \pm 6.9 years). All students felt prepared for culturally responsive physiotherapy practice as a student (68.9\% strongly agree; $31.1 \%$ agree). However, there were incongruences between perceptions of preparedness and beliefs about how culture impacts upon the therapeutic interaction. This was reflected by responses to the Dogmatism Scale as $18.2 \%$ of students scored as closed-minded and potentially less tolerant to cultura differences. Students felt the most useful education preparation for culturally responsive practice was clinical placements (91.1\%), learning from peers (84.4\%) and physiotherapy practical class (64.4\%). Reading textbooks (6.7\%) and completing a specific unit on culturally diversity $(6.7 \%)$ were rated as least useful.

Conclusion: Third year physiotherapy students perceive themselves culturally responsive. However, there was some naivety about how culture impacts on clinical interaction. Students perceive their learning about culturally responsiveness comes from interactions with culturally diverse people rather than in a dedicated unit.

\section{Key Practice Points:}

- Specific units designed to prepare physiotherapy students for culturally responsive interactions are not perceived as useful sources of education by students.

- Weaving cultural examples into practical classes may provide more effective methods to enhance learning.

\section{THE EFFECTS OF LASER ON NERVES}

\section{Chow R}

Brain and Mind Research Institute, University of Sydney

The effect of light on retinal neurons is a well known example of light sensitivity within the nervous system. What is less well known is the light sensitivity of nerves of the somatosensory system. These nerves are predominantly the unmyelinated C fibres and thinly myelinated A $\delta$ fibres, which convey noxious stimulation from skin to spinal cord. The terminals of these nerves are well within the penetration depths of many lasers used therapeutically. Light must be absorbed by the neuron to have a biological effect. Absorption occurs by photoacceptors the most well known of which is cy tochrome Coxidase present in all mitochondria. In neurons, ATP-rich mitochondria travel the length of the axon from the cell body to peripheral terminals along the cytoskeleton delivering ATP as required. When laser is absorbed by the mitochondria and other photoacceptors in periphera nerves conduction block occurs and the cytoskeleton is disrupted slowing transport of neurotrophic factors from the periphery at sites of injury and inflammation to the dorsal horn. Disruption of the cytoskeleton leads to down-regulation of synaptic activity at the dorsal horm, which modulates pain long term. Light has profound effects on the peripheral nervous system which is the basis for its pain relieving effects.

\section{Key Practice Points:}

- Understand that nerves can absorb light which profoundly alters bioelectrical and neurotrophic function.

- In a clinical context understand that one of the mechanisms for pain reduction with laser relates to changes in nerve function. 


\section{PAIN - WHAT IT IS AND HOW IT IS INFLUENCED BY LASER}

\section{Chow R}

Brain and Mind Research Institute, University of Sydney

Pain is defined by the International Association for the Study of Pain as "an unpleasant sensory and emotional experience associated with actual or potential tissue damage or described in terms of such damage". Pain is experienced as a culmination of the biological, psychological and social elements that occur when an illness or injury occurs. This is described as the Biopsychosocial Model of pain. In any one individual the importance of each of these elements will vary so that pain management strategies will also vary. Laser at intensities which do not produce heat or tissue damage, referred to as photobiomodulation, addresses the biological aspect of pain though several mechanisms. Laser causes conduction block in small unmyelinated $C$ and thinly myelinated $A \delta$ nerve fibres which convey noxious stimuli from the periphery to the spinal cord. Laser also reduces muscle spasm by modulating neurotransmitter release at the neuromuscular junction. Laser has anti-inflammatory effects in some studies equivalent to commonly used drugs. Laser stimulates the motoricity of lymph vessels which reduces oedema. Laser also has tissue repair effects by increasing the activity of cells involved in healing such as fibroblasts and macrophages. Changes in the dorsal horn which occur as a result of tissue changes in the periphery can lead to long term pain relief and reduction in central sensitisation though the neuroplasticity of the nervous system. The optimal clinical application of laser for pain requires the correct wavelength at the right energy density for the correct duration for the specific condition.

\section{Key Practice Points:}

- Pain is not just nociception, it is an emotional experience.

- Laser has multiple mechanisms of action relevant to pain relief

- The choice of wavelength, dose and duration of application are critical to achieving optimal results in clinical application

\section{THE FIRST STEP TOWARDS STANDARDIZING PAEDIATRIC TERMINOLOGY IN AUSTRALIA: A SYSTEMATIC REVIEW}

\section{Clark $\mathbf{R}^{1,2}$, Locke $\mathrm{M}^{1,2,3}$, Bialocerkowski $A^{1,2}$}

${ }^{1}$ Menzies Health Institute Queensland, Griffith University, Gold Coast ¿School of Allied Health Sciences, Griffith University, Gold Coast

3Movement Solutions, Coorparoo

Questions: What paediatric terms and age definitions are used within the Australian health and health education context? Is Australian paediatric terminology comparable to existing US models? What is a suggested standard paediatric terminology framework for use within Australia?

Design: Systematic review using a novel domain-specific hierarchical method to review grey literature and Australian health and health education websites published from 2009 to 2014.

Participants: Australian health and health education webpages.

Outcome Measures: Paediatric terms with an associated age range definition.

Results: 1794 identified paediatric terms, reduced to 34 common terms, showed a Pareto language distribution (21\% terms, accounted for $72 \%$ of cumulated terms). When combined with an age range, we identified 197 unique definitions. Paediatric terminology was inconsistent with semantic ambiguities, (19 terms with multiple definitions), and lexical ambiguities, (129 definitions with multiple terms). Age definitions were clustered into four statistically significant groups ( $F=245.3, p<.001)$

Conclusion: No standard set of Australian paediatric terminology exists, with ambiguous terms and definitions identified within Australian health and health education websites. Ambiguities were resolved based on age definitions from a decision tree analysis combined with high frequency terms from the Pareto language distribution. Comparison to an existing US model suggests Australian paediatric terminology require broader age definitions. We recommend the following paediatric terms and definitions: "infant: 0 up to 1 year", "early childhood: 1 up to 5 years", "child: 5 up to 13 years", "young person: 13 up to 22 years".

\section{Key Practice Points:}

- No consistent use of paediatric terminology within Australian health and health education exists.

- We recommend using "infant: 0 up to 1 year", "early childhood: 1 up to 5 years", "child: 5 up to 13 years", "young person: 13 up to 22 years" until further work refines paediatric terminology.

- Using a standard paediatric terminology will improve future research translation to strengthen assessment and intervention studies.

- Other nations can use this innovative method to explore paediatric terminology before developing international standards.

\section{ARE PAEDIATRIC LOWER LIMB NEUROLOGICAL TESTS CLINIMETRICALLY SOUND? A SYSTEMATIC REVIEW}

Clark R $\mathbf{R}^{1,2}$, Locke $\mathrm{M}^{1,2,3}$, Hill B ${ }^{1,2,4}$, Bialocerkowski $A^{1,2}$

${ }^{1}$ Menzies Health Institute Queensland, Griffith University, Gold Coast 2School of Allied Health Sciences, Griffith University, Gold Coast ${ }^{3}$ Movement Solutions, Coorparoo

${ }^{4}$ Epworth Monash Rehabilitation Medicine Unit, Melbourne

Questions: Do clinimetrically-sound lower limb neurological examinations exist for paediatric individuals aged under 18 years with a neurological condition? Design: Systematic review with clinimetric properties from studies critically appraised and synthesised.

Participants: Primary studies focussed on the clinimetric evaluation of lower limb neurological tests for muscle strength, sensation, or reflexes for children and young people with a neurological condition.

Outcome Measures: The methodological quality of each primary study was rated from poor to excellent using a critical appraisal tool. Clinimetric properties for each neurological test were then synthesised.

Results: Eight clinimetric studies were identified. All evaluated the reliability of lower limb muscle strength. Strength, however, was evaluated using a variety of different tests. The quality of these studies was rated as fair to good and variable reliability was found. Hand-held dynamometer had the greatest volume of evidence in children and young people with cerebral palsy. However, the evidence appeared too variable to support its use.

Conclusion: The limited clinimetric properties found for lower limb muscle strength tests in children with a neurological condition demonstrates that additional high quality research is required for neurological examinations of the lower limb in paediatric individuals with a neurological condition.

\section{Key Practice Points:}

- No clinimetrically-sound paediatric lower limb sensation or reflex outcomes measures were identified

- Limited clinimetric properties were identified for the hand held dynamometer as a measure of lower limb muscle strength in children with a neurological condition

- Recommendations for a gold standard lower limb neurological examination require population specific clinimetrically-sound tests

- Further high quality evidence is needed for recommendations of a clinimetrically-sound paediatric lower limb neurological examination in children with a neurological condition

\section{CONSENSUS BETWEEN ASSESSORS REGARDING RATINGS OF COMPETENCY OF PHYSIOTHERAPY STUDENTS: IS IT POSSIBLE?}

Clark $\mathrm{C}^{1}$, Kirwan $\mathrm{G}^{1}$, Dalton $\mathrm{M}^{2,3}$

${ }^{1}$ Menzies Health Institute, Griffith University, School of Allied Health Sciences, Gold Coast

¿Monash University, Faculty of Medicine, Nursing and Health Science, Melbourne ${ }^{3}$ Central Queensland University, School of Human, health and Social Science, Rockhampton

Question: Is there assessor consensus when rating scripted videos of student performance using the Assessment of Physiotherapy Practice (APP) instrument?

Participants: Clinical educators ( $n=156)$ from Australia and New Zealand with a minimum of 3 years clinical experience and 1 year as a clinical educator. Intervention: Three levels of student performance (not adequate, adequate and good/excellent) were scripted and filmed for four clinical areas: musculoskeletal, neurorehabilitation, cardiorespiratory and orthopaedics. Scripts were written by Griffith University staff. An expert panel reviewed the vignettes to ensure validity $(n=8)$. Participants reviewed one randomly allocated vignette rating the performance on the global rating scale. Participants were blinded to the vignette performance level.

Outcome Measure: A priori percentage agreement of 75\% between the participant rating and the performance level was set

Results: There was 100\% agreement rating the inadequate vignette in cardiorespiratory ( $n=11)$, neurological $(n=5)$ and orthopaedics $(n=11)$ and the good/excellent vignettes in cardiorespiratory $(n=5)$ and orthopaedics $(n=5)$. One respondent passed the inadequate musculoskeletal $(n=7)$ and one passed the good/excellent neurological $(n=8)$ and musculoskeletal $(n=7)$ vignettes. Overall $>75 \%$ of assessors rated the adequate vignettes as adequate however wide variability existed.

Conclusions: Assessors seem equipped to assess student performance at a fail or good/excellent level. Vignettes at a pass level have a wider variability in rating. Consensus between assessors regarding student performance rating is possible however variability exists when educators rate a performance scripted at an adequate level.

\section{Key Practice Points:}

- Variability exists when educators assess student performance at an adequate level.

- The vignettes depict student performance at the level they were set. 


\section{HEALTHY ATHLETES WITHIN NORMAL WEIGHT RANGE ARE SUSCEPTIBLE TO DVT: 5 CASE STUDIES}

Claus $\mathrm{A}^{1,2}$, Mellor $\mathrm{R}^{1,3}$, McLean $\mathrm{R}^{4}$

${ }^{1}$ University of Queensland, School of Health \& Rehabilitation Sciences

¿Peter Dornan Physiotherapy

3 University of Queensland, Sports Injuries Rehabilitation \& Prevention

for Health

${ }^{4}$ X Physiotherapy

Question: Is there enough clinician awareness of the possibility of deep venous thrombosis (DVT) in runners presenting with calf pain?

Design: Case studies

Participants: Case history of a marathon runner who presented with lower limb pain that mimicked peripheral neural sensitivity on examination, 4 cases of runners with DVT identified by QId physiotherapists, and published case studies.

Intervention: Management of DVT in athletes varied widely according to medical practitioner preference with anticoagulant medication, activity level/return-to-sport advice and follow-up investigations,

Outcome Measures: Diagnostic ultrasound demonstrated resolution of DVT, but impacts on psychosocial factors were not typically addressed by medical practice.

Results: There is poor awareness of risk and wide discrepancy in management of DVT in runners.

Conclusion: Physical activity does not exclude the risk of DVT occurrence. Clinical presentation of DVT may mimic other musculoskeletal conditions, emphasizing the importance of investigating suspicious presentations. Further research is needed to identify the epidemiology of DVT in physically active people, to inform acute and chronic management planning.

\section{Key Practice Points:}

- Consider DVT in differential diagnosis for athletic patients with atypical calf pain, and refer for diagnostic ultrasound investigations promptly.

- Psychosocial support may be beneficial in medium to long-term management of DVT.

- In the absence of sensitive and specific diagnostic physiotherapy tests, administrative and cost barriers to direct physiotherapy referral for ultrasound investigation of DVT should be removed, in the interest of patient safety and cost to the health system.

\section{USING EVIDENCE TO INFORM THE IMPLEMENTATION OF A WARM WATER EXERCISE PROGRAM IN A COMMUNITY SETTING}

Clavisi $0^{1}$, Barker A², Morello R², Talevski J2, Nolan G1 , Briggs A , de Silva $\mathrm{R}^{1}$, King $\mathrm{J}^{1}$, Sackville $\mathrm{G}^{1}$

${ }^{1}$ Arthritis and Osteoporosis Victoria

${ }^{2}$ Falls and Bone Health Team, Health Services Research Unit, Department of Epidemiology and Preventative Medicine, Monash University Arthritis and Osteoporosis Victoria (A\&OV) is a peak organisation representing people with musculoskeletal (MSK) conditions. From 1977 - 2014 A\&OV administered the Waves program: a peer-led, warm water exercise program. To determine whether the program aligned with current evidence concerning the effectiveness of warm water exercise for people with chronic MSK conditions, A\&OV partnered with Monash University to undertake a phased research program:

1. A systematic review evaluating the effectiveness of warm water exercise for people with MSK conditions.

2. Facilitated workshops with Waves program leaders to i) disseminate the review findings and ii) address evidence-practice gaps.

3. An observational cohort study to identify the impact of the Waves program on key self-reported measures.

The review showed that warm water exercise significantly improved pain, physical functioning and quality of life (QOL) in people with MSK conditions; although further studies are needed to establish long-term effects and optimal content and dosage. Two evidence-practice gaps were identified: i) the provision of an education component and, ii) inclusion of balance exercises. The observational study showed that Waves participants experienced significant QOL improvements, although no significant improvements in symptoms or functional impairment were observed at 12-week follow-up. Participants also reported high levels of satisfaction with the program.

\section{Key Practice Points:}

- Warm water exercise is associated with an improvement in pain, physical functioning and QOL in MSK conditions.

- Community-based warm water exercise programs have been shown to improve consumers' QOL.

- Warm water therapy administered by physiotherapists can be augmented by community-based programs such as the Waves program.

\section{THE GROWING ROLE OF PHYSIOTHERAPISTS IN INFLUENCING WORK RELATED OUTCOMES}

\section{Coburn P}

WorkSafe Victoria and the Transport Accident Commission

Physiotherapists are commonly engaged in returning their patients to work following an injury. However, the evidence for the effectiveness of this activity has not been clearly demonstrated. There is an argument of ten mounted by physiotherapists, that their impact on return to work is nullified by the actions or inactions of others within the compensable setting including employers, insurance agents, occupational rehabilitation providers, and other health care providers. But this assertion has recently been challenged by the results from a study on a network of private practitioners working across a state of Australia. This group, provided with training and support, demonstrated an improvement in return to work outcomes over a group of their peers who did not have access to the same resources

In this presentation, the key principles of the training of these physiotherapists will be discussed. Central to the discussion will be the role of the physiotherapist influencing, and where possible writing, the certificate of capacity. The benefits of extending certificate writing authorisation by physiotherapists in the various legislations will be considered.

\section{PHYSIOTHERAPISTS PROVIDED WITH TRAINING AND SUPPORT, IMPROVE RETURN TO WORK OUTCOMES AND CONTAIN OVERALL MEDICAL COSTS IN A COMPENSABLE}

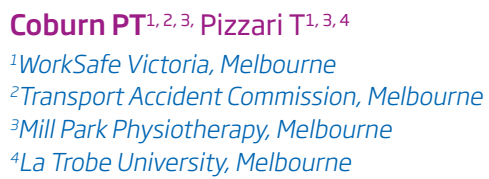

Question: Can a network of physiotherapists provided with training and support, improve return to work outcomes and contain overall medical costs in a compensable scheme?

Design: Cohort study

Participants: 105 workers compensation claims treated by a network physiotherapist compared with 1136 claims treated with standard-care physiotherapy.

Intervention: Physiotherapists providing network care received specific training in the management of injured workers and return to work strategies including the identification of barriers to appropriate certification or work capacity.

Outcome Measures: Average and median medical costs for a claim at 12 months, income supplementation spend, return to work rate at 6 months and risk ratios comparing likelihood of returning to work at 6 months.

Results: The average and median medical and like cost for a claim at 12 months for standard-care physiotherapy claims was $\$ 5462$ and $\$ 3568$, whereas for network treated claims it was $\$ 4239$ and $\$ 2429(p=0.02)$. The average and median income supplementation for standard-care physiotherapy claims was $\$ 5124$ and $\$ 1902$ and for network treated claims it was $\$ 2846$ and $\$ 1389(p=0.46)$. At 6 months post commencement of physiotherapy $98 \%$ of network treated claims had returned to work compared with $90 \%$ of standard-care claims $(p=0.02)$. Workers were 3.3 times more likely to return to work by 6 months if treated by a network physiotherapist.

\section{Key Practice Points:}

- Physiotherapists have an active role in compensable system, improving return to work and controlling medical costs

- Training and support can be effective in assisting physiotherapists in their performance in a compensable scheme 


\section{TEACHING MANUAL HANDLING TO UNDERGRADUATE PHYSIOTHERAPY STUDENTS, WHERE ARE WE STARTING FROM?}

Coffee J, Boucaut R, Milanese S

University of South Australia

Background: Universities teach physiotherapy (PT) students manua handling (MH) principles and practise in readiness for their clinical practice. The delivery of this teaching content is predicated on the assumption that students are prepared to assimilate the content at a particular point in their program of education. The Transtheoretical model of change (TTMOC) has been promoted as a framework for developing interventions to promote health behaviour change (such as $\mathrm{MH}$ ). It describes temporal stages that an individual follows in the course of changing their behaviour. The traditional $\mathrm{MH}$ teaching approach assumes the PT cohort is homogenous, but is this realistic?

Question: To establish the risk profile and stage of change of undergraduate (UG) and graduate entry (GE) PT students in their first year of study.

Design: Cross sectional Cohort

Participants: Two cohorts of first year physiotherapy students were surveyed ( $U G=62, G E=31$ ) in the first study period of their program prior to the delivery of any instruction in $\mathrm{MH}$

Outcome Measures: On- line survey The questions established subject demographics, past experience of $\mathrm{MH}$ training, and the risk awareness of the individual with regard to $\mathrm{MH}$. Ethics approval granted by UniSA.

Results: Differences were found between the cohorts which appeared related to years of age and previous experience (both work and training).

Conclusion: PT students are not a homogenous group when considering their risk profile and are therefore at different stages of risk awareness.

\section{Key Practice Points:}

- Academics should consider the challenges this presents in design and delivery of education in manual handling for this cohort.

PREVALENCE AND FACTORS ASSOCIATED WITH RADIOGRAPHIC PATELLOFEMORAL OSTEOARTHRITIS IN YOUNG TO MIDDLE-AGED ADULTS WITH CHRONIC PATELLOFEMORAL PAIN

Collins $\mathbf{N}^{1,2}$, Vicenzino $\mathrm{B}^{1}$, Macri $\mathrm{E}^{1,3}$, Crossley KM ${ }^{1,4}$

${ }^{1}$ The University of Queensland, St Lucia, Australia

${ }^{2}$ The University of Melbourne, Melbourne, Australia

University of British Columbia, Vancouver, Canada

${ }^{4}$ La Trobe University, Bundoora, Australia

Question: How prevalent is radiographic patellofemoral osteoarthritis in young to middle-aged adults with chronic patellofemoral pain, and what clinical factors are associated with osteoarthritis severity?

Design: Cross-sectional cohort study.

Participants: 80 participants (aged $26-50$ years) with patellofemoral pain 33 months duration

Outcome Measures: Patellofemoral and tibiofemoral osteoarthritis severity were graded from weight-bearing anteroposterior, lateral and skyline radiographs. Established osteoarthritis was defined as Kellgren \& Lawrence $(K \& L)$ grade ${ }^{32}$, and early osteoarthritis as grade 1 . Multivariate linear regression evaluated association of demographics, patient-reported outcomes, clinical tests and patellar alignment with K\&L grade.

Results: 20 participants had established osteoarthritis, 34 had early osteoarthritis, and 26 had no osteoarthritis. Older age (unstandardised b coefficient $0.04,95 \% \mathrm{Cl} 0.02$ to 0.07$)$, higher BMI $(0.05,0.01$ to 0.1$)$, lower hip external rotation isometric torque $(-1.38,-2.64$ to -0.11$)$ and higher tibiofemoral K\&L grade $(0.46,0.19$ to 0.72$)$ were significantly associated with patellofemoral $K \& L$ grade (adjusted $\mathrm{R}^{2}=0.4$ ).

Conclusion: One-quarter of young to middle-aged adults with chronic patellofemoral pain had established osteoarthritis, most commonly in the patellofemoral joint, and almost half had early osteoarthritis. BM and hip strength are modifiable factors that explained patellofemoral osteoarthritis severity, and may represent effective targets for intervention.

\section{Key Practice Points:}

- Chronic patellofemoral pain is common and persistent in young to middleaged adults.

- Physiotherapists should be aware of the likelihood of radiographic patellofemoral osteoarthritis in this population.

- Interventions to reduce BMI and increase hip external rotation strength may help to slow the onset and progression of radiographic patellofemoral osteo

\section{EXPERIENCES OF FATIGUE FOLLOWING CRITICAL ILLNESS: A MIXED METHODS STUDY}

Colman $\mathrm{Z}^{1}$, Johnston $\mathrm{C}^{1}$, Ashby $\mathrm{S}^{1}$, Mackney $\rfloor^{1}$

${ }^{1}$ The University of Newcastle, Callaghan

Question: Do survivors of critical illness experience fatigue a year or more post intensive care unit discharge? How do survivors of critical illness experience fatigue?

Design: Mixed methods design.

Participants: Five survivors of critical illness, 2 female and 3 male, aged (mean [SD]) 59 [10] years who were discharged from intensive care 2.3 $[0.5]$ years prior to participation following an intensive care length of stay of 10 [2] days

Outcome Measures: Fatigue Impact Scale and details of care in the intensive care unit. Semi-structured interviews analysed using Colaizzi's interpretation of the phenomenological method to identify key themes.

Results: Fatigue Impact Scale scores showed that fatigue impacted on four of five participants to varying degrees. Participants described four key themes relating to their experiences of fatigue post critical illness: multifaceted fatigue, lack of information, strategy formation, and role loss. Participants described physical and cognitive dysfunction that worsened with increased fatigue, and their parental, worker, spousal and social roles were altered as a result of fatigue in their recovery. Information offered to participants regarding fatigue and recovery was limited.

Conclusions: The impact of fatigue in survivors of critical illness is profound, and can continue for several years after discharge from intensive care. Recovery may be aided by improved education and appropriate management strategies.

\section{Key Practice Points:}

- Fatigue in survivors of critical illness may be profound.

- Improved assessment and education regarding fatigue pre-discharge, and the provision of fatigue management strategies for critical illness survivors may reduce the impact of fatigue on recovery.

\section{PERCEPTUAL FUNCTION OF CHILDREN WITH CEREBRAL PALSY: EXPLORING THE CONNECTIONS BETWEEN VISION AND TACTILE DYSFUNCTION}

Conroy $\mathrm{A}^{1}$, Auld $\mathrm{ML}^{2,1}$, Hankin $\mathrm{N}^{1}$, Tan $X \mathrm{Y}^{1}$, Johnston $\mathrm{LM}^{1}$

${ }^{1}$ School of Health and Rehabilitation Sciences, The University of Queensland, Brisbane

${ }^{2}$ Cerebral Palsy League, Brisbane

Question: Do children with cerebral palsy (CP) experience global sensoryperceptual problems?

Design: Cross sectional study.

Participants: Participants were 8-12-year-old children from the inTOUCH study, including 20 children with CP (median nine years; 10 males: GMFCS$E \& R I=4 ; \|=9 ; \quad I V=5 ;$ MACS I=10; $\|=5 ; \quad\| \mid=4$; hemiplegia=6, diplegia=7, quadriplegia=4, ataxia=3) and 27 typically developing children (median 10 years; 16 males; 23 right-handed).

Outcome Measures: Assessments included tactile registration (Semmes Weinstein Monofilaments; spatial perception (Single Point Localisation (SPL), static Two-Point Discrimination (S2PD)); haptic perception (common objects, manual forms, geometric shapes); and visual perception (Motorfree Visual Perception Test (MVPT)). Descriptive statistics, relationships within groups (correlations), and differences between groups (Wilcoxon rank-sum tests) were calculated.

Results: Typical children showed superior visual $(p<0.001)$ and tactile performance ( $p<0.035$ ) with correlations between visual (MVPT) and tactile perception (SPL: $\mathrm{p}=0.002$; S2PD: $\mathrm{p}<0.034$ ). Children with CP demonstrated deficits in visual perception (70\%), tactile function (85\%) or both visual and tactile function (70\%). Visual perception deficits (MVPT) were correlated with deficits in tactile registration $(p=0.008)$, spatial perception (SPL: $p<0.024$ ) and haptic perception (common objects: $p=0.003)$.

Conclusion: Most children with CP demonstrated visual and tactile deficits, suggesting they may experience global sensory-perceptual problems. Research is required to understand the source of globa sensory-perceptual deficits, plasticity of sensory-perceptual function and possible interventions.

\section{Key Practice Points:}

- Many children with CP have concomitant deficits in visual and tactile function

- Comprehensive sensory-perceptual assessment is essential for adequate management 
PHYSIOTHERAPY IS A COST-

EFFECTIVE ALTERNATIVE TO

CORTICOSTEROID INJECTION FOR

LATERAL EPICONDYLALGIA: ECONOMIC

EVALUATION OF A RANDOMISED CONTROLLED TRIAL

Coombes BK ${ }^{1,2}$, Connelly $\mathrm{L}^{3}$, Bisset $\mathrm{L}^{4}$, Vicenzino $\mathrm{B}^{1}$

${ }^{1}$ The University of Queensland, School of Health and Rehabilitation Sciences, Brisbane

2The University of Queensland, School of Biomedical Sciences, Brisbane

${ }^{3}$ The University of Queensland, Faculty of Health and Behavioural Sciences,

Brisbane

${ }^{4}$ Menzies Health Institute, Griffith University, Gold coast

Question: What is the cost-effectiveness of corticosteroid injection, physiotherapy and a combination of these interventions for treating lateral epicondylalgia.

Design: Randomised control trial with economic evaluation from a societal perspective.

Participants: 165 adults meeting a clinical diagnosis of unilateral lateral epicondylalgia of longer than six weeks duration from Brisbane.

Intervention: Saline injection (placebo), corticosteroid injection, saline injection plus eight sessions of elbow manipulation and exercise delivered by a physiotherapist (physiotherapy) or corticosteroid injection plus physiotherapy.

Outcome Measures: Costs to society and health-related quality of life (estimated by EuroQol-5D) over one year follow-up were used to generate incremental cost per quality-adjusted life year (QALY) ratios for each intervention relative to placebo.

Results: Intention to treat analysis was possible for $93 \%$ of trial participants. Physiotherapy was more costly, but was the only intervention that produced a statistically significant improvement in quality of life relative to placebo (MD, 95\% CI 0.035, 0.003 to 0.068). Similar cost/QALY ratios were found for physiotherapy (AUD\$29,343) and corticosteroid injection $(\$ 31,750)$, however the probability of being more cost-effective than placebo at a pre-defined threshold (of $\$ 50$ 000/QALY) was $81 \%$ for physiotherapy and only 53\% for corticosteroid injection. Cost/QALY was far greater for a combination of corticosteroid injection and physiotherapy $(\$ 228,000)$

Conclusion: Physiotherapy was a cost-effective treatment of lateral epicondylalgia, while the cost-effectiveness of corticosteroid injection was more uncertain. A combination of corticosteroid injection and physiotherapy was both clinically- and cost-ineffective.

\section{Key Practice Points:}

- Despite higher intitial treatment costs, eight sessions of physiotherapy provides a cost-effective alternative to a single corticosteroid injection.

- Practitioners and policymakers should consider both clinical and economic effects of treatments when choosing interventions for lateral epicondylalgia.

\section{ASSESSING FITNESS AND PHYSICAL ACTIVITY: HOW TO GET BETTER COMPLIANCE FROM YOUR PATIENTS}

\section{Coombes JS ${ }^{1}$}

${ }^{1}$ Centre for Research in Exercise, Physical Activity and Health, School of Human Movement and Nutrition Sciences, The University of Queensland The relationship between physical activity levels and cardiorespiratory fitness is not strong, indicating that these are two different constructs. Assessing the physical activity levels of clients has been made easier with wrist worn personal devices and smart phone applications. Measuring fitness levels has also been revolutionised with the use of non-exercise fitness tests based on algorithms from large studies with objectively measured cardiorespiratory fitness values. This presentation will discuss the strengths and limitations of these approaches and how they may be used to improve the physical activity and fitness levels of your patients.

\section{Key Practice Points:}

- Participants will be given knowledge on the use and effectiveness of devices and applications to assess the physical activity and fitness levels of their clients
BUT THEY DON'T 'JUST DO IT' - HOW TO GET YOUR PATIENTS EXERCISING

\section{Coombes JS}

${ }^{1}$ Centre for Research in Exercise, Physical Activity and Health, School of Human Movement and Nutrition Sciences, The University of Queensland Despite much effort to market and promote exercise, the majority of people are still not regularly active. Physiotherapists are well positioned to improve the physical activity levels of their clients, and a better understanding of the multifactorial causes of inactivity and various approaches to change behaviour may assist in achieving this outcome. This session will focus on working with individual clients. A framework to understand exercise adherence will be presented along with proactive strategies to promote exercise adoption and maintenance. This will include understanding self efficacy, the client's context, self regulation, support and satisfaction. The presentation will encourage self reflection on current practices to promote behaviour change.

\section{Key Practice Points:}

- Participants will be given a framework to help understand exercise adherence and strategies to promote physical activity behaviour change and adaption

\section{CRITICAL PHYSIOTHERAPY FORUM: A PHILOSOPHICAL, ETHICAL AND CREATIVE CONVERSATION ABOUT HOW PHYSIOTHERAPY IS WAS AND CAN BE}

\section{Corrigan $\mathrm{R}^{1}$, Doran $\mathrm{B}^{2}$, Edwards $\mathrm{I}^{3}$, Hiller $\mathrm{A}^{4}$, Nicholls DA ${ }^{5}$,} Owen $\mathrm{G}^{6}$, Setchell $]^{7}$

${ }^{1}$ School of Community Health, Charles Sturt University, Albury/Wodonga.

2 The Royal Children's Hospital, Melbourne

${ }^{3}$ School of Health Sciences, University of South Australia, Adelaide

${ }^{4}$ Department of Physiotherapy, Melbourne School of Health Sciences, The University of Melbourne, Melbourne

${ }^{5}$ School of Clinical Sciences, Faculty of Health and Environmental Sciences, Auckland University of Technology, New Zealand

${ }^{6}$ Cardiff School of Physiotherapy, Cardiff University, Wales

${ }^{7}$ School of Psychology, Department of Health and Behavioural Science, The University of Queensland, Brisbane

This session provides a platform to explore topics from the emerging field of 'critical physiotherapy'. Critical physiotherapy investigates and highlights philosophical, historical, ethical, political and social aspects of the profession. It critiques the status quo of physiotherapy, considering and challenging assumptions and norms of practice. Critical physiotherapy also looks externally at how physiotherapy does, or can, affect the world. Therefore, in this forum, speakers (and the audience) have the opportunity to discuss, and make explicit, how physiotherapy is, was and can be, encouraging a conscious understanding, theorising and development of the profession. We include a diverse range of speakers in our platform presentations, and encourage debate through a panel discussion that includes opportunity for audience participation. This session will comprise of three sections: 1) Introduction: a brief explanation of critical physiotherapy and an outline of the scope of the session, 2) Platform presentations: six speakers will deliver individual 10 minute presentations that highlight different critical insights into physiotherapy practice and theory. These include: an exploration of reflexivity in research, phenomenological reflections on physiotherapy ethical codes of conduct, embodiment as an important conceptual framework for exploring physiotherapy, critical deconstruction of evidence based practice, clinical applications of narrative developed from dramatic "psychological realism", and a consideration of the future of physiotherapy as luxury, and 3) Panel discussion: Speakers from the platform presentations will participate in a panel discussion that will address philosophical issues within physiotherapy and issues raised in the platform presentations. Audience questions/comments will be encouraged. 


\section{EFFECTS OF POSTURAL CONTROL INTERVENTIONS ON SCHOOL- RELATED FUNCTION IN CHILDREN: A SYSTEMATIC REVIEW}

Cota $\mathrm{L}^{1}$, Milne $\mathrm{N}^{1}$, Pope $\mathrm{R}^{1}$, Hunt $\mathrm{P}^{2}$

${ }^{1}$ Faculty of Health Sciences and Medicine, Bond University,

Robina, Queensland

2Department of Education and Training, Queensland

Question: What effect do postural control interventions have on the school-related functions of: physical activity; reading; handwriting; and behaviour and attention?

Design: Systematic review of intervention studies

Participants: School-aged children between 5-18 years; typically or atypically developing.

Intervention: Postural control interventions were defined as interactions of an individual with a task and environment that challenge the physiological systems that control static and dynamic balance, coordination, and visual tracking.

Outcome Measures: Physical activity measures included Gross Motor Function Measure-(GMFM)-88/66, Pediatric Balance Scale, Movement Assessment Battery for Children (MABC-2). Reading-related measures included the Dyslexia Screening Test (DST) and national (US) academic tests

Results: Of 643 potential articles, five studies with fair to good methodological quality (mean modified Downs and Black score of 75\%: range $64-82 \%$ with $100 \%$ scoring agreement) were evaluated. The five studies utilised diverse postural control interventions, with four resulting in positive physical activity outcomes and one resulting in improved reading outcomes. No studies were identified which reported on the outcomes of handwriting, behaviour or attention.

Conclusion: Postural control interventions, delivered in a variety of modalities can positively affect physical activity-related outcomes and potentially reading outcomes. Further high quality research is required to determine the impact of postural control interventions on school-related functions such as reading, handwriting, behaviour and attention.

\section{Key Practice Points:}

- Limited research exists examining the effects of postural control interventions on school-related functional outcomes.

- Further high quality research is needed to investigate the effects of postural control interventions on school-related function, including reading, handwriting, behaviour and attention.

\section{REHABILITATION AFTER ELECTIVE TOTAL HIP REPLACEMENT - A RANDOMISED CONTROLLED TRIAL}

Coulter $\mathrm{C}^{1,2}$, Neeman $\mathrm{T}^{2}$, Scarvell $]^{3}$, Smith $\mathrm{PN}^{1,2}$

Canberra Hospital, Canberra

Australian National University, Canberra

University of Canberra, Canberra

Question: To determine the benefit of a supervised or unsupervised program of rehabilitation exercise on patient outcomes following total hip replacement.

Design: Randomised controlled trial.

Participants: Ninety eight unilateral hip replacement patients were randomised.

Ethics approval: ACT Health Human Research Ethics Committee (HREC) and Australian National University HREC approved this study.

Intervention: Following discharge, a supervised rehabilitation group completed four weekly circuit classes supervised by a physiotherapist. An unsupervised group was provided instructions to continue their exercise program at home with phone support.

Outcome Measures: Western Ontario and McMasters University Osteoarthritis Index (WOMAC), Short form 36 (SF-36) and Timed Up and Go test (TUG) were completed pre-operatively and at five, twelve and twenty-six weeks post-operatively. Assessors were blind to intervention. Linear mixed models analyses were used to compare outcomes between the two groups.

Results: Both groups showed improved WOMAC scores (supervised group improved 9.5 points and unsupervised 6.7 points, but with no between-group difference $(p=0.62)$. The two interventions were not clinically significant (8 points; $95 \% \mathrm{Cl}-6.75$ to 5.73). SF-36 and TUC scores also demonstrated improvements over time, with no between group differences

Conclusion: Rehabilitation outcomes post total hip replacement were similar clinically and statistically whether the rehabilitation was supervised or not. This study lacked a no-intervention control group.

\section{Key Practice Points:}

- Hip replacement patients without complications may be discharged home to manage independently with their exercise program.

- After discharge physiotherapy can be re-directed to those with additional rehabilitation requirements.

- Consistently research indicates that key components for effective programs include abductor strengthening and weight bearing exercise.

\section{TACTILE PERCEPTION IN THE HAND PREDICTS MOTOR FUNCTION IN CHILDREN WITH DEVELOPMENTAL COORDINATION DISORDER}

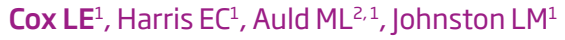

${ }^{1}$ Division of Physiotherapy, School of Health and Rehabilitation Sciences, The University of Queensland, St Lucia

Division of Research and Innovation, Cerebral Palsy League, Brisbane

Question: Do children with Developmental Coordination Disorder (DCD)

have tactile deficits in the hands? Is tactile dysfunction related to deficits in upper limb motor function?

Design: Cross-sectional observational study

Participants: Children aged eight to 12 years with $D C D(n=20)$ or typical development (TD) ( $n=16)$ both confirmed using the Movement Assessment Battery for Children, second edition.

Outcome Measures: A comprehensive tactile-motor battery including: tactile registration (Semmes Weinstein Monofilaments); tactile spatial perception (Single Point Localisation (SPL) and two-point discrimination (2PD)); haptic perception (Stereognosis); speed of simple everyday manual tasks (Jebsen-Taylor Test of Hand Function (JTTHF)); and handwriting speed and accuracy (Evaluation Tool of Children's Handwriting (ETCH)).

Results: Compared to children with TD, children with DCD demonstrated poorer tactile spatial perception (SPL non-dominant hand $p=0.04$ ), slower speed of alphabet writing (ETCH $p<0.05$ ) and less legible handwriting $(\mathrm{ETCH} p<0.01)$. Localisation of touch in the hand $(\mathrm{SPL})$ predicted handwriting legibility (ETCH $r=0.11$ ) and speed of functional tasks (JTTHF $r=0.33)$.

Conclusion: Localisation of touch in the hand is a significant predictor of fine motor accuracy and speed. Tactile assessment should be performed to determine reasons for upper limb motor difficulties experienced by children with DCD

\section{Key Practice Points:}

- Children with DCD show poorer tactile perception than children with TD.

- Tactile performance predicts fine motor accuracy and speed in children with DCD.

- Routine tactile assessment should be performed for children with DCD who display fine motor deficits.

\section{A CLINICAL CASE STUDY ON THE USE OF THE EQUIBAND TM SYSTEM AND DRY NEEDLING TO IMPROVE RANGE OF MOTION AND SPINAL POSTURE}

\section{Cruickshank SE}

Vetphysio Limited, New Zealand

This single case study was designed to examine the effectiveness of the use of the Equiband ${ }^{T M}$ System and dry needling incorporated into a specific prescribed exercise programme for a 22 year old Dutch Warmblood gelding who was 'turned out' (had not received any exercise or riding) for over 3 months.

The Equiband ${ }^{\mathrm{TM}}$ System was developed to strengthen the core musculature of the horse and to improve the horse's ability to work in a round outline. It also reduces the risk of pain and injury due to extension of the back in the ridden horse and or instability of the spinal joints by specifically stimulating receptors in the skin and hair follicles and thereby activating the abdominal, sub lumbar and deep neck and back muscles.

Dry needling is a treatment technique that is well described in physiotherapy literature to treat muscular trigger points, improve range of motion and reduce pain, however very little exists with regards to the treatment of horses with dry needling. In this clinical case study dry needling was used as the treatment technique to ensure that the equine subject could achieve full spinal range of motion and had no active or latent trigger points through the spine prior and during the completion of a specific prescribed exercise programme utilising the Equiband ${ }^{\mathrm{TM}}$ System The programme was prescribed by a physiotherapist to improve and strengthen the core musculature and improve the horses ability to work and maintain a rounded outline, and to improve the horses resting spinal flexion and spinal range of motion. 


\section{COMPARISON OF THE PELVIC LIMB MUSCULATURE AND MOMENT ARMS OF THE QUARTER HORSE AND THE ARAB}

Crook TC, Cruickshank SE, McGowan CM, Stubbs N, Wakeling JM, Hodson-Tole E, Wilson AM, Payne RC

The Royal Veterinary College and University of Liverpool, UK Vetphysio Limited, New Zealand

Due to the differing breed characteristics of the Quarter Horse (bred for rapid acceleration) and Arab (bred for endurance) it was hypothesised that a difference would be demonstrated in muscle architecture and that Quarter Horse hind limb extensor muscles would have longer moment arms when compared to the Arab, conferring a greater potential for torque generation at the hip, stifle and tarsus during limb extension. Studies into the form and function of the leg muscles in human sprint and endurance runners have demonstrated that differences exist in their muscle architecture. It is not known whether similar differences exist in the horse. Rapid acceleration as seen in Quarter Horse racing requires fast powerful muscle contraction and generation of large joint torques, particularly by the hind limb muscles. Six Quarter Horse and six Arab hind limbs were dissected and examined to determine muscle mass and architecture of seven hindlimb muscles. Specifically, muscle mass, fascicle length and pennation angle were quantified and physiological cross-sectional area (PCSA) and maximum isometric force were estimated. Muscle moment arm lengths were determined for six hindlimb muscles. The hind limb muscles of the Quarter Horse were of a significantly greater mass, but had similar fascicle lengths and pennation angles when compared with those of the Arab; this resulted in the Quarter Horse hind limb muscles having greater PCSAs and hence greater isometric force potential. This study suggests that the Quarter Horse possesses large strong hind limb muscles, with the potential to accelerate their body mass more rapidly than those of the Arab. The moment arms of biceps femoris (acting at the hip) and gastrocnemius lateralis (acting at the stifle) were significantly longer in the Quarter Horse, although the length of the remaining muscle moment arms were similar in both breeds of horse. All the Quarter Horse muscles were capable of generating greater muscle moments owing to their greater physiological cross-sectional area (PCSA) and therefore greater isometric force potential, which suggests that PCSA is a better determinant of muscle torque than moment arm length in these two breeds of horse and that Arab hind limb muscles are optimised to function at maximum economy rather than maximum power output.

\section{ULTRASOUND IMAGING: RELIABILITY OF MEASUREMENTS OF ABDOMINAL AND MULTIFIDUS MUSCLE THICKNESS AND MULTIFIDUS CROSS-SECTIONAL AREA OF ADULTS AGED 50-79 YEARS}

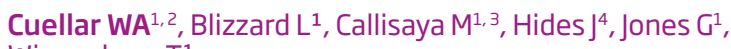
Winzenberg $\mathrm{T}^{1}$

1Menzies Institute for Medical Research, University of Tasmania, Hobart 2School of Medicine, University of Tasmania, Hobart

${ }^{3}$ Southern Clinical School, Monash Medical Centre, Monash University, Clayton ${ }^{4}$ Australian Catholic University, Brisbane

Question: What is the procedural reliability of the measurement of abdominal and multifidus muscle thickness and cross-sectional area from ultrasound imaging of older adults?

Design: Intra-examiner procedural reliability study with repeated measurements one week apart.

Participants: Twenty-three older adults (70\% male, age range 5175 years) participating in a randomised controlled trial of vitamin D supplements for knee osteoarthritis.

Outcome Measures: Ultrasound images of abdominal and multifidus muscle thickness and multifidus cross-sectional area

Results: Reliability of abdominal muscle thickness measurements both at rest and contracted, and of multifidus cross-sectional area, were substantial (ICC 0.81-1.0). Reliability of multifidus measurements of muscle thickness at rest and contracted ranged from fair to substantial (ICC 0.55-0.86).

Conclusion: Abdominal muscle thickness and multifidus cross-sectional area of older adults can be measured using ultrasound imaging with similar reliability to that for younger adults. Reliability of multifidus muscle thickness at L4-5 level was lower than that reported previously for older adults.

\section{Key Practice Points:}

- It is possible for an experienced examiner to measure with high consistency the abdominal muscle thickness and multifidus crosssectional area of older adults using ultrasound imaging.

- Reliability of measurement of multifidus muscle thickness was lower than previously reported for L4-5 spinal levels of older adults.

- These results support the use of ultrasound imaging as a reliable tool for the assessment of the abdominal and multifidus muscles of older adults in clinical practice and for research.

\section{OVERCOMING BARRIERS TO THE MOBILISATION OF PATIENTS IN AN INTENSIVE CARE UNIT: A FOUR-PART QUALITY IMPROVEMENT PROJECT}

Dafoe $\mathrm{S}^{1}$, Chapman $\mathrm{M}^{2}$, Edwards $\mathrm{S}^{3}$, Stiller $\mathrm{K}^{4}$

IPrincipal Physiotherapist, Acute Care and Surgery, Physiotherapy Department, Royal Adelaide Hospital, Central Adelaide Local Health Network, Adelaide

2Director of Research and Senior Consultant Intensive Care Unit, Royal Adelaide Hospital, Central Adelaide Local Health Network, Adelaide ${ }^{3}$ Statistician, Data Management and Analysis Centre, University of Adelaide, Adelaide

${ }^{4}$ Allied Health Research Co-ordinator, Central Adelaide Local Health Network, Adelaide

Questions: Can the frequency of mobilisation of patients in an intensive care unit (ICU) setting be increased with the implementation of simple strategies?

Design: A four-part quality improvement project was undertaken. Parts 1 and 4 involved prospective audits documenting mobilisation frequency Part 2 was a staff survey. Part 3 involved identifying barriers potentially amenable to short-term change and the implementation of strategies to overcome these.

Participants: Audit participants (Parts 1 and 4 ) comprised all patients admitted to the Royal Adelaide Hospital ICU over two four-week periods. For the survey (Part 2) participants comprised ICU staff. Part 3 involved ICU staff.

Interventions: Strategies to address perceived barriers to mobilisation. Outcome Measures: Mobility outcomes were compared between audits.

Results: Patient- and institutional-related factors were perceived by staff as having the greatest impact on the mobilisation of patients in our ICU. Inadequate staff education, inter-disciplinary communication and leadership were identified as barriers to mobilisation that were potentially amenable to short-term change and, over a three-month period, various strategies were implemented to address these. Multivariable analyses revealed that mobility outcomes did not significantly change between the baseline and follow-up audits.

Conclusion: Relatively simple strategies to improve staff education, interdisciplinary communication and leadership were ineffective in the shortterm at improving mobility outcomes of patients in an ICU setting that already had a proactive stance regarding mobilisation. Other strategies (e.g. changing sedation practices, increased staffing/resources) may be required to improve mobility outcomes.

Trial registration: ANZCTR 12613001092785

\section{Key Practice Points:}

- Patient- and institutional-related barriers most often prevented mobilisation of patients in an ICU setting.

- Strategies to improve staff education, inter-disciplinary communication and leadership were ineffective in the short-term at improving mobility outcomes in an ICU with a relatively proactive stance regarding mobilisation.

- Other strategies, such as changing sedation practices and/or increased resources (e.g. staffing, equipment) may be necessary to increase the frequency and level of mobility. 


\section{STAFF PERCEPTIONS OF THE BARRIERS TO MOBILISING PATIENTS IN AN INTENSIVE CARE UNIT: A SURVEY}

Dafoe $\mathbf{S}^{1}$, Stiller $\mathrm{K}^{2}$, Chapman $\mathbf{M}^{3}$

${ }^{1}$ Principal Physiotherapist, Acute Care and Surgery, Physiotherapy Department, Royal Adelaide Hospital, Central Adelaide Local Health Network, Adelaide

${ }^{2}$ Allied Health Research Coordinator, Central Adelaide Local Health Network, Adelaide

${ }^{3}$ Director of Research and Senior Consultant, Intensive Care Unit, Royal Adelaide Hospital, Central Adelaide Local Health Network, Adelaide

Questions: What barriers do intensive care unit (ICU) staff perceive as impacting on the mobilisation of patients in an ICU setting? Do patientrelated, institutional-related or other barriers have the most impact on mobilisation?

Design: A survey was undertaken as part of a larger quality improvement project.

Participants: 93 permanent/semi-permanent medical, nursing and physiotherapy staff working in the Royal Adelaide Hospital ICU.

Outcome Measures: A purpose-designed survey that investigated staff perceptions of the barriers to the early progressive mobilization of patients in an ICU setting was developed. The survey mainly comprised closed statements requiring responses using a visual analogue scale. Barriers to mobilisation were divided into patient-related, institutional-related and other

Results: Overall, patient-related barriers (e.g. haemodynamic instability, reduced level of consciousness, sedation, agitation, impending medical procedure) were perceived as most frequently impacting adversely on the mobilisation of patients in an ICU setting. Institutional-related barriers (e.g. staff/equipment availability, time constraints) were also perceived as frequently preventing mobilisation. Other barriers to mobilisation (e.g. poor ICU culture, lack of leadership, poor inter-disciplinary communication) were less frequently perceived as impacting adversely on mobilisation.

Conclusion: ICU staff perceived that patients' medical condition and resource limitations most frequently impacted adversely on the mobilisation of patients in an ICU setting.

Trial registration: ANZCTR 12613001091796

\section{Key Practice Points:}

- Barriers to the mobilisation of patients in an ICU setting are multi-factorial.

- Patient-related barriers were perceived as most often preventing mobilisation, followed closely by institutional-related factors.

\section{ADVANCED PRACTICE MUSCULOSKELETAL PHYSIOTHERAPY COMPETENCIES FOR PAIN SERVICE DELIVERY}

\section{Daly $A E^{1}$}

${ }^{1}$ Pain Service, Health and Independence Programs, Austin Health, Heidelberg, VIC, Australia

Background and Aims: The Australian Physiotherapy Association describes advanced practice as 'a role that is within the currently recognised scope of practice that may require additional training as well as significant professional experience and competency development' Advanced practice roles present an opportunity for pain Services to take full advantage of existing musculoskeletal physiotherapists who specialise in pain management. The aims of this presentation are to demonstrate the expected breadth of 'pain' knowledge of advanced practice musculoskeletal physiotherapists and to introduce a competency standard, cumulative assessment tool and self-directed learning modules, thereby creating a learning hierarchy for physiotherapists working within or towards an advanced practice role in pain services.

Methods: The competency standard and cumulative assessment tool were determined by combining the Advanced Practice Musculoskeletal Physiotherapy: A Clinical Education and Competency Framework (2012) with the Australian Standards for Physiotherapy (2006) and National Common Health Capability Resource (2013) and included a review of relevent literature. The self directed learning modules were developed from the International Association for the Study of Pain Curricula (2012 2014) and also included an extensive review of the relevant literature. Where appropriate, multidisciplinary subject matter experts to reviewed the content of all documents.

Results: All documents are available on the Victorian Department of Health and Human Services website. These documents are dynamic and open to review.

Conclusions: The role that appropriately experienced and credentialed musculoskeletal physiotherapists can fulfil in Pain Services provides affordable and accessible opportunities to promote excellence in patient care, improve job satisfaction for individual physiotherapists, staff retention and succession planning for health services.

\section{Key Practice Points:}

- Advanced Practice roles demand additional training as well as significant professional experience, development of new competencies and demonstration of existing ones.

- For physiotherapists with an interest in Pain Service delivery, a set of documents now exist to guide their professional development.

\section{THE DEVELOPMENT OF A PERSISTENT PAIN STRATEGY FOR COMPENSABLE CLIENTS IN VICTORIA: OPPORTUNITIES FOR BOTH PUBLIC AND PRIVATE SECTOR PHYSIOTHERAPISTS}

\section{Daly $\mathrm{AE}^{1}$}

${ }^{1}$ Transport Accident Commission and WorkSafe Victoria, Health and Disability Strategy Group, Melbourne

Background and Aims: Persistent pain has a high prevalence amongst compensable injuries and has a significant impact on the recovery of TAC clients and injured workers; with up to $21 \%$ of clients impacted by persistent pain. Along with the financial, emotional and social costs to the person, there is an average claim liability cost more than double the average of all claims costs. The purpose of the Persistent Pain Strategy is to describe the strategic goals and initiatives necessary to improve the health, work and independence outcomes of TAC clients and injured workers at risk of, or with persistent pain. The aims of this presentation are to present the overall strategy and specifically the rehabilitation treatment suite to colleagues, provide updated data on existing initiatives within the strategy, specifically Network Pain (multidisciplinary pain management) and to highlight the opportunities for Physiotherapists to adopt key roles in this work.

Methods: Key activities that informed the Strategy's development included consultations with 40+ internal and external stakeholders, environmental scanning, review of evidence provided by stakeholders and extensive internal data review across both organisations.

Results: Five strategic themes (involving 16 separate initiatives) were formulated that informed an enhanced rehabilitation treatment suite. The Network Pain initiative was found to be cost neutral within 12 months.

Conclusions: A Persistent Pain strategy aimed at optimising the recovery and independence of TAC clients and injured workers in Victoria has been developed after broad consultation. Dynamic implementation and evaluation will be key to the strategy's success in achieving the desired outcomes.

\section{Key Practice Points:}

- Persistent pain is common, but not inevitable, following injuries sustained in compensable circumstances.

- A suite of services have been identified and, in part, implemented that match individual client's needs at appropriate periods in their rehabilitation.

- Opportunities exist for physiotherapists to be major contributors to the health, work and independence outcomes of compensable clients with persistent pain 
THE REVIEW AND DEVELOPMENT OF THE ACCREDITATION STANDARD FOR ENTRYLEVEL PHYSIOTHERAPY IN AUSTRALIA: A PROJECT REPORT

Davidson $\mathrm{M}^{1}$, Nickson WM${ }^{1}$, Chipchase LS²

${ }^{1}$ Health Workforce Consulting Pty Ltd, Melbourne

${ }^{2}$ Australian Physiotherapy Council Limited, Melbourne

Project Objectives: To review the existing standards and to develop a contemporary, stakeholder supported set of accreditation standards for entry-level physiotherapy programs in Australia.

Project Governance: The Australian Physiotherapy Council Limited (The Council) is appointed by the Physiotherapy Board of Australia as the external accreditation authority for physiotherapy under the Health Practitioner Regulation National Law Act 2009. Health Workforce Consulting Pty Ltd was engaged by The Council to conduct the project, guided by an External Reference Group.

Project Method: A consultation paper was released to explain the project, to gauge satisfaction with the current standards and to canvass opinions on relevant issues. Wide-ranging consultation was conducted and feedback, together with a desktop review of accreditation standards for higher education, registered health disciplines in Australia, and physiotherapy internationally, was used to inform the writing of the first draft of the new Accreditation Standards. Stakeholder feedback on the first draft was used to further refine the standards. Feedback on the second draft informed the final version of the Accreditation Standards to be approved by The Council.

Project Outcomes: The Accreditation Standards for Entry-Level Physiotherapy are consistent with the Standards for Higher Education and are aligned with the Physiotherapy Practice Thresholds in Australia and Aotearoa New Zealand. The new standards reflect contemporary expectations of quality education and to ensure that graduates of entrylevel physiotherapy programs meet the Physiotherapy Practice Thresholds. The new standards are organised to facilitate efficient preparation and assessment of the evidence required for accreditation of entry-level physiotherapy programs.

\section{EXPLORING THE FACTORS INFLUENCING JOB SATISFACTION AND CAREER DEVELOPMENT OF PHYSIOTHERAPISTS WORKING IN PRIVATE PRACTICE}

Davies JM, Debenham J, Edgar S

'University of Notre Dame, Fremantle

Question: What factors influence the job satisfaction of physiotherapists in private practice, and do these factors change based on the career stage of the individual?

Design: Qualitative case study design utilising focus groups.

Participants: Sixteen participants allocated to 3 focus groups including new graduates $(n=6)$, post graduates $(n=5)$ and practice owners $(n=5)$,

Outcome Measures: Focus groups were transcribed verbatim and a thematic analysis was undertaken to determine themes and subthemes from each focus group.

Results: Each group had differing views in regards to issues surrounding peer support and mentoring, professional development and career progression. In particular, issues surrounding the new graduate experience in private practice were explored, with all groups noting lack of support and financial pressures were of concern.

Conclusion: Results identified the need for universities to consider weighting the physiotherapy curriculum more towards private practice education given that participants felt new graduates were unprepared to work in this sector. Results also highlighted that the career progression of private practice physiotherapists is extremely varied and appears to lack any structured career pathway, suggesting a need to streamline the profession.

\section{Key Practice Points:}

- Peer support, mentoring and professional networks improve job satisfaction

- The ethical dilemma of making money versus delivering best patient care is difficult to navigate

- New graduates need support upon entering the private practice workforce

- There should be consideration of a structured career pathway for physiotherapists working in private practice
HOW TO: INTEGRATE THE PRINCIPLES OF GOOD WORK DESIGN THROUGHOUT THE HEALTHCARE FACILITY PROJECT LIFECYCLE

\section{Davies KN}

Children's Health Queensland Hospital and Health Service, Brisbane

Background: The Lady Cilento Children's Hospital with 359 beds, is the largest paediatric hospital in Australia. In November 2014, the purpose built hospital brought together two existing hospitals which shared more than 200 years of experience providing paediatric care.

Objectives: The purpose of this 'how to' session is to share professional learning and analysis of how to achieve good work design, for future application by physiotherapists advising within healthcare facility projects.

Approach: This is a post-occupancy case study providing retrospective analysis of complex interactions, timeframes and requirements so as to propose a strategy to assist end user organisations to achieve safe, healthy and productive work and people, in the operational phase.

Conclusion: Good work design and human factors practice can be strategically embedded in each phase of the healthcare facility project lifecycle, to be addressed at the right time, by the right people.

\section{Key Practice Points:}

- Good work design protects workers from harm, promotes their health and wellbeing and optimises human performance, job satisfaction and productivity.

- Occupational health physiotherapists are well placed to provide expert advice to organisations to support good work design.

- Effective design of work considers the work; the physical working environment and the workers.

- The healthcare facility project lifecycle is prolonged and complex and therefore requires a strategic approach to good work design and human factors throughout.

\section{'WHAT COULD PHYSIOTHERAPY LOOK LIKE IN AGED CARE IN 2020' - Q \& A}

\section{Dawson $\mathrm{R}^{1}$, Turner $\mathrm{C}^{2}$, Grimley $\mathrm{R}^{3}$, Fu S}

${ }^{1}$ Agewell Physiotherapy

${ }^{2}$ Allied Connect

${ }^{3} 4$ F Project (Fits, Faints, Falls and Funny Turns)

${ }^{4}$ Mater Hospital community care representative

APA members will be able to hear the panel members views on the current aged care system, highlighting its strengths and weakness and outline their hopes for the future of physiotherapy practice in aged care.

\section{Points To Consider:}

- How can we address excellence and consumer led management in the current climate?

- New directions and initiatives

- What could the future of aged care look across all sectors?

- How can evidence based research influence APA advocacy efforts in this area? 


\section{AMOUNT OF PHYSICAL ACTIVITY VARIES DURING REHABILITATION ACCORDING TO HEALTH CONDITION, GROUPS AND TIMING OF REHABILITATION}

Dean $\mathrm{B}^{1}$, Gallagher $\mathrm{S}^{1}$, Scobie $\mathrm{E}^{2}$, Scrivener $\mathrm{K}^{1}$, Jones $\mathrm{TM}^{1}$, Dean $\mathrm{CM}^{1}$ ${ }^{1}$ Department of Health Professions, Macquarie University ${ }^{2}$ Royal Rehab, Ryde

Question: Does amount and intensity of physical activity during rehabilitation differ according to health condition, groups or timing of rehabilitation?

Design: Observational study of multidisciplinary rehabilitation which included group session (mobility, hydrotherapy, circuit) delivered over 7 days.

Participants: Six participants, 3 with orthopaedic and 3 with neurological conditions.

Outcome Measures: Amount of physical activity was measured as duration of upright time (min/day and \% day) and number of steps (\#/day and \%step goal). Intensity was measured as average heart rate (\%max) and rate of perceived exertion (RPE)

Results: In terms of amount of physical activity, neurological participants spent $8 \% /$ day (95\% Cl-16 to 0) less time upright, but achieved $22 \%$ (95\% CI 3 to 41 ) more of their expected steps than the orthopaedic participants During the mobility group, participants were upright 18\% (95\% Cl 37 to -1) more of the time than during the circuit groups and took 1986 (4182 to 209) more steps/hr than during the hydrotherapy group. Participants were upright for 261 (SD 73) and 236 (SD 97) min/day on weekdays and weekends respectively.

Conclusion: Neurological participants took more steps than orthopaedic participants for a similar upright time/day. The mobility group produced the highest amount of physical activity. There was no effect of health condition, group or timing of rehabilitation on intensity of physical activity.

\section{Key Practice Points:}

- Physical activity in rehabilitation meets current health guidelines.

- Health condition and group session both influence physical activity levels

- Physical activity data should be collected and used to guide service improvements.

\section{NO LONGER ALONE AND INACTIVE, HIGH ACTIVITY LEVELS OBSERVED IN A NEW REHABILITATION SERVICE}

Gallagher S1, Dean B ${ }^{1}$, Scobie E ${ }^{2}$, Scrivener $\mathrm{K}^{1}$, Jones TM${ }^{1}$, Dean $\mathrm{CM}^{1}$ ${ }^{1}$ Department of Health Professions, Macquarie University

${ }^{2}$ Royal Rehab

Question: What are the physical, cognitive and social activity levels of inpatients in a new transdisciplinary, private mixed rehabilitation service?

Design: Observational study using behavioural mapping techniques to record location, people present, body position and activities from 7am-8pm on two weekdays and one weekend day.

Participants: Eight participants, 3 with surgical, 3 with orthopaedic and 2 with neurological conditions.

Outcome Measures: Percentage of observations spent in any activity or physical, cognitive and social activities and percentage of observations both alone and inactive

Results: On average participants performed activity for $82 \%$ (SD 10), physical for $49 \%$ (SD 13), cognitive for $26 \%$ (SD 10), social for $40 \%$ (SD 19) of the day. Mean activity levels were higher on weekend (87\%) than weekdays (80\%). Overall, participants were observed both alone and inactive for $16 \%$ (SD 10 ) of the time, $17 \%$ of weekdays and $12 \%$ on the weekend.

Conclusion: The activity levels observed in this new rehabilitation service are higher than previously reported. This new transdisciplinary rehabilitation service which involves family/visitors and volunteers was able to promote high levels of activity.

\section{Key Practice Points:}

- High activity levels in rehabilitation are achievable.

- Alone and inactive observations can be reduced to less than $20 \%$

- Behaviour mapping data can be used to guide service improvements

\section{INTERVENTIONS TO IMPROVE AMBULATION AFTER STROKE}

\section{Dean $\mathrm{CM}^{1}$}

${ }^{1}$ Department of Health Professions, Faculty of Medicine and Health Sciences, Macquarie University

Being able to walk independently is a major determinant of whether an individual returns home following a stroke and has long lasting implications for their quality of life, community participation and physical activity levels. Physiotherapists frequently implement interventions to establish walking in non-ambulatory and improve walking in ambulatory stroke survivors. Research over the last two decades is providing clearer evidence that interventions need to be targeted. For example for the non-ambulatory person mechanically assisted walking with partial weight support via an overhead harness provides the opportunity to complete large amounts of walking practice. More evidence has become available that this form of intervention is effective at establishing more walking without detriment to the quality of walking. For those who can already walk, there is evidence from systematic reviews that treadmill walking and cueing of cadence are effective in improving walking speed and distance. There is also evidence that provision of walking sticks can increase walking speed. However there is emerging evidence that walking interventions are not equally effective, with some more effective in faster walkers. In this session, the key research findings and suggestions on how to use the latest evidence to guide intervention will be presented.

\section{Key Practice Points:}

- One size does not fit all - interventions to improve walking are not equally effective for all stroke survivors

- Interventions need to be tailored to the individual

- Walking speed should be used to tailor intervention

\section{PD WARRIOR PROGRAM EVALUATION: PARTICIPANTS' SATISFACTION, FEEDBACK AND HEALTH STATUS SURVEY}

Popiolkiewicz V1르. McConaghy $\mathrm{M}^{2}$, Scrivener $\mathrm{K}^{1}$, Dean $\mathrm{CM}^{1}$

${ }^{1}$ Department of Health Professions, Macquarie University ${ }^{2}$ Advanced Rehab Centre

Question: What is the level of satisfaction, feedback and health status of people with early stages of Parkinson's disease who commenced the PD Warrior Program?

Design: Survey

Participants: 68 participants aged 64 (SD 9) diagnosed with PD 4.8 years ago and who commenced the PD warrior program at Advanced Rehab Centre after 1 October 2013

Intervention: The PD Warrior program is a evidence-based, group exercise program specifically designed to be fun and target the impairments present in Parkinson's disease.

Outcome Measures: Satisfaction with and feedback on PD warrio program, current health status including falls, physical activity and selfefficacy.

Results: $87 \%$ of participants were either very satisfied or satisfied with the program, and $11 \%$ were neutral. 98\% reported the program was worth their time, $71 \%$ reported higher or much higher confidence in physical activity and $94 \%$ of participants would recommend the program. Health status and QoL were rated highly, however $42 \%$ were fallers and $20 \%$ had a high fear of falling. Participants completed 30 hrs/wk of total physical activity, but participants reported 4 (95 Cl 0 to 7 ) hrs/wk less incidental and 1.3 (0.2 to 2.6) hrs/week more planned activity than health elderly.

Conclusion: Participants reported high levels of satisfaction with the PD warrior program. Falls are an ongoing issue for participants with mild PD.

Key Practice Points:

- Participants of the PD Warrior program report high levels of satisfaction

- Almost all participants (94\%) would recommend the program

- Health, falls and activity screening data can guide program improvements 


\section{MENTAL HEALTH AND PHYSIOTHERAPY: WHAT CAN PHYSIOTHERAPISTS DO?}

\section{Dear B}

eCentreClinic, Department of Psychology, Macquarie University, Sydney, Australia

Dr Blake Dear will expand on the mental health consequences of health and disability related worklessness. He will provide some 'clinic ready' tips to assist your clients to prepare for entry back into the workforce whilst managing chronic health conditions.

Anxiety and depression are very common with approximately 1 in 5 Australians experiencing significant symptoms each year. These conditions are more prevalent among adults with chronic health conditions and are a significant cause of disability and worklessness themselves. Unfortunately, research also indicates very few adults with these conditions seek treatment. This presentation will briefly the importance of anxiety and depression to physiotherapy practice and some practical 'tips' for identifying and supporting patients with anxiety and depression.

\section{INCREASING ACCESS TO PAIN MANAGEMENT PROGRAMS USING THE INTERNET}

\section{Dear B}

eCentreClinic, Department of Psychology, Macquarie University, Sydney Australia

Dr Blake Dear will contribute as a Psychologist with special expertise in the development and delivery of innovative online e-health programs for anxiety, depression and persistent pain.

There is strong evidence that timely access to multidisciplinary pain management can significantly reduce the disability, anxiety and depression associated with chronic pain. However, access to multidisciplinary pain management programs is significantly limited, both nationally and internationally. Common barriers to these programs include the direct and indirect costs, mobility limitations, long waitlists, distance and their decreasing availability outside of metropolitan areas. This has led severa research groups to explore the potential of using the internet to deliver the psychological component of pain management programs as a way of increasing access to treatment. This presentation will briefly describe the results of several Australian clinical trials examining the efficacy of an internet-delivered pain management program.

\section{HIP MUSCLE STRENGTH IN PEOPLE WITH KNEE OSTEOARTHRITIS: A SYSTEMATIC REVIEW WITH META-ANALYSIS}

Deasy M, Leahy E, Semciw A

Question: Is hip muscle strength weaker in people with knee OA when compared to a healthy control?

Design: Systematic review with meta analysis of cross-sectional data.

Participants: Participants with radiological and symptomatic osteoarthritis were included. Only studies with a healthy control were included.

Outcome Measures: Muscle strength dynamometry and isokinetic machines that measured isometric and isokinetic peak strength respectively.

Results: Five studies were identified and three could be included in meta-analysis. Pooled results from three studies indicate significantly weaker peak isometric hip abduction torque in patients (SMD 0.62,95\%

$\mathrm{Cl} 0.16$ to 1.08); and data from 2 studies indicate no difference in isometric peak adduction torque (SMD 0.34,95\% Cl -0.89 to 1.57). Data could not be pooled for other isometric hip actions. Pooled data from 2 studies indicate significantly weaker isokinetic hip strength in all directions $\mathrm{p}<0.002$ ).

Conclusion: Hip muscle weakness is present in people with knee OA Further work is required to identify if targeted strategies can improve and/ or prevent hip weakness in people with knee OA.

\section{Key Practice Points:}

- Hip muscle strength should be assessed in people with Knee OA

- Targeted hip strengthening programs may should be prescribed in people with knee $\mathrm{OA}$

\section{AN INTRODUCTION TO BLOOD FLOW RESTRICTION TRAINING: WHAT IT IS, WHAT IT ISN'T AND WHY PHYSIOTHERAPISTS SHOULD CARE}

\section{Demase B]}

Background: Physiotherapists often look to increase patients' muscle tone and strength as these are key components of maintaining a healthy physical capacity. Traditionally the scientific literature has shown that only exercise performed against high levels of resistance produces any meaningful gains in skeletal muscle size and strength. This of ten presents a problem for the Physiotherapist as large sectors of the patient population are unable to perform such exercise for various reasons. Therefore, any method of exercise which could produce similar increases in muscle size and strength to that of high load training, yet only required extremely low levels of resistance which almost any patient could perform, would be of immense value to physiotherapy as an intervention. Blood flow restriction training provides a cutting edge and uniquely beneficial mode of exercise producing strength gains using low loads which are analogous to those normally seen only with high load training.

Aim: To introduce the concept of BFR as an important new intervention in physiotherapy.

Approach: A how-to workshop is to be performed giving attendees both the evidence-based theories behind blood flow restriction training, along with practical instructions and demonstrations as to how to perform this type of training with different patients in the clinic.

\section{Key Practice Points:}

- Exercise against extremely low levels of resistance can produce similar results to those of high levels of resistance when performed under conditions of blood flow restriction

- Blood flow restriction training has over forty five supporting studies in the peer-reviewed literature

\section{MACHINES THAT GO "PING" MAY IMPROVE BALANCE BUT MAY NOT IMPROVE MOBILITY OR REDUCE RISK OF FALLS: A SYSTEMATIC REVIEW}

Dennett $\mathrm{AM}^{1,2}$, Taylor NF2

${ }^{1}$ Eastern Health, Allied Health Clinical Research Office, Box Hill ${ }^{2}$ La Trobe University, Faculty of Health Sciences, Bundoora

Question: Do computer-based electronic devices that provide feedback improve mobility, balance and reduce falls?

Design: Systematic review with meta-analysis of randomised trials.

Participants: Adults, including young healthy or community dwelling older people with or without a primary health condition (eg.stroke)

Intervention: Computer-based electronic devices that provide some form of feedback.

Outcome Measures: A variety of clinical and computerised tests was used to measure balance, activity limitation, falls efficacy and falls risk. These included, but were not limited to the Clinical Test of Sensory Integration of Balance, 10 m walk test, Falls Efficacy Scale and Physical Profile Assessment Score.

Results: Thirty trials were included. Computerised devices improved dynamic balance in people with a neurological condition compared with no therapy (Standardised Mean Difference 0.28, 95\% Cl 0.01 to 0.55). Computerised devices may have no significant effect on mobility, falls efficacy and falls risk in any populations when compared to no therapy or physiotherapy modalities.

Conclusion: There is high quality evidence that computerised devices that provide feedback may be useful in improving balance in people with neurological conditions when compared to no therapy but may not be as effective in causing more meaningful changes to mobility and falls risk.

\section{Key Practice Points:}

- Computer-based electronic devices maybe useful for improving dynamic balance in people who have a neurological condition.

- Computer-based electronic devices may not be useful for improving balance, mobility, confidence and falls risk in other populations and thus should not be used exclusively for these purposes for rehabilitation. 


\section{SECONDARY PREVENTION OF CHRONIC HEALTH CONDITIONS IN THOSE WITH MULTI-MORBIDITY - WHAT CAN PHYSIOTHERAPISTS DO?}

\section{Dennis SM}

Discipline of Physiotherapy, Faculty of Health Sciences, University of Sydney

The prevalence of multi-morbidity is increasing in Australia and is a burden for both the Australian health care system and patients and their carers.

The health system has been designed to manage single conditions and this can add to the difficulties that patients with multi-morbidity face. Research has also focused on single conditions and patients with complex health problems have often been excluded. Physiotherapists have an important role to play in the secondary prevention and management of people with multi-morbidity. Often, this has been provided by physiotherapists in the hospital and community setting. Increasingly multi-morbidity is managed in primary care by general practitioners where access to adequate allied health services is limited. The challenge for physiotherapy is design and test interventions to address the problems faced by people with multimorbidity to improve patient outcomes and to explore how physiotherapy can be better integrated into primary care to prevent expensive hospital admissions for people with multi-morbidity.

\section{Key Practice Points:}

- To consider the impact of multi-morbidity on patients in the context of a health system designed around the needs of single conditions.

- To discuss the growing emphasis on multi-morbidity in primary care and the opportunities for physiotherapy to improve patient outcomes.

\section{DEEP CERVICAL EXTENSOR MUSCLE DIMENSIONS CHANGE UNDER CONTRACTION AND CAN BE MEASURED RELIABLY USING VIDEO REAL TIME ULTRASOUND}

Desa VG르, Leaver $A^{1}$, Shirley $D^{1}$, Clarke ${ }^{2}$, Rebbeck $T^{1}$ ${ }^{1}$ Discipline of Physiotherapy, Faculty of Health Sciences, The University of Sydney

${ }^{2}$ Discipline of Medical Radiation Sciences, Faculty of Health Sciences, The University of Sydney

Question: How reliable is it to measure the deep cervical extensor muscles using video real time ultrasound and how do the muscle dimensions change under contraction in asymptomatic participants?

Design: Observational study with assessor blinding.

Participants: Twenty-two participants without neck pain.

Intervention: The deep cervical extensors were imaged using real time ultrasound at the C4 level at rest and under a low-load contraction. Thickness and width of these muscles were subsequently measured by independent raters for right and left sides.

Outcome Measures: Reliability was determined using Intraclass correlation coefficients. Mean differences and percent change between relaxed and contracted states were calculated.

Results: Thickness measurements of the deep cervical extensors displayed excellent intra-rater reliability in both the relaxed and contracted states (e.g. left ICC 0.91; Cl 0.78 to 0.96) and good to excellent inter-rater reliability for the relaxed and contracted states (e.g. right relaxed ICC 0.93; Cl 0.86 to 0.97). Width measurements of the deep cervical extensors displayed poor intra-rater and inter-rater reliability for both states (e.g. left relaxed ICC $0.58 ; \mathrm{Cl}-0.04$ to 0.83 ). Thickness increased significantly with contraction up to $7.25 \%$ on the right $(p=0.01)$. Width dimensions did not significantly change.

Conclusion: Thickness of the deep cervical extensors can be reliably measured between and within raters, and appears to increase under low load contraction in asymptomatic individuals. Width measurements were not reliable with this protocol, hence revision is required.

\section{Key Practice Points:}

- Clinicians can use video ultrasound to measure the change in thickness of cervical deep extensors under contraction.

- Clinicians should not yet use this protocol for measuring change in width. - The thickness increases in asymptomatic individuals by up to $7 \%$.
SENSORIMOTOR CONTROL IN PEOPLE WITH INSIDIOUS ONSET NECK PAIN AND HEALTHY INDIVIDUALS: A SYSTEMATIC REVIEW

de Zoete RMJ' ${ }^{1}$, Osmotherly PG ${ }^{1}$, Rivett DA ${ }^{1}$, Farrell SF${ }^{1}$, Snodgrass SJ ${ }^{1}$ ${ }^{1}$ School of Health Sciences, The University of Newcastle, Callaghan

Question: Can sensorimotor control be identified and quantified in people with insidious onset neck pain compared to healthy individuals?

Design: Systematic review of English and Dutch language observational and intervention studies, identified through searching the databases MEDLINE, CINAHL, EMBASE, AMED and SportDiscus.

Participants: Individuals with insidious onset neck pain and healthy individuals.

Outcome Measures: Sensorimotor control is measured through various tests: joint position error (JPE), postural sway, subjective verticality/horizontality, continuous linear movement, The Fly ${ }^{\circledR}$, head steadiness and smooth pursuit.

Results: In included studies ( $n=51$ ), sensorimotor control was most commonly assessed by JPE (expressed as angular or distance error) and postural sway (expressed as sway area or distance). Means for neck rotation JPE ranged from 2.2-9.8 and 4.52-5.21 cm in people with neck pain, and $1.36-6.0^{\circ}$ and $2.69-$ $4.5 \mathrm{~cm}$ in healthy individuals. Means for flexion-extension JPE ranged from 5.3-6. $3^{\circ}$ and 5.55-5.62 cm in people with neck pain, and $1.2-5.1^{\circ}$ and $2.54-4.9 \mathrm{~cm}$ in healthy individuals. Mean sway areas for eyes open ranged from 4.85 $10.5 \mathrm{~cm}^{2}$ (neck pain) and 3.50-6.6 $\mathrm{cm}^{2}$ (healthy), and $2.51-16.6 \mathrm{~cm}^{2}$ (neck pain) and $2.74-10.9 \mathrm{~cm}^{2}$ (healthy) for eyes closed. Mean sway distance was reported for 54 people with neck pain in one study $(0.45 \mathrm{~cm}$ anteroposterior; $0.20 \mathrm{~cm}$ mediolateral, eyes open). In healthy individuals, anteroposterior distance ranged from $0.40-2.33 \mathrm{~cm}$ (eyes open) and from $0.44-3.0 \mathrm{~cm}$ (eyes closed), and one study reported a mediolateral distance of $0.15 \mathrm{~cm}$ (eyes open and closed). Methods varied considerably in the remaining 16 studies.

Conclusion: JPE and postural sway testing show poorer scores for people with insidious onset neck pain compared to healthy individuals.

\section{Key Practice Points:}

- Sensorimotor control in people with insidious onset neck pain is most commonly assessed through JPE and postural sway testing, with greater mean scores (i.e., worse sensorimotor control) in people with insidious onset neck pain compared to healthy individuals.

- Greater JPE and postural sway mean scores suggest sensorimotor control is disturbed in people with insidious onset neck pain.

- Other tests for sensorimotor control are not sufficiently evaluated to be recommended in clinical practice at this time.

\section{EARLY INTERVENTION INJURY PREVENTION PROGRAM FOR WORK- RELATED MUSCULOSKELETAL DISORDERS IN POULTRY MEAT PROCESSING WORKERS: DOES IT AFFECT COMPENSATION OUTCOMES?}

Donovan $\mathrm{M}^{1}$, Khan $\mathrm{A}^{1}$, Johnston $\mathrm{V}^{1}$

${ }^{1}$ The University of Queensland, Brisbane

Question: Does an onsite early intervention injury prevention program reduce compensation outcomes from work-related musculoskeletal disorders in poultry meat processing workers?

Design: Secondary pre-post analysis of the meatworks' compensation data from 1 July 2008 to 30 June 2012 with the intervention commencing 1 July 2010. Participants: Employees at a meatworks plant in Queensland during the study period who lodged workers' compensation claims for work-related musculoskeletal disorders $(n=595)$

Intervention: An onsite early intervention program which included immediate reporting, reassurance, multidisciplinary participatory consultation, workplace modifications and onsite physiotherapy.

Outcome Measures: Costs per claim, work days absent and rate of claims per million working hours at an individual claim level.

Results: Generalized linear model revealed a significant reduction in average costs per claim of $\$ 831$ (OR $=0.74 ; 95 \%$ CI 0.59 to 0.93; $p=0.009$ ) at post-intervention period. Median days absent was reduced by $37 \%(p=$ 0.024 ) with an $18 \%$ reduction in claims per million hours ( $p=0.017)$

Conclusion: The introduction of an early intervention injury prevention program demonstrated positive effects on compensation outcomes for work-related musculoskeletal disorders in poultry meat processing workers. Prospective studies are needed to confirm the findings of the present study within other industries.

\section{Key Practice Points:}

- Onsite physiotherapy involvement within a workplace-based intervention helped reduce compensation rates and costs and accelerate return to work. - Onsite physiotherapy assisted with early triage of musculoskeletal disorders.

- Further study is warranted to quantify physiotherapy contribution to such interventions. 


\section{LOAD MANAGEMENT IN ATHLETES: WHAT IS THE EVIDENCE AND WHERE DOES IT FIT IN AN INJURY PREVENTION PARADIGM?}

\section{Drew MK}

Department of Physical Therapies, Australian Institute of Sport,

Canberra, Australia

Australian Centre for Research into Injury in Sport and its Prevention (ACRISP) Federation University Australia, Ballarat Australia

Department of Physiotherapy, Faculty of Health, University of Canberra, Australia Training load has long been associated with injury risk. This presentation will present the results of a systematic review of the literature to qualify this statement. These findings will be presented in an epidemiological classification of injury prevention. Classification of prevention measures are either based on clinically-focussed pathology prevention or risk factor identification and mitigation. Clinically-focused pathology prevention encompasses primary, secondary and tertiary prevention which are aligned to the natural history of disease (the progression of the injury if the patient had not sought treated). Prevention based on risk factors can be administered in parallel through three categories. Universal prevention is targeted at eliminating generic risk factors for all individuals participating in a sport. Selective prevention is aimed at risk factors of a particular asymptomatic sub-group (for example, sex and age) with indicated prevention provided to non-symptomatic individuals exhibiting factors that increase their risks of injury above baseline levels. Load management when applied in injury free populations should encompass traits of primary prevention (pre-participation screening) in combination with universal and selective prevention measures. Where biological onset has occurred, is detectable and the individual is asymptomatic, secondary prevention and indicated prevention strategies are required. When symptomology, disability or incapacity has developed the individual is in the clinical phase and tertiary prevention measures are indicated.

\section{Key Practice Points:}

The learning outcomes from this session will contribute to participants practice by

- Providing the evidence for the relationship between training load and injury

- Establish a framework for clinicians to implement training load management as an injury prevention measure

\section{AUSTRALIAN FOOTBALL PLAYERS EXHIBIT PRIMARY HYPERALGESIA OF THE ADDUCTOR TENDON WHEN EXPERIENCING GROIN PAIN}

Drew MK ${ }^{1,2}$, Palsson TS ${ }^{3}$, Lovell G ${ }^{4}$, Chiarelli P1 ${ }^{1}$ Osmotherly PG ${ }^{1}$

${ }^{1}$ School of Health Sciences, Faculty of Health and Medicine, University of Newcastle, Australia

Department of Physical Therapies, Australian Institute of Sport, Canberra, Australia

${ }^{3}$ Laboratory for Musculoskeletal Pain and Motor Control, Center for Neuroplasticity and Pain (CNAP), Department of Health Science and Technology, Faculty of Medicine, Aalborg University, Denmark ${ }^{4}$ Department of Sports Medicine, Australian Institute of Sport, Canberra, Australia

Question: Do Australian (Rules) Football players have hyperalgesia when experiencing groin pain?

Design: Case-control design

Participants: Professional $(n=66)$ and semi-professional $(n=9)$ Australian football players were recruited with and without current or previous groin injuries were recruited during the pre-season through two AFL clubs.

Outcome Measures: Pain pressure thresholds (PPTs) were assessed at regional and distant sites using a handheld pressure algometer across four sites bilaterally (adductor longus tendon, pubic bone, rectus femoris, tibialis anterior muscle)

Results: Hyperalgesia was observed in the adductor longus tendon site (OR=16.27,95\%Cl 1.86 to 142.02). The ROC area under the curve of the regression model was fair ( $\mathrm{AUC}=0.76,95 \% \mathrm{Cl} 0.54$ to 0.83 ).

Conclusion: This study has shown that hyperalgesia exists in Australian football players experiencing groin pain indicating the need to assess mechanical thresholds as a component of the clinical assessment. Future research should evaluate other quantitative sensory testing parameters in athletes with experiencing groin pain in larger cohorts so subgrouping on diagnoses is possible.

\section{Key Practice Points:}

- Hyperalgesia of the adductor tendon exists in Australian Football players experiencing groin pain

- Pain pressure thresholds can discriminate players who are currently symptomatic with fair powe

- Clinicians working with Australian Football players should include pain pressure testing as a component of their screening protocols
INSPIRATORY MUSCLE TRAINING

FACILITATES WEANING FROM

MECHANICAL VENTILATION IN PATIENTS

IN THE INTENSIVE CARE UNIT: A SYSTEMATIC REVIEW

\section{Elkins $\mathrm{M}^{1}$, Dentice $\mathrm{RL}^{2}$}

${ }^{1}$ Department of Respiratory Medicine, Royal Prince Alfred Hopital, Sydney ¿Department of Physiotherapy, Royal Prince Alfred Hopital, Sydney

Questions: Does inspiratory muscle training improve inspiratory muscle strength in adults receiving mechanical ventilation? Does it improve the duration or success of weaning? Does it reduce length of stay, reintubation, tracheostomy, mortality, or the need for post-extubation non-invasive ventilation?

Design: Systematic review of randomised controlled trials.

Participants: Adults receiving mechanical ventilation.

Intervention: Inspiratory muscle training versus sham or no training.

Outcome Measures: Inspiratory muscle strength, breathing pattern, weaning success and duration, duration of mechanical ventilation, reintubation, tracheostomy, length of stay, use of non-invasive ventilation after extubation, survival and tolerability.

Results: Ten eligible studies involving 394 participants were identified. Meta-analyses showed that the training significantly improved maximal inspiratory pressure (MD $7 \mathrm{cmH20}, 95 \%$ Cl 5 to 9), weaning duration (MD 2.1 days, $95 \% \mathrm{Cl} 0.3$ to 4.0 , ) and weaning success (RR 1.34, 95\% Cl 1.02 to 1.76). Although only assessed in individual studies, significant benefits were also reported for the time on non-invasive ventilation after weaning (MD 16 hours, 95\% Cl 13 to 18), length of stay in ICU (MD 4.5 days, 95\% Cl 3.6 to 5.4) and length of stay in hospital (MD 4.4 days, $95 \% \mathrm{Cl} 3.4$ to 5.5). The other outcomes were not significantly affected.

Conclusion: Inspiratory muscle training in selected ICU patients facilitates weaning, with potential reductions in length of stay and duration of noninvasive ventilatory support after extubation.

\section{Key Practice Points:}

- Threshold devices and adjustment of ventilator trigger sensitivity are effective training methods.

- Training results hastens weaning from mechanical ventilation.

- Training shortens length of stay.

\section{AN ACTION RESEARCH PROJECT TO EXPLORE, IMPLEMENT AND EVALUATE THE USE OF REHABILITATION GUIDELINES FOR PHYSIOTHERAPY IN THE CRITICALLY ILL}

\section{Elliott $\mathbf{S}^{1}$}

${ }^{1}$ Medway NHS Foundation Trust, UK

Purpose: This study aims to investigate existing rehabilitation guidelines through a literature review, implement rehabilitation guidelines into critica care and evaluate their use.

Relevance: Rehabilitation in critical care has the potential to restore lost function and improve quality of life on discharge but patients are often viewed as 'too sick' to participate in physical rehabilitation. There is a need to standardise pathways for clinical decision making in early rehab so interventions are safe, timely and consistent.

Methods: Action research was selected as a methodology, and included several phases including assessing feasibility of using critical care guidelines, a literature review, implementing and evaluating the use of rehabilitation guidelines before modifying for future use.

Analysis: Evaluation of the implementation of the rehabilitation guidelines included simple statistics for quantitative data gained from the questionnaire. Content analysis was utilised for analysis of focus groups.

Results: All participants acknowledge that the use of rehabilitation guidelines in critical care are advantageous in supporting clinical reasoning and are especially useful for less experienced physiotherapists and decided to implement Stiller at al (2007) guidelines. However, six months after implementation $82 \%$ of the physiotherapists felt that the guidelines needed to be modified and made more specific to the hospital and should act as a prompt / checklist rather than strict guidelines that couldn't be deviated from.

Conclusion: Modified critical care rehabilitation guidelines have been developed for use by the physiotherapists. The physiotherapy team are now more confident in delivering a safe and consistent approach to rehabilitation for the critically ill patient. 
HOW TO IMPLEMENT STRATEGIES TO REDUCE HAND PAIN DURING MANUAL THERAPY TECHNIQUES

Evans $\mathbf{K}^{1,2}$, Tuttle $\mathbf{N}^{1,2}$

${ }^{1}$ School of Allied Health Sciences, Griffith University

2Menzies Health Institute Queensland

Background: A large number of physiotherapists develop pain in their thumbs or hands particularly associated with manual therapy assessment and treatment.

Aims / objectives: The aim of the session is to provide participants with strategies to reduce or prevent these symptoms. By the end of the session, the participants will 1) have strategies to reduce the force applied during assessment and treatment techniques and 2) perform one technique in a variety of ways to make it more suitable for individual therapists or patients.

Approach: Evidence will be presented for how less force than the average values reported in the literature can be effective in assessment and treatment of the cervical spine and that when therapists undergo training to become more precise in their techniques that the force employed can be reduced. A technique can be considered as a means to achieve an intention such as producing a particular movement or effect. The standard form of a technique including aspects such as hand position and posture provide a starting point for each technique. From that starting point, using one technique, participants will perform and modify that standard form in a variety of ways to suit their own (or their student's or patients) particularities to best achieve the intention with the minimum strain on their hands and thumbs.

\section{Conclusion / Key Practice Points:}

Participants will have strategies to

- Modify techniques to achieve their intention with less load on their hands

- Reduce the force applied during techniques while potentially increasing their effectiveness.

\section{CERVICAL MENISCOID MORPHOLOGY IN WHIPLASH ASSOCIATED DISORDER: A PRELIMINARY COMPARATIVE ANALYSIS}

Farrell SF${ }^{1}$, Osmotherly PG ${ }^{1}$, Cornwall $]^{2}$, Lau P ${ }^{3}$, Rivett DA ${ }^{1}$

${ }^{1}$ Discipline of Physiotherapy, School of Health Sciences, The University of Newcastle, Newcastle

2Departments of Anatomy and Physiology, and Faculty of Law, University of Otago, Dunedin, New Zealand

3Hunter New England Imaging and Department of Clinical Research and Pain Management, Newcastle

Question: This study investigated the size and composition of cervical meniscoids in people experiencing chronic whiplash associated disorder (WAD).

Design: Comparative observational study.

Participants: Seven volunteers (three female; mean age 40.7 years, SD 15.3 years) with grade II WAD lasting more than three months; seven age/ gender matched pain-free controls.

Outcome Measures: Participants underwent cervical spine magnetic resonance imaging. Each lateral atlantoaxial and zygapophyseal joint (C1/2-C6/7) was inspected for meniscoids. Meniscoid anterior-posterior length was measured and composition (adipose/fibrous/fibroadipose) inferred based upon signal intensities. Data were analysed using contingency tables and Wilcoxon signed-rank tests $(p \leq 0.05)$

Results: Meniscoids were identified in the WAD ( $\mathrm{n}=112$ ) and control ( $\mathrm{n}$ $=90)$ groups. At the lateral atlantoaxial joints, median meniscoid length was greater in the control group $(6.8 \mathrm{~mm})$ than WAD group $(5.7 \mathrm{~mm} ; \mathrm{p}=$ $0.014)$. There was a greater proportion of fibrous meniscoids in the WAD group, whereas the control group had a greater proportion of fibroadipose meniscoids (Fisher's exact $<0.001$ ).

Conclusion: Cervical meniscoids may be affected by a fibrotic process following WAD, which may contribute to the chronicity of this condition.

\section{Key Practice Points:}

- Cervical meniscoids are thought to contribute to pain in WAD, however pathoanatomical changes in these structures have not been previously investigated.

- Meniscoids in people following WAD appear more frequently composed of fibrous tissue, whereas in their age-matched counterparts meniscoids appear more frequently fibroadipose in composition.

- A possible fibrotic process of cervical meniscoids may contribute to the clinical presentation of chronic WAD.
VISUALISATION OF INTRA-ARTICULAR

SPINAL MENISCOIDS USING E12

SHEET PLASTINATION - A TOOL

FOR PHYSIOTHERAPY CLINICAL ANATOMY EDUCATION

Farrell SF${ }^{1}$, Osmotherly PG ${ }^{1}$, Rivett DA ${ }^{1}$, Cornwall ] ${ }^{2}$

${ }^{1}$ Discipline of Physiotherapy, School of Health Sciences, The University of Newcastle, Newcastle

2Departments of Anatomy and Physiology, and Faculty of Law, University of Otago, Dunedin, New Zealand

Question: Intra-articular meniscoids are thought to be involved in some spinal pain presentations. Meniscoids have been identified using dissection and imaging, however no studies have identified these structures using E12 sheet plastination. Moreover, these modalities are not generally available in physiotherapy clinical anatomy education. This study therefore sought to examine the presence of intra-articular meniscoids in the spines of cadaver sections that had undergone E12 sheet plastination to investigate their potential use as an educational aid.

Design: Observational anatomical study.

Participants: Two female cadavers (65, 86 years) that had undergone E12 sheet plastination ( $2 \mathrm{~mm}$ sagittal and transverse sections)

Outcome Measures: All 195 sections were photographed on a light box. Lateral atlantoaxial, cervical, thoracic and lumbar zygapophyseal joints were inspected under magnification to identify meniscoids.

Results: Ninety-two zygapophyseal and four lateral atlantoaxial joints were identified on 281 photographs. Meniscoids were observed in all cervical joints, $79 \%$ of thoracic joints and $80 \%$ of lumbar joints. In sagittal sections, 24 meniscoids were observed in cervical joints, and 32 meniscoids in the thoracic spine. In transverse sections, 14 meniscoids were identified in the thoracic spine and 12 meniscoids in the lumbar spine.

Conclusion: Meniscoids were visualised in all spinal regions on E12 sheet plastinates, at rates of prevalence comparable to previous reports using dissection and imaging. E12 sheet plastinates are cheaper and more accessible than dissection or imaging; this study confirms their potential usefulness as an educational tool in physiotherapy clinical anatomy education to convey the form and location of intra-articular spinal meniscoids.

\section{Key Practice Points:}

- Intra-articular spinal meniscoids can be effectively visualised on E12 sheet plastinates in sagittal and transverse sections.

- Intra-articular spinal meniscoids were identified at rates of prevalence comparable to previous reports using dissection and imaging.

- E12 sheet plastination can be utilised in physiotherapy clinical anatomy education to effectively convey the form and location of these clinically important structures. 
WHAT TOOL SHOULD BE USED TO

ASSESS DISABILITY ASSOCIATED

WITH GREATER TROCHANTERIC PAIN

SYNDROME (GLUTEAL TENDINOPATHY

AND BURSA PATHOLOGY)?

Fearon $\mathrm{AM}^{1,3}$, Ganderton $\mathrm{C}^{2}$, Scarvell $]^{1,3}$, Smith $\mathrm{P}^{1}$, Nash $\mathrm{C}^{4}$, Cook $]^{4}$

${ }^{1} T O R U, A N U @ T C H$

¿La Trobe University

3University of Canberra

${ }^{4}$ Monash University

Question: Is the VISA-G a valid and reliable instrument to measure the disability associated with greater trochanteric pain syndrome (GTPS).

Design: A four phase qualitative and quantitative study.

Participants: Symptomatic and asymptomatic participants meeting

eligibility criteria were enrolled. The first draft was tested on 72

symptomatic participants. The second draft was tested on 52 naïve

symptomatic and 31 asymptomatic participants.

Outcome Measures: Phase one: Literature review and item generation via in-depth interviews of stakeholders. Participants reviewed draft one for item and format clarification.

Phase two: Item reduction was undertaken via principal component analysis, scree plots and Spearman Rho correlation.

Phase three: Draft two was compared with existing scores for tendinopathies

Phase four: Draft two was tested for reliability (inter class co-efficient, ICC), internal consistency (Cronbach's Alpha), and construct validity (Spearman Rank correlation co-efficient) via comparison with the Harris Hip Score (HHS) and the Oswestry disability index (ODI).

Results: The 22 item first draft was reduced to an eight item second draft. The second draft had:

- a reliability of ICC2,1 (95\% Cl) of 0.827 (0.638 to 0.923)

- internal consistency of 0.809

- measured different constructs to the HHS and ODI (0.020 and 0.0205 respectively)

- demonstrated no floor or ceiling effects

Conclusion: Clinicians and researchers should use the VISA-G to measure the severity of disability associated with GTPS - gluteal tendon/ bursa pathology.

\section{Key Practice Points:}

The VISA-G is

- reliable and valid

- evaluates disability associated with GTPS

- evaluates different constructs than tools previously used to assess GTPS - the HHS and ODI

\section{EXERCISE VS. NON-PHARMACOLOGICAL INTERVENTIONS FOR PAIN AND QUALITY OF LIFE IN ADULTS WITH CHRONIC TENSION HEADACHES: A SYSTEMATIC REVIEW}

Ferrar $\mathrm{K}^{1,2}$, Gower $\mathrm{A}^{2}$, Graetz $]^{2}$, Guirguis $\mathrm{R}^{2}$, Hallbach $\mathrm{M}^{2}$, Harris $\mathrm{L}^{2}$ ${ }^{1}$ Alliance for Research in Exercise, Nutrition and Activity, Sansom Institute, University of South Australia, Adelaide

2School of Health Sciences, University of South Australia, Adelaide

Question: Chronic tension-type headache is a serious chronic pain condition with few successful treatment options. No synthesis of studies has been conducted to investigate the effects of exercise on this headache type. The aim of this study was to systematically review all evidence to determine the effectiveness of prescribed exercise on pain and quality of life in adults with chronic tension-type headache compared with other non-pharmacological interventions.

Design: Systematic review and narrative synthesis.

Participants: Adults with chronic tension-type headaches.

Intervention: Any prescribed exercise intervention compared to any nonpharmacological intervention.

Outcome Measures: Effect of exercise in terms of headache pain and/or quality of life.

Results: Five studies were included in the review (total $n=293$ ); with various study designs and low risk of methodological bias. Exercise interventions were disparate, including yoga, specific neck exercise, and aerobic exercise. Overall the five studies showed prescribed exercise had a positive effect on either headache pain and/or their reported quality of life.
Conclusion: Prescribed exercise could potentially be an effective intervention for patients with chronic tension headaches. Individual preference may dictate the choice of exercise, as positive benefits were shown across different exercise types. The small number of studies and the lack of standardised treatment protocols clearly communicate the need for further robust research in this area.

\section{Key Practice Points:}

- Prescribed exercise could be an effective intervention for patients suffering chronic tension-type headache

- All exercise types appear equally beneficial

- Research in this field is limited, and high quality evidence is needed to guide practice

\section{TRENDS AND VARIATIONS IN HOSPITAL CARE FOR VERTEBRAL COMPRESSION FRACTURE}

Ferreira $\mathrm{ML}^{1,2}$, March L², $\mathrm{Li}^{1}{ }^{1}$, Ferreira $\mathrm{PH}^{3}$

${ }^{1}$ The George Institute for Global Health

2Institute of Bone and Joint Research, Sydney Medical School, The University of Sydney

${ }^{3}$ Discipline of Physiotherapy, Faculty of Health Sciences, The University of Sydney

Question: What is the frequency, volume, nature and cost of hospitalisations for vertebral compression fracture in Australia?

Design: The NSW Admitted Patient Data Collection and the Emergency Department Data Collection were used to identify hospital admissions for vertebral compression fracture between 2003 and the last available record; and provide information on patient demographics, source of referral, service referred, procedures, mode of separation and episodes of care.

Population: patients with a diagnosed vertebral compression fracture in lumbar or thoracic sites.

Intervention: all hospital care provided to patients with vertebral compression fracture.

Outcomes: health care outcomes of hospitalisations included hospital length of stay, costs, rehospitalisation, transfers, adverse outcomes and mortality.

Results: a total of 195,828 hospital separations for all lower back pain, including a diagnosis of vertebral compression fracture, have been identified between $1^{\text {st }}$ of January, 2003 and $30^{\text {th }}$ of September, 2014. An additional 204,916 records for emergency department presentations for low back pain were also identified. Specific analysis of trends and variations of hospital care for vertebral compression fracture is underway and will be completed in June/2015 and presented in September.

Conclusion: the results of this study will provide robust evidence on the incidence, variations, costs and contributors at the person, geographic and service-level of hospital care for vertebral compression fracture. This will inform better planning of primary care to provide improved management and decrease unnecessary hospitalisations for a highly prevalent condition. Key Practice Points:

- The management pathway of VCF is still largely unknown.

- Although the effectiveness of surgical management of VCF is debatable, the number of hospital separations for vertebral compression fracture is substantial. 
QUALITY OF LIFE IN ANTERIOR CRUCIATE LIGAMENT DEFICIENT INDIVIDUALS: A SYSTEMATIC REVIEW

Filbay SR ${ }^{1}$, Culvenor $\mathrm{AG}^{1}$, Ackerman IN², Russell T'1 , Crossley KM ${ }^{1,3}$ ${ }^{1}$ Division of Physiotherapy, School of Health and Rehabilitation Sciences, The University of Queensland, Brisbane

2Melbourne EpiCentre, The University of Melbourne, Melbourne

${ }^{3}$ The College of Science, Health and Engineering, La Trobe University, Melbourne

Question: Is longer-term quality of life (QOL) impaired in anterior cruciate ligament $(A C L)$ deficient individuals? How does this compare with $A C L$ reconstructed groups?

Design: Systematic review with meta-analysis.

Participants We systematically identified and methodologically appraised all studies reporting QOL in ACL-deficient people 5-20 years following ACL rupture. Outcome Measures: QOL scores in ACL-deficient and ACL-reconstructed groups were pooled and compared using a random-effects meta-analysis

Results: Eleven studies reported QOL in $473 \mathrm{ACL}$-deficient participants at a mean 10 (range 5-23) years following ACL rupture. Knee Injury and Osteoarthritis Outcome Score (KOOS)-QOL mean scores (eight studies) ranged from 54 to 77 (best possible score, 100), these were impaired compared to reference groups. SF-36 scores (five studies) were similar to population norms, but impaired compared to active populations. Metaanalysis revealed no significant differences in KOOS-QOL (mean difference [95\% CI], 2.9 [-3.3 to 9.1]) and SF-36 scores (for all SF-36 domains except Vitality) between ACLD and ACLR groups. A range of biases were identified with potential to influence $\mathrm{OOL}$ estimates in this patient population. including baseline activity levels, advice regarding activity modification, and surgical treatment of meniscus injuries in ACL-deficient cohorts.

Conclusions: Knee-related QOL in ACL-deficient individuals 5 - 25 years after ACL rupture is impaired compared to population norms and similar to ACL-reconstructed groups.

\section{Key practice points:}

- OOL impairments are evident after ACL rupture, irrespective of management strategy.

- This warrants the development of targeted strategies to improve QOL after ACL rupture.

- These findings can be used by clinicians to educate patients about realistic long-term outcomes.

\section{RETURN TO SPORT EXPLAINS VARIABILITY IN OUALITY OF LIFE 5 TO 20 YEARS FOLLOWING ANTERIOR CRUCIATE LIGAMENT RECONSTRUCTION}

Filbay SR ${ }^{1}$, Ackerman IN², Russell T11, Crossley KM ${ }^{1,3}$

${ }^{1}$ School of Health and Rehabilitation Sciences, The University of Queensland, Brisbane

¿Melbourne EpiCentre, The University of Melbourne, Melbourne ${ }^{3}$ The College of Science, Health and Engineering, La Trobe University, Melbourne

Question: What factors are associated with quality-of-life (QOL) in individuals with knee symptoms after ACL-reconstruction?

Design: Analysis of baseline data from a longitudinal study.

Participants: 162 participants 5-20 years following ACL-reconstruction with self-reported knee pain, stiffness or activity limitations were recruited from surgeons' records and community advertisements.

Outcome Measures: Participants completed the ACL-QOL, Knee injury and Osteoarthritis Outcome Score (KOOS), and a demographics questionnaire. Multivariable linear regression identified factors explaining variability in QOL scores.

Results: Participants aged $38 \pm 9$ (mean $\pm S D$ ) years completed questionnaires $9+4$ years following ACL-reconstruction. Over half were overweight/obese (56\%), 39\% returned to pre-injury level of sport, $28 \%$ returned to a lower level and $32 \%$ did not return to sport after ACL-reconstruction (79\% reported that this was due to their knee). Average KOOS-QOL (55 \pm 20 ) and ACL-QOL scores (57 \pm 21 ) were impaired compared to population norms. Return to sport explained the greatest amount of variability in QOL scores. Not returning to sport after ACL- reconstruction was associated with poorer $\mathrm{KOOS}-\mathrm{QOL}(\beta=.27$, $p=0.003)$ and $A C L-Q O L(\beta=.47, p<0.001)$ scores. Subsequent surgery, increased $\mathrm{BMI}$ and undergoing contralateral ACL-reconstruction were also associated with poorer QOL scores.

Conclusion: QOL is impaired in people with knee symptoms 5-20 years after ACL-reconstruction. Individuals who do not return to sport after ACLreconstruction may be at heightened risk of poor long-term QOL.

\section{Key Practice Points:}

- Individuals who cease sport after ACL-reconstruction may be at risk of poor longer-term QOL.

- Clinicians should facilitate transition to an active lifestyle following sport cessation.

- Targeted interventions to improve QOL after ACL-reconstruction are warranted.

\section{AN IN-DEPTH EXPLORATION OF OUALITY OF LIFE FOLLOWING ANTERIOR CRUCIATE LIGAMENT RECONSTRUCTION - A QUALITATIVE STUDY}

Filbay SR ${ }^{1}$, Ackerman IN², Crossley KM1, 3

${ }^{1}$ Division of Physiotherapy, School of Health and Rehabilitation Sciences, The University of Queensland, Brisbane

¿Melbourne EpiCentre, The University of Melbourne, Melbourne

${ }^{3}$ The College of Science, Health and Engineering, La Trobe

University, Melbourne

Question: What factors are associated with knee-related quality of life (QOL) 5-20 years following anterior cruciate ligament reconstruction (ACLR)?

Design: Qualitative study.

Participants: Seventeen individuals with knee symptoms 5 -20 years following ACLR and high $(n=8)$ or low $(n=9)$ OOL scores were recruited from a larger longitudinal study

Outcome Measures: Semi-structured interviews were used to capture the full array of experiences impacting QOL since ACL rupture. Inductive coding and thematic analysis was performed.

Results: All participants experienced fear of re-injury after ACLR. Three recurrent themes emerged; fear-adaptation: individuals reduced fear of re-injury by modifying movement patterns to allow participation in desired activities, this resulted in a satisfactory QOL; fear-avoidance: individuals avoided sport due to fear of re-injury, this was associated with poor QOL among those with a strong preference for competitive sport; fear-suppression: individuals suppressed or overcame fear of re-injury to continue unrestricted sport, this resulted in satisfactory QOL despite a heightened prevalence of subsequent knee injury. ACL re-rupture and progressive deterioration of knee function. These individuals commonly described poor QOL after sport cessation.

Conclusion: These findings highlight key factors that influence QOL in people with knee symptoms after ACLR. This information can be used to develop strategies aimed at optimising QOL after ACLR.

\section{Key Practice Points:}

- Activity preferences and fear of re-injury influence QOL following ACLR.

- Individuals who do not enjoy recreational exercise may be at heightened risk of poor QOL outcomes after ACLR.

- Education and support regarding activity modification and re-injury fears should be incorporated into post-operative rehabilitation.

\section{KEY ELEMENTS OF EFFECTIVE SUPERVISION FOR PHYSIOTHERAPISTS}

Redpath $\mathrm{A}^{1}$, Gill S1 , Finlay $\mathbf{N}^{1}$, Brennan $\mathrm{F}^{1}$, Hakkennes $\mathrm{S}^{2}$

${ }^{1}$ Physiotherapy Department, Barwon Health, Geelong

${ }^{2}$ Knowledge and Information Services, Barwon Health, Geelong

Question: What are the key elements considered essential for effective clinical supervision in physiotherapy?

Design: Qualitative focus groups collected data using an emergentsystematic focus group design. Data were analysed using principles of thematic analysis and constant comparison methods. The Barwon Health human research ethics committee approved this study.

Participants: Forty six physiotherapists and six physiotherapy assistants from a large regional health service attended one of seven focus groups.

Outcome Measures: Physiotherapists' and assistants' preferences regarding supervision.

Results: Professional supervision was the term preferred by participants to describe a supervision program encompassing both clinical and nonclinical activities. Supervision must be individualised and responsive to the supervisee's needs. Participants highly valued informal supervision addressing needs as they arise, rather than regular scheduled sessions. Supervision formats identified as highly effective, incorporated contextual learning elements. Participants recognised the value of formal systems and structures to ensure supervision is accessible, resourced and sustainable.

Conclusion: This study identified key elements associated with an effective supervision program in physiotherapy and supports others suggestions that much workplace learning occurs informally and contextually. Based on these findings we propose that supervision models emphasising scheduled reflective discussion alone may not provide effective supervision for physiotherapists.

\section{Key Practice Points:}

- Professional supervision better describes a model that encompasses both clinical and non-clinical activities

- Professional supervision programs should acknowledge informal supervision in meeting supervision needs for physiotherapists

- Formal systems and structures are required to ensure professional supervision programs are effective and sustainable. 


\section{INTERVENTIONS FOR ANTERIOR CHRONIC EXERTIONAL COMPARTMENT SYNDROME (CECS); A SYSTEMATIC REVIEW}

Fisher B ${ }^{1}$

${ }^{1}$ Ministry of Defence, London, UK

Question: How effective is the treatment of anterior CECS?

Design: Systematic review with narrative synthesis.

Participants: All studies concerning adult patients with a diagnosis of anterior chronic exertional compartment syndrome (CECS), either via compartment pressure testing or by clinical diagnosis.

Interventions: The patient had a surgical or conservative treatment for CECS.

Outcome Measures: A clearly defined and patient-related outcome measure, including the percentage of patients who improved in each study.

Study appraisal and synthesis methods: Included studies were appraised for risk of bias using the tool developed by the Effective Public Health Practice Project (EPHPP). Due to heterogeneity of studies, a narrative synthesis was undertaken

Results: Fourteen studies were included. Study quality (risk of bias) was globally weak in all fourteen included studies. Studies with comparatively lower risk of bias reported lower patient outcomes, and higher amounts of adverse events. Compartment pressure levels (used to diagnose CECS) varied between studies, and the methodologies were unreliable. Success rates, as percentage of patients improved, ranged from 52\% to 95\% for surgery, and $70 \%$ to $80 \%$ for conservative interventions. Pre-operative conservative treatment was rarely reported. Post-operative rehabilitation was poorly reported or defined.

\section{Conclusions and Key Practice Points:}

- Emerging non-operative treatments (e.g. running re-education, conditioning) ae encouraging, but require further research with more robust study methodologies.

- Adverse events and poor outcomes from surgical interventions may be under-reported.

- There is no accepted post-op protocol for patients after surgical treatment for CECS

\section{IT'S NOT ALWAYS ABOUT WEAK PELVIC FLOORS}

\section{Fitzgerald J, Samuels A, Diggles A}

Active Rehabilitation Physiotherapy, South Brisbane

Mars Clinic for Children's Continence, Annerley

Two case reports of female athletes referred to physiotherapy for sports related stress incontinence. Case Report One: Fourteen year old female, high level gymnast, referred by GP for small volumes of daytime incontinence with physical activity. Symptoms commenced 12-months prior to presentation. Assessment included subjective questioning, real-time ultrasound, non-invasive uroflowmetry with pelvic floor EMG recording and urinalysis. Assessment indicated no leakage with cough and sneeze, dehydration, mild constipation, voiding postponement at school and a high tone pelvic floor at rest leading to an onset of bladder overactivity. Intervention was focused on improving fluid intake, managing constipation, improving voiding frequency and biofeedback for pelvic floor relaxation (not squeezing!). Patient reported being accident free in one week. Case Report Two: Forty-five year old semi-professional triathlete, referred by their team physio for incontinence, urgency and abdominal pain during training. Symptoms gradually worsened over the last year. She was informed that she had stress incontinence and required pelvic floor strength training. Assessment included subjective questioning, realtime ultrasound and internal examination involving measurement of the vaginal length and perineum. Assessment indicated no leakage with cough, moderate constipation, and noticeable leakage in squat, good pelvic floor strength but descent of the anterior wall on bearing down. Intervention needed to address the symptoms of prolapse, modification to training times, constipation management, functional bracing pelvic floor training and internal support for triathlon events. Patient reported no symptoms with modified training schedule and better control during weight training. For events, she no longer had any symptoms once using support.

\section{Key Practice Points:}

- Not all urinary incontinence in female athletes is due to stress incontinence and weak pelvic floors

- An accurate and targeted assessment, followed by simple treatment (such as managing fluid intake, voiding habits, bowel habits), can have a huge impact on continence issues

- If you are not sure, refer for further investigation. Real time ultrasound only shows movement on pelvic floor - absence of movement does not indicate that the muscle is not working.

- Strategic partnerships between medical specialists and physiotherapy, such as extended scope continence services, improve patient outcomes.

\section{MEASURING FRAILTY IN ELDERLY} CARDIAC SURGERY PATIENTS

Fitzgerald $\mathrm{S}^{1}$, Yap $\mathrm{CH}^{2}$, Wynne $\mathrm{R}^{1}$, Denehy $\mathrm{L}^{1}$, El-Ansary $\mathrm{D}^{1}$

${ }^{1}$ The University of Melbourne, Australia

2Monash University, Australia

Question: What is the prevalence of frailty in older adults undergoing cardiac surgery? Does measurement of frailty predict short term postoperative outcomes after cardiac surgery?

Design: Prospective observational study

Participants: One hundred and eleven participants over the age of 65 undergoing elective cardiac surgery after attending a pre-operative clinic for frailty assessment.

Outcome Measures: Frailty was measured pre-operatively using the Fried frailty criteria. Short term post-operative outcomes including 30 day mortality, 30 day readmission to hospital and incidence of post-operative complications were compared between frail and non-frail individuals.

Results: Seventeen (15.3\%) participants were frail. Frailty was not associated with an increased incidence of 30 day mortality ( $p=0.153)$, 30 day readmission to hospital $(p=0.14)$, or post-operative complications with the exception of prolonged ventilation time $>24$ hours $(95 \% \mathrm{Cl} 1.21$ to 11.22, $p=0.016)$. Frail patients were more likely to be discharged to rehabilitation 35\% vs 19\% (OR 2.3, 95\% CI 0.75 to 7.01) however this was not statistically significant $(p=.137)$.

Conclusion: The prevalence of frailty identified in this study supported previous literature findings. Discharge destination and intervention to target frailty should be measured in future studies.

\section{Key Practice Points:}

- Screening for frailty is important in the cardiac surgery population given the increasing age (Mean $=70$ years) and prevalence of comorbidities

- It is safe and feasible to measure frailty in the outpatient setting using the Fried frailty criteria

- Frail patients may be more likely to be discharged to a rehabilitation setting

\section{INCREASING ACCESS TO PHYSIOTHERAPY IN AN AMBULATORY SETTING: THE IMPOSSIBLE CHALLENGE?}

\section{Fitzhenry SK ${ }^{1}$, Bew $\mathrm{P}^{2}$}

${ }^{1}$ Caloundra Hospital, Sunshine Coast Health Service District,

Queensland Health

${ }^{2}$ Brighton Rehabilitation Hospital, Metro North Health District Queensland Health

Question: In an ambulatory setting can access to physiotherapy services for neurological patients be increased with a changed model of service delivery?

Design: Retrospective Cohort Study

Participants: 12 outpatients (8female, 4male) with neurological conditions, average age of 55.8(14-75).

Intervention: Following review of current practice, routine one-to-one sessions were changed to a circuit class format. With up to 9 patients in sessions, attendance was for 3 hours a day, twice a week for 4 weeks, plus a home exercise program. This program was developed within existing resources.

Outcome: Process evaluation included service utilization, with the dose of therapy represented by therapy time.

Results: In the corresponding time of the preceding 3months, $430 c c a s i o n s$ of physiotherapy service were provided totally 38hours of therapy. In the first 3months of the circuit program 105 occasions of service were provided, totalling 315 hours of physiotherapy. This represented an $828 \%$ increase in therapy time. Average repetitions per session per patient were 528. Non-attendance rates for both programs were comparable. There was a trend towards reducing waiting time from 28(SD 22.1) to 22.5days (SD 19)

Conclusion: Changing the format of therapy from one-to-one sessions to a circuit class design format can increase access to physiotherapy services in an ambulatory setting. This was feasible with no additional resources.

\section{Key Practice Points:}

- In an ambulatory setting introduction of circuit classes increased the amount of physiotherapy for patients.

- Treating therapists need to consider means of translating research findings into clinical services.

- Pragmatic research within the clinical setting brings with it numerous challenges. 


\section{PROGNOSTIC FACTORS FOR PEOPLE WITH LOW BACK PAIN RECEIVING PHYSIOTHERAPY}

Ford $\mathrm{JJ}^{1}$, Richards $M \mathrm{C}^{1}$, Hahne $A \mathrm{~J}^{1}$, Surkitt $\mathrm{LD}^{1}$, Chan $A \mathrm{Y}^{1}$, Slater $\mathrm{SL}^{1}$, Hinman $\mathrm{R}^{2}$, Taylor $\mathrm{NF}^{1}$

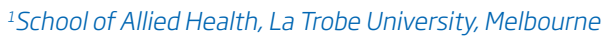

2School of Health Sciences, The University of Melbourne

Question: What are the prognostic factors for back and leg pain in patients with low back pain

Design: A secondary analysis from a randomised controlled trial (ACTRN12609000834257)

Participants: 300 patients with a primary complaint of low back and/or referred leg pain

Intervention:Patients received either 10 -sessions of individualised physiotherapy combined with guideline-based advice or 2-sessions of physiotherapist-delivered advice alone.

Outcome Measures:Primary outcomes were activity limitation (Oswestry Disability Index) and numerical rating scales for back and leg pain.

Prognostic factors included sociodemographics, treatment related factors, subjective/physical examination and standardised questionnaires. Univariate analysis followed by a generalised estimating equation were used to identify a multi-variate prognostic model.

Results: Fifty-eight prognostic factors progressed to the multivariate stage where 15 showed significant $(p<0.05)$ associations with at least one of the three outcomes. Positive prognostic factors were subgroup membership, paraesthesia below waist, walking as an easing factor and low transversus abdominis tone. Negative prognostic factors were both parents being born overseas, deep leg symptoms, higher sick leave duration, high multifidus tone, clinically determined inflammation, higher back and leg pain severity, lower Oswestry lifting capacity, lower work capacity and higher pain drawing area.

Conclusion: The identified multi-variate model has potential implications for clinicians and researchers.

\section{Key Practice Points:}

- The results suggest that practitioners should consider a range of biomedical and psychosocial factors in the management of low back pain

- The results challenge the notion that biomedical factors are less relevant than psychosocial factors in persistent pain

- Further research is required to confirm these findings

\section{INDIVIDUALISED PHYSIOTHERAPY}

VERSUS ADVICE FOR PEOPLE WITH LOW BACK DISORDERS: A 2-YEAR FOLLOW-UP OF A RANDOMISED CONTROLLED TRIAL

Ford $]^{1}{ }^{1}$, Hahne A ${ }^{1}$, Surkitt LD ${ }^{1}$, Slater SL ${ }^{1}$, Richards $M C^{1}$, Chan AYP ${ }^{1}$, Hinman RS', Taylor NF

${ }^{1}$ Department of Physiotherapy, La Trobe University, Melbourne

2Department of Physiotherapy, Melbourne School of Health Sciences, The University of Melbourne

Question: What are the 2-year outcomes of individualised physiotherapy relative to advice for people with low back disorders?

Design: Two-year follow-up of a randomised controlled trial (ACTRN12609000834257)

Participants: 300 patients with a primary complaint of low back and/or referred leg pain

Interventions: Patients received either 10 -sessions of physiotherapy that was individualised based on pathoanatomical, psychosocial, and neurophysiological barriers to recovery combined with guideline-based advice or 2-sessions of physiotherapist-delivered advice alone.

Outcome Measures: Primary outcomes were activity limitation (Oswestry Disability Index) and numerical rating scales for back and leg pain.

Results: Between group differences for Oswestry favoured specific physiotherapy at 2-years (3.1; 95\% Cl 0.2 to 6.0) consistent with 1-year results. Patients receiving individualised physiotherapy were significantly more likely to be have a $10 \%$ reduction in Oswestry (risk difference $=22 \%$ : $95 \% \mathrm{Cl} 11 \%$ to $33 \%$ ) and be "much improved on the global rating of change scale (risk difference=15\%; $9 \% \%$ CI 3\% to 26\%). There were no significant between group differences for back or leg pain.

Conclusion: Individualised physiotherapy leads to greater reduction in activity limitation across a 2 -year follow-up period compared to guidelinerecommended advice. These differences appear to be clinically important.

\section{Key Practice Points:}

- Our physiotherapy classification and individualised treatment protocol provides clinically important benefits compared to advice at 2-years

- Practitioners should consider pathoanatomical, psychosocial and neurophysiological barriers to recovery when planning individualised treatment

- Individualised physiotherapy should be considered for the management of low back disorders in addition to guideline-based advice

\section{THE EFFECTIVENESS OF SPECIFIC MANUAL THERAPY COMPARED TO ADVICE FOR LUMBAR ZYGAPOPHYSEAL JOINT PAIN: A 2-YEAR FOLLOW-UP OF A RANDOMISED CONTROLLED TRIAL}

Ford $\mathrm{JJ}^{1}$, Slater SL${ }^{1}$, Hahne AJ ${ }^{1}$, Surkitt LD ${ }^{1}$, Richards MC ${ }^{1}$, Chan AYP ${ }^{1}$, Hinman RS², Taylor NF ${ }^{1}$

${ }^{1}$ Department of Physiotherapy, La Trobe University, Melbourne

Department of Physiotherapy, Melbourne School of Health Sciences, The University of Melbourne

Question: What are the 2-year outcomes of specific manual therapy relative to advice for people with low back pain considered to be of lumbar zygapophyseal joint origin?

Design: Two-year follow-up of a randomised controlled trial

Participants: 64 patients with a primary complaint of low back and/or referred leg pain and clinical features of lumbar zygapophyseal joint (sixweek to six-month duration)

Interventions: Patients received either 10-sessions of physiotherapy with specific manual therapy combined with guideline-based advice or 2-sessions of advice alone.

Outcome Measures: Primary outcomes were activity limitation (Oswestry Disability Index) and numerical rating scales for back and leg pain.

Results: Between group differences for Oswestry favoured specific manual therapy at 2-years (5.9: 95\% Cl 0.4 to 11.2) consistent with 1-year results. Patients receiving specific manual therapy were significantly more likely to have a 10\% reduction in Oswestry (risk difference=29\%; $95 \% \mathrm{Cl}$ $1.1 \%$ to $51 \%$ ). There were no significant between group differences for back or leg pain

Conclusion: Specific manual therapy leads to greater reduction in activity limitation across a 2 -year follow-up period compared to guideline-based advice.

Key Practice Points:

- Our specific manual therapy protocol provides clinically meaningful benefits compared to advice at 2-years

- Specific manual therapy should be considered for low back disorders with clinical features of zygapophyseal joint pain in addition to guidelinebased advice 


\section{EDUCATING OLDER PATIENTS TO ENGAGE IN FALLS PREVENTION STRATEGIES WHILE IN HOSPITAL: MUTUAL UNDERSTANDING IS REQUIRED}

Hill AM1, 2, MCPhail SM $M^{3,4}$, Francis-Coad J $^{1,2}$, Waldron $\mathrm{N}^{5,6}$, Etherton-Beer $\mathrm{C}^{7}$, Flicker $\mathrm{L}^{7}$, Ingram $\mathrm{K}^{8}$, Haines $\mathrm{TP}^{9,10}$

${ }^{1}$ School of Physiotherapy, The University of Notre Dame Australia, Fremantle 2Institute for Health Research, The University of Notre Dame Australia, Fremantle

${ }^{3}$ Centre for Functioning and Health Research, Metro South Health Queensland, Brisbane

${ }^{4}$ Institute of Health and Biomedical Innovation and School of Public Health \& Social Work, Queensland University of Technology, Brisbane

${ }^{5}$ Department of Rehabilitation and Aged Care, Armadale Kelmscott Memorial Hospital, Perth

${ }^{6}$ Health Strategy and Networks, Strategic System, Policy \& Planning,

Department of Health, Government of Western Australia, Perth

'WA Centre for Health and Ageing, Royal Perth Hospital Unit, School of Medicine \& Pharmacology and Centre for Medical Research, University of Western Australia Perth

${ }^{8}$ Department of Rehabilitation and Aged Care Sir Charles Gairdner Hospital, Perth

9Physiotherapy Department, Monash University, Melbourne

${ }^{10}$ Allied Health Research Unit, Monash Health, Melbourne

Question: Patient and staff education delivered by trained educators significantly reduced falls and injurious falls in an older rehabilitation population. What were the educators perspectives about how the program worked to prevent falls?

Design: A qualitative exploratory study using a focus group, written educator notes and reflective researcher field notes.

Participants: $\mathrm{n}=10$ educators who delivered the individualised education program to over 750 patients on rehabilitation wards

Intervention: Multimedia education with individualised follow-up was provided to older patients to facilitate engagement in falls prevention strategies. Staff were provided with education to support patient actions.

Outcome Measures: Educators' perspectives about the barriers and facilitators to providing the program. Data were thematically analysed and presented using a conceptual framework.

Results: Falls prevention was conceptualised by educators as an effective three way interaction between staff actions, patient actions and the ward environment. This interaction was facilitated by the educators. Educators perceived that they assisted to reconcile differences that emerged between patients and staff about falls prevention, by providing education for patients and feedback from patients to staff. This encouraged the patient and staff to arrive at a mutual understanding about the patient's level of function and mobility.

Conclusion: Providing individualised patient education effectively provides patients who receive it with the capability and motivation to develop and undertake falls prevention strategies in hospital.

\section{Key Practice Points:}

- Individualised patient education facilitates patients to engage in falls prevention strategies.

- Staff support is essential for facilitating patient actions.

- Trained educators facilitate effective falls prevention action on rehabilitation wards.

\section{TO DETERMINE THE FEASIBILITY OF IMPLEMENTING AND OPERATING A VIRTUAL FALLS PREVENTION COMMUNITY OF PRACTICE ACROSS MULTIPLE RESIDENTIAL CARE SITES}

Francis-Coad ] ${ }^{1,2}$, Etherton-Beer $\mathrm{C}^{3}$, Bulsara $\mathrm{C}^{4}$, Nobre $\mathrm{D}^{5}$, Hill A-M $\mathrm{M}^{1,2}$ ${ }^{1}$ School of Physiotherapy, The University of Notre Dame Australia, Fremantle Institute for Health Research, The University of Notre Dame Australia, Fremantle

WA Centre for Health and Ageing Royal Perth Hospital Unit School of Medicine \& Pharmacology and Centre for Medical Research, University of Western Australia, Perth

${ }^{4}$ School of Nursing \& Midwifery, The University of Notre Dame

Australia, Fremantle

${ }^{5}$ Brightwater Care Group, Brightwater House, Osborne Park

Question: Is it feasible to implement and operate a virtual falls prevention community of practice (CoP) across multiple sites within a residential aged care organisation?

Design: Cross-sectional survey and prospective observation
Participants: Multidisciplinary staff $(n=20)$ self-nominating as CoP members, representing 13 sites.

Intervention: The CoP was established using determinants of success for CoPs identified in the literature. Participants received training on the CoP concept followed by a four-week asynchronous virtual communication trial using the organisation's intranet platform.

Outcome Measures: Online survey evaluating capability, opportunity and motivation to engage online. Researcher journaling recorded participant intranet activity and feedback

Results: Participants were capable of using the intranet (Strongly Agree $60 \%$, Agree 20\%) and had opportunity to use the intranet (Strongly Agree $75 \%$, Agree 25\%) in their work practices. Motivation to engage centred on anticipated benefits of gaining knowledge to apply at their sites. Only 6 (30\%) had previously used a blog format. All participants communicated by posting asynchronously, but the highest frequency of virtual engagement ( 2 posts, 2 article downloads) by any participant was low. Prompts using email alerts were identified as a behavioural change strategy to improve virtual engagement.

Conclusion: It was feasible to implement and operate a virtual falls prevention CoP across multiple sites of a residential aged care organisation. Participant feedback resulted in intranet modifications for facilitating behaviour change to improve virtual engagement.

\section{Key Practice Points:}

- Virtual CoPs could facilitate the translation of evidence into practice through interdisciplinary collaboration.

- Virtual CoP membership can connect physiotherapists working in isolation.

\section{TOWARDS COMMON AGREED STANDARDS FOR ABORIGINAL AND TORRES STRAIT ISLANDER HEALTH CURRICULUM ACROSS THE ALLIED HEALTH PROFESSIONS}

Francis-Cracknell A ${ }^{1}$, Palermo $\mathrm{C}^{1}$, Adams $\mathrm{K}^{1}$, Gilby R ${ }^{1}$, MurrayM ${ }^{1}$, Keating $\rfloor^{1}$

IMonash University, Faculty of Medicine, Nursing and Health Sciences, Clayton, Australia

Question: To review Committee of Deans of Australian Medical Schools (CDAMS) Indigenous health curriculum framework and Health Workforce Australia (HWA) National Indigenous health curriculum project to determine applicability to other health professions.

Design: Combined key representatives from Departments within Monash University to form a working group. The eight disciplines collaborated to develop endorsed entry-level health professional curriculum. The CDAMS and HWA documents informed the development of our consensus learning objectives

Participants: Multi-disciplinary representatives participated on a working group. Representatives from ten disciplines/departments were included. HWA National Indigenous health Curriculum project members were included.

Outcome Measures: This was a qualitative action research project using a "look, think and act" approach, to enhance teaching and learning practice, build on existing knowledge and create new knowledge (Stringe 1996). Working Group members were consulted using a modified Delphi methodology to refine learning outcomes and identify when to introduce them into the curriculum - at the 'Novice', 'Intermediate' or 'Advanced' learner level.

Results: Key outcomes included the development of new partnerships between Indigenous and non-Indigenous Australians, between University programs and endorsed Indigenous health curriculum, including key learning outcomes for 'Novice', 'Intermediate' and 'Advanced' learners.

Conclusion: Most of CDAMS framework was agreed appropriate for allied health professions. Some items were re-worded/removed to obtain endorsement of allied health professions.

\section{Key Practice Points:}

- Most of the CDAMS framework was agreed as appropriate for allied health professions

- Interprofessional collaboration promotes coordinated implementation of Aboriginal and Torres Strait Islander health curriculum

- Identifying learning objectives as Novice, Intermediate or Advanced may be useful 
HIGHER GLUTEUS MEDIUS MUSCLE ACTIVITY DURING RUNNING IS A RISK FACTOR FOR HAMSTRING INJURIES IN ELITE AFL PLAYERS

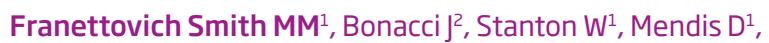
Hides $J A^{1}$

${ }^{1}$ Centre for Musculoskeletal Research, Mary MacKillop Institute for Health Research, ACU, Brisbane

2School of Exercise and Nutrition Sciences, Deakin University Australia, Geelong

Question: Is volume and function of the gluteus medius and gluteus maximus muscles a risk factor for hamstring injuries in elite AFL players?

Design: Prospective observational study.

Participants: Twenty-six players from a professional AFL club.

Outcome Measures: At the beginning of the season bilateral gluteus medius and gluteus maximus muscle volume was measured with magnetic resonance imaging and electromyographic recordings were obtained during walking and running. Pre-season and season incidence of hamstring injuries were determined from club injury reports.

Results: Nine individuals incurred a hamstring injury during the season. Higher gluteus medius muscle activity during running was a risk factor for hamstring injury, $\mathrm{p}=0.03$

Conclusions: This study identified that greater activation of gluteus medius during running is predictive of hamstring injuries. Gluteus medius muscle volume was not a risk factor and suggests that the observed increase in muscle activity may be more related to a neuromotor control issue. Future studies are required to understand this alteration in neuromotor control, for example, evaluating size and function of other muscles of the trunk/pelvic/hip region and evaluating alterations in trunk/ lower limb kinematics.

\section{Key Practice Points:}

- Higher activation of gluteus medius during running is predictive of hamstring injuries in elite AFL players

- Muscle size was not a risk factor for injury and suggests that higher activation of gluteus medius is likely related to neuromotor control

- Gluteus medius activity during running but not walking was related to hamstring injury and highlights the importance of assessments that are task and sport specific

\section{BALANCE AND GAIT IN YOUNG SUBJECTS WITH INSIDIOUS ONSET NECK PAIN FROM PROLONGED FLEXION}

\section{Fraser $\mathrm{RM}^{1}$, Reid SA}

${ }^{1}$ School of Physiotherapy, Australian Catholic University, North Sydney

Question: Do subjects with insidious onset neck pain linked to prolonged flexion of the cervical spine while looking down at mobile devices (termed 'Text Neck') have deficits in balance and gait compared to healthy controls?

Design: Observational study.

Participants: Forty young adults with $(n=20)$ and without $(n=20)$ suspected 'Text Neck' pain.

Outcome Measures: Subjects performed a series of balance tests with different visual conditions, stances and types of surfaces measured with the Humac Balance. Subjects completed a gait assessment using the GAITRite system at different paces.

Results: Subjects with 'Text Neck' displayed significantly $(p=0.02)$ poorer balance compared to controls under challenging balance conditions. Subjects with neck pain had a mean centre-of-pressure path length of $193 \mathrm{~cm}($ SD $32 \mathrm{~cm}$ ) while standing on a soft surface with eyes closed and $68 \mathrm{~cm}(\mathrm{SD} 22 \mathrm{~cm}$ ) while standing on one leg with cervical spine flexion compared to the asymptomatic control group's $139 \mathrm{~cm}($ SD $32 \mathrm{~cm})$ and $45 \mathrm{~cm}$ $(S D 18 \mathrm{~cm})$ respectively. However, there were no significant differences between the two groups with gait.

Conclusion: The results indicate that subjects suspected of 'Text Neck' have deficits in balance but not gait compared to controls. Additionally, altered balance in the neck pain group was most evident in challenging postural positions.

\section{Key Practice Points:}

- Subjects with suspected 'Text Neck' demonstrate impairments in balance but not gait.

- Clinicians can use simple tests to evaluate balance deficits in people with 'Text Neck'.

- This may facilitate optimal treatment to minimise impairments.

Ethics: Approved by ACU Research Committee (2014344N).
DOES PELVIC FLOOR MUSCLE TRAINING HELP FEMALE SEXUAL FUNCTION?

\section{Frawley $\mathbf{H}^{1,2}$}

${ }^{1}$ Associate Professor Physiotherapy, Department of Rehabilitation, Nutrition and Sport, School of Allied Health, La Trobe University, Bundoora VIC 2Head of Centre for Allied Health Research and Education, Cabrini Institute. Malvern VIC

Female sexual function may be affected by biological, psychosexual or contextual factors. Treatment of female sexual dysfunction may be addressed by any or a combination of these factors. Pelvic floor muscle training (PFMT) has been proposed to improve female sexual function via the biological pathway. The effect of (PFMT) on female sexual function has received little attention from robust studies to date. This topic will be addressed with reference to a recently published systematic review (Ferreira et al 2015) which explored findings from eight randomised controlled trials published since 1997. While studies were heterogeneous and of low-moderate quality, there is some evidence that PFMT may improve at least one variable or aspect of sexual function. Most studies explored the relationship between PFMT and sexual function in women with existing pelvic floor dysfunction, therefore little is known regarding the effect of PFMT on sexual function in women without pelvic floor dysfunction. This presentation will also consider the mechanisms of how PFMT may affect sexual function, the association between adherence to PFMT and change in sexual function, and the association between improvement in sexual function and change in PFM function.

\section{Key Practice Points:}

- Through an understanding of the mechanism of how PFMT may improve female sexual function and an integration with the evidence from a recent systematic review, participants will be better equipped to apply and evaluate the effect of PFMT as an intervention to treat female sexual dysfunction in their clinical practice.

\section{REHABILITATION FOLLOWING TREATMENT FOR PELVIC CANCER}

\section{Frawley $\mathrm{H}^{1,2}$}

${ }^{1}$ Head of Centre for Allied Health Research and Education, Cabrini Institute, Malvern VIC; and on behalf of Cabrini Allied Health Pelvic Oncology Research Project team

${ }^{2}$ Associate Professor Physiotherapy, Department of Rehabilitation, Nutrition and Sport, School of Allied Health, La Trobe University, Bundoora VIC

This presentation will consider rehabilitation following surgical treatmen for urological, gynaecological and colorectal cancer. Although improved surgical techniques and adjuvant therapies result in higher survival rates following surgery for pelvic cancer, patients often report lower levels of physical activity compared to pre-diagnosis, suffer from physical and psychological morbidity, and complain of impaired pelvic floor function. Evidence is emerging regarding the effect of physical activity on cancer recurrence and survival in many tumour streams, hence international guidelines recommend cancer patients engage in regular and moderate intensity physical activity following treatment. However this is not routinely implemented and has not been evaluated in gynaecological and colorectal populations in Australia. Further, the effect of a general physical activity program on pelvic floor function in pelvic cancer cohorts has not been evaluated. We decided to investigate the feasibility of a multidisciplinary general oncology rehabilitation program for patients following surgery for pelvic cancer in a private health care setting. Secondarily, we were interested in the effect of this program on functional capacity, muscle strength, psychological distress, level of physical activity, self-efficacy, and health-related quality of life. This is a prospective pre-post cohort study. Recruitment target is 80 participants, expected to be reached by mid-late 2016 . The study protocol is registered with the Australian New Zealand Clinical Trials Registry (ANZCTR):12614000580673.

Results: Results of feasibility to date (patient referral rates, enrolment, attendance, adherence and participant evaluation of program) will be presented, as will clinical outcome data for participants who have completed their 6-month assessment time-point.

\section{Key Practice Points:}

- Participants will gain an understanding of the current evidence and guidelines for oncology rehabilitation, and an awareness of how these guidelines have been implemented in a clinical setting. 


\section{PERINEAL MUSCLE STIFFNESS IN WOMEN WITH AND WITHOUT VULVODYNIA: RELIABILITY OF MEASUREMENT AND DIFFERENCES IN MUSCLE STIFFNESS}

Davidson $\mathrm{MJ}^{1}$, Bryant $\mathrm{AL}^{2}$, Frawley $\mathrm{HC}^{3,4}$

${ }^{1}$ Remarkable Physios, Queenstown, New Zealand 2 The University of Melbourne ${ }^{3}$ La Trobe University, Melbourne ${ }^{4}$ Cabrini Health, Melbourne

Question: can perineal muscle stiffness be reliably measured and does it differ in women with and without vulvodynia?

Design: A cross-sectional observational study.

Participants: Women aged 18 - 50 years, asymptomatic of pelvic floor dysfunction (asymptomatic cohort) and symptomatic of vulvodynia (symptomatic cohort)

Intervention: intra-rater and inter-rater reliability of muscle stiffness using a myometer, the MyotonPro ${ }^{T M}$ and a cross-sectional comparison of perineal muscle stiffness in asymptomatic and symptomatic cohorts.

Outcome Measures: reliability was measured using intra-class correlation coefficient (ICC, 95\% CI); perineal muscle stiffness was measured using the MyotonPro ${ }^{\mathrm{TM}}$

Results: In the asymptomatic cohort, intra-rater reliability was very good (left: ICC = 0.80, 95\% CI 0.62 to 0.89; right: ICC = 0.85, 95\% CI 0.73 to 0.92 ) and inter-rater reliability was good (left: ICC $=0.77,95 \%$ CI 0.41 to 0.91 ; right: ICC $=0.70,95 \%$ CI 0.23 to 0.88). In the symptomatic cohort, intrarater reliability was very good (left: ICC $=0.88,95 \% \mathrm{Cl} 0.75$ to 0.94 ; right: ICC $=0.91,95 \%$ CI 0.81 to 0.96 ); inter-rater reliability was very good (left: ICC=0.86, 95\% CI 0.57 to 0.96; right: ICC $=0.83,95 \% \mathrm{Cl} 0.46$ to 0.94). The symptomatic cohort demonstrated significantly higher stiffness values than the asymptomatic cohort on both left and right sides ( $<<001)$

Conclusions: Perineal muscle stiffness can be reliably and objectively measured using the MyotonPro ${ }^{T M}$. Women with vulvodynia demonstrate increased stiffness in the perineal muscles.

\section{Key Practice Points:}

- Reliable measures of perineal muscle stiffness will assist clinicians to measure the effect of their interventions aimed at altering muscle tension.

\section{CORRELATION BETWEEN MUSCLE STIFFNESS AND PAIN IN WOMEN WITH VULVODYNIA}

Davidson $\mathrm{MJ}^{1}$, Bryant $\mathrm{AL}^{2}$, Frawley $\mathrm{HC}^{3,4}$

${ }^{1}$ Remarkable Physios, Queenstown, New Zealand

${ }^{2}$ The University of Melbourne

${ }^{3}$ La Trobe University, Melbourne

${ }^{4}$ Cabrini Health, Melbourne

Question: In women with vulvodynia, do perineal muscle stiffness and pain correlate?

Design: A cross-sectional observational study

Participants: Women aged 18 - 50 years

Outcome Measures: Perineal muscle stiffness was measured using myometer, the MyotonProTM. Pain was measured using the Short Form McGill Pain Questionnaire (SFMPQ), Numeric Rating Scale (NRS) and vulval Q-tip examination test.

Results: The mean (SD) stiffness values were $(R)$ perineal side $=142.85$ (25.65), (L) side = 155.05 (28.99); mean (SD) of the SFMPO was 12.34 (7.06); median (IQR) of the Q-tip NRS was 3.00 (2) and averaged NRS was 0.40 (1). Correlation analyses revealed small, non-significant, negative correlations between stiffness values and SFMPQ values (R side $r=-0.26, p=0.14$; $L$ side $r=-0.26, p=0.16$ ); $Q$-Tip NRS scores (R side $r=-0.15, p=0.40$; $L$ side $r=-0.25$ $p=0.16$ ); and averaged NRS scores (R side $r=-0.19, p=0.30$; $L$ side $r=-0.21$, $p=0.25$ ).

Conclusion: No correlation was found between perineal muscle stiffness scores and vulval pain, using either the SFMPQ or the NRS scores. In this cohort, pain duration was prolonged and pain severity scores were moderately - very low. The cluster of low severity pain scores may have created insufficient spread in the data to detect an association between stiffness and pain

\section{Key Practice Points:}

- If pain levels were not correlated with stiffness levels, what is the explanation for higher stiffness values in women with vulvodynia?

- The findings from this study do not support the notion that altering stiffness levels may attenuate symptoms of pain.
CAN HIP EXTENSION STRENGTH PREDICT FUNCTIONAL PERFORMANCE DURING A STEP OUT LUNGE?

Freke $\mathrm{MD}^{1,2}$, Crossley KMㄹ, ${ }^{2}$, Russell T2 ${ }^{2}$, Sims KJ ${ }^{2}$

${ }^{1}$ Enoggera Health Centre, Gallipoli Barracks, Enoggera, Brisbane

2Physiotherapy, School of Health and Rehabilitation Sciences, University of Queensland

${ }^{3}$ School of Allied Health, College of Science, Health and engineering, La Trobe University, Melbourne

Question: Can hip extension strength predict functional performance during step-out lunge?

Design: Cross-sectional

Participants: Thirty-three healthy volunteers (18 men, 15 women) were recruited (mean age 32 , mean height $174 \mathrm{~cm}$, mean weight $75 \mathrm{~kg}$ ). Limb dominance was preferred leg to kick a football.

Outcome Measures: Excessive anterior trunk lean, medial knee movement and ankle dorsiflexion during step-out lunge are considered deviations from ideal technique. The aim of this paper was to determine whether hip extension strength was related to trunk, knee and ankle kinematics during the step out lunge utilising Microsoft Kinect ${ }^{T M}$ and SportsCoach video analysis. Thirty-three healthy and physically active volunteers (18 men) were recruited (mean age $32 \pm 9$, height $1.74 \mathrm{~m} \pm 0.07$, weight $75 \mathrm{~kg} \pm 15)$. Limb dominance was determined as the preferred leg to kick a football. Hip extension strength was measured using a handheld dynamometer. The Microsoft Kinect ${ }^{\text {TM }}$ captured sagittal and frontal plane movement during the step out lunge. Linear trunk, hip and knee movements were determined from the three-dimensional positional change of the lower limb and spine during the lunge. Ankle dorsiflexion was measured using onscreen goniometry. Correlation analysis revealed that greater hip extension strength was moderately associated with a more upright trunk during lunge for the non-dominant leg $(r=-0.539 ; p=0.002$ ) but not for the dominant leg $(r=-0.148 ; p=0.428)$. For knee movement in the frontal plane, there was a fair correlation between hip extension strength and medial deviation in the non-dominant leg $(r=-0.351 ; p=0.049)$ but not in the dominant leg ( $r=0.067 ; p=0.715)$. Hip extension strength was not significantly correlated to dorsiflexion range for dominant ( $r=$ -0.076, $p=0.69$ ) or non-dominant legs $(r=0.051, p=0.787)$. Hip extension strength has a fair to moderate correlation with trunk lean and knee alignment during step-out lunge in healthy individuals in the non-dominant limb only. No relationship exists in the dominant limb or with dorsiflexion on either limb.

Results: Correlation analysis revealed that greater hip extension strength was significantly associated with a more upright trunk during lunge for the non-dominant leg $(r=-0.539 ; p=0.002)$ but not for the dominant leg ( $r=$ -0.148: $p=0.43$ ). For knee movement in the frontal plane, a mild correlation was found between hip extension strength and less medial deviation in the non-dominant leg $(r=-0.351 ; p=0.05)$ but no correlation was found in the dominant leg $(r=0.067 ; p=0.715)$. Hip extension strength was not significantly correlated to dorsiflexion amount for dominant $(r=-0.076$ $p=0.69)$ or non-dominant legs $(r=0.051, p=0.79)$

Conclusion: Exercise prescribers should consider that isolated strengthening of hip extension may not improve lunge performance.

This study was approved by the University of Queensland Human Research Ethics committee (HREC - 2013000285). All participants signed a written informed consent. 


\section{DIFFERENCES IN JOINT RANGES \\ OF MOTION IN COMPETITIVE AND RECREATIONAL SURFERS}

Furness $]^{1}$, Hing $\mathrm{W}^{1}$, Pope $\mathrm{R}^{1}$, Climstein $\mathrm{M}^{1,2}$

${ }^{1}$ Water Based Research Unit, Institute of Health \& Sport, Faculty of Health Science, Bond University, Gold Coast

Exercise Health \& Performance Faculty Research Group, Faculty of Health Sciences, The University of Sydney

Question: What is known about the actual and optimal range of motion in a surfing population?

Design: Cross-sectional study of competitive and recreational surfers.

Participants: 35 male surfers were evaluated; 16 competitive surfers, ranked in the top 100 in the world and 19 classed as recreational surfers (mean ages of 26 and 27 years respectively).

Outcome Measures: Standardised clinical tools (inclinometers, cervical ROM brace, tape measure) and methods were used to determine ROM in spine, shoulder, hip and ankle. Independent $t$ tests were used to determine differences between competitive and recreational surfers.

Results: Competitive surfers had significantly greater thoracic rotation $\left(64^{\circ}\right.$ versus $56^{\circ} ; p=0.01$ ), lumbar extension ( $37 \mathrm{~cm}$ versus $33 \mathrm{~cm} ; p=0.01$ ), hip internal rotation $\left(30^{\circ}\right.$ versus $\left.24^{\circ} ; p=0.03\right)$ and ankle dorsiflexion $(17 \mathrm{~cm}$ versus $13 \mathrm{~cm}$; $\mathrm{p}$ < 0.001). No differences existed between shoulder and cervical ROM or thoracic ROM in the sagittal plane.

Conclusion: Significantly greater ROM in thoracic rotation, lumbar extension, hip IR and ankle DF are attributes of competitive surfers

\section{Key Practice Points:}

- A profile of ROM in both a recreational and competitive cohort has been provided which can be used as a guideline for rehabilitation or to potentially enhance performance.

- Competitive surfers clearly show greater joint ROM in several regions. The significance of these findings is yet to be determined from a performance and injury prevention perspective.

\section{TRUNK MOBILITY IN THE SAGITTAL AND HORIZONTAL PLANES: CLINICAL METHODS TO QUANTIFY MOVEMENT IN AN ELITE SURFING POPULATION}

Furness $\mathrm{J}^{1}$, Hing $\mathrm{W}^{1}$, Pope $\mathrm{R}^{1}$, Climstein $\mathrm{M}^{1,2}$

${ }^{1}$ Water Based Research Unit, Institute of Health \& Sport, Faculty of Health Science, Bond University, Gold Coast

'Exercise Health \& Performance Faculty Research Group, Faculty of Health Sciences, The University of Sydney

Question: What is the intra-rater reliability of two measures of thoracic ROM? Do elite surfers possess greater thoracic ROM than age- and gendermatched controls?

Design: Clinical measurement intra-rater reliability (repeated measures) studies and matched cohort study.

Participants: 30 participants ( $30.84 \pm 10.96$ years, female $n=16$, male $n=14$ ) to determine reliability of existing thoracic rotation measure; 27 participants ( $31.69 \pm 11.52$ years, female $n=15$, male $n=12$ ) to determine reliability of new method to assess thoracic mobility in the sagittal plane; 15 elite surfers $(26.47 \pm 4.59$ years, male $n=15)$ and 11 age- and gendermatched control participants (drawn from the reliability study) for thoracic ROM study.

Outcome Measures: Movement excursion distance, joint angles measured by an inclinometer.

Results: Intra-rater reliability (within and between sessions) intraclass correlation coefficient (ICC) values ranged between 0.95 - 0.99 for thoracic ROM measures. There was no significant difference in sagittal plane thoracic ROM between groups; however the elite surfers had significantly greater rotation than the control participants (mean rotation $63.57^{\circ}$ versus $40.80^{\circ}$, respectively, $\mathrm{p}<0.001$ )

Conclusion: The thoracic ROM measures have good intra-rater reliability Elite surfers exhibit greater thoracic rotation ROM than matched controls.

\section{Key Practice Points:}

- Further research is warranted to assess the prognostic value of thoracic rotation ROM for surfing performance and injury risk.

- The described thoracic ROM measures can be reliably used by a single clinician to assess changes and differences in thoracic ROM, but further research is required to assess their inter-rater reliability.
HOW TO - GET PAID AS A PHYSIO BY QUANTIFYING EFFECTIVENESS

\section{Gabel CP1,2}

${ }^{1}$ Coolum Physiotherapy - Sunshine Coast

¿University of the Sunshine Coast

Background: Practitioners face the dilemma of quantifying the effectiveness of their interventions to patients, themselves and third party payers, but can simplify this through the three stage protocol of: initial risk assessment, concurrent baseline functional measurement and subsequent re-measurement. Assumed knowledge: the biopsychosocial health model, risk and functional measurement, and some self-report tools.

Aims/objectives: Quantification of biopsychosocial risk factors that delay recovery and concurrent demonstration of functional status and its changes that validate chosen interventions and justify service costs and management decisions.

Learning outcomes:

- Improved knowledge: how to integrate risk recognition and functional status measurement;

- Acquire skills: choosing and interpreting tools, scores and constructs affecting risk, status and recovery:

- Techniques: constructing integrated time-status charts within the riskrecovery paradigm;

- Competent: information assessment and management decision processes for intervention proposals and costings.

Approach: Short lecture; interactive participatory learning through knowledge sharing, consensus, written and computer/net-based measurement tools and facilitated individualised and computerised decision support systems

Materials: Workbook and summary tool resource including net-based links, supporting research and evidence that enables immediate clinical and research based implementation.

\section{Key Practice Points:}

- Immediate use and implementation of the demonstrated tools within the clinic and research settings

- Identification of global and individual construct risks that influence delayed patient recovery

- Provision of evidence that supports their selected current management and intervention methodology

- Documentation of the individual patient's predicted, current and projected risk and recovery time lines

- Quantification and ensuing justification of current and projected costs and required payments. 
HOW TO COMPLETE INDIVIDUAL

CLINICAL RESEARCH - CONTEMPLATE, INITIATE, UNDERTAKE AND PUBLISH

\section{Gabel CP1,2}

${ }^{1}$ Coolum Physiotherapy - Sunshine Coast

2University of the Sunshine Coast

Background: Clinicians hold extraordinary untapped resources of knowledge and experience with potential research and evidence implications-provided simplified processes are readily available to passage this to published domains.

Aims/objectives: simplified pathways that assist clinicians transferring knowledge from experience to research.

Learning outcomes:

- Knowledge: structured progressive processes that facilitate clinical research efficiency and effectiveness

- Acquire skills: conceptualising research from personal experience and interest, information sourcing and pathway planning focused on recognition of pitfall, available resources and project completion

- Techniques: understanding progression from concept to hypothesis, ethics, measurement tool acquisition and/or development, data collection, analysis, writing, submission and review responses to publication

- Competent: in abilities to initiate, proceed and publish individua clinical research.

Approach: short lecture; interactive participatory learning through knowledge sharing, consensus approaches and brainstorming for individual/group projects including:

- Project identification and hypothesis clarification

- Ethics submissions and requirements

- Strategy recognition to facilitate approval including peer support, coauthorship and external review

- Measurement and assessment tool determination, acquisition and/or development to facilitate task requirements

- Data collection including software and spread sheets

- Statistical analysis approached that are individually learned or peersupported

-Writing, submitting and responding to reviews Materials work-sheets, published examples including: letters to editors, case studies and prospective investigations.

\section{Key Practice Points:}

- Resources for immediate implementation of strategies to initiate and complete clinical based research

- A simple progressive pathway for private clinical research from initiation to publication

- Confidence in how to progress alone or seek peer support and available resources.

\section{A MULTI-MODAL APPROACH OF LASER- THERAPY, ECCENTRIC EXERCISES, ACUPUNCTURE, MASSAGE AND ELECTROTHERAPY IS EFFECTIVE IN MANAGING SYMPTOMATIC ACHILLES TENDONS}

\section{Gabel CP1,2}

${ }^{1}$ Coolum Physiotherapy - Sunshine Coast

¿University of the Sunshine Coast

Question: Can symptomatic achilles tendon injuries in active genera populations be effectively managed with multi-modal therapy-laser therapy (LLLT), eccentric exercises and electrotherapy.

Design: prospective repeated measures with graded intervention introduction and progressive increasing modality dose.

Participants: Consecutive de-identified fee-paying private patients $(n=63$, age $=48.8 \pm 17.7$, male $=68 \%$ ) with $<6$ weeks symptomatic tendoachilles, mid-insertional. Exclusion-rupture, age<16 years.

Intervention: Multi-modal therapy provided 2-1 x weekly: LLLT [1W Cluster-probe:5x200mw-810nm, spot-size $1 / \mathrm{e}^{2}=1.14 \mathrm{~mm} \times 3.24 \mathrm{~mm}-$ elliptical; Power Density $=5.96 \mathrm{~W} / \mathrm{cm}^{2}$, Energy Density $=6 \mathrm{~J} / \mathrm{cm}^{2}, 120-240$ //session dependent area, continuous-mode, direct-contact parallel bilaterally, local only], interferential current (12 min,10-150Hz), acupuncture (tendon-achilles threading, calf trigger-points and Sp6,9,GB34,UB58) plus eccentric-loading exercises (3x15reps daily 2-1x) til discharge (5.8 \pm 6.7 weeks).
Outcome Measures: functional-status (VISA-A and LLFI), NRS, Global perceived status, patient-specific status and Advise-Rehab decision support software.

Data: baseline and 1-2 weekly for all outcomes till discharge/cessation with 'intention to treat' analysis.

Results: outcomes showed significant improvement (t-test, $p<0.0001$ ) with participants positive change-range scores: NRS $=54 \%$, LLFI-10=78\%, VISA-A $=79 \%, \mathrm{PSI}=86 \%, \mathrm{LLFI}=89 \% \mathrm{DSS}=95 \%$. The $6 \mathrm{~J} / \mathrm{cm}^{2}$ was greater than other studies $\left(3 \mathrm{~J} / \mathrm{cm}^{2}\right.$ ) and more sensitive outcome measures (LLF and DSS >VISA-A) improved response evidence.

Conclusion: multi-modal therapy provided positive functional gains and pain reduction. Limitations were no control group. Outcome tool sensitivity reflects change levels and variations in previous studies results with lessresponsive measures

\section{Key Practice Points:}

- Multi-modal therapy used strategically can benefit sporting general population with symptomatic achilles tendons

- Dose progression and variation should be considered in response to patient feedback

- Measurement tool sensitivity can effect study results

- In a fee paying active private population a multi-modal program is feasible and effective.

\section{WATER RUNNING DEEP VERSUS TETHERED - ADVANTAGES AND DISADVANTAGES OF GROUND TOUCH AND REACTION FORCES}

\section{Gabel CP1,2}

${ }^{1}$ Coolum Physiotherapy - Sunshine Coast

¿University of the Sunshine Coast

Question: Is there an advantage to either deep water (DWR) or tethered running (TR); in what circumstances; and injury conditions?

Design: a literature and experienced based review of the advantages and disadvantages DWR versus TR.

Participants: literature based and case series reviews from individual running sports to team sports.

Outcome Measures: athlete performance, individual subjective feedback and literature reviewed values of ground reaction forces (GRF), neural feedback and reported sensory feedback from qualitative research.

Results: TR is preferred to DRW (with/without shoes in-water) due to: depth varying capacity; lower depth and hydrostatic pressure reduces heart rate and VO2(max) which approximates land running; leg blood flow increases from weight bearing and arm water-resistance is minimal; lower lactate levels as anaerobic metabolism and perceived exertion reduce; and activity specificity is improved with GRFs. A combination of both DWR and TR may be optimal with time preference toward TR when training effects are desired with DWR used for warm-up/down or where toe-touch or load must be excluded (eg calf injury, achilles or fractures).

Conclusion: TR is often misunderstood and not frequently used as an aquatic based training and rehabilitation technique. The advantages over tradition DWR include the capacity to graduate GRF, improve sport specificity and improve training effect both physiologically, neurologically and through perceived exertion.

\section{Key Practice Points:}

Recognition of:

- Technique differences between DWR and TR including shoes in-water

- Physiological differences between DWR and TR

- Neurological and physical advantages of shoes in-water flexibility of TR techniques and higher simulation to land-based running. 


\section{WHICH OF 10 DIFFERENT UPPER LIMB OUTCOME TOOLS IS OPTIMAL?}

\author{
Gabel CP ${ }^{1,2}$ \\ ${ }^{1}$ Coolum Physiotherapy - Sunshine Coast \\ ¿University of the Sunshine Coast
}

Question: Which of 10 patient reported outcomes (PROs) is optimal for upper-limb measurement?

Design: Observational, prospective.

Participants: 229 with 495 responses.

Outcome Measures: Disabilities-Arm-Shoulder-Hand (DASH), Quick-DASH (11-items) and Quick-DASH-9, Upper-Extremity-Functional-Scale (UEFS), Upper-Extremity-Functional-Index (UEFI), Upper-Limb-Functional-Index (ULFI) and ULFI-10, Advise-Rehab-Global-Scale (ARGS) software, PatientSpecific-Functional-Scale (PSFS) and global status numerical-ratingscale (NRS). Summary performance was assessed by the 'Measurement of Outcome Measures' (MOM) providing criterion validity (Pearson's-r), test-retest reliability (ICC2.1), internal consistency (Alpha), sensitivity/ Error (MDC90), responsiveness (ES-SRM) and factor structure. Practicality included missing responses, completion and scoring time and readability (Flesch-Kincaid reading-ease and reading-grade).

Results: All tools demonstrated: criterion validity $(0.70-0.98)$, test-retest reliability (ICC2:1>0.92), internal consistency (Alpha $>0.80<0.95$-except the DASH $=0.96$ and $\mathrm{UEFI}=0.98$ ), responsiveness (ES=0.9-1.2 and $\mathrm{SRM}=1.2-2.2$ ), sensitivity (MDC90 $=6 \%-13 \%$ ) and single Factor structure (except the QuickDASH, UEFS, UEFI and PSFS). Missing responses varied $(0 \%=U L F I$, ULFI-10, PSFS, ARGS and NRS to 34\%=DASH). Completion and scoring $=45 \mathrm{sec}(\mathrm{ULFI})$ to $300 \mathrm{sec}(\mathrm{DASH})$ while reading graded 6-9.5 with ease 50\%-68\%. The MOM scale demonstrated ULFI=100\%, ULFI-10 and ARGS $=96 \%$, Q-DASH- 9 and $\mathrm{Q}-\mathrm{DASH}=88 \%$, NRS, PSFS and UEFI $=84 \%$, DASH and UEFS $=68 \%$.

Conclusions: The ULFI, ULFI-10 and ARGS were preferred with broader constructs plus improved completion and scoring times with the QuickDASH-9 less sensitive. All others showed psychometric flaws questioning validity or summated scores.

\section{Key Practice Points:}

- Many commonly advocated and investigated upper-limb PROs have questionable validity

- Net-based, mobile-device PROs are effective and efficient and should be adopted

- Clinicians and researchers must verify PRO reliability from independent researchers and not suffer the 'Cassandra Effect', 'assuming suitability because everyone else uses them

\section{DEVELOPING A RESEARCH PROJECT: DN \& ACUPUNCTURE, USING ACHILLES TENDON AS A TEMPLATE}

\section{Gabel CP ${ }^{1}$}

${ }^{1}$ Coolum Physiotherapy - Sunshine Coast

Question: Can acupuncture and Dry needling therapy (ADNT) practitioners develop research projects within their clinical settings?

Background: Individual clinicians' knowledge base offers an extensive resource for potential research. Often a pathway and understanding of research principles is required for clinically focused research. The process of knowledge extraction to enable project formation and eventual publication may be considered onerous and not contemplated-resulting in a knowledge loss. To encourage this, clinical research examples are provided through case series, cohort and case controls including ADNT for Achilles injuries.

Aims / objectives: To introduce participants to structured progressive processes that facilitate clinical research: from concept to completion, conclusion and final publication. To highlight the obstacles and common failures, and provide simple strategies for resource recognition that enhance research efficacy.

Design: A narrative of research options including case control and repeated measures designs.

Conclusion: ADNT Clinicians can facilitate the provision of clinical based research through recognition of their own available resources. This assists the immediate implementation of strategies that enable research to be initiated and completed.

\section{Key Practice Points:}

- Simple progressive pathways facilitate the process of project recognition, hypothesis formation, subsequent sampling and analysis.

- Interpretation and implications of research can be determined and publication or presentation to a larger forum considered.

- With confidence and experience in researching alone or with peer support, the knowledge base of physiotherapy can be expanded and evidence of different intervention techniques effective can be gained.
THE ROLE OF EXERCISE IN SPINAL CORD INJURY MANAGEMENT

\section{Galea $\mathrm{MP}^{1}$}

${ }^{1}$ Department of Medicine, Royal Melbourne Hospital, The University of Melbourne, Parkville, Victoria

Spinal cord injury (SCI) is an extreme example of deconditioning or movement deprivation, which has profound effects on the neuromusculoskeletal system. Rehabilitation after SCI has traditionally involved teaching compensatory strategies for identified impairments and deficits in order to improve functional independence. There is some evidence that regular and intensive activity-based therapies, directed at activation of the paralysed extremities, promotes neurological recovery and improvement of walking ability in people with incomplete $\mathrm{SCl}$. However more research is needed to determine those for whom this type of intervention will lead to meaningful clinical benefit. Physical activity in general appears to be a critical factor in the maintenance of the health of the person with $\mathrm{SCl}$, as well as maintaining major organ systems. The evidence to date shows that regular physical activity is effective in improving fitness, and that muscle and arterial atrophy can be reversed through functional electrical stimulation (FES)-assisted exercise of the paralysed limbs. However there is a need for further research to determine optimum dosage of interventions to achieve specific goals, for example, prevention of muscle atrophy and osteoporosis, and preservation of neural function. Barriers to exercise, such as a lack of access to suitable facilities and equipment, or the burden associated with using modalities such as FES, need to be addressed. Physical rehabilitation after SCI needs to move beyond the goal of maximizing independence to focus on maintenance of optimum health and fitness, as well as maintenance of target system function below the level of injury.

\section{Key Practice Points:}

- Activity-based therapies, directed at activation of the paralysed extremities in people with incomplete $\mathrm{SCl}$, have been shown to promote neurological recovery

- There is strong evidence for the benefit of regular physical activity for people with SCl

- Lack of access to suitable facilities and equipment is a significant barrier to regular exercise

\section{SPINAL CORD REPAIR AND REGENERATION}

\section{Galea MP}

${ }^{1}$ Department of Medicine, Royal Melbourne Hospital, The University of Melbourne, Parkville, Victoria

Advances in spinal cord injury research fall into two major categories: 1) protective and restoration strategies focused on the injury site, and 2) plasticity of spinal networks below the level of lesion. Many strategies have been tested and found to be successful in animal studies, but few have been tested in human clinical trials. At the lesion site, neuroprotective strategies to limit secondary damage, to replace damaged cells and to stimulate sprouting and axonal regeneration have been investigated. The earliest neuroprotective strategies include the reduction of compressive injury through early surgical decompression of the injured spinal cord or hypothermia. Other strategies target aspects of the injury cascade, such as hypoxia/ischaemia and excitotoxicity at the acute stage, inflammation and cell death at the intermediate stage and loss of myelin and scarring at the chronic stage. The second group of strategies is concerned with reawakening the spinal networks using electrical and pharmacological stimulation, and exercise. Exercise increases concentrations of neurotrophic factors in spinal and muscle tissue and reduces inflammation around the lesion site. Importantly activity is important in preventing deterioration of neuronal circuitry below the level of injury. However many questions remain about the appropriate timing of exercise interventions. Epidural stimulation with locomotor training has led to initiation of voluntary movement, standing and stepping in people with complete $\mathrm{SCl}$. The addition of monoaminergic replacement therapies to facilitate locomotion is currently being investigated. It is increasingly clear that combined approaches are needed, and timing of application of different therapies appears to be crucial.

\section{Key Practice Points:}

- Improved acute management of SCI has the potential to improve functional outcomes

- Many pharmacological interventions for neuroprotection are being translated into human clinical trials

- Activity-based therapies, directed at activation of the paralysed extremities, have been shown to preserve neural circuits below the level of injury and promote neurological recovery 


\section{SUPPORT MY SPINE ASAP! A RURAL TELE-HEALTH CARE MODEL FOR PATIENTS BEING MANAGED WITH A THORACIC LUMBAR SACRAL ORTHOTIC (TLSO)}

Gallagher $\mathbf{R}^{1}$, Henderson $]^{1}$, Giles $\mathrm{M}^{2}$, Morison ${ }^{3}$

${ }^{1}$ Physiotherapy department John Hunter Hospital, Newcastle, Hunter New England Local Health District

${ }^{2}$ Nursing and Midwifery Research Centre, Hunter New England Local Health District

${ }^{3}$ Neurosurgical department John Hunter Hospital, Newcastle, Hunter New England Local Health District

Question: Does a Telehealth model of care facilitating treatment in rural hospitals for patients requiring a TLSO eliminate the need for transfer to a tertiary referral hospital?

Design: Quantitative pre and post intervention design.

Participants: Physiotherapists, nursing and medical staff at rural hospital pilot sites.

Intervention: Pre and post intervention data collection provided patient demographics, and length of stay data around model of care implementation. Pre intervention staff surveys identified knowledge gaps informing educational packages which were provided with a mobile Telehealth link.

Outcome Measures: Primary: Number of patients managed under the new model of care.

Secondary: Length of stay, travel distance eliminated and complications. Results: 30 patients fitted with TLSO's and managed under this model of care to date. Length of stay has reduced from 9.14 days pre intervention to 3.8 days post intervention. Approximate savings of $\$ 6840$ per patient have been identified, with over $6000 \mathrm{kms}$ of patient travel eliminated. No complications have occurred. Rural Physiotherapists have been successfully up skilled in managing patients requiring TLSO fitting.

Conclusion: This intervention successfully created a model of care to facilitate local management of patients requiring TLSO fitting with Telehealth support from a tertiary referral hospital. Considerable savings have occurred from this model of care for patients and the health district. This model is currently being implemented district wide.

Key Practice Points:

- Telehealth model of care can be successfully implemented to Physiotherapy practice

- Physiotherapists critical role in redesigning models of care

- Telehealth increase the efficiency of healthcare resources

\section{INPH QUEST STUDY: QUANTIFYING A BATTERY OF GAIT, COGNITIVE AND RADIOLOGICAL EXAMINATIONS TO IMPROVE SHUNT RESPONSE FROM THE LUMBAR PUNCTURE TAP TEST - INTERIM RESULTS}

Gallagher $\mathbf{R}^{1,2}$, Chiarelli PE², Marquez J2 , Osmotherly PG ${ }^{1}$ Affliation: Physiotherapy department John Hunter Hospital, Newcastle, Hunter New England Local Health District

${ }^{2}$ Affiliation: School of Health Sciences, University of Newcastle

Question: Are changes identified on a lumbar puncture tap test(TT) in a battery of gait and balance outcome measures predictive of response to ventricular peritoneal (VP) Shunt insertion in idiopathic normal pressure hydrocephalus (iNPH)?

Design: Prospective study, examining the ability of a battery of outcome measures to predict change after VP shunt insertion.

Participants: 34 patients being considered for VP Shunt insertion. Intervention: Patients undergoing a TT for consideration of a VP shunt underwent a battery of outcome measures before and after a TT and again post-surgery.

Outcome Measures: Berg balance scale (BBS), Tinetti assessment, timed up and go (TUG), $10 \mathrm{~m}$ walk test.

Results: 15 patients were deemed responders, 11 underwent a VP Shunt. From response status, statistically significant differences were identified after TT for: Tinetti, 10m walk, TUG and BBS ( $p<0.05)$. Significant differences in change scores were identified between baseline and surgery for the Tinetti and BBS $(p<0.01)$. The Tinetti assessment has demonstrated an ability to predict response to surgery OR $0.56 \mathrm{p}<0.01$ adj $R^{2} 33.3 \%$.

Conclusion: A combination of balance and gait assessments can identify change in patients undergoing a TT. There may be ability to predict outcome to VP Shunt insertion based on TT response. Further study is required to confirm these findings.

\section{Key Practice Points:}

- A combination of tests can identify change in patients undergoing a TT with iNPH

- Outcome measures aid in identify responders for surgery

- Prognostic ability may exist for the Tinetti assessment to predict response to surgery

\section{OUANTIFYING STANDARD CARE IN}

ANN ACUTE STROKE UNIT (ASU): WHAT ARE THE INFLUENCES AND OUTCOMES AROUND COMMENCING MOBILISATION?

\section{Gallagher R ${ }^{1}$, Newphry A}

${ }^{1}$ Physiotherapy department, John Hunter Hospital, Newcastle, Hunter New England Local Health District

${ }^{2}$ Acute Stroke team, John Hunter Hospital, Newcastle, Hunter New England Local Health District

Question: What are the barriers to and outcomes around mobilisation in thrombolised patients admitted to an ASU in a tertiary hospital? Design: Retrospective analysis of ASU admissions over 2 years Participants: 100 thrombolised ASU admissions.

Intervention: Retrospective analysis of time and type of mobilisation, stroke severity, disability levels and barriers to first mobilisation.

Outcome Measures: time to first mobilisation, modified Rankin score (mRS), National institute health stroke scale (NIHSS), mobilisation type.

Results: No significant differences were present between measures by year. Significant differences were identified between mean time to first mobilisation by mobilisation type: walked (1.8days SD 0.65), sling transfer (3.11 days SD 1.41), standing transfer (2.42 days SD 1.63). Medical instability and investigations were the primary reason for delays in commencing mobilisation. Patients who were admitted over a weekend were more likely to be mobilised later than those admitted on a weekday. A moderate to strong correlation existed between days to $1^{\text {st }}$ mobilisation and NIHSS $(0.411 \mathrm{p0.01})$ at 24 hours and days to $1^{\text {st }}$ mobilisation and mRS at 7 days (0.411 p0.01) and 90 days (0.413 p0.01).

Conclusion: Unstable medical conditions and investigations in the first 48 hours represent the most significant barriers mobilisation in an ASU. Differences are present in time to, and type of mobilisation. Stroke severity also impacts mobilisation times. Correlation exists for time to first mobilisation and mRS at 7 and 90 days.

Key Practice Points:

- Mobilisation times and type are influence by stroke severity

- Medical instability and investigations are main barriers to mobilisation 
A SYSTEMATIC REVIEW OF PREVALENCE, INCIDENCE AND RISK FACTORS FOR SHOULDER AND NECK DYSFUNCTION AFTER NECK DISSECTION

Gane $\mathrm{EM}^{1,2}$, Michaleff $\mathrm{ZA}^{3}$, Jaber $\mathrm{K}^{4,5}$, McPhail SM2,6, Hatton $\mathrm{AL}^{1}$, Panizza $\mathrm{B}^{5,7}, \mathrm{O}^{\prime}$ Leary $\mathrm{S}^{8,9}$

${ }^{1}$ School of Health and Rehabilitation Sciences, The University of Queensland, Brisbane, Australia

2Centre for Functioning and Health Research, Metro South Hospital and Health Service, Brisbane, Australia

${ }^{3}$ The George Institute for Global Health, University of Sydney, Musculoskeletal Division, Sydney, Australia

${ }^{4}$ Department of Orthopaedics, Ipswich Hospital, Ipswich, Australia ${ }^{5}$ School of Medicine, The University of Queensland, Brisbane, Australia ${ }^{6}$ Institute of Health and Biomedical Innovation and the School of Public Health and Social Work, Queensland University of Technology, Brisbane, Australia ${ }^{7}$ Department of Otolaryngology, Head and Neck Surgery, Princess Alexandra Hospital, Brisbane, Australia

${ }^{8}$ Department of Physiotherapy, Royal Brisbane and Women's Hospital, Brisbane, Australia

${ }^{9}$ Centre of Clinical Research Excellence in Spinal pain, injury and health, The University of Queensland, Brisbane, Australia

Question: What are the prevalence, incidence and risk factors for shoulder and neck dysfunction after neck dissection (ND) surgery?

Design: Systematic review.

Participants: Eligible studies were cross-sectional, case-control or cohort in design featuring adult patients undergoing ND for head and neck cancer. Results were reported as prevalence, incidence or risk factors for outcomes of the shoulder or neck.

Intervention: Electronic search of Pubmed, CINAHL, Embase, The Cochrane Library, NYAM and OpenGrey.eu.

Outcome Measures: Methodology quality was assessed with The Critical Review Form - Quantitative Studies.

Results: Sixty-four papers were included in this review. The majority (40, $62.5 \%$ ) were cross-sectional in design and reported on the prevalence of shoulder dysfunction. Radical ND was consistently reported to have a higher prevalence of shoulder dysfunction followed by selective ND of levels II-V and modified radical ND. Risk factors for shoulder dysfunction include surgical, physical and psychosocial results. There was significant variability in the way in which dysfunction was defined.

Conclusion: The variability in prevalence and incidence reported may reflect inconsistent definition of "shoulder dysfunction", as well as the influence of radiotherapy. The review highlighted the lack of prospective studies investigating risk factors for dysfunction and the lack of investigation of neck outcomes in this population.

Key Practice Points:

- Prevalence of dysfunction is higher when the accessory nerve is resected

- When the nerve is preserved, clearance of nodes from around the accessory nerve does not always lead to poor outcome

- Clinicians are prompted to consider radiotherapy and psychosocial status of the patient

\section{AQUATIC PHYSIOTHERAPY STRATEGIES AND POOLSIDE ASSESSMENTS TO HELP BABIES AND CHILDREN LEARN ACTIVE LATERAL WEIGHT SHIFT - VIDEO CASE STUDIES}

\section{Gavin $\mathrm{S}^{1}$}

'Splash Physiotherapy, Melbourne

Poolside pre and post assessments allow us to see active change in a child's movement within and between sessions. This, along with independent practise of new movement patterns, facilitates carryover of skills learned in the aquatic environment onto land for function and participation goals. Approaches from Neuro Developmental Treatment, Halliwick, and swimming teaching, using the properties of the water, are combined to develop treatment strategies aimed at active lateral weight shift with elongation on the weight bearing side, in a variety of positions and movement transitions, related to goals. Video case studies of a range of babies and children will demonstrate ideas for poolside assessments and treatment strategies.
HOW DOES THE PRESENCE OF ASTHMA AFFECT SPORTING PARTICIPATION OF ACTIVE 12-15 YEAR OLDS?

Gaylor H, Crawford R, Smith M

Objective: The aim of this study was to develop an understanding of sporting participation among adolescents who experience asthma during sport.

Methods: Eight, 12 to 15 year olds, were interviewed on questions surrounding the topics of asthma, sporting participation and asthma management during sport.

Results: The results of this study identified that all participants regularly participated in sport and felt asthma was not the limiting factor to this participation. Participants were aware of the progression of their symptoms yet had minimal knowledge surrounding the management of asthma during sport. There was no limitation in the ability of participants to take up new sports; however, participants felt limited when stepping outside their comfort zone.

Conclusions: This study identified that asthma does not affect participation in sport. Furthermore, this study has revealed that there is a reduction in the delivery of asthma management education to adolescents, particularly when they are progressing from junior to elite level sport.

\section{Key Practice Points:}

- Adolescents living with asthma can participate in sport to the same level as those without asthma

- Adolescents are unaware of techniques to manage asthma when increasing training intensity for advancement to high level sport

- Further high quality studies are needed to develop research strategies, targeting how learning about asthma occurs in this age group to enable enhanced participation in higher level sports

\section{GROWING YOUR ANIMAL PHYSIO BUSINESS THROUGH VETS}

\section{Gelderman D}

Turbo Charge Your Practice

Growing your animal physiotherapy business can be difficult and challenging. You know there is a'need' by patients out there. But many of the pet-patient owners don't perceive that need and if and when they do they have no idea how to fulfil it. Typically they'll turn to their primary pet health-care provider, their Veterinarian for advice and treatment. Make no mistake, these dedicated professionals ARE good at what they do. But animal physiotherapy is not what they do and it's certainly not what they're good at. In fact, many of them have no idea that this modality even exists - after all, they did not learn about it at Uni. What's more, many of them are ingrained and even 'narrow' in their paradigmed thinking and are not in many cases interested in referring their pets on to you - for MANY reasons. All that can be changed. You just need to know, understand and address THEIR reasons. When you know what vets want (and need) to know about what you do, what vets want in communications, what help vets need from you to refer to you - then your potentially greatest referral source does become your GREATEST referral source. When you know how best to market animal physio to vets, you'll see your client numbers explode.

\section{Key Practice Points:}

- Identify what vets want (and need) to know about what you do

- Know what vets want in communications

- Learn what help vets need from you to refer to you 
EFFECTS OF AQUATIC THERAPY AND LAND-BASED THERAPY VERSUS LANDBASED THERAPY ON RANGE OF MOTION, OEDEMA AND FUNCTION AFTER HIP OR KNEE REPLACEMENT

\section{Gibson $\mathbf{A J}^{1}$, Shields $\mathbf{N}^{1,2}$}

${ }^{1}$ Department of Physiotherapy, Northern Health, Melbourne

2Department of Rehabilitation, Nutrition and Sport, School of Allied Health, La Trobe University, Melbourne

Question: Does aquatic therapy in combination with land-based therapy improve patient outcomes after hip or knee arthroplasty compared with land-based therapy alone?

Design: Systematic Review and Meta-analysis.

Participants: Hip or knee arthroplasty patients.

Methods: Six online databases (MEDLINE, CINAHL, AMED, EMBASE,

Cochrane, and PEDro) were searched from the earliest date available until September 2013. Controlled trials were included if they were published in English in a peer-reviewed journal and (1) participants had undergone any type of hip or knee arthroplasty; (2) participants were $\geq 18$ years old; (3) the study evaluated aquatic therapy in combination with land-based therapy; (4) the comparison group completed land-based therapy alone. Trial quality was assessed using the PEDro scale. Data were presented as standardized mean differences (SMDs), their associated 95\% Cls. Meta-analyses were conducted.

Results: Three small trials of moderate quality were included. Metaanalysis of two studies found moderate-quality evidence that aquatic therapy in combination with land-based therapy improves functional outcomes (SMD = 0.53; 95\% Cl, 0.03-1.03), knee range of motion (measured in knee or hip arthroplasty; SMD = 0.78; 95\% Cl, 0.27-1.29), and oedema (SMD $=-0.66 ; 95 \% \mathrm{Cl}$, -1.16 to -0.15 ) compared with land-based therapy alone. The changes in functional outcomes were not considered clinically significant.

Conclusion: The use of aquatic therapy in combination with land-based therapy, over land-based therapy alone for rehabilitation after hip or knee replacement cannot be supported because of the small number of studies of limited quality available and the modest differences found. An economic evaluation alongside a randomized controlled trial is needed to examine the cost-effectiveness of these clinical outcomes.

\section{Key Practice Points:}

- Aquatic therapy and land-based therapy are used after orthopaedic surgery to improve function, pain, oedema, muscle strength, and joint ROM

- This systematic review and meta-analysis suggests that there is currently not enough evidence to support or refute the hypothesis that aquatic therapy in combination with land-based therapy provides a clinically meaningful advantage over land-based therapy alone for rehabilitation after hip or knee replacement

- Further high-quality studies are needed to inform clinical practice.

\section{PAIN MANAGEMENT GUIDELINES IN THE ELDERLY}

\section{Gibson S]}

National Ageing Research Institute

Caulfield Pain Management and Research Centre

The world's population is ageing rapidly and the number of people over 80 years of age is expected to triple by the middle of this century. Bothersome, persistent pain is a major threat to overall health, function, psychological well-being and quality of life; affecting almost $50 \%$ of older adults. However, the older population is probably the most heterogeneous in terms of health status, cognitive and functional capacity. As a result, approaches to pain management are likely to vary considerably according to the specific capacities and needs of the older individual. The current presentation will explore relevant age-related differences in the approach to pain management, emphasising the diverse needs of the older population. Current best practice guidelines will be reviewed and synthesised to highlight key issues. A demonstration project on guideline implementation will be provided for a specific sub-group of older adults: namely, those living in residential aged care facilities. It will be shown that adopting best practice guidelines for the management of pain can lead to demonstrable improvements in pain, mood and function in this highly vulnerable and dependent group.

\section{Key Practice Points:}

- Participants will have greater insight into the diversity of specific needs and capacities of older adults suffering from persistent pain.

- Participants will develop a better understanding of best practice guidelines for the management of pain in older persons.

- Participants will be informed about a research demonstration project on the implementation of best practice guidelines into residential aged care facilities and the associated improvements in pain management practice that can result.
SUGAR GLIDER REHABILITATION POST SPINAL FRACTURE

Goff L, Sheard BJ

Question: Is it possible to return a Sugar Glider to functional capacity after spinal injury?

Design: A case study

Participants: One female Sugar Glider. Chosen for a case management due to the neurological deficits associated with the injury.

Intervention: Physiotherapy intervention was used at symptomatic, part practice and full task levels and treatment took place for approximately

4 months. A home based program with the utmost compliance was also adhered to.

Outcome Measures: Functional task completion; including walking, climbing and feeding.

Results: The sugar glider never returned to the wild but her motor control and function allowed her to be able to survive in a safe environment.

Conclusion: It is therefore possible to assume that physiotherapy interventions that target the pathology and symptoms of injury can be applied to different animals. It is important to be reminded of the scope of practice as a limitation to treatment and ensure that the goal of each therapeutic technique is constantly being evaluated.

\section{Key Practice Points:}

- Reminder that symptomatic, part and full practice are the keys to achieving functional tasks

- If you keep within your scope of practice you will do no harm

- Keeping an open line of communication with an owner explaining the goals and techniques of treatment will often give an opportunity to treat an animal and gain a positive result.

\section{APPLICATION OF ULTRASONOGRAPHY TO EXAMINE MUSCLE RECRUITMENT IMBALANCE BETWEEN SUPERFICIAL AND DEEP CERVICAL FLEXOR DURING CRANIO-CERVICAL FLEXION TEST (CCFT)}

Goo $\mathrm{MR}^{1}$, Jun $\mathrm{DH}^{2}$, Kim SG ${ }^{3}$

${ }^{1}$ Department of Physical Therapy, Daegu University, Korea

¿School of Health and Rehabilitation Sciences, The University of Queensland, Brisbane

${ }^{3}$ Department of Physical Therapy, Uiduk University, Korea

Question: Is it applicable to apply ultrasonography to detect imbalance of muscle recruitment in cervical flexor during CCFT and to contribute to clinical suggestion?

Design: A cross-sectional study

Participants: 18 adults with non-specific neck pain scored 5 points or greater in Neck Disability Index (NDI)

Outcome Measures: Muscle thickness of Sternocleidomastoid (SCM) and Deep Cervical Flexor unit (DCF) during CCFT were measured at $20 \mathrm{mmHg}$ (A reference point), $22 \mathrm{mmHg}, 24 \mathrm{mmHg}, 26 \mathrm{mmHg}, 28 \mathrm{mmHg}$, and $30 \mathrm{mmHg}$ by ultrasonography. Muscle imbalance at each pressure was calculated as a ratio of the magnitude change of SCM and DCF (muscle thickness on target pressure - muscle thickness on the reference pressure) / muscle thickness on the reference pressure). Repeated measures analysis of variance (ANOVA) was used for analysis.

Results: The significant differences of the ratio of magnitude change of both muscles across the level of pressures were found $(p<0.05)$. The lowest ratio was $1.33 \pm 0.42$ in $24 \mathrm{mmHg}$, followed $1.47 \pm 0.47$ in $26 \mathrm{mmHg}$ High ratio represented great imbalance of muscle recruitment between SCM and DCF, which appears high recruitment of SCM and low recruitment of DCF.

Conclusion: Ultrasonography is applicable to examine muscle recruitment imbalance of cervical flexor by identifying the ratio of magnitude change of muscles. The minimum ratio was in $24 \mathrm{mmHg}$ which was recommended as an optimal pressure for adequate DCF exercise.

\section{Key Practice Points:}

- The applicability of ultrasonography to evaluate the imbalance of cervical flexors was suggested.

- People with neck pain have great imbalance of muscle recruitment between SCM and DCF.

- The optimal pressure for DCF exercise might be established on the pressure level, $24 \mathrm{mmHg}$. 


\section{COMPARISON BETWEEN THE EFFECT OF BALANCE TRAINING USING A REACHING TASK IN SITTING AND STANDING}

Kim SG' ${ }^{1}$, Goo MR², Park JH²

${ }^{1}$ Department of Physical Therapy, Uiduk University, Korea

2Department of Physical Therapy, Daegu University, Korea

Question: Is balance training in sitting as effective as training in standing?

Design: Non-randomised controlled trial

Participants: 30 women aged over 65 years, without a history of the falls within the last year, or diseases which can affect their gait

Intervention: Participants were divided into two groups ( $n=$ each 15 ). Both performed 20 min balance training using a reaching task 3 times per week for 8 weeks in sitting and standing.

Outcome Measures: Balance was measured in both groups before and after intervention using Short Form of the Berg Balance Scale (BBS-SF) Timed 10-Meter Walk Test (10MWT) and Timed Up \& Go test (TUG). Fear of falling was assessed using the Falls Efficacy Scale (FES). Paired t-test and independent t-test were used for analysis.

Results: Balance training was effective for both groups, with improvement across in BBS-SF, 10MWT, and TUG $(p<0.05)$. However, only the standing group had a significant difference in reduction of fear of falling $(p<0.05)$. In the comparison between the groups after the intervention, only 10MWT showed that it is more improved in the standing group $(p<0.05)$

Conclusion: Balance training in both standing and sitting positions using a reaching task for the elderly were found to improve balance. Standing is more effective in reducing fear but sitting can be an option if falls risk is significant.

\section{Key Practice Points:}

- Balance training in sitting is effective to improve balance.

- Balance training in sitting may can be suggested as an alternative training for people in high falls risk

- Future research should apply this effectiveness to people with reduced balance.

\section{CANCER AND PHYSICAL ACTIVITY (CAPACITY) STUDY: IMPLEMENTATION OF THE PHYSICAL ACTIVITY GUIDELINES IN LUNG CANCER}

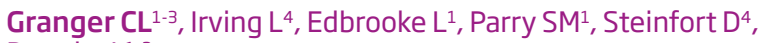
Denehy $L^{1,3}$

${ }^{1}$ Department of Physiotherapy, The University of Melbourne, Melbourne 2Department of Physiotherapy, Royal Melbourne Hospital, Melbourne Institute for Breathing and Sleep, Melbourne

${ }^{4}$ Department of Respiratory and Sleep Medicine, Royal Melbourne Hospital, Melbourne

Questions: Is a physical activity (PA) self-management program feasible when implemented in lung cancer management? Can it prevent decline in patients' PA levels, which occurs post-surgery?

Design: Prospective case series.

Participants (preliminary analysis): 18 patients with newly diagnosed operable lung cancer.

Intervention: PA program based on the cancer PA guidelines. This included education about PA and prescription of an unsupervised home exercise program. Information was delivered by an initial outpatient physiotherapy appointment, followed by weekly telephone consultations. Commenced either pre-surgery (if recruitment occurred $>7$ days prior to surgery) or post-surgery (if recruitment occurred $\leq 7$ days prior to surgery); and continued for all patients to 8-weeks post-surgery.

Outcome Measures: Feasibility of the intervention. Patient outcomes, including self-reported PA, function, symptoms, self-efficacy, mood and health-related quality of life, assessed pre-surgery and 8-weeks post-surgery.

Results: $17 \%$ of patients commenced the intervention pre-surgery; the remaining 83\% commenced post-surgery. Median [IQR] number of intervention sessions delivered (face-to-face or telephone) was 4 [3 to 5] per patient (out of a maximum possible of 6). There was no change in PA levels, function or dyspnoea from pre-surgery to 8-weeks post-surgery ( $p>0.05)$.

Conclusion: A PA program is feasible when implemented in the postsurgical setting. Compared with published literature that shows decline, patients maintained their PA levels 8-weeks post-surgery.

Funding: National Health and Medicine Research Council (Translating Research Into Practice Fellowship); Cancer Australia.
Key Practice Points:

- Current models of care do not address PA levels in patients with lung cancer

- Implementation of a PA program is feasible in the post-surgical setting

WHAT ARE THE BARRIERS TO

IMPLEMENTATION OF THE PHYSICAL ACTIVITY GUIDELINES IN LUNG CANCER? A OUALITATIVE STUDY INVOLVING EXPERT CLINICIANS

Granger $\mathrm{CL}^{1-3}$, Denehy L $\mathrm{L}^{1,3}$, Remedios $\mathrm{L}^{1}$, Parry $\mathrm{S}^{1}$

${ }^{1}$ Department of Physiotherapy, The University of Melbourne, Melbourne 2Department of Physiotherapy, Royal Melbourne Hospital, Melbourne IInstitute for Breathing and Sleep, Melbourne

Question: What are the barriers and enablers to implementation of the physical activity (PA) guidelines in lung cancer management from the perspective of treating clinicians?

Design: Qualitative study with focus groups and semistructured interviews.

Participants: Purposeful sampling. Seventeen clinicians from five hospitals in Melbourne (eight physiotherapists, three respiratory physicians, two thoracic surgeons, two oncologists, two nurses).

Qualitative methodology: Focus groups and interviews were conducted by independent moderators. Semi-structured questions were used to explore attitudes, beliefs and experiences. Thematic analysis was used to interpret data.

Results: Barriers at a hospital system level included: lack of standardised protocols; lack of knowledge; limited staffing and resources; limited time and lower prioritisation compared to delivery of other medical information particularly at time of diagnosis. Barriers at a patient level included: low motivation / fear to exercise; lacking knowledge regarding benefits; and external barriers (environment, social support, symptoms). Perceived enablers to implementation of PA included: adoption of a standardised framework; allocation of funding and resources; and individualisation at a patient level to enable behavioural change to increase PA.

Conclusion: Key barriers to implementation of the PA guidelines in lung cancer exist at both a hospital and patient level.

\section{Key Practice Points:}

- There is a gap between evidence-based PA guidelines for cancer and current clinical practice.

- Barriers to implementation exist at a hospital and patient level.

- Research targeted at bridging this gap is required in attempt to improve outcomes in lung cancer. 


\section{PONSETI CASTING AND THE EFFECT ON PASSIVE DORSIFLEXION RANGE OF MOTION IN CHILDREN WITH RELAPSED CLUBFOOT: A RETROSPECTIVE DATA ANALYSIS}

Marquez $\mathrm{EL}^{1}$, Pacey $\mathrm{V}^{1,2}$, Chivers $\mathrm{A}^{2}$, Gibbons $\mathrm{P}^{2}$, Gray $\mathrm{K}^{1,2}$

1Macquarie University, Sydney

2The Children's Hospital at Westmead, Sydney

Question: How much improvement in passive ankle dorsiflexion range of motion is achieved after Ponseti casting in children presenting with idiopathic relapsed clubfoot?

Design: Retrospective data analysis

Participants: Fifty-three children (13F, mean age 6.8 years) with idiopathic clubfoot initially managed with the Ponseti technique, presenting with relapse between 2005 and 2014. Children with atypical or syndromal clubfoot, and children not solely managed at The Children's Hospital at Westmead were excluded.

Outcome Measures: Passive ankle dorsiflexion range, passive abduction range, and Dimeglio scores.

Results: Of 170 relapse cases, 27 and 26 cases of unilateral and bilateral clubfoot respectively met the inclusion criteria. Of the 26 children with bilateral clubfoot, 10 presented with unilateral relapse. Children received an average of 4.3 casts (range $1-9$ ) during relapse management. In bilateral relapse cases, one foot was randomly selected to meet statistical independence requirements. Data was tested for normality and appropriate statistical tests were conducted. The median improvement between baseline and immediately post-casting passive dorsiflexion range in both unilateral and bilateral clubfoot $(n=45)$ was $15.0^{\circ}(95 \% \mathrm{Cl}$ 12.5 to $17.5^{\circ}, p \leq 0.05$ ). Median improvement in passive abduction range (n = 36) was $10.0^{\circ}\left(95 \% \mathrm{Cl} 7.5\right.$ to $\left.15.0^{\circ}, p \leq 0.05\right)$, and mean improvement in Dimeglio score ( $n=11$ ) was $2.27 / 20(95 \% \mathrm{Cl} 1.11$ to $2.44, p \leq 0.05)$. Sixteen participants experienced minor adverse events, predominantly skin redness.

Conclusion: Ponseti casting is an effective conservative treatment technique for children with idiopathic relapsed clubfoot, improving passive ankle dorsiflexion, abduction, and Dimeglio scores.

\section{Key Practice Points:}

- Idiopathic relapsed clubfoot may be successfully managed in a paediatric population with Ponseti casting.

- Significant improvements are noted in passive dorsiflexion and abduction ranges of motion, as well as Dimeglio scores.

- On average, 4.3 casts are necessary to anticipate these changes.

\section{IMPROVING COMMUNITY-BASED}

PHYSIOTHERAPY FOR INDIGENOUS

CHILDREN WITH GROSS MOTOR

DELAY OR PHYSICAL DISABILITY:

A CONTINUOUS OUALITY

\section{IMPROVEMENT APPROACH}

Greenstein $\mathrm{C}^{1}$, Lowell $\mathrm{A}^{2}$, Thomas $\mathrm{D}^{3}$

${ }^{1}$ Menzies School of Health Research

${ }^{2}$ Charles Darwin University

${ }^{3}$ Menzies School of Health Research

Question: Could a continuous quality improvement approach improve services provided by a community-based paediatric physiotherapy service to Indigenous children with gross motor delay or physical disability?

Design: A continuous quality improvement process based on Audit and Best Practice for Chronic Diseases, a successful program used within Indigenous health care, was adapted for physiotherapy.

Participants: One community-based child physiotherapy service in Northern Territory.

Intervention: The continuous quality improvement process involved a clinical audit; a workshop where clinicians assessed their health care systems, identified weaknesses and strengths, and developed goals and strategies for improvement; and reassessment through a second audit and workshop.

Outcome Measures: Achievement of 3 goals at the second audit related to: documentation for clients with complex needs, hip surveillance, and goal setting and outcomes.

Results: Goals were met for clients with complex needs and hip surveillance. Goal setting and outcome goals were not met. We identified improvements in all health care system areas with the biggest improvements in the area of information systems and decision support and self-management support.
Conclusion: This continuous quality improvement process provided a practical method: 1) to gain insight into client needs, access to services and the quality of physiotherapy care 2) for staff reflection on issues such as cultural competence and external influences 3) to develop measurable outcomes for physiotherapists working in Indigenous health care.

\section{Key Practice Points:}

- This continuous quality improvement process can improve service provision

- This continuous quality improvement process reflects measurable change in the health care system

- Time and resources must be allocated for maximum benefit APPROVED BY THE: Human Research Ethics Committee of the Northern Territory Department of Health and Menzies School of Health Research and the Indigenous Health Sub-committee (HREC-2012-1912)

\section{COMMUNICATION AND CONTEXT ARE IMPORTANT TO INDIGENOUS CHILDREN WITH PHYSICAL DISABILITY AND THEIR CARERS AT A COMMUNITY-BASED PHYSIOTHERAPY SERVICE}

Greenstein $C^{1}$, Lowell $A^{2}$, Thomas $D^{3}$

${ }^{1}$ Menzies School of Health Research

${ }^{2}$ Charles Darwin University

3Menzies School of Health Research

Question: What are the experiences of Indigenous children with physical disability and their carers with their community-based physiotherapy service? What factors influence their experiences with the physiotherapy service and how could the service be improved?

Design: A qualitative study using in-depth, semi-structured open-ended interviews consistent with the interpretivist perspective of the researcher and ethical principles of Indigenous health research. Interviews were audio-recorded, transcribed, and coded for themes using inductive analysis. Each interview was then checked with the participant to confirm themes.

Participants: Nine parents and foster carers of children with physica disability aged 0-21 years and five children and youth with physical disability aged 8 -18 years were selected using purposive and theoretical sampling technique.

Results: Three themes emerged from the data and informed practice: carers of children with physical disability experience multiple, complex demands in their lives; relationships involving caring, consistency and communication are important; being Indigenous influences children's and carers' experiences in ways that may not be obvious to non-Indigenous service providers. The issue of communication underpinned the participant experiences throughout these themes.

Conclusion: The research highlighted the importance of effective communication, developing relationships, viewing the child holistically and recognising the influence of being Indigenous on health care experiences.

\section{Key Practice Points:}

- Effective communication skills are essential in community-based physiotherapy practice.

- Community Physiotherapists should be aware that being Indigenous may influence lives in ways that are not visible to service providers.

- Community Physiotherapists working in similar environments should consider context-specific, person/family centred practices.

APPROVED BY THE: Human Research Ethics Committee of the Northern Territory Department of Health and Menzies School of Health Research and the Indigenous Health Sub-committee (HREC-2012-1912) 


\section{CLINIMETRIC PROPERTIES OF GROSS MOTOR ASSESSMENT TOOLS FOR PRESCHOOL AND PRIMARY SCHOOL AGE CHILDREN: A SYSTEMATIC REVIEW}

Griffiths A $^{1,2,3}$, Spittle $A^{2}$, Morgan $\mathrm{P}^{3}$

'Monash University, Melbourne

2The Murdoch Children's Research Institute, Melbourne

${ }^{3}$ The Royal Children's Hospital, Melbourne

Question: What are the clinimetric properties of assessment tools for evaluation of gross motor skills in children $2-12$ years?

Design: Systematic review of the validity, reliability and clinical utility of gross motor assessment tools

Participants: Children aged 2-12 with or without a diagnosis

Outcome Measures: Assessment tools were included if they were discriminative, evaluative or predictive of gross motor function, assessed $>2$ components of gross motor function and a gross motor score was able to be meaningfully extracted. All papers were assessed for methodological quality using the COSMIN checklist.

Results: Eight assessment tools met inclusion criteria: Bayley Scale of Infant and Toddler Development III (Bayley-III), Bruininks-Oseretsky Test of Motor Proficiency 2 (BOT-2), Movement Assessment Battery for Children 2 (MABC-2), McCarron Assessment of Neuromuscular Development (MAND), Neurological Sensory Motor Developmental Assessment (NSMDA), Peabody Developmental Motor Scales 2 (PDMS-2), Rapid Neurodevelopmental Assessment Tool and Test of Gross Motor Development 2 (TGMD-2). Content and construct validity and internal consistency of most tests was good to excellent (Cronbach's $\otimes 0.5-0.99$ ). The BOT-2 has excellent test-retest reliability (ICC 0.80-0.99) supported by excellent methodology. The MABC-2 has excellent test-retest (ICC 0.83 0.96 ) and variable inter-rater reliability (ICC 0.63-0.98) supported by poor to good methodology. The TGMD-2 is supported by poor to good quality publications for test-retest (ICC 0.81-0.92), intra-rater (ICC 0.92-0.99) and inter-rater (ICC 0.75-0.93) reliability. There is limited evidence of reliability for other assessment tools in this age group.

Conclusion: Most identified assessments are supported by good-excellent content and construct validity. The BOT-2, MABC-2, and TGMD-2 have the most evidence for reliability in this age group.

\section{Key Practice Points:}

- Selection of assessment tools should be guided by evidence of validity and reliability

- All assessment tools have an element of error which should be taken into consideration when interpreting results

- The BOT-2, MABC-2 and TGMD-2 have the most evidence for reliability in children 2-12 years

\section{UTILITY OF CLINICAL TESTS IN PATIENTS WITH LATERAL HIP PAIN TO DIAGNOSE MRI-IDENTIFIED GLUTEAL TENDINOPATHY}

Grimaldi A ${ }^{1,2}$, Mellor R², Bennell $\mathrm{K}^{3}$, Hodges P², Vicenzino $\mathrm{B}^{2}$

IPhysiotec Physiotherapy, Brisbane

2The University of Queensland, Brisbane

${ }^{3}$ The University of Melbourne, Melbourne

Question: What is the diagnostic utility of clinical tests for gluteal tendinopathy in patients with lateral hip pain?

Design: Cross-sectional diagnostic utility/accuracy study.

Participants: Sixty-five participants reporting lateral hip pain.

Intervention: A mix of novel and commonly used pain provocation tests applying compressive and tensile loads on the gluteal tendons and aiming to reproduce pain over the greater trochanter (e.g., flexion adduction external rotation \pm isometric internal rotation, side-lying hip adduction \pm isometric abduction, single leg stance sustained for 30 seconds, palpation) were used to clinically diagnose gluteal tendinopathy.

Outcome Measures: MRI of the hip served as the reference standard, where intratendinous high signal intensity was required for the diagnosis of gluteal tendinopathy.

Results: Palpation had highest sensitivity (80\%) and lowest specificity (47\%), whereas all other clinical tests had high specificity (87-100\%) but low sensitivity (20-44\%) in detecting MRI-identified gluteal tendinopathy. The combined test battery returned a sensitivity of $60 \%$ and a specificity of $73 \%$. Twenty participants (31\%) had gluteal tendinopathy on MRI but were negative clinically.

Conclusion: Palpation is most useful for ruling out gluteal tendinopathy, while clinical tests involving contraction in positions that load the gluteal tendons are most useful for ruling it in.

\section{Key Practice Points:}

- A patient with lateral hip pain who is not palpably tender over the greater trochanter is unlikely to have gluteal tendinopathy.

- A patient who develops lateral hip pain within 30 seconds of single-legstanding is very likely to have gluteal tendinopathy.

- Clinical tests with an active muscle contraction have greatest diagnostic utility.

\section{ANZAC RELATIONSHIPS AND THE SHARED PHYSIOTHERAPY PRACTICE THRESHOLDS FOR AUSTRALIA AND AOTEAROA NEW ZEALAND}

Shinkfield P1, Grudzinskas K², Bell A², Mueller J3 , Grbin M $^{4}$

${ }^{1}$ Chair, Physiotherapy Board of Australia

2Physiotherapy Board of Australia

${ }^{3}$ Chair, Physiotherapy Board of New Zealand

${ }^{4}$ Physiotherapist, New Zealand

Question: The objectives of both regulatory authorities were to develop and publish shared, binational, stakeholder supported, evidence informed entry level physiotherapy practice statements.

Design: The project established methodology to ascertain current stakeholder satisfaction with existing qualifying statement documents, to identify the most appropriate and contemporary format and nomenclature for new statements, and to identify best practice approaches to undertaking the development of new statements.

Participants: Project manager (ARETE Pty Ltd), Expert Reference Group, stakeholders including patients/consumers, professional associations, registration boards, accreditation bodies, education providers, students and physiotherapists

Outcome Measures: Following an extensive consultation process, including more than 200 physiotherapists engaged in focus groups and refinement workshops, the Physiotherapy Practice Thresholds in Australia and Aotearoa New Zealand is a format adapted from a framework for physicians in Canada ('the CanMeds approach').

Results: The Threshold Statements in both countries were formally launched on May $1^{\text {st }}$ 2015. Implementation will be a phased process and will include accommodating differences in the regulatory environment across both countries.

Conclusion: The new Thresholds represent the first internationally shared statements entry level competency to be established for the physiotherapy profession.

\section{Key Practice Points:}

- Threshold statements provide a benchmark for accreditation functions, practitioners to assess their own competence, and Board registration decisions, professional conduct/competence matters and supervision arrangements

- Shared threshold statements represent a common baseline of competency for physiotherapists in Australia and Aotearoa New Zealand practising under the Trans Tasman Mutual Recognition Arrangement. 


\section{DEVELOPING A RESOURCE FOR PHYSIOTHERAPY ANTENATAL GROUP EDUCATION}

Guyatt SL, Hickey L

Physiotherapy Department, Mater Health Services, South Brisbane Continence and Women's Health Physiotherapy Network, Queensland Health

Question: Antenatal physiotherapy education: What should be included and what form should this take?

Design: Review of current literature on physiotherapy for childbearing women and effective adult education strategies.

Participants: Physiotherapists facilitating antenatal education and pregnant women.

Interventions: Applying evidence from systematic reviews, randomised controlled studies, clinical guidelines and recognised texts on physiotherapy interventions for childbearing women and effective education strategies to antenatal physiotherapy education.

Outcome: Develop a resource for physiotherapists.

Results: There is varied skill and knowledge between physiotherapists facilitating antenatal education. Queensland clinical guidelines recommend pregnant women receive antenatal education on pelvic floor muscle training (PFMT) from a physiotherapist and information on perineal massage. Physiotherapy is effective for preventing and managing low back pain and pelvic girdle pain (PGP) in pregnancy. Skills and knowledge of relaxation, stress management, good bladder / bowel habits and physical activity are beneficial to childbearing women. Effective education is practical, uses problem solving and a variety of communication styles. Women seek health knowledge about pregnancy, birth and postnatal recovery from a range of sources.

Conclusion: Quality physiotherapy antenatal education is practical, problem based and simple. Antenatal physiotherapy education should include teaching practical skills for management of key areas of impact in pregnancy, childbirth, postnatal recovery and parenthood with supporting written and/or web based information.

\section{Key Practice Points:}

- Antenatal education should include PFMT, perineal massage, selfmanagement of low back / PGP, exercise, stress management and relaxation.

- Physiotherapists can teach these as practical skills effectively.

- Web based resources are a useful adjunct to antenatal education.

\section{INDIVIDUALISED PHYSIOTHERAPY IS COST-EFFECTIVE RELATIVE TO ADVICE FOR PEOPLE WITH LOW BACK DISORDERS: ECONOMIC ANALYSIS WITHIN A RANDOMISED CONTROLLED TRIAL}

Hahne A ${ }^{1}$, Ford $\mathrm{JJ}^{1}$, Surkitt LD ${ }^{1}$, Slater SL ${ }^{1}$, Richards MC ${ }^{1}$, Chan AYP ${ }^{1}$, Hinman RS², Taylor NF ${ }^{1}$

1Physiotherapy, La Trobe University, Melbourne

'Department of Physiotherapy, Melbourne School of Health Sciences, The University of Melbourne

Question: Is it cost-effective to deliver 10 sessions of individualised physiotherapy versus two sessions of guideline-endorsed advice?

Design: A within trial cost-utility analysis was conducted from the healthcare perspective within a randomised controlled trial (ANZCTR12609000205235).

Participants: 300 participants with a primary complaint of low back and/ or referred leg pain.

Interventions: Participants received either 10 sessions of individualised physiotherapy or two sessions of guideline-endorsed advice over a 10 week period.

Outcome Measures: Cost data included the intervention, cointerventions, and medication over a 12-month period. Quality adjusted life years (QALY) were calculated using validated EuroQol health state utilities. The incremental cost effectiveness ratio (ICER) measured the costeffectiveness of individualised physiotherapy relative to advice.

Results: Healthcare costs (trial physiotherapy, co-interventions and medication) were similar for both groups: mean difference \$41.63 (95\% Cl: $-\$ 308.45$ to $\$ 391.70)$. Health benefits were significantly greater with individualised physiotherapy: incremental QALY $=0.06$ ( $95 \%$ Cl: 0.02 to 0.10). The ICER was $\$ 649.80$ per QALY gained. Additionally, costs associated with work absence were significantly lower in the individualised physiotherapy group relative to advice: \$-3073.08 (95\% Cl: -\$5924.43 to -\$221.73)

Conclusion: For people with low back disorders, individualised physiotherapy is cost-effective relative to guideline-endorsed advice.

\section{Key Practice Points:}

- Advice is typically seen as an effective and cheap treatment for low back disorders.

- Spending more money to provide 10 sessions of individualised physiotherapy was not only more effective than advice, but also led to cost savings relating to other interventions, medication and work absences over a 12-month period.

\section{INDIVIDUALISED PHYSIOTHERAPY VERSUS ADVICE FOR PEOPLE WITH LUMBAR DISC HERNIATION AND ASSOCIATED RADICULOPATHY: TWO- YEAR FOLLOW-UP OF A RANDOMISED CONTROLLED TRIAL}

Hahne A ${ }^{1}$, Ford $\mathrm{JJ}^{1}$, Surkitt LD ${ }^{1}$, Slater $\mathrm{SL}^{1}$, Richards $\mathrm{MC}^{1}$, Chan AYP ${ }^{1}$, Hinman RS², Taylor NF ${ }^{1}$

${ }^{1}$ La Trobe University, Melbourne

Department of Physiotherapy, Melbourne School of Health Sciences, The University of Melbourne

Question: What are the 2-year outcomes of individualised physiotherapy relative to advice for people with lumbar disc herniation and associated radiculopathy?

Design: Two-year follow-up of a randomised controlled trial (ANZCTR12609000205235).

Participants: Fifty-four participants with clinical and radiological evidence of lumbar disc herniation with associated radiculopathy (six-week to sixmonth duration).

Interventions: Participants received either 10 sessions of individualised physiotherapy or two sessions of guideline-recommended advice over a 10-week period.

Outcome Measures: Primary outcomes were activity limitation (Oswestry Disability Index), leg pain and back pain. Global rating of change was a secondary outcome.

Results: Significant primary outcomes that favoured individualised physiotherapy at earlier timeframes were not sustained at 2-years. However, participants in the individualised physiotherapy group were still significantly more likely to be "much improved" on the global rating of change scale (risk difference=28\%; $95 \% \mathrm{Cl} 3 \%$ to $49 \%$ ), and were more likely to achieve a $50 \%$ reduction in leg pain from baseline (risk difference=33\%; 95\% Cl 6\% to 54\%).

Conclusion: People with lumbar disc herniation and associated radiculopathy achieve similar activity limitation, back pain and leg pain outcomes by 2 -year follow-up with either individualised physiotherapy or guideline-recommended advice. Individualised physiotherapy appears to provide superior benefits in relation to other clinically important secondary outcomes

\section{Key Practice Points:}

- For people with lumbar disc herniation and associated radiculopathy, the primary benefits of providing individualised physiotherapy over advice seem to diminish at 2-year follow-up.

- Individualised physiotherapy continues to provide some clinically important secondary benefits over advice even after 2 -years. 


\section{WHO BENEFITS MOST FROM INDIVIDUALISED PHYSIOTHERAPY \\ VERSUS ADVICE FOR LOW BACK DISORDERS: EFFECT MODIFIER ANALYSIS OF RANDOMISED CONTROLLED TRIAL DATA}

Hahne A $J^{1}$, Ford $]^{1}$, Surkitt $L D^{1}$, Slater SL ${ }^{1}$, Richards $M C^{1}$, Chan AYP ${ }^{1}$, Hinman RS' ${ }^{2}$, Taylor NF ${ }^{1}$

${ }^{1}$ La Trobe University, Melbourne

'Department of Physiotherapy, Melbourne School of Health Sciences, The University of Melbourne

Question: Which participants with low back disorders benefit most from individualised physiotherapy relative to guideline endorsed advice.

Design: Pre-planned secondary analysis of the Specific Treatment of Problems of the Spine (STOPS) randomised controlled trial (ANZCTR12609000205235).

Participants: 300 patients with a primary complaint of low back and/or referred leg pain

Interventions: Participants received either 10 sessions of individualised physiotherapy or two sessions of guideline-recommended advice over a 10-week period.

Outcome Measures: Primary outcomes were activity limitation (Oswestry Disability Index), leg pain and back pain, measured over 12-months. Ten potential effect modifiers were selected a-priori based on plausible mechanisms.

Results: Higher baseline back pain, higher Orebro score, longer duration of symptoms and worse coping were associated with significant $(p<.05)$ additional benefits of individualised physiotherapy over advice. Multivariate models led to the elimination of coping as a significant effect modifier, but the other variables remained. The size of the effect modification was clinically important.

Conclusion: People with low back disorders derive additional benefit from individualised physiotherapy over advice if they have higher baseline back pain, higher Orebro scores and longer duration of symptoms.

\section{Key Practice Points}

- These results offer clinicians an opportunity to target their treatment towards people with low back disorders who are most likely to benefit.

- People with high back pain scores, high Orebro scores, and longer duration of symptoms should be given more intensive individualised physiotherapy rather than advice.

- Those with very low scores on these baseline measures are likely to do equally well with advice.

\section{WHAT IS THE EFFECTIVENESS OF WEEKEND ALLIED HEALTH SERVICES ON ACUTE MEDICAL AND SURGICAL WARDS FOR REDUCING LENGTH OF STAY, READMISSIONS AND ADVERSE EVENTS IN HOSPITAL? RESULTS OF TWO RANDOMISED TRIALS}

Haines $\mathrm{T}^{1,2}$, Mitchell $\mathrm{D}^{2}$, Bowles $\mathrm{K}^{1}$, May K2, Markham $\mathrm{D}^{2}$, Chiu $T^{3}$, Philip $K^{4}$, $\mathrm{O}^{\prime}$ Brien $\mathrm{L}^{1}$, Plumb $\mathrm{S}^{5}$, Sarkies $\mathrm{M}^{2}$, Ghaly $\mathrm{M}^{3}$, Juj $\mathrm{G}^{5}$, McDermott $\mathrm{F}^{1,2}$, Skinner $\mathrm{E}^{3}$

1Monash University, Clayton, Australia

¿Monash Health, Clayton, Australia

${ }^{3}$ Western Health Service, Footscray, Australia

${ }^{4}$ Department of Health and Human Services, Melbourne, Australia

${ }^{5}$ Melbourne Health, Melbourne, Australia

Question: What is the effectiveness of weekend allied health services on acute medical and surgical wards for reducing length of stay, readmissions and adverse events in hospital?

Design: Two stepped-wedge randomised controlled trials. The first was a disinvestment (roll-in) stepped wedge of the existing model of service, while the second was a conventional (roll-out) stepped wedge of a new, stakeholder-driven model.

Participants: Admissions to 12 acute medical and surgical wards across two metropolitan teaching hospitals in Melbourne, Victoria over a 14 month period across 2014/2015

Intervention: 1) Existing weekend allied health services were inclusive of physiotherapy and occupational therapy at both sites, with speech pathology, dietetics and social work at one site, 2) A new, stakeholderdriven model of weekend service designed to be tailored to the individual needs of wards in the trial. This was examined during the roll-out trials.

Outcome Measures: Patient adverse events were collected using routine administrative data collection systems combined with daily ward visits and hand-over note reviews. Length of stay and readmissions within 28 days were captured using administrative data collection systems.

Results: The two trials have been completed in April 2015 with over 13,000 patients involved. Interim safety analyses have demonstrated no adverse impact of removing the current weekend allied health service on patient length of stay, readmission or adverse event outcomes (all multilevel, generalised linear models p-values >0.05).

Conclusion: Removal of the weekend allied health services from acute medical and surgical wards did not affect patient flow or safety outcomes. Key Practice Points:

- There is need to ensure that resources are allocated to areas that will generate the greatest benefit.

- Weekday and weekend services in other areas (eg. rehabilitation wards, ICU) all compete with weekend services provided on acute medical and surgical wards.

- There is need to consider where these resources can be allocated to have the greatest effect.

\section{DO FALLS AND EXERCISE IMPACT ON DEPRESSION IN OLDER ADULTS RECENTLY DISCHARGED
FROM HOSPITAL?}

Haines $T^{1,2}$, Lalor $A^{1}$, Robins $L^{1}$, Lee $D C A^{1,2}$, Brown $T^{1}$, Russell $G^{1}$, Stolwyk R ${ }^{1}$, McDermott $\mathrm{F}^{1,2}$, Williams $\mathrm{C}^{3}$, Johnson $\mathrm{C}^{1}$, O'Connor $\mathrm{D}^{1,2}$ 'Monash University, Clayton, Australia

¿Monash Health, Clayton, Australia

3Peninsula Health, Frankston, Australia

Question: Do falls and reduced physical activity impact lead to depressive symptoms in older adults recently discharged from hospital?

Design: Mixed-methods study; prospective cohort with in-depth qualitative interviews.

Participants: Older adults admitted to wards at Monash Health and Peninsula Health for a period of 2 weeks or more. Participants with cognitive impairment were excluded. Quota sampling was used to ensure that $50 \%$ of our sample was male and at least $25 \%$ were from culturally and linguistically diverse backgrounds.

Outcome Measures: Symptoms of depression and anxiety were measured using the Geriatric Depression Scale Short Form and Geriatric Anxiety Inventory respectively. Physical activity was measured using the PhoneFITT scale. Falls were measured using telephone or face-to-face interview. Each measure was collected prior to hospital discharge, then monthly for 6 months with baseline, 3 and 6 month assessments being face-to-face, and those between being telephone-based. Qualitative interviews were conducted at the 6 month assessment with a subsample of participants who reported encountering clinically significant symptoms of depression or anxiety over the course of the follow-up. They provided a narrative account of what they felt contributed to their symptoms of depression or anxiety.

Results: The prospective cohort study recruited $n=300$ participants. The time-course of symptoms of depression and anxiety did not follow patterns originally anticipated by the investigators, with a high proportion exhibiting a "spike" above the clinically significant threshold followed by remission of these symptoms. Falls in some instances preceded these spikes and narrative accounts revealed broader psychological and social impacts of the fall that may have later contributed to depressive symptoms. Engagement in physical activity and community activities was viewed as being of benefit, though a broader narrative of loss, concern for the future, social isolation and support was also evident.

Conclusion: It appears that falls and physical activity levels are two of a number of important factors that impact upon the development of symptoms of depression and anxiety in older adults recently discharged from hospital.

\section{Key Practice Points:}

- Older adults encounter a period of substantial change when transitioning home after an extended period of hospitalisation.

- Symptoms of depression and anxiety are relatively common and may appear some time following the hospitalisation.

- Falls can precipitate concern about the future by the older adult and their family and a change in self-perception that can then lead on to feelings of anxiety and depressio 


\section{ELECTROMYOGRAPHIC ANALYSIS OF HIP DURING SINGLE-LEG SQUAT EXERCISES IN SUBJECTS WITH GLUTEUS MEDIUS WEAKNESS}

Han $\mathrm{HR}^{1}$, Lim OB ${ }^{1}$, Son $\mathrm{JI}^{1}, \mathrm{YiCH}^{2}$, Cynn HS², You SH${ }^{2}$

${ }^{1}$ Dept. of Physical Therapy, The Graduate School, Yonsei University, Wonju, Republic of Korea

2Dept. of Physical Therapy, College of Health Science, Yonsei University, Wonju, Republic of Korea

Question: How do the three different single-leg squat exercises affect the gluteus medius (GMED), tensor fasciae latae (TFL), and adductor longus $(A L)$ in subjects with gluteus medius weakness?

Design: Randomized, within-participants, experimental study

Participants: Fifteen adults with gluteus medius weakness

Methods: Participants performed three different single-leg squat exercises with the supporting leg's knee flexed to $60^{\circ}$. The three singleleg squat exercises were the unilateral wall squat, lateral step down, and frontal step down.

Outcome Measures: Surface electromyography (EMG) was used to measure GMED, TFL, and AL activity and to assess the GMED:TFL and GMED:AL ratios. One-way repeated analysis of variance was used to compare GMED, TFL, and AL muscle activity and the GMED/TFL and GMED/ $A L$ ratios. Bonferroni correction was used to determine differences among the exercises.

Results: TFL EMG activity significantly decreased during the unilateral wal squat compared to the lateral step down and frontal step down $(p<0.05)$. The GMED:TFL ratio significantly increased during the unilateral wall squat compared to the lateral step down $(p<0.05)$

Conclusion: These data indicated that the unilateral wall squat exercise could be used clinically to enhance GMED muscle activity.

Key Practice Points:

- Single-leg squat exercises represent one of the most commonly used hip-strengthening exercises.

- Single-leg squat activities require GMED activation of the weight-bearing side to control the pelvis.

- This study has implications for physical therapists supporting hip abductor strengthening exercises to treat patellofemoral pain syndrome.

\section{DOES RIGID TAPING ALTER LUMBAR RANGE OF MOVEMENT?}

Harding $\mathrm{AL}^{2}$, Langford $]^{2}$, Cowan $\mathrm{SM}^{1,2,3}$

${ }^{1}$ Clifton Hill Physiotherapy, Melbourne

2St Vincent's Hospital, Melbourne

${ }^{3}$ The University of Melbourne, Melbourne

Question: Does rigid taping alter lumbar movement in asymptomatic subjects?

Design: Within subject, repeated measures experimental study design.

Participants: Ten healthy adults $171(8) \mathrm{cm}$ tall, weighing $71(11) \mathrm{kgs}$ and aged 34(9) years old, with no recent (past six months) history of low back pain.

Intervention: Data were collected during functional movement under two randomised conditions; with and without lumbar taping. Lumbar taping consisted of a fixomull underlay and rigid (leukotape) overlay, applied as two vertical strips between the thoraco-lumbar junction and posterior superior iliac spine with one cross intersecting at L3.

Outcome Measures: Lumbo-pelvic motion was assessed using DorsaV movement sensor technology during lumbar active movement (flexion, extension, lateral flexion), functional movement (inline lunge, hurdle step overhead squat, step down, single leg balance) and during sitting posture (usual, slouched, upright)

Results: Paired t-tests demonstrated lumbar taping significantly reduced lumbar lateral flexion range of movement $(p<0.05)$ and trended towards significance for decreasing lumbar flexion range $(p=0.05)$. Taping was also found to significantly increase lumbar extension $(p=0.05)$. During the step down task taping decreased lateral flexion $(p<0.05)$ but had no effect on the other functional movements or sitting postures ( $p>0.05$ )

Conclusion: Rigid taping significantly altered lumbar range of movement in asymptomatic individuals.

\section{Key Practice Points:}

- Rigid taping altered lumbar movement patterns in an asymptomatic population

- These findings provide mechanistic support for the common clinical practice of taping painful lower backs.

- Further research should investigate the effect of lumbar taping on subjects with low back pain.

\section{ARE WE 'ABOVE PAR' WITH A \\ VICTORIAN STATE-WIDE INITIATIVE OF INTRODUCING PHYSIOTHERAPY-LED POST ARTHROPLASTY REVIEW CLINICS?}

Harding $\mathrm{P}^{1}$, Walter $\mathrm{K}^{1}$, Page $\mathrm{C}^{2}$, Phan $\mathrm{U}^{3}$, Terrill $\mathrm{D}^{4}$

${ }^{1}$ Department of Physiotherapy, The Alfred, Melbourne

2Department of Physiotherapy, St Vincent's Hospital, Melbourne

${ }^{3}$ Department of Physiotherapy, The Royal Melbourne Hospital, Melbourne

${ }^{4}$ Department of Health and Human Services, Melbourne

Question: What is the value add of introducing a physiotherapy-led post arthroplasty review clinic?

Design: Prospective, observational, multicentre evaluation of a new model of care

Participants: Post arthroplasty review clinics from eight Victorian public hospitals.

Outcome Measures: Cost efficiency (cost of occasions of service post implementation compared with baseline); cost effectiveness (increased surgeon capacity); safety and quality (adverse events, percentage of patients with outcome measures completed, communication with General Practitioners, and patient and staff satisfaction).

Results: The cost of an occasion of service averaged \$66 (\$34 - \$114) less per site compared to baseline. The average cost effectiveness of increasing surgeon capacity was $\$ 7,185(\$ 1,649-\$ 19,690)$. Safety and quality was demonstrated with the consistent use of outcome measures, increased communication with general practitioners, and no adverse events were recorded. Of significance, $96 \%$ of patients were satisfied with their experience, and $83 \%$ of the workforce expressed they were very satisfied/ satisfied in understanding the scope of practice for the role.

Conclusion: Physiotherapy-led post arthroplasty review clinics are a safe, cost effective and efficient adjunct to the traditional surgeon-led clinics. These services can contribute to managing the increasing demand on orthopaedic outpatient clinics.

\section{Key Practice Points:}

- New models of care provided by musculoskeletal physiotherapists can be cost effective and efficient compared to traditional medical models of care.

- Physiotherapy-led post arthroplasty review clinics increase capacity for surgeons to review patients with urgent and/or complex needs.

- The collection of patient outcome measure across multiple hospitals provides a unique opportunity to measure outcomes for future research.

\section{VISUAL PERCEPTION AND UPPER \\ LIMB FUNCTION IN CHILDREN WITH DEVELOPMENTAL COORDINATION DISORDER}

Harris EC ${ }^{1}$, Cox LE ${ }^{1}$, Auld ML1,2, Johnston LM ${ }^{1,2}$

${ }^{1}$ The University of Queensland, Brisbane, Australia

Cerebral Palsy League, Brisbane, Australia

Question: Do children with Developmental Coordination Disorder (DCD) have deficits in global visual perception? Does global visual perception predict upper limb function in children with DCD?

Design: Cross-sectional observational study

Participants: 32 children aged six to 12 years, 17 with DCD and 15 typical developing (TD) children.

Outcome Measures: Visual perception (Motor-Free Visual Perception Test-3 (MVPT));object handling speed (Jebsen-Taylor Test of Hand Function (JTTHF-objects)); handwriting accuracy (Evaluation Tool of Children's Handwriting (ETCH-accuracy)) and speed (JTTHF-handwriting; ETCH speed); and manual dexterity (Movement Assessment Battery for Children, $2^{\text {nd }}$ edition (MABC2))

Results: Children with DCD demonstrated significantly poorer scores on global visual perception than TD children $(p=0.03)$. Visual perception was most predictive of performance on object handling speed for the dominant $(p=0.005)$, and non-dominant hands $(p=0.008)$ and manual dexterity tasks $(p=0.003)$. Visual perception also predicted handwriting accuracy for legibility of letters $(p=0.009)$ and words $(p<0.05)$. Visual perception was only related to handwriting speed for one subtest of the ETCH; uppercase alphabet writing $(p=0.03)$

Conclusion: Visual perception makes a large contribution to upper limb function for children with DCD, especially for dexterity and speed-based upper limb tasks involving hand trajectories and grasp.

Key Practice Points:

- Some children with DCD have visual perception deficits

- Visual perception should be formally assessed in children with DCD

- Visual perception is predictive of upper limb function tasks especially hand trajectories and grasp at speed. 


\section{FIBROCARTILAGENOUS EMBOLISM (FCE)}

\section{Harrison L}

Question: How difficult is FCE to Rehabilitate?

Design: A case study

Participants: Single case study of a 5 year-old neutered female whippet

Intervention: Discussion of neurological rehabilitation strategies implemented to aid recovery including gait facilitation, standing balance, NMES and taping of the carpus to prevent knuckling and proprioception retraining.

Outcome Measures: The following outcome measures were implemented:

- 4 point standing stability - level of support required, timed symmetrical stance

- Gait assessment - timed walk test, quality of movement (video)

- Postural sets - quality, ease of changing postural sets, symmetry (video)

- Muscle bulk - tape measure

Results: The dog steadily improved over the next 3 months, although complications were encountered due to owner compliance. By week 10 the dog was able to walk and have correct foot placement $95 \%$ of the time. However the quicker she moved, the less able she was to achieve a successful foot strike

Conclusion: The dog was delayed from receiving some key interventions due to inappropriate management at home but overall, she progressed wel although failed to achieve $100 \%$ recovery.

\section{Key Practice Points:}

- An explanation of what FCE is.

- Examples and explanations of treatment techniques used

- Communication issues with owners - problems encountered.

\section{A PILOT RANDOMISED CONTROLLED TRIAL OF EARLY GOAL DIRECTED MOBILISATION VERSUS STANDARD CARE IN INTENSIVE CARE UNITS}

Hodgson C (Chair) ${ }^{1}$, Bailey $\mathrm{M}^{1}$, Bellomo R², Berney $\mathrm{S}^{2}$, Buhr $\mathrm{H}^{3}$, Denehy $L^{4}$, Gabbe $B^{1}$, Harrold $M^{5}$, Higgins $A^{1}$, Iwashyna $\left.T\right]^{1}$,

Presneill $]^{6}$, Saxena $M^{7}$, Skinner $E^{8}$, Tipping $C^{9}$, Webb $S^{10}$, Young $P^{11}$

${ }^{1}$ Australian and New Zealand Intensive Care Research Centre,

Melbourne, Australia

${ }^{2}$ Austin Health, Melbourne, Vic, Australia

${ }^{3}$ The Royal Prince Alfred Hospital, Sydney, NSW, Australia

${ }^{4}$ University of Melbourne, Melbourne, Vic, Australia

${ }^{5}$ Curtin University, Perth, WA, Australia

${ }^{6}$ The Mater Hospital, Brisbane, QLD, Australia

'The George Institute, Sydney, NSW, Australia

${ }^{8}$ Western Hospital, Melbourne, VIC, Australia

${ }^{9}$ The Alfred Hospital, Melbourne, Vic, Australia

${ }^{10}$ Royal Perth Hospital, Perth, WA, Australia

${ }^{11}$ Wellington Hospital, Wellington, New Zealand

Question: What is the feasibility and safety of delivering early goal-

directed mobilisation compared with standard care?

Design: Pilot, randomised controlled study conducted in five intensive care units (ICUS)

Participants: Fifty critically ill adults, mechanically ventilated for greater than 24 hours.

Intervention: Patients were randomly assigned to early goal-directed mobilisation (intervention) or to standard care (control). Early goal-directed mobilisation included functional rehabilitation treatment conducted at the highest level of activity possible for that patient assessed by the ICU mobility scale (IMS).

Outcome Measures: The primary objective of the pilot study was to determine if the intervention could be delivered with increased maximal levels of activity during the ICU stay. We recorded IMS, strength, ventilation duration, ICU and hospital length of stay and total inpatient (acute and rehabilitation) stay

Results: Fifty patients were enrolled (mean age 61 years; $60 \%$ male). Time from ICU admission to randomisation was 3 days. Separation was achieved with a significant difference in the highest level of activity (IMS) recorded during the ICU stay between the intervention and control groups $7.3 \pm 1.9$ versus $5.8 \pm 3.0$, respectively, $p=0.05$. There was a significant difference in the proportion of patients who walked in ICU (intervention $\mathrm{N}=19(66 \%)$ versus control $\mathrm{N}=8(38 \%), p=0.05)$. There was no difference in total inpatient days between the intervention 20 [15-35] and control groups (34 [18-43], $p=0.37)$. There were no adverse events

\section{Conclusion / Key Practice Points:}

- Delivery of early goal-directed mobilisation was feasible, safe and achieved early activity including walking in ICU.

\section{INTERACTIVE COMPUTER GAME- BASED BIOFEEDBACK IN CHILDHOOD FUNCTIONAL OUTLET OBSTRUCTION}

Burgess C, Bradley T, Hardy S, Hart S, Connor F

The Mars Clinic for Children's Continence. South Brisbane Lady Cilento Children's Hospital. South Brisbane

Question: Is physiotherapy-directed use of a computer game-based biofeedback system a useful adjunct in the treatment of functional outlet obstruction (dyssynergic defecation)?

Design: Prospective observational study.

Participants: 36 children between March 2010 and December 2014. Paediatric Gastroenterologist referrals for all patients presenting with functional outlet obstruction were examined.

Intervention: Intervention included standard therapy, computer gamebased biofeedback and non-invasive uroflowmetry. Computer game-based biofeedback uses surface electromyography to facilitate visualisation and control of pelvic floor muscle activity through a computer game. Uroflowmetry technology enables simultaneous recording of surface electromyography and urine flow, with visual display of pelvic floor muscle activity, flow rate and volume.

Outcome Measures: Quantitative data comparisons are made between initial and final treatment transverse rectal diameters, pelvic floor activity, voiding pattern flow rates and episodes of soiling. Qualitative data comparisons are made to assess perceived severity of constipation and impact of constipation on quality of life, measured via visual analogue scales.

Results: Ages ranged from 5 - 12 years. Average number of treatment sessions was five (range 2 - 14). Thirty-three subjects (92\%) demonstrated improvement in constipation and soiling. Statistically significant $(p<0.01)$ improvements were observed in transverse rectal diameters, pelvic floor activity and quality of life scores.

Conclusion: Physiotherapy-directed use of a computer game-based biofeedback system is demonstrated to be a useful adjunct to standard therapy in the management of functional outlet obstruction.

\section{Key Practice Points:}

- Strategic partnerships between medical specialists and physiotherapy, such as the Mars Clinic, improve patient outcomes.

- Computer game-based biofeedback in children improves patient compliance. 


\section{PROPRIOCEPTION DEFICITS IN NECK PAIN DETECTED USING THE PROPRIOCEPTION INCONGRUENCE DETECTION TEST}

Harvie DS ${ }^{1,7}$, Hillier SL'1 , Madden V $]^{1}$, Meulders $A^{4,5}$, Smith RT ${ }^{3}$, Broecker M³, Moseley $\mathrm{GL}^{1,2,6}$

1Sansom Institute for Health Research, University of South Australia

2PainAdelaide, Adelaide, Australia

${ }^{3}$ Wearable Computer Lab, University of South Australia, Australia

${ }^{4}$ Research Group on Health Psychology, Department of Psychology, University

of Leuven, Belgium

${ }^{5}$ Center for Excellence on Generalization Research in Health and

Psychopathology, University of Leuven, Belgium

${ }^{6}$ Neuroscience Research Australia

${ }^{7}$ CRE in Road Traffic Injury, CONROD Injury Research Centre, Menzies Health Institute Queensland, Griffith University

Question: We aimed to determine whether proprioceptive imprecision could be detected in people with neck pain by testing their ability to identify incongruence between true head motion and a false visua reference - the Proprioception Incongruence Detection test (PID test).

Design: Cross-sectional study.

Participants: Twenty-four people with persistent neck pain recruited from local physiotherapy clinics and twenty-four matched controls.

Outcome Measures: Subjects repeatedly rotated to specific markers within a virtual world and indicated if their true head rotation was more or less than the rotation suggested by the visual feedback. Visual feedback was manipulated at six corrections, ranging from $60 \%$ of true movement to $140 \%$ of true movement. A standard repositioning error test was also undertaken for comparison.

Results: Healthy controls were better able to detect incongruence between vision and true head rotation $(M(S D)=75.6(8.5) \%)$ than people with neck pain were $(M(S D)=69.6(12.7) \%): p=0.03$. Repositioning error score was not different between groups. The Proprioception Incongruence Detection test score related to self-reported pain intensity but did not relate to repositioning error test score.

Conclusion: Proprioceptive precision for neck movement appears worse in those with neck pain than in those without.

\section{Key Practice Points:}

- Detecting proprioceptive deficits is challenging

- The PID test appears to be a more sensitive test than the RPE test

- Deficits relate to pain severity

- Clinical assessment of proprioception should be considered

\section{HOW CAN I MEASURE NECK PROPRIOCEPTION IN THE CLINIC?}

Harvie DS ${ }^{1,3}$, Treleaven $]^{2}$, Basteris A $^{1}$, Pedler $A^{1}$

${ }^{1}$ CRE in Road Traffic Injury, CONROD Injury Research Centre, Menzies Health Institute Queensland, Griffith University

2School of Health and Rehabilitation Sciences, University of Queensland ${ }^{3}$ Body in Mind research group, University of South Australia

Background: Precision in the proprioceptive system is widely considered to be a potential player in persistent neck pain. Moreover, treatment programs that reduce neck pain frequently include proprioception training. Despite this, proprioception is rarely subject to formal assessment outside of the research context. New technology is allowing new methods that can be used to clinically assess proprioception.

Aims / objectives: This session aims to summarise and demonstrate the tests of proprioception available to clinicians, and how technology can help. Attendees will gain an understanding of the properties of a range of tools and will gain competency in the use of some easily accessible tests of proprioception.

Approach: The session will involve discussion and practical demonstration. Session notes with relevant links and references will be provided to facilitate clinical translation. We will provide a concise review of proprioception and its relevance to neck pain. We will then introduce and demonstrate some new methods of assessing proprioception and will present and contrast their clinometric properties to more traditional methods. Finally, we will demonstrate a freely available, webcam-based test of proprioception, which is easily deployed in the clinical context.

\section{Conclusion / Key Practice Points:}

- By the end of the session attendees will have a current understanding of the role of proprioception in neck pain, and will have the knowledge and access to resources to enable them to conduct basic clinical assessments of proprioception. Clinicians will also be aware of the limitations of the tests and will be empowered for their judicial use.
AMOUNT (ACTIVITY AND MOBILITY USING TECHNOLOGY) REHABILITATION

TRIAL: MOBILITY LIMITATIONS

ADDRESSED AND TECHNOLOGIES UTILISED IN THE FIRST SIX MONTHS

Hassett $\mathrm{L}^{1,2}$, van den Berg $\mathrm{MEL}^{3}$, Chagpar $\mathrm{S}^{1}$, Weber $\mathrm{H}^{3}$, Wong $\mathrm{S}^{4}$, Vargas JP1, Schurr $\mathrm{K}^{5}$, McCluskey MA², Smith $\mathrm{S}^{6}$, Bongers $\mathrm{B}^{7}$, Lindley $\mathrm{R}^{1}$, Crotty $\mathrm{M}^{3}$, Sherrington $\mathrm{C}^{1}$

${ }^{1}$ The George Institute for Global Health, University of Sydney, Sydney, NSW ${ }^{2}$ Faculty of Health Sciences, University of Sydney, Sydney, NSW

${ }^{3}$ Flinders University, Adelaide, SA

${ }^{4}$ Liverpool Brain Injury Rehabilitation Unit, NSW

${ }^{5}$ Banktown-Lidcombe Hospital, Sydney, NSW

EUniversity of the Sunshine Coast, OLD

?University of Technology Sydney, NSW

Question: What mobility limitations were addressed and what technologies were utilised in an inpatient setting during the first six months of recruitment to the NHMRC-funded AMOUNT rehabilitation trial? Design: Intervention description from a randomised controlled trial.

Participants: Forty participants, predominantly male (65\%) with a mean (SD) age of 72 (17) years; 53\% had mobility limitations from neurological conditions.

Intervention: The intervention is additional to standard care, and prescribed according to a protocol which matches eight technologies and games/exercises to seven mobility limitations. Technologies include commercially available devices and android/iOS applications (Nintendo Wii; Xbox kinect: Fitbit: Runkeeperapp), rehabilitation-specific devices (Humac Fysiogaming), and technologies developed for the trial (Stepping Tiles; exercise iPAD apps)

Results: Participants completed a median (IQR) of 9 (5 to 13) intervention sessions, in addition to usual therapy. All seven mobility limitations were addressed, with $85 \%$ of participants using technologies to address standing limitations. Each participant used technology to address a mean (SD) of 4 (1) different mobility limitations. Seven of the eight technologies described in the protocol were used with the Humac as the first introduced technology for $50 \%$ of participants. A mean (SD) of 3 (1) different technologies were utilised per participant with the Humac used most (73\%), and the commercial gaming systems used least (Xbox kinect $18 \%$; Nintendo Wii 20\%).

\section{Conclusion and Key Practice Points:}

- A range of mobility limitations can be addressed using tailored prescription of technology to match the individuals' limitations.

- Rehabilitation-specific devices may be preferable to commercially available devices in inpatient rehabilitation. 


\section{FUNCTIONAL OUTCOMES AND \\ QUALITY OF LIFE IN PATIENTS \\ UNDERGOING EXTRACORPOREAL \\ MEMBRANE OXYGENATION PRE OR \\ POST HEART TRANSPLANT - AN \\ OBSERVATIONAL STUDY}

Hayes $K^{1}$, Holland $A E^{1,2}$, Pellegrino $\mathrm{VA}^{3}$, Leet $A S^{4,5}$, Fuller $\mathrm{LM}^{1,2}$, Hodgson $\mathrm{CL}^{1,6}$

${ }^{1}$ Department of Physiotherapy, The Alfred Hospital , Melbourne

2Department of Physiotherapy, La Trobe University, Melbourne

3Intensive Care Department, The Alfred Hospital, Melbourne

${ }^{4}$ Heart Transplant Unit, The Alfred Hospital, Melbourne

${ }^{5}$ Baker Heart Institute, Melbourne

${ }^{6}$ Department of Epidemiology and Preventive Medicine, Monash University, Melbourne

Question: What were the functional outcomes, quality of life scores and leg complications in patients that received extracorporeal membrane oxygenation (ECMO) pre or post heart transplant?

Design: Retrospective observational study.

Participants: Twenty five patients that received ECMO pre or post heart transplant.

Outcome Measures: Physical function was assessed using the Medical Research Council sum-score (MRC) and the ICU mobility scale (IMS) at ICU and hospital discharge. Six minute walk distance (6MWD) was assessed at hospital discharge and 3 months post discharge. Vascular and neurological leg complications and quality of life (SF-36) were measured at hospital discharge.

Results: At ICU discharge, strength and mobility were poor (mean MRC $46 \pm 7$, median IMS 7 [IQR 4 - 7]). Both measures significantly improved by hospital discharge $(p<0.001$ ) despite lower limb complications reported in $45 \%$ of patients. There was a significant improvement in 6MWD from hospital discharge to 3 months post discharge (mean improvement 203 m. 95\% Cl 140 to 265). Quality of life scores were significantly lower than Australian norms ( $p<0.05)$

Conclusion: In patients requiring ECMO pre or post heart transplant, physical function was impaired at ICU discharge and a moderate number had leg complications. Physical function improved by hospital discharge and continued to improve over time, whilst quality of life requires longer term evaluation.

\section{Key Practice Points:}

- First study describing functional outcomes in patients undergoing ECMO

- High incidence of leg complications warrants further investigation

- Potential early role for physiotherapy to improve functional outcomes and quality of life

\section{THE COCHRANE SYSTEMATIC REVIEW AND MRS O: APPLYING SUMMARY FINDINGS ABOUT PELVIC FLOOR MUSCLE TRAINING FOR PARTICULAR PATIENTS}

\section{Hay-Smith EJC $\mathrm{C}^{1,2}$}

${ }^{1}$ Rehabilitation Teaching and Research Unit, Department of Medicine, University of Otago, Wellington

'Department of Women's and Children's Health, Dunedin School of Medicine, University of Otago, Dunedin

There are several Cochrane systematic reviews of the effectiveness of pelvic floor muscle training (PFMT) ${ }^{1}$. Taking advantage of systematic reviews that have done the work of finding then synthesising the multiple PFMT trials, busy clinicians can instead spend their time thinking about two key questions - do I trust the review and how do I apply it? The first question prompts thought about how sound the review process is. If poorly done you are less certain about the review results. Critiquing the internal validity (quality of the review methods), is essential. There is a saying 'garbage in, garbage out', so if the process is rubbish then you are unable to trust the conclusions. Another saying, 'you're so heavenly minded you're no earthly good' (a Johnny Cash lyric), also applies. Just as important as trustworthy findings is the question of whether those findings are generalizable (close to real life). Is there external validity? Can you apply the findings for your patients and setting? Glasziou et al $(1998)^{2}$ posed four key questions to help apply systematic review findings for an individual patient (a) Is my patient so different from those in the review that results cannot be applied? (b) Is the treatment feasible in my setting? (c) What are the likely benefits and harms from treatment? (d) How will my patient's values influence the decision? The presentation will examine systematic reviews of PFMT, and individualise the findings for a hypothetical yet typical case of a woman with stress urinary incontinence.

\section{Key Practice Points:}

- It is as important to consider the external validity (or generalisability) as the internal validity (or trustworthiness) of systematic reviews when applying findings in practice

- When you look at the summary statistic in a meta-analysis, use both the point estimate and the $95 \%$ confidence intervals to think about the likely effect and whether that is clinically important

- If the underlying cause (patho-biology) of symptoms is likely to be the same or similar between your patient and those in the review, then the findings probably apply although the amount of benefit (or risk of harm) might be altered by a particular characteristic of your patient.

\section{IS PELVIC FLOOR MUSCLE TRAINING A PHYSICAL OR BEHAVIOURAL THERAPY?}

\section{Hay-Smith EJC $\mathrm{C}^{1,2}$}

${ }^{1}$ Rehabilitation Teaching and Research Unit, Department of Medicine, University of Otago, Wellington

'Department of Women's and Children's Health, Dunedin School of Medicine, University of Otago, Dunedin

Is limiting the physiotherapy profession's conceptualisation of exercise as a physical therapy a major contributor to the poor exercise adherence we see in everyday practice? Exercise to rehabilitate or maintain health requires planned, structured, repetitive physical movement (or, at least, muscle contraction). Physiotherapists "are experts in movement and function" and apply this expertise to prevent and treat "disease and disability through physical means"1. The profession undoubtedly considers exercise, such as pelvic floor muscle training (PFMT), a physical therapy. There is good evidence for the structure (e.g. slow and fast contractions) and repetitions (e.g. how many contractions per set, and sets per week) characteristic of an effect PFMT programme. However, I would be a rich woman if I got a dollar each time a patient said something like "I find it so hard to remember to do them" in response to my enquiry about her home PFMT. Doing the exercises (adherence) is problematic for many patients and appears to decrease over time. If a patient is asked to do exercise, then the physiotherapist is prescribing a behaviour; behaviour is about actions, what we do. Thinking of exercise as a behaviour therapy offers exciting possibilities for practice - there is considerable theory and evidence about behaviour change that physiotherapists can apply in practice to support better exercise adherence in their patients. The presentation covers health behaviour theory and behaviour change techniques ${ }^{2}$ applied to the prescription of PFMT. Specific examples are drawn from a recent synthesis of patient experiences of PFMT. ${ }^{3}$

\section{Key Practice Points:}

- Choosing a physiologically sound exercise training programme and incorporating strategies to address training behaviour are equally important in effective pelvic floor muscle training

- More 'education' will not be sufficient to help most patients adhere to exercise

- Increasing your skill in the application of behaviour change techniques may support increased exercise adherence in your patients both in the short and longer term. 
RELIABILITY, VALIDITY AND NORMATIVE

DATA OF ACTIVE AND PASSIVE HAMSTRING FUNCTION USING VIMOVE SENSOR TECHNOLOGY

Heerey $\mathrm{J}^{1}$, Cowan $\mathrm{SM}^{1,2,3}$

${ }^{1}$ Clifton Hill Physiotherapy, Melbourne

${ }^{2}$ The University of Melbourne, Melbourne

${ }^{3}$ St Vincents Hospital Melbourne, Melbourne

Question: Is the Askling $\mathrm{H}$-test reliable and accurate when assessed with ViMove sensor technology and if so what are normative scores?

Design: Intra-rater reliability, validity and normative data study.

Participants: 74 community AFL players and 9 healthy normals.

Outcome Measures: 11 participants were tested on two occasions a week apart to determine intra-rater reliability using ViMove sensor technology. Construct validity was assessed in nine participants using both ViMove sensor technology and VICON movement analysis. A further 63 participants (community AFL players) were tested to determine normative data.

Results: Reliability: Paired t-tests revealed no significant differences between the two testing sessions ( $p>0.5$ ). Intra-class correlationcoefficients ranged from .88 to .98. Construct validity: Vicon testing and ViMove testing were highly correlated $(p<0.01)$. No significant differences between the two measures for active and passive straight leg raise and peak movement velocity were identified ( $p>0.7)$. Normative data: Active flexibility for the $\mathrm{H}$-test averaged (SD) 100 (11) degrees and was greater than passive flexibility 79(13) degrees. No significant differences were observed between the right and left legs ( $p>0.05$ )

Conclusion: This study demonstrates that ViMove sensor technology is a reliable and accurate way to assess the Askling $\mathrm{H}$-test and provides normative data of community AFL players.

\section{Key Practice Points}

- ViMove sensor technology provides a quick, simple and reliable objective measure of the Asklings H-test.

- The reliability and normative data are comparable to previously published literature.

- Future research should evaluate populations with hamstring injuries and the usefulness of ViMove sensor technologies in predicting readiness to return to sport.

\section{A CLINICAL AUDIT AND \\ SERVICE EVALUATION OF A \\ NEW ROLE IN PRIMARY CARE: \\ MUSCULOSKELETAL PRACTITIONER}

\section{Hensman-Crook AS}

Windermere Health Centre, UK

Question: With growing patient and financial demands on Primary Care, and the need for relevant referral into secondary care, the musculoskeleta (MSK) care pathway was identified as an area that could be streamlined to address these issues. Can a new role be created to benefit the patient, GP and secondary care?

Design: The MSK practitioner role was created to provide patients direct access to a highly specialised service for the assessment, diagnosis and triage for MSK problems.

Intervention: This evaluates the role as it was introduced into a general practice.

Outcome Measures: Data collection over the first three months of service implementation focusing on capacity, source of referral, assessment outcome and patient satisfaction.

Participants: All MSK patients at the general practice.

Results: Direct referral and General Practitioner use of the service increased. 81\% of all patients seen had no general Practitioner contact. creating 168 available consultation spaces over the three month period. Referral into secondary care had a $99 \%$ conversion rate. A Patient satisfaction survey stated $90 \%$ excellent for the overall service.

Conclusion: The MSK practitioner role has provided an effective, efficient and a potentially financially viable role within primary care. It addresses both primary and secondary care service requirements, and provision of an easily accessible, highly specialised MSK service for patients.

\section{Key Practice Points:}

- The new role creates capacity for GPs to focus on medical conditions

- The role reduces referral into secondary care

- Patients were very satisfied with direct access into a specialized MSK service.
NEUROMAGNETIC AND LOW LEVEL LASER TREATMENT OF A TWO YEAR OLD COLT FOLLOWING EYE SURGERY TO DEPENDANT OEDEMA

\section{Hermans D}

A two year old colt was referred to Animal Physiotherapy following eye surgery in November 2014. The following hours post op presented with dependant oedema of near forefoot lameness of the thoroughbred colt. The horse was sedated for pain relief and had significant oedema of near fore leg. Treatment consisted of Low level Laser and Neuromagnetic devices over the next three days. Resulting in the horse not being euthanized returning to training as a racing thoroughbred.

\section{REHABILITATION AT THE END OF LIFE}

\section{Hewitt B ${ }^{1}$}

IPhysiotherapy Department, Liverpool Hospital, Liverpool, NSW Working with people as they approach the end of their life can prove daunting to therapists. Some of the concerns that have been raised include: the dilemma of realistic goal setting while still maintaining hope; managing the expectations of patients and their families and the subtleties of adapting treatment sessions when a patient's medical condition deteriorates. This presentation aims to address these concerns while exploring the role of physiotherapy in Palliative Care. It will outline some of the challenges and opportunities of working with this particular patient group. Case studies will be utilised to provide examples of how physiotherapy interventions can positively impact a patient's physical, spiritual and psychosocial wellbeing.

\section{Key Practice Points:}

- Goal setting within the context of Palliative Care

- Maintaining realistic hope; Flexible treatment progression/ regression

- Development of self-care strategies

\section{SPORTS AQUATIC PHYSIOTHERAPY: PRACTICAL OPTIONS FOR EARLY MOBILITY AND LOW LOAD EXERCISE THROUGH TO USING PLYOMETRICS IN REHABILITATION}

\section{Heywood SE $\mathrm{E}^{1,2}$}

${ }^{1}$ Australian Catholic University, Melbourne

2Melbourne Sports Medicine Centre, Melbourne

Aquatic physiotherapy with athletes covers a wide range of acute through to chronic management options. There is a lack of evidence in the literature related to acute rehabilitation of joint sprains and haematomas. However, there are effective outcomes due to hydrostatic pressure and lower load functional movement options. Early mobility can be addressed with slow dynamic movement in water which is may be of more value than static stretching. Follow up after aquatic sessions to maximise any improvements in swelling can include finishing with cold immersion as well as compression. Aquatic rehabilitation can involve plyometric options with skipping, bounding, landing and jogging prior to return to running on land. Aquatic plyometric training may offer similar loading in the push off phase with less joint impact in the landing phase. This has benefits for the lower limb joint with an effusion, pain or poor control with loading on land. Spinal rehabilitation offers a different challenge and many athletes have difficulty adapting to slower, efficient movement in water. Over recruitment of trunk muscles can be monitored to limit maladaptive stabilising strategies in water and basic level or initial stabilising is best completed on land. Hand-on mobilising of the thoracic spine can be valuable for athletes with heavy weights programs, thoracic stiffness or chronic shoulder pain. Functional carryover to improved performance on land is the main goal of aquatic physiotherapy and outcomes can be assessed poolside pre and post treatment or following a number of sessions. Individual preferences for the type of aquatic intervention, time and temperature immersed also plays a role.

\section{Key Practice Points:}

- Despite limited research evidence acute sprains, strains and haematomas can be effectively managed in water

- Aquatic plyometrics reduces joint impact

- Close monitoring of muscle activity with aquatic exercise is required to limit over recruitment 
AQUATIC PHYSIOTHERAPY GRADUATE TEACHING: DETERMINING PRIORITIES FOR CONTENT AND PREPARATION FOR CLINICAL PLACEMENTS

Heywood SE ${ }^{1,2}$, Duguid $L^{3}$, Cope L ${ }^{4}$, Maloney $S^{4}$

${ }^{1}$ Australian Catholic University, Melbourne

2Melbourne Sports Medicine Centre, Melbourne

3Peninsula Health, Frankston

${ }^{4}$ Monash University, Frankston

Questions: What are the priorities for the content of direct teaching in aquatic physiotherapy? How effective is aquatic physiotherapy graduate teaching?

Design: Mixed method design via anonymous survey and practical session tasks

Participants: Undergraduate year three physiotherapy students

Intervention: Teaching priorities were determined by experienced aquatic physiotherapists. Theory content (three hours) covered applied physics and load with exercise, applied physiology and screening, evidence based practice and clinical reasoning. Additionally, a multimedia online learning activity covered risk management. Practical sessions (six hours) included buoyancy and drag in exercise for case studies, monitoring cardiovascula exercise, manual strengthening and mobilising techniques, handling skills facilitation of movement, rotation control and pool rescue.

Results: 50 (64\%) of the 78 students invited to participate completed the survey. Following the teaching, $98 \%$ of students felt they had the skills and knowledge to plan an exercise program and manage risks on their upcoming clinical placements. 56\% felt they could complete a screening and referral form and $44 \%$ were unsure. $90 \%$ of students could appropriately identify risks. All students successfully completed the practical session skills

Conclusions: Undergraduate students felt competent with managing risks and designing an aquatic physiotherapy program for upcoming clinical placements. More time is needed discussing screening and referral processes. Further evaluation while on placement is indicated.

\section{Key Practice Points:}

- Physiotherapy students gain skills and knowledge from direct teaching hours in aquatic physiotherapy

- Physiotherapy students may feel confident with designing a clinica

program but could need more assistance with screening

\section{FALL PREVENTION EDUCATION IN HOSPITAL AND AFTER DISCHARGE}

\section{Hill A-M ${ }^{1}$}

${ }^{1}$ The University of Notre Dame Australia

Falls remain one of the most frequent adverse events in hospitals. Individualised patient falls prevention education which is supported by training and feedback for staff is effective in reducing falls. The underlying concepts of how this patient education is delivered uses principles of health behaviour change. The program when provided in addition to usual care reduced rates of falls and injurious falls by older patients in rehabilitation hospital units. This is the first intervention which has shown a reduction in injurious falls in this setting. Patients have been positive about receiving clear education about how they can assist their own recovery. The initial translation of this program into WA Health settings will also be described. The program has the potential to be rolled out widely across hospital rehabilitation units that admit older patients as an effective tool to reduce falls in these high risk settings. Communicating with patients about how they can undertake safe strategies while they are in hospital allows them to be actively involved in their own recovery efforts. Older people who have been recently discharged from hospital are at increased risk of falls and functional decline is a problem for up to one third of patients during this period. An overview of a novel education program which is being tested in a large RCT in this population will also be presented.

\section{Key Practice Points:}

- Individualised patient falls prevention education which is supported by training and feedback for staff is effective in reducing falls and injurious falls across rehabilitation wards.

- Older patients find receiving this education a positive and empowering experience.
SURVEY OF PREGNANT WOMEN IN WESTERN AUSTRALIA TO EVALUATE THEIR KNOWLEDGE, BELIEFS ABOUT AND ENGAGEMENT IN PELVIC FLOOR MUSCLE EXERCISES

Wilson $\mathrm{M}^{1}{ }^{1}$, Berlach $\mathrm{RG}^{2}$, Hill A-M ${ }^{1,2}$

${ }^{1}$ School of Physiotherapy, The University of Notre Dame Australia, Fremantle Institute for Health Research, The University of Notre Dame Australia, Fremantle

Question: What are pregnant women's knowledge; beliefs about pelvic floor muscle exercise programmes; attendance at antenatal education; urinary incontinence; and usage of the Internet?

Design: Cross-sectional survey.

Participants: Pregnant women $(n=633)$ over 18 years of age in Western Australia.

Outcome Measures: Pregnant women's levels of knowledge of and beliefs about pelvic floor muscle exercise (PFME) programmes; attendance at antenatal education; urinary incontinence; and usage of the Internet. Data were summarised using descriptive statistics, cross-tabulations of Chisquare, Fisher's Exact Test and graphs.

Results: Pregnant women surveyed ( $n=633$ ), English speaking respondents $(82 \%)$ were significantly more knowledgeable about PFM; significantly more likely to practice PFME; significantly more likely to attend antenata education compared to respondents who spoke languages other than English (18\%). Urinary incontinence was reported by $49 \%$ of respondents. Fewer than $50 \%$ of primiparae women attended antenatal education.

Conclusions: Surveying pregnant women identified that large numbers of primiparae women do not attend antenatal education. These women reported low levels of engagement in PFME and a high frequency of urinary incontinence. Women reported that web-based antenatal education may be a feasible alternative for providing antenatal education so future research should evaluate this intervention for education about PFM and PFME.

\section{Key Practice Points:}

- Data related to PFM dysfunction are not currently collected in WA

- Collection of data related to PFM dysfunction may change services for women and reduce chronic problems and costs in the future

- Research into alternative methods of antenatal education are recommended.

\section{WEB-BASED PELVIC FLOOR MUSCLE} EDUCATION FOR PRIMIPARAE WOMEN: A PILOT RANDOMISED CONTROLLED STUDY

Wilson $\mathrm{JM}^{1}$, Berlach RG ${ }^{2}$, Hill A-M $\mathbf{M}^{1,2}$

'School of Physiotherapy, The University of Notre Dame Australia, Fremantle zInstitute for Health Research, The University of Notre Dame

Australia, Fremantle

Question: What is the effect of delivering a web-based pelvic floor muscle education intervention in addition to usual antenatal care on primiparae women's knowledge; beliefs about engaging in and adhering to a pelvic floor muscle exercise programme compared to provision of usual antenatal care alone?

Design: Two-group blinded pilot RCT.

Participants: Primiparae women $(n=70)$ over 18 years of age and 22 to 27 weeks' gestation with poor knowledge of pelvic floor muscles (PFM) in Western Australia.

Intervention: Web-based PFM education programme and usual care. Outcome Measures: Primary outcome measures were participants' levels of knowledge of PFM exercises (PFME); belief to participate in and adherence to a PFME programme.

Data were collected with 3 surveys; baseline (22 - 27 weeks' gestation); post-intervention; follow-up (35 weeks' gestation); and a semi-structured interview. Changes in levels of knowledge, beliefs and adherence to PFME were compared using McNemar's test; cross-tabulations of Chi-square Fisher's Exact Test; and generalised estimating equations.

Results: Participants in the intervention group had significantly increased knowledge; belief about; and adherence to a PFME programme [Odds ratio $19.1 ; 95 \%$ Confidence Interval $(1.8,196), p=.013$ ] at follow-up compared to the control group.

Conclusions: Providing web-based antenatal education was a feasible and effective method of increasing knowledge, belief about and adherence to PFME. Future research should further evaluate this intervention.

\section{Key Practice Points:}

- Web-based PFM education for primiparae women is effective for increasing knowledge and adherence to PFME.

- Alternative methods of antenatal education are required.

- Larger RCT is recommended. 


\section{ASSESSMENT OF THE CONTENT VALIDITY AND RESPONSIVENESS OF THE REVISED HIGH-LEVEL MOBILITY ASSESSMENT TOOL FOR TRAUMATIC MULTI-TRAUMA ORTHOPEDIC INJURIES}

Hill B ${ }^{1}$, Kahn M$^{1}$, Pallant $]^{2}$, Williams $G^{1,3,4}$

${ }^{1}$ Epworth Monash Rehabilitation Medicine Unit, Epworth HealthCare,

Melbourne, Victoria, Australia

${ }^{2}$ The University of Melbourne, Melbourne, Victoria, Australia ${ }^{3}$ Department of Physiotherapy, The University of Melbourne, Victoria, Australia

${ }^{4}$ Department of Physiotherapy, LaTrobe University, Melbourne, Victoria, Australia

Question: Is the revised High-Level Mobility Assessment Tool (HiMAT) a valid and responsive measure of advanced mobility skills following multitrauma orthopaedic trauma?

Design: Cross sectional sample of convenience

Participants: 106 people with lower limb multi-trauma orthopaedic injuries within 12 weeks of full weight bearing

Intervention: One complete revised HiMAT was analyzed using Rasch analysis. A convenience sample of 43 participants also completed the motor Functional Independence measure (FIM) and Lower extremity Functional Scale (LEFS) at weight bearing, 6 and 12 weeks.

Results: Rasch analysis of the revised HiMAT showed adequate overall fit to the model $(P=0.29)$ with no misfitting items (fit residual SD $=$ 0.69 ) or persons (fit residual SD = 0.62). The scale showed good internal consistency (Person Separation Index $=0.91$ ), was unidimensional, with no differential item functioning for gender or age, and well targeted across all ability levels. The correlation between the revised HiMAT and the motor FIM was moderate $(r=0.49 ; P<.001)$, partly induced by a ceiling effect in the motor FIM. The correlation with the revised HiMAT and LEFS was weak $(r=0.39 ; P=.012)$, indicating a weak relationship between self-reported mobility problems and actual performance. The revised HiMAT was more responsive than the motor FIM and LEFS

Conclusion: The revised HiMAT is a uni-dimensional measure of advanced mobility skills for males and females recovering from multi-trauma orthopaedic lower limb injuries. It has poor-to-moderate concurrent validity with and is more responsive to change than the motor FIM and LEFS.

\section{Key Practice Points:}

- The High-level Mobility Assessment Tool is a unidimensional measure of advanced mobility skills following multi-trauma orthopaedic injury

- The revised High-level Mobility Assessment Tool has poor-to-moderate concurrent validity with the LEFS and motor FIM suggesting they may be measuring different aspects of mobility

- The motor FIM demonstrates a high ceiling effect in this population

\section{A NOVEL METHOD OF ITEM GENERATION FOR A NEW PATIENT-REPORTED OUTCOME MEASURE}

Hill B ${ }^{1,2}$, Williams $G^{2}$, Olver $]^{2}$, Bialocerkowski $A^{1}$

1Menzies Health Institute, Queensland, Australia

¿Epworth Monash Rehabilitation Medicine Unit, Epworth HealthCare, Melbourne, Victoria, Australia

Question: Can the Comprehensive Core Set for Hand Conditions (CCS$\mathrm{CH}$ ) provide a framework to generate items for a new patient-reported outcome measure? Does the CCS-HC reflect a condition that affects the whole upper limb?

Design: A cross-sectional design using qualitative content analysis Participants 21 adults with a traumatic Brachial Plexus Injury and 30 expert clinicians

Intervention: Participants attended either a nominal group or 1:1 interview. Data collection continued to saturation. Two independent reviewers classified all reported activities into the CCS-CH activity categories using existing linking criteria

Results: A total of 522 items were generated. The inter-rater reliability for classification to core set domains was excellent $(k=0.94,95 \% \mathrm{Cl}$ $0.92-0.96)$. Activities reported by patients and clinicians represented all 29 CCS-CH activity domains. Five low-level activities (2\%) could not be classified to any International Classification of Functioning, Disability and Health category. A further 6 items (2\%) were classified to a non CCS-HC ICF category - 'd335 producing nonverbal messages'

Conclusion: This study validates the use of the CCS-CH activity categories as a framework to determine activities that are undertaken by people with traumatic Brachial Plexus Injury, an injury that affects the whole upper limb. This is evidenced by the large number of activities reported and classification to all 29 CCS-HC activity categories. Activities identified that could not be classified to any ICF category were related to very 'low-level' tasks undertaken by those with minimal to no arm use.

\section{Key Practice Points:}

- The Comprehensive Core Set for Hand Conditions provides an appropriate framework for item generation

- The Comprehensive Core Set for Hand Conditions appears to reflect conditions that affect the whole upper limb

- We recommend the inclusion of ICF category 'd335 producing nonverbal messages' in the Comprehensive Core Set for Hand Conditions

Ethics approval: Griffith University, Australia (PES / 12 / 13 HREC)

\section{DEVELOPING TRUST WITH ELDERLY AND CHRONIC PAIN POPULATIONS THROUGH COMMUNICATION}

\section{Hiller $\mathbf{A}^{1}$}

${ }^{1}$ Department of Physiotherapy, School of Health Sciences, The University of Melbourne

Communication is an essential and under-recognised component of all physiotherapy treatments. Effective communication has been demonstrated to increase patient satisfaction and improve treatment outcomes and is also a tool to assist the development of a trusting relationship between therapist and patient. A trusting relationship is particularly pertinent for physiotherapists interacting with the elderly and those with chronic pain due to the prolonged nature of treatment and the need for active patient involvement. This presentation will explore and outline key aspects of communication that facilitate trust and the subsequent positive relationship between patient and practitioner. Data and results from qualitative research that explored the patient-physiotherapist interaction through observation of treatment encounters and interviews with patients and physiotherapists will be utilised to support the discussion. Examples of techniques that enable the development of a trusting relationship are the use of touch, active listening and verbal acknowledgement of specifics about the individual patient. Most importantly, the ability to relate to elderly patients as individuals is essential to achieving trusting therapeutic relationships that enable optimal pain management to occur.

\section{Key Practice Points:}

- Developing trust through effective communication is important and beneficial to optimising pain management treatments for the elderly. - An ability to relate to patients individually is essential to achieving a trusting relationship.

- Specific communication skills, such as the use of touch, can assist with building trust with elderly patients.

\section{ELITE RUGBY LEAGUE ATHLETES AND TENDON TAP / STRETCH REFLEXES: A MATCHED COHORT STUDY}

Maurini JR ${ }^{1}$, Ohmsen PD², Pope RP ${ }^{1}$, Hing $\mathbf{W}^{1}$

${ }^{1}$ Bond University, Gold Coast

¿Gold Coast Titans, Gold Coast

Questions: Do elite national rugby league athletes have altered tendon tap / stretch reflexes when compared to an age- and gender-matched control group? Also, does the length of the player's career, history of injury or a history of surgical intervention have any effect on tendon tap reflexes?

Design: Matched cohort study

Participants: 54 healthy males (Athlete $n=25$ and Control $n=29$ ).

Outcome Measures: Biceps, triceps, patellar and achilles tendons were assessed using clinical guidelines and results were compared between groups.

Results: Clinically and statistically significant reductions ( $p<0.005)$ were found in the biceps, triceps, patella and achilles reflex responses of the athlete group. High correlations were found between right and left tendon reflexes, as well as significant negative correlations ( $p=0.01$ ) between the reflex response of each tendon and the number of years playing professional sport. Biceps reflexes demonstrated the largest difference between groups when compared with the triceps $(p=0.004)$, patella $(p=$ 0.001 ) and achilles ( $p<0.001)$ tendons.

Conclusion: Elite rugby league athletes have diminished and absent tendon tap reflexes when compared to an age- and gender-matched control group. Negative correlations were observed between length of playing career and reflex response.

Ethics approval: Bond University Human Research Ethics Committee R01905

\section{Key Practice Points:}

- Athletes have diminished and absent tendon tap reflexes

- Moderate negative correlation between length of the players career and reflex responses 


\section{DOES OBJECTIVE MONITORING OF CUFF PRESSURES RESULT IN LESS ADVERSE EFFECTS? : A SYSTEMATIC REVIEW}

Hockey C $^{1}$, Paratz $]^{1,2,3}$, Van Zundert ${ }^{3,4}$

'Physiotherapy Department, Royal Brisbane and Women's Hospital, Queensland ªriffith University, Queensland

${ }^{3}$ Burns, Trauma \& Critical Care Research Centre, University of

Queensland, Queensland

${ }^{4}$ Department of Anaesthetics and Perioperative Surgery, RBWH, Queensland

Question: Does use of objective measurement of cuff pressures as compared to no measurement or estimation techniques result in less adverse effects and maintain a more accurate cuff pressure?

Design: Systematic review with meta-analysis of randomised trials.

Participants: Mechanically ventilated patients both within the intensive care unit and the operating theatre with either an endotracheal or tracheostomy tube.

Intervention: Objective cuff pressure measurement such as using a manometer or pressure volume loop closure was compared to no measurement or estimation techniques such as pilot balloon palpation, and measuring but not adjusting the cuff pressure within an appropriate range.

Outcome Measures: Primary outcomes included sore throat, hoarse voice cough, lesions of the trachea and incidences of silent aspiration at various time points post extubation. Secondary outcomes were cuff pressures within the recommended range.

Results: 9 studies were identified involving 1077 patients. 19 effect sizes from 7 studies were included in meta-analysis. The review found preliminary evidence that objective measurement as compared to no or subjective measurement is beneficial in preventing cough at two hours (OR 0.42 , Cl 0.23 to 0.79, $p=0.007$ ), hoarseness at 24 hours (OR 0.49, CI 0.31 to $0.76, p<0.002$ ), sore throat (OR 0.73, CI 0.54 to $0.97, p<0.03$ ), lesions of the trachea/bronchus and incidences of silent aspiration ( $p=0.001)$ as well as maintaining accurate cuff pressures (Hedges g 1.61, Cl 2.69 to 0.53, $p=0.003)$

Conclusion: Subjective or no cuff pressure measurement may lead to patient related adverse effects and inaccurate cuff pressures. It is recommended that an objective form of measurement be used.

\section{Key Practice Points:}

- The use of objective cuff pressure measurement prevents the development of patient related adverse events and is effective at maintaining an accurate cuff pressure.

- An objective form of cuff pressure measurement should be used to monitor cuff pressures both in the intensive care unit and in the operating theatre.

\section{JOP ORATION - CONNECTED: DISCOVERING THE UNEXPECTED BY CHALLENGING THE INTERFACE BETWEEN PHYSIOTHERAPY SPECIALTIES}

\section{Hodges PW}

NHMRC Centre of Research Excellence in Spinal Pain, Injury and Health

School of Health and Rehabilitation Sciences

The University of Queensland, Brisbane

Physiotherapy as a profession has involved a tug-of-war between the maturation of our sub-disciplines/specialties (e.g. musculoskeletal; cardiopulmonary; neurological physiotherapy; etc), and the necessity to draw on our unique multidimensional understanding of body systems to consider the "whole person" in the derivation of treatment. Research in the mainstream of each specialty is making great advances for the evidence base of physiotherapy. By necessity, much of this work must ignore or exclude consideration of other domains (e.g. patients with comorbid conditions are generally excluded from clinical trials). Yet sometimes we can make exciting new discoveries and conceptual leaps if we peer ove the divisions between our sub-disciplines, if we apply findings from one sub-discipline to another, or if we wear the glasses of one sub-discipline when looking at the problems/challenges of another. Some examples of such leaps that have spearheaded a host of new opportunities for rehabilitation include: application of contemporary understanding of neuroplasticity from neurology to musculoskeletal conditions; application of biomechanics and neuromechanics from musculoskeletal physiotherapy to challenges in continence; and application of understanding of pelvic floor and breathing disorders to management of back pain. There are many more examples. Whichever way we progress as professionals, we can only benefit from maintaining a dual focus on specialisation and our underlying skills as generalists.

\section{THE MALE PELVIC FLOOR}

Hodges P, Stafford R

NHMRC Centre of Research Excellence in Spinal Pain, Injury and Health School of Health and Rehabilitation Sciences

The University of Queensland, Brisbane Qld

Understanding of male urinary continence is surprisingly incomplete and largely based on inaccurate assumptions of anatomy and function. This is likely to underpin the conflicting evidence of efficacy of pelvic floor muscle training for management of post-prostatectomy incontinence. Unfortunately contemporary clinical practice is focused on assessment of anal contraction and training, which has little to do with urinary continence. Using new recording techniques we are developing a new understanding of urinary continence and incontinence in men. This includes development of new assessments using transperineal ultrasound imaging that directly evaluate urethral control mechanisms and methods to directly assess activation of the striated urethral sphincter muscles. These methods are providing new insight into the interaction between the multiple muscle mechanisms of the pelvic floor (striated urethral sphincter levator ani, bulbocavernosus, and smooth muscle of the bladder neck and urethra) for the maintenance of continence during a range of functional activities (voluntary and involuntary coughing, postural tasks, speaking) and during voluntary contractions. These studies have: (i) identified novel patterns of coordination between multiple striated muscle complexes during tasks that raise intra-abdominal pressure, (ii) validated the interpretation of measures made with transperineal ultrasound imaging regarding activation of the different muscle complexes, (iii) clarification of the function of the striated urethral sphincter muscle, (iv) identified differences in coordination between men that may have relevance for post-prostatectomy incontinence, and (v) provided insight into changes in function after prostatectomy. This new understanding provides a foundation for more specific assessments and interventions of men with urinary incontinence.

\section{DIASTASIS RECTUS ABDOMINIS}

Hodges $\mathrm{P}^{1,2,3}$, Lee $\mathrm{D}^{4}$

${ }^{1}$ NHMRC Centre of Research Excellence in Spinal Pain, Injury and Health

¿School of Health and Rehabilitation Sciences

${ }^{3}$ The University of Queensland, Brisbane Qld

${ }^{4}$ Diane Lee \& Associates, South Surrey, Canada

Rehabilitation of diastasis rectus abdominis (DRA) generally aims to reduce inter-rectus distance (IRD) as it is considered restoration of anatomy should optimise function. However, an alternative view is that reduced separation between the rectus muscles may slacken the linea alba and compromise force transfer across between sides and control of the abdominal contents. Thus, it may be advantageous contract lateral abdominal muscles to tension the linea alba. Although this may widen the IRD, it may optimise force transfer. To test this hypothesis we completed a series of studies to investigate novel measures of the tension in the linea alba and the outcomes of different muscle contraction strategies. The behavior of the linea alba during curl-ups performed naturally (Autocurl) and with pre-activation of transversus abdominis (TrA), which pulls laterally with potential to widen the IRD, was studied in women with DRA and controls. Ultrasound images were recorded above the umbilicus. Ultrasound measures were the IRD and a novel measure of distortion of the linea alba (distortion index [DI]). A separate study compared the DI and tissue stiffness measured using supersonic shear velocity imaging. When women with DRA performed the Auto-curl the IRD reduced, but the linea alba was distorted by protrusion of abdominal contents. Although pre-activation of TrA lessened the reduction of IRD during the curl-up, linea alba distorted less. These observations show that IRD narrowing in women with DRA "slackens" the linea alba and allows greater protrusion of abdominal contents. IRD widening by TrA contraction might improve functional and cosmetic outcomes. 


\section{DO SHOULDER PHYSICAL \\ CHARACTERISTICS DIFFER IN ELITE \\ SWIMMERS WITH AND WITHOUT \\ SHOULDER PAIN?}

Holt $\mathrm{K}^{1}$, Boettcher $\mathrm{C}^{2}$, Halaki $\mathrm{M}^{3}$, Ginn $\mathrm{KA}^{3}$

${ }^{1}$ Australian Institute of Sport

${ }^{2}$ Regent Street Physiotherapy

${ }^{3}$ The University of Sydney

Question: In elite swimmers to determine: the degree of humeral torsion \& shoulder rotation range \& strength characteristics: whether changes in these physical characteristics and/or training load are associated with shoulder pain.

Design: Descriptive cross-sectional study

Participants: Seventy (male, $n=41$; female, $n=29$; age , >16) elite Australian swimmers

Outcome Measures: Differences in internal \& external rotation ROM \& strength normalised to body weight were examined using two factor ANOVA. Associations between humeral torsion, ROM and strength were investigated using Pearson's correlation coefficient ( $r$ ). The association between shoulder pain and training load over one year was investigated with Spearman's Rho.

Results: A high level of humeral antetorsion was demonstrated in this elite swimming cohort (dominant: $26.9 \pm 9.7^{\circ}$; non-dominant: $30.8 \pm 10.0^{\circ}$ ). There were significant differences in rotation ROM measured passively and actively in prone and supine $(F 2,138 \geq 96.6, p<0.001) \&$ internal \& external rotation strength $(F 1,67=244.39, p<0.001)$. The ratio between external to internal rotation strength was 1:1.5 bilaterally. In general, increased humeral antetorsion was positively associated with increased internal \& decreased external rotation ROM $(r \geq 28)$. Shoulder pain was not associated with humeral torsion, rotation $R O M$, strength $\left(R^{2}=0.058, p=0.46\right)$ or training load (Rho=0.19,p>0.05)

Conclusion: Humeral torsion, shoulder ROM and strength ratios were within normal population parameters. Shoulder pain was not associated with these musculoskeletal parameters or training load in these elite swimmers.

\section{Key Practice Points:}

- Humeral structure is not changed as a result of elite swimming - No one factor is predictive of shoulder pain in each individual swimme highlighting the multifactorial nature of painful swimmers' shoulde

\section{PHYSIOTHERAPISTS' PERCEPTIONS OF PAIN MANAGEMENT IN POST CORONARY ARTERY BYPASS GRAFT SURGERY PATIENTS IN AUSTRALIAN AND NEW ZEALAND HOSPITALS}

\section{Hong SS $^{1,2}$, Milross $\mathrm{MA}^{2}$, Alison JA $\mathrm{A}^{2,3}$}

${ }^{1}$ Physiotherapy Department, Liverpool Hospital, Sydney

2Discipline of Physiotherapy University of Sydney, Sydney

${ }^{3}$ Physiotherapy Department, Royal Prince Alfred Hospital

Question: What are physiotherapists' perceptions of pain severity and pain management in coronary artery bypass graft surgery (CABG) patients? What do physiotherapists believe limit patients' walking distances post CABG?

Design: Cross-sectional study.

Participants: Physiotherapists who treat CABG patients in all hospitals in Australia and New Zealand that perform cardiac surgery.

Interventions: Self-administered questionnaires

Outcome Measures: Perception of pain severity with $100 \mathrm{~mm}$ visual analogue scale (VAS); adequacy of pain management in relation to physiotherapy treatments and factors limiting walking distance post CABG

Results: Fifty-four hospitals were identified from national databases. Fifty-four questionnaires were sent, 42 were returned (response rate $78 \%$ ) and 41 were analysed. One questionnaire was excluded as the site no longer performed cardiothoracic surgery. Physiotherapists perceived that patients experienced most pain on Day 1 post CABG (mean \pm standard error VAS $41 \mathrm{~mm}, \pm 3$ ) and this reduced by Day 4 post CABG (VAS $15 \mathrm{~mm}, \pm 2)$, and that patients' pain was significantly higher after physiotherapy sessions on Days 1,2 and 3 post CABG ( $p<0.00, p<0.00, p<0.04$, respectively). Thirtyseven respondents ( $90 \%$ ) believed that patients' pain was well managed for chest physiotherapy treatment. A majority of the respondents $(n=28$, $68 \%$ ) believed that pain was not a limiting factor in patients' walking distance post CABG, with general fatique believed to be the limiting factor ( $n=37,90 \%)$.

Conclusion: Physiotherapists perceive that pain in post CABG patients reduces over time but is higher after physiotherapy treatment sessions, that current pain management is adequate, and that general fatigue limits walking distance more than pain. Trial ID: ACTRN 12615000050550

\section{Key Practice Points:}

- Physiotherapists perceive that patients post CABG experience greatest pain on postoperative day 1 but this reduces by postoperative day 4

- Physiotherapists perceive that patients experience higher pain after physiotherapy sessions

- Physiotherapists believe that general fatigue, more than pain, is a limiting factor for distance walked post CABG.

\section{EFFECT OF CONTINUOUS \\ LOCAL ANAESTHETIC IN POST CARDIAC SURGERY PATIENTS: A SYSTEMATIC REVIEW}

Hong SS ${ }^{1,2}$, Milross MA², Alison JA2,3

${ }^{1}$ Physiotherapy Department, Liverpool Hospital, Sydney

Discipline of Physiotherapy, University of Sydney, Sydney

3Physiotherapy Department, Royal Prince Alfred Hospital, Sydney

Question: Does continuous local anaesthetic (CLA) infusion around the sternal wound post cardiac surgery reduce pain, time to ambulation, length of hospital, length of intensive care (ICU) stay, time to extubation, and total narcotic consumption?

Design: Systematic review with meta-analysis of randomised controlled trials.

Participants: Adults undergoing cardiac surgery via sternotomy. Intervention: Studies were included if CLA infusion around sternotomy was compared to placebo or usual care.

Outcome Measures: Pain scores with visual analogue scale (VAS) at any time point; time to ambulation or any other activity measures; length of hospital and ICU stay; time to extubation and total narcotic consumption.

Results: 7 eligible trials with a total of 426 participants were identified and 5 had data that could be included in meta-analyses. CLA infusion significantly reduced the total mean VAS pain score at 72 hours (MD -12 $\mathrm{mm}, 95 \% \mathrm{Cl}-18$ to -6$)$; reduced time to ambulate (MD -2.8 hours, $95 \% \mathrm{Cl}$ -5.2 to -0.4 ); reduced time to ambulate to chair (MD - 1.7 hours, $95 \% \mathrm{Cl}-4.1$ to 0.6); reduced the length of hospital stay (MD -0.5 days, $95 \% \mathrm{Cl}-0.9$ to -0.2 ) with no difference in length of ICU stay (MD 0.9 hours, $95 \% \mathrm{Cl}-3$ to 5) or time to extubation (MD 0.5 hours, $95 \% \mathrm{Cl}-0.1$ to 0.9 ). There was insufficient data to perform a meta-analysis on total narcotic consumption. Conclusion: CLA infusion after cardiac surgery reduces pain score at 72 hours, shortens time to ambulate and reduces length of hospital stay. PROSPERO CRD42014010188

\section{Key Practice Points:}

- CLA infusion around sternal wound after cardiac surgery reduces pain, time to ambulate, time to ambulate to a chair and length of hospital stay.

- Continuous local anaesthetic infusion around sternal wound does not reduce time to extubation or length of stay in ICU.

- Effect of continuous local anaesthetic infusion around sternal wound on total narcotic consumption is unclear. 
WOMEN WITH AKP EXHIBIT POOR LOWER LIMB DYNAMIC POSTURAL CONTROL

Horan $\mathrm{S}^{1,2}$, Connolly $\mathrm{M}^{2}$, Arnold $\mathrm{B}^{1,2}$, Weeks $\mathrm{B}^{1,2}$

${ }^{1}$ Centre for Musculoskeletal Research, Menzies Health Institute Queensland ${ }^{2}$ School of Allied Health Sciences, Griffith University

Question: Does dynamic postural control contribute to performance in lower-limb functional movement tests in women with AKP?

Design: Observational laboratory based study.

Participants: Ten women with AKP (23.2 \pm 2.8 years) and ten women free from musculoskeletal impairment (23.1 \pm 1.8 years).

Outcome Measures: Dynamic postural control was examined using the Star Excursion Balance Test (SEBT) with scores normalised to leg length, summed for an overall score, and separated into scores for 'away' and 'toward' the affected leg. Participants also performed three lower-limb functional movements, including a single leg squat (SLS), single leg hop (SLH) and drop vertical jump (DVJ). Each movement was captured using 2D video and rated for quality using a validated global 10 -point ordinal scale by 10 physiotherapists. Data were analysed using one-way ANOVA and linear regression.

Results: Total SEBT score $(345 \pm 32 \mathrm{~cm}$ vs $377 \pm 19 \mathrm{~cm}, \mathrm{p}=0.015)$ and SEBT score 'away' from the affected leg $(134 \pm 10 \mathrm{~cm}$ vs $150 \pm 8 \mathrm{~cm}, \mathrm{p}=0.002)$ were lower for those with AKP compared to controls. There was no difference in SEBT score 'toward' the affected leg for the AKP group compared to the controls $(121 \pm 15 \mathrm{~cm}$ vs $31 \pm 9 \mathrm{~cm}, p=0.114)$. Total SEBT score predicted variance in SLS performance (R2 $=0.525, p<0.001)$; but not SLH ( $p=0.861)$ or DVJ performance $(p=0.335)$

Conclusion: Young adult women with AKP exhibit poorer lower limb dynamic postural control than healthy controls. Further, balance is positively related to single leg squat performance.

\section{Key Practice Points:}

- Predictably, women with AKP exhibit poorer dynamic postural control

- Postural control measured by SEBT performance predicts variance in SLS performance in young women

- SLH and DVJ performance have less clinical utility than SLS performance in the assessment of women with AKP

\section{HOW ACCURATE ARE SURFACE MEASURES FOR CHANGE IN SPINAL POSTURE? A NOVEL APPROACH THAT QUANTIFIED SKIN ARTEFACT WITH ULTRASOUND IMAGING}

Hu $E^{1}$, Claus $A^{1}$, Hodges $P^{1}$

${ }^{1}$ The University of Queensland, Centre of Clinical Research Excellence in Spinal Pain, Injury and Health, School of Health and Rehabilitation Sciences, Brisbane, QLD, Australia

Question: Surface measures of spinal posture are used in laboratories and clinical practice, to quantify movement of the underlying spinous processes, but error due to skin artefact has not been previously reported. How much does the skin move in the sagittal plane relative to the lumbar spinous processes with changes in posture?

Design: Participant experimental study.

Participants: Twelve healthy adult participants.

Outcome Measures: Ultrasound imaging was used to mark the skin overlying spinous processes from L1 to S1. This was repeated in slumped sitting, standing and in prone lying. Sagittal plane displacement of the skin relative to spinous processes was measured in the cranio-caudal direction and depth.

Results: With slumped sitting or standing relative to prone lying, the median skin displacement ranged from $1.6 \mathrm{~mm}$ caudal to $17.1 \mathrm{~mm}$ cranial, and from $1.1-9 \mathrm{~mm}$ increase in spinous process depth. The interquartile range cranio-caudally was $-9.7-22.4 \mathrm{~mm}$, which approaches the typical distance between two spinous processes, and the depth from 1.1 -

$29.2 \mathrm{~mm}$

Conclusions: Changes in spinal posture commonly displaced surface markers up to $17 \mathrm{~mm}$ relative to spinous processes, and sometimes as far as adjacent spinal levels.

\section{Key Practice Points:}

- For laboratory and clinical measures for change in spinal posture, skin artefact relative to spinous processes limits ability to infer the position of individual spinal segments.

- Surface markers can be displaced as far as adjacent spinal levels.

- Measures across a series of spinal segments offer more

reasonable validity.
THE ROLE OF THE PHYSIOTHERAPIST IN SHAPING CULTURE AND INTEGRITY IN AUSTRALIAN SPORT

\section{Hughes D}

Australian Institute of Sport

Over the past 50 years, sport has changed in Australia and around the world from a recreational pastime to a vast economic, media and cultural phenomenon. Sport-focussed articles frequently dominate not only the sports section of the media but substantial portions of mainstream current affairs content. Since early 2013 there have been numerous assaults upon the integrity of sport including the Australian Crime Commission findings on organised crime and drugs in sport, Lance Armstrong's admission of doping offences, the Southern Stars match fixing scandal and, more recently, the dramatic arrests of senior FIFA officials over bribery and corruption allegations. With immense sums of money, political reputations and personal ambition in play, preserving integrity in sport can at times seem a daunting and overwhelming assignment. The physiotherapist occupies a unique and influential position in the Australian sporting landscape. Sporting organisations are often subtly subdivided into functional silos comprising the athletes, the medical staff, the coaches and the management staff. The physiotherapist will often be the only individual within a sports team or organisation who holds a position of respect across all of those groups. This position of trust provides the physiotherapist with an opportunity to influence culture within their organisation and, through culture, to shape the integrity underpinning all that is good in sport. An understanding of the role of culture and an appreciation of universal principles of good governance can provide the physiotherapist with the armamentarium required to optimise power and influence in flexing their 'integrity-muscle', for the betterment of Australian sport.

\section{Key Learning Objectives:}

- Understand the integrity threats to Australian sport including doping, match fixing and corruption

- Understand the new obligations placed upon team support staff (including physiotherapists) as a result of the changes to the 2015 WADA Code

- Understand the key principles of good governance as they apply to sporting organisations

- Appreciate how application of governance principles will protect integrity in sport

- Understand the role of organisational culture in strengthening or undermining organisational integrity

- Gain insight into the potential influence of the physiotherapist on culture and integrity in sport

\section{SPORTS ON FIELD DILEMMAS: A PRACTICAL SESSION}

Hughes $D^{1}$, O'Sullivan-Pippia L², Staples K³ , Brown M4, Mellifont $\mathrm{D}^{5}$, Sartori $\mathrm{S}^{6}$, Lisle $\mathrm{K}^{7}$

${ }^{1}$ Australian Institute of Sport

2Twelve9teen Sports Physiotherapy

${ }^{3}$ RHP Physiotherapy / Australian Catholic University

${ }^{4}$ Sports Medicine Australia / Griffith University

${ }^{5}$ Sunshine Coast University

${ }^{6}$ North Queensland Cowboys Rugby League

${ }^{7}$ Football Federation of Australia Socceroos

In this 1 hour session participants will rotate through 3 different stations, each of which will involve a structured examination of the optimal approach to managing different types of acute sports injuries in a field of play setting. The presenters will outline a case study of an acute on-field injury and assist the participants to problem solve the best approach to its immediate and subsequent management while integrating consideration of current evidence informed guidelines as well as the legal, ethical and scope of practice issues and the sports specific rules that can complicate the role of the Sports Physiotherapist in a field of play setting when quick decision making is necessary.

\section{Key Practice Points:}

- The learning outcomes from this session should provide participants with the ability to prepare for and approach a case that requires quick decision making while incorporating clinical reasoning skills and guidelines for a complex scenario 


\section{MIND YOUR STEP - PREVENTING FALLS IN DEMENTIA}

\section{Hunter SW}

${ }^{1}$ School of Physical Therapy, University of Western Ontario, London, Ontario, Canada

People with dementia have twice the annual fall risk of the cognitively normal and fallers with dementia are five times more likely to be institutionalized than people with dementia who do not fall. The risk of major fall-related injuries, such as hip fractures, and death are also increased among adults with dementia. Current programs that address modifiable fall risk factors, such as weakness, balance and gait problems, decrease the number and rate of falls in cognitively normal communitydwelling older adults. However, these strategies have not translated successfully to community or institution-dwelling people with dementia. Successful rehabilitation interventions for people with dementia are desperately needed and this is an emerging area of research and clinical interest. Physiotherapists regularly come into contact with older adults with dementia in their clinical practice. This presentation will present information on the importance of understanding how the underlying disease process impacts physiotherapists' ability to deliver effective treatment, ranging from how to modify the way we speak to clients to accommodate the language changes of the disease to the choice of the best clinical assessment scale to evaluate gait and balance problems. The constellation of fall risk factors that are shared with cognitively normal older adults and unique risk factors that are not present in cognitively normal adults will be reviewed. Lastly, the presentation will summarize the findings of fall prevention studies for people living with dementia.

\section{Key Practice Points:}

- An understanding of the disease process will highlight the importance of modifying how physiotherapists deliver treatments (e.g., how to accommodate language and memory changes) and will provide suggestions for modifications.

- An understanding of range of fall risk factors among older adults with dementia will provide a framework for the assessment of older adults with dementia that addresses their unique health care needs.

- An appreciation of the results from fall prevention trials in older adults with dementia will inform treatment practices across care settings.

\section{TIPPING POINTS - FALLS PREVENTION IN HIGH FUNCTIONING OLDER ADULTS}

\section{Hunter SW}

${ }^{1}$ School of Physical Therapy, University of Western Ontario, London, Ontario, Canada

The most prominent fall prevention guidelines recommend a simple two-step screening algorithm for identifying and stratifying fall risk in older adults. The guidelines recommend people who report no falls in the previous 12 months or have a single fall, but no balance or gait problems, do not require interventions. Unfortunately, the guidelines do not provide direction for how best to assess balance or gait and do not factor in a person's functional level in the clinician's choice of balance test. Considering it takes only one fall to produce deleterious effects, high functioning older adults are not well-served by current fall prevention guidelines. This presentation will demonstrate that healthy communitydwelling older adults without a history of falls in the previous 12 months have a risk of falling that is not insignificant, roughly $16 \%$ to $49 \%$. Additionally, the majority of people who transition from non-fallers to fallers possessed modifiable fall risk factors before falling. To prevent falls in this group of older adults, the choice of balance scales is important as is the need for a consensus on the level of future fall risk we are willing to accept before initiating fall prevention interventions. Physiotherapists are well positioned to improve the identification of fall risk and prevent future falls in this group. This presentation will highlight the epidemiology of their fall risk and how to assess risk in this population of older adults.

\section{Key Practice Points:}

- The absence of a fall history does not mean an absence of modifiable fall risk factors and therefore targeted clinical evaluation is needed before "no intervention" is an appropriate treatment option.

- To facilitate client uptake of information among high functioning older adults, the use of the screening process does not have to be limited to fall prevention, but to the maintenance of autonomy, healthy aging and prevention of functional decline in general.

- Recognize that the choice of clinical balance assessment tool should be scaled to the functional level of the person being assessed to identify problems. Therefore clinicians need to have a repertoire of balance tests that can be scaled to the functional level of the individual.

\section{ASSESSING FUNCTIONAL EXERCISE CAPACITY USING TELEHEALTH: IS IT RELIABLE AND VALID IN PATIENTS WITH CHRONIC HEART FAILURE?}

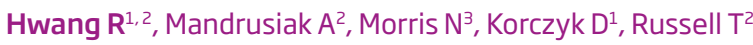
${ }^{1}$ Princess Alexandra Hospital, Metro South, Brisbane, Australia ¿School of Health and Rehabilitation Sciences, The University of Queensland, Brisbane, Australia

${ }^{3}$ School of Allied Health Sciences, Griffith University and Menzies Health Institute Queensland, Gold Coast, Australia

Question: Is video-based telerehabilitation assessment reliable and valid compared with traditional face-to-face assessment in patients with heart failure?

Design: A nested study within a randomised controlled trial of heart failure telerehabilitation. Video-based telerehabilitation assessments of three functional tests (timed up and go, six minute walk, grip strength) were compared with traditional face-to-face assessments at week 12 (post-program)

Participants: Sixteen participants in the telerehabilitation group undertook post-program assessments via telerehabilitation and on the same day, face-to-face. The assessment order was randomised and conducted by independent assessors.

Outcome Measures: Three functional tests: timed up and go (time), six minute walk (distance), grip strength; system usability scale to rate participant experience with telerehabilitation assessment; and number of technical issues encountered. Inter- and intra-rater reliabilities and validity of telerehabilitation assessment were examined using intra-class correlation coefficients

Results: Preliminary analysis was undertaken on 15 participants (mean 69 years, $87 \%$ males). Inter- and intra-rater correlations for all functional tests were excellent (all >0.98). Similarly these tests were strongly associated with face-to-face assessments, with correlations between 0.89 and 0.94 . The mean (standard deviation) system usability scale score was 85 (16)/100. Some incidences of internet drop-outs, video freezing and auditory fading occurred.

Conclusion: The use of telehealth in assessing functional exercise capacity appears to be reliable and valid in patients with heart failure

\section{Key Practice Points:}

- These functional measures assessed via telerehabilitation are reliable and valid in a sample of patients with heart failure.

- Studies with larger sample sizes and other settings are required in this emerging field.

\section{“LOAD”, WHAT ARE WE MEASURING? - AN ANALYTICAL REVIEW OF CURRENT METHODS OF MEASUREMENT}

Jhala $\mathrm{P}^{1,2}$, Raper $\mathrm{D}^{3}$

${ }^{1}$ Spring Street Sports \& Spinal Physiotherapy, Chatswood

2Hawkesbury Cricket Club, Sydney

${ }^{3}$ Geelong Cats Football Club

Question: The purpose of this review was to identify methods of measuring "load" in the literature and assess their relation to bone and tendon injuries

Design: Analytical Review

Participants: Athletes

Outcome Measures / Methods: A systematic search of the Medline, PubMed and SPORTDiscus databases to identify methods of measuring "load", using the terms athlete, monitor*, measur*, evaulat*, quantify* and load*

Results: Several methods were identified, separated into two categories. Internal "load" included physiological measurements such as heart rate, ventilation, rate of perceived exertion and hormonal changes. External "load" included distance run, accelerations, ' $g$ ' forces or number of events specific to that sport (e.g. tennis strokes).

Discussion / Conclusion: "Load" is the centre of bone and tendon injury models. Thus measuring "load" becomes pivotal to injury prevention and management in elite sport. This review found that methods to measure "load" investigated multiple physiological variables or variables specific to an individual sport. While authors generally acknowledged the role of "load", none specifically defined the term "load" or discussed the relationship between parameters measured and the development of injuries. Consequently there seems to be a disparity between the use of the term "load", its relationship to the pathogenesis of injury and the measurement of "load" in the field of sports science.

\section{Key Practice Points:}

- Identify methods of measuring "load"

- Analyse the role of "load" in bone and tendon injury models

- Evaluate if current methods of measuring "load" assist in injury prevention and rehabilitation of bone and tendon injuries 


\section{THE IMPACT OF SUPERVISING CHALLENGING STUDENTS ON CLINICAL PLACEMENT}

Johnston $\mathrm{CL}^{1}$, Newstead CJ ${ }^{1}$, MacDonald-Wicks L2

${ }^{1}$ Discipline of Physiotherapy, School of Health Sciences, The University of Newcastle, Callaghan

2Discipline of Nutrition and Dietetics, School of Health Sciences, The University of Newcastle, Callaghan

Question: What is the impact of supervising challenging physiotherapy students on the clinical educator, the workplace and workplace culture?

Design: Cross sectional written survey

Participants: Physiotherapy clinical educators ( $n=307$ ) and managers $(\mathrm{n}=81)$ listed with the University of Newcastle physiotherapy clinical placement database.

Outcome Measures: Purpose designed anonymous surveys were sent to identified participants. The survey consisted of a mix of categorical and open free text response options relating to participant characteristics and the impact of supervising challenging students on the clinical educator, workplace culture and dynamics.

Results: Response rates were $54 \%(n=166)$ and $53 \%(n=43)$ for clinical educators and managers respectively. Most clinical educators ( $n=144$ $87 \%$ ) and managers ( $n=40,93 \%)$ reported having supervised, or worked with, a challenging student in the clinical setting. Respondents indicated that supervising challenging students most adversely affected clinical educator workload and stress levels. Managers and educators also reported that challenging students had a negative impact on staff attitudes towards student supervision. Written survey comments indicated that challenging students: may improve educator skills; are time consuming, fatiguing and stressful; require experience to successfully manage; negatively impact capacity for placements, workload and other students and require early identification. Clinical educators and managers reported that increased training and support was required.

Conclusion: Supervising challenging students has an adverse impact on physiotherapy clinical educators and workplace culture and dynamics. Additional training and support is required to enable educators to more effectively manage challenging students on clinical placement.

\section{Key Practice Points}

- The impact of supervising challenging students has not been specifically investigated in physiotherapy.

- This study demonstrated that supervising challenging students on clinical placement was of concern to physiotherapy clinical educators and managers.

- Supervising challenging students may have an adverse impact on clinical educator workload and stress levels and, in addition, may lead to negative staff attitudes towards student supervision.

- Further research is required into how best to prepare and support physiotherapy educators to manage challenging students in the clinical setting.

\section{'FAILURE TO FAIL' IN PHYSIOTHERAPY CLINICAL EDUCATION}

Johnston $\mathrm{CL}^{1}$, Newstead C] ${ }^{1}$, MacDonald-Wicks L ${ }^{2}$

${ }^{1}$ Discipline of Physiotherapy, School of Health Sciences, The University of Newcastle, Callaghan

2Discipline of Nutrition and Dietetics, School of Health Sciences, The University of Newcastle, Callaghan

Question: Do physiotherapy clinical educators consider that there is a 'failure to fail' poorly performing/unsafe students on clinical placement? What are the factors which contribute to students who are poorly performing/unsafe on clinical placement being passed or graded as competent?

\section{Design: Cross sectional written survey}

Participants: Physiotherapy clinical educators $(n=307)$ listed with the University of Newcastle physiotherapy clinical placement database.

Outcome Measures: Purpose designed anonymous surveys were sent to identified participants. The survey consisted of a mix of categorical and open free text response options relating to participant characteristics and the issue of 'failure to fail' underperforming students on clinical placement.

Results: The response rate was 54\% ( $n=166)$. Most respondents ( $n=120$, $72 \%$ ) considered that there was a 'failure to fail' underperforming students on clinical placement. Forty one clinical educators (25\%) indicated that they had previously passed a student who, on reflection, they should have failed. The most commonly cited factors influencing these educators' decisions not to fail underperforming students were: empathy/sympathy, fear of repercussions for the student; a perception of having inadequate documentation or not having given enough feedback, lack of time for accurate assessment and lack of confidence in decisions. Educators written comments indicated that inexperience, lack of confidence and support contributed to poor decision making in relation to grading underperforming students.
Conclusion: Physiotherapy clinical educators consider that there is a 'failure to fail' underperforming students on clinical placement. Improved training and support of clinical educators, particularly novices, is necessary to improve the assessment and grading of underperforming students.

\section{Key Practice Points:}

- The issue of a 'failure to fail' underperforming students has been reported in other health professions including nursing and medicine but, to date, this issue has not been specifically investigated in physiotherapy.

- Accurate assessment and grading of underperforming/unsafe students on placement is important. Poor identification and management of these students may result in risks to patient and public safety, substandard patient care and may adversely impact on professional standing.

- Further research is required into how to best prepare and support clinical educators (particularly novices) to manage underperforming/unsafe students in the clinical setting.

\section{FORUM: PREPARING, PROGRESSING AND PROMOTING PAEDIATRIC PHYSIOTHERAPY}

\section{Johnson LM}

The University of Queensland, Brisbane, Australia

Please bring your ideas, enthusiasm and tablets/ smart phones to our final group session which will be an interactive forum discussion around PREPARING entry level Physio students, PROGRESSING towards a career working with children and PROMOTING the role of paediatric physiotherapists within future healthcare initiatives such as the NDIS. The forum will be facilitated by Dr Leanne Johnston and will also include invited stakeholder panel members and audience participation with interactive polling. The forum will commence with a "desk audit" of current teaching approaches used to meet Entry Level Paediatric Standards at universities around the country. The session will then move on to discuss a current "framework model" of clinical reasoning to prepare entry level students be confident clinicians working with younger patients. The panel and audience will discuss the clinical competency expectations of entry level physiotherapists and the various mechanisms for training and recognition as a physiotherapist who chooses to focus on paediatric clients and opportunities for expanded scope.

Key Practice Points:

- The forum will follow on from the 2013 conference great debate Paediatric Education - core ingredient or icing on the cake?

- The forum aims to identify which "ingredients are essential for the cake to rise".

\section{IMPACT OF VISUAL PERCEPTION ON UPPER-LIMB MOTOR PERFORMANCE IN CHILDREN WITH CEREBRAL PALSY}

Hankin $N^{1}$, Auld $\mathrm{ML}^{2}$, Conroy $\mathrm{A}^{1}$, Cox $\mathrm{L}^{1}$, Harris $\mathrm{E}^{1}$, Tan $\mathrm{XY}^{1}$, Johnston LM ${ }^{1}$

${ }^{1}$ The University of Queensland, Brisbane, Australia

${ }^{2}$ Cerebral Palsy League, Brisbane, Australia

Question: How does visual perception impact upper-limb motor performance in children with cerebral palsy (CP)?

Design: Cross-sectional study

Participants: Children aged 8-12 years from the inTOUCH study, including 20 with CP (median nine years: 10 males: GMFCS-E\&R $=5, \|=9$, IV=6: MACS $|=10| \mid,=6$, |||=4; hemiplegia=6, diplegia=7, quadriplegia=4, ataxia=3) and 27 typically developing (TD) (median 10 years; 16 males; 19 right-handed).

Outcome Measures: Visual perception (Motor-free Visual Perception Test (MVPT)); object handling speed (Jebsen-Taylor Test of Hand Function (JTTHF-objects)); handwriting accuracy (Evaluation Tool of Children's Handwriting (ETCH-accuracy); and speed (JTTHF-speed, ETCH-speed); and unilateral movement accuracy (Melbourne Assessment of Unilateral Upper Limb Function (MUUL)).

Results: Children with CP demonstrated poorer performance than TDC on all tests $(p<0.005)$ except ETCH-accuracy. Visual deficits were correlated with slower object handling for the total group $(p<0.01)$, especially children <10 years ( $p<0.062$ ); and slower handwriting in children >10 years $(p=0.019)$. Poorer visual perception was correlated with slower object handling in both hands of children with MACS-II $(p<0.01)$ and the non-dominant hand of children with MACS-I $(p=0.02)$.

Conclusion: Visual perception is strongly related to object handling speed, especially for younger children and those with MACS Level II. It is moderately correlated with handwriting speed in older children.

\section{Key Practice Points:}

- Visual perception deficits are common in children with CP

- Visual perception deficits impair mainly speed of hand function

- Visual perception should be routinely assessed and managed in children with CP 


\section{CHILDHOOD MOBILITY FUNCTION PREDICTS PARTICIPATION POTENTIAL IN YOUNG ADULTS WITH CEREBRAL PALSY}

Johnston LM ${ }^{1}$, Halloran $\mathrm{T}^{1}$, Lacasse $\mathrm{N}^{1}$, Law $\mathrm{M}^{1}$, Rutkowski $\mathrm{S}^{1}$, Singer $\mathrm{L}^{1}$, Louca $\mathrm{C}^{2}$, deLacy $\mathrm{M}^{2}$

${ }^{1}$ School of Health \& Rehabilitation Sciences, The University of Queensland, Brisbane

${ }^{2}$ Queensland Cerebral Palsy Register

Question: Can childhood mobility function predict participation of young adults with cerebral palsy (CP)?

Design: Retrospective cohort

Participants: Participants were 100 young adults with CP aged 18-21 years (mean 19.41 years; 62 males; 57 ambulant, 11 marginally ambulant, 32 non-ambulant) with childhood data on the Queensland Cerebral Palsy Register.

Outcome Measures: included current mobility level (ambulant, marginally ambulant, non-ambulant); mobility at five and 15 years (GMFCS-E\&R); and participation diversity, frequency and environmental facilitators/barriers according to ICF domains. Participation predictors were determined using multiple regression

Results: A mobility-participation matrix was developed. Highest participation was in public (86\%) or private leisure/recreation (86\%), medical appointments (85\%) and shopping/banking (83\%). Lowest participation was in employment (37\%), education (51\%) and sport/ physical activity (58\%). Poor current mobility restricted participation in education ( $p=0.007)$, employment $(p<0.001)$ and physical activity ( $p$ $=0.005$ ). Poor childhood mobility (GMFCS-E\&R $=29,\|=22,\| \|=24, I V=12$ $\mathrm{V}=12$ ) at age 15 predicted restricted adult participation in education ( $\mathrm{p}=$ $0.018)$, employment ( $<0.001$ ) and physical activity $(p=0.003)$; and at age five predicted restricted adult participation in employment $(p<0.001)$ and physical activity $(p=0.031)$

Conclusion: Poor mobility in young adulthood restricts participation in education, employment and physical activity. Poor childhood mobility was found to predict adult participation restriction in these domains.

Key Practice Points:

- Childhood mobility of children with CP is a strong early indicator of adult participation in education, employment and physical activity

- Childhood mobility classification should guide support planning required to optimise participation potential

\section{SUPERVISOR COMPETENCIES FOR SUPPORTING RETURN TO WORK: A MIXED-METHODS STUDY}

\section{Johnston V}

School of Health and Rehabilitation Sciences, Faculty of Health and Behavioural Sciences, The University of Queensland, Brisbane

Question: What knowledge, skills and behaviours do supervisors need to effectively support staff returning to work after a mental health or musculoskeletal condition?

Design: Five focus groups with supervisors and a survey of rehabilitation professionals

Participants: Experienced supervisors ( $n=29)$ from five Australian industries and Human resources (HR), occupational health and safety (OHS) and rehabilitation professionals (RP) $(n=344)$

Outcome Measures: Following a multi-stage analysis of focus group data, return to work competencies were developed and incorporated into an online survey administered to HR, OHS and RPs. The level of importance of each competency was scored on a five point scale from not important to essential.

Results: Supervisors cited more differences than similarities in comparing support needs between workers returning after a mental health condition or musculoskeletal injuries. 84 generic competencies, eight specific to mental health conditions, and two to musculoskeletal disorders were identified. Nearly all rehabilitation professionals agreed that supervisors should receive training to support return to work. Over $50 \%$ of respondents rated 90 of 94 competencies as very important or essential. The highest ratings were for competencies relating to personal attributes, knowledge of return to work processes, and empathetic support of the worker

Conclusion: There was widespread agreement that supervisors need a range of knowledge, skills, and personal characteristics to effectively support staff returning to work which can be provided with training.

\section{Key Practice Points:}

- Supervisors need additional knowledge, skills and behaviours to effectively support staff returning to work with a mental health or musculoskeletal condition;

- Face-to-face training was preferred by most supervisors.
THE EFFECTIVENESS OF AQUATIC PHYSIOTHERAPY FOLLOWING TOTAL HIP REPLACEMENT

Jones $\mathrm{L}^{1}$

${ }^{1}$ Donvale Rehabilitation Hospital

Question: What is the efficacy of aquatic physiotherapy following total hip replacement surgery?

Design: Systematic review of randomised controlled trials synthesised in a narrative format.

Participants: Patients who had undergone a primary total hip replacement for osteoarthritis.

Intervention: Aquatic physiotherapy intervention versus land based intervention or control.

Outcome measures: Patient activity limitation and impairment measures.

Results: The review identified three studies including 415 participants One good quality study compared an early aquatic intervention six days post-operatively versus a later aquatic intervention of 12 days. One poor quality study compared a conventional gym program to aquatic intervention over 10 days. The third study of good quality that described the aquatic intervention in detail, compared additional ward physiotherapy to a general water exercise intervention or a specific aquatic physiotherapy program over 10 days. This study suggested a trend $(p=0.55)$ of increased hip abductor strength in the aquatic physiotherapy group. Due to conflicting results between the three studies it cannot be concluded that aquatic exercise has an effect on self-reported disability.

Conclusion: Despite evidence supporting improvements in function and quality of life with aquatic intervention in other musculoskeletal conditions, the evidence following total hip replacement was inconclusive. Future research should consider how the properties of water can be optimised and progressed in aquatic physiotherapy.

\section{Key Practice Points:}

- Aquatic physiotherapy is commonly used in the treatment of total hip replacement patients but evidence is inconclusive.

- High quality randomised trials of aquatic therapy and detailed program reporting post total hip replacement are required.

\section{IS THE LOWER EXTREMITY FUNCTIONAL SCALE AN APPROPRIATE OUTCOME MEASURE FOR OUTPATIENT REHABILITATION PATIENTS?}

Jones $\mathrm{L}^{1}$, Blair $\mathrm{M}^{1}$, de Morton $\mathrm{N}^{1}$

${ }^{1}$ Donvale Rehabilitation Hospital

Questions: Is the Lower Extremity Functional Scale (LEFS) a valid measure of activity limitation in a hospital outpatient setting? How do the measurement properties of the LEFS compare to the Lawton's Activities of Daily Living Scale?

Design: Prospective observational study.

Participants: 359 consecutive outpatients were included; the mean patient age was 71.0 (sd 10.53) years, 67\% were female.

Outcome measures: LEFS and Lawton's assessments were conducted at admission and discharge physiotherapy outpatient sessions. A direct headto-head comparison was conducted between the LEFS and Lawton's.

Results: A ceiling effect was identified at discharge for the Lawton's with $51.3 \%$ of patients scoring the highest possible score. Evidence of known groups, convergent and discriminant validity were obtained for the LEFS. High responsiveness to change for the LEFS was identified (Effect Size Index 1.7) and the minimal clinically important difference was six points.

Conclusion: The LEFS was demonstrated to be a valid measure of activity limitation in an outpatient population. The LEFS showed a broad scale width and the Lawtons had a ceiling effect showing it is unable to accurately measure changes in activity limitation for more than half the population.

\section{Key Practice Points:}

- The Lawton's Activities of Daily Living Assessment is currently applied in outpatient settings for national benchmarking however clinicians and managers report that it has significant limitations in this setting

- The LEFS has a broad scale width and items that capture the range of abilities that typically exist in a community outpatient rehabilitation setting. 


\section{AFTER-HOURS OR WEEKEND \\ REHABILITATION IMPROVES OUTCOMES \\ AND INCREASES PHYSICAL ACTIVITY BUT DOES NOT AFFECT LENGTH OF STAY}

Scrivener $\mathrm{K}^{1}$, Jones $\mathrm{TM}^{1}$, Schurr K², Graham $\mathrm{PL}^{3}$, Dean $\mathrm{CM}^{1}$

${ }^{1}$ Department of Health Professions, Macquarie University, Sydney

2Physiotherapy Department, Bankstown-Lidcombe Hospital, Sydney ${ }^{3}$ Department of Statistics, Macquarie University, Sydney

Question: In adults undergoing inpatient rehabilitation, does additional after-hours rehabilitation (AAHR) decrease length of stay (LOS) and improve functional outcome, activities of daily living (ADL) performance and physical activity (PA)?

Design: Systematic review with meta-analysis of randomised trials.

Participants: Adults participating in inpatient rehabilitation.

Intervention: Additional rehabilitation provided after hours

Outcome Measures: Functional measures, e.g. Motor Assessment Scale, 10-m walk test; ADL measures, e.g. Functional Independence Measure; LOS measured in days; PA measured as step count or time spent upright. Standardised mean differences (SMD) or mean differences (MD) were used to combine these outcomes. Adverse events were summarised using relative risks (RR)

Results: Seven trials met inclusion criteria. Among the measures of function, only balance showed a significant effect: MD was 14 points bette (95\% CI 5 to 23) with AAHR. Improvement in ADL performance with AAHR was of borderline statistical significance (SMD 0.10, 95\% CI 0.00 to 0.21). LOS did not differ significantly (MD -1.8 days, $95 \% \mathrm{CI}-5.1$ to 1.6). Those receiving AAHR had significantly higher step counts and spent significantly more time upright. Overall, the risk of adverse events was not increased with after-hours or weekend rehabilitation (RR 0.87, 95\% Cl 0.70 to 1.10),

Conclusion: AAHR can increase physical activity and may improve ADLs, but does not seem to affect the hospital LOS.

\section{Key Practice Points}

- Additional after-hours rehabilitation can increase physical activity in adults undergoing inpatient rehabilitation.

- Additional after-hours rehabilitation does not appear to impact hospital length of stay.

- Additional after-hours rehabilitation may improve ADLs.

\section{EXPLORING INTEREST IN AN INTERNET \\ DELIVERED SELF-MANAGEMENT PROGRAM FOCUSED ON PHYSICAL ACTIVITY AFTER ACQUIRED BRAIN INJURY}

Jones $\mathrm{TM}^{1,3}$, Dean $\mathrm{CM}^{1,3}$, Dear BF${ }^{2,3}$, Hush JM $\mathrm{M}^{1,3}$, Titov $\mathrm{N}^{2,3}$

${ }^{1}$ Department of Health Professions, Macquarie University, Sydney ${ }^{2}$ Centre for Emotional Health, Department of Psychology, Macquarie University, Sydney

${ }^{3}$ Centre for Physical Health, Macquarie University, Sydney

Question: Are Australian adults with ABI interested in an internetdelivered self-management program aimed at increasing physical activity. Design: Online survey

Participants: Australian adults with non-degenerative ABI living in the community ( $n=59)$.

Outcome measures: Information was collected about demographic characteristics; general health; emotional well-being; mobility and physical activity status, and satisfaction; barriers to physical activity; confidence in overcoming barriers, and; interest in an internet self-management program. Chi-square tests and independent-samples t-tests for continuous data were used to analyse interest.

Results: Over $58 \%$ of respondents were not satisfied with their current physical activity status and over $40 \%$ reported some level of psychological distress. The most frequently reported barriers were pain/discomfort $(36.6 \%)$, fatigue $(29.3 \%)$ and fear $(26.8 \%)$, and confidence to overcome these barriers was very low. Interest in an internet-delivered selfmanagement program was high (74\%) and not related to the amount of physical activity $(t(50)=0.24, p=.981)$, satisfaction with physical activity $(X 2(4)=4.474, p=.346)$ or total number of barriers $(t(53)=0.759, p=.451)$ Those reporting higher levels of psychological distress were more likely to be interested $(t(46.895)=2.701, p=.010)$.

Conclusion: Australian adults with $\mathrm{ABI}$ are interested in accessing an internet-delivered self-management program aimed at improving physical activity levels.

\section{Key Practice Points:}

- Psychological distress and low satisfaction with physical activity is common in Australian adults with AB

- Barriers to physical activity, such as pain, fatigue and fear, need to be addressed.

Survey results have been used to inform development of an internetdelivered self-management program to increase physical activity after $A B I$.

\section{A SYSTEMATIC REVIEW OF THE EFFICACY OF SELF-MANAGEMENT PROGRAMS FOR INCREASING PHYSICAL ACTIVITY IN COMMUNITY-DWELLING ADULTS WITH ACQUIRED BRAIN INJURY}

Jones $\mathrm{TM}^{1,3}$, Dean $\mathrm{CM}^{1,3}$, Hush JM ${ }^{1,3}$, Dear $\mathrm{BF}^{2,3}$, Titov $\mathrm{N}^{2,3}$

${ }^{1}$ Department of Health Professions, Macquarie University, Sydney ¿Centre for Emotional Health, Department of Psychology, Macquarie University, Sydney

${ }^{3}$ Centre for Physical Health, Macquarie University, Sydney

Question: Do self-management programs improve physical activity in adults with acquired brain injury (ABI)?

Design: Systematic review with data from randomised or quasirandomised controlled trials synthesised in narrative format.

Participants: Community-dwelling adults with non-degenerative ABI. Intervention Self-management program with at least one component focused on increasing physical activity

Outcome measures: Measure of physical activity (objective or selfreport) and/or physical activity specific outcome, such as physical activity self-efficacy.

Results: Five studies met the inclusion criteria. Studies were widely heterogeneous with respect to content, delivery characteristics, and outcomes, although all utilised behavioural change principles. Four studies examined interventions in which physical activity was a component of a multifaceted intervention, where depth of physical activity specific content could not be clearly established. Three studies showed favorable physical activity outcomes following self-management interventions for stroke, however risk of bias was high and overall efficacy remains unclear. Remote delivery via telephone was the predominant form of delivery in two studies with support for its inclusion in self-management programs for individuals following stroke.

Conclusion: The efficacy of self-management programs in increasing physical activity levels in community-dwelling adults following ABI is still unknown. However, promising results indicate further examination of selfmanagement programs specifically aimed at improving physical activity after $\mathrm{ABI}$, including those utilising remote delivery, is warranted.

\section{Key Practice Points:}

- Self-management programs appear to have a favourable effect on physical activity after stroke, however efficacy cannot be established.

- Self-management programs delivered remotely warrants further investigation.

- Depth of physical activity specific content in multifaceted programs was unclear. 
HYDRATION TRENDS AMONGST PLAYERS IN AUSTRALIA'S PROFESSIONAL FOOTBALL (SOCCER) LEAGUE

Jordan $\mathbf{W}^{1}$, Hing WA ${ }^{1}$, Pope RP ${ }^{1}$, Oostenbroek $T^{2}$

${ }^{1}$ Faculty of Health Science and Medicine, Bond University, Gold Coast Brisbane Roar Football Club, Brisbane

Question: Empirically, what levels of fluid loss and associated risk are observed in professional football players during training and competition sessions across seasons?

Design: Retrospective, repeated measures, single subject design

Participants: Six professional players (age 28.83 \pm 5.21 , body mass 75.48 $\pm 7.20 \mathrm{~kg}$, height $1.78 \pm 0.07 \mathrm{~m}$ )

Outcome Measures: Three seasons of session hydration data, as measured by percentage body mass loss (BML), were retrospectively analysed. Based on previous research and to assess risk, cognitive deficits and physical performance deficits were assumed to commence at 1-2\% body mass loss and greater than $2 \%$ mass loss, respectively. Accompanying the hydration data was data on types and intensity of exercise documented by strength and conditioning staff.

Results: On average, players exhibited fluid loss equivalent to 1-2\% BML and sufficient to cause degradation in cognitive performance in $40 \%$ of sessions, and greater fluid loss, sufficient to cause degradation in physical as well as cognitive performance, in a further $39 \%$ of sessions. Individuals varied in mean per-session fluid loss.

Conclusion: This research is the first to monitor hydration fluctuations in professional football players over a prolonged period of time. The results indicate substantial fluctuations in hydration levels and considerable associated risks related to hydration-related degradation in cognitive and physical performance.

\section{Key Practice Points:}

- Session fluid loss distributions, in addition to player mean values, is a method that can be used to assess individual hydration trends and associated risk

- Access to fluid and fluid intake should be emphasized during and following high-intensity training sessions and football matches.

\section{PHYSICAL RISK FACTORS FOR THE DEVELOPMENT OF NECK PAIN IN OFFICE WORKER: A SYSTEMATIC REVIEW}

Jun $\mathrm{D}^{1}$, Michaleff $Z^{2}$, Johnston $\mathrm{V}^{1}$, O'Leary $\mathrm{S}^{1,2}$

'School of Health and Rehabilitation Sciences, The University of Queensland, Brisbane

2Physiotherapy Department, Royal Brisbane \& Women's Hospital, Australia

Question: Do office-based physical risk factors have a cause-effect relationship with the onset of neck pain?

Design: A systematic review of prospective cohort studies.

Participants: Computer-based office workers

Intervention: No intervention proposed.

Outcome Measures: Incidence of non-specific neck pain

Results: Twelve selected studies; 2 preventive randomized control studies, nine high quality and one low quality prospective studies revealed 36 potential physical risk factors. These were classified into five groups; work type (14), ergonomic (10), workplace environment (6), physical capacity (4), and physical activity (2). One study demonstrated limited evidence for the effect of workplace environment or physical capacity. Conflicting evidence for several other factors (ergonomic, work type, and physical activity) was evident with studies showing inconsistent direction of relationships.

Conclusion: Inconsistencies in the literature is potentially due to a high dependence of studies on self-report outcomes. Overall there is a dearth of prospective studies using valid objective measures to investigate the association between physical factors and the development of neck pain in office workers.

\section{Key Practice Points}

- No physical factors were identified as a risk for the development of neck pain in office workers

- Generally the literature on this topic suffers from a lack of quality studies and methodological issues.

- Prospective studies are needed to better investigate the relationship between physical factors and the development of neck pain in office workers.
JOB STRAIN AND COPING STRATEGIES INFLUENCE DEPRESSION AND STRESS IN OFFICE WORKERS; FROM TWO WORKSITES, BRISBANE AND SOUTH KOREA

Jun $\mathrm{D}^{1}$, Johnston $\mathrm{V}^{1}$, O'Leary $\mathrm{S}^{1,2}$

${ }^{1}$ School of Health and Rehabilitation Sciences, The University of Queensland, Brisbane

2Physiotherapy Department, Royal Brisbane \& Women's Hospital, Australia Question: What is the impact work related psychosocial factors and psychological distress in office workers?

Design: Cross-sectional study

Participants: 170 office workers ( 80 men and 90 women) aged $38.62 \pm 10.97$ years from the sites in Brisbane and South Korea.

Outcome Measure: Psychological distress was measured with the abbreviated Depression, Anxiety and Stress Scale. Work related psychosocial factors was measured with the; 1 ) Job Content Questionnaire for job strain, job insecurity, and job support, 2) Latack Coping Scale for coping strategies to workplace stress.

Results: Job strain and negative coping strategies (avoidance behaviours and alcohol consumption) were significantly positively correlated with depression ( $\beta$ coefficient 050 and 1.378) and stress symptoms ( $\beta$ coefficient 0.64 and 1.363). In multiple regression analysis, job strain, coping strategies with other predictors explained $33 \%$ and $20 \%$ of the variance in depression and stress symptoms (depression; $F(1,200)=$ 8.088, stress; $\mathrm{p}<0.00 ; F(2145)=4.033, \mathrm{p}<0.00)$

Conclusion: Negative coping strategies at work, and job strain, are significantly associated with depression and stress in office workers in Australia and Korea.

\section{Key Practice Points:}

- Work related psychosocial factors may have an influence on a workers' general psychological distress.

- Similar findings in both Korean and Australian office workers suggest that these findings may be generalizable to office workers.

- Individual strategies for coping with workplace stressors should be considered in the assessment of office workers. 


\section{MEASUREMENT OF ASSOCIATED \\ REACTIONS OF THE UPPER LIMB IN \\ PEOPLE WITH ACQUIRED BRAIN INJURY: A SYSTEMATIC REVIEW}

Kahn M1, Clark R², Bower K², Mentiplay B², Williams $G^{1,3,4}$

${ }^{1}$ Epworth Healthcare, Australia

${ }^{2}$ Australian Catholic University, Australia

3University of Melbourne, Australia

${ }^{4}$ LaTrobe University, Australia

Question: What methods are used to assess associated reactions (ARs) in the hemiplegic upper limb in people with acquired brain injury (ABI)?

Design: Two stage systematic search of 10 databases. Review synthesised in a narrative format.

Participants: Adults with ABI who have ARs.

Outcome Measures: Methods that quantify ARs of the hemiplegic upper limb were identified for stage one Clinimetric properties and clinical utility of the AR assessment methods were examined for stage two.

Results: Eighteen papers were included for review. The methods used to evaluate ARs were electromyography(11), standard goniometry(5), electrogoniometry(1), dynamometry(5), subjective clinician(2), and patient rating forms(2). Electromyography, electrogoniometry and dynamometry mostly tested ARs in stationary, seated testing positions using maximal voluntary contractions of the intact upper limb to induce the hemiplegic upper limb AR. Standard goniometry most frequently tested ARs dynamically, using a mobility task to provoke the AR. There was limited clinimetric data on any AR assessment method. Only half of the assessment methods were clinically feasible. The most commonly used methods to quantify ARs were laboratory-based.

Conclusion: There was no dynamic, clinically feasible, objective method for evaluating the ARs that occur during provocative functional tasks, such as walking. Current ARs assessment methods predominantly include subjective rating scales or laboratory-based, stationary-seated assessments. Given that ARs are effort-dependent and occur dynamically, stationary assessment methods have poor ecological validity, and may fail to reflect the extent of the problem that is observed when a person is moving. There is little clinimetric information available for any AR assessment method.

\section{Key Practice Points:}

- Associated reactions are a common focus of physiotherapy interventions in people with $A B I$

- Majority of the assessment methods in the literature are laboratorybased, stationary, seated assessments with minimal clinimetric data available

- Further research is required to develop a clinically feasible objective assessment tool to dynamically measure ARs in the hemiplegic upper limb

\section{CASE REPORTS ON ATHLETES WITH DISABILITY}

\section{Kahn V ${ }^{1}$, Wallis M² $^{2}$ Staples K \\ ${ }^{1}$ OPSMC \\ ${ }^{2}$ AlS Physical Therapies, Canberra \\ ${ }^{3}$ RHP Physiotherapy, Brisbane}

A series of $5 \times 5$ report studies will be presented to highlight the diversity of injuries, and challenges with sports physiotherapy for athletes with a disability. The reports will be from wheelchair basketball, equestrian, and winter sport. These reports are designed to illustrate the amazing returns when working with this specialised sports group.

\section{Key Practice Points:}

- Advanced clinical reasoning and ability to think laterally is required for physios working in this field. A clinical reasoning approach will be highlighted.
WHOSE PAIN IS IT ANYWAY? COMPARABILITY OF PAIN REPORTS
FROM CHILDREN AND THEIR PARENTS

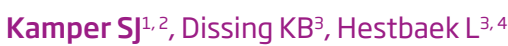

${ }^{1}$ The George Institute for Global Health, University of Sydney, Sydney, Australia

¿EMGO+ Institute, VU University Medical Centre. Amsterdam, The Netherlands IInstitute of Sports and Clinical Biomechanics, University of Southern Denmark. Odense, Denmark

${ }^{4}$ Nordic Institute for Chiropractic and Clinical Biomechanics. Odense. Denmark

Questions: How well do parents' reports of the presence of

musculoskeletal pain in their children match their children's own report?

Do gender, age, pain intensity and pain duration influence how well they match?

Design: Parents were asked by SMS whether their child had pain over the past week, the children underwent assessment at school including questions about the presence of pain in the past week.

Participants: The study included 354 children aged from 10 to 14, and their parents.

Outcome Measures: Parents received an SMS asking whether their child had musculoskeletal pain during the last week. The children filled out a body chart marking any pain experienced in the last week and rated its intensity and duration.

Results: Percentage agreement between the parent and the child report was 52.4\% (Kappa 0.20). The low agreement was almost entirely due to children reporting pain and the parent not. Child age and gender did not influence the rate of agreement but better agreement was related to higher pain intensity and possibly longer duration.

Conclusion: Agreement between children and their parents was generally poor, children often reported pain when their parents did not. When pain intensity is greater the reports matched better.

\section{Key Practice Points:}

- Parents' and their child's report of pain should not be considered interchangeable.

- Children often report pain that is not reported by their parents, but the reverse is rarely the case.

- The age or gender of the child does not influence matching, but pain intensity and pain duration likely do.

\section{HOW TO MEASURE OUTCOME IN YOUR PRACTICE}

\section{Kamper $\mathrm{SJ}^{1}$, Williams $\mathrm{CM}^{2}$}

${ }^{1}$ The George Institute for Global Health, University of Sydney ${ }^{2}$ Hunter Medical Research Institute, University of Newcastle and Hunter New England Local Health District

Background: The limitations and biases associated with human memory are well-accepted. This means that accurate measurement and documentation of outcomes is a fundamental requirement if physiotherapists are to record and assess their own practice. However outcome measurement represents a whole field of study of itself and understanding what and how to measure requires particular skills. Physiotherapists also face practical barriers to integrating measurement into their daily practice.

\section{Aims / objectives:}

- To understand the key features of good measurement instruments and practices

- To learn about the different types of measures and when their use is appropriate.

- To discuss the practical requirements of outcome measurement in practice.

Approach: The workshop will involve delivery of material based on the learning objectives via short presentations. This will be supplemented by information made available to participants after the workshop. During the workshop there will be facilitated discussion led by the presenters focused on the third objective. Discussion will centre on specific barriers that participants have faced in the past in implementing outcome measurement in practice and issues that arise based on the information delivered in the presentations.

Conclusion / Key Practice Points:

- Participants will be introduced to the concepts necessary to assess the quality of outcome measures.

- Participants will learn about the different types of measures and what to consider when determining which are appropriate for their practice.

- Participants will be given practical advice for integrating measurement into their day-to-day practice. 


\section{EFFECTS OF A TREADMILL-BASED \\ EXERCISE INTERVENTION ON \\ SYMPTOMS ASSOCIATED WITH PRIMARY \\ DYSMENORRHEA: PRELIMINARY \\ RESULTS OF A RANDOMIZED \\ CONTROLLED TRIAL}

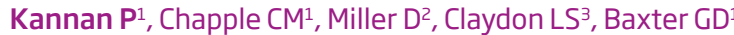

${ }^{1}$ Centre for Health, Activity and Rehabilitation Research, University of Otago, Dunedin, New Zealand

'Department of Women's and Children's Health, Dunedin School of Medicine, Dunedin, New Zealand

${ }^{3}$ Department of Allied and Public Health, Anglia Ruskin University, Chelmsford, UK

Question: Is exercise an effective intervention for the management of primary dysmenorrhea?

Design: A single blind (assessor), prospective, two-arm randomized controlled trial.

Participants: Seventy non-pregnant women in the age group 18-43 years with primary dysmenorrhea were randomly assigned to either the exercise group $(n=35)$ or

usual care control group ( $n=35$ ).

Intervention Treadmill-based exercise intervention for 7 months beginning with one month training period at the clinic followed by a home exercise programme for 6 months.

Outcome Measures: Menstrual pain intensity was measured with visual analogue scale and quality of life measured using 12-Item short form health survey at baseline (week 0 ), week 4 and week 16. Data were analyzed using Mann Whitney U test and Friedman's ANOVA.

Results: There was a significant difference in mean ranks for pain intensity between groups at week $4(p=0.001)$ and week 16 ( $p<$

$0.001)$. A statistically significant difference was also observed in mean ranks for quality of life between groups at week $4(p<0.001)$ and week $16(p<0.001)$

Conclusion: These preliminary results demonstrate that exercises are effective in reducing pain intensity and improving quality of life in women with primary dysmenorrhea.

\section{Key Practice Points:}

- Evidence for the effectiveness of exercise for primary dysmenorrhea.

- Evidence to support the use of exercise in alleviation of symptoms associated with primary dysmenorrhea.

- A low-cost intervention for the long term management of primary dysmenorrhea.

\section{ASSESSING THE RELIABILITY OF USING ULTRASOUND IMAGING TO EXAMINE RADIAL NERVE EXCURSION}

Kasehagen $\mathrm{B}^{1}$, Ellis $\mathrm{R}^{2}$, Mawston $\mathrm{G}^{2}$, Allen $\mathrm{S}^{3}$, Hing $\mathrm{W}^{1}$

${ }^{1}$ Bond University, Faculty of Health Sciences and Medicine, Bond University Institute of Health and Sport, Gold Coast, Robina, Queensland 4226, Australia ${ }^{2}$ Health and Rehabilitation Research Institute, School of Clinical Sciences, Auckland University of Technology, Auckland, 1020, New Zealand ${ }_{3}^{3}$ Sound Experience, Clinic 41, Unitec Gate 3139 Carrington Road, Mt Albert. Auckland 1025, New Zealand

Question: Is ultrasound imaging a reliable method for quantifying radial nerve excursion

Design: Controlled laboratory cross-sectional study using a single-group within-participant comparison of nerve excursions under different conditions and test-retest reliability assessment.

Participants: Thirty healthy participants

Intervention: Active and passive movements were examined for two wrist movements (extension-flexion and radial deviation-ulnar deviation) used to induce radial nerve excursion. These movements were performed in both a supinated and pronated forearm position.

Outcome Measures: Longitudinal nerve excursion was quantified using frame-by-frame cross-correlation analysis. Muscle activity was measured using EMG and expressed as percentage of maximal voluntary isometric contraction. Wrist range of motion was measured using an electrogoniometer.

Results: Assessment of the reliability of measuring longitudinal radial nerve excursion was moderate to high (ICC $=0.63-0.86$, SEM 0.19-0.48) Radial nerve excursion ranged from 0.41 to $4.03 \mathrm{~mm}$ with wrist extensionflexion and 0.28 to $2.91 \mathrm{~mm}$ with radial deviation-ulnar deviation. Forearm supination produced larger overall nerve excursion $(1.41 \pm 0.32$ $\mathrm{mm})$ when compared with forearm pronation $(1.06 \pm 0.31 \mathrm{~mm})(p<0.01)$ Passive movement produced a mean $0.39 \pm 0.49 \mathrm{~mm}(p<0.01)$ greate nerve excursion and significantly less muscle activation $(p<0.01)$ than active movement.
Conclusion: Real-time ultrasound is a reliable, cost effective, in vivo method for analysis of radial nerve excursion.

\section{Key Practice Points:}

- Real-time ultrasound is a reliable, cost effective, in vivo method for analysis of radial nerve excursion

- Passive wrist movements produced significantly greater radial nerve excursion than active movements

- Current literature is limited to selected nerves and generally in healthy populations.

\section{CAN A CULTURE CHANGE MAKE AGED CARE A MORE ATTRACTIVE ROTATION FOR JUNIOR PHYSIOTHERAPY STAFF?}

Kay J

Melbourne Health, Melbourne

Question: Can Aged Care rotations be attractive for junior Physiotherapists?

Design: A staff satisfaction survey was conducted in February 2012 followed by a team-building planning day. The newly appointed team leade identified poor team culture, through observation and informal feedback from the Aged Care team.

Participants: Aged Care Physiotherapy team, at a large metropolitan hospital in Melbourne, Australia (approximately fourteen Physiotherapists and Allied Health Assistants).

Intervention: Following the survey and planning day, a multiple strategy approach included shifting Physiotherapy from gym-based to ward-based interventions; caseload reallocation to relieve pressure on junior staff; allowing Physiotherapists to discharge patients when intervention is futile and no goals identified; and the allocation of patients' with increased complexity to senior staff, or to junior staff with extra support, including senior presence during family meetings.

Outcome Measures: Staff were re-surveyed using the original survey in March 2015

Results: Staff listed senior support and teamwork as the best features of working in the Aged Care team. Caseload pressure was not listed as a positive or negative. Overall team culture and the relationship with ward staff has improved. Grade One rotation preferences selecting Aged Care increased over the past three years.

Conclusion: Positive outcome from implementing suggestions from within the Aged Care Physiotherapy team.

\section{Key Practice Points:}

- Junior Physiotherapy staff need increased support in Aged Care rotations, particularly with patients and families who are grieving the loss of function and independence.

- Junior Physiotherapy staff need guidance and support for discharging patients when intervention is futile. 


\section{CONNECTING KNEE INSTABILITY TO OSTEOARTHRITIS: THE RELATIONSHIP BETWEEN JOINT INSTABILITY AND OSTEOARTHRITIS IN ANTERIOR CRUCIATE LIGAMENT-DEFICIENCY 12 YEARS AFTER INJURY}

Keays SL ${ }^{1,2}$, Bullock-Saxton $]^{3,4}$, Newcombe PA5 ${ }^{5}$, Keays $A C^{2}$, Savage $]^{4}$

University of the Sunshine Coast

2Private Practice, Nambour

${ }^{3}$ Australian Catholic University

${ }^{4}$ Private Practice, Brisbane

${ }^{5}$ The University of Queensland

Question: Do patients with stable knees (copers), unstable knees (noncopers) or equivocal knee stability (adaptors) develop more osteoarthritis $(O A), 12$ years after anterior cruciate ligament $(A C L)$ injury?

Design: Prospective cohort study

Participants: 52 ACL-injured patients, (32 males, 20 females, mean age 40 years) were recruited from a cohort of $297 \mathrm{ACL}$-injured patients. Patients with a ruptured $A C L$ on $M R I$, arthroscopy or orthopaedic examination and without reconstructive surgery were included. 34 patients had meniscal injuries.

Intervention: Patients were treated with physiotherapy and followed-up 12 years post injury.

Outcome Measures: Stability measures included subjective, instrumented (KT 1000) and clinical testing. Patients were grouped according to stable, unstable and equivocally stable knees. Tibiofemoral (TF) and patellofemoral (PF) OA on radiograph were graded by a radiologist blinded to group. ANOVA was conducted to compare the incidence and severity of OA between groups.

Results: 14 patients had stable ACL-deficient knees, 15 patients had unstable and 23 had equivocally stable knees. Overall $62 \%$ of patients developed TFOA and $44 \%$ developed PFOA. $42 \%$ of stable knees, $80 \%$ of unstable knees and $63 \%$ of equivocally stable knees developed TFOA with significant differences between the stable and unstable group ( $p=0.017$ ). No differences were found for the development of PFOA

Conclusion: Twelve years post injury, there exists an ACL-injured group that remains stable and develops significantly less TFOA compared to an unstable group. Detailed assessment of ACL-injured patients before making surgical decisions is important as more stable knees treated conservatively may develop less OA than if treated surgically.

\section{Key Practice Points:}

- Patients who rupture their ACL present with very varying levels of stability.

- Patients with more stable knees develop significantly less tibiofemoral OA

- Preventing ACL injuries is the optimal strategy to prevent the development of knee $O A$

\section{GENERALISED JOINT LAXITY IN SIBLINGS WITH AND WITHOUT ANTERIOR CRUCIATE LIGAMENT (ACL) INJURY?}

Keays $\mathrm{SL}^{1,2}$, Newcombe $\mathrm{PA}^{3}$, Keays $A C^{2}$

${ }^{1}$ University of the Sunshine Coast

${ }^{2}$ Private Practice, Nambour

${ }^{3}$ The University of Queensland

Question: Are siblings with ACL injuries more likely to share generalized joint laxity (GJL) than uninjured matched siblings.

Design: Controlled cohort study

Participants: 24 siblings with ACL injuries from 10 families were matched for age, gender, sporting level and family composition with 24 siblings with healthy knees.

Intervention: Siblings were assessed using modified Beighton's criteria for hypermobility: fifth finger metacarpophalangeal hyperextension $>90^{\circ}$; wrist hyperflexion with the thumb reaching the volar forearm surface;

knee hyperextension $>5^{\circ}$; elbow hyperextension $>10^{\circ}$; trunk flexion with flat palms to the floor. Incidence of GJL was compared between injured and healthy siblings and injured and healthy sibling pairs.

Outcome Measures: GJL was graded 0-5 according to the presence or absence of hypermobility in five joints. Siblings were positive for GJL if they scored >3.

Results: Of the injured siblings 58\% were positive for metacarpophalangeal hyperextension; $66 \%$ for wrist hyperflexion; 38\% elbow hyperextension; 83\% knee hyperextension; 17\% trunk hypermobility. $58 \%$ were positive for GJL. Of the uninjured siblings $8 \%$ were positive for metacarpophalangeal extension; $8 \%$ for wrist hyperflexion; 17\% elbow hyperextension; 21\% knee hyperextension; 20\% trunk hypermobility. $17 \%$ were positive for GJL. In the injured group, six (60\%) sibling pairs were matched for GJL compared with only one $(10 \%)$ pair in the control group, $X^{2}(1, N=20)=5.50, p=0.019$

Conclusion: A significant difference exists in the number of sibling pairs with GJL in the injured compared to the uninjured group. Most likely GJL has a genetic underpinning and contributes to the high incidence of ACL injuries in siblings.

\section{Key Practice Points:}

- Hypermobility is common in siblings who have a ruptured ACL

- Siblings with GJL, in particular hyperextending knees, may be prone to ACL injuries

- Siblings, and possibly all athletes, should be screened for hypermobility and undergo prevention training in order to reduce ACL injuries.

\section{OUADRICEPS STRENGTHIS AN INDEPENDENT PREDICTOR OF PHYSICAL ACTIVITY POST- HEART TRANSPLANTATION}

Kelly RL ${ }^{1,4}$, Walsh JR ${ }^{1,2,4}$, Paratz JD ${ }^{4}$, Yerkovich ST ${ }^{2,3}$, McKenzie S $^{2,3}$, Morris NR 2,4

${ }^{1}$ Physiotherapy Department, The Prince Charles Hospital, Brisbane zHeart Lung Institute, The Prince Charles Hospital, Brisbane ${ }^{3}$ School of Medicine, The University of Queensland, Brisbane ${ }^{4}$ School of Allied Health Sciences, Griffith University, Gold Coast

Question: Determine levels of physical activity post-heart transplantation and identify factors that predict physical activity level.

Design: Prospective observational cross-sectional study.

Participants: Medically stable adults; $\geq 6$ months post-heart transplantation.

Setting: Single-institution outpatient clinic. Inclusion criteria: Compliance of multi-sensor device $\geq 22$ hours/day for $\geq 3$ days. Exclusion criteria: Reduced left ventricular function, recent rejection episode, injury or illness.

Outcome Measures: Physical activity was assessed using a SenseWear Pro 3 armband. Physical activity level was defined as total energy expenditure in 24 hours/basal metabolic rate derived from the sleeping metabolic rate. Quadriceps strength was measured using a hand-held dynamometer. 6-minute walk test was performed per standardised protocol. Participants' demographics, time post-heart transplantation, quadriceps strength and 6-minute walk distance were assessed to determine predictors of physical activity level.

Results: 76 subjects; 21 females, age (mean \pm SD) $56.0 \pm 14.4$ years. 27 participants were classified very sedentary, 27 sedentary and 22 $\geq$ moderately active. Physical activity level was correlated with quadriceps strength $(r=0.414, p<0.001)$, body mass index $(r=-0.380, p<0.001)$ and 6 -minute walk distance $(r=0.329, p=0.004)$. In a multivariate linear regression, only quadriceps strength $(\beta=0.00395, p<0.001)$ was identified as an independent predictor of physical activity level $\left(r^{2}=0.152\right)$.

Conclusion: Post-heart transplantation physical activity level appears to be largely independent of most outcome measures except quadriceps strength. We hypothesise that peripheral muscle dysfunction impacts physical activity level.

\section{Key Practice Points:}

- Recovery in physical activity post-heart transplantation is complex.

- Muscle strength is a good outcome measure to predict physical activity level.

- If we target muscle strength recovery, we may improve physical activity level. 
HIP FUNCTIONAL IMPAIRMENTS EXIST

BILATERALLY, ARE RELATED TO HIP

STRENGTH AND PREDICT PATIENT-

REPORTED OUTCOMES IN PATIENTS WITH CHONDROLABRAL PATHOLOGY

Kemp JL $L^{1,6}$, Risberg MA², Schache $\mathrm{AG}^{3}$, Makdissi $\mathrm{M}^{3}$, Pritchard MG ${ }^{4}$, Crossley $\mathrm{KM}^{5,6}$

${ }^{1}$ Australian Centre for Research into Injury in Sport and its Prevention,

Federation University Australia

¿Department of Sport Medicine, Norwegian School Sport Sciences, Oslo Norway

\& Department of Orthopedic Surgery, Oslo University Hospital, Oslo Norway

3University of Melbourne

${ }^{4}$ Wellington Orthopaedics, Hobart

${ }^{5}$ La Trobe University

EUniversity of Queensland

Question: In people with chondrolabral pathology 1-2 years post hip arthroscopy: (i) what are between-limb differences in functional performance tests compared to age matched healthy controls; (ii) what

is the relationship between functional performance and hip strength and (iii) are measures of functional performance associated with patientreported outcomes.

Design: Cross-sectional study

Participants: 71 chondrolabral patients; 60 age-matched controls with no history of hip surgery or pain.

Outcome Measures: International Hip Outcome Tool (iHOT-33) patientreported outcome (PRO); functional performance tests (single leg hop (hop), side bridge (bridge), one leg rise (rise)); and hip muscle strength.

Results: Chondrolabral group had significantly poorer performance compared to controls on (i) number of rises for both surgical (MD $(95 \% \mathrm{Cl})$

14 (8-19); and non-surgical sides (14 (8-19)); (ii)the hop for both surgical (32

$(21-42) \mathrm{cm})$ : and non-surgical sides $(37(27-47) \mathrm{cm})$ : (iii) the bridge on surgical

(23(10-36)s) and non-surgical sides (24(11-37)s). Greater hip abduction (adjusted r2 0.310 p<0.001); adduction (adjusted r20.547 p<0.001); and extensor strength (adjusted r20.407-0.613 p<0.001) were associated with better functional performance. In addition, better performance on the bridge and hop tests (adjusted r2 0.285-0.481 p<0.001), was associated with better PRO scores.

Conclusion: Post-hip arthroscopic rehabilitation programs could include hip strength exercises and functional performance retraining in order to optimise outcomes.

\section{Key Practice Points}

- Greater strength in hip abduction, adduction and extension were associated with better functional performance. Better bridge and hop tests were associated with better PROs.

- This may guide clinicians in designing targeted rehabilitation programs.

\section{MEASURING OLDER ADULTS' COMMUNITY INTEGRATION FOLLOWING TRAUMATIC BRAIN INJURY}

Kent $\mathrm{J}^{1}$, Wright-St Clair $\mathrm{V}^{1}$, Kersten $\mathrm{P}^{2}$

${ }^{1}$ Physio Professionals, Sunshine Coast

${ }^{2}$ Auckland University of Technology, New Zealand

Question: To explore community integration from the perspective of olde adults following mild traumatic brain injury, and compare findings with results from two community integration outcome measures.

Design: Mixed method study.

Participants: Four older adults who had experienced mild traumatic brain injury within the last year and had resided back in their community for at least three months post-injury.

Outcome Measures: Qualitative descriptive methods were used to explore participants' experiences through semi-structured interview followed by implementing two outcome measures: the Community Integration Questionnaire and the Community Integration Measure Qualitative data were thematically analysed and the findings for each participant then compared with outcome measure results.

Results: Traumatic brain injury caused major disruption in community reintegration, with participants describing a battle to maintain their preinjury independence and being challenged with the consequences of losing some autonomy following injury. Setting up or maintaining good support networks was identified as vital to reintegration into the community. Neither standardised measure wholly reflected participants' subjective community integration experience.

Conclusions: Community integration presents a major challenge following traumatic brain injury. Care should be taken when interpreting the results from these outcome measures for older adults following mild traumatic brain injury. Further research is needed to assess their validity within this population.

\section{Key Practice Points:}

- Successful community integration should be the primary goal of physiotherapy rehabilitation.

- Supplement the Community Integration Questionnaire or Community Integration Measure with open questions exploring everyday experiences of older adults' community integration following mild traumatic brain injury.

\section{"HOME REHABILITATION" FOLLOWING HIP FRACTURE REPAIR FOR NURSING HOME RESIDENTS IMPROVES MOBILITY OUTCOMES: A SINGLE BLIND RANDOMISED CONTROL TRIAL}

Killington $\mathrm{M}^{1}$, Davies $\mathrm{O}^{1}$, Walker $\mathrm{R}^{2}$, Miller $\mathrm{M}^{3}$, Cameron $\mathrm{I}^{4}$, Kurrle $\mathrm{S}^{4}$, Chehade $\mathrm{M}^{5}$, Crotty $\mathrm{M}^{1}$

${ }^{1}$ Rehabilitation, Aged \& Extended Care, (Flinders Clinical Effectiveness), School of Health Sciences, Flinders University, and Repatriation General Hospital, Adelaide, Australia

¿Southgate Institute for Health, Society and Equity, Flinders University, Adelaide, Australia

${ }^{3}$ Flinders Clinical \& Molecular Medicine, Flinders University, Adelaide, Australia ${ }^{4}$ John Walsh Centre for Rehabilitation Research, Kolling Institute, University of Sydney, Sydney, Australia

${ }^{5}$ Orthopaedics and Trauma Unit, University of Adelaide, Adelaide, Australia

Question: Does "home rehabilitation" following hip fracture repair improve mobility for nursing home residents.

Design: Previously walking nursing home residents $(n=240)$ with hip fractures received 4 weeks of home rehabilitation in their nursing home or usual care. In parallel, families and nursing home staff for the first 30 participants were invited to share their perceptions of the journey for residents at interviews/focus groups.

Intervention: Physiotherapy (minimum three sessions week for 4 weeks) focused on restoration of transfers and limited mobility.

Outcome Measures: The Nursing Home Life Space Diameter (NHLSD), mobility status including level of independence and qualitative data organised as a thematic analysis with the assistance of NVivo 10 were collected.

Results: Nursing home residents who received "home rehabilitation" mobilised further and more frequently as assessed by the NHLSD ( $p<$ 0.0001). More "home rehabilitation" participants were reported to have regained independent mobility with a lower burden of care compared to usual care participants. Acute care staff struggled to provide people with dementia mobility retraining and nursing home staff were ill-equipped to provide post-operative care including mobility retraining and pain management on their return home.

Conclusion: Following hip fracture surgery "Home rehabilitation" for nursing home residents was feasible and improved independence with mobility was achieved.

Key Practice Points:

- Strategies need to be developed to engage people who have dementia in early rehabilitation in the acute setting.

- Mobile nursing home residents with dementia who fracture their hips can improve their mobility levels in response to in reach physiotherapy. 
MANAGEMENT OF PATIENTS

BROUGHT IN BY AMBULANCE (BIBA)

TO THE EMERGENCY DEPARTMENT

(ED): THE ROLE OF THE ADVANCED

MUSCULOSKELETAL PHYSIOTHERAPIST (AMP)

Kinsella $\mathbf{R}^{1}$, Collins $\mathrm{T}^{1}$, Sayer $]^{2}$, Shaw B ${ }^{1}$, Cary B ${ }^{1}$, Cowan $\mathrm{S}^{1,3}$

${ }^{1}$ St. Vincent's Hospital, Melbourne

2The Alfred Hospital, Melbourne

${ }^{3}$ The University of Melbourne

Aim: To evaluate the AMP's role in managing patients with musculoskeletal complaints BIBA to the ED.

Design: Dual-centered observational study.

Participants: Patients aged 16-65, BIBA to two Melbourne hospitals ove a 12 month period and seen by the AMP, were compared with a matched group seen by other ED medical staff.

Outcome Measures: Primary outcomes: waiting time (WT) and length of stay (LOS). Secondary outcome: representation back to ED (<24 hours).

Results: Data from 1441 patients BIBA within the Australian Triage Scale (ATS) categories 3-5, with musculoskeletal complaints were included. 172 were managed by the AMP and 1269 by ED medical staff. No baseline differences were found for age or gender but AMPs were more likely to see patients within ATS category 4. Mean WT and LOS were significantly shorter for those managed by the PCP than for matched patients managed by other ED medical staff. On average patients were seen 18 minutes earlier by AMPs and their average LOS was 72 minutes less $(p<0.001)$ There were no representations back to ED within 24 hours.

Conclusion: This study demonstrates that patients BIBA to ED and managed by AMPs are likely to wait less time and be discharged more quickly than if seen by other ED medical staff.

Key Practice Points:

- The first study to investigate the AMPs' role in managing patients BIBA in a primary capacity and provides evidence that AMPs may positively impact ambulance presentations.

- Supports the growing body of evidence demonstrating that AMPs improve patient flow in ED.

\section{DEVELOPMENT OF A TRAINING \\ RESOURCE TO ASSIST IN THE} ASSESSMENT OF PHYSIOTHERAPY STUDENTS USING THE ASSESSMENT OF PHYSIOTHERAPY PRACTICE (APP)

Kirwan $\mathrm{G}^{1}$, Clark $\mathrm{C}^{1}$, Dalton $\mathrm{M}^{2,3}$

${ }^{1}$ Menzies Health Institute, Griffith University, School of Allied Health Sciences, Gold Coast

2Monash University, Faculty of Medicine, Nursing and Health

Science, Melbourne

${ }^{3}$ Central Queensland University, School of Human, health and Social Science, Rockhampton

Question: Despite the recognised validity and reliability, anecdotal student and educator feedback indicates that the interpretation and application of the APP in student assessment may be inconsistent among some physiotherapy clinical educators. Therefore, the aims of the study were to firstly determine if consensus exists between experienced assessors when rating a video vignette of a student performance and secondly, to develop a resource to improve consistency in the interpretation of the APP. The study was conducted in four clinical areas, however only the results from the cardiorespiratory setting will be discussed in this presentation Participants: Physiotherapists $(n=41)$ from Australia and New Zealand with a minimum 3 years clinical experience, who have undertaken student supervision within past 12 months and identified cardiorespiratory as their primary clinical area were included in the study

Intervention (if applicable): Three distinct levels of student performance (not adequate, adequate and good to excellent) were scripted and filmed for the clinical areas of cardiorespiratory. Participants each reviewed one randomly allocated vignette, rated the student performance on the global rating scale using the APP and provided a rationale for their decision. Participants were blinded to the designated vignette performance level.

Outcome Measures: An a priori percentage agreement of $75 \%$ among assessors was considered acceptable

Results: Based on the APP global rating, 100\% of assessors agreed that one performance was not adequate $(n=11)$ and another was good - excellent ( $n=5)$. In the remaining video, $46 \%$ of assessors rated the performance as adequate and $30 \%$ rated the performance as good ( $n=26)$, therefore, $76 \%$ of assessors rated the video as adequate to good.

Conclusion: Assessors are better equipped to assess student performance at a not adequate or good to excellent level. As the level of the vignette approached the level of a pass, wider variability in performance ratings was observed. Importantly, there appeared to be clear consensus between an adequate and not adequate performance, which is arguably the most critical decision for assessing performance on clinical placement.

\section{Key Practice Points:}

- There appears to be consensus among experienced cardiorespiratory clinical educators when rating a student performance using the global rating scale

- Greater variability in rating was apparent when the student performance approached the level of adequate. However, 96\% of educators agreed on a performance that was adequate or better and 100\% agreed on an inadequate performance, which is a crucial decision in assessing a student performance.

- Based on the results of the study, the videos provide a valuable training tool to assist new educators in assessing student performance using video exemplars.

PHYSICAL ACTIVITY IN THE ACUTE WARD FOLLOWING HIP FRACTURE SURGERY IS ASSOCIATED WITH A LOWER DEGREE OF FEAR OF FALLING

Kronborg $\mathrm{L}^{1}$, Bandholm $\mathrm{T}^{1,2,3}$, Palm H${ }^{3}$, Kehlet $\mathrm{H}^{4}$, Kristensen $\mathrm{MT}^{1,3}$ ${ }^{1}$ Physical Medicine and Rehabilitation Research - Copenhagen (PMR-C), Department of Physiotherapy, Copenhagen University Hospital, Hvidovre, Denmark

${ }^{2}$ Clinical Research Centre, Copenhagen University Hospital, Hvidovre, Denmark ${ }^{3}$ Department of Orthopedic Surgery, Copenhagen University Hospital, Hvidovre, Denmark

${ }^{4}$ Section for Surgical Pathophysiology, Rigshospitalet, Copenhagen University, Denmark

Question: How is physical activity (time spent upright) the first week after hip fracture surgery related to functional performance and fear of falling at discharge?

Design: Prospective observational study

Participants: Thirty-seven patients (mean 80 years \pm 8.4 ) admitted to the acute ward after hip fracture surgery was recruited from 40 patients enrolled in parallel study.

Outcome Measures: The 24-hour upright time and daily upright events (sitting to standing) were measured using a thigh-worn accelerometer the first week after surgery. The Short Falls Efficacy Scale International (Short FES-I) was used to measure fear of falling at discharge.

Results: Upright time increased from median 13 (IQR 6;31) minutes and 7 $(4 ; 16)$ upright events at day 2 , to $46(11 ; 107)$ minutes and $18(8 ; 34)$ upright events at day 7 . More upright time at discharge was significantly associated with a lower degree of fear of falling $(r=-0.48, p=0.01, n=27)$

Conclusion: Patients with a hip fracture following an enhanced recovery program increase the time spent upright during the first week postoperatively. The association between the time spent upright and fear of falling at discharge indicate a need to further explore how to empower these patients and promote their confidence in physical activity shortly after surgery

\section{Key Practice Points:}

- The amount of physical activity in the acute ward after hip fracture surgery is low.

- Inter-professional focus on physical activity in the acute ward may benefit the patient.

- Focus on fear of falling may optimize the rehabilitation pathway. 
HOW TO CONDUCT PROGRESSIVE STRENGTH TRAINING IN THE ACUTE WARD AFTER HIP FRACTURE SURGERY

Kronborg $\mathrm{L}^{1}$, Bandholm $\mathrm{T}^{1,2,3}$, Palm H ${ }^{3}$, Kehlet $\mathrm{H}^{4}$, Kristensen $\mathrm{MT}^{1,3}$

${ }^{1}$ Physical Medicine and Rehabilitation Research - Copenhagen (PMR-C), Department of Physiotherapy, Copenhagen University Hospital, Hvidovre, Denmark

${ }^{2}$ Clinical Research Centre, Copenhagen University Hospital, Hvidovre, Denmark ${ }^{3}$ Department of Orthopedic Surgery, Copenhagen University Hospital, Hvidovre, Denmark

${ }^{4}$ Section for Surgical Pathophysiology, Rigshospitalet, Copenhagen University, Denmark

Background: Patients with a hip fracture loose more than $50 \%$ kneeextension strength in the fractured limb within one week of surgery ${ }^{1}$. Hence, immediate progressive strength training following hip fracture surgery may be rational and has been found feasible. The clinical efficacy is being tested in a randomized controlled design at the moment (NCT00848913).

Aims / objectives: To disseminate the skills of conducting systematical, progressive strength training in the acute ward following hip fracture surgery, based on knowledge from a published study?.

Approach: The workshop will be focused on practical demonstration of how to conduct a simple and systematic program of daily, progressive knee-extension strength training for the fractured limb, using ankle weight cuffs in 3 sets of 10 repetition maximum loadings. Issues of pain and other limitations for completing sessions will be discussed. Examples of related documentation forms will be provided. The participants are invited to try out the approach and to implement the knowledge in own clinical practice. The approach is considered applicable for almost any elderly person in the in- or outpatient setting.

Conclusion: Progressive knee-extension strength training of the fractured limb commenced in the acute ward is feasible and may reduce strength asymmetry between limbs without hip pain interfering.

\section{Key Practice Points:}

- Reduction of knee-extension strength asymmetry may improve balance and reduce the risk of falls.

- This strength training approach is low cost, feasible and welcomed by the elderly patient.

- Reduced loss of knee-extension strength may provide better prognosis for regaining prefracture physical functional.

\section{EXERCISE AND ABDOMINAL PRESSURE: HOW MUCH DO WE REALLY KNOW?}

\section{Kruger $\mathbf{J}^{1}$}

${ }^{1}$ Auckland Bioengineering Institute, University of Auckland. New Zealand This presentation will deal with the concept of abdominal pressure variation in response to exercise and what is currently known in the area. The association between abdominal pressure, pelvic floor (PF) disorders and exercise intensity is poorly understood. Currently, women who are at risk of pelvic organ prolapse (POP) or urinary incontinence are advised to avoid certain activities that might precipitate these conditions (1) For instance, exercises have been categorized as PF safe (e.g. walking, wall push-ups, narrow squats) because it is assumed that these types of activities develop lower abdominal pressure variation than other exercises (e.g. running, full push-ups and wide squatting). However, there is little supporting evidence for this categorisation. This is, in part, due to a lack of methods to accurately measure these physiological parameters. The paucity of accurate data on abdominal pressure development has led to large inconsistencies in post-operative advice and restrictions given to women following surgery. These restrictions include the inability to work, which often imposes a large financial burden. There is also little quantitative evidence upon which to recommend which types of exercise are suitable for women at risk of POP. Recent work by our group, has seen the development of an intra-vaginal pressure sensor, capable of wirelessly measuring abdominal pressure during exercise. I will be presenting some preliminary results, from an exercise study comparing abdominal pressure variation between PF 'safe' exercises, and similar more conventional exercise. I will also be showing a short video illustrating abdominal pressure variations during some activities of daily living which are typically advised against post operatively.

\section{Key Practice Points:}

- The importance of evidence based practice/advice

- How different instrumentation can often give highly variable results

\section{THE EFFECT OF ALTERING SIX- MINUTE WALK TEST COURSE LENGTH ON DISTANCE WALKED AND PHYSIOLOGICAL RESPONSE}

Kwiatkowski SL ${ }^{1,3}$, Kuys $\mathrm{S}^{1,2}$, Laakso $L^{1}$, Morris NR ${ }^{1}$ ${ }^{1}$ Griffith University and Menzies Health Institute, Southport ${ }^{2}$ Australian Catholic University, Brisbane 3Metro South Hospital and Health Service, Brisbane

Question: To determine the impact of course length for six-minute walk tests (6MWT) on distance walked or physiological response in participants with severe chronic obstructive pulmonary disease (COPD).

Design: Repeated measures observational study.

Participants: 11 community-dwelling adults over 40 years with severe or very severe COPD were recruited from outpatient respiratory medicine clinics and pulmonary rehabilitation programs.

Intervention: Each participant completed two 6MWT's were conducted at three different course lengths $(5 \mathrm{~m}, 10 \mathrm{~m}$ and $30 \mathrm{~m})$ over three separate testing occasions on room air. Each course length was measured at least one week apart in random order

Outcome Measures: Six-minute walk distance (6MWD), nadir oxygen saturations (SpO2), peak heart rate (HR) and highest oxygen uptake (VO2) measured across the six-minutes of the walk test.

Results: All 11 participants completed the three testing sessions. Whilst there were significant differences in 6MWD between tests (repeated measures ANOVA, $\mathrm{F}=23.802, \mathrm{p}=0.01$ ), no significant differences in the highest VO2 or HR achieved during the test nor the nadir SpO2 were detected. Post hoc analysis showed differences between the means of the $30 \mathrm{~m}$ vs $10 \mathrm{~m}(65.27 \mathrm{~m}, \mathrm{p}<0.01)$ and $30 \mathrm{~m}$ vs $5 \mathrm{~m}(117.37 \mathrm{~m}, \mathrm{p}<0.01)$ course lengths.

Conclusions: 6MWT course length affects 6MWD however the physiological response appears independent of course length.

\section{Key Practice Points:}

- Reduction in 6MWT course length is associated with a reduction in 6MWD.

- Despite a reduction in 6MWD, physiological measures of highest V02, nadir SpO2 and peak HR do not differ across course lengths.

\section{LASER AND THE CENTRAL NERVOUS SYSTEM}

\section{Laakso E-L}

School of Allied Health Sciences, Griffith University

When the appropriate dose parameters are utilised, Low Level Laser Therapy (LLLT) has a robust evidence base for modulating pain in a number of conditions including chronic and acute neck pain, tendinopathy, frozen shoulder and OA knee pain. An important aspect regarding laser phototherapy for pain management is to understand the effect mechanisms in order to determine the most appropriate clinical applications. This presentation summarises the outcomes of our research investigating central mechanisms and dosing parameters. Our investigations have demonstrated that the sympathetic nervous system plays a partial role in the treatment response for laser photobiomodulation; and the effects in different conditions are dependent on dose, wavelength and power output. Our research has identified that when appropriate parameters of laser photobiomodulation are used, it is possible to influence moment-to-moment changes in physiological responses.

\section{Key Practice Points:}

- Understand the evidence-base for LLLT in pain management

- Consider the possible mechanisms of effect of laser photobiomodulation of pain

- Understand the need to choose the correct dose parameters in order to obtain clinical outcomes 


\section{EVIDENCE INFORMED PRACTICE: TIME TO DEBUNK THE PELVIC ASYMMETRY MODEL OF SIJ DYSFUNCTION}

\section{Lambridis $\mathrm{T}^{1}$}

${ }^{1}$ Clinic Director, Spinal Synergy Physiotherapy, Cremorne, Sydney

Question: Is the pelvic asymmetry model of SII dysfunction evidence informed practice?

Introduction: Various therapeutic models have been proposed in identifying and treating SIJ dysfunction. One such widely used model depends on identifying asymmetry of pelvic bony landmarks in order to determine a positional fault or misalignment. Based on identified pelvic positional faults treatment is directed towards correcting the misalignment and achieving positional symmetry.

Aim: To present research findings which bring into question the continued use of this treatment method and challenge physiotherapist to adopt a more evidence informed practice when treating SIJ dysfunction.

Results: A review of research questions the reliability of using bony landmarks to determine pelvic asymmetry and also brings into question the link between pelvic asymmetry and SIJ dysfunction. It would appear that bony landmarks are not reliable in determining asymmetry and that so-called asymmetry is unrelated to SIl dysfunction and hence there is low validity in using this approach as a clinical therapeutic model.

Conclusions: Evidence informed practice questions the reliability and validity of using this method and questions the continued use of positional misalignment as a way of identifying SIJ dysfunction. In the absence of any substantiating evidence it would seem surprising that the method of positional faults based on bony palpations continues to be used by physiotherapists.

\section{Key Practice Points:}

- Lack of evidence of pelvic asymmetry associated with SIJ dysfunction - Question the reliability and validity of pelvic bony landmark assessment - Challenge the 'Osteopathic' conceptual model of SIJ dysfunction

\section{HOW TO APPLY MUSCLE ENERGY TECHNIQUE (MET) FOR TREATING CERVICAL DYSFUNCTION}

\section{Lambridis $\mathrm{T}^{1}$, McGovern $\mathrm{B}^{2}$}

${ }^{1}$ Clinic Director, Spinal Synergy Physiotherapy, Cremorne, Sydney ¿Senior Practice Clinician, Spinal Synergy Physiotherapy, Cremorne, Sydney

Background: The practical application of Muscle Energy Technique (MET) for the cervical spine

Aims / objectives: Present instruction on applying Muscle Energy Technique (MET) for assessment and treatment of cervical spine presentations. Delegates will be given a brief overview and historical background of this method with specific details of how restricted joint segments are identified, naming of the diagnosis and the application of this method. The aim is to provide delegates with the practical skills needed to safely apply Muscle Energy technique to the cervical spine.

Approach: Power Point slides, practical demonstration and course handout. The instructive portion covers brief overview of MET naming of restricted joint segments based on Osteopathic principles and the theory of spinal coupling motion. Delegates will be provided with printed handout to also include safe clinical practice guidelines and references applicable to MET. The practical session will involve a patient/volunteer demonstration followed by a group participation of this method. Delegates will have the opportunity to practice MET of the cervical spine while instruction is provided by the two presenters. The aim would be to have a sufficient number of portable plinths to allow delegates to work in pairs to practice using MET. (Alternative: use of exercise mats and pillows on the floor).

\section{Conclusion / Key Practice Points:}

- Understand the principles of Muscle Energy Technique (MET)

- How to safely apply MET to the cervical spine

- Provide instruction on safe use of manual therapy for treating cervical dysfunction

\section{AMBULATORY ACTIVITY IN PEOPLE WITH PARKINSON'S DISEASE}

Lamont RM ${ }^{1,2}$, Morris $\mathrm{ME}^{3}$, Woollacott $\mathrm{MH}^{4}$, Brauer SG

${ }^{1}$ School of Health and Rehabilitation Sciences, University of Queensland ${ }^{2}$ Asia-Pacific Centre for Neuromodulation, Centre for Clinical Research,

University of Queensland

${ }^{3}$ School of Allied Health, LaTrobe University

${ }^{4}$ Institute of Neuroscience, Department of Human Physiology, University of Oregon

Question: What personal and disease-related factors are associated with participation in moderate and high intensity daily ambulatory activity in people with mild to moderate Parkinson disease (PD)?

Design: Cross-sectional, observational study

Participants: Fifty community-dwelling people with mild-moderate PD.

Outcome Measures: The dependent variable was average daily time spent performing moderate intensity ambulatory activity measured over three days using the ActivPAL activity monitor. Explanatory variables including personal factors, disease characteristics, gait and cognitive capacity were measured.

Results: People with PD spent an average of 77 minutes walking per day, mostly at a moderate intensity resulting in a median of 6300 steps/day. Disease severity predicted time spent in moderate ambulation bouts $\left(\mathrm{R}^{2}\right.$ $=0.116, p=0.017$ ). Gait (Timed Up and Go Test) and executive function together predicted engagement in high intensity ambulatory activity $\left(R^{2}>\right.$ $0.170, p<0.022$ ).

Conclusion: People with mild to moderate PD are able meet physical activity guidelines with respect to their levels of moderate intensity ambulatory activity. While disease severity, gait performance and executive function were predictive of engagement in moderate and high intensity walking activity, additional personal and social factors should be considered and are likely to also strongly impact on activity levels.

Key Practice Points:

- People with mild to moderate PD are capable of achieving physical activity guidelines with respect to their levels of moderate intensity ambulatory activity

- Level of disease severity, gait and executive function capacity should be considered when attempting to increase or maintain levels of ambulatory activity

- Additional person and social factors also need to be considered.

\section{CONNECTING THE PAEDIATRIC THERAPEUTIC MANAGEMENT OF CONCURRENT AUTOSOMAL RECESSIVE CONDITIONS, MOROUIOS (MPS 4A) AND CYSTIC FIBROSIS (CF)}

\section{Lazzarin $\mathrm{T}^{1}$}

${ }^{1}$ Lady Cilento Children's Hospital, Brisbane

Questions: How do we optimise respiratory function and physical activity within the limitations of skeletal deformity and frequent orthopaedic surgery? Will participation in Morquios clinical trial positively affect mobility and respiratory function?

Design: Retrospective case study

Participant: 12 year old with Morquios (with widespread skeleta abnormalities, restrictive lung disease, and limited exercise tolerance) and moderate obstructive CF lung disease with bronchiectasis

Outcome Measures: Pre and post Morquios trial audit of hospita admissions, physiotherapy interventions, lung function (FEV1, FVC) and exercise capacity (6MWD)

Results: Following 10 hospital admissions in 11 years ( 5 for major orthopaedic surgery) patient maintained an ability to mobilise short distances without an aid (6MWD 186m) and FEV1 was maintained (over 80\%). After involvement in Morquios trial patient has maintained FEV1 and FVC and increased 6MWD to 251m, physical ability (can jump two feet for the first time) and quality of life (can attend school for 3.5days/wk).

Conclusion: Prior to the Morquios clinical trial, physiotherapy management goals included optimisation of respiratory function, mobility and exercise capacity. With the ongoing drug trial, if mobility and exercise capacity improves, should there be a rehabilitation focus, especially in light of ongoing orthopaedic surgery?

\section{Key Practice Points:}

- In the presence of complex co-morbidities, individualisation and adaptation of interventions is essential

- Outcome measures require individualisation to demonstrate change in this unique presentation.

- A coordinated and proactive approach is needed for musculoskeletal and respiratory interventions prior to and after orthopaedic surgery. 


\section{PATIENT-REPORTED OUTCOME QUESTIONNAIRES FOR WHOLE SPINE DYSFUNCTION - A SYSTEMATIC REVIEW}

Leahy $\mathrm{E}^{1,2}$, Davidson $\mathrm{M}^{1}$, Benjamin $\mathrm{D}^{1}$, Wajswelner $\mathrm{W}^{1}$

${ }^{1}$ La Trobe University, Melbourne

${ }^{2}$ Northern Health, Melbourne

Question: Which patient-reported outcome questionnaires should be used to evaluate the function of people with pain in any area of the spine?

Design: Systematic review.

Participants: Included patient-reported outcome questionnaires evaluated dysfunction of all spinal regions. Only original studies that evaluated the measurement properties of these questionnaires were included.

Outcome Measures: Internal consistency, reliability, measurement error content validity, construct validity and responsiveness.

Results: Fifty-nine questionnaires were screened for inclusion by 2 reviewers. Two reviewers independently screened 238 studies for eligibility, performed data extraction and evaluated the methodological quality of included studies. Three questionnaires fulfilled the inclusion criteria and 17 articles evaluated their measurement properties. The Functional Rating Index was most commonly evaluated, with positive results for 4 measurement properties, a negative result for measurement error and conflicting results for reliability. The Extended Aberdeen Back Pain Scale had negative results for 2 measurement properties and the Spinal Functional Index had positive results for 5 measurement properties.

Conclusion: The Functional Rating Index is provisionally recommended as the most psychometrically sound questionnaire when a whole of spine functional assessment is required. A limitation of this review is the pooling of results from mainly fair quality studies with differing subject samples with regards to language spoken and areas of spine pain

\section{Key Practice Points:}

- Three questionnaires exist for functional assessment of patients with pain in any spine area.

- Functional Rating Index is provisionally recommended for whole of spine functional assessment.

- There is a paucity of high quality research in this area.

\section{GLOBAL LEARNING PARTNERSHIP: AN INTERCULTURAL AND INTERDISCIPLINARY GLOBAL CLINICAL PLACEMENT}

Lees $]^{1,2}$, Remedios $L^{1,2}$, Webb $G^{1,2}$, Coulston F ${ }^{1}$, Smart $A^{1}$

${ }^{1}$ University of Melbourne, Melbourne

¿Universitas 21 Health Sciences UNMDG Group, Canada

Primary Objective: To evaluate the effectiveness of a new model for a global clinical placement that fosters a cooperative relationship between students and academics from Universitas 21 network to promote working toward the achievement of the United Nations Millennium Development Goals.

Design: A pilot group of 15 physiotherapy students from Kathmandu University, and 15 students representing 6 health disciplines and 8 U21 universities participated in a shared learning experience in Nepal. The clinical placement including a one week university based workshop on leadership and need analysis theory and a three week community based experience.

Outcome Measures: A multi-phase, mixed method design was selected for the evaluation of the GLP model, and utilises a combination of focus groups and questionnaires to assess cultural competency and UNMDG learning outcomes.

Results: Focus group data gathered from students and academics, pre and post questionnaire data gathered from students in July, 2015 and project evaluation data will be provided in the presentation.

Conclusion: The GLP model proposes a project around the fundamental concept of engagement and sharing between students and academics across universities and cultural contexts to build capacity through education, while capitalising on strengths of existing global health placements, and by focusing on the inclusion of host-country students and academics in this international learning exchange.

\section{Key Practice Points:}

- Collaborations between academics from Universities from developed, research intensive universities and Universities from developing countries are essential to promoting global health.

- Global clinical placement models should be designed to reduce the risks of paternal voyeurism which have only temporary benefits for host communities and visiting students.
HEAD ORIENTATION PREFERENCE AND ITS ASSOCIATION WITH POSITIONAL PLAGIOCEPHALY IN YOUNG HEALTHY INFANTS

Leung $\mathrm{A}^{1,2}$, Mandrusiak $\mathrm{A}^{1}$, Johnston LM ${ }^{1}$

${ }^{1}$ School of Health \& Rehabilitation Sciences, The University of Queensland, Brisbane

${ }^{2}$ Child Development Service, Children's Health Queensland Hospital and Health Service, Brisbane

Questions: How does head orientation preference change with age in healthy infants? Is orientation preference or child positioning associated with positional plagiocephaly?

Design: Prospective observational study.

Participants: Ninety-four healthy, full term infants aged three weeks, recruited from child health clinics, antenatal clinics and the general community

Outcome Measures: Head orientation preference (duration and timeto-turn from right, left or midline positions) and child positioning were measured at age three, six and nine weeks. Positional plagiocephaly was examined at nine weeks using the modified Cranial Vault Asymmetry Index.

Results: Lateral orientation preference predominated at three weeks (right=39.98 $\pm 21.73 \%$; left=40.59 $\pm 21.45 \%$; midline=19.33 $\pm 19.85 \%$ ) Lateral orientation decreased bilaterally in favour of midline positioning at nine weeks (right $=29.48 \pm 22.25 \%$; left $=24.15 \pm 22.04 \%$; midline $=46.1 \pm 27.02 \%$; right, $p=0.002$; left and midline, $p<0.001$ )

Greater orientation symmetry at nine weeks was associated with parents performing active counter-positioning at three weeks $(p=0.05)$. Positional plagiocephaly at nine weeks was not associated with orientation preference at any age. Instead, it was associated with an increase in the combined time spent in supine when awake or asleep at six weeks $(p<0.001)$

Conclusion: Healthy infants show progressive change from symmetrical lateral head orientation to midline orientation from three to nine weeks. Time spent in supine significantly contributes to development of positional plagiocephaly.

\section{Key Practice Points:}

- Typical infants show lateral head orientation under the age of nine weeks.

- Early active counter-positioning is associated with greater head orientation symmetry and absence of plagiocephaly at later age. - Non-supine positioning should be encouraged.

\section{HOW TO ASSESS PLAGIOCEPHALIC HEAD} SHAPE IN INFANTS?

Leung $\mathrm{A}^{1,2}$, Mandrusiak $\mathrm{A}^{1}$, Johnston LM ${ }^{1}$

${ }^{1}$ School of Health \& Rehabilitation Sciences, The University of Queensland, Brisbane

${ }^{2}$ Child Development Service, Children's Health Queensland Hospital and Health Service, Brisbane

Background: Infants with asymmetrical head shape are often referred to paediatric physiotherapy services. A common approach in busy clinics is observation of the head shape. It is important for the physiotherapist to systematically assess head shape to identify possible causes and severity of the asymmetry. An efficient and economical tool to measure head asymmetry is paramount to guide clinical management.

Aims / objectives: The aims of this session are to outline the differential diagnosis of positional versus synostotic causes of head asymmetry, and to introduce and demonstrate an objective measurement tool (modified Cranial Asymmetry Index) to measure head shape asymmetry. A paper on this measurement tool was published by the presenter in 2013. The participants should understand the different presentations of positional and synostotic causes of head asymmetry and the rationale, procedure, technique and application of the measurement tool.

Approach: PowerPoint presentation for theories, video to demonstrate techniques of measuring the modified Cranial Asymmetry Index and hands on practice for participants. Handouts of the PowerPoint will be provided.

Conclusion / Key Practice Points: It is expected that on completion of this session, participants will be able to:

- Increase accuracy in assessment of infants with asymmetrical head shape

- Learn a measurement tool that is efficient and economical to apply in clinics and home environments

- Use an objective measure to monitor progress of head asymmetry and guide clinical management

- Have more confidence in providing best practice to this population. 


\section{CANINE AND EQUINE RESEARCH UPDATE}

\section{Levine $\mathrm{D}^{1,2}$}

${ }^{1}$ The University of Tennessee, Chattanooga

¿University of Tennessee College of Veterinary Medicine

During this session, David Levine PhD, will spend half an hour each on the latest research from around the world in the fields of canine and equine physiotherapy. David is the research committee chair for the International Association of Veterinary Rehabilitation and Physical Therapy and so has his finger on the pulse of the latest work by postgraduate students and research centres in the US, South Africa, Europe and the United Kingdom. He will present a review of the current themes of research in each species, as well as discuss future trends - what ss on the horizon in the world of animal physiotherapy? Dr Levine will also discuss resources and technology and give a 5 minute strategy to keep up with any new research in our field, every week - for free!

\section{Key Practice Points:}

- Learn what is the latest in equine and canine research

- Know where animal physio trends are heading

- A 5 minute weekly strategy to keep up to date with unfolding research

\section{ANIMAL ASSISTED THERAPY}

\section{Levine $\mathrm{D}^{1,2}$}

IProfessor of Physical Therapy, The University of Tennessee, Chattanooga ${ }^{2}$ Adjunct Professor, University of Tennessee College of Veterinary Medicine Animal Assisted Therapy (AAT) is a goal-directed intervention directed and/or delivered by a health (human) service professional with specialized expertise, and within the scope of practice of his/her profession. AAT is designed to promote improvement in human physical, social, emotional, and/or cognitive functioning. This lecture will provide an introduction to AAT with an emphasis on physiotherapy. Participants will become familiar with terminology, organizations and certification processes, research supporting AAT, and AAT specifically in use in physiotherapy. Specifically to our stream of animal physiotherapy, we will consider the 'full circle' of AAT. Most people have heard of hippotherapy and seen the research showing that interactions with dogs can help reduce human blood pressure and improve mood. So we will focus on the use of dogs in physiotherapy, as well as the effect of this work on the dog, describing ongoing research into dogs' cortisol levels while performing their duties. We will also watch videos of dogs assisting in physiotherapy for humans with neurological impairments, for example, and workshop the physical implications of this work on the dog and how animal physiotherapists may be able to help keep these dogs comfortably doing their duties for longer. The lecture will conclude with advice for any physios keen to get started in this field.

\section{Key Practice Points:}

- Understand what animal assisted therapy is and it's terminology

- Learn how to become involved in animal assisted therapy

- Review the literature on animal assisted therapy for various diagnoses

\section{SMALL STEPS: EFFECTIVENESS AND FEASIBILITY OF AN INCREMENTAL GOAL- SETTING INTERVENTION TO REDUCE SITTING TIME IN OLDER ADULTS}

Lewis LK ${ }^{1}$, Rowlands $A^{1}$, Gardiner $\mathrm{P}^{2}$, Standage $\mathrm{M}^{3}$, English $\mathrm{C}^{1}$, Olds $\mathrm{T}^{1}$

${ }^{1}$ Alliance for Research in Exercise, Nutrition and Activity (ARENA), Sansom Institute for Health Research, University of South Australia, Adelaide 2The University of Queensland, School of Public Health, Faculty of Medicine and Biomedical Sciences, Brisbane

${ }^{3}$ Department for Health, University of Bath, Bath, United Kingdom

Question: What is the effectiveness and feasibility of a program to reduce sitting time in older adults?

Design: Pre-post study

Participants: Thirty non-working adults ( $\geq 60$ years) were recruited.

Intervention: Participants attended a one hour face-to-face session and were guided through: a review of their sitting time; normative feedback on sitting time; and guided goal setting to reduce both total sitting time and bouts of prolonged sitting. Participants chose six goals and integrated one per week incrementally for six weeks.

Outcome Measures: Sitting time and bouts of prolonged sitting (>30 minutes) were measured objectively for seven days (activPAL3c inclinometer) pre and post-intervention. During these periods, a 24-hour time recall instrument was administered by computer-assisted telephone interview.

Results: Twenty-seven participants completed the assessments (71.7 \pm 6.5 years). Post-intervention, objectively measured total sitting time was significantly reduced by 51.5 minutes $(p=0.006)$ and bouts of prolonged sitting by 0.8 per day $(p=0.002)$. Participants spent 34 minutes less per day watching a screen $(p=0.004)$ and engaged in more light $(p=0.01)$ and moderate $(p=0.02)$ physical activity. Participants were highly satisfied with the program.

Conclusion: The 'Small Steps' program is a feasible and promising avenue for behavioural modification to reduce sitting time in older adults.

Key Practice Points:

- Sitting time has been linked to adverse health outcomes and mortality.

- This study evaluated a goal-setting intervention to reduce sitting time in older adults.

- The findings may inform a larger clinical trial of an intervention to reduce sitting time.

\section{DIAGNOSED WITH TRIGEMINAL}

NEURALGIA, DESTINED FOR PAIN AND SUFFERING FOR LIFE: WHAT SHOULD I DO AND HOW? A SINGLE-CASE STUDY

\section{Lim M}

Maxvale Physiotherapy

Introduction: Physiotherapists have been well-trained in terms of diagnosing and managing physical and emotional aspects of the patient. However, there lies a missing link to ensure successful treatment outcome and prevention of recurrences for patients who have frequent relapses of their presenting problem, faulty beliefs and negative emotional baggage. This case study will illustrate this missing link and the 'soft skills' in physiotherapists to complete the biopsychosocial management of a patient.

Purpose: 1) Illustrating the relationship of emotional drivers and synaesthetic experiences in a lady diagnosed with trigeminal neuralgia and how and why relapses occurs.

2) Utilisation of the biopsychosocial approach, manual therapy, exercise and neuro-linguistic programming to let go of the major emotional baggage inhibiting successful recovery.

3) Algorithmic prescription of exercise programs, coping strategies, relaxation techniques and prevention of relapses and dis-eases.

Methods: 62 year old lady diagnosed with trigeminal neuralgia for four years and features of central processing dysfunction and synaesthetic experiences was managed with manual therapy, exercise and neurolinguistic programming over six physiotherapy sessions. During the process, learning and decision-making strategies, beliefs, values and emotional-behavioural responses were elicited. Physical and cognitive emotional states of the patient was matched and incorporated into the management plan. Manual therapy, exercise programs, coping strategies and relaxation techniques were anchored with positive emotional states. Patient was discharged with a list of prevention and coping strategies.

Results: There was a complete resolution of pain and synaesthetic experiences, decrease in medication use, complete recovery of function and change in mindset when the biopsychosocial approach incorporating neuro-linguistic programming was taken in the management of the patient.

Conclusion: A successful clinical outcome was achieved utilising a biopsychosocial approach incorporating neuro-linguistic programming in the management of a previously diagnosed trigeminal neuralgia patient with no hope. 


\section{EFFECTS OF DIFFERENT SITTING POSTURES ON GLUTEAL PRESSURE AND PELVIC INCLINATION ANGLE DURING COMPUTER USE}

$\operatorname{Lim} \mathrm{OB}^{1}$, Han $\mathrm{HR}^{1}, \mathrm{Kim} \mathrm{YH}^{2}, \mathrm{YiCH}^{3}$

${ }^{1}$ Dept, of Physical Therapy, The Graduate School, Yonsei University, Wonju, Republic of Korea

${ }^{2}$ Dept. of Biomedical Engineering, College of Health Science, Yonsei University, Wonju, Republic of Korea

${ }^{3}$ Dept. of Physical Therapy, College of Health Science, Yonsei University, Wonju, Republic of Korea

Question: Do four different sitting postures affect gluteal pressure and pelvic inclination angle during computer use?

Design: Randomised, within-participants experimental study

Participants: Twenty-nine healthy adults

Methods: Participants sat in four different postures: (A) with legs uncrossed and both feet on the floor, (B) with legs crossed so that the right knee was over the left knee, (C) with legs crossed so that the right ankle was on the left knee, and (D) with the right ankle crossed over the left ankle.

Outcome Measures: A Tekscan system measured gluteal pressure, and a palpation meter measured the pelvic inclination angle. One-way repeated ANOVA with Bonferroni's post-hoc test was used to compare uncrossed and cross-legged sitting postures

Results: In posture $B$, right gluteal pressure significantly increased compared to $A(p=0.001)$ and was lower in $D$ than in $A(p<0.001)$. Left gluteal pressure significantly decreased in posture B compared to $A$ $(p<0.001)$ and was lower in $C$ than in $A(p<0.001)$. The right pelvic inclination angle was significantly greater in posture $A$ compared to the others $(B, C, D$ : $p<0.001)$. The left pelvic inclination angle was significantly lower in posture A compared to the others (B, C, D: $p<0.001)$

Conclusion: The findings suggest that prolonged cross-legged sittings may cause different gluteal pressure. Also, deformation of pelvic inclination may alter body mechanics and increase strain of soft tissues, consequently cause musculoskeletal pain.

\section{Key Practice Points:}

- Cross-legged sitting may be a detrimental position for workers.

- Workers need a well-informed sitting posture.

- Workers should take frequent breaks.

\section{EXERCISE INTERVENTIONS FOR PATIENTS WITH GYNAECOLOGICAL CANCER: A SYSTEMATIC REVIEW AND META-ANALYSIS}

Lin $\mathrm{K}-\mathbf{Y}^{1}$, Frawley $\mathrm{HC}^{2,3}$, Denehy $\mathrm{L}^{1}$, Feil $\mathrm{D}^{3}$, Granger $\mathrm{CL}^{1,4}$ ${ }^{1}$ Department of Physiotherapy, School of Health Sciences, The University of Melbourne, Melbourne

2Physiotherapy, School of Allied Health, La Trobe University, Melbourne ${ }^{3}$ Cabrini Health, Melbourne

${ }^{4}$ Department of Physiotherapy, Melbourne Health, Melbourne

Question: Are exercise interventions effective for patients with gynaecological cancer?

Design: A systematic review and meta-analysis of randomised controlled trials.

Participants: Patients diagnosed with gynaecological cancer. Intervention: Exercise interventions or education regarding exercise.

Outcome Measures: Functional exercise capacity, health-related quality of life, physical activity levels, muscle strength, fatigue, body composition, and depressive symptoms. Pooled data presented as weighted mean differences (MD).

Results: A total of seven randomized controlled trials involving 221 participants were included. Meta-analysis demonstrated that, compared to control groups, the exercise intervention groups showed significantly greater improvements in physical activity levels $(\mathrm{MD}=12.92: 95 \% \mathrm{Cl} 6.34$ to 19.51: $<$ 0.001) and body mass index (MD $=-1.57: 95 \% \mathrm{Cl}-2.32$ to -0.82 $p<0.001)$. No significant effects were found for fatigue, depression, and health-related quality of life. A meta-analysis of functional exercise capacity and muscle strength was not possible due to insufficient data in the included trials.

Conclusion: Exercise intervention for patients with gynaecological cancer appears to be effective at improving physical activity levels and body mass index. The majority of studies provided exercise intervention as a part of the multi-component intervention programs. Therefore, further research is required to examine the effects of exercise intervention alone in this population.

\section{Key Practice Points:}

- Exercise interventions may be effective in improving physical activity levels and body mass index in patients with gynaecological cancer

- Health care professionals may provide some form of exercise

intervention to this population.

- The optimal exercise type and dosage remain unknown.

\section{THE USE OF GPS IN REHABILITATION - A CASE STUDY}

\section{Lion $\mathrm{P} 123$}

${ }^{1}$ APA Sports Physiotherapist

${ }^{2}$ Brisbane Lions Australian Football Club, Brisbane, Queensland ${ }^{3}$ Queensland Sports Medicine Centre, Brisbane, Queensland

GPS can be integrated into the return to play programming of injured athletes. It can be used as part of the decision making picture in ensuring athletes have trained at the appropriate load and intensity to return to the training and competitive environment.

This presentation will use the return to play of an elite AFL player to highlight the possible clinical uses of GPS technology in this process.

\section{Key Practice Points:}

- A clinical application of how clinicians can use technology to connect to the athlete in the sporting environment.

\section{WHOSE CLASS IS IT ANYWAY?}

\section{STAKEHOLDER FEEDBACK FROM A FALLS REHABILITATION PROGRAM}

Liston $\mathbf{M}^{1,2,3}$, Borghesi $\mathrm{M}^{4}$, Kennedy $\mathrm{B}^{4}$

${ }^{1}$ School of Science and health, University of Western Sydney, Sydney 2Faculty of Health, Social Care and Education, St George's University of London, London

${ }^{3}$ Centre of Human and Aerospace Physiological Sciences, Kings College London, London

4St George's University Hospitals NHS Foundation Trust, London

Question: What are the perceptions of older adult fallers in relation to how a Falls Rehabilitation Programme meets their needs?

Design: Qualitative focus group study and thematic analysis.

Participants: Seventeen clients (aged 60-80 years) attending Physiotherapy led falls rehabilitation classes in London, UK.

Outcome Measures: Focus Group data that has been transcribed verbatim and analysed using thematic content analysis.

Results: A disconnect between individual's perceived specific needs and anxieties, and their understanding of the purpose of generalised exercises offered in the classes, emerged as the major theme. Participants reported concerns related to functional balance activities such as standing in the shower and crossing the road as being of most importance to them, but perceived a lack of patient choice and customisation in the programme design for attending to these. Participants valued how the class had improved physical function and how the peer group structure benefitted their psychological and social wellbeing.

Conclusion: Group classes are valued by participants but Falls

Rehabilitation Programmes require flexibility to tailor them to meet individual needs, as well as ensure that the rationale for exercises is contextually meaningful. Programmes particularly need to address anxieties related to outdoor mobility.

\section{Key Practice Points}

- Falls rehabilitation classes need to be customisable.

- Providers need to ensure that class participants can identify how prescribed exercises are addressing their specific anxieties and needs. 


\section{OUTCOMES OF PHYSIOTHERAPY MANAGEMENT OF PELVIC MUSCLE PAIN: RESULTS OF A PRACTICE AUDIT}

Locke L ${ }^{1}$, Neumann $\mathrm{P}^{2}$, Thompson $]^{1}$, Briffa $\mathrm{N}^{1}$

${ }^{1}$ Curtin University, Perth

IInternational Centre for Allied Health Evidence, University of South Australia, Adelaide

Question: What are the outcomes of biopsychosocial physiotherapy management for women with persistent pelvic floor muscle pain?

Design: Clinical practice audit.

Participants: Consenting women with pelvic floor muscle pain, referred by a gynaecologist/pain physician within a multidisciplinary pelvic pain clinic.

Intervention: a course of psychologically informed pelvic floor physiotherapy incorporating pain science education.

Outcome Measures: Visual Analogue Scales for 10 aspects of pain, Pelvic Pain Screening Questionnaire, Pelvic Floor Bother Questionnaire, Female Sexual Function Index, Patient Global Impression of Improvement. Baseline screening: The Depression, Anxiety and Stress Scale.

Results: Of 42 consenting women, 11 completed treatment and provided complete data sets. Participants reported moderate levels of psychosocial dysfunction at baseline. Following treatment, there were clinically and statistically relevant changes in Visual Analogue Scales for pain, statistically significant changes were found for the Pelvic Pain Screening Questionnaire ( $p=.011$ ) and the Pelvic Floor Bother Questionnaire ( $p$ $=.012$ ) but not the Female Sexual Function Index ( $p=.722) .100 \%$ of participants improved on the Patient Global Impression of Improvement, with $72 \%$ "very much better" or "much better".

Conclusion: Audit results support a biopsychosocial approach for the physiotherapy management of persistent pelvic muscle pain in women.

\section{Key Practice Points:}

- This psychologically informed approach to the physiotherapy management of pelvic floor muscle pain, incorporating pain science education, shows promise

- Psychosocial screening may help to personalise interventions and stratify patients

- More robust studies are needed to explore the most beneficial aspects of treatment

\section{EFFECTS OF ACUPUNCTURE AND MINDFULNESS ON THE LIMBIC AND PREFRONTAL CORTEX: A WORKING HYPOTHESIS FOR PAIN AND ANXIETY MANAGEMENT}

\section{Longbottom J}

University of Hertfordshire, UK

Within the field of chronic and /or perceptual pain within the UK, physiotherapists are increasingly required to assess and manage complex pain, anxiety and depression presentations within restricted time constraints and with increasing emphasis of patient self-management. This presentation will address the underlying patho-physiology of anxiety and depression, together with acupuncture interventions and the subsequent effects on the Limbic and Pre-frontal cortex in patients presenting with complex pain states.

\section{Key Practice Points:}

This presentation will offer delegates:

- A theoretical model underpinning of the patho-physiological changes within the Limbic and Pre-frontal cortex contributing to;

- Anxiety

- General depression

- Pain.

- A theoretical model underpinning of physiological process during depression with relevance to the sympathetic and parasympathetic systems.

- Integration of acupuncture with CBT/ Mindfulness as a clinically reasoned treatment plan

- Discussion of recent research evidence to support the development of clinical protocols to facilitate the management of complex pain presentations and facilitate patient self management

\section{ACUPUNCTURE FOR OVERACTIVE BLADDER SYNDROME (OABS): COST- EFFECTIVE AND CLINICALLY EFFECTIVE}

\section{Longbottom ]}

Lecturer University of Hertfordshire, UK

Current UK quidelines from the National Institute for Clinical Excellence (NICE 2014) support the use of Percutaneous Tibial Nerve Stimulation (PTNS) for "Women" presenting with OABS. This intervention costs the UK NHS more than 1 million pounds per year and requires extensive training for nurses; extensive treatment interventions (12-18), followed by six monthly "top-up." Acupuncture and TENS have an equal action on OABS when used by physiotherapist offering a cost effective and patient centred intervention... to both men and women.

\section{Key Practice Points:}

This presentation will offer delegates;

- A critical overview of the recent research to support the use of acupuncture in OABS

- Provide evidence from pilot study research for the use of acupuncture (initially) and TENS (home management) as an integrated service for the management of OABS

- Offer an effective argument for the inclusion of acupuncture as a cost effective, clinically effective intervention, enhancing physiotherapy management in bladder retraining programmes

- Provide delegates with clinical skills for a future multi-centre RCT comparing PTNS, acupuncture and TENS

\section{PROXIMAL HAMSTRING TENDINOPATHY: A CASE REPORT}

\section{Low A}

Ascend Physiotherapy, Curtin University

Tendinopathy (pain and pathology within a tendon) is prevalent amongst athletes. It has been reported 30 - $50 \%$ of all sports related injuries are due to overuse and increased athletic load may be a factor in the development of an overuse tendinopathy. Once a tendon becomes painful there is a reduction in the athlete's ability to utilise the energy storage capacity within the tendon, thereby compromising function and performance. Excessive load may come in the form of stretch shortening cycle loading and tensile stress. More recently there has been increased awareness of the deleterious effect of compressive load on the undersurface of tendons particularly at their insertion. Compression of a tendon results in alteration of the structure and function of the extracellullar matrix and these changes appear to be implicated in pathology. Animal models show greater tendon injury when overload is combined with compressive forces. Proximal hamstring tendinopathy (PHT) is a common injury amongst distance runners and athletes performing either saggital plane activities (such as sprinters/hurdlers) or changes of direction activities. Hockey players not only perform multiple short sprints and changes of direction, but they also frequently reach down and forward with their sticks moving into increased trunk and hip flexion to gain control of the ball. Hockey is a game always played with the stick in the right hand, therefore the left hip is often placed more frequently into positions of greater than 90 degrees flexion during skills such as slap hitting or a tomahawk. These positions theoretically increase the compression of the proximal hamstring insertion/enthesis against the ischial tuberosity. Reactive on degenerative tendinopathy of the proximal hamstring tendon (PHT) can be easily confused with other conditions. The purpose of this case report is to describe a hockey player presenting with pain in the proximal hamstring region during a high pressure finals and selection campaign. An initial hypothesis of fatigue induced muscle disorder was made, but with continued loading the diagnosis of reactive on degenerative proximal hamstring tendinopathy became apparent.

\section{Key Practice Points:}

- Discussion on current thoughts on pathophysiology of tendinopathy, including both tensile and compressive loads

- Discussion on loading of the proximal hamstring tendon in a hockey player

- Discussion on managing a talented junior athlete with multiple coaches and conflicting periodisation schedules 


\section{FACE VALIDITY OF A UNIQUE PATIENT FOCUSSED SYMPTOMS SEVERITY INDEX FOR ASSESSING INFLAMMATORY CONDITIONS OF THE LACTATING BREAST}

Cooper $\mathrm{M}^{1}$, Lowe $\mathrm{H}^{2}$, Garth $\mathrm{B}^{3}$, McArdle $\mathrm{A}^{4}$

IInForm Physiotherapy formerly Marshall Street Practice

${ }^{2}$ Through Life Physiotherapy

${ }^{3}$ Southern GP Training and Melbourne University

${ }^{4}$ Monash University

Question: Is this unique patient focussed symptoms severity index appropriate to measure the primary symptoms when assessing inflammatory conditions of the lactating breast

Design: Assess face validity using the Delphi method

Participants: This study involved three stages, two online questionnaires and one focus group. For the first and second questionnaires 29 and 22 patients, 23 clinicians (physiotherapists or lactation consultants) and five and four academics from related research fields respectively participated. The focus group comprised two patients, three clinicians and two academic facilitators

Intervention: A patient focussed symptoms severity index used for assessing inflammatory conditions of the lactating breast. The symptom domains in the tool included nociception, erythema, size, tension, local temperature and sickness.

Outcome Measures: This Delphi method aimed at reaching a consensus about the face validity of each symptom domain

Results: Consensus was achieved with $95 \%$ of participants agreeing that the index was appropriate for asking patients about their primary symptoms associated with inflammatory conditions of the lactating breast. Minor changes were made to the language and administrative instructions to facilitate the tool's application in clinical settings.

Conclusion: Patient focussed outcome measures are increasingly important to support therapeutic processes. This unique symptom severity index has strong face validity, which appropriately assesses the primary inflammatory symptoms of the lactating breast when used as a patient focussed outcome measure.

\section{Key Practice Points:}

- Quickly and comprehensively assesses and measures change of primary symptoms

- Facilitates further validity and reliability investigation

- Can be used in clinical practice and research

\section{THE CLINICAL COURSE OF PATIENTS} WITH SCIATICA TREATED SURGICALLY: A SYSTEMATIC REVIEW WITH METAANALYSIS OF COHORT STUDIES

Machado $\mathrm{GC}^{1}$, Witzleb AJ ${ }^{1}$, Fritsch $\mathrm{C}^{2}$, Maher $\mathrm{CG}^{1}$, Ferreira $\mathrm{PH}^{3}$, Ferreira $\mathrm{ML}^{1,4}$

${ }^{1}$ The George Institute for Global Health, Sydney Medical School, University of Sydney, Sydney

2Department of Physiotherapy, Universidade Federal de Ciências da Saúde de Porto Alegre, Porto Alegre, RS, Brazil

${ }^{3}$ Discipline of Physiotherapy, Faculty of Health Sciences, University of Sydney, Sydney

${ }^{4}$ Institute of Bone and Joint Research, The Kolling Institute, University of Sydney, Sydney

Question: What is the course of surgery in terms of pain and disability for patients with sciatica?

Design: We conducted a systematic review with meta-analysis

MEDLINE, EMBASE and CINAHL were searched from inception to April 2015. We screened for prospective cohort studies investigating pain or disability outcomes.

Participants: Patients with sciatica.

Intervention: Surgery.

Outcome Measures: Means and standard deviations for pain and disability were extracted and converted to a 0 to 100 scale. Fractional polynomia regression analysis was used to generate pooled estimates across 5 years after surgery.

Results: We included 40 records including a total of 13,883 patients with sciatica. Before surgery pooled leg pain score was 75.2 (95\% CI 68.1 to 82.4) and patients experienced large improvements after 3 months (15.3, $95 \% \mathrm{Cl} 8.5$ to 22.1). Patients never fully recovered in the long-term and pain intensity increased to $21.0(95 \% \mathrm{Cl} 12.5$ to 29.5$)$ at 5 years. The pooled estimate for disability before surgery was 51.7 (95\% Cl 49.7 to 53.7 ) and this decreased to 15.2 ( $95 \% \mathrm{Cl} 13.4$ to 17.0 ) at 3 moths and further reduced to 11.4 ( $95 \% \mathrm{Cl} 9.4$ to 13.5$)$ at 5 years.
Conclusion: Patients still experience mild/moderate pain intensity 5 years after surgery.

\section{Key Practice Points:}

- Physiotherapists should be aware of the prognosis of surgery for sciatica

- Most patients have a substantial improvement in the first 3 months, however, patients never seem to fully recover after surgery

- The course of surgery for sciatica is not as favorable as previously suggested by clinical trials

\section{TRIGGERS FOR AN EPISODE OF PERSISTENT LOW BACK PAIN: 12-MONTH FOLLOW-UP OF A CASE- CROSSOVER STUDY}

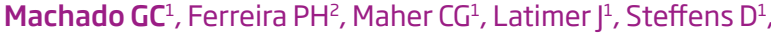
Koes BW ${ }^{3}$, Li ${ }^{1}$, Ferreira $\mathrm{ML}^{1,4}$

${ }^{1}$ The George Institute for Global Health, Sydney Medical School, University of Sydney, Sydney

${ }^{2}$ Faculty of Health Sciences, University of Sydney, Sydney

${ }^{3}$ Department of General Practice, Erasmus Medical Centre, Rotterdam, the Netherlands

${ }^{4}$ Institute of Bone and Joint Research, The Kolling Institute, Sydney Medical School, University of Sydney, Sydney

Question: Is transient physical and psychosocial activities associated with the risk of developing persistent low back pain?

Design: Case cross-over study

Participants: A sample of 999 patients with acute low back pain presenting to 300 primary care clinics in Sydney, Australia were followed up for 12 months. Participants were interviewed by telephone and asked whether they had recovered and if so the date of recovery.

Outcomes: Exposure to physical and psychosocial factors during the two hours preceding onset of a persisting episode of back pain (case window) was compared to that in the 2 -hour period 24 hours before the onset (control window). Persistent back pain was defined as pain not recovered within six weeks from onset and expressed as odds ratios (OR) and 95\% confidence intervals (C).

Results: We successfully followed up 832 participants (83\%). Manua tasks involving awkward positioning (OR 11.7, 95\% CI 5.4 to 25.3), heavy loads (OR 6.6, 95\% CI 3.2 to 13.9) or live people/animal (OR 5.0,95\% Cl 1.1 to 22.8) were strongly associated with developing persistent low back pain. Exposure to vigorous physical activity (OR 3.5, 95\% CI 1.6 to 7.7) was slightly more hazardous than exposure to moderate physical activity (OR $3.2,95 \% \mathrm{Cl} 1.9$ to 5.6). Being fatigued during a task or activity was less strongly associated (OR 2.9, 95\% Cl 1.3 to 6.4).

Conclusion: This is the first study to quantify the risk in developing persistent low back pain according to transient factors. Our findings could enable new preventive management strategies.

\section{Key Practice Points:}

- Patients with persistent low back pain contribute substantially to disease burden and treatment costs

- Risk factors associated with a persistent episode are only linked to patients' psychosocial characteristics

- Exposure to transient physical and psychosocial factors are highly associated with the development of persistent low back pain 


\section{THE EFFICACY OF CONSERVATIVE TREATMENT OF OSTEOPOROTIC COMPRESSION FRACTURES ON ACUTE PAIN RELIEF: A SYSTEMATIC REVIEW WITH META-ANALYSIS}

Rzewuska $\mathrm{M}^{1}$, Ferreira $\mathrm{ML}^{1,2}$, McLachlan $\left.\mathrm{A}\right\rfloor^{3}$, Machado $\mathrm{G}^{1}$, Maher $\mathrm{C}^{1}$

${ }^{1}$ The George Institute for Global Health, Sydney Medical School, The University of Sydney

${ }^{2}$ The Institute of Bone and Joint Research, The Kolling Institute, The University of Sydney

${ }^{3}$ Faculty of Pharmacy, The University of Sydney

Question: Are first-line conservative treatments of acute pain related to osteoporotic vertebral compression fractures effective?

Design: We conducted a systematic review with meta-analysis of randomised clinical trials. The GRADE criteria were used to assess strength 0 evidence and the Physiotherapy Evidence Database (PEDro) scale used to assess methodological quality of included studies.

Population: patients with a diagnosed vertebral compression fracture in lumbar or thoracic sites

Intervention: eight electronic bibliographic databases were searched for randomized controlled trials (RCTs) examining the effects of first-line pain relieving medications, passive physical therapies, bed-rest or orthoses.

Outcome Measures: pain, activity/participation outcomes and adverse events were extracted.

Results: Five RCTs (total $n=350$ ) were identified including one placebocontrolled and four controlled trials examining pain medications (2 studies) and orthoses (3). PEDro scores ranged from 4 to 7. The overall quality of evidence ranged from very low, to low. Immediate and short-term pain effects of analgesic medications differed between a placebo-controlled trial (standardized mean difference (SMDs): 0.09, 95\% confidence interval (CI) $-0.45,0.62$ to $0.32,95 \% \mathrm{Cl}-0.23,0.87$ ) and a controlled trial (SMD: - 1.17 , $95 \% \mathrm{Cl}-2.32,-0.03$ to $-1.66,95 \% \mathrm{Cl}-2.88,-0.44)$. Fixed effect meta-analyses of two trials examining spinal orthoses, demonstrated significant mediumterm pain relief (pooled SMD: $-1.47,95 \% \mathrm{Cl}-1.82,-1.13 ; \mathrm{I}^{2}=0 \%$ ) and disability reduction (pooled SMD: $-1.73,95 \% \mathrm{Cl}-2.09,-1.37 ;\left.\right|^{2}=0 \%$ ).

Conclusion: Low/insufficient statistical power of trials, additional treatment applied and potential conflict of interest might have influenced the results. There is a need for large, well-designed RCTs to inform first-line conservative care for acute pain related to $\mathrm{VCF}$.

\section{Key Practice Points:}

- The short-term benefits of analgesic medication for pain relief in patients with vertebral compression fractures are still unknown.

- There is low quality evidence showing spinal orthoses are effective for pain and disability in the medium-term

- The evidence in this field is in general of low quality.

\section{FUTURE WAYS OF WORKING? EVALUATION OF ERGONOMIC EDUCATION AND ENVIRONMENTAL INTERVENTIONS ON SITTING BEHAVIOUR IN OFFICE WORKERS}

\section{Mackey $\mathbf{M}^{1}$}

${ }^{1}$ Discipline of Physiotherapy, The University of Sydney

Introduction: Sustained sitting, a specific form of sedentary behaviour, is known to be associated with increased risk of morbidity, independent of leisure time physical activity. Workplace sitting may add to this risk but the threshold for safe exposure is not yet known. The aim of this presentation is to report on recent studies by the author which evaluated the impacts of ergonomic, education and environmental interventions on sedentary. behaviours, musculoskeletal discomfort and work ability of office workers.

Methods: Research designs consisted of a randomised trial and observational study. Participants were recruited from government and private industry and performed predominately computer-based office tasks. Interventions evaluated included:

- Education-based ('Happy Body at Work' program)

- Ergonomic-based (Sit-stand workstations)

- Environmental design-based (Activity based working - ABW)

Outcomes: Outcomes were average daily sedentary time during work hours (Actigraph accelerometer), self-reported sitting behaviour (Occupational Sitting and Physical Activity Questionnaire), musculoskeletal discomfort (Nordic Musculoskeletal Questionnaire), and work ability (Work Ability Index)

Results: Self-reported sitting time was respectively 63 and 91 minutes ( $p<0.01$ for both) less per day for participants exposed to a combined education/sit-stand workstation intervention and an ABW environment. Low back musculoskeletal discomfort was 50\% lower in ABW and work ability was rated as good. Objectively determined sedentary behaviour was not different between conditions as less sitting time was replaced with more (sedentary) standing, rather than walking behaviour.

\section{Key Practice Points}

- Activity permissive workstations and ABW environments promote potentially health enhancing reductions in sitting behaviour

- Education alone is unlikely to promote sustained behaviour change

\section{CONNECTING THE BRAIN, THE HAND AND THE FOOT: AN INTEGRATED APPROACH TO REHABILITATION OF THE INJURED SHOULDER IN THE THROWING ATHLETE}

\section{Magarey ME}

${ }^{1}$ Flex Rehabilitation Clinic, Norwood SA

¿University of South Australia

Sports physiotherapists are always looking for new ways to enhance management of the injured athlete. Research is teaching us a lot how treatment targeted at brain function can potentially enhance our management of the injured athlete. The influence of pain on the ability to facilitate muscle function, use of 'brain-related' strategies to reduce pain and enhance control and strength training will all be explored. Peter Blanch always said that 'the shoulder begins at the foot'. We now know far more about the impact of a lack of kinetic chain involvement has on shoulder impairment. To enhance the management of the athlete with a disabled throwing shoulder, we need to connect the research evidence across many fields with clinical experience. In this presentation, the factors contributing to pain in the disabled throwing shoulder will be explored specifically with a view to incorporating new knowledge into management and a suggestion for how to connect clinical experience and research in management of the throwing athlete.

Conclusion: Evidence-informed clinical experience incorporating attention to the multifactorial contributions to pain in the shoulder in the throwing athlete will enhance the response to management. Looking beyond the shoulder to make the connection between multiple aspects of our knowledge is essential in a contemporary approach to management of the patient presenting with a disabled throwing shoulder.

\section{Key Practice Points:}

For a patient presenting with a disabled throwing shoulder, the participant will:

- Understand how to integrate research knowledge from the pain sciences into evaluation

- Appreciate the contribution of multiple factors beyond the shoulder

- Appreciate how to integrate all factors into management 


\section{GEOFFREY DOUGLAS MAITLAND \\ MBE: CONNECTING HIS LEGACY TO \\ THE FUTURE OF MUSCULOSKELETAL PHYSIOTHERAPY EDUCATION}

\section{Magarey ME}

${ }^{1}$ Flex Rehabilitation Clinic, Norwood SA

¿University of South Australia

Geoffrey Douglas Maitland MBE was a leader, not only in the field of manipulative physiotherapy (now musculoskeletal physiotherapy), but also in advocating many aspects of physiotherapy that Australian physiotherapists now take for granted but which, at the time when Geoff first talked about them, were novel, far sighted and outside the convention for physiotherapists of the day. With the demise of many of the hallmark university-based programs in musculoskeletal physiotherapy, the question must be asked - where to from here? It is difficult to believe that a self-directed educational program such as that required to achieve titled membership of MPA or other national groups could possibly achieve the standards expected of a graduate of a formal masters program. For Australia to remain at the forefront of musculoskeletal physiotherapy education, research and practice, such issues must be considered.

How do we cope with the changing world of physiotherapy postgraduate education and still maintain standards? How can the standards demanded by IFOMPT be met by a self-directed program? Is the model currently in use within the APA and through the accreditation/evaluation process of postgraduate programs the best option available? Do we need to create new options for consideration? In this paper, the legacy left by Geoffrey Maitland in relation to physiotherapy standards, education and patient centred-management will be explored in the context of contemporary musculoskeletal physiotherapy education and where such education may go in the future.

\section{Key Practice Points:}

- Achieve a new insight into the history of education within musculoskeletal physiotherapy

- Be provided with an understanding of the development of postgraduate education within musculoskeletal physiotherapy

- Be provided with a proposal for consideration for the future development of education in musculoskeletal physiotherapy

\section{PHYSIOTHERAPISTS' KNOWLEDGE ABOUT DEMENTIA}

Maggs PK, Kay J, Baker J

Physiotherapy Department, Melbourne Health, Melbourne

Question: Dementia and physiotherapists-What knowledge gaps exist?

Design: Cross-sectional survey of clinical staff

Participants: 50 physiotherapists working across acute and sub-acute campuses of a major metropolitan Melbourne health organisation.

Outcome Measures: The Dementia Knowledge Assessment Tool version 2, a 21 item questionnaire examining knowledge about dementia, administered in a single assessment.

Results: $100 \%$ ( $N=50)$ of surveyed physiotherapists knew that dementia occurs because of changes in the brain and $96 \%(\mathrm{~N}=48)$ were aware that exercise can be beneficial in this patient population. Only $58 \%(\mathrm{~N}=29)$ were aware that dementia is likely to limit life expectancy despite $94 \%(N=47)$ knowing that the brain changes are often progressive. In the late stages of dementia, $66 \%(N=33)$ of physiotherapists showed knowledge that swallowing difficulties may be present, $72 \%(\mathrm{~N}=36)$ knew that movement is limited and $76 \%(\mathrm{~N}=38)$ knew that it is possible to establish if a person is in pain.

Conclusion: Whilst some basic understanding about dementia exists in physiotherapy staff, the survey results highlight that there are some key areas of the disease that are poorly understood. In particular, physiotherapists showed limited knowledge of the disease trajectory, swallowing difficulties, movement implications and assessment of pain.

\section{Key Practice Points:}

- There are key knowledge gaps within physiotherapy regarding dementia.

- Physiotherapists' lack of knowledge regarding dementia may have implications for clinical decision making and may be associated with adverse outcomes and costly futile interventions.

- Further research is required to develop specific education to increase physiotherapists' knowledge to ensure best practice care
PROSPECTIVE ACCELEROMETER AND GPS MEASUREMENT OF COMMUNITY AMBULATION ACROSS THE FIRST SIX MONTHS FOLLOWING STROKE

Mahendran $\mathbf{N}^{1,2}$, Kuys $\mathrm{SS}^{3,4}$, Brauer SG

${ }^{1}$ Division of Physiotherapy, School of Health and Rehabilitation Sciences, University of Queensland, Brisbane

2Discipline of Physiotherapy, Faculty of Health, University of

Canberra, Canberra

${ }^{3}$ School of Physiotherapy, Faculty of Health Sciences, Australian Catholic University, Brisbane

${ }^{4}$ Griffith Health Institute, Griffith University, Brisbane

Questions: How do people with stroke recover community ambulation at one, three and six months after hospital discharge post-stroke?

Design: Prospective, observational study.

Participants: 34 subacute stroke survivors (mean age: 71.6 SD 13.8, $70.6 \%$ male), independent with gait and no cognitive impairment.

Outcome Measures: Measures of community ambulation were collected by the ActivPAL ${ }^{\text {TM }}$ accelerometer, Garmin Forerunner 910XT GPS and activity diary. These included: volume (step count; time spent in the community, lying/sitting, standing and walking); frequency (number of community trips; number of and time in short, medium, long duration bouts); intensity (number of and time at low, moderate, high intensity bouts); and trip type at one, three and six months following hospital discharge.

Results: At one-month, participants took on average one trip, spending 137 SD 113 minutes per day in the community. Overall, most community ambulation was spread across long duration (mean = 11.3 SD 14.1 minutes) and moderate intensity bouts (mean = 14.0 SD 16.1 minutes). When measures of community ambulation were transformed and adjusted for age and discharge gait speed, there was no change over time except for a greater number of ( $p=0.01,95 \%$ Cl 1.7 to 77 ) and time ( $p=0.03,95 \% \mathrm{Cl}$ 3.5 to 22.7) spent in long ambulation bouts at six-months. There was no change in community ambulation trip type $(p=0.30)$ over time.

Conclusions: Community ambulation does not change over the first six months following hospital discharge post-stroke, aside from an increase in long duration ambulation bouts at six months.

\section{Key Practice Points}

- People with stroke do not increase total daily community ambulation despite meeting criteria for independence at hospital discharge.

- Review of stroke survivors at six-months following hospital discharge is suggested, as this is when changes in community ambulation may first be observed.

- Increasing post-stroke community ambulation may result in greater time spent in long duration and moderate intensity ambulation per day. 
WHAT FACTORS AT HOSPITAL

DISCHARGE PREDICT COMMUNITY

AMBULATION OUTCOMES ACROSS THE FIRST SIX MONTHS OF RETURNING HOME AFTER STROKE?

Mahendran $\mathrm{N}^{1,2}$, Kuys SS $\mathrm{S}^{3,4}$, Brauer $\mathrm{SG}^{1}$

${ }^{1}$ Division of Physiotherapy, School of Health and Rehabilitation Sciences, University of Queensland, Brisbane

Discipline of Physiotherapy, Faculty of Health, University of Canberra, Canberra

${ }^{3}$ School of Physiotherapy, Faculty of Health Sciences, Australian Catholic University, Brisbane

${ }^{4}$ Griffith Health Institute, Griffith University, Brisbane

Questions: What factors at hospital discharge predict community ambulation outcomes at one, three and six months later in people with stroke?

Design: Prospective prediction study.

Participants: 30 subacute stroke survivors from a tertiary rehabilitation unit.

Outcome Measures: Discharge measures of fatigue, mood, executive function, gait speed, endurance, perceived recovery, ambulation selfefficacy, pre-stroke activity and perceived health outcomes were collected. At one, three and six months, community ambulation was measured using an ActivPAL ${ }^{\text {TM }}$ accelerometer, Garmin GPS and activity diary. Highly correlated factors were entered into a stepwise linear regression model for each outcome at all time points.

Results: All measures of community ambulation at one month were only predicted by discharge gait endurance $\left(r^{2}=0.29\right.$ to $\left.0.38, p<0.007\right)$, except for frequency of community trips, which was also predicted by age $\left(r^{2}=\right.$ $0.40, p=0.04$ ). Beyond one month, volume of community ambulation was not predicted by any factor. Frequency of community ambulation at three and six months was predicted by either age $\left(r^{2}=0.22\right.$ to $\left.0.34, p<0.03\right)$ or pre-stroke activity $\left(r^{2}=0.19, p=0.04\right)$. Intensity of community ambulation was predicted by a combination of gait endurance, executive function and pre-stroke activity at three months $\left(r^{2}=0.82, p<0.001\right)$ and gait endurance and executive function at six months $\left(r^{2}=0.39, p<0.026\right)$

Conclusions: Gait endurance at discharge predicted community ambulation at one month, however after this time, age, pre-stroke activity and attention were predictive of community ambulation outcomes.

\section{Key Practice Points}

- Discharge gait endurance predicts community ambulation outcomes early after hospital discharge.

- Beyond one month, factors other than walking ability contribute to community ambulation outcomes after stroke.

- Factors including age, pre-stroke activity behaviours, and attention also impact community ambulation outcomes after one month of returning home.

\section{THE AUSTRALIAN COLLEGE OF PHYSIOTHERAPISTS - AN UPDATE AND INSIGHT INTO PATHWAYS TO FELLOWSHIP}

\section{Maher $\mathrm{C}^{1}$, Morris $\mathrm{M}^{2}$, Nitz J ${ }^{3}$, Ryan $\mathrm{M}^{4}$}

${ }^{1}$ The George Institute for Global Health, The University of Sydney

'School Allied Health, College of Science, Health \& Engineering,

La Trobe University

${ }^{3}$ School of Health and Rehabilitation Sciences, University of Queensland

${ }^{4}$ President, Australian College of Physiotherapists

The Australian College of Physiotherapists (ACP) has undergone a period of considerable change and improvement over the past decade, with the development of the Training Program towards Specialisation and refinement of the process leading to Fellowship by Original Contribution. The ACP continues to work with all stakeholders to ensure that clinical and academic career pathways exist and flourish in the physiotherapy profession. This session will provide an update from ACP President, Mr Mike Ryan, on the College's activities and strategic direction and will present an overview of the research of three Fellows by Original Contribution: Professor Chris Maher, whose research area focused on primary care management of low back pain, Dr Jenny Nitz who will summarise her research on management of older adults in residential aged care settings and Professor Meg Morris who will speak about her extensive work in the area of physiotherapy approaches to the management of Parkinson's Disease
PRIMARY CONTACT PHYSIOTHERAPY PRACTITIONER REDUCES WAITING

TIMES AND LENGTH OF STAY

FOR PATIENTS WITH ISOLATED MUSCULOSKELETAL CONDITIONS IN THE EMERGENCY DEPARTMENT

Maka $\mathrm{K}^{1}$, Alkhouri $\mathrm{H}^{2}$, Murray $\mathrm{M}^{2}$, McCarthy $\mathrm{S}^{2}$

${ }^{1}$ Physiotherapy Department, Westmead Hospital, Westmead, NSW ¿Emergency Care Institute, Agency for Clinical Innovation, Chatswood, NSW

Question: What is the impact of the primary contact physiotherapist on length of stay (LOS) and waiting times (WT) for patients presenting to the emergency department (ED) with musculoskeletal (MSK) conditions.

Design: A mixed method prospective observation study

Participants: All patients who presented with isolated MSK conditions seen by physiotherapist working in either a primary or a secondary contact role or by a doctor during September to November 2014 in 4 EDs in WSLHD. Intervention: A single episode of physiotherapy service

Outcome Measures: Patient LoS and WT till initial medical or physiotherapy assessment

Results: The primary contact physiotherapist role resulted in significant reduction in patient LoS by $108+4$ min (mean + SER): $p<0.001$ and WT by $10 \pm 2$ min; $p<0.01(n=626)$ compared to those seen only by doctors during the same working hours $(n=1000)$. Patients who received care by a doctor first and then physiotherapist $n=387$ had prolonged LoS compared to all other patients groups.

Conclusion: Management of patients with isolated MSK injuries by a primary contact physiotherapist was associated with reduced LoS and WT compared to those who are managed by a doctor only or a doctor followed by physiotherapist.

Key Practice Points:

- Primary contact physiotherapists can manage significant number of MSK patients presenting to ED

- This model of care reduces LoS and WT for patients with MSK conditions

- The results provide evidence that having physiotherapists working in primary contact roles may be one strategy to improve MSK patient flow in ED

\section{IMPLEMENTATION OF AN EXERCISE PROGRAM IN BREAST CANCER REHABILITATION TOIMPROVE SHOULDER STRENGTH: A PILOT STUDY}

\section{Ceprnja D ${ }^{1}$, Maka $\mathbf{K}^{1}$}

${ }^{1}$ Westmead Hospital, Sydney

Question: Does an exercise group program for patients who had undergone breast cancer surgery reduce pain and disability and improve strength for shoulder flexion and abduction?

Design: Observation single-centre pilot study. The study was approved by Western Sydney Local Health District Human Research Ethics Committee.

Participants: Twenty-six patients who had undergone breast cancer surgery and were receiving physiotherapy intervention at a tertiary level hospital outpatient physiotherapy department.

Intervention: Participants attended a weekly supervised group exercise session for 8 weeks. The exercise program consisted of strengthening exercises for major muscle groups and was individually tailored. Exercises were progressed to accommodate increases in strength gained over the course of the 8 week program.

Outcome Measures: The primary outcome measure was shoulder pain and disability index (SPADI). The secondary outcome measure was strength of shoulder flexion and abduction taken with a hand held dynamometer

Results: The exercise group intervention resulted in a significant reduction in SPADI scores $(p<0.001)$ and small but significant increases in shoulder flexion strength $(\mathrm{p}<0.001)$ and shoulder abduction strength $(p=0.001)$

Conclusion: This study provides some empirical evidence that an 8 week group exercise program for patients post breast cancer surgery can be effectively implemented in the clinical setting with small increases in shoulder strength, and an improvement in pain and disability.

\section{Key Practice Points:}

- A group exercise program can be effectively implemented in the clinic

- Small but statistically significant gains in shoulder muscle strength

- Reduction in reported pain and disability

- No adverse effects were reported. 
PATIENT AND STAFF SATISFACTION WITH THE ROLE OF THE EMERGENCY PHYSIOTHERAPY PRACTITIONER IN THE EMERGENCY DEPARTMENT

Alkhouri $\mathrm{H}^{1}$, Maka $\mathrm{K}^{2}$, Murray $\mathrm{M}^{1}$, McCarthy $\mathrm{S}^{1}$

${ }^{1}$ Emergency Care Institute, Agency for Clinical Innovation, Chatswood, NSW 2Physiotherapy Department, Westmead Hospital, Westmead, NSW

Question: To determine the level of patient satisfaction with the role of the emergency physiotherapy practitioner and how is this role perceived by clinical staff?

Design: A single episode of physiotherapy service

Participants: Across 7 NSW emergency departments (ED), 618 patients who presented with isolated musculoskeletal (MSK) injuries and seen by a physiotherapist completed a patient satisfaction survey and within the same facilities 360 clinical staff completed a staff satisfaction survey.

Intervention: A single episode of physiotherapy

Outcome Measures: Patient satisfaction and ED clinical staff perception of the physiotherapy service provided.

Results: More than $95 \%$ of patients managed by a primary contact physiotherapy practitioner strongly agreed (64\%) or agreed (31\%) that they were satisfied with the overall management of their condition and understood the advice given and discharge information. At least 90\% of emergency staff who completed the survey were positive about the role, skills and knowledge of the physiotherapists working in ED. The two items with the highest positive responses were that the physiotherapy role will make the ED team more effective (96\%) and will improve access to emergency care (97\%).

Conclusion: This multicentre survey approach revealed high levels of satisfaction of the physiotherapy role in ED by patients and clinical staff

Key Practice Points:

- Patients who received care by the physiotherapy practitioner in the ED felt that they received good management and advice about their condition.

- Clinicians perceived positively the role of the primary contact physiotherapy practitioner

- High levels of satisfaction expressed by patients and ED staff.

\section{SEASONAL VARIATION IN CERVICAL ARTERIAL DISSECTION IN THE HUNTER NEW ENGLAND REGION, NEW SOUTH WALES: A RETROSPECTIVE COHORT STUDY}

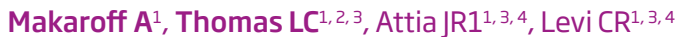

${ }^{1}$ University of Newcastle

¿University of Queensland

${ }^{3}$ Hunter Medical Research Institute

4ohn Hunter Hospital

Question: Does cervical arterial dissection show seasonal variation in the Hunter New England Region, NSW?

Design: Retrospective cohort study. The study examined seasonal variation in cervical arterial dissection in a temperate climate as this has been described in northern hemisphere regions with extremes of temperature.

Participants: Patients $18-60$ years with radiologically confirmed cervical artery dissection identified from the Cardiac and Stroke Outcomes Unit between 2006-2013.

Intervention: Digital case record review.

Outcome Measures: Demographic details, date of dissection, risk factors including exposure to minor neck trauma or infection, clinical variables including blood pressure and full blood count were collected from digita case records. Clinical variables were compared between autumn-winte and spring-summer and site of dissection.

Results: 61 cases of dissection were identified, 37 involving the vertebral artery, 37 participants were male. There was a strong trend for dissection in autumn and winter compared to spring and summer $(p=0.054)$. Exposure to minor trauma was more common in vertebral artery dissection $(p=0.023)$ and white blood count was also elevated (0.041) in comparison to internal carotid arterial dissection. Exposure to minor trauma was more common in the autumn-winter group but was not significant ( $p=0.128)$.

Conclusion: Cervical arterial dissection appears to occur more commonly in autumn and winter and suggests differing trigger mechanisms between dissection sites.

\section{Key Practice Points:}

- Seasonal variation suggests different trigger mechanisms between ICAD and VAD perhaps reducing the contribution from manipulation as a causative factor

- Consider greater possibility of dissection in autumn and winter

- Ask about recent exposure to minor trauma, recent infection
SOWING SEEDS, GRASSROOTS AND HARVEST: UO INTERPROFESSIONAL CLINICAL PLÁCEMENT MODEL IN VIETNAM

Mandrusiak $A^{1}$, Dunwoodie $\mathrm{R}^{1}$, Fagan $\mathrm{A}^{1}$, Quinlan $\mathrm{T}^{2}$, Hill $\mathrm{A}^{3}$

${ }^{1}$ Physiotherapy, School of Health and Rehabilitation Sciences, The University of Queensland, Brisbane

2Occupational Therapy, School of Health and Rehabilitation Sciences, The University of Queensland, Brisbane

${ }^{3}$ Speech Pathology, School of Health and Rehabilitation Sciences, The University of Queensland, Brisbane

Question: What is an effective model for providing interprofessional, intercultural clinical experiences in an international context?

Design: A clinical placement in Vietnam developed by the School of Health \& Rehabilitation Sciences at The University of Queensland, in partnership with the Office of Genetic Counselling and Disabled Children (Hue).

Participants: Students and clinical educators from physiotherapy, occupational therapy and speech pathology (two students from each profession in each placement), and in-country partners.

Intervention: Students work in interprofessional teams to provide sustainable management for children who have physical, communication and learning needs, and their families and facility staff. Experiences are across diverse settings, including schools, centres, orphanages and rural villages. Prior to leaving for Vietnam, students participate in a suite of workshops to develop readiness to practice in interprofessional and intercultural contexts.

Outcome Measures: A range of evaluation strategies have been employed to investigate outcomes and to refine this model, exploring perspectives from students, educators, and in-country partners.

Results: This successful placement partnership has delivered sustained positive outcomes since 2011, and a strong foundation for continued growth.

Conclusion: This model provides opportunities for students to build interprofessional and intercultural skills in an international and diverse context.

\section{Key Practice Points:}

- This is an effective model of interprofessional education to provide students with intercultural experiences in an international context

- Clinical supervision delivered face-to-face and supported remotely provides an effective and feasible model.

- A focus on sustainable, grassroots management approaches is vital, enriching the children and communities in Vietnam, and our students' professional learning.

\section{THE APPLICATION OF PUBLIC HEALTH INTERVENTIONS TO TACKLE WORK- RELATED MUSCULOSKELETAL DISORDERS}

\section{Mangharam $\rfloor^{1}$}

${ }^{1}$ WorkSafe, Western Australia

Work-related musculoskeletal disorders (WMSDs) have and continue to create heavy burden on Australian companies and communities. The burden associated with work injuries can be measured in multiple ways, including financial cost, lost time from work and disability. In Western Australia (WA) alone, the total direct cost (such as weekly payments, lump sum payments, treatments, legal costs, transport) of lost time injury workers' compensation claims between 2004/05-2008/09 amounted to approximately $\$ 2.4$ billion. Preventing and managing WMSDs are complex, owing to the interaction of multiple risk factors, including workplace physical, workplace psychosocial and individual risk factors. This talk will present evidence that an array of primary, secondary and tertiary prevention level interventions such as ergonomic intervention, risk management, wellness programs, early and appropriate clinical intervention and return to work rehabilitation programs have been tested. This talk will highlight that wide scope public health influencers exist in several forms, including education, stakeholder partnership, financial incentive programs and legislation. Evidence for the effectiveness of the various interventions in their various forms of delivery has been mixed. Collectively, there are adequate signs to indicate that public health strategies for this condition require a multidisciplinary and multifaceted approach that can be sustained over a period of time.

\section{Key Practice Points:}

- The need for all disciplines and stakeholders, including physiotherapy professionals, to realize their importance and to work within a widescope framework that may be applied within and across Australian states. 


\section{ASSESSMENT OF INDIVIDUAL MUSCLE ACTIVITY DURING A RAPID WRIST EXTENSION TASK IN PEOPLE WITH CHRONIC LATERAL EPICONDYLALGIA: A CASE-CONTROL STUDY}

Manickaraj $\mathbf{N}^{1}$, Bisset $\mathrm{L}^{1}$, Kavanagh $\mathrm{JJ}^{1}$, Ryan $\mathrm{M}^{1}$

IMenzies Health Institute Queensland, Griffith University, Gold Coast

Question: Does the timing of individual forearm extensor muscle activity differ between people with chronic lateral epicondylalgia and healthy controls during a rapid wrist extension task?

Design: A case-control observational design.

Participants: 11 lateral epicondylalgia and 11 matched healthy subjects.

Methods: Intramuscular electromyography from extensor carpi radialis brevis, extensor digitorum, extensor carpi ulnaris and anconeus muscles were recorded during rapid wrist extension in response to an audio cue. The data were de-identified and visually inspected. The reaction time (from the cue to the start of wrist extension), pre-motor time (from the cue to the start of electromyographic activity) and motor time (from the start of electromyographic activity to wrist extension) were assessed using a twoway factorial ANOVA.

Results: Reaction time was significantly slower in the lateral epicondylalgia group by 34 ms (95\% Cl 1-66). Extensor carpi radialis brevis, extensor digitorum and extensor carpi ulnaris demonstrated increased motor time by $18 \mathrm{~ms}$ (95\% Cl 6-31), $12 \mathrm{~ms}$ (95\% Cl2-23) and $28 \mathrm{~ms}$ (95\% C $9-46)$ respectively. There were no between group differences in pre-motor time.

Conclusion: People with lateral epicondylalgia have slower reaction time compared to healthy people, which can be explained by the increased motor time of the forearm extensor muscles.

\section{Key Practice Points:}

- People with lateral epicondylalgia have slower reaction time in wrist extension compared to healthy people.

- It takes longer for movement to occur after activation of forearm muscles in people with lateral epicondylalgia, implying increased tissue compliance.

- Local tendon pathology may explain the delayed reaction time.

\section{SUBACROMIAL CORTICOSTEROID INJECTION BY A PHYSIOTHERAPIST COMPARED TO AN ORTHOPAEDIC SURGEON: A NON-INFERIORITY RANDOMISED CONTROLLED TRIAL}

Marks $D^{1,2}$, Bisset $L^{2}$, Comans $T^{2,3}$, Thomas $M^{1}$, O'Leary $\mathrm{S}^{4,5}, \mathrm{Ng} \mathrm{SK}^{2}$, Conaghan $\mathrm{PG}^{6}$, Scuffham $\mathrm{PA}^{2}$

${ }^{1}$ Gold Cost Hospital and Health Service, Gold Coast

2Menzies Health Institute Queensland, Griffith University, Gold Coast

${ }^{3}$ Metro North Hospital and Health Service, Brisbane

${ }^{4}$ School of Health and Rehabilitation Sciences, University of Queensland, Brisbane

5Physiotherapy Department, Royal Brisbane and Women's Hospital, Brisbane ${ }^{6}$ Leeds Institute of Rheumatic \& Musculoskeletal Medicine, University of Leeds, \& NIHR Leeds Musculoskeletal Biomedical Research Unit, UK

Question: Can a physiotherapist prescribe and deliver subacromial corticosteroid injection for adult shoulder pain at least as clinically and cost effectively as an orthopaedic surgeon?

Design: Double-blind (patient, assessor) non-inferiority randomised controlled trial with economic analysis.

Participants: Two hundred and seventy-eight adults with shoulder pain referred to hospital Orthopaedics were assessed by a physiotherapist and separately by an orthopaedic surgeon.

Intervention: Sixty-four eligible participants were randomised to receive subacromial corticosteroid with local anaesthetic injection from the physiotherapist or orthopaedic surgeon.

Outcome Measures: The primary outcome was total Shoulder Pain and Disability Index score at baseline, 6 and 12 weeks, with a 15-point noninferiority margin. Secondary outcomes included quality of life, perceived improvement, pain, satisfaction and costs.

Results: Non-inferiority of injection by the physiotherapist was declared at 6 and 12 weeks (upper limit of the two-sided 90\% Cl: 13.34 and 7.17 at 6 and 12 weeks respectively). There were no significant differences between groups at 6 or 12 weeks on any other outcome measure. The physiotherapist was less expensive from the health funder perspective. Conclusion: A trained physiotherapist can prescribe and deliver subacromial corticosteroid injections for adult shoulder pain, at least as clinically effectively and less expensively than an orthopaedic surgeon.

\section{Key Practice Points:}

- Trained physiotherapists can safely, effectively, and cost effectively provide subacromial corticosteroid injection

- Regulations may need to be reconsidered regarding the scope of this practice for appropriately trained physiotherapists

- Additional physiotherapist training and accreditation will be required to implement this practice on a larger scale

\section{MEDICAL SUBSTITUTION IN THE MANAGEMENT OF MUSCULOSKELETAL DISORDERS: A SYSTEMATIC REVIEW}

Marks $\mathrm{D}^{1,2}$, Comans $\mathrm{T}^{2,3}$, Bisset $\mathrm{L}^{2}$, Scuffham PA ${ }^{2}$

${ }^{1}$ Gold Cost Hospital and Health Service, Gold Coast

${ }^{2}$ Menzies Health Institute Queensland, Griffith University, Gold Coast 3Metro North Hospital and Health Service, Brisbane

Question: What is the impact of medical substitution in the management of adult musculoskeletal disorders?

Design: Systematic review of the literature.

Participants: Non-medical health care professionals and doctors providing care for adults with non-emergency, non-inflammatory musculoskeletal disorders.

Intervention: Care provided by any non-medical professional, compared with usual medical care.

Outcome Measures: Health outcomes, care processes, resource utilization and costs.

Results: Sixteen studies met the inclusion criteria, most were of low methodological quality. Heterogeneity of conditions and outcome measures precluded meta-analysis. There is moderate evidence of good correlation between physiotherapist and orthopaedic consultant diagnostic and management decisions for selected knee, hip and shoulde presentations. Only five studies reported specific health outcomes, three quality of life and two performed economic evaluations. Neither medical substitution, nor usual medical care produced consistently superior health outcomes, satisfaction, resource usage or value for money, in primary or secondary care. Investigation of extended scope activities is sparse and there has been no direct reporting of non-medical prescribing compared to usual medical care.

Conclusion: Research into the impact of medical substitution for musculoskeletal disorders is lacking and does not consistently report better, or worse outcomes than usual medical care.

\section{Key Practice Points:}

- Despite growing popularity, there is a lack of research comparing doctors with alternative professionals in this patient population

- The dearth of evidence surrounding prescribing and extended scope activities may hinder the regulatory and health service uptake of innovative care models

- Further high quality research is needed to inform professional roles in musculoskeletal care pathways

WHAT CAN PHYSIOTHERAPISTS DO ABOUT PREVENTION AND TREATMENT OF OBESITY - INDIVIDUAL AND SOCIETAL APPROACHES

\section{Martin}

'Obesity Policy Coalition

This presentation will outline the problem of overweight and obesity in Australia, covering trends in adults and children, the impacts on health and on quality of life. It will detail the importance of prevention for physiotherapists utilising techniques such as brief interventions; strategies to raise the importance of maintaining a healthy body weight: and relevant resources. More broadly, population based approaches such as mass media education campaigns will be covered. An outline of what elements need to be addressed at a societal level to reduce overweight and obesity will be explored. It is very important to create an environment where the healthy choice becomes the easy choice; otherwise it is very unlikely that individuals will be able to successfully change their behaviour in the long-term. Physiotherapists can play an influential role in supporting environmental change through involvement in policy discussions via peak bodies; more direct individual engagement; and leadership within the community. A number of advocacy strategies will be discussed, including using the media to influence change. 


\section{THE EFFECT OF GENDER, CHRONICITY, AGE OR LATERALITY IN A THREE YEAR FOLLOW-UP STUDY OF PATIENTS WITH PATELLOFEMORAL PAIN}

Mason $\mathrm{M}^{1}$, Keays $\mathrm{SL}^{1,2}$, Newcombe $\mathrm{PA}^{3}$

1 Private Practice Nambour

¿University of the Sunshine Coast

${ }^{3}$ The University of Queensland

Question: Does gender, chronicity, age or laterality of patients with patellofemoral pain (PFP) affect the 3-year outcomes after a 1-month physiotherapy program?

Design: Prospective cohort study

Participants: Thirty-seven participants (55 knees), screened for PFP, were assessed before and three years following one month of physiotherapy. Characteristics: 14 males, 23 females; chronicity (mean six years); age 13 82 years; 19 unilateral, 18 bilateral.

Intervention: Fortnight 1: local quadriceps strengthening, quadriceps stretching, taping. Fortnight 2: Addition of individualised, global treatment Discharge with self-management advice, exercise.

Outcome Measures: Paired sample t-tests compared the differences between initial assessment and three years using eight measures: Quadriceps strength, quadriceps length, eccentric knee-control angle, Kujala score and pain walking upstairs, downstairs, during controlled step-down and a self-selected activity. Independent-sample t-tests analyses compared improvement scores in outcome measures across Gender, Chronicity ( $\leq 1$ or $>1$ year), Age $(<40,>40)$, Laterality (uni, bilateral)

Results: All measures improved over time: $p<0.001$, with no significant differences across Gender or Chronicity. Older participants improved significantly more with self-selected activity pain $p=0.012$, than younger participants but significantly less for the Kujala scores $p=0.023$. Significant greater pain reductions were reported by the unilateral than the bilateral participants across all pain measures ps $\leq 0.024$, and Kujala score $p=0.016$, but significantly less quadriceps length improvement $p=$ 0.014 .

Conclusion: Laterality and to a lesser extent age affected outcomes. The greatest gains in improvement were evident in the unilateral group of participants

\section{Key Practice Points:}

- Patients with bilateral PFP don't perform as well as unilateral ones over three years.

- Patients with bilateral PFP need more attention to quadriceps stretches.

- Age > 40 years affects the Kujala score.

- Neither gender nor chronicity affect the improvement

outcomes significantly

\section{TREATMENT OF PATELLOFEMORAL PAIN ACCORDING TO INDIVIDUAL PRESENTATION OR PHYSICAL SUBGROUPS: A 3-YEAR FOLLOW-UP STUDY OF INDIVIDUALISED TREATMENT}

Keays SL ${ }^{1,2}$, Mason $\mathrm{M}^{2}$, Newcombe $\mathrm{PA}^{3}$

${ }^{1}$ University of the Sunshine Coast

2Private Practice Nambour

${ }^{3}$ The University of Queensland

Question: Should patients with patellofemoral pain (PFP) be treated individually or according to physical subgroups?

Design: Prospective cohort study.

Participants: Thirty-seven participants (55 knees), screened for PFP, were assessed before and three years after following a 1-month physiotherapy program.

Intervention: Fortnight 1: local quadriceps strengthening, quadriceps stretching, taping. Fortnight 2: Addition of individualised, global exercises directed by the assessment of stance, sitting/movement patterns, biarticular muscle length, patellofemoral osteoarthritis (PFOA); identification of participants into physical subgroups based on individual presentation. One month: discharge with self-management advice and exercises.

Outcome Measures: Quadriceps strength, quadriceps length, eccentric knee-control, four pain measures, Kujala score were assessed initially and at three years. Paired sample t-tests compared the differences in these measures over time. Independent-sample t-tests compared the improvement of those participants falling into each subgroup compared to those not doing so.

Results: All participants were classified according to presence or absence of: hypermobile stance (27\% present); faulty movement pattern (73\%); hypomobile (38\%); PFOA (32\%). All eight measures improved over time, p
$<0.001$. Participants without PFOA improved significantly more than those with PFOA in quadriceps strength $(p=0.034)$ and quadriceps length $(p=$ 0.045 ). There were no significant outcome measure differences within each of the other subgroups.

Conclusion: Individualised treatment leads to highly significant improvement in PFP. The subgroup similarity in improvement could be due to (i) overlap between subgroups and (ii) individualised treatment according to presenting deficits. The current trend to physical subgrouping PFP is useful for assessment but treatment should be individualised.

\section{Key Practice Points}

- One month of individualised physiotherapy improves PFP, strength and function over three years.

- Physical subgrouping is helpful for determining individualised treatment.

- Pain improves in all subgroups, including the PFOA subgroup.

\section{CLINICAL PREDICTORS OF \\ TREATMENT SUCCESS FOR PATIENTS WITH PATELLOFEMORAL PAIN: A SYSTEMATIC REVIEW}

Matthews $\mathrm{M}^{1}$, Claus $\mathrm{A}^{1}$

${ }^{1}$ The University of Queensland, School of Health and Rehabilitation Science, Queensland

Question: Can patient characteristics predict a successful outcome to specific treatments for patellofemoral pain?

Design: Systematic review of quantitative intervention studies.

Participants: Patients with patellofemoral pain.

Intervention: Non-surgical and non-pharmacological interventions.

Outcome Measures: Studies were assessed with a methodological quality checklist. Likelihood ratios and odds ratios were extracted.

Results: Six electronic databases were searched (June 2014). Ten studies on prognosis and nine evaluating outcome to a specified treatment (including five clinical prediction rules) were included. Three prognostic studies determined that a longer duration of patellofemoral pain was associated with greater risk of an unsuccessful outcome regardless of treatment. Nine studies identified 23 patient characteristics that were associated with successful outcomes after specific treatment with foot orthoses, lumbopelvic manipulation, or patellar taping. It is unclear whether these patient characteristics predicted response to a specific treatment, or the prognosis for improvement regardless of treatment selected, because the studies lacked comparator treatments.

Conclusion: Clinicians can use the current evidence to help identify patients who are at risk of an unsuccessful outcome regardless of treatment, but not predict a preferential response to a specific treatment. Clinicians ought to be circumspect in their application of these findings until such time as there are higher quality randomized controlled trials that compare relevant treatments.

\section{Key Practice Points}

- Longer duration of patellofemoral pain was associated with an unsuccessful outcome, regardless of treatment.

- Patient characteristics currently cannot identify patients with patellofemoral pain who will respond better to a specific treatment than to other potentially appropriate treatments. 
DO MEASURES OF BALANCE, MOBILITY, MOOD AND BALANCE CONFIDENCE DIFFER BASED ON SELF-PERCEIVED PHYSICAL STATUS AT DISCHARGE HOME FROM REHABILITATION?

Mattin $\mathrm{S}^{1,2}$, Kuys $\mathrm{S}^{1}$, Donovan $J^{2}$, Stanton $\mathrm{W}^{1}$, Low Choy $\mathrm{N}^{1,3}$ ${ }^{1}$ Australian Catholic University, Brisbane

2Princess Alexandra Hospital, Woolloongabba

${ }^{3}$ The Prince Charles Hospital, Chermside

Question: Do measures of balance, mobility, function, mood and balance confidence differ at discharge to home from rehabilitation based on patient-perceived physical status?

Design: Prospective observational study.

Participants: Older adults ( $n=101,56.9 \%$ females, mean age $75.59 \pm 9.96)$ diagnosed with neurological, ortho-geriatric and general debility completing inpatient rehabilitation at a tertiary hospital.

Outcome Measures: Self-reported Modified Readiness for Hospital Discharge Scale (MRHDS) categorized patients as high ( $>7)$ or low (<7) readiness for discharge. Balance Outcome Measure for Elder Rehabilitation (BOOMER), Functional Independence Measure - Motor (FIM-M), Activities Specific Balance Confidence (ABC) Scale, and Geriatric Depression Scale (GDS) were completed at discharge. ANOVA determined differences in BOOMER, FIM-M, ABC and GDS scores at discharge for MRHDS groups.

Results: Participants with neurological diagnoses were younger (68 \pm 15$)$ and stayed longer in hospital (65 days) than ortho-geriatric and general-debility groups. No other between-group differences were observed, with data pooled for further analysis. Most participants (84\%) indicated readiness for discharge (>7 MRHDS score). Based on perceived physical status, those reporting higher readiness for discharge scored higher on the BOOMER ( $p=0.032)$. FIM-M ( $P=$ $0.039), \operatorname{ABC}(p=0.013)$, and lower GDS ( $p=0.017)$ scores

Conclusion: Patients at discharge from inpatient rehabilitation reporting higher physical status demonstrated better balance, mobility and confidence, with lower risk of depression, than those rating a lower level of physical status. Physiotherapy treatment regimes may need to consider patient perceived physical status at discharge along with optimizing balance and mobility.

\section{Key Practice Points:}

- Patients with higher self-reported physical status demonstrated better balance, mobility and confidence, with lower risk of depression, than those with lower self-reported physical status.

- The MRHDS may be useful in assessing patient perceived physical status at discharge in geriatric rehabilitation populations

\section{LITERATURE REVIEW OF OUTCOMES IN DOGS FOLLOWING SURGICAL REPAIR OF CRANIAL CRUCIATE LIGAMENT INSUFFICIENCY - WHAT IS THE EVIDENCE FOR PHYSIOTHERAPY?}

\section{McCarthy EA}

Question: Is there research supporting the use of physiotherapy in improving outcomes in dogs post-surgical repair of cranial cruciate ligament insufficiency?

Design: Literature review

Participants: Dogs having undergone surgical repair of cranial cruciate ligament insufficiency, including a review of those who have participated in physiotherapy.

Intervention: Research evaluating outcomes post-surgery included intracapsular, extracapsular and osteotomy techniques.

Outcome Measures: Including lameness scoring, kinematic and kinetic gait analysis, joint range of motion, muscle strength, functional ability and quality of life.

Results: Significant differences in stifle range of motion and thigh circumference has been found $1-5$ years post-surgery. A loss of stifle range is correlated with greater lameness score - osteoarthritic changes are associated with loss of extension in particular. Dogs who undergo physiotherapy immediately after surgical extracapsular stabilization demonstrated better functional gait recovery. Physiotherapy has been shown to increase thigh circumference as well as induce functional improvement in range of motion.

Conclusion: Research indicates that early physiotherapy intervention is beneficial and should be considered as part of the postoperative management plan.

Key Practice Points:

- Physiotherapy focusing on restoring full stifle range of motion may reduce the onset of osteoarthritis.

- Research suggests that there are ongoing chronic impairments in stifle range of motion as well as muscle strength in up to $50 \%$ of cases. Physiotherapy may have a role to play in improving outcomes.
THE EFFICACY OF ACUPUNCTURE AND DRY NEEDLING: PHYSIOTHERAPY INTEGRATIVE PATIENT MANAGEMENT

\section{McCutcheon L}

Bond University, Queensland

Physiotherapists in 'western' countries, including Australia, the UK New Zealand, Canada, the US and South Africa are increasingly using Dry Needling and Western Acupuncture. Western Acupuncture has its foundations in neurophysiological clinical reasoning and combines local, segmental and extra-segmental needling points. Dry needling will be discussed considering Travell \& Simons, Gunn and Baldry approaches. Clinical reasoning combining both Western Acupuncture and Dry Needling will be presented along with relevant literature from the current evidence base for needling in migraine, TTHA, neck pain, whiplash, LBP, pelvic girdle pain, shoulder conditions, lateral epicondylalgia, knee pain, OA plantar fasciitis, TMD, tendinopathy, fibromyalgia, women's health, cancer, neurology and sports enhancement

\section{Key Practice Points:}

-When to implement needling therapies in relation to evidence based medicine and clinical guidelines

- When to incorporate acupuncture and dry needling into multimodal care of the physiotherapy patient

- Conditions that have not been researched - where to from here?

NEUROPHYSIOLOGY AND THE EFFECTS OF ACUPUNCTURE AND DRY NEEDLING IN THE CHRONIC AND NEUROPATHIC PAIN POPULATION

\section{McCutcheon L}

Bond University, Queensland

Clinical reasoning for the use of acupuncture and dry needling relies on both animal and human research and in more recent years includes functional MRI research to consider the neurophysiological effects which occur at local, segmental and extrasegmental levels.

In particular when considering acupuncture as a treatment modality for the neuropathic pain population research highlights reduced glutamate release and NMDA channel down regulation and forebrain and limbic system deactivation. Additionally acupuncture has been shown to have a synergistic relationship with tricyclic antidepressants (TCAs) which may be beneficial when treating neuropathic pain and depression. In contrast and worthy of note is that aggressive styles of dry needling (pecking release styles) should be avoided in neuropathic patients due to activation of forebrain centres and the limbic system. In the area of pain management the use of acupuncture and dry needling for migraine, tension type headache, chronic LBP, TMD, adhesive capsulitis, fibromyalgia, depression and dysmenorrhoea has moderate support at systematic review and meta-analysis levels. There is mixed evidence in the areas of WAD, knee OA lateral epicondylgia, peripheral joint OA and rotator cuff disorders.

\section{Key Practice Points:}

- To consider the effect of acupuncture and dry needling in the dorsal horn in relation to pain modulation

- To assess changes that occur in the higher centres of the CNS in relation to activation or de-activaton patterns associated with needling therapies - what needling techniques to employ and which to avoid when treating the neuropathic patient

- To examine the activation of the descending pain inhibitory systems in relation to acupuncture and dry needling and the modulation of pain 
THE EFFECTIVENESS OF VESTIBULAR REHABILITATION IN THE ADULT, COMMUNITY-DWELLING POPULATION OF PEOPLE WITH SYMPTOMATIC

UNILATERAL PERIPHERAL VESTIBULAR DYSFUNCTION

McDonnell MN ${ }^{1}$, Hillier SL ${ }^{1}$

IInternational Centre for Allied Health Evidence, Sansom Institute for Health Research, School of Health Sciences, University of South Australia, Adelaide

Question: What is the evidence for vestibular rehabilitation to treat symptomatic, unilateral peripheral vestibular dysfunction in adults?

Design: Systematic review of the literature from 2010 to Jan 2014. This presentation reports on the update of our 2010 Cochrane review.

Participants: Adults living in the community, diagnosed with symptomatic unilateral peripheral vestibular dysfunction.

Intervention: Exercise regimes designed to address motionprovoked dizziness.

Outcomes: Primary outcome measures were those measuring change in the frequency and severity of dizziness. Secondary outcomes were measures of visual disturbance, quality of life, physiological status such as changes in balance impairment, function, and measure/s of physiological status with known functional correlation.

Results: We included 39 studies involving 2441 participants with unilateral peripheral vestibular disorders. Individual and pooled analyses of the primary outcome of interest, frequency of dizziness, showed a statistically significant effect in favour of vestibular rehabilitation over control or no intervention (OR 2.67, 95\% Cl 1.85 to 3.86)

Conclusion: There is moderate to strong evidence that vestibular rehabilitation is a safe, effective management for unilateral peripheral vestibular dysfunction, based on a number of high quality randomised controlled trials. There is moderate evidence that vestibular rehabilitation provides a resolution of symptoms and improvement in functioning in the medium term.

\section{Key Practice Points:}

- There is moderate evidence that vestibular rehabilitation is effective at reducing dizziness and improving function in adults with unilateral vestibular dysfunction.

- However, physical based manoeuvres are more effective for benign paroxysmal positional vertigo (BPPV).

- There is insufficient evidence to discriminate between differing forms of vestibular rehabilitation.

\section{ASSOCIATION BETWEEN TELEVISION VIEWING TIME AND RISK OF INCIDENT STROKE IN A GENERAL POPULATION: RESULTS FROM THE REGARDS STUDY}

McDonnell MN ${ }^{1}$, Hillier SL ${ }^{1}$, Judd $S^{2}$, Yuan $\mathrm{Y}^{2}$, Howard VJ ${ }^{3}$

IInternational Centre for Allied Health Evidence, Sansom Institute for Health Research, School of Health Sciences, University of South Australia Adelaide, Australia

2Department of Biostatistics, School of Public Health, University of Alabama at Birmingham, Birmingham, AL, USA

${ }^{3}$ Department of Epidemiology, School of Public Health, University of Alabama at Birmingham, Birmingham, AL, USA

Question: Is there a relationship between prolonged sitting time, as a measure of sedentary behavior, and risk of incident stroke in a large prospective cohort of men and women?

Design: Observational cohort study with a cross-sectional analysis

Participants: This analysis involved 22257 participants from the The REasons for Geographic And Racial Differences in Stroke (REGARDS) study who reported the amount of time spent watching television/video daily.

Outcomes: Suspected stroke events were identified at 6-monthly telephone calls and were physician-adjudicated. Cox proportional hazards models were used to examine risk of stroke.

Results: During 7.1 years of follow-up, 727 incident strokes occurred. In a multivariable adjusted model, watching TV/video $\geq 4 \mathrm{~h} /$ day (30\% of the sample) was associated with a $37 \%$ increased risk of all stroke ( $95 \%$ confidence interval, 1.10-1.71) and incident ischemic stroke (hazard ratio 1.35, confidence interval 1.06-1.72). This association was attenuated by socioeconomic factors such as employment status, education and income, but was independent of physical activity and other health factors.

Conclusion: These results provide support for benefits of decreasing sedentary behaviour to reduce the incidence of stroke.

\section{Key Practice Points:}

- Watching TV/video for $\geq 4$ h/day was associated with a 37\% increased risk of incident stroke in a multivariable model.

- When adjusting for socioeconomic factors like education and income this association was attenuated.

- Primary prevention strategies could target reducing sedentary behaviours to reduce stroke risk.

\section{INVESTIGATION OF BODY SCHEMA IN ADULTS POST STROKE}

Williams $\mathrm{L}^{1}, \mathrm{McDonnell} \mathrm{MN}^{1}$, Moseley GL ${ }^{2}$, Hillier SL ${ }^{1}$

IInternational Centre for Allied Health Evidence, Sansom Institute for Health Research, School of Health Sciences, University of South Australia, Adelaide, Australia

Body in Mind Research Group, Sansom Institute for Health Research, University of South Australia, Adelaide, Australia

Question: Is body schema distorted post stroke, and is it associated with light touch sensation, proprioception or unilateral neglect?

Design: Observational study with a cross-sectional analysis and longitudinal follow-up.

Participants: Fifteen participants were recruited from a major metropolitan hospital within two weeks of a first-ever hemispheric stroke, provided they had sufficient communication and cognition. Nine participants were available for a follow-up assessment three weeks later.

Outcomes: Participants were assessed at the bedside. The primary outcome was body schema, assessed using a left/right hand judgement task with the Recognise ${ }^{\mathrm{TM}}$ app (Neuro Orthopaedic Institute) and recording contralesional and ipsilesional hand judgement accuracy and reaction time. Secondary outcomes included assessing personal neglect, light touch sensation and proprioception. Associations between somatosensory deficits, unilateral neglect and distorted body schema were explored.

Results: A significant association was identified with impairment of proprioception and a distorted body schema $(p=0.03)$ in the acute stage post stroke. There was no significant difference in body schema between contralesional and ipsilesional sides of the body or from the acute to subacute stage post stroke.

Conclusion: A distorted body schema was associated with impaired proprioception in adults post stroke.

\section{Key Practice Points:}

- Upper limb proprioception, measured with the Distal Proprioception Test early after stroke may assist in highlighting people with impairments of body schema

- The RecogniseTM app was feasible to use by the beside to assess body schema, but was not related to neglect in this small sample

- Proprioceptive deficits may contribute to movement execution post-stroke 
AN INVESTIGATION OF CORTICAL

NEUROPLASTICITY FOLLOWING

STROKE IN ADULTS: IS THERE EVIDENCE

FOR A CRITICAL WINDOW FOR

REHABILITATION? A PROTOCOL

McDonnell MN ${ }^{1}$, Koblar $\mathrm{S}^{2}$, Ward $\mathrm{N}^{3}$, Rothwell J $\mathrm{C}^{3}$,

Hordacre $\mathrm{B}^{4}$, RiddingBC ${ }^{4}$

IInternational Centre for Allied Health Evidence, Sansom Institute for Health Research, School of Health Sciences, University of South Australia Adelaide, Australia

¿South Australian Health and Medical Research Institute and The Queen Elizabeth Hospital, Adelaide, Australia

Institute of Neurology, University College London, London, England

${ }^{4}$ Neuroplasticity, Development and Repair Research Group, The University of Adelaide, Adelaide, Australia

Question: Is there a "critical window" of enhanced neuroplasticity in patients following stroke?

Design: Observational study within three stroke units.

Participants: We will recruit participants recently admitted to the Stroke Unit of major metropolitan hospitals who have had an ischaemic stroke and can provide informed consent. Participants will be excluded if they have any contraindications to Transcranial Magnetic Stimulation. We will perform functional and neurophysiological measures as soon as participants are medically stable, then at regular intervals until one year post-stroke. We will also compare neurophysiological outcomes in an age-matched healthy control group.

Outcomes: The primary outcome is the change in the motor evoked potential (MEP) amplitude in a hand muscle, after the administration of a plasticity-inducing paradigm to the affected hemisphere. Secondary outcomes include measures of cortical excitability, intracortical inhibition and arm function.

Results: Results are expected in 2016

Conclusion: The data from this trial will clarify whether there is a critical window for neuroplastic change in the brain following stroke. If so, intensive rehabilitation during this period could be more effective, reducing long-term disability and the cost burden of stroke.

Key Practice Points:

- Evidence in animal stroke models suggests that neuroplasticity takes place maximally in a specific time window after an ischaemic lesion, which may coincide with the optimal time to intervene with rehabilitation.

- In adults post-stroke, the optimal period to induce functionally-relevant neuroplastic change in the brain is unknown.

- This study will assist us to understand the motor cortical reorganization that underpins functional recovery following stroke.

\section{GRADUATING PHYSIOTHERAPISTS}

PERCEPTIONS OF EVIDENCE BASED PRACTICE (EBP) UNIVERSITY TRAINING AND IMPACT IN THE WORKPLACE: A MIXED METHODS STUDY

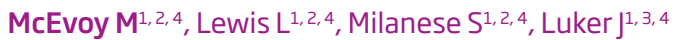

${ }^{1}$ School of Health Sciences, University of South Australia

${ }^{2}$ The International Centre for Allied Health Evidence (iCAHE)

${ }^{3}$ Alliance for Research in Exercise, (ARENA)

${ }^{4}$ Sansom Institute for Health Research
}

Question: What changes occur in self-reported EBP knowledge, attitudes and behaviours and actual knowledge of physiotherapy students across three EBP courses? What are students' perceptions of the EBP training undertaken and impact it will have on their first year in the workforce?

Design: Mixed methods, pre-post design with focus groups

Participants: Physiotherapy students who undertook three EBP courses, completed Bachelor of Physiotherapy December 2013 and questionnaires both prior to the first course and after the final EBP course.

Intervention: All physiotherapy students were invited to complete EBP self-report and knowledge questionnaires before and after three EBP courses (2010, 2012, 2013). In November 2013 students were invited to participate in focus groups.

Outcome Measures: The Evidence Based Practice Profile questionnaire addresses five EBP domains: Relevance, Terminology, Confidence, Practice, Sympathy; the Knowkedge-REC instrument addresses a sixth domain: actual EBP knowledge.

Results: Pre-EBP1 questionnaires completed by 98 of 127 students. PostEBP3 questionnaires completed by 109 of 125 students. Matched datasets for 56 participants of which 21 participated in focus groups.
Improvements in all EBP domains were significant: Relevance $p=<0.001$, ES 2.29, Terminology $p=0.001$, ES 3.13, Confidence $p=0.001$, ES 1.67, Practice $p=0.001$, ES 1.8, Sympathy $p=0.008$, ES 0.49, Knowledge (actual) $p=0.001$, ES 4.3.

\section{Key Practice Points:}

- Greatest changes occurred in EBP knowledge and relevance, with less in confidence practice and sympathy

- Students did not anticipate the focus on EBP, looked for role models and gained EBP confidence by graduation

- Perceptions for EBP use in the workforce related to time, resource access and workplace culture

HOW TO ASSESS AND TREAT THE UPPER TORSO MUSCULOSKELETAL SYMPTOMS EXPERIENCED BY FEMALE PATIENTS WITH LARGE BREASTS

McGhee DE ${ }^{1}$, Steele JR ${ }^{1}$

${ }^{1}$ University of Wollongong, New South Wales, Australia

Background: Women with large breasts present to physiotherapists with musculoskeletal symptoms in the upper torso secondary to their breast size.

Aims / objectives: This session provides evidence-based guidelines on assessments and treatment interventions to relieve these symptoms, prevent their progression and promote physical activity in women with large breasts. Learning outcomes include: (i) applied knowledge and understanding of the fundamental components of assessment and treatment (manual therapy and exercise interventions that target structural and functional changes and physical activity recommendations based on breast biomechanics; and (ii) skill and competency in breast support and bra fit assessment and bra design critiquing.

Approach: Didactic learning will be used for evidenced-based assessments, manual therapy, exercise interventions and physical activity recommendations. Visual and simulated learning will be used for breast support and bra fit assessments. This will be followed by active participatory learning of these assessments, including bra design critiquing, where participants will examine and critique, in an open discussion environment, a wide range of bras currently available for larger breasts. Learning materials provided will include assessment sheets and free patient educational resources (paper and electronic format) on breast support and bra fit.

Conclusion / Key Practice Points: This session will provide physiotherapists with:

- Clinical knowledge and skills to assess and treat the musculoskeletal symptoms associated with large breasts, including suitable forms of physical activity and bra designs to recommend to women with large breasts so they can exercise in comfort.

- Assessment sheets and free patient education resources on breast support and bra fit. 
DOES BREAST SIZE AFFECT MEASURES

OF UPPER TORSO PAIN AND

MUSCULOSKELETAL STRUCTURE AND FUNCTION?

McGhee DE ${ }^{1}$, Coltman KA ${ }^{1}$, Riddiford-Harland $\mathrm{DL}^{1}$, Steele $\mathrm{R}^{1}$

'University of Wollongong, New South Wales, Australia

Question: Does breast size affect upper torso pain and musculoskeletal structure and function?

Design: Cross-sectional study, Ethics: HE13/053.

Participants: 27 women with large breasts (bilateral breast volume > $1200 \mathrm{ml}$ ) and 26 women with small breasts (bilateral breast volume $<800$

$\mathrm{ml})$. Matched for age to represent the normal population.

Outcome Measures: Upper torso pain (Visual Analogue Scores), flexion torque (three-dimensional scanning), thoracic kyphosis (Flexicurve ruler) shoulder and thoracic range of motion (goniometry), and thoracic extensor muscle strength (during upright row using a load cell).

Results: Women with large breasts reported a higher upper torso pain score (46.6, 95\%Cl 33.3-58.0 versus 24.1,95\% Cl 12.5-37.8), accompanied by a larger flexion torque $(5.9 \mathrm{Nm}, 95 \% \mathrm{Cl} 4.5-5.8 \mathrm{Nm}$ versus $0.9 \mathrm{Nm}, 95 \% \mathrm{Cl}$ $0.8-2.4 \mathrm{Nm})$, greater thoracic kyphosis $\left(34^{\circ}, 95 \% \mathrm{Cl} 31-38^{\circ}\right.$ versus $27^{\circ}$

$\left.95 \% \mathrm{Cl} 24-31^{\circ}\right)$ decreased shoulder flexion range-of-motion $\left(160^{\circ}, 95 \% \mathrm{Cl}\right.$

$158-163^{\circ}$ versus $\left.169^{\circ}, 95 \% \mathrm{Cl} 166-172^{\circ}\right)$ and decreased thoracic extensor endurance-strength (511 s, 95\%Cl 362-691 s versus 876 s, 95\%Cl 6921028 s) compared to the women with small breasts.

Conclusion: Breast size negatively affects the structure and function of the upper torso. Results provide insight into the underlying causes of the musculoskeletal pain experienced by women with large breasts.

\section{Key Practice Points:}

Evidence-based assessments and fundamental components of physiotherapy treatment to relieve pain and prevent symptom progression in female patients with large breasts include:

- Using upper torso pain scales to quantify symptoms

- Breast support and bra fit assessment and patient education

- Shoulder range-of-motion assessment and treatment

- Thoracic extensor endurance strength assessment and treatment

\section{HOW TO ASSESS BREAST SUPPORT AND BRA FIT FOR FEMALE ATHLETES AND PATIENTS}

\section{McGhee $\mathrm{DE}^{1}$, Steele JR}

${ }^{1}$ University of Wollongong, New South Wales, Australia

Background: Insufficient breast support is a potential barrier to physical activity in women due to breast discomfort and embarrassment, and can negatively affect sporting performance. Physiotherapists are in an ideal position to educate women on this sensitive topic when treating musculoskeletal disorders associated with large breasts and during sports coverage of female athletes/teams.

Aims / objectives: The aim of this session is to provide physiotherapists with the knowledge, clinical skills and evidence-based resources to assess the breast support and bra fit of female athletes/patients. Learning outcomes: Applied knowledge, understanding and skills to assess breast support and bra fit for women of varying age, bra size and level of physica activity. Competency to critique bra design and organise breast support and bra fit education sessions for female athletes/teams.

Approach: Visual and simulated learning of breast of support and bra fit assessments, followed by active participatory learning to practice these assessments and physically critique the level of breast support provided by a range of sports bras for women of varying age, bra size and activity level. Open discussion of strategies and challenges in implementing educational sessions to specific groups of female sporting teams/athletes. Learning materials of assessment sheets and free patient educational resources (pamphlet and web-based) on breast support and bra fit.

\section{Conclusion / Key Practice Points:}

Provide physiotherapists with:

- Clinical skills to assess bra fit and breast support and critique bra design.

- Assessment sheets and free patient educational resources.

- Strategies to implement educational sessions to female sporting teams/athletes.

\section{POST SURGICAL PHYSICAL THERAPY IN DOGS UNDERGOING HEMILAMINECTOMY FOR INTERVERTEBRAL DISC DISEASE}

\section{McGhie JA ${ }^{1}$}

${ }^{1}$ School of Veterinary Science, University of Queensland

Intervertebral Disc Disease is a common cause of neurologic dysfunction in dogs ( $2 \%$ of canine population). Chondrodystrophic canine breeds are most frequently affected with Dachshunds accounting for $45 \%-73 \%$ of all cases of acute disc extrusion. Hemilaminectomy surgery is routinely performed to achieve spinal cord decompression. The prognosis for recovery is good (>85\%) in dogs with deep pain sensation intact before surgery. The average time to ambulation post surgery is recorded in days rather than weeks and is an important part of the decision making process for owners when considering surgical treatment. Physical therapy in post hemilaminectomy dogs starts in hospital. Treatments such as cryotherapy start within hours of the dog returning to the ICU. Massage and passive range of motion exercises are introduced within 24-48 hours of surgery, progressing to muscle strengthening and balance exercises. Underwater treadmill/swimming, cavaletti exercises, incline work and TENS therapy may all become part of the post surgery therapy regime as dogs improve. Physical therapy in dogs carries a unique set of challenges related to the physical size and temperament of the dog, owner compliance and commitment, and access to trained therapy staff, equipment and facilities.

\section{Key Practice Points:}

- Gain an insight into the current role of physical therapy in dogs undergoing hemilaminectomy for intervertebral disc disease

- How access to trained animal physical therapists could improve our patient care and recovery.

\section{EVALUATING THE EFFECTS OF INCREASING PHYSICAL ACTIVITY IN HOSPITALIZED OLDER ADULTS: MOVE TRIAL UPDATE}

Said $C^{1,2,3}$, Morris $\mathrm{M}^{2}$, McGinley ${ }^{3}$, Szoeke $\mathrm{C}^{3}$, Workman $\mathrm{B}^{4,5}$ Liew $D^{3,6}$, Hill K $K^{7}$, Woodward $M^{1}$, Wittwer $]^{2}$, Churilov $L^{8}$, Bernhardt $]^{8}$ ${ }^{1}$ Austin Health, Heidelberg

¿La Trobe University, Bundoora

${ }^{3}$ The University of Melbourne, Parkville

${ }^{4}$ Monash Health, Cheltenham

${ }^{5}$ Monash Ageing Research Centre, Cheltenham

6Melbourne Health, Parkville

${ }^{7}$ Curtin University, Perth

${ }^{8}$ The Florey Institute of Neuroscience and Mental Health, Heidelberg

Question: Does providing increased physical activity to older people receiving hospital based rehabilitation lead to better mobility outcomes at discharge?

Design: Approved by relevant ethics committees. Single blind, multisite randomized controlled trial, blinded assessment of outcome, intention to treat analysis.

Participants: People aged > 60 years undergoing in-patient rehabilitation to improve mobility were recruited from geriatric rehabilitation units at two Australian hospitals.

Intervention: Participants received usual care; in addition they were randomized to either spend time daily performing additional physical activities to improve mobility (intervention), or an equal amount of social activities with minimal impact on mobility (control)

Outcome Measures: Self-selected gait speed using a 6-meter walk test at discharge (primary outcome) and 6 months follow up (secondary outcome). Additional measures of mobility, function and quality of life are secondary outcomes at discharge and tertiary outcomes at 6 months follow up.

Results: Recruitment is completed with 198 participants randomized (mean age 81 years, 43\% male); 4 participants have withdrawn; 188 participants have completed the discharge assessment; 86 have completed the six month assessment. An additional 4455 sessions have been delivered ( $85 \%$ of intended sessions).

Conclusion: Results indicate this inpatient program is feasible with good compliance and retention. Establishing the efficacy of increased physical activity will assist development of evidenced based inpatient rehabilitation programs for older people.

Trial Registration: ACTRN12613000884707

Funding: NHMRC App1042680

Key Practice Points:

- Older people undergoing inpatient rehabilitation can increase physical activity.

- We do not know whether increased physical activity improves mobility in this group. 


\section{MOTOR FUNCTION AND GAIT IN CHILDREN WITH AUTISM SPECTRUM DISORDER}

McGinley JL ${ }^{1}$, Murphy $A^{2}$, Enticott P3 , Tonge $B^{4}$, Bradshaw $\rfloor^{4}$ Papadopoulos $\mathrm{N}^{3}$, May $\mathrm{T}^{3}$, Rinehart $\mathrm{N}^{3}$

${ }^{1}$ Physiotherapy, School of Health Sciences, The University of Melbourne, Carlton

${ }^{2}$ Clinical Research Centre for Movement Disorders and Gait, Monash Health, Cheltenham

${ }^{3}$ Deakin Child Study Centre, School of Psychology, Deakin University, Burwood ${ }^{4}$ Centre for Developmental Psychology and Psychiatry, Monash

University, Clayton

Question: How does motor function and gait of children with autism spectrum disorder (ASD) compare to typically developing children? Is motor function associated with emotional-behavioural symptoms in children with ASD?

Design: Prospective observational cohort study

Participants: Sample of convenience of children aged 7 - 12 years with ASD; Asperger's Disorder (AD) $(n=27)$, High functioning autism (HFA) $(n=$ 23) and a typically developing (TD) group ( $n=24)$.

Outcome Measures: A 3-dimensional motion analysis system recorded full body joint kinematics as children walked at varied speeds. The Movement Assessment Battery for Children-2 evaluated motor proficiency, and the Developmental Behavioural Checklist measured symptoms of emotional/behavioural disturbance.

Results: Children with ASD walked at comparable speeds to TD children, yet showed subtle differences, such as greater forward trunk tilt and range, and altered timing of ankle motion (all $p<0.05$ ). Children with HFA performed worse than TD with manual dexterity tasks ( $p=0.005)$, ball skills ( $p<0.001$ ) and balance $(p<0.001)$. Higher motor proficiency was significantly associated with less communication disturbance and severity of autistic symptoms ( $r=0.37,033$ respectively)

Conclusion: Many children with ASD have impaired motor function that may contribute to activity limitations and participation restrictions. The role of early motor assessment and intervention warrants urgent evaluation.

\section{Key Practice Points:}

- ASD is very common, affecting approximately 1 in 100 Australian children

- Many children with ASD have impaired motor skills or subtle

movement problems

- Motor symptoms are a core element of the clinical profile of children with ASD

\section{PHYSICAL ACTIVITY OF AUSTRALIANS WITH PARKINSON'S DISEASE}

McGinley JL1, Danoudis $\mathrm{M}^{1,2}$, Bilney B ${ }^{1}$, Morris ME ${ }^{3}$, Higgins $\mathrm{R}^{1,4,5}$ ${ }^{1}$ Physiotherapy, School of Health Sciences, University of Melbourne ${ }^{2}$ Clinical Research Centre for Movement and Gait, Monash Health, Melbourne ${ }^{3}$ Physiotherapy, School of Health Sciences, La Trobe University, Melbourne ${ }^{4}$ Heart Research Centre, Melbourne

${ }^{5}$ Cabrini Health, Melbourne

${ }^{6}$ Psychology, Faculty of Health, Deakin University, Melbourne

Question: How physically active are Australians living with Parkinson's disease (PD), and what factors are associated with physical activity?

Design: Observational cross-sectional survey

Participants: A sample of 346 community dwelling ambulant people with PD completed an online or mailed survey. Participants were $60 \%$ male, mean age 68.6 (8.8), with PD duration of 6.1years (6.97), with mild moderate disability (median Hoehn and Yahr stage 2).

Outcome Measures: The survey evaluated Physical Activity (International Physical Activity Questionnaire, total weekly PA (mins)), exercise selfefficacy, disability, falls history and falls self-efficacy, and a range of motor (e.g., freezing of gait) and non-motor (e.g., fatigue, apathy, depression, anxiety) symptoms.

Results: Thirty-two percent of participants reported a low level of PA [category 1], 53.5\% a moderate level [category 2], and 14.5\% a high level [category 3]. Weekly PA varied markedly; with a median of 527 minutes [IQR: 210 - 1040]. Factors associated with greater PA included higher exercise self-efficacy, higher falls self-efficacy, and lower levels of freezing of gait, fatigue, apathy, anxiety, depression and disability (all $p<0.5$ ).

Conclusion: Identification of factors associated with PA may guide the development of interventions. Furthermore, the presence of psychologica factors as predictors of exercise, indicate the potential importance of addressing mental health in interdisciplinary programs to increase PA

\section{Key Practice Points:}

- Many people with PD do not meet the guidelines for recommended levels of PA

- Modifiable factors associated with PA may represent targets for interventions

- Non motor symptoms including psychological factors may be important targets for intervention

\section{SCIENCE IN ANIMAL TRAINING}

\section{McGreevy $\mathrm{P}$}

The University of Sydney

The lengthy association of humans with horses and dogs has established traditional training techniques that have served military needs well. However, many of these approaches fail to recognise what we know about the ethology (natural behaviour) of these important species. Dog training has generally been swift to embrace more recent approaches that are based on scientific principles. Most professional dog trainers are fluent in the language of learning theory and this is inspiring veterinarians to do so too. The correct terminology allows professionals to demystify virtually all learned behaviours; both welcome and unwelcome. In contrast, many equestrians continue to apply dominance-based training philosophies and regard the horse as a willing participant without questioning the consequence of this approach when horses fail to respond as required. Although effective, these traditions have by-passed the research findings of modern psychologists, who developed the fundamentals of learning theory. That said, the pools of equestrian debate are far from stagnant. The latest wave of horse whisperers has offered some refinements and some novel interpretations of the motivation of horses undergoing training. Nevertheless, many still question the welfare of the ridden horse since it is largely trained using negative reinforcement (the removal of pressure), has to respond to pressure-based signals and is seldom asked to work for positive rewards. These are some of the topics addressed by equitation science, an emerging discipline that combines learning theory, physics and ethology to examine the salience and efficacy of horsetraining techniques.

\section{Key Practice Points:}

- How best practice in animal training and animal handling has a strong evidence base and is already improving the health and welfare of Australian dogs.

- From an equestrian perspective, it will also introduce you to the emerging discipline of equitation science, a movement with proudly Australian roots. 


\section{HYPERFLEXION OF HORSES' NECKS: A META-ANALYSIS AND COST- BENEFIT EVALUATION}

McGreevy P $^{1}$, König von Borstel U², Kienapfel K³ , McLean $A^{4}$, Wilkins $C^{5}$, Evans D

${ }^{1}$ Faculty of Veterinary Science, University of Sydney, NSW 2006, Australia ¿Department of Animal Breeding and Genetics, University of Goettingen, Albrecht-Thaer-Weg 3, 37075, Goettingen, Germany

${ }^{3}$ Department of Animal Ecology, Evolution and Biodiversity, Ruhr Universität Bochum, Bochum, Germany

${ }^{4}$ Australian Equine Behaviour Centre, Broadford, VIC 3658, Australia

Question: How does the scientific literature dealing with the effects of equine head and neck postures (HNP) on welfare and gymnastic outcomes such as kinematics, muscle activity, respiratory related issues or overall workload justify the use of hyperflexion of the neck in the training and warm-up of sport horses?

Design: The meta-analysis, a dataset containing overall results of the individual studies, coded positive (1), contradictory or insignificant (0) or negative (-1) influences on a) welfare and b) gymnastics. Information on the reported features of hyperflexion (e.g., degree and duration) and the horses (e.g., level of dressage training) was also integrated into the dataset

Participants: There were 55 articles dealing with the effects of equine head and neck postures (HNP) on welfare $(n=42)$ and/or gymnastic outcomes ( $n=35)$

Outcome Measures: This was a meta-analysis that focused on evidence for the popular technique influencing gymnastic outcomes or horse welfare. Desired gymnastic effects included higher dressage scores, a larger range of motion in the back or legs or an increased overall workload, while undesired gymnastic effects included lower dressage scores, increased activation of lower neck muscles and reduced oxygen supply due to obstruction of upper airways. Reasons for compromised welfare impairment included impeded ventilation, pathological changes in the structures of the neck, impaired forward vision, and stress and pain due to these factors as well as the rider intervention necessary to achieve the posture.

Results: The significant majority of studies (88\%; Z= 4.94; $P<0.0001)$ indicated that a hyperflexed HNP compromises welfare. Only one study suggested positive effects on welfare. An across-study analysis using a mixed model revealed that the probability of a study reporting negative welfare effects was unrelated to any of the investigated factors such as horses' familiarity with the posture, level of dressage training, duration of the HNP or size of the study (all P>0.1). While gymnastic benefits were described in $26 \%$ of the studies, a similar proportion of studies $(23 \%$, P>0.1) detected undesirable gymnastic consequences of a hyperflexed HNP. The remaining studies (46\%) described insignificant or contradictory effects on gymnastics. Studies conducted on highest level dressage horses and in horses familiar with a hyperflexed HNP were more likely to describe gymnastic benefits than studies conducted on non-dressage horses or those unfamiliar with the posture (both $\mathrm{P}<0.05$ ).

Conclusion: These findings question whether any desirable effects of this training method rely on learned response rather than effects based solely on biomechanical relationships.

\section{Key Practice Points:}

- The presumed gymnastic benefits of training horses in a hyperflexed head and neck posture are by far outweighed by both undesired gymnastic effects and reduced equine welfare.

- Nnegative effects on welfare prevail regardless of the circumstances under which hyperflexion is practiced.

\section{PHYSICAL ACTIVITY LEVELS IN PEOPLE WITH COPD WHO EXPERIENCE EXERCISE- INDUCED OXYGEN DESATURATION}

McKeough $Z^{1}$, Leung $\mathrm{R}^{1}$, McDonald $\mathrm{CF}^{2,3}$, Jenkins $\mathrm{S}^{5,6}$, Holland $\mathrm{A}^{3,4}$, Hill K ${ }^{5}$, Morris N7, Hill $C^{2,3}$, Lee $A^{4}$, Cecins $N^{6}$, Seale $H^{8}$, Spencer $L^{9}$, Wootton $\mathrm{S}^{1}, \mathrm{Ng} \mathrm{C}^{10}$, Alison $]^{1}$

${ }^{1}$ Physiotherapy, University of Sydney, NSW

${ }^{2}$ Respiratory and Sleep Medicine, Austin Health, VIC

IInstitute for Breathing \& Sleep, VIC

${ }^{4}$ Physiotherapy, Alfred Health, VIC

5Physiotherapy and Exercise Science, Curtin University, WA

'Physiotherapy, Sir Charles Gairdner Hospital, WA

${ }^{7}$ Menzies Health Institute, Griffith University, QLD

${ }^{8}$ Physiotherapy, Prince Charles Hospital, QLD

9Physiotherapy, Royal Prince Alfred Hospital, NSW

10Physiotherapy, Singapore General Hospital, Singapore

Question: Is there a difference in physical activity (PA) levels and

functional exercise capacity in people with COPD with exercise-induced oxygen desaturation (EID) (nadir SpO2 < 90\% during a six minute walk test (6MWT)) and without EID?
Design: Observational, comparison study.

Participants: People with COPD, with and without EID

Outcome Measures: PA assessed and reported as the average of (i) number of steps per day, and (ii) time spent in sedentary ( 0 to < 1.5 METs) and moderate activity ( 3 to <6 METs), using a multi-sensor accelerometer (SenseWear, MF); Spirometry as a measure of disease severity; functional exercise capacity via two 6MWTs.

Results: 65 participants were recruited to the EID group (mean (SD) age 70 (7) years, FEV1 50 (20) \%predicted, nadir SpO2 84 (4)\%) and 77 participants to the no EID group (age 69 (8), FEV1 48 (14) \%predicted). PA levels were lower in the EID group compared to the no EID group with a lower average steps per day (2976 (2120) vs 5686 (2944), p<0.01), less time spent in moderate activity (23 (29) mins vs 50 (34) mins, $p<0.01$ ) and more time spent sedentary (19.7 (2) hrs vs 18.8 (2) hrs, p<0.05). Functional exercise capacity was lower in the EID group compared to the no EID group (6MWD 406 (97)m vs 479 (81)m, mean difference (95\% CI) 73m (43 to 102), $\mathrm{p}<0.01)$.

Conclusion: PA levels and functional exercise capacity were lower in people with COPD who had EID compared to those without EID, despite similar age and disease severity

Supported by: NHMRC project grant: 1019989

Key Practice Points:

- Oxygen saturation is an important measure during the 6WMT to identify people with EID

- Clinicians should be aware that people with EID could have a lower physical activity level compared to people without EID

- Clinicians should be aware that people with EID could have a lower functional exercise capacity compared to those without EID

\section{HOW TO USE A HAND-HELD DYNAMOMETER TO RELIABLY MEASURE SHOULDER STRENGTH}

McLaine SJ ${ }^{1}$, Ginn $\mathrm{KA}^{2}$, Shing $\mathrm{CM}^{1}$, Fell JW${ }^{1}$, Bird $\mathrm{ML}^{1}$

${ }^{1}$ School of Health Sciences, University of Tasmania, Launceston 2Discipline of Biomedical Science, Sydney Medical School, University of Sydney

Background: Quantitative testing of muscle strength can provide a useful foundation for clinical assessment, diagnosis, rehabilitation and research. Due to the portability and relatively low cost, the hand-held dynamometer is becoming a popular and useful tool in the clinic which places an increased importance in developing and teaching reliable testing protocols. Factors that may influence the results of hand-held dynamometer strength tests include: the type of test employed, strength and experience of the tester, patient position and stabilisation of the patient and tested limb.

Aims / objectives: We aim to share knowledge, experience and research findings relevant to the reliable use of a hand-held dynamometer and demonstrate a reliable protocol for using the hand-held dynamometer to test shoulder strength based on research outcomes. Upon completion of the workshop participants will: be familiar with factors that influence the reliability of hand-held dynamometer strength testing, perform reliable shoulder strength testing using a hand-held dynamometer and determine the magnitude of strength change that is meaningful.

Approach: A power point presentation will outline the literature and provide information relevant to using a hand-held dynamometer such as clinical application, reliability and limitations. A practical demonstration and an opportunity to practise will be offered.

\section{Conclusion / Key Practice Points:}

- Shoulder strength testing can be performed reliably using a hand-held dynamometer provided the technique is sound.

- Using evidence based practice, optimal positions for strength testing to enhance reliability can be chosen.

- With reliable strength testing a meaningful change in measures can be determined. 


\section{PROGRAM DEVELOPMENT AND MONITORING}

\section{McLellan $\mathrm{C}^{12}$}

${ }^{1}$ Bond University Institute of Health and Sport, Gold Coast, Queensland ${ }^{2}$ Brisbane Lions Australian Football Club, Brisbane, Queensland

The integration of portable monitoring strategies to determine athlete preparedness for training and competition has become commonplace in professional sport. During training and competition athletes regularly participate in high-intensity physical efforts, including running, repeated sprint activities, high speed changes of direction, accelerations, decelerations, jumping and landing. Team sport athletes in professional football codes and combat sports will experience frequent bouts of blunt force trauma associated with tackling and collisions. Sustained participation in training and competition over an extended in-season period may contribute to the manifestation of physiological and perceptual fatigue, increased injury risk and a subsequent decrement in performance. A systematic approach to athlete monitoring using subjective and objective strategies is key to the quantification of the demands of training, competition and recovery on a daily basis. Global Positioning Systems (GPS) and integrated tri-axial accelerometry are universally considered the predominant portable training and competition load analysis system in sport and exercise. Limitations exist with respect to isolated integration of GPS / accelerometry data to establish an individualised model of physiological preparedness for optimal sports performance. A more robust quantification of the influence of training and competition on daily activities may be provided via the incorporation of athlete monitoring strategies to identify the neuromuscular, endocrine and immunological profile characteristics of individuals to inform sports science and performance practitioners and facilitate individualised training interventions to optimise performance. This presentation will provide insight into current athlete monitoring techniques to determine the impact of training on daily activities in team sport athletes.

\section{Key Practice Points:}

- Understand the role GPS plays in the planning and monitoring of elite sport programs

- Gain insight into where GPS fits into the High Performance program and the monitoring of elite athletes

\section{HOW IS CULTURAL COMPETENCE BEING BUILT INTO PHYSIOTHERAPY EDUCATION? A SYSTEMATIC REVIEW}

McLoughlin $\mathrm{E}^{1}$, Phillips $\mathrm{C}^{1}$

${ }^{1}$ Australian National University, Canberra

Question: Where and how is cultural competence being incorporated into physiotherapy education? Should this be a part of future education? And how do we go about it?

Design: Systematic review of papers on teaching cultural competence in Physiotherapy synthesised into narrative format.

Outcome Measures: Narrative analysis / synthesis of qualitative data. Key data reviewed includes working definition of cultural competence and style/ model of teaching used.

Results: 14 articles met the inclusion criteria. No consensus about a best teaching model was reached. A number of the articles suggested a transformative learning approach that involved some sort of didactic teaching component and a placement component.

Conclusion: According to current literature the integration of cultural competency in physiotherapy education is poor. Factors contributing to this include limited understanding of the concept and excessive focus on clinical interaction. Without addressing these factors and underlying myths about culture it does not matter which teaching model is chosen. In order to successfully incorporate cultural competence into any physiotherapy curriculum we first need to reflect on our own culture and we need to develop educators who are experts in this field.

\section{Key Practice Points:}

- Understand a working definition of cultural competence

- Awareness of the relevance of cultural competence to

health professionals

- Awareness of advantages/ disadvantages of models of teaching cultural competence

- Awareness of ways to start incorporating greater cultural awareness into own practice/ teaching
COMMUNITY-BASED EXERCISE TRAINING IS

FEASIBLE, EFFECTIVE AND WELL ACCEPTED BY PEOPLE WITH CHRONIC RESPIRATORY AND CHRONIC CARDIAC DISEASE

McNamara RJ ${ }^{1}$, Dallimore $\mathrm{JT}^{2}, \mathrm{McKeough} \mathrm{Z}^{3}$, Dennis SM${ }^{3}$

IPrince of Wales Hospital, Sydney

Eastern Sydney Medicare Local, Sydney

3The University of Sydney, Sydney

Question: Is a community-based exercise program for people with chronic respiratory and/or chronic cardiac disease feasible and effective in improving exercise capacity? What is the level of participant and physiotherapist satisfaction?

Design: Prospective pilot study.

Participants: People with chronic respiratory and/or chronic cardiac disease referred to a hospital-based pulmonary rehabilitation program who attended an initial assessment and were offered community-based rehabilitation.

Intervention: Physiotherapist-supervised exercise program conducted in a local council gymnasium for 60 minutes, twice a week for eight weeks.

Outcome Measures: Eligibility and acceptance rates; adverse events; participant and physiotherapist satisfaction measured via a survey and semi-structured interview; 6-minute walk distance (6MWD); pulmonary rehabilitation waiting time.

Results: Of 76 people screened for eligibility for community-based exercise training, $67(88 \%)$ met the eligibility criteria. The community venue was chosen for exercise training by 32 people (48\%) (mean (SD) age $73(11)$ years; $n=21$ (66\%) female), with the remainder choosing the hospital venue. No adverse events occurred at the community venue. High satisfaction with the community venue was reported by participants and physiotherapists. Mean (SD) change in 6MWD was 41 (47) m compared to 43 (16) $\mathrm{m}$ in the comparison (hospital-based exercise training) group ( $p=$ 0.93). The median waiting time to access pulmonary rehabilitation over the six month trial of the additional community-based program decreased from 59 days to 21 days.

Conclusion: Supervised community-based exercise training for people with chronic respiratory and/or chronic cardiac disease is feasible, effective and well accepted by participants and health providers.

Funding: Eastern Sydney Medicare Local

\section{Key Practice Points:}

- A physiotherapist-supervised community-based exercise training for people with chronic respiratory and/or chronic cardiac disease is effective in improving exercise capacity.

- A community gymnasium is a well-accepted venue for exercise training by physiotherapists and people with chronic respiratory and/or chronic cardiac disease.

- Community gymnasiums could be utilised as an alternative venue to hospital-based gymnasiums for exercise training in people with chronic respiratory and/or chronic cardiac disease. 


\section{DEFINING THE SMALLEST WORTHWHILE EFFECT OF LAND-BASED AND WATER- BASED PULMONARY REHABILITATION FOR CHRONIC OBSTRUCTIVE PULMONARY DISEASE}

McNamara RJ ${ }^{1}$, Elkins $\mathrm{MR}^{2}$, Ferreira $\mathrm{ML}^{3}$, Spencer $\mathrm{LM}^{4}$, Herbert RD ${ }^{5}$ ${ }^{1}$ Prince of Wales Hospital, Sydney

2The University of Sydney, Sydney

${ }^{3}$ George Institute for Global Health \& Institute for Bone and Joint Research, Sydney

${ }^{4}$ Royal Prince Alfred Hospital, Sydney

${ }^{5}$ Neuroscience Research Australia (NeuRA), Sydney

Question: What is the smallest worthwhile effect of pulmonary rehabilitation on the 6-minute walk distance among people with chronic obstructive pulmonary disease (COPD)? Is the smallest worthwhile effect different when the exercise training component of pulmonary rehabilitation is water-based instead of land-based?

Design: Benefit-harm trade-off method.

Participants: 100 people with COPD referred to outpatient pulmonary rehabilitation who had completed two baseline 6 -minute walk tests at the commencement of pulmonary rehabilitation.

Intervention: Participants were presented with two scenarios: 8 weeks of land-based and 8 weeks of water-based pulmonary rehabilitation. Each scenario included the associated costs, risks and inconvenience. Participants were guided through an iterative process on a computer that allowed them to progressively refine their estimates of the smallest improvement in 6-minute walk distance due to each form of pulmonary rehabilitation that would outweigh the costs, risks and inconvenience.

Outcome Measures: 6-minute walk distance (metres).

Results: One hundred people with COPD participated (mean (SD) age 72 (9) years; FEV1 54 (16) \% predicted; baseline 6-minute walk distance 377 (101) metres). For land-based pulmonary rehabilitation, the median smallest worthwhile effect was 20 metres ( $95 \% \mathrm{Cl} 15$ to 37 ). For waterbased pulmonary rehabilitation, the median smallest worthwhile effect was 26 metres (95\% Cl 15 to 33). These estimates did not differ significantly $(p=0.10)$.

Conclusion: People with COPD typically perceive that pulmonary rehabilitation would be worthwhile if it increased the 6-minute walk distance by about six percent. The smallest worthwhile effects of landbased and water-based pulmonary rehabilitation were similar.

Funding: Physiotherapy Research Foundation Tagged Aquatic Grant Key Practice Points:

- The smallest worthwhile effect for the 6-minute walking test in people with COPD is 20 metres for land-based pulmonary rehabilitation and 26 metres for water-based pulmonary rehabilitation. These estimates provide the most valid and interpretable estimates to date of the smallest worthwhile effect of the 6-minute walk test following pulmonary rehabilitation.

- In the opinions of people with COPD, the costs, risks and inconvenience of water-based exercise training as part of pulmonary rehabilitation are not substantially greater than those of land-based exercise training. Given there is no significant difference between the two approaches in terms of effect on the 6 -minute walk distance, the current findings support the conclusion that water-based exercise training could be offered as part of a pulmonary rehabilitation program.

\section{REMOTE-COMMUNICATION TECHNOLOGIES TOIMPROVE ADHERENCE TO EXERCISE AND PHYSICAL ACTIVITY RECOMMENDATIONS FROM PHYSIOTHERAPISTS: WHICH TECHNOLOGIES DO PATIENTS WITH MUSCULOSKELETAL DISORDERS PREFER?}

\section{McPhail SM ${ }^{1,2}$, Schippers $M^{1,2}$, Marshall $A^{5,6}$}

${ }^{1}$ Centre for Functioning and Health Research, Metro South Health, Cnr of Ipswich Road and Cornwall Street, Buranda, Brisbane, QLD Institute of Health and Biomedical Innovation and School of Public Health and Social Work, Oueensland University of Technology, Victoria Park Road, Kelvin Grove, Brisbane, QLD

Question: What are patients' with musculoskeletal disorders preference ratings for five possible modalities of receiving remote-communication support for the uptake of recommended exercise and physical activity?

Design: Cross-sectional preference rating study.

Participants: Participants ( $n=221,75 \%$ of eligible patients) from three hospital outpatient physiotherapy clinics were recruited to include a representative sample from the target clinical population.
Outcome Measures: The main outcomes were preference ratings for "how much" patients wanted to receive physical activity support via each of the five potential communication modalities. The five ratings were generated on the same horizontal analogue rating scale where 0 represented "not at all" and 10 represented "very much". Generalized linear modelling was used to examine patient factors associated with preference ratings.

Results: Printed communications had the highest median preference rating (7/10), followed by email and telephone (both 5/10), text messaging $(1 / 10)$ and private social network messages (0/10). Desire to be referred to a physical activity intervention was associated with higher preference ratings for printed materials (coefficient $=2.739, p<0.001$ ), telephone calls (coefficient $=3.000, p<0.001$ ) and email (coefficient $=2.059, p=0.02$ ). Older age was associated with lower preference for email (coefficient= $-0.100, p<0.001$ ), texting (coefficient $=-0.096, p<0.001$ ), and social network private messages (coefficient $=-0.065, p<0.001$ )

Conclusion: Promoting exercise adherence via print materials, email or telephone calls are worthy of further investigation. A limitation of this study is that it only examined patient preferences not effectiveness.

\section{Key Practice Points:}

- Most ( $n=155,70 \%$ ) patients wanted support to be more physically active.

- Patients' typically preferred the least novel communication modalities.

- Printed materials, telephone calls and email were the most preferred communication modalities.

\section{HOW TO INCORPORATE HEALTH ECONOMICS INTO YOUR RESEARCH OR SERVICE EVALUATION}

McPhail SM1,2, Comans TA ${ }^{3,4}$, Haines TP5, 6

${ }^{1}$ Centre for Functioning and Health Research, Metro South Health

Institute of Health and Biomedical Innovation, Queensland University of Technology

${ }^{3}$ Menzies Institute Queensland, Griffith University

${ }^{4}$ Metro North Hospital and Health Service

${ }^{5}$ School of Physiotherapy, Monash University

${ }^{6}$ Allied Health Research Unit, Monash University

Background: Determining the value for money of health services is a key consideration in today's resource constrained environment. The inclusion of appropriate economic evaluations in research, service evaluation and model of care planning contexts has become a necessity for those who wish to influence policy or practice, including the development or refinement of clinical services. Physiotherapy interventions are typically 'low cost' relative to other health interventions. The appropriate generation and use of economic data will enhance the translation of cost-effective interventions and service models into clinical practice and influence healthcare policy.

Aims / objectives: The objective of this practical session is to introduce and learn about the key types of economic evaluations, and 'how to' go about incorporating health economics into research or health service evaluations and planning activities.

Approach: This session will be an interactive workshop format comprising of a brief lecture introducing the key principals of economic evaluation in healthcare contexts with a specific focus on 'cost-effectiveness'. This will be follow by practical material, including planning a simple mock economic evaluation. This session will conclude with a brief interactive 'panel' discussion with opportunity for delegates to ask specific questions. Delegates will also be provided with 'take home' written resources summarising the content of the workshop.

\section{Conclusion / Key Practice Points:}

- Understand the most common types of economic evaluations and how they are used.

- Know how to interpret 'cost-effectiveness' information.

- Gain familiarity with the fundamental steps required for inclusion of health economics in research and health service evaluation contexts. 
HOW TO USE CONTEMPORARY

SMARTPHONE TECHNOLOGIES TO

ENHANCE CARE BY PROMOTING

POSITIVE LIFESTYLE BEHAVIOURS,

EXERCISE ADHERENCE AND AUTOMATE

REMOTE-MONITORING FUNCTIONS

\section{McPhail SM ${ }^{1,2}$}

${ }^{1}$ Centre for Functioning and Health Research, Metro South Health 2Institute of Health and Biomedical Innovation, Queensland University of Technology

Background: Smartphone ownership in Australia continues to reach unprecedented levels. Contemporary smartphones contain a range of technologies that have potential application in clinical practice. This not only includes potential for remote-telecommunication, but automated monitoring of a range of clinical factors and lifestyle behaviours as well as opportunities to enhance patient motivation and adherence to clinical recommendations. Research evidence supporting potential uses of smartphones in clinical settings is rapidly advancing.

Aims / objectives: This practical session will introduce delegates to a selection of readily accessible smartphone functions and apps that have potential for use in clinical practice.

Approach: This session will be a practical and interactive workshop comprising of discussions, demonstrations and a number of practical 'walk-through' opportunities for the potential use of smartphone apps and hardware. The focus of the session will be on how to use smartphone technologies to support patients' adherence to clinical recommendations in the context of patients' day-to-day lives between clinic sessions. This will include use of hardware as well as freely available apps for iOS (iPhone) and Android devices. There will be opportunity for delegates to share their own experiences, practical tips and discuss the latest research literature.

\section{Conclusion / Key Practice Points:}

- Delegates will learn how smartphone functions (hardware and software dependent) can be incorporated into their clinical practice.

- Delegates will gain familiarity with useful and accessible smartphone apps that have potential to enhance patients' lifestyle behaviours.

- Delegates will learn how to use freely available software and webinterfaces for remote-monitoring (with and without automation).

HOW TO EVALUATE MEANINGFUL

\section{CHANGE IN HEALTH-RELATED QUALITY OF LIFE AMONG OLDER ADULT POPULATIONS}

\section{McPhail SM ${ }^{1,2}$}

${ }^{1}$ Centre for Functioning and Health Research, Metro South Health Institute of Health and Biomedical Innovation, Queensland University of Technology

Background: Physiotherapy interventions for older adults typically endeavour to improve patients' health-related quality of life in a meaningful way. Use of patient reported outcomes, including healthrelated quality of life questionnaires, has become commonplace in research and clinical practice in the field gerontology. Yet a number of unique challenges and potential for bias exist when attempting to evaluate change in health-related quality of life among older adults.

Aims / objectives: This workshop will explain and explore evidence based approaches to evaluating meaningful change in health-related quality of life among older adults receiving healthcare interventions.

Approach: This session will be an interactive workshop including an entertaining but informative exploration of potential challenges that exist when evaluating health-related quality of life among older adults. This will include an initial brief discussion of potential biases when evaluating meaningful change in quality of life, interactive practical case scenarios, opportunity for delegates to experience simulated biases during mock quality of life evaluations and a concluding discussion regarding the interpretation of patient-reported health-related quality of life information and its potential use in research and clinical practice.

\section{Conclusion / Key Practice Points:}

- Delegates will learn how to select an appropriate patient-reported outcome measure to evaluate health-related quality of life among older adults.

- Delegates will learn how to interpret health-related quality of life scoring (and change scores) in the context of their practice.

- Delegates will learn how to identify and avoid biases that threaten valid evaluation of health-related quality of life among older adults.
HOW TO SELECT AND IMPLEMENT THE BEST PATIENT-REPORTED OUTCOME MEASURES FOR CLINICAL PRACTICE AND RESEARCH AMONG PATIENTS WITH NEUROLOGICAL CONDITIONS

\section{McPhail SM ${ }^{1,2}$}

${ }^{1}$ Centre for Functioning and Health Research, Metro South Health 2Institute of Health and Biomedical Innovation, Queensland University of Technology

Background: Use of patient-reported outcome measures has become a core part of neurological physiotherapy research and practice. Patientreported outcome measures have potential to enable clinicians and researchers to gain important insight into the patients' perspective. The number of patient reported outcomes available for use in clinical practice has been growing exponentially. Jargon-filled reports of questionnaire measurement properties can be confusing for the uninitiated and key considerations for their valid use among people with neurological conditions are often not considered.

Aims / objectives: This workshop will explore key practical and evidenced based considerations for selecting appropriate patient reported outcomes for use among people with neurological conditions.

Approach: An interactive workshop format will include a discussion of important considerations when selecting questionnaires for use in clinica practice and research contexts. This will include typical and atypical case scenarios that will provide delegates with opportunity to work together to select'the best' patient reported outcome measures in a variety of contexts relevant to the practice of neurological physiotherapy. The session will conclude with an interactive discussion, where delegates will have opportunity to share their experiences and favourite (or least favourite) patient reported outcomes for use among patients with neurological conditions.

\section{Conclusion / Key Practice Points:}

- Delegates will gain an understanding of the essential and desired measurement properties of questionnaires.

- Delegates will learn key tips and tricks for interpreting whether an outcome measure is suitable for use in clinical practice.

- Delegates will have opportunity to learn from one another's experience using patient reported outcomes among people with neurological conditions.

\section{DRAINING THE SWAMP - PREVENTING WORK-RELATED MUSCULOSKELETAL DISORDERS}

\section{McPhee B}

Jim Knowles Group, Kurri Kurri NSW

There is a saying: 'It's hard to think about draining the swamp when you are up to your neck in crocodiles'. For me the swamp is prevention and the crocodiles are the floods of preventable disorders that treating physiotherapists and others are seeing every day in clinics all over Australia. I believe that there are three areas of major challenge in the prevention of musculoskeletal at work disorders - and therefore for physiotherapists - in $21^{\text {st }}$ century Australia: sedentary work and work postures, demanding and poorly designed manual tasks and exposure to harmful Whole-body Vibration (WBV) in mobile machinery.There is excellent research being done on sedentary work and the impact on health and the musculoskeletal system. Prevention strategies include: workplace and task design and work organisation. Designing out unsafe manual tasks has been the focus of Codes of Practice, legislation and extensive activity in Australia for the past 30 years. In some respects we have succeeded in getting stakeholders to recognise the manual tasks that will cause harm and sometimes we have addressed these successfully.

Exposure to WBV on the other hand is only now being recognised as a major contributor to back and neck pain. Usually symptoms arise slowly and are non-specific. The cause-effect relationship makes sense but until recently we have had no evidence of the nature and scale of the problem and even less evidence on its contribution to spinal damage and symptoms. However there are now a range of tools to help health practitioners identify and deal with harmful WBV exposures.

\section{Key Practice Points:}

- Prevention of musculoskeletal disorders at work

- Whole-body Vibration and back pain. 


\section{MOVING BEYOND "KICK FASTER": \\ WHAT IS THE CLINICAL UTILITY OF SPORTS BIOMECHANICS FOR PARALYMPIC SWIMMERS?}

\section{Mellifont $\mathrm{D}^{1}$, Formosa $\mathrm{D}^{2}$, Burkett $\mathrm{B}^{1,3}$}

${ }^{1} \mathrm{CHI}$ - Cluster for Health Improvement, University of the Sunshine Coast, Queensland, Australia

Paralympic Sports Science \& Sports Medicine Coordinator,

Swimming Australia

${ }^{3}$ Paralympic Performance Scientist, Swimming Australia \& Professor of Sports Biomechanics

The elite nature of Paralympic swimming, heterogeneity of athletes and the aquatic environment combine to pose enormous challenges for the development of high level research evidence. However, Paralympic swimming case vignettes provide an invaluable opportunity to explore the unique adaptive biomechanics of athletes with disabilities. Enhanced understanding of these adaptations assists clinicians in targeting strategies for performance enhancement, injury prevention and management. Furthermore, quantitative sports biomechanics may provide clinically important insights that may be missed by even the most discerning qualitative movement analysis.

\section{Key Practice Points:}

- Biomechanical analysis of athletes with disabilities assists in the understanding of unique adaptive movement strategies. Common patterns of movement strategies can be observed in different groups (classification and disability) of Paralympic swimmers. However, individual quantitative and qualitative analysis of movement assists clinicians in targeting tailored strategies for performance enhancement, injury prevention and management.

\section{THE DIGITALLY CONNECTED PATIENT: PARTICIPATORY HEALTH AND PHYSIOTHERAPY}

\section{Merolli M}

${ }^{1}$ Health and Biomedical Informatics Centre, Melbourne Medical School, The University of Melbourne

Participatory health continues to be shaped by the evolution of the Internet and digital media. This is creating a shift in patient-centric care. Increased connectivity, access to information, and social networking are at the centre of a new paradigm in patient management. The ePatient: engaged, empowered, enthusiastic and electronic is keen to be an active partner in managing their conditions and participatory health enabling technologies (PHETs) such as: social media, mobile devices and apps, and self-quantification tools are allowing them to do so. PHETs relevant to physiotherapists might include: condition specific social networks, patient-led communities, self-management apps and, self-tracking devices. Familiarity with PHETs will allow physios to: understand their current place in patient management, converse with patients on a more technological level, and explore innovative tools with the potential to influence practice in years to come.

\section{Key Practice Points:}

- The Internet and digital media continue to influence patient-centric care

- Participatory Health Enabling Technologies (PHETs) are enabling ePatients to be more active partners in their own health management

- Examples of PHETs in physiotherapy might include: condition specific social networks, patient-led communities, self-management apps and, self-tracking devices

- Familiarity with PHETs will allow physios to: understand their place in patient management, converse with patients on a more technological level, and explore innovative tools with the potential to influence management.

\section{IS POSTURAL ALIGNMENT IMPAIRED DURING MOBILITY FOR ADULTS AFTER ACQUIRED BRAIN INJURY? A SYSTEMATIC REVIEW}

Mills S] ${ }^{1}$, Mackintosh SF², McDonnell MN², Thewlis D2

${ }^{1}$ South Australian Brain Injury Rehabilitation Service

ZUniversity of South Australia

Design: Systematic review of the literature.

Participants: Adults with acquired brain injury, including stroke, traumatic brain injury, hypoxic brain injury.

Results: 12 studies were included from the search. Studies were rated using the National Health \& Medical Research Council Levels of Evidence; due to the low volume of evidence, all levels were included. There is some evidence to demonstrate that postural alignment is impaired after acquired brain injury, during sit-to-stand, standing, and walking. Increased forward inclination of the trunk in standing, and increased amplitude of trunk motion during walking have been demonstrated. A stable, upright trunk during running has been observed consistently in one study, potentially indicating that this is a key factor to achieve running after acquired brain injury.

Conclusion: There is limited evidence to show that postural alignment is impaired after acquired brain injury. Despite the high number of studies of biomechanics during mobility, most of them studying walking, many of these limit their analysis to hips, knees and ankles. The lack of studies including alignment of trunk and head leaves a relative gap in understanding how the whole body operates during mobility after acquired brain injury

\section{Key Practice Points}

- Lack of stable upright posture may be associated with balance and mobility impairment after acquired brain injury

- The evidence that currently exists suggests that analysis of postural alignment in clinical practice and in biomechanical research is warranted

- Further research is needed to explore the relationship between postural alignment and mobility after acquired brain injury

\section{THE RELATIONSHIP BETWEEN MOTOR PROFICIENCY AND READING ABILITY IN YEAR 1 CHILDREN: A PROSPECTIVE COHORT STUDY}

Milne $\mathbf{N}^{1}$, Cacciotti $\mathrm{K}^{1,2}$, Davies $\mathrm{K}^{2}$, Orr R

${ }^{1}$ Faculty of Health Sciences and Medicine, Bond University Department of Education, Training and Employment, QLD

Question: Is there a relationship between motor proficiency and reading skills in Year 1 children?

Design: Prospective cohort study.

Participants: A single class of Year 1 students $(n=24)$ aged 5 to 7 years (females $n=11$; males $n=13$, mean age $=6.07 \pm 0.35$ years).

Outcome Measures: The Process Assessment of the Learner (PALII) (reading components only); the Bruininks-Oseretsky Test of Motor Proficiency $-2^{\text {nd }}$ Edition; parent-reported height/weight measurements; and retrospective academic reports from Preparatory year were assessed during regular class time.

Results: For children with high-to-very-high English grades, moderate negative associations were identified between total motor proficiency and reading skills (silent reading fluency SSF: $r=-.682, p=0.01$, sentence comprehension SSA: $r=-.596, p=0.03$ ). For children with average-to-low English grades, a stronger negative relationship existed between total motor proficiency and silent reading fluency (SSF: $r=-.815, p=0.48$ ), however moderate positive relationships were also found between total motor proficiency and reading skills (phonological decoding fluency: $r=0.716, p=0.02$ and phonological decoding accuracy: $r=670, p=0.34$ ) for this group.

Conclusion: These study results suggest that a relationship between motor proficiency and key reading skills exists, particularly in children with average-to-low English grades. Further research is warranted to investigate if interventions focused on motor skill development can enhance reading ability for Year 1 children.

\section{Key Practice Points:}

- Positive associations exist between physical activity and increased academic performance.

- Investigating the links between children's motor proficiency and reading outcomes may help provide optimal academic outcomes, particularly for those struggling to read.

- This study suggests a relationship between motor proficiency and reading exists in young children. 


\section{MODIFIED SHUTTLE TEST-PAEDS: A VALID CARDIORESPIRATORY FITNESS MEASURE FOR CHILDREN}

Milne $\mathrm{N}^{1}$, Simmonds $\mathrm{MJ}^{1,2}$, Hing $\mathrm{W}^{1}$

${ }^{1}$ Faculty of Health Sciences and Medicine, Bond Institute of Health and Sport, Bond University, Gold Coast, Australia

2Heart Foundation Research Centre, Griffith University, Gold Coast, Australia

Questions: What is the concurrent and predictive validity of the Modified Shuttle Test-Paeds (MSTP); a measure of cardiorespiratory fitness in children that does not require 'drop-out' for completion? What is the strength of the relationship between the MSTP and VO2peak, compared to that of the commonly used 20m Multi-Stage-Running-Test (20m-MSRT)?

Design: A concurrent validation study.

Methods: A convenience sample of 25 school-aged children (age: 6-16 yr male/female: 19/5; BMl: $21 \pm 9 \mathrm{~kg} / \mathrm{m}^{2}$ ) participated in this study. Physical measures included: Bruininks-Oseretsky Test of Motor Proficiency-2 ${ }^{\text {nd }}$ Edition (BOT2), VO2peak, 20m-MSRT and MSTP, body composition and basic anthropometry.

Results: Mean cardiorespiratory fitness results included: V02 peak: 43.8 $\pm 11.2(\mathrm{~mL} / \mathrm{kg} / \mathrm{min}) ; 20 \mathrm{~m}$ MSRT: 5.48 \pm 2.96 (level); MSTP: $22.10 \pm 3.05$ (no.). A significant and strong predictive relationship existed between V02peak and MSTP $\left(r^{2}=0.749, p<0.01\right)$. The relationship between V02peak and 20m MSRT was significant and moderately strong $\left(r^{2}=0.486, p<0.001\right)$

Conclusions: The MSTP is a valid measure of cardiorespiratory fitness with a high predictive validity for estimating VO2peak in children, using a simple equation that is clinically applicable.

\section{Key Practice Points:}

- Alternative measures of cardiorespiratory fitness that do not support a 'drop-out' nature to testing are sought in QLD schools.

- The MSTP is a fun and engaging cardiorespiratory fitness measure that is valid with a strong ability to predict a child's peak oxygen consumption level from a 3-minute field test.

- The MSTP should be considered as an alternative measure for predicting V02peak, especially in environments where there are sensitivities to measuring cardiorespiratory fitness in children with diverse fitness abilities (e.g. school environments)

\section{EXAMINATION OF SPASTICITY AND WEAKNESS IN PEOPLE WITH FRIEDREICH ATAXIA}

Milne SC1,2,3, Yiu E ${ }^{1}$, Tai G ${ }^{1}$, Georgiou-Karistianis $N^{3}$ Delatycki $\mathrm{MB}^{1,4}$, Corben LA ${ }^{1,3,5}$

${ }^{1}$ Bruce Lefroy Centre for Genetic Health Research, Murdoch Childrens Research Institute, Melbourne

2Physiotherapy Department, Kingston Centre, Monash Health, Melbourne

${ }^{3}$ School of Psychological Sciences, Monash University, Melbourne

${ }^{4}$ Department of Clinical Genetics, Austin Health, Melbourne

${ }^{5}$ Occupational Therapy Department, Monash Medical Centre, Monash

Health, Melbourne

Question: What is the prevalence and character of upper and lower limb spasticity in people with Friedreich ataxia?

Design: Cross-sectional study.

Participants: Forty-six individuals with Friedreich ataxia.

Intervention: Three physiotherapists and an occupational therapist used the Modified Tardieu Scale to examine spasticity. The occupational therapist also tested muscle strength to examine the upper limb.

Outcome Measures: Modified Tardieu Scale measured muscle length and spasticity in the gastrocnemius, soleus, flexor digitorum profoundus/ superficialis and flexor pollicis longus. The Manual Muscle Test was used to measure strength of the intrinsic musculature of the hand. The Functional Independence Measure quantified functional ability.

Results: In the lower limbs all participants had spasticity in at least one muscle examined, and 69\% of non-ambulant participants had contracture in one or both of their gastrocnemius muscles. The Functional Independence Measure demonstrated significant correlations with gastrocnemius muscle length and angle of catch. $73 \%$ of participants demonstrated spasticity in wrist and finger flexors, 46\% had contracture in at least one joint in their hands, $86 \%$ demonstrated weakness in the intrinsic musculature of the hand.

Conclusion: There is a significant incidence of lower limb and upper limb spasticity in people with Friedreich ataxia.

\section{Key Practice Points:}

- Spasticity in the lower limb muscles is related to a reduction in function and therefore should be assessed in people with Friedreich ataxia.

- Weakness in the upper limbs is present in people with Friedreich ataxia and therefore should be treated appropriately.

- Spasticity management in people with Friedreich ataxia should be considered to ensure optimal function.
THE VICTORIAN ASSISTANT WORKFORCE MODEL, ALLIED HEALTH- THE RIGHT TASK, THE RIGHT WORKER

Milne $\mathrm{SC}^{1}$, Davis $A \mathrm{M}^{1}{ }^{1}$, Somerville $\mathrm{L}^{2}$, Thomson $\mathrm{A}^{2}$, Philip $\mathrm{K}^{3}$

IMonash Health, Melbourne

${ }^{2}$ Alfred Health, Melbourne

${ }^{3}$ Victorian Department of Health and Human Services, Melbourne

Question: Are allied health assistants utilised to their full scope of practice? Does the Victorian Assistant Workforce Model provide a successful framework to increase physiotherapy workforce capacity?

Design: Qualitative data collected via focus groups and a quantitative staff survey to scope the capacity for the assistant workforce.

Participants: Fifty-one health care organisations across Victorian rural/regional, metropolitan health services, and community and ambulatory services.

Intervention: Quantification surveys distributed to physiotherapists to identify time spent on tasks that could be reallocated to allied health assistants. Staff satisfaction surveys and focus groups provided qualitative information in regard to barriers, issues and opportunities for an assistant workforce.

Outcome Measures: Current physiotherapy and assistant workforce staffing profiles, staff job satisfaction and confidence in delegation were measured. Physiotherapy full time equivalent that could be delegated to an allied health assistant was measured.

Results: Rural and regional health services identified $21.9 \%$ of

physiotherapy full time equivalent that could be reallocated. Metropolitan tertiary health services identified $16.9 \%$ and metropolitan community and ambulatory services identified $18.0 \%$ of full time equivalent that could be allocated to an allied health assistant.

Conclusion: The Victorian Assistant Workforce Model enables service redesign so that the physiotherapy workforce can be suitably configured with physiotherapists and assistants to match the right task to the right worker.

\section{Key Practice Points:}

- Workforce capacity building ensures health workforce skills are delivered in the most optimal context.

- There is significant opportunity to reform the current physiotherapy workforce.

- The Victorian Assistant Workforce Model is a successful framework that assists health care organisations strategically align their workforce into the future. 


\section{AQUATIC PHYSIOTHERAPY FOR PEOPLE WITH FRIEDREICH ATAXIA}

Milne $\mathrm{SC}^{1,2,3}$, Yiu $\mathrm{E}^{1}$, Murphy $\mathrm{A}^{4,5}$, Georgiou-Karistianis $\mathrm{N}^{3}$, Corben LA ${ }^{1,3,6}$, Delatycki MB ${ }^{1,7}$

${ }^{1}$ Bruce Lefroy Centre for Genetic Health Research, Murdoch Childrens Research Institute, Melbourne

2Physiotherapy Department, Kingston Centre, Monash Health, Melbourne

${ }^{3}$ School of Psychological Sciences, Monash University, Melbourne

${ }^{4}$ MONARC, Faculty of Medicine, Nursing and Health Sciences, Monash University, Melbourne

${ }^{5}$ Clinical Research Centre for Movement Disorders and Gait, Kingston Centre,

Monash Health, Melbourne

${ }^{6}$ Occupational Therapy Department, Monash Medical Centre, Monash Health, Melbourne

'Department of Clinical Genetics, Austin Health, Melbourne

Question: Does rehabilitation consisting of aquatic physiotherapy improve the function of people with Friedreich ataxia?

Design: Single-blinded randomised controlled trial with wait-list control.

Participants: Thirty-two people, both ambulant and wheelchair dependent, aged 15 and above, with genetically confirmed Friedreich ataxia, will be recruited.

Intervention: Six weeks of three days per week of land-based and aquatic physiotherapy. Rehabilitation will be broken down into seven domains: coordination and control exercises, functional mobility, strengthening, cardiovascular fitness and muscle endurance, core-stability, balance training, and stretching. The control group will be wait-listed to receive rehabilitation after a six week delay.

Outcome Measures: The Functional Independence Measure, Berg Balance Scale, spatiotemporal gait parameters and the Patient Global Impression of Change will be assessed; at baseline, six weeks and 12 weeks.

Results: Preliminary results will be presented. Physiotherapy techniques and exercises are performed utilising the physical properties of water, such as buoyancy, heat, turbulence and increased viscosity. Individuals can exercise without having to work against the force of gravity; complete balance exercises with decreased speed of balance reactions; progress strengthening exercises gradually from a level lower than gravity; and increase flexibility of their muscles.

Conclusion: The hydrotherapy pool provides a suitable environment to achieve therapeutic goals in individuals with Friedreich ataxia. In addition the manual handling requirement by the physiotherapist is reduced in this environment.

\section{Key Practice Points:}

- Aquatic physiotherapy should be considered in the rehabilitation of people with Friedreich ataxia.

- Properties of water maximise therapeutic gains.

- Aquatic physiotherapy provides benefit to ambulant and non-ambulant people with Friedreich ataxia.

\section{DIFFERENTIAL ACTIVATION OF THE HIP ADDUCTOR MUSCLES DURING A SIMULATED WEIGHT-BEARING TASK}

Hides $]^{2}$, Beall $\mathrm{P}^{1}$, Franettovich Smith $\mathrm{MM}^{2}$, Stanton $\mathrm{W}^{2}$, Miokovic $\mathrm{T}^{2}$, Richardson $\mathrm{C}^{1}$

1 Division of Physiotherapy, School of Health and Rehabilitation Sciences, St Lucia Campus, The University of Queensland, Brisbane, Australia ${ }^{2}$ Centre for Musculoskeletal Research, Mary Mackillop Institute for Health Research, Australian Catholic University, Brisbane, Australia

Question: To investigate and quantify the pattern of muscle activation of the individual hip adductor muscles (adductor magnus and adductor longus) during a standardised simulated unilateral, weight-bearing task.

Design: A repeated measures design.

Participants: 20 healthy individuals (11 females, 9 males) ranging in age from 20 to 25 years participated in the study.

Outcome Measures: Surface electromyography (EMG) recordings from adductor magnus and adductor longus muscles were taken at levels representing 10 to $50 \%$ of body weight during a simulated weightbearing task. EMG data were normalised to maximal voluntary isometric contraction.

Results: The adductor magnus was recruited at significantly higher levels than the adductor longus muscle during a simulated weight-bearing task performed across 10 to $50 \%$ of body weight ( $p<0.01$ ).

Conclusions: Adductor magnus and adductor longus muscles are recruited to different extents during a simulated weight-bearing task. The results of this study may assist in improving exercise selection and specificity in the development of prevention and management strategies for groin strains.

\section{Key Practice Points:}

- Slow, controlled weight-bearing through the heel, with the hip, knee and ankle in good alignment, recruited the adductor magnus muscle to a greater extent than the adductor longus muscle across all levels of percentage body weight tested.

- Closed chain exercises with weight-bearing through the lower limb are more likely to recruit the adductor magnus muscle over the adductor longus muscle.

\section{BRIEF BIOPSYCHOSOCIALLY INFORMED EDUCATION IMPROVES INSURANCE WORKERS' PAIN BELIEFS AND CLAIM MANAGEMENT BEHAVIOR - IMPLICATIONS FOR IMPROVING OUTCOMES FOR INJURED WORKERS}

Mitchell $\mathrm{T}^{1}$, Beales $\mathrm{D}^{1}$, Pole $\mathrm{N}^{2}$, Weir $\mathrm{J}^{2}$

${ }^{1}$ School of Physiotherapy and Exercise Science, Curtin University, Western Australia

2CGU Insurance Limited

Question: Does biopsychosocially informed education about musculoskeletal conditions for insurance workers have a positive impact on their pain beliefs and their claims management behaviors?

Design: A pre-, post and medium term follow-up of an education intervention.

Participants: Insurance workers (claims managers and injury management advisors) operating in the Western Australian workers' compensation system.

Intervention: Two 90-minute sessions of biopsychosocially informed education focusing on understanding and identifying barriers to recovery of injured workers with musculoskeletal conditions from an evidencebased perspective.

Outcome Measures: Pain beliefs were assessed pre-education immediately post-education and at three-month follow-up (Back Beliefs Questionnaire \& Health Care Providers' Pain and Impairment Relationship Scale). Self-reported and Injury Management Advisor-reported assessment of change in claims management behaviours were also collected at the three-month follow-up.

Results: There were positive changes in the Health Care Providers Pain and Impairment Relationship Scale $(p=.009)$ and Back Beliefs Questionnaire $(p=.049)$ immediately following the education that were sustained at the three-month follow-up. Positive changes in claims management behaviours were supported by the qualitative data.

Conclusion: This study provides evidence that a brief biopsychosocially informed education program can positively influence insurance workers' beliefs regarding musculoskeletal conditions.

\section{Key Practice Points:}

- Insurance workers are important stakeholders in the rehabilitation of injured workers with musculoskeletal conditions.

- Brief education can positively influence insurance workers' beliefs and is associated with positive changes in claims management behaviours.

- This intervention has potential to facilitate improvements in claims management outcomes for injured workers. 


\section{EVALUATION OF COMMUNITY EXERCISE CLASSES TARGETING INDIVIDUALS WITH CARDIOVASCULAR DISEASE}

Moghaddas $\mathrm{D}^{1,2}$, Snodgrass S $]^{1,2}$, Callister R ${ }^{2,3}$

${ }^{1}$ Discipline of Physiotherapy, School of Health Sciences, The University of Newcastle, Newcastle

2Priority Research Centre for Physical Activity and Nutrition, The University of Newcastle, Newcastle

3University of Newcastle, School of Biomedical Sciences and Pharmacy, The University of Newcastle, Newcastle

Question: What are the features of community exercise classes for individuals with long-term cardiovascular disease (CVD), including class components, exercise intensity and characteristics of class participants?

Design: Cross-sectional observation study.

Participants: Exercise class participants $(n=82)$ attending 12 community exercise classes identified by their listing on the National Heart Foundation (NHF) website as providing Heartmoves ${ }^{\oplus}$, a community-based cardiac rehabilitation program developed by the NHF.

Outcome Measures: Class components observed, exercise intensity: mean and highest heart rate as a percentage of age-predicted maximum (HR) for each class component, and 10-point perceived exertion scale (RPE), and class participant characteristics (questionnaire). Data were compared to international guidelines (NHF, American Heart Association, Scottish Intercollegiate Guidelines Network) for community exercise classes for individuals with CVD.

Results: All classes included guideline-recommended components of warm-up, cool down, strength and aerobic conditioning; $58 \%(n=7)$ of classes incorporated strength and aerobic conditioning in a circuit. Class participants exercised at low to moderate intensities as indicated by HR (mean warm-up 55 bpm, SD 11; cool down 52, SD 11; strength 59, SD 11; aerobic conditioning 58, SD 12) and overall RPE (6+2). Class participants' mean age was 70 years (SD 8); $27 \%(n=24)$ were referred by health professionals, $73 \%(n=61)$ attended for fitness, $61 \%(n=50)$ had CVD risk factors, and $21 \%(n=17)$ reported diagnosed CVD.

Conclusion: Community-based exercise classes for individuals with CVD comply with international guidelines for recommended components and intensities for ongoing cardiac rehabilitation. Only a small proportion of class participants reported diagnosed CVD.

\section{Key Practice Points:}

- Community-based exercise classes designed for individuals with CVD are safe for these participants, as they meet international guidelines for appropriate class components and exercise intensities for long-term community-based cardiac rehabilitation.

- Community-based exercise classes for individuals with CVD include participants with CVD risk factors and other chronic health-related conditions, as well as healthy older participants wishing to maintain general fitness.

- As only a small proportion of class participants were referred by health professionals, there is an opportunity for physiotherapists to refer patients with CVD to community-based exercise classes as these are a safe environment for patients with CVD.

\section{COMPARISON OF SUPRASPINATUS}

MUSCLE ARCHITECTURE DURING THREE DIFFERENT SHOULDER STRENGTHENING EXERCISES USING CROSS-SECTIONAL ANALYSIS BY ULTRASONOGRAPHY

Moon IY1 ${ }^{1} \mathrm{Lim} \mathrm{OB}^{1}, \mathrm{Cynn} \mathrm{HS}^{2}, \mathrm{YiCH}^{2}$

${ }^{1}$ Dept. of Physical Therapy, The Graduate School, Yonsei University, Wonju, Republic of Korea

2Dept. of Physical Therapy, College of Health Science, Yonsei University, Wonju, Republic of Korea

Question: How does the supraspinatus muscle change under external load ( $5 \%$ of the body mass) during three different isometric rehabilitation exercises?

Design: Randomized, cross-sectional laboratory study

Participants: Sixteen healthy adults

Methods: Participants performed three different exercises: (A) empty-can (EC; the arm was maintained at $60^{\circ}$ abduction with full internal rotation in the sitting position), (B) full-can (FC: the arm was maintained at $60^{\circ}$ abduction with full external rotation in the sitting position), and (C) prone full-can (PFC; the arm was maintained at $60^{\circ}$ abduction with full external rotation in the prone position).

Outcome Measures: Ultrasonography was used to measure the muscle thickness (MT), pennation angle (PA), and fiber bundle lengths (FBL) of the supraspinatus. One-way repeated ANOVA with Bonferroni's post-hoc test was used to compare between the three exercises and the initial position of each exercise.
Results: There were significant difference for $M T, P A$, and $F B L(P=.001$ $P<.001 ; P<.001)$. Compared with each initial position, the MT and FBL showed greatest difference in the FC compared to the EC and PFC, and the PA significantly increased in the EC.

Conclusion: The findings suggest that each exercise (EC, FC, and PFC) has its own advantages for rehabilitation programs.

\section{Key Practice Points:}

- The EC, FC, and PFC exercises were used clinically to strengthen the supraspinatus.

- The supraspinatus is a complex muscle so research is needed to understand its function.

- The data provides shoulder rehabilitation guideline.

\section{SINGLE-LEG BRIDGE AND SINGLE- LEG SOUAT ELICIT HIGH MUSCLE ACTIVITY LEVELS IN ALL SEGMENTS OF GLUTEUS MEDIUS, AND GLUTEUS MINIMUS POSTERIOR}

Moore $D^{1}$, Semciw $A^{2}$, Wajswelner $\mathrm{H}^{1}$, McClelland $\mathrm{J}^{1}$, Pizzari $\mathrm{T}^{1}$

${ }^{1}$ LaTrobe University, Melbourne

${ }^{2}$ The University of Queensland, Brisbane

Question: 1) Quantify activity levels of gluteus medius and minimus during a single-leg bridge and single-leg squat. 2 ) Is there a difference in gluteus medius and minimus activity between the single-leg bridge and singleleg squat?

Design: Cross-sectional

Participants: Ten healthy adults (mean age (range) $=23.8(22-26)$ years females $=4$ )

Intervention: Three sets, six repetitions of each exercise timed to a beat of a metronome. Order randomly assigned.

Outcome Measures: Fine-wire electromyography (EMG) electrodes recorded activity from gluteus medius anterior, middle and posterior segments, and the gluteus minimus anterior and posterior segments on the stance limb during each exercise. 1) Average EMG amplitude was normalised to maximum isometric voluntary contraction (MVIC) and quantified for each muscle during each exercise. 2) Activity between exercises were compared with paired $t$-tests $(\alpha=0.05)$

Results: 1) Mean (standard deviation) single leg bridge activity was high for gluteus medius anterior (43.23 (16.18)), middle (41.79 (12.68)) and posterior (37.83 (19.51)); and gluteus minimus posterior (37.81 (29.39)) but not anterior (17.43 (13.69)). Mean (SD) single leg squat activity was high for gluteus medius anterior (46.68 (21.97)), middle (42.81 (21.31)) and posterior (41.80 (24.40)); and gluteus minimus posterior (48.16 (27.60)) but not anterior (27.71 (10.80)). There were no significant difference in average amplitude between exercises $(p>0.05)$ for all segments of the gluteus medius and gluteus minimus.

Conclusion: Single leg bridge and single leg squat could be equally applied in practice to induce muscle hypertrophy for all segments except gluteus minimus anterior.

\section{Key Practice Points:}

- Single leg bridge and single leg squat could be equally applied in practice for gluteus medius hypertrophy.

- Other exercises should be considered if targeting gluteus minimus anterior hypertrophy.

- Single leg bridge and single leg squat could be equally applied in practice for gluteus minimus posterior hypetrophy. 


\section{THE EFFECTIVENESS OF THERAPY ON OUTCOME FOLLOWING BOTULINUM NEUROTOXIN INJECTION FOR FOCAL SPASTICITY IN ADULTS WITH NEUROLOGICAL CONDITIONS - SYSTEMATIC REVIEW}

Moore $E^{1,2}$, Banky $M^{1}$, Olver J, 5,6, Bryant $A L^{2}$, Williams $G^{1,2,3}$

1Physiotherapy Department, Epworth Healthcare

2Physiotherapy Department, The University of Melbourne

${ }^{3}$ Physiotherapy Department, Latrobe University

${ }^{4}$ Rehabilitation Medicine, Epworth HealthCare

SRehabilitation Medicine, Monash University

Epworth Monash Rehabilitation Unit (EMReM)

Question: Does therapy following botulinum neurotoxin injection for spasticity improve rehabilitation outcomes in adults with neurological conditions?

Design: Systematic review

Participants: Adults with a neurological condition presenting with focal spasticity injected with botulinum neurotoxin.

Intervention: Adjunctive therapies were defined as treatments that were provided by physiotherapists or occupational therapists in conjunction with the botulinum neurotoxin, such as serial casting and stretching.

Outcome Measures: Studies were classified in terms of the clinical indications (i.e. active or passive) for which botulinum neurotoxin was prescribed. Effect sizes were expressed as mean differences or standardized mean differences with 95\% confidence intervals.

Results: The search identified 12 studies that reported on the effects of eight different adjunctive therapies. No high level evidence was identified. Only one study reported data for a between-group difference, however this study did not find a significant between-group difference. The assessment of the risk of bias found serious limitations with design and implementation of most of the studies. Although several studies reported significant effects, the effectiveness of adjunctive therapies could not be interpreted due to limitations with methodological quality and a lack of data reporting the between-group differences.

Conclusion: There was insufficient evidence to support or refute the effectiveness of any of the commonly prescribed adjunctive therapies following botulinum neurotoxin injection for focal spasticity in adults.

\section{Key Practice Points:}

- Limited evidence exists supporting any specific treatment protocols following botulinum neurotoxin injection.

- Clinical expertise guides the application of adjunctive therapies following botulinum neurotoxin; therapists should continue to provide therapy in conjunction with botulinum neurotoxin to optimize rehabilitation outcomes.

\section{THE INFLUENCE OF THERAPY ON OUTCOME FOLLOWING BOTULINUM NEUROTOXIN INJECTION FOR FOCAL SPASTICITY IN ADULTS WITH NEUROLOGICAL CONDITIONS}

Moore $E^{1,2}$, Olver J,5,6, Bryant AL ${ }^{2}$, Williams $G^{1,2,3}$

${ }^{1}$ Physiotherapy Department, Epworth Healthcare 2Physiotherapy Department, The University of Melbourne

3Physiotherapy Department, Latrobe University

${ }^{4}$ Rehabilitation Medicine, Epworth HealthCare

${ }^{5}$ Rehabilitation Medicine, Monash University

${ }^{6}$ Epworth Monash Rehabilitation Unit (EMReM)

Question: Does therapy following botulinum neurotoxin improve rehabilitation outcomes in adults with neurological conditions?

Design: Prospective observational study

Participants: Thirty-one adults with spasticity injected with botulinum neurotoxin.

Outcome Measures: The proportion of participants that achieved their predetermined goals. Expected outcomes were documented using the 'GAS-light' method. Goal attainment was measured on a dichotomous scale as successful $(0$ to +2$)$ or unsuccessful $(-1$ to -2$)$. A tool was designed to measure adherence to a prescribed therapy program. Goals were linked to the therapies that would optimise goal attainment.

Results: Thirty-one participants had 76 goals. Goals were classified into four groups. Upper limb passive ( $n=12)$, lower limb passive ( $n=3)$, upper limb active $(n=18)$ and lower limb active $(n=43)$. Overall, there was a weak correlation between goal attainment and adherence to therapy $(r=0.21$ $p=0.06$ ). However, there was a significant relationship between therapy adherence and goal attainment for individuals with active lower limb goals $(n=43, r=0.04, p=0.008)$
Conclusion: The most common goals related to active indications for the lower limb, which were significantly correlated to adherence to a prescribed therapy program. Further investigations are required in a larger sample to examine the relationship between the effectiveness of therapy and other indications for botulinum toxin.

Key Practice Points:

- Therapy influences outcome for people with active lower limb goals

- Indications for botulinum neurotoxin were primarily to the lower limb and provided to improve 'active' function

- Preliminary results indicate that therapy provided in conjunction with botulinum neurotoxin may be more effective when targeting the lower limb.

\section{PELVIC SUPPORT BELT VERSUS COMPRESSION SHORTS: WHICH IS SUPERIOR IN THE MANAGEMENT OF PREGNANCY RELATED PELVIC GIRDLE PAIN (PGP) WITH REDUCED FORCE CLOSURE?}

\section{Moore J}

Fitwise Physiotherapy

Design: Randomised controlled Pilot study with 3 arms, pretestposttest design.

Control was "usual treatment" which included the allocation of a pelvic support belt.

Participants: 30 pregnant women with established pregnancy related PGP were selected from a Women's Health Private Physiotherapy practice. None of these subjects had received any previous treatment for their complaint and all took part on a voluntary basis.

Intervention: Outcome measures were assessed then participant provided with a randomly assigned support garment (pelvic belt or pregnancy compression shorts) along with standardized education and manual treatment program. Outcome measures were reassessed one week later.

Outcome Measures: Pain was measured using a 10-point visual analogue scale (VAS). Disability was measured using the Pelvic girdle questionnaire (PGQ)

Results: The belt group had a greater reduction in pain levels after one week $(p=0.047)$ (mean DVAS $=4.92 \pm 5.41)$, compared to the shorts group (mean DVAS=1.36 \pm 3.81$)$. There was no significant difference $(p=0.293)$ between groups for change in disability (mean belt DPGQ $=10.63 \pm 17.06$ and mean shorts DPGQ $=3.77 \pm 16.24)$.

Conclusion: The results of this study show that the pelvic support belts are superior to the shorts at reducing pain levels after a one-week intervention. Due to the small sample size and short duration of the intervention, results should be interpreted with caution. Further studies with larger samples are needed to support the findings of this pilot study, and participants should be followed up through the duration of their pregnancy.

\section{Key Practice Points:}

- Mitton pelvic support belt appears to be superior when compared with pregnancy support shorts in providing pain relief in pregnancy related PGP

- Support belts should be used preferentially in the management of pregnancy related PGP with reduced force closure.

- Further high quality evidence with higher- powered studies are required to additionally validate these findings 


\section{A RANDOMISED CLINICAL TRIAL FOR THE EFFICACY OF PHYSIOTHERAPY INTERVENTION FOR EARLY-ONSET HIP OSTEOARTHRITIS}

Moore $\mathbf{K}^{1}$, Kemp $\mathrm{LL}^{1,2}$, Fransen $\mathbf{M}^{3}$, Russell TG ${ }^{4}$, Crossley KM ${ }^{4,5}$

${ }^{1}$ Bodysystem Physiotherapy, Hobart, Tasmania

${ }^{2}$ Australian Centre for Research into Injury in Sport and its Prevention, Federation University Australia

${ }^{3}$ Faculty of Health Sciences, University of Sydney

${ }^{4}$ University of Queensland

${ }^{5}$ La Trobe University

Question: What is the feasibility of conducting a randomised controlled trial investigating a targeted physiotherapy intervention for younger people with early-onset hip osteoarthritis?

Design: A randomised assessor- and participant-blind, controlled trial, conforming to the SPIRIT guidelines.

Participants: Seventeen volunteers ( 9 female; age $32+8$ years; height $1.71 \pm 0.08$ metres; weight $77 \pm 17 \mathrm{~kg}$ ), recruited 14 months post-hip arthroscopy and diagnosed with chondropathy and/or labral pathology were randomised to a physiotherapy intervention group or control group.

Intervention: Semi-standardised physiotherapy including (i) manual therapy; (ii) strengthening and functional retraining; and (iii) health education sessions. Control: health education sessions. Eight treatment sessions provided over three months (weekly for four weeks, then fortnightly for eight weeks)

Outcome Measures: Primary outcome was feasibility (percentage of eligible participants enrolled, adherence to the intervention, study dropouts and losses to follow-up at 12 weeks). Secondary outcomes included the Hip disability and Osteoarthritis Outcome Score and the International Hip Outcome Tool patient-reported outcomes.

Results: Seventeen patients (31\% of eligible) agreed to be randomised. There were no losses to follow-up at 12 weeks. All patients attended all scheduled treatment sessions, meaning $>75 \%$ adherence to the intervention was 100\% for both the physiotherapy intervention and control groups.

Conclusion: Enrolment, adherence to the intervention and limited loss to follow-up at 12 weeks supports the feasibility of a full-scale randomised controlled trial, to determine the efficacy of physiotherapy in early hip osteoarthritis.

\section{Key Practice Points:}

- A full scale randomized controlled trial is feasible and important.

- Future studies will determine best practice physiotherapy for early hip osteoarthritis.

\section{ADDED CHALLENGES IN TRAINING THE FEMALE RUGBY PLAYER}

\section{Moran MG'1,}

${ }^{1}$ Australian Rugby Union

¿Sydney Stadium Sports Physiotherapy

Rugby Sevens is a high paced, high impact sport played by both Men and Women, and is a full participation sport at the Rio Olympics in 2016. Apart from the typical impact injuries sustained across Rugby Union, and specific load associated injuries related to high speed repetitive effort training specific to Rugby Sevens, we have encountered issues that are gender based to the Women's Program. In particular stress urinary incontinence was reported in one athlete which led us to question the prevalence within the squad and how we are approaching the management of these issues in a high impact sport. This presentation will demonstrate the methods of identification of incontinence issues, and the current methods of intervention for this group of high speed, high impact athletes.

\section{Key Practice Points:}

- Improve awareness of stress urinary incontinence in the female athlete

- Identify specific sport related incontinence issues

- Propose methods for identification of stress urinary incontinence in the female athlete

- Create an intervention program based on the specific needs of a target sporting population.

\section{ENRICH EARLY- ADVANCING MOTOR OUTCOMES FOR INFANTS WITH CEREBRAL PALSY}

\section{Morgan C}

Cerebral Palsy Alliance Research Institute

Although cerebral palsy (CP) by definition starts early in life it is usually not diagnosed until about 18 months of age. Late detection often means that early neurorehabilitation is not accessed until motor impairment is evident. Consequently the dose of active intervention during the critical period for brain plasticity is inadequate. Little evidence exists for the effectiveness of early intervention protocols for infants with CP. In particular, interventions that take a motor learning approach and focus on the task and environment as well as the child, have not been rigorously researched in this population. A knowledge translation project to embed the use of the General Movements Assessment, the most predictive assessment for a later diagnosis of CP, was carried out in NSW. Pooled outcome data from 5 sites demonstrated that accurate detection of CP was possible in an Australian context with sensitivity and specificity values similar to established international rates. Two randomised controlled trials of a novel early enrichment programme "GAME" (Goals, Activity and Motor Enrichment) were conducted of infants 3-4 months of age with abnormal general movements and at high risk of CP. Results demonstrated advanced motor and cognitive outcomes when compared to standard care.

\section{Key Practice Points:}

- Infants with cerebral palsy can be identified earlier than is commonly practiced

- Interventions that enrich the environment have a small but measurable benefit on motor outcomes of infants with CP

- Task-specific motor training conducted in an enriched home environment can advance the motor outcomes of infants with CP

\section{PULMONARY HYPERTENSION: RISKS AND BENEFITS OF EXERCISE}

\section{Morris N}

School of Allied Health Sciences, Griffith University

Menzies Health Institute of Queensland

Queensland Lung Transplant Service, The Prince Charles Hospital, Queensland

Pulmonary hypertension is a complex disease characterised by a progressive increase in breathlessness, limited functional capacity, poor quality of life and, if left untreated, right sided heart failure and death. Whilst recent advances in medical therapy have improved patient outcomes, long term prognosis remains poor. Until recently, exercise has been discouraged in pulmonary hypertension for fear it would worsen symptoms and negatively impact on right heart function. Highlighting these risks, we recently reported the first ever serious adverse events during a six minute walk test in three patients with pulmonary hypertension. Prof Norman Morris is currently collaborating with clinicians and researchers at The Prince Charles Hospital in Brisbane examining both the acute and chronic responses to exercise in pulmonary hypertension. In this presentation he will examine the relationship between measurements of gas exchange taken during the six minute walk test and disease severity in a range of patients with pulmonary hypertension. Given the potential impact of exercise on right heart function, the research team has recently commenced studies examining the impact of exercise training on right heart function using state-of-the art cardiac magnetic resonance imaging (CMRI) techniques. He will present CMRI data contrasting the acute responses to exercise in patients with pulmonary hypertension and control subjects and the impact of exercise training in this patient group. 


\section{INTENSITY OF EXERCISE TRAINING: OLD DOGS, INTERVALS AND NEW TRICKS}

\section{Morris N}

School of Allied Health Sciences, Griffith University

Menzies Health Institute of Queensland

Queensland Lung Transplant Service, The Prince Charles Hospital, Queensland

Typically we consider intensity of exercise training to be a key component of any exercise training program. In younger healthy individuals, a higher exercise training intensity is thought to result in a greater training adaptation. However in older individuals, we have found that adaptations to exercise training may be independent of intensity, particularly when the volume of exercise training is controlled over 10-12 week period. In chronic lung disease patients, it is not clear if higher intensity training results in greater physiological adaptation. Confusing this issue has been the use of interval training, a training mode that alternates periods of exercise with periods of rest or low intensity exercise. We have shown that compared to high intensity continuous exercise, individuals are able to perform a greater volume of interval exercise with lower oxygen uptake, ventilation, heart rate and blood lactate response. In the history of athletic performance, some of greats even used interval training with outstanding results. It was reported that the Dr Eric Bannister used interval training to conquer the four-minute mile barrier. What about the effect of interval training on clinical populations, can they break any world record's with this approach? One of the confounding issues with this research has been the fact that studies typically have manipulated both the mode and the intensity of exercise when comparing different outcomes. This approach makes it difficult to evaluate the impact of exercise training mode (interval vs continuous) and training intensity (high vs low) on adaptations. This presentation will examine the research comparing high vs low intensity exercise and interval training. Maybe it is easier than we think.

\section{YEAR IN REVIEW - ICU REHABILITATION AND POSTINTENSIVE CARE SYNDROME}

\section{Morris P}

University of Kentucky, Lexington, KY, USA

This session will provide a summary of the key literature across three categories: intensive care rehabilitation, muscle wasting and mechanistic pathways, and outcome measurement to evaluate functional recovery which have been published in the past 2 years. The late breaking results of the largest RCT, TARGET, to be conducted in critical care will also be presented in this session.

\section{Key Practice Points}

- Inform clinicians of the latest evidence based research which will be relevant to practice and implementation of best patient centred care

\section{CURRENT AND FUTURE REHABILITATION STRATEGIES FOR THE CRITICALLY ILL}

\section{Morris P}

University of Kentucky, Lexington, KY, USA

An overview of the current delivery of rehabilitation within the intensive care unit will be the foundation from which a discussion of future challenges and research directions will be conducted.

\section{Key Practice Points:}

- Develop understanding of the importance of rehabilitation for critically ill

- Appreciation of the complexity of rehabilitation interventions in critical care

- Strategies to address challenges and guide new research directions for clinicians and researchers

\section{PAIN IN ATHLETES - MANAGEMENT OUTSIDE OF THE NORMAL BOX}

Moseley L $\mathrm{L}^{1,2}$, Bisset $\mathrm{L}^{3}$, Caneiro JP $\mathrm{P}^{4,5,6}$

${ }^{1}$ NOI Group, Adelaide

2School of Health Sciences, University of South Australia, Adelaide

${ }^{3}$ School of Allied Health Sciences, Griffith University, Brisbane

${ }^{4}$ Body Logic Physiotherapy, Perth

${ }^{5}$ Curtin Unviersity of Technology, Perth

${ }^{6}$ Rowing Australia

Chronic pain and stress affects up to $20 \%$ of the general population, however the prevalence amongst athletes, both elite and recreational, is unknown. Whilst evidence continues to grow in favour of a biopscyhosocial approach to dealing with chronic pain, many athletes continue to search for a pathoanatomical cause for their chronic pain. As a result, there is sparse treatment of athletes playing sport via a pain sciences approach. This session brings together three physiotherapists with expert knowledge and experience in treating chronic pain. The session will aim to enhance the capabilities of sports physiotherapists to treat athletes in chronic pain when a structural, functional or biomechanical model does not apply. A key component of this will be focussing on the importance of anti- and pro-nociceptive mechanisms which will help to inform athlete prognosis and assist the clinician in identify appropriate treatments to target these mechanisms. The presentations will assess the existing evidence for the neurophysiological mechanisms associated with pain, discuss clinically relevant methods for assessing the presence of altered nociceptive processing, and propose how the clinician might use this information to individualise treatment for the athlete experiencing chronic pain. The session will specifically focus on pain modulation, the use of quantitative sensory testing, cognitive functional testing

\section{PAIN AND THE BRAIN: THEORETICAL AND CLINICAL CONSIDERATIONS}

\section{Moseley $\mathrm{L}^{1}$}

${ }^{1}$ University of South Australia

That the brain is a critical player when it comes to pain is now well established and widely, although not universally, accepted. As a result, the way we interpret many clinical interventions has changed and new interventions have emerged. Not surprisingly, the massive advances in our understanding of the role of the brain in pain have highlighted how little we really know. This has triggered a renewed focus on theoretical models and the development of novel experimental approaches with which to test them. In this talk, I will present some contemporary theories that serve as a kind of road map along which we are pursuing a better understanding of why some people get chronic pain and others do not, how we can interrupt this process and how we can treat those who have already made the transition to chronicity. I will focus on theories that relate to the human as a complex system, rather than theories based on any particular molecular, genetic or end-organ dysfunction. 


\section{THE PHYSIOTHERAPY SPECIALIST IN AOTEAROA NEW ZEALAND: REGULATION IN PRACTICE}

Mueller $]^{1}$, Woltman-Black $\rfloor^{2}$

${ }^{1}$ Chair, Physiotherapy Board of New Zealand

${ }^{2}$ Chief Executive, Physiotherapy Board of New Zealand

Question: In late 2012 the Board set an international physiotherapy regulation precedent announcing the establishment of a regulated Physiotherapy Specialist scope of practice under the HPCA Act 2003. It provides consumers and the sector with a transparent mechanism to identify clinical physiotherapy specialists.

Design: The Board engaged multiple stakeholders during wide-ranging public consultations to develop the framework. The Board defined the scope and required qualifications (required by the Act), determined the clinical areas of specialisation, practice competencies, assessment criteria and re-certification processes. A two-stage assessment process of candidates is used; a panel of assessors complete an initial portfolio assessment, followed by face-to-face meeting to discuss with candidates.

Participants: Board secretariat, Board members, stakeholders including patients/consumers, professional associations, funders, employers, other regulatory authorities, healthcare providers, and physiotherapists.

Outcome Measures: 370 individual respondents and organisations responded during the consultations(s) processes to develop the Physiotherapy Specialist proposed scope of practice. Review of the first year of implementation is currently underway.

Results: In May 2015 there were three Specialists-Musculoskeletal on the public register.

Conclusion: Implementation of a new scope of practice takes time, requires robust consultation, an evidence-based framework and buy in from consumers and the profession.

\section{Key Practice Points:}

- The regulated scope of practice 'Physiotherapy Specialist' acknowledges the sub-specialty nature of advanced clinical practice and is a mechanism for consumers to independently recognise and access specialist physiotherapy expertise.

- It provides a clinical structure to support practitioners who require second opinions and or an avenue for referral for complex patients.

\section{HOW OSTEOARTHRITIC KNEE}

PAIN IS MANAGED IN AUSTRALIA: VARIATION, DELIBERATION AND MAYBE AN OPERATION

\section{Mulcahy A}

Australian Commission on Safety and Quality in Healthcare, Sydney

Question: Geographical variation in healthcare, including physiotherapy, has been observed for decades. Variation not related to patient need or preference, raises questions about appropriateness of care. Do Australians with osteoarthritic knee pain appropriately receive surgery or physiotherapy?

Design: This is an observational study. The Australian Commission on Safety and Quality in Healthcare and the Australian Institute of Health and Welfare analysed 2010-11 hospital admission and Medicare Benefits Schedule data for knee replacements and knee arthroscopies. This was reported at Medicare Local level and compared with Organisation for Economic Cooperation and Development (OECD) data.

Results: Australia's admission rate for total knee replacement was the highest of any country in the OECD. Rates for total knee replacements in Medicare Locals ranged from 140 admissions per 100,000 population to 330 admissions, a 2.4 fold variation. There was also high, significant variation in admission rates for knee arthroscopy. Rates across Medicare Locals ranged from 232 admissions per 100,000 population to 726 admissions per 100,000, a three-fold variation. Four out of five admissions for knee arthroscopy occurred in private hospitals.

Conclusion: This study has a huge impact for physiotherapists. Australia does more total knee replacements and knee arthroscopies than any other OECD country. This is despite strong evidence that arthroscopy is of little benefit for osteoarthritis. Musculoskeletal physiotherapists are vital to ensure that patients with osteoarthritic knee pain receive appropriate management.

\section{Key Practice Points:}

- Given the variation in care, there is enormous potential for musculoskeletal physiotherapists to triage appropriate alternative pathways to surgery.
INTENSIVE CARE EARLY

REHABILITATION, LESSONS FROM LEADING INTERNATIONAL FACILITIES

\section{Mullins RM}

Sir Charles Gairdner Hospital, Perth

Question: How do leading international facilities optimally implement intensive care early mobilization programs?

Design: Winston Churchill fellowship researching intensive care early mobilization programs at leading international facilities.

Participants: Intensive care staff St Thomas's Hospital, London, John Radcliffe Hospital, Oxford, Foch Hospital and Cochin Hospital, Paris, UZ Leuven Gathuisberg Hospital, Belgium, Toronto General Hospital, Canada, New York Presbyterian/Columbia University Medical Centre, New York and John Hopkins Hospital, Baltimore.

Interventions: Investigator met with medical and physiotherapy professional leads and observed early mobility practices in intensive care.

Outcome Measures: Protocol availability, staffing allocation, patient acuity, patient selection, de-sedation practices, multi-disciplinary collaboration and equipment utilization to facilitate early mobility.

Results: Implementation of early mobility programs is successfully achieved in high acuity cohorts through excellent multi-disciplinary teams and novel ideas to facilitate and combat barriers. Team collaboration facilitates and streamlines physiotherapy interventions. De-sedation practices vary amongst facilities and are successfully more ambitious than standard Australian practice.

Conclusion: Leading international intensive cares prioritize mobilization over standard care, facilitated by strong medical support and multidisciplinary collaboration.

\section{Key Practice Points:}

- Multi-disciplinary team prioritization of mobility

- Physiotherapy focus on mobilization

\section{PROGRESSIVE RESISTANCE TRAINING IN ACUTE STROKE: IS IT SAFE AND DOES IT IMPROVE FUNCTION? A SYSTEMATIC REVIEW AND META-ANALYSIS}

Musovic A, Salter K, Taylor N

Eastern Health, Melbourne

Question: Progressive resistance training in acute stroke: Is it safe and does it improve function? A systematic review and meta-analysis

Design: Systematic review with meta-analysis of randomised trials Participants: People who have suffered stroke within 3 months Outcome Measures: Adverse Events. Upper limb function and mobility.

Results: Data for the primary outcome of safety was synthesised descriptively and meta-analyses for other outcomes were conducted. The quality of the 6 included studies ranged from fair to excellent. For the trials investigating adverse events, none reported any significant increase in events after PRT. There was high level evidence that PRT had little or no effect on). There were no significant benefit for upper limb function and mobility after PRT compared with controls.

Conclusion: There was no evidence reported that PRT is unsafe in people with acute stroke, although there was a lack of reporting about adverse events. The lack of demonstration of effect in improving muscle strength and activity suggests there is insufficient evidence to recommend the prescription of PRT for people in the acute phase after stroke

\section{Key Practice Points:}

- Progressive resistance training in acute stroke has shown no increase in adverse events.

- Current studies in progressive resistance training in acute stroke demonstrated limited intensity with exercises.

- From current studies there was no measurable benefit of PRT on strength, upper limb function or mobility.

- Further studies are needed with appropriate intensity and progressions to assess the effects of PRT in acute stroke. 


\section{EXPLORING THE MINIMAL, MODERATE AND MAXIMAL IMPORTANT DIFFERENCES FOR THE 6MWT POST TOTAL KNEE ARTHROPLASTY: WHICH IS THE APPROPRIATE CHANGE METRIC?}

Naylor JM 1,2,3, Buhagiar $\mathrm{M}^{2,4}$, Mills $\mathrm{K}^{5}$, Wright $\mathrm{R}^{6}$, Fortunato $\mathrm{R}^{7}$

Whitlam Orthopaedic Research Centre, Liverpool Hospital, Sydney

2South West Sydney Clinical School, UNSW, Sydney

Ingham Institute of Applied Medical Research, Sydney

${ }^{4}$ HammondCare, Sydney

5Macquarie University, Sydney

${ }^{6}$ Fairfield Hospital

${ }^{7}$ Campbelltown Hospital, Sydney

Question: Is the minimal, moderate or maximal important difference (MID, MoID, MalD) the most appropriate change metric for the 6 minute walk test (6MWT) post total knee arthroplasty (TKA)?

Design: Data from a combined randomised trial and observational study were utilized.

Participants: 243 TKA recipients

Method: Individuals performed a 6MWT 4 weeks before and 26 weeks after TKA. Post-operatively, each was asked to rate their perceived improvement in mobility on a transition scale anchored with "much better" and "much worse".

Outcome Measures: The change thresholds were identified using receiver operating curves on data dichotomised into improved or not, with the classification of 'improvement' varying across methods. Area under the curve (AUC) sensitivity and specificity of each threshold were calculated, with AUC comparisons made around the predictive ability of the three classifications.

Results: MID - 31.5 m [Sensitivity: 71.54\% (95\% Cl 63.41 to 79.67), Specificity: $66.67 \%$ (46.67 to 90.71)]; MolD - $74.5 \mathrm{~m}$ [52.21\% (41.59 to 61.53), 84\% (68 to 96)]; MalD - $36.8 \mathrm{~m}$ [75\% (65 to 85), 34.48 (36.8 to 46.55)]. There was no significant difference in AUC between the classification strategies. All were poor-to-fair predictors of improvement $(67.34 \%, 67.49 \%, 60.24 \%)$

Conclusion: Thresholds for important improvements were obtained, however, no threshold classification method was a good predictor of change.

\section{Key Practice Points:}

- A patient-perceived important change threshold may not be a possible metric for the 6MWT post-TKA

- Identifying important change remains challenging for clinician and researcher

- Linking a change in 6MWT to a functional change may be more relevant

\section{IS THERE A ROLE FOR REHABILITATION STREAMING FOLLOWING TOTAL KNEE ARTHROPLASTY?}

Naylor JM1, 2,3 ${ }^{\text {, Crosbie J }}{ }^{4}, \mathrm{Ko} \mathrm{V}^{1}$

${ }^{1}$ Whitlam Orthopaedic Research Centre, Liverpool Hospital, Sydney

¿South West Sydney Clinical School, UNSW, Sydney

IIngham Institute of Applied Medical Research, Sydney

${ }^{4}$ UWS, Sydney

Question: Do total knee arthroplasty (TKA) recipients demonstrating poor mobility at entry to rehabilitation and who receive supervised therapy, demonstrate better mobility after rehabilitation than those who receive less supervision?

Design: Retrospective analysis of randomized trial data. The original RCT demonstrated that 1-to-1 therapy was not superior to group-based or home-based therapy across a range of outcomes.

Participants: TKA recipients randomized to supervised $(n=159)$ or homebased therapy $(n=74)$. Groups were dichotomized based on mean target 6 -min walk test (6MWT) pre-therapy.

Intervention Supervised therapy - 12 sessions of either group or 1-to-1 therapy; Unsupervised therapy - a monitored home program. Physiotherapists from four physiotherapy outpatient departments participated.

Outcome Measures: Absolute and change in 6MWT amongst low performers in the supervised ( $n=89$ ) and unsupervised $(n=36)$ groups were compared, as were high performers in the supervised $(n=70)$ and unsupervised ( $n=38$ ) groups.

Results: Low performers in the unsupervised compared with the supervised group demonstrated significantly poorer 6MWT scores (absolute $\delta=8.5 \%, p=0.003$; change $\delta=8.1 \%, p=0.007$ ) when therapy ceased (10 weeks post-surgery). No differences in 6MWT were observed between the high performing subgroups.
Conclusion: Individuals manifesting comparatively poor mobility at the commencement of physiotherapy may recover their mobility more quickly if streamed to supervised therapy.

Key Practice Points:

- Physiotherapy post TKA can be streamed based on baseline mobility

- Some TKA patients require supervision whilst other do not

- Streaming may be a cost-effective strategy for overburdened services

\section{TEACHING PELVIC FLOOR EXERCISES FOR RADICAL PROSTATECTOMY - WHAT IS THE RIGHT APPROACH}

\section{Neumann $\mathbf{P}^{1}$ \\ ${ }^{1}$ Pelvic Floor Clinic}

This presentation will evaluate the current literature on pelvic floor muscle training (PFMT) for men undergoing radical prostate surgery. It is challenging to say what the right approach is when two Cochrane Reviews in 2011 and $2015^{1}$ both concluded that there is limited evidence for PFMT in this patient group. Close scrutiny of the literature, however, reveals some conflicting results from good quality RCTs not addressed by the Cochrane reviewers. Some studies have shown positive outcomes ${ }^{2-4}$ while others have been equivocal ${ }^{5}$ or shown even worse outcomes for the intervention group ${ }^{6}$. This conflicting evidence will be analysed and explanations proposed. One factor may be the use of anal palpation to assess the pelvic floor muscles, and the use of anal biofeedback and electrical stimulation for training, providing men with erroneous feedback. Recent research has demonstrated the complexity of the male continence mechanism and the effect of different verbal instructions on its activation ${ }^{7}$. The instructions to "shorten the penis" or "stop the flow of urine" have been shown to target the urethral sphincter mechanism preferentially and are thus more likely to benefit men with PPI. Men with prostate cancer are often fit men in their middle years so the same training protocols that have worked for women after childbirth cannot reasonably be applied to these men. Alternative training protocols may be required. The presentation will cover the optimal instructions, positions and training regimens for men with PPI, with practical tips for clinicians, based on the best available evidence.

\section{INSTRUCTIONS TO "STOP THE URINE FLOW" PRIOR TO RADICAL PROSTATE SURGERY PRODUCES QUANTIFIABLE BLADDER NECK ELEVATION USING TRANSPERINEAL ULTRASOUND}

Neumann $\mathrm{P}^{1,2}$, Panuccio $\mathrm{C}^{3}$, Fuller $\mathrm{A}^{4}$, Sutherland $\mathrm{P}^{4}$

IInternational Centre for Allied Health Evidence, University of South Australia

${ }^{2}$ The Pelvic Floor Clinic, Norwood, SA 5067

3Specialist Imaging Partners, North Adelaide, SA

${ }^{4}$ South Terrace Urology, Adelaide, SA

Questions: Does the verbal instruction to "stop the flow of urine" result in appropriate cranial displacement of the bladder neck in men undergoing radical prostate surgery? Can this movement be reliably quantified by a trained physiotherapist using transperineal ultrasound in the clinic?

Design: prospective observational study

Participants: 26 consecutive consenting men who received one hour of physiotherapy prior to radical prostate surgery including verbal instructions to "stop the flow of urine"

Outcome measure: Bladder neck displacement, quantified using transperineal ultrasound and performed by the physiotherapist in the clinic was compared with measures obtained 1-2 weeks later by an accredited sonographer on a sophisticated ultrasound system.

Results: All men achieved cranial displacement of the bladder neck. Mean (SD) displacement measured by the ultrasonographer was 10.9 (4.23) $\mathrm{mm}$. This was not statistically significantly different from those of the physiotherapist $(p=0.29$.).

Conclusion: Prior to radical prostate surgery, verbal instructions to contract the pelvic floor muscles "as if stopping the flow of urine" resulted in cranial bladder neck displacement which could be accurately quantified in the clinical setting by transperineal ultrasound performed by a trained physiotherapist. The relevance of this instruction to focus on urethral, compared with anal, sphincter activation on continence outcomes is not known and warrants further research.

Key Practice Points:

- Instructions to "stop the flow of urine" resulted in bladder neck elevation in all men

- Transperineal ultrasound can provide quantifiable and accurate measures of pelvic floor movement in pre-prostatectomy men in the clinical setting 


\section{CLINICAL EDUCATION SUPERVISION MODEL FOR THE ACUTE NEUROSCIENCES PHYSIOTHERAPY TEAM}

\section{Nguyen $T^{1}$}

iMelbourne Health

Background: The Acute Neurosciences Physiotherapy Team at The Roya Melbourne Hospital evaluates its clinical education program annually, according to feedback from students. In 2014, this process evolved to include the experiences of educators. Following this evaluation, evidence from the literature was utilised to design a new supervision model. The finalised model involves one clinical educator supervising two students during morning sessions throughout a placement. The afternoons were dedicated to observational and learner driven sessions with other members of the team.

Method: The new model was implemented in two phases. Phase 1 involved two students with one educator while Phase 2 involved four students with two educators. A mixed method qualitative and quantitative evaluation occurred between the phases, where feedback was sought. The clinical educators also participated in semi-structured interviews to expand on themes surrounding the quality of supervision provided. Evaluation was repeated following the completion of Phase 2 . Feedback and interview responses were thematically analysed.

Results: The qualitative findings supported the use of this new model. Greater ownership of the students' learning was reported by the clinical educators. Communication and planning were strong features that supported the delivery of education. Students reported that the placements enhanced their learning and were flexible to meet their needs.

Conclusions: A supervision model involving one educator with two students associated with a team teaching approach results in an improved learning experience. Continual evaluation and reference to evidence will assist in designing placements. This method may inform other areas in the delivery of clinical education.

\section{FUNCTIONAL IMPAIRMENT AND MOTOR PERFORMANCE IN CHILDREN WITH SPECIFIC LANGUAGE IMPAIRMENT}

Nicola $\mathbf{K}^{1}$, Watter $\mathrm{P}^{1}$

${ }^{1}$ Physiotherapy, The University of Queensland, Brisbane

Question: Is functional impairment related to motor performance in children with specific language impairment?

Design: Experimental study

Participants: Children aged 7 years and above with severe specific language impairment ( $\mathrm{N}=30$, males $=20, \mu$ age $=118$ months).

Outcome Measures: Height, weight, body mass index, one-leg stance (left and right leg), sit and reach, vertical jump, seated basketball throw, and the Movement Assessment Battery for Children 2nd Edition.

Results: Children were at risk or had definite problems on the Movement Assessment Battery for Children ( $\mu$ \%ile= 5.42). One-leg stance, vertical jump and sit-and-reach correlated with Movement Assessment Battery for Children scores. Older children performed better on most tests, but not on aiming and catching, and balance subtests of the Movement Assessment Battery for Children. No gender differences were seen. Body mass index positively correlated with throwing distance and negatively with sit and reach. Jump height and throw distance correlated, as did right and left leg stance time. Qualitative descriptors for one-leg stance did not predict stance time.

Conclusion: Children with severe language impairment, present with significant motor impairment. A relationship is present between functional measures of balance, strength and flexibility, and motor performance. One-leg stance may prove to be a good clinical measure to determine motor performance.

\section{Key Practice Points:}

- Physiotherapists should evaluate motor performance in children with language impairment.

- Physiotherapists now have more specific knowledge about relationships between the measures of strength, flexibility, balance and motor performance in language impairment.

- This will translate into improved practice by guiding future assessment and treatment directions.
THE RELATIONSHIP BETWEEN

VISUAL PERCEPTUAL SKILLS

AND MOTOR PERFORMANCE IN

CHILDREN WITH SEVERE SPECIFIC

LANGUAGE IMPAIRMENT

Nicola $\mathrm{K}^{1}$, Watter $\mathrm{P}^{1}$, Dalton $\mathrm{D}^{1}$, Tracy $\mathrm{G}^{1}$

${ }^{1}$ Physiothearpy, The University of Queensland, Brisbane

Question: Is there a relationship between visual perceptual and motor performance in children with specific language impairment?

Design: Retrospective chart audit

Participants: Children 4 years and above with specific language impairment only were included ( $N=102$, males=78)

Outcome Measures: The Beery-Buktenica Developmental Test of Visual-Motor Integration $3^{\text {rd }}, 4^{\text {th }} 5^{\text {th }}$ Editions, Developmental Test of Visual Perception-2 $2^{\text {nd }}$ Edition, and the Bruininks-Oseretsky Test of Motor Proficiency $1^{\text {st }}$ Edition.

Results: Age and gender did not affect scores. Gross motor, fine motor and overall motor scores were positively correlated with both the BeeryBuktenica Developmental Test of Visual-Motor Integration [gross motor $(r=0.544, p=0.000)$; fine motor $(r=0.528, p=0.001)$; overall motor $(r=0.510$, $p=0.001)]$, and the Developmental Test of Visual Perception-2 Visual-Motor Integration Quotient [gross motor ( $r=0.652, p=0.001)$; fine motor $(r=0.748$, $p=0.000)$; overall motor $(r=0.499, p=0.021)]$. Gross $(r=0.586, p=0.007)$ and fine motor $(r=0.657, p=0.002)$ subtests correlated positively with the Developmental Test of Visual Perception-2 overall score. There was no relationship between pure visual perception and motor performance.

Conclusion: Pure visual perception does not relate to motor performance. However, a relationship exists between the ability to integrate visual perceptual and motor systems, and motor performance. Therefore, motor performance may be predicted by screening a child's visualmotor integration.

\section{Key Practice Points:}

- Physiotherapists need to evaluate visual-motor integration in children with specific language impairment.

- Physiotherapists now have more specific knowledge about the relationships between visual perception and motor performance in this cohort.

- This knowledge will translate into improved practice by guiding future assessment and treatment directions.

\section{ALTERING PAIN MEMORIES BY USING EXERCISE THERAPY FOR PATIENTS WITH CHRONIC MUSCULOSKELETAL PAIN}

\section{Jo Nijs $^{1,2,3}$}

${ }^{1}$ Pain in Motion International Research Group

'Department of Physiotherapy, Human Physiology and Anatomy, Faculty of Physical Education \& Physiotherapy, Vrije Universiteit Brussel, Belgium ${ }^{3}$ Department of Physical Medicine and Physiotherapy, University Hospital Brussels, Belgium

Even though nociceptive pathology has often long subsided, the brain of patients with chronic musculoskeletal pain has typically acquired a protective (movement-related) pain memory. Exercise therapy for patients with chronic musculoskeletal pain is often hampered by such pain memories. Musculoskeletal therapists can alter pain memories in patients with chronic musculoskeletal pain by integrating pain neuroscience education with exercise interventions. The latter includes applying graded exposure in vivo principles during exercise therapy, for targeting the brain circuitries orchestrated by the amygdala (the memory of fear centre in the brain). Before initiating exercise therapy, a preparatory phase of intensive pain neuroscience education is required. Next, exercise therapy can address movement-related pain memories by applying the 'exposure without danger' principle. By addressing patients' perceptions about exercises, therapists should try to decrease the anticipated danger (threat level) of the exercises by challenging the nature of, and reasoning behind their fears, assuring the safety of the exercises, and increasing confidence in a successful accomplishment of the exercise. This way, exercise therapy accounts for the current understanding of pain neuroscience, including the mechanisms of central sensitization. 


\section{APPLYING MODERN PAIN NEUROSCIENCE IN PHYSIOTHERAPY PRACTICE: DIFFERENTIAL DIAGNOSIS BETWEEN NOCICEPTIVE, NEUROPATHIC AND CENTRAL SENSITIZATION PAIN}

\section{Jo Nijs $\mathbf{s}^{1,2,3}$}

${ }^{1}$ Pain in Motion International Research Group

'Department of Physiotherapy, Human Physiology and Anatomy, Faculty of Physical Education \& Physiotherapy, Vrije Universiteit Brussel, Belgium ${ }^{3}$ Department of Physical Medicine and Physiotherapy, University Hospital Brussels, Belgium

The awareness is growing that central sensitization is of prime importance for the assessment and management of chronic pain, but its classification is challenging clinically since no gold standard method of assessment exists. In order to select an effective and preferably also efficient treatment in daily clinical practice, pain patients should be classified clinically as either predominantly nociceptive, neuropathic or central sensitization pain. Therefore a body of evidence from original research papers was used by 18 pain experts from seven different countries to design the first classification criteria for central sensitization pain. It is proposed that the mechanism-based classification of pain entails two major steps: the diagnosis or exclusion of neuropathic pain and the differential classification of predominant nociceptive versus central sensitization pain. For the former, the International Association for the Study of Pain diagnostic criteria are available for diagnosing or excluding neuropathic pain. For the latter, clinicians are advised to screen their patients for three major classification criteria, and use them to complete the classification algorithm for each individual patient with chronic pain. The first and obligatory criterion entails disproportionate pain, implying that the severity of pain are disproportionate to the nature and extent of injury or pathology (i.e. tissue damage or structural impairments). The two remaining criteria are: 1) the presence of diffuse pain distribution (i.e. neuroanatomical illogical pain pattern), allodynia and hyperalgesia; and 2) hypersensitivity of senses unrelated to the musculoskeletal system (defined as a score of at least 40 on the Central Sensitization Inventory). Although based on direct and indirect research findings (i.e. several original research findings including a Delphi survey, a study of a large group of low back pain patients, and validation studies of the Central Sensitization Inventory), the classification algorithm requires experimental testing in future studies. Clinicians can use the proposed classification algorithm for differentiating predominant neuropathic, nociceptive and central sensitization pain. The classification criteria were recently adopted to the low back pain population and cance survivors suffering from pain (submitted).

\section{THINKING BEYOND ANATOMY AND PHYSIOLOGY: THERAPISTS' AND PATIENTS' ATTITUDES AND BELIEFS REGARDING CHRONIC PAIN ARE KEY TO APPLYING EFFECTIVE TREATMENT}

\section{Jo Nijs $\mathbf{s}^{1,2,3}$}

1Pain in Motion International Research Group

2Department of Physiotherapy, Human Physiology and Anatomy, Faculty of Physical Education \& Physiotherapy, Vrije Universiteit Brussel, Belgium ${ }^{3}$ Department of Physical Medicine and Physiotherapy, University Hospital Brussels, Belgium

Persistent pain is highly prevalent in various areas of physiotherapy, including manual therapy, oncology, sports, neurology, rheumatology, pediatrics and geriatrics. Although many physiotherapists have moved on in their thinking and apply a broad biopsychosocial view with regard to chronic pain disorders, the majority of clinicians have received a biomedical-focused training/education. Such a biomedical training is likely to influence the therapists' attitudes and core beliefs toward chronic pain. Therapists should be aware of the impact of their own attitudes and beliefs on the patient's attitudes and beliefs. As patient's attitudes and beliefs influence treatment adherence, physiotherapists should be aware that focusing on the biomedical model for chronic pain is likely to result in poor compliance with evidence based treatment guidelines, less treatment adherence and a poorer treatment outcome. Clinicians may want to apply a 5-step approach toward effective and evidence-based care for patients with chronic pain. The starting point entails self-reflection: therapists can easily self-assess their attitudes and beliefs regarding chronic musculoskeletal pain. Once the therapist holds evidence-based attitudes and beliefs regarding chronic pain, assessing patients' attitudes and beliefs will be the natural next step. Such information can be integrated in the clinical reasoning process, which in turn results in individually-tailored treatment programs that specifically address the patients' attitudes and beliefs in order to improve treatment adherence and outcome.
TEMPOROMANDIBULAR JOINT IN SMALL ANIMALS A VETERINARY PERSPECTIVE

\section{Norton U}

Eclipse Park Veterinary Surgery

There are not a great amount of studies on the temporomandibular joint in Small animal. This presentation is a review of the joint itself how it differs between cats and dogs and the most common problems seen in these animals. Often disorders of the TMJ in small animals are associated with more than one pathology however the most common disorder is osteoarthritis and this is often under diagnosed due to the lack of obvious clinical signs and then the difficulties associated with imaging the area. Small animals in the consultation room are also not as compliant as human patients when it comes to the examination of the jaw this is especially the case in cats. Some of the common problems with the area will be looked at and also some situations in practice that may predispose to pain in this area and what may be seen by the examiner.

\section{STEM CELL TOURISM: HYPE OR HOPE?}

\section{Novak $\left.\right|^{1,2}$}

${ }^{1}$ Cerebral Palsy Alliance, Brain Mind Research Institute, University of Sydney ¿School of Medicine, University of Notre Dame

As many as 1 in 3 might benefit from regenerative medicine, and stem cells are unquestionably the new frontier of medicine. Stem cell interventions offer enormous hope to people with serious illnesses and disabilities, but are also sold as hype to hopeful patients in unregulated, unmonitored, and unethical experimental conditions, known as "stem cell tourism". The media has reported numerous investigative journalism stories of "cures" but also of tragic scandals where hopeful patients (including children) have died after buying stem cell interventions overseas. For some of these patients it has been discovered at autopsy that the transplanted stem cells were not even human cells. How do we know if something is being blown out of proportion (hype) and discern hype from very exciting advances in the field that defy our current understanding of medicine but might be potential cures (hope)?

\section{Key Practice Points:}

- Acquired a general knowledge of stem cells

- Obtained a pragmatic and non-judgemental questioning method that they can use within clinical practice to help patients objectively appraise stem cell tourism marketing

- Acquired an understanding of the scientific rationale and state of the science for stem cell intervention as promising empirical treatment option for children and adults with neurological conditions

- Obtained an understanding of the potential future clinical role that physiotherapists might play within stem cell intervention for children and adults with neurological conditions

\section{"WHERE TO FOR SECRETION RETENTION: SHOULD WE BOTHER?"}

\section{Ntoumenopoulos G}

Australian Catholic University, St Vincent's Hospital Sydney, Guy's and St Thomas' NHS Foundation Trust UK

Intubation and mechanical ventilation can impair secretion clearance and lead to lung collapse, pneumonia and prolong time on mechanical ventilation. The role of chest physiotherapy for the management of secretion retention is unclear. There is conflicting evidence regarding the impact of chest physiotherapy on patient-centred outcomes in the intubated and mechanically ventilated patient. This provides challenges for physiotherapists in intensive care to justify treatments for secretion clearance. Ventilator flow rates, mucus viscosity and location(s) of mucus may affect detection of secretion retention. Changes in mechanical ventilator signs such as raised peak pressure in volume control modes or loss of tidal volume may only become evident with central airway obstruction. The "sawtooth" expiratory flow waveform pattern combined with added sounds on tracheal auscultation is currently the most accurate method available for bedside detection of secretion retention. If secretion retention leads to lung collapse then the physiotherapists reliance on chest radiograph findings may not provide sufficient diagnostic accuracy. As the evidence base for chest physiotherapy is conflicting, the focus of physiotherapy in intensive care is now directed to early rehabilitation. However, with sub-standard diagnostic accuracy of respiratory bedside assessments, the role of chest physiotherapy in intensive care requires further evaluation. Earlier landmark trials on the role of chest physiotherapy and bronchoscopy for the management of acute lobar collapse used chest radiograph criteria to evaluate treatment effectiveness. Hence these studies may have included patients with additional or alternative diagnoses such as pleural effusion or pulmonary oedema and impact on treatment outcome.

\section{Key Practice Points:}

- The evidence base surrounding secretion retention will be addressed including the diagnosis, detection, management and outcomes. 


\section{EXTENDED SCOPE OF PRACTICE: DIAGNOSTIC THORACIC ULTRASOUND FOR RESPIRATORY PHYSIOTHERAPY?}

\section{Ntoumenopoulos G}

Australian Catholic University, St Vincent's Hospital Sydney, Guy's and St Thomas' NHS Foundation Trust UK

This presentation will outline the basic concepts/technique of diagnostic thoracic ultrasound. Ultrasound findings with normal lung, pulmonary oedema, pneumothorax, pleural effusions, consolidation/collapse and diaphragm function will be highlighted. The issues surrounding training, clinical governance and extended-scope roles will be addressed.

\section{Key Practice Points:}

- The potential role of diagnostic thoracic ultrasound in respiratory physiotherapy will be covered including image findings, training and governance issues

\section{EFFECT OF VELOCITY AND FAMILIARISATION ON THE RELIABILITY OF ISOKINETIC DYNAMOMETRY IN HEALTHY ADULTS: A TEST-RETEST RELIABILITY STUDY}

\section{Nugent EP1, Snodgrass S $]^{1}$, Callister R2}

${ }^{1}$ Discipline of Physiotherapy, School of Health Sciences, The University of Newcastle, Callaghan

2Priority Research Centre in Physical Activity and Nutrition, The University of Newcastle, Callaghan

Questions: What is the test-retest reliability of the HUMAC-NORM isokinetic dynamometer? How much familiarisation is required to minimise the effect of practice-based improvement (PBI) on isokinetic measures at different velocities?

Design: Test-retest reliability study.

Participants: Convenience sample of seventy healthy adults.

Outcome Measures: Using the HUMAC-NORM participants completed five repetitions of concentric knee flexion and extension at 60, 120, 180 and $240 \%$ s on four occasions, each one week apart. Peak torque, angle of peak torque, total work and average power were recorded. Reliability was determined using percentage change in mean (group reliability), typical error (within-subject reliability) and intraclass correlation coefficients (ICC; rank-order reliability).

Results: Group reliability was excellent after two trials for knee extension (e.g. change in mean for angle of peak torque 60\% Trials 1-2:13.2\%, 95\% Cl 8.3-18.3; Trials 2-3: 1.0\%, 95\% Cl-3.1-5.3) but was more variable for knee flexion $>120 \%$ s. Within-subject reliability was acceptable after two trials, but again was more variable at higher velocities, particularly knee flexion. ICCs were acceptable after two trials at all velocities.

Conclusion: The HUMAC-NORM demonstrated excellent test-retest reliability when assessing isokinetic strength of groups, and reasonable reliability for individuals, after repeated testing. At least one familiarisation session is recommended prior to testing to minimise the impact of PBI.

\section{Key Practice Points:}

- The HUMAC-NORM demonstrates excellent test-retest reliability, after appropriate familiarisation.

- One familiarisation session is recommended prior to testing to minimise $\mathrm{PBI}$ in groups of healthy adults.

- Additional familiarisation is beneficial at higher velocities, particularly when testing individuals and knee flexion.

\section{A SURVEY OF AUSTRALIAN AND NEW ZEALAND PHYSIOTHERAPY PRACTICES WITH PASSY-MUIR VALVES IN INTENSIVE CARE UNITS}

O'Connor LR ${ }^{1,2}$, Smith, M1

${ }^{1}$ Faculty of Science, Charles Sturt University, Albury, Australia

2Physiotherapy Department, Gold Coast University Hospital, Southport, Australia

Question: The purpose of this study was to examine the current practices with Passy-Muir valves in intensive care units across Australia and New Zealand, primarily investigating factors influencing physiotherapy involvement, initiation, funding and access to Passy-Muir valves, and secondly, whether Passy-Muir valves are used specifically for physiotherapy treatment.

Design: Cross-sectional survey

Participants: One physiotherapist from each public hospital with an intensive care unit in Australia and New Zealand was invited to participate in this study.

Outcome Measures: A 22 question electronic survey was designed and sent to 100 ICU physiotherapists (response rate 66\%). The survey comprised of fixed-response questions with free text options, and openended questions. Participants were given four weeks to complete the survey. Fixed-response data was analysed using descriptive statistics, and open-ended questions analysed thematically.

Results: Passy-Muir valves were reported as being used in $83 \%$ of hospitals surveyed, and $52.7 \%$ of physiotherapists reported using Passy-Muir valves. In the 26 hospitals where physiotherapists do not use Passy-Muir valves, most commonly it was related to the involvement by other disciplines. In relation to funding, initiation and access to Passy-Muir valves, the results indicate that physiotherapists have a secondary role. However, $29 \%$ of physiotherapists surveyed reported using Passy-Muir valves specifically for treatment, for indications including, improving cough effectiveness, oxygenation and tracheostomy weaning.

Conclusion: This study indicated that speech pathologists have the lead role with Passy-Muir valves, however emerging evidence suggests that Passy-Muir valves may be a valuable adjunct to physiotherapy treatment, and it is recommended that multidisciplinary roles for the management of Passy-Muir valves be investigated.

\section{Key Practice Points:}

- Passy-Muir valves were originally used to improve communication in patients with tracheostomies, and therefore have traditionally been the role of speech therapists.

- There is emerging evidence to suggest that Passy-Muir Valves can improve oxygenation, ventilation, secretion management, ventilator weaning and postural control, all of which are relevant to physiotherapy practice.

- Although physiotherapists are involved in using Passy-Muir valves, and furthermore some reported using Passy-Muir valves specifically for treatment, formal workplace guidelines are limited, and the extent to which physiotherapists are involved in using Passy-Muir valves varies from unit to unit.

\section{INCONTINENCE IN FEMALE ATHLETES: THE UNSPOKEN PROBLEM}

\section{O’Dwyer M}

Female athletes experiencing urinary incontinence (UI) may not seek treatment for this all too common condition perhaps due to embarrassment or acceptance. Leaking urine should not be considered a normal outcome of athletic performance. Conservative treatment utilizing pelvic and musculo-skeletal physiotherapy is a legitimate first intervention strategy and should be considered before referring for pharmacological or surgical treatment. Urinary incontinence is typically associated with pelvic floor muscles failing to control intra abdominal pressure. This failure may be caused by weakness or by short, tight muscles failing to effectively contract with loading. It's relevant to assess more than the pelvic floor, as there may be other contributing factors to $\mathrm{UI}$ in the female athlete. Failure to address secondary factors may be a barrier to recovery. The goal of this presentation is to enhance understand of pelvic floor issues with elite female athlete incontinence along with practical suggestions to help unravel the sometimes interconnected layers of dysfunction influencing continence control.

\section{Key Practice Points:}

- This presentation will facilitate more effective understanding of the sometimes-complex nature of female incontinence and enhanced treatment outcomes through musculo skeletal interventions 
HIP AND KNEE ARTHROPLASTY FOLLOWUP COMPLETE IN 3 MONTHS UNDER PAR

O'Keeffe $\mathbf{D}^{1}$, Tran $\mathrm{T}^{2}$, Zeman $\mathrm{B}^{3}$, Gibbs $\mathrm{A}^{4}$

${ }^{1}$ Advanced Musculoskeletal Physiotherapy Clinic Lead, Monash Health 2Director of Orthopaedics, Monash Health

${ }^{3}$ Clinical Manager of Physiotherapy, Casey and Dandenong Hospitals, Monash Health

${ }^{4}$ Advanced Musculoskeletal Physiotherapist, Monash Health

Question: What are the outcomes of an Advanced Musculoskeletal Physiotherapist (AMP) led Post Arthroplasty Review (PAR) Clinic where patients are discharged at 3 months post-operatively?

Design: Prospective observational study

Participants: 128 Patients post hip or knee arthroplasty in Monash Health, AMPs, Orthopaedic doctors and clinic staff.

Intervention: Patients underwent post-operative outpatient reviews with an AMP in the PAR Clinic. Each patient was discussed with an Orthopaedic Consultant. The standard Model of Care (MOC) includes discharge from the Orthopaedic Unit three months post-operatively.

Outcome Measures: PAR Clinic appointment outcomes were recorded. Records of discharged patients were reviewed for re-presentations. Patient satisfaction surveys were conducted. RiskMan was monitored for adverse events.

Results: $81 \%$ of 185 patient appointment outcomes have adhered to the MOC. No discharged patients were referred back to Monash Health. 100\% of 33 patients surveyed were satisfied with their experience in the PAR Clinic. No clinical incidents were reported.

Conclusion: This MOC has the earliest standard time-point for discharge from orthopaedic outpatient follow-up in Victoria. AMPs can be effectively utilised to implement a MOC with outpatient discharge at three months post arthroplasty. Long-term outcomes of discharged PAR Clinic patients is needed. This project was possible due to funding made available by the Victorian Department of Health.

\section{Key Practice Points:}

- This MOC has the earliest standard time-point for discharge from orthopaedic outpatient follow-up in Victoria.

- AMPs can be effectively utilised to implement a MOC with outpatient discharge at three months post arthroplasty.

- Long-term outcomes of discharged PAR Clinic patients is needed.

\section{COMPARATIVE STRENGTH AND \\ ENDURANCE PERFORMANCE OF THE CERVICAL EXTENSOR AND FLEXOR MUSCLES IN FEMALES WITH AND WITHOUT MECHANICAL NECK PAIN}

O'Leary $\mathrm{S}^{1,2}$, Hoogma $\mathrm{C}^{1}$, Solberg $\mathrm{OM}^{1}$, Sundberg $\mathrm{S}^{1}$

'School of Health and Rehabilitation Sciences, The University of Queensland, Brisbane

2Physiotherapy Department, Royal Brisbane and Women's Hospital, Brisbane

Question: Is strength and endurance performance of the flexor and extensor muscles of the neck different in females with and without mechanical neck pain?

Design: Cross-sectional comparison study

Participants: Females with and without mechanical neck pain Intervention: Nil

Outcome Measures: Strength and endurance (50\% maximal) performance, including performance ratios, of the craniocervical and cervicothoracic flexor and extensor muscles.

Results: Performance was recorded in 53 females with, and 36 females without, mechanical neck pain. Reduced strength values were observed for the cervicothoracic flexor $(p<0.05)$ and extensor $(p=0.001)$ muscle groups of the neck pain group compared to the control group. Reductions in endurance were also evident in the craniocervical flexor $(p=0.01)$ and extensor $(p=0.04)$, and cervicothoracic flexor $(p=0.02)$, muscle groups. The neck pain group also demonstrated significantly different values to the control group in three of the six strength ratios examined but in none of the endurance ratios.

Conclusion: These findings of substantial deficits, and altered performance ratios, of cervical muscle strength and endurance in females with mechanical neck pain further support clinical recommendations for the inclusion of exercise in the management of these individuals.

\section{Key Practice Points:}

- Deficits in performance were evident in both craniocervical and cervicothoracic muscles, as well as flexors and extensors.

- Normal flexor/extensor performance ratios may be altered in individuals with neck pain.

- The findings further support the prescription of exercise within the overall management of mechanical neck pain.
AUSTRALIAN ARMY RECRUIT TRAINING: COURSE LENGTH AND RECRUIT INJURIES

Dawson GME ${ }^{1}$, Broad R², Orr R

${ }^{1}$ Bond University, Health Sciences and Medicine, Gold Coast Department of Defence, Wagga Wagga

Question: Does the length of a recruit training program influence injury rates?

Design: Retrospective cohort study. Data were recorded within a oneyear period during two different Army recruit training course types - the Australian Recruit 80 day Course (ARC), and the Australian Soldier 100 day Course (ASC).

Participants: Participants were 267 Australian Regular Army recruits undertaking Basic Training in 2013 (ARC $n=194$ : ASC $n=73$ ).

Intervention: Exposure to Army recruit training programs of varying lengths, with minimal other differences.

Outcome Measures: Reported rates, types and mechanisms of injuries were collected. Rates included injury prevalence (number of reported injuries/number of personnel completing respective course $x 100$ ) and injury incidence (number of injuries/100 recruits completing the respective course/course length in days $\times 100$ ).

Results: Injury prevalence for ASC recruits was $17.8 \%$ and for ARC recruits 13.9\%. Injury incidence for the ASC and ARC were 17.8 injuries and 17.4 injuries /100 soldiers/100 days respectively. While the majority of injuries for both courses were sprains and strains, the leading mechanisms of injury differed.

Conclusions: Longer, less intensive recruit training programs may be associated with similar rates of injury per unit time as shorter, more intensive programs. As such, greater proportions of recruits may be injured across longer programs.

\section{Key Practice Points:}

- Lengthening a recruit training program with the aim of making it less intensive may not reduce the proportion of recruits injured - in fact a higher proportion is likely to be injured due to the longer period of exposure to training.

\section{SELF-REPORTED LOAD CARRIAGE INJURIES IN AUSTRALIAN REGULAR ARMY SOLDIERS}

Orr RM ${ }^{1}$, Pope $\mathrm{R}^{1}$, Coyle J ${ }^{2}$, Johnston $\mathrm{V}^{3}$

${ }^{1}$ Bond University, Gold Coast

2Charles Sturt University, Albury

3University of Queensland, Brisbane

Question: What injury risks are associated with load carriage in Australian soldiers?

Design: Online survey

Participants: Australian Regular Army soldiers from selected Corps with high exposure to load carriage.

Intervention: Exposure to Army load carriage tasks

Outcome Measures: Self-reported injury incidence, types, body sites and sources, associated with military load carriage

Results: Of 338 respondents, 34\% reported at least one load carriage injury over their military career $(9.4 \pm 7.4$ years of service) of which $8 \%$ were female and $92 \%$ were male. Female soldiers reported 1.21 (Cl 0.71 to 2.04 ) times as many injury incidents per capita as males. Of the $42 \%$ of injured soldiers who reported sustaining more than one injury, $43 \%$ reinjured the same body site. The majority of injuries (61\%) affected the lower limbs, with bones and joints the most frequently injured body structures (39\%). Endurance marching was the activity accounting for most (38\%) injuries.

Conclusions: Occupational load carriage is a source of soldier injuries. Once injured, soldiers are at high risk of subsequent load carriage injury. The body sites and natures of self-reported injuries are akin to those of formally reported injuries and those reported by other military forces.

\section{Key Practice Points:}

- Soldiers are required to carry loads as part of their occupation and these loads may predispose them to injuries

- Once injured soldiers are at an increased risk of subsequent load carriage injuries

- When treating a soldier for musculoskeletal injuries, developing load carriage resilience prior to return to work is a priority. 


\section{EFFECT OF THERAPEUTIC MASSAGE ON PAIN IN DEMENTIA AND ADVANCED DEMENTIA: A PILOT STUDY}

\section{Kapoor $\mathrm{Y}^{1}$, Orr RM ${ }^{1}$}

${ }^{1}$ Bond University, Gold Coast

Question: Is routinely administered therapeutic massage effective in relieving pain in aged care residents with dementia/advanced dementia.

Design: A randomized controlled pilot study.

Participants: Aged care residents ( $n=10$; age $=82 \pm 7.05$ years, length of stay $=36 \pm 23$ months), recruited from a residential aged care facility with a diagnosis of dementia/advanced dementia.

Intervention: The intervention group received 10 minutes of therapeutic massage four times per week for four weeks. Techniques used were effleurage, kneading and trigger point therapy on an area of chronic pain with a non-fragrant hypoallergenic cream as the medium. The contro group received no massage. Both groups continued with current pain management plans.

Outcome Measures: The PAINAD (Pain Assessment in Advanced Dementia) observational scale administered pre and post intervention.

Results: Following the intervention, no significant changes in mean PAINAD scores were found within groups (Control $=5.2+3.49$, Intervention $=5.0+3.49: p=0.456)$. However, results suggest that the intervention group had a greater magnitude of change $(1.200+1.78)$ compared to the control group $(0.800+2.16)$, which shows that massage may have had a greater effect in relieving pain than current treatment protocols.

Conclusion: Therapeutic massage may provide a useful adjunct to the current pain management in patients with Dementia/Advanced Dementia.

\section{Key Practice Points}

- Massage is known to be a useful in treating stress and agitation associated with dementia/ advanced dementia.

- Massage can be an adjunct to chronic pain management in these patients.

- Massage was not found to have any side effects in treating chronic pain in patients with dementia/advanced dementia.

\section{A FUNCTIONAL MOVEMENT SCREEN PROFILE OF AN AUSTRALIAN POLICE FORCE}

Orr RM ${ }^{1}$, Pope R ${ }^{1}$, Stierli M², Hinton B $^{2}$

${ }^{1}$ Bond University, Gold Coast

${ }^{2}$ New South Wales Police, Sydney

Question: What is the Functional Movement Screen (FMS) profile of police recruits and officers?

Design: Cross-sectional research design.

Participants: Female and male recruits and attested officers of an Australian police force $\left(o^{\top} n=1155\right.$, mean $( \pm S D)$ age $=31.34 \pm 8.41$ years: $ᄋ$ $n=357$, mean age $=27.99 \pm 8.02$ years).

Intervention(s): Qualified Police Physical Training Instructors conducted FMS testing of participants in their workplace gymnasium.

Outcome Measures: The FMS was selected due to its value as a predictor of injury risk in tactical populations and its high inter- and intrarater reliability.

Results: Significantly higher mean FMS scores were found for recruits (15.23 2 2.01 points) when compared to attested officers (14.57 \pm 2.96 points; $\mathrm{p}<.001)$ and for females $(15.24 \pm 2.35$ points) when compared to males (14.84 \pm 2.55 points, $p=.008)$. A FMS score of $\leq 14$ points, predictive of higher injury risk, was observed in $43 \%$ of male police officers, $41 \%$ of female officers, $36 \%$ of male recruits and $33 \%$ of female recruits. The components of poorest performance, being the hurdle step and rotary stability, correspond to the leading sites of injury in this population, being knee and back.

Conclusions: Generally, attested police officers have a lower functional movement capability when compared to recruits, with a greater percentage scoring $\leq 14$ points on the FMS

\section{Key Practice Points:}

- The FMS is a useful outcome measure for police officers.

- FMS movements with poorest performance correspond to injuries typically sustained in a police population.

- Specific conditioning programs to improve performance in movements identified with poorer performance may reduce injuries in police officers.

\section{PROFILE OF POLICE OFFICERS}

THAT ATTEND WORKPLACE REHABILITATION SERVICES

Orr RM ${ }^{1}$, Hua, $\mathrm{M}^{1}$, Stierli $\mathrm{M}^{2}$

${ }^{1}$ Bond University, Gold Coast

${ }^{2}$ New South Wales Police, Sydney

Question: What are the typical presentations of police officers who attend a newly established workplace rehabilitation service?

Design: A retrospective observational study.

Participants: Injured police officers (male $n=30$ : mean age $43.3 \pm 9.56$ years: female $n=12$, mean age $38.2 \pm 6.39$ years) attending workplace rehabilitation (August to December 2014).

Outcome Measures: Reported injury numbers and types, numbers of treatments, attendance rates, and comparative costs.

Results: Of 340 booked treatment sessions, 296 (87\%) of were attended. Lumbar spine injury was the most commonly reported work-related musculoskeletal disorder, accounting for $40.5 \%(n=17)$ of all injuries and $119(40.2 \%)$ treatment sessions. Lumbar spine injuries occurred across all ranks and years of service, and a higher proportion of affected officers wore a hip holster than wore a thigh holster $(44.4 \%, n=12$ vs. $33.3 \%, n=5)$ Female officers showed a higher average number of treatments attended per injury than male officers ( $8.25 \pm 5.12$ vs. $6.57 \pm 4.03)$

Conclusion: A workplace rehabilitation service for injured police officers presents as a viable service with lower back injuries the primary presentation type.

\section{Key Practice Points:}

- Injuries to the lumbar spine were the most common presentation in a police workplace rehabilitation service and were more often associated with wearing a hip holster than wearing a thigh holster, though cohortlevel research would be needed to examine the strength of any possible causal link.

- Workplace rehabilitation services for injured police officers can limit lost productivity and travel costs associated with travel to external services during work time.

\section{ROTATION STRESS TESTING FOR THE ALAR LIGAMENTS; NORMAL ROTATION RANGE AND THE INFLUENCE OF AGE}

Osmotherly PG ${ }^{1}$, Folbigg $\mathrm{SL}^{1}$, Symonds $\mathrm{T}^{1}$

${ }^{1}$ School of Health Sciences, The University of Newcastle

Question: What is the variation in measured range during rotation stress testing for the alar ligaments in a clinical setting? Is rotation range during testing affected by patient age?

Design: Cross-sectional study.

Participants: 88 people between the ages of 18 and 86 with no current neck problems and no known risk factors for craniocervical instability.

Intervention: Participants underwent rotation stress testing for the alar ligaments performed to each direction in neutral, flexion and extension in both sitting and supine.

Outcome Measures: Cervical rotation range (degrees) during testing was recorded using an electromagnetic movement tracking system (Liberty, Polhemus, USA). Range was assessed overall and compared by 10 -year age groups. Rotation range and age was assessed using Spearman's Rank correlation. Reliability of measurements was assessed by estimation of ICCS.

Results: Mean angles of upper cervical rotation ranged between $8.86^{\circ}$ (SD $2.76^{\circ}$ ) to $17.2^{\circ}$ (SD $5.24^{\circ}$ ). Overall measured rotation ranged from $2.34^{\circ}$ to $33.22^{\circ}$. A low negative correlation was found between range of rotation and age, particularly in sitting (rho ranging from -0.15 to -0.47). Older age groups displayed less rotation, however, this was rarely statistically significant.

Conclusion: Normal range of rotation on stress testing for the alar ligament varies widely between individuals. Whilst all values fell within recommended ranges for ligament integrity, interpretation of the test will be problematic for people with inherently little rotation before the onset of a clinical disorder

\section{Key Practice Points:}

- Considerable variation exists in the amount of craniocervical rotation occurring during the imposition of this stress test.

- Measured ranges for people without alar ligament compromise all fell below the ranges recommended to flag possible instability.

- It should not be inferred that findings of low range of rotation necessarily represent normal ligament integrity. 


\section{EARLY STAGE PROSTATE CANCER RADIOTHERAPY WITH OR WITHOUT NEOADJUVANT ANDROGEN DERIVED THERAPY TREATMENT OUTCOMES ON PELVIC FLOOR MUSCLE FUNCTION}

Chiarelli PE ${ }^{1}$, Osmotherly $\mathrm{PG}^{1}$, Vaughan $\mathrm{BL}^{1}$

${ }^{1}$ School of Health Sciences, The University of Newcastle

Question: Will pelvic floor muscle training improve urinary and bowel outcomes in men receiving radiotherapy with or without hormone therapy treatment for prostate cancer?

Design: RCT Pilot study with blinded assessment and randomised allocation.

Participants: 40 men with early stage localised prostate cancer receiving external beam radiotherapy with or without androgen derived therapy (Lucrin)

Intervention: Four groups divided between radiotherapy alone and radiotherapy and androgen deprivation therapy to receive current treatment with or without a continence promotion and pelvic floor muscletraining program.

Outcome Measures: Validated continence questionnaire instruments measured lower urinary tract and bowel symptoms. Pelvic floor muscle function assessed using ultrasound imaging and anal perineometer at baseline, immediately after, then 1, 3, 6 months after radiation therapy. Men receiving hormone therapy had additional follow up 6 months after beginning hormone therapy to assess possible additional effects.

Results: An improved mean external anal sphincter squeeze pressure was found in men in the ADT intervention group compared to baseline at most follow-up points. Faecal urgency showed a statistical significant difference between radiotherapy groups at early follow up $(p=0.01)$. No other difference within or between treatment groups were found.

Conclusion: Men in the ADT intervention group showed significant improvement in average squeeze pressures at follow up measurements providing support for the efficacy of the intervention.

\section{Key Practice Points:}

- Evidence of bowel symptoms over time following radiation and hormone treatment.

- Increasing survival rates raise issues of long-term quality of life.

- Pelvic floor muscle training may improve functional outcomes and quality of life.

\section{PAEDIATRIC HYPERMOBILITY AND SPORTS}

\section{Pacey $\mathbf{V}^{1}$, Sinclair $\mathbf{M}^{2}$, McGough A $^{3}$}

${ }^{1}$ Department of Health Professions, Macquarie University and Kids Rehab, The Children's Hospital, Westmead

2Twelve9teen Sports Physiotherapy, Albion and Cirque Du Soliel "Corteo" ${ }^{3}$ Clem Jones Physiotherapy and QAS artistic gymnastics

Generalised joint hypermobility, greater than normal movement in multiple joints, is of ten asymptomatic. However, chronic joint pain, instability and other associated multi-system involvement, such as gastrointestinal dysfunction, fatigue and dysautonomia, causes significant functiona difficulties in approximately one third of all hypermobile children. Physiotherapy plays a pivotal role in the multidisciplinary management of symptomatic hypermobility. A growing evidence base suggests that physiotherapy can effectively reduce pain and improve mental health in this population. Andrew and Michelle will add to this session by sharing their clinical experience of working with young athletes, circus performers and gymnasts where generalised joint hypermobility can be advantageous for their chosen sport. Strength and neuromuscular control are key to high performance and injury prevention.

\section{Key Practice Points:}

- This talk aims to provide delegates with the current best-available evidence base and clinical how-to for assessing and treating children hypermobile children presenting to physiotherapy

- Emerging research results identifying which children are most likely to decline during childhood and adolescence will also be discussed
NEUROMUSCULAR DEFICIT PROFILES ASSOCIATED WITH ACL INJURY RISK IN A LARGE COHORT OF ADOLESCENT FEMALE ATHLETES

Pappas E ${ }^{1,2}$, Shiyko MP³, Ford KR ${ }^{4}$, Myer GD ${ }^{5,6,7,8}$, Hewett TE5, 6,7 ${ }^{1}$ Discipline of Physiotherapy, Faculty of Health Sciences, University of Sydney, Lidcombe, NSW, Australia

Orthopaedic Sports Medicine Center of Ioannina, Department of Orthopaedic Surgery, School of Medicine, loannina, Greece

${ }^{3}$ Department of Counseling Psychology, Bouve School of Health Sciences, Boston, MA, USA

${ }^{4}$ Department of Physical Therapy, School of Health Sciences, High Point University, High Point, NC, USA

${ }^{5}$ Department of Pediatrics, College of Medicine, University of Cincinnati, Cincinnati, OH, USA

${ }^{6}$ Division of Sports Medicine, Cincinnati Children's Hospital Medical Center, Cincinnati, OH, USA

'OSU Sports Medicine, The Sports Health \& Performance Institute, Departments of Physiology \& Cell Biology, Orthopaedic Surgery, Family Medicine and Biomedical Engineering, The Ohio State University, Columbus, $\mathrm{OH}$, USA

${ }^{8}$ The Micheli Center for Sports Injury Prevention, Waltham, MA, USA

Question: To identify the most common risk profiles for ACL injury in adolescent female athletes. Factors related to ACL injury risk can be categorized into four theories: Ligament dominance (excessive knee valgus), trunk dominance (poor trunk control), quadriceps dominance (excessive quadriceps recruitment), and leg dominance (motion asymmetries between the two lower extremities)

Design: A population study design was utilized. All high school female soccer, basketball and volleyball players in a midwestern county in the USA were tested in the biomechanics laboratory.

Participants: Seven hundred twenty one (721) female athletes.

Outcome Measures: Trunk and lower extremity kinetics and kinematics were collected with a 10-camera system and two force plates during an unanticipated sidestep cutting task. Latent profile analysis was utilized to detect the most prevalent profiles.

Results: The most prevalent profiles consisted of: a) no neuromuscular deficits (40\%), b) combination of quadriceps and leg dominance deficits (24\%), c) combination of trunk and leg dominance deficits and to a lesser extent ligament dominance deficits (22\%), and d) very high ligament dominance deficits only (14\%)

Conclusion: Approximately $60 \%$ of female athletes belong to one of the defined high-risk profiles. With the exception of the ligament dominance profile, the current analysis indicates that risk profiles consist of a combination of neuromuscular deficits.

\section{Key Practice Points:}

- Injury prevention programs should be tailored to the most common deficits

- Neuromuscular deficits are additive and cooperative

- $40 \%$ of adolescent female athletes exhibit no neuromuscular deficits and may be at low risk for ACL injury 


\section{DOES BURN INJURY INCREASE LONG TERM CARDIOVASCULAR DISEASE RISK?}

Paratz JD ${ }^{1,2,3}$, Leung $B^{1}$, Younger ${ }^{3}$, Muller $\mathrm{M}^{1,3}$, Stockton $\mathrm{K}^{1,4}$

${ }^{1}$ Burns, Trauma \& Critical Care Research Centre, University of Queensland Brisbane, ${ }^{2}$ Griffith University, Brisbane

${ }^{3}$ Royal Brisbane \& Women's Hospital, Brisbane

${ }^{4}$ Centre for Children's Burns and Trauma Research

Question: Burn patients have prolonged derangements in metabolic,

endocrine, haemodynamic and psychosocial systems, potentially impacting on their cardiovascular health. There are no investigations on the risk or incidence of cardiovascular disease post-burn injury. The aim of our study was to record lipid values and evaluate cardiovascular disease risk in adult burn survivors.

Design: A cross sectional observational study

Participants: Adults from 2-20 years post burn injury between $15-80 \%$ total burn surface area with no known cardiovascular disease contacted via an existing data base.

Outcome Measures: Total cholesterol, low density lipoprotein, high density lipoprotein, glucose and triglycerides were measured via a point of care unit (Alere Cholestech LDX). Means were compared to optimal ranges. Multivariate regression models were performed to assess the association of lipids with percentage of total burn surface area, age, years post-burn, physical activity level, diet pattern, family history and smoking. A p value $<0.05$ was considered significant. The Framingham General Cardiovascular Risk Score was calculated.

Results: Fifty patients were included in the study. Compared to optimal values, patients had low high density lipoprotein and high triglycerides. Greater percentage of total burn surface area was associated with statistically significant elevation of triglycerides $(p=0.007)$ and total cholesterol/high density lipoprotein ratio $(p=0.03)$. The median Framingham General Cardiovascular Risk Score was 3.9\% (low) 10-year risk of cardiovascular disease with $82 \%$ of patients in the low-risk category. A medium/high level of physical activity was shown to be associated with optimal values of high density lipoprotein, total cholesterol/high density lipoprotein, glucose and triglycerides. There was no association with years post burn, age, family history, diet, or smoking.

Conclusion: Adult burn survivors had alterations in lipid profile proportional to percentage of total burn surface area which could be modified by physical activity.

\section{Key Practice Points:}

- Subjects up to 20 years post burn injury had adverse alterations in their lipid profile associated with the initial percentage of total body surface area.

- These abnormalities in lipid profile could be modified by an increased leve of physical activity

\section{BARRIERS AND ENABLERS TO THE IMPLEMENTATION OF EARLY REHABILITATION IN CRITICAL CARE: A OUALITATIVE STUDY WITH EXPERT CLINICIANS}

Parry SM ${ }^{1-3}$, Granger $\mathrm{CL}^{1-2}$, Denehy $\mathrm{L}^{1}$, Knight LD², Beach LJ ${ }^{2}$, Rollinson $\mathrm{TC}^{3}$, Berney $\mathrm{S}^{3}$, Remedios $\mathrm{L}^{1}$

${ }^{1}$ Department of Physiotherapy, The University of Melbourne, Melbourne ¿Department of Physiotherapy, Royal Melbourne Hospital, Melbourne ${ }^{3}$ Department of Physiotherapy, Austin Health, Melbourne

Question: What are the barriers and enablers to implementation of early rehabilitation in the intensive care unit (ICU) from the perspective of treating clinicians?

Design: Qualitative study involving focus groups.

Participants: Purposeful sampling. Twenty-six clinicians (16 physiotherapists, six intensive care physicians and four intensive care nurses) who work in ICU across two acute tertiary hospitals in Melbourne.

Qualitative methodology: Focus groups were conducted by independent moderators. Semi-structured questions were used to explore attitudes, beliefs and experiences. Data were transcribed and thematic analysis was used to interpret data.

Results: Institutional barriers included: culture of the ICU; perceived environmental and patient barriers to safely undertake rehabilitation; limited resources (equipment and staffing); level of experience and confidence of staff; poor communication between disciplines; and daily care planning to enable prioritisation of rehabilitation. Major patient barriers identified were: medical instability; excessive sedation; and delirium. Major enablers for rehabilitation included: coordinated effort across the multidisciplinary team to prioritise rehabilitation in the daily care plan; designation of a champion or a leader; structured mentoring of junior staff to improve confidence; and access to staffing, resources and equipment.
Conclusion: This study has highlighted the barriers at an institutional and patient level and potential strategies to address to enable early rehabilitation in ICU.

\section{Key Practice Points:}

- Early rehabilitation is perceived as challenging in ICU.

- Clinicians highlight that barriers exist at an institution and patient level.

- Research targeted at bridging this evidence-practice gap is required to improve provision of rehabilitation in ICU.

\section{PHYSICAL FUNCTION IN CRITICAL CARE (PACIFIC): AN OBSERVATIONAL STUDY}

Parry SM ${ }^{1,2}$, Granger $\mathrm{CL}^{1-3}$, Denehy $L^{1,3}$, de Morton $N^{4}$, Baldwin $\mathrm{C}^{5,6}$, Knight LD2

${ }^{1}$ Department of Physiotherapy, The University of Melbourne, Melbourne 2Department of Physiotherapy, Royal Melbourne Hospital, Melbourne IInstitute for Breathing and Sleep, Melbourne

${ }^{4}$ Donvale Rehabilitation Hospital, Ramsay Health, Donvale International Centre for Allied Health Evidence (iCAHE), University of South Australia, Adelaide

${ }^{6}$ Flinders Medical Centre, Adelaide

Questions: Can the de Morton Mobility Index (DEMMI) and Physical Function in Intensive care Test scored (PFIT-s) be transformed into a single measure to evaluate function in intensive care (ICU) survivors? What is the clinical utility of these tests when used in isolation across the hospital admission?

Design: Multi-site prospective observational study.

Participants: Adult ICU patients mechanically ventilated $>48$-hours.

Outcome Measures: PFIT-s and DEMMI on awakening, ICU and hospital discharge.

Results: Data presented in this abstract are from preliminary analyses of the first 11 participants (Question 2 only). On awakening mean \pm SD PFIT-s was $4.6 \pm 3.0$ (out of 10 ) and DEMMI was $18.4 \pm 20.7$ (out of 100). The PFIT-s had a small floor effect (18\%) and DEMMI had a larger floor effect (36\%).

The PFIT-s had small ceiling effects on awakening (9\%) and ICU discharge (11\%), but a larger ceiling effect at hospital discharge (62\%). There was no ceiling effect for the DEMMI at any time-point. Strong correlations existed between the PFIT-s and DEMMI on awakening $(r=0.88, p<0.005)$ and ICU discharge $(r=0.96, p<0.005)$. Full analyses will be presented at the conference.

Conclusion: The DEMMI and PFIT-s have limitations (floor and ceiling effects) when used in isolation.

\section{Key Practice Points:}

- Development of one functional outcome that can be used until hospital discharge is urgently needed

- The PFIT-s performs strongly in ICU and the DEMMI performs strongly on the ward

- A combined version of these two tests may be a promising tool to evaluate function across the acute hospital continuum 


\section{MINIMUM STANDARDS OF CLINICAL PRACTICE FOR PHYSIOTHERAPISTS WORKING IN AUSTRALIAN AND NEW ZEALAND CRITICAL CARE SETTINGS: A MODIFIED DELPHI TECHNIQUE}

Skinner $\mathrm{EH}^{1-3}$, Thomas $\mathrm{P}^{4}$, Reeve $\mathrm{JC}^{5}$, Patman $\mathrm{S}^{6}$

${ }^{1}$ Western Health, Melbourne

2Monash University, Melbourne

${ }^{3}$ The University of Melbourne, Melbourne

${ }^{4}$ Royal Brisbane and Women's Hospital, Brisbane

${ }^{5}$ AUT University, Auckland

¿University of Notre Dame Australia, Fremantle

Questions: What are the minimum standards of clinical practice required by physiotherapists working in critical care settings in Australia and New Zealand?

Design: A modified Delphi technique, consisting of three prospective survey rounds, was used to obtain consensus on 217 items grouped into 19 themes reflecting common aspects of physiotherapy practice in critical care.

Participants: An expert panel of cardiorespiratory clinicians, academics and specialists $(n=61)$ was invited from a pool of eligible Australian and New Zealand physiotherapists $(n=93)$. Eligibility criteria were defined a-priorion the basis of possession of expertise and experience in the practice and teaching of critical care physiotherapy.

Outcome Measures: Questionnaires were developed using empirical resources including landmark textbooks and manuscripts on the physiotherapy role in critical care. Questionnaires were piloted, refined and disseminated electronically (via email, using SurveyMonkey ${ }^{\circledR}$ ).

Participants ranked items using response options 'Essential/Unsure/Not Essential'. Consensus was defined as achieved in favour or against when items were ranked 'Essential' or 'Non Essential' by more than $70 \%$ of participants, respectively.

Results: Fifty physiotherapists consented and participated in the initial round. Forty-five (90\%) completed all rounds. Consensus was reached on 199 (89\%) items. The panel agreed that 132 (58\%) items were 'Essential' items for inclusion in the final framework. Essential items included provision of mobilization in ventilated patients and manual hyperinflation.

Conclusion: This is the first study to develop an expert consensus framework of minimum standards of practice for critical care physiotherapists. The clinical and education utility of this framework requires further assessment.

\section{Key Practice Points:}

- No studies have previously defined the clinical knowledge and skills required by physiotherapists working independently in critical care settings.

- A Delphi technique has enabled the development of a framework for minimum standards of practice for physiotherapists working in the critical care environment

- The expert consensus framework of 132 items forms an important professional resource that will guide education and training needs across Australia and New Zealand

Trial registration: ACTRN12613000753752.

\section{POST-OPERATIVE PHYSIOTHERAPY MANAGEMENT OF PATIENTS UNDERGOING OPEN HEART SURGERY: A SURVEY OF CURRENT PRACTICE IN AUSTRALIAN PUBLIC HOSPITALS}

\section{Paton $\mathrm{M}^{1}$, Miles $\mathrm{P}$}

${ }^{1}$ Monash Health Physiotherapy Department, Clayton, Victoria

Question: What is standard physiotherapy care for patients undergoing open heart surgery in Australian public hospitals?

Design: A survey assessing staffing levels and routine treatment for patients undergoing open heart surgery was designed and reviewed by senior clinicians and distributed to appropriate hospitals.

Participants: Twenty-two Australian public hospitals were identified from the Australian Society of Cardiac and Thoracic Surgeons web site and internet searching as performing open heart surgery.

Outcome Measures: Results were collated via survey monkey for analysis.

Results: There was a response rate of $72.7 \%$. Post-operative length of stay was 4-7 days (mean 5.6). All respondents stated physiotherapy intervention started on day 1 post-operatively, with $100 \%$ sitting out of bed, $62.5 \%$ mobilising, and $62.5 \%$ prescribed deep breathing exercises. On day 2 mobilisation increased to $87.5 \%$. After day 6, physiotherapy intervention was not provided by $75 \%$ of respondents. Physiotherapists were the main instigators of patient mobility, with increased nursing involvement day 2 , and patient initiated mobilisation by day 3. Allied Health Assistants performed mobility in two hospitals, and cardiac education at four. Exercises Physiologists were used in one hospital for cardiac education only.

Conclusion: Disparity exists in physiotherapy treatment for patients following open heart surgery across Australian public hospitals, with hospitals still providing treatments that are not evidence-based.

\section{Key Practice Points:}

- Re-enforcement of the lack of evidence for provision of breathing exercises post open heart surgery.

- Enforcement of the benefits of mobilisation post-operatively.

- Increased utilisation of Allied Health Assistants and Exercise Physiologists for patients on standard care pathways

\section{DESIGN OF WORK FOR HEALTH}

\section{Pazell S}

Viva! Health at Work

As we navigate our way through safety management systems, legislative framework, and the implementation of wellness strategies in the workplace, we may ask: what are the intersections among work, equipment, environment, organisation, and health? The presentation will help us consider our approach to safety, health, and wellness from a perspective of human-centred design. A human-factors engineering and lifecycle design approach helps us consider a multitude of activities that may contribute to a robust and resilient workforce strategy. It speaks of systemic leadership and organisational design where value-laden judgement is set aside, vulnerability is permitted for ideas-generation, language focuses on successes and opportunities rather than failures and risks, and authentic analysis and learning can take shape to support business innovation.

\section{Key Practice Points:}

- Participants will be inspired to implement best-practice human-centred design in workplaces

- Participants will learn to apply systems-thinking in analysis of work opportunities through to safety investigations

- Participants will discover ways to link safety initiatives with health and wellness programming

\section{FATTY INFILTRATES IN PATIENTS WITH WHIPLASH: SYSTEMIC OR LOCAL PHENOMENA?}

Pedler $\mathbf{A}^{1}$, Durbridge $\mathrm{G}^{2}$, McMahon $\mathrm{K}^{2}$, Galloway $\mathrm{G}^{2}$, Sterling $\mathrm{M}^{1}$ ${ }^{1}$ CRE in Road Traffic Injury, CONROD Research Injury Centre, Menzies Health Institute QId, Griffith University, Gold Coast, Australia

2Centre for Advanced Imaging, The University of Queensland, Brisbane, Australia

Question: Are fatty infiltrates present in muscles remote from the cervical spine in patients with whiplash associated disorders? If so are these changes related to the presence of symptoms of posttraumatic stress in these patients?

Design: Cross-sectional observational study.

Participants: Thirty-two patients with chronic (> 3 months) whiplash and 15 asymptomatic controls were recruited.

Outcome Measures: Using a Siemens TRIO 3T MRI scanner (Siemens, Germany) a 2-point Dixon method was used to acquire images of the cervical spine and right calf musculature. Regions of interest (ROI) were created over the bilateral cervical multifidus at the C3 level and mid-belly of the soleus and tibialis anterior. Fatty infiltrates (MFI) were calculated based on the signal intensity within the ROI. Participants with WAD completed self-report measures of disability (Neck Disability Index: NDI) and symptoms of PTSD (Posttraumatic Stress Diagnostic Scale: PDS).

Results: There were no significant differences in MFI between groups for the calf musculature. Significantly higher MFI was observed in the C3 multifidus of patients with whiplash $(p<0.001)$. There was no significant correlation between MFI and PDS or MFI and NDI scores.

Conclusion: Fatty infiltrates are not universally present in skeletal muscles of patients with whiplash and are not associated with symptoms of posttraumatic stress.

\section{Key Practice Points:}

- MFI in the cervical spine is a feature of patients with whiplash

- These changes are not present in remote muscles suggesting a local mechanism

- These changes are not associated with levels of posttraumatic stress indicating usual rehabilitative approaches may be appropriate 
MUSCLE STRENGTH IN ADULTS WITH

SPINAL CORD INJURY: SYSTEMATIC

REVIEW OF MANUAL MUSCLE

TESTING, ISOKINETIC AND HAND HELD

DYNAMOMETRY CLINIMETRICS

\section{Peek $\mathrm{K}^{1,2}$}

${ }^{1}$ Health Behaviour Research Group, University of Newcastle

Joanna Briggs Institute, University of Adelaide

Design: Systematic review with data from quantitative studies synthesised in a narrative format

Participants: Adults with spinal cord injury (SCl)

Intervention: Muscle strength testing using manual muscle testing (MMT), isokinetic (ID) or hand held dynamometry (HHD).

Outcome Measures: The method used to assess muscle strength will be considered within the clinimetric domains of reliability, validity, responsiveness and interpretability.

Results: Eleven studies met the inclusion criteria of this systematic review. The results demonstrated that MMT showed varying inter-tester reliability over 10 muscle groups tested, ID demonstrated good reliability for the shoulder but not the elbow, HHD showed good reliability and validity for the upper limb and trunk, as well as good results for responsiveness and interpretability. Positive correlations were seen between MMT, ID and HHD particularly at the lower MMT grades. However, change in muscle strength scores seen on ID and HHD testing were not always correlated with changes in MMT grade. Significant overlapping of scores was seen between MMT and HHD particularly for grades 4 and 5, with MMT unable to detect subtle changes in muscle strength compared with dynamometry.

Conclusions: When considering the clinimetrics of the 3 methods for assessing muscle strength in adults with SCI there does appear to be support in the literature to recommend the wider application of HHD compared with MMT and ID.

\section{THE IMPACT OF ONSITE WORKPLACE HEALTH-ENHANCING PHYSICAL ACTIVITY INTERVENTIONS ON WORKER PRODUCTIVITY: A SYSTEMATIC REVIEW}

Pereira $\mathrm{MJ}^{1}$, Coombes BK${ }^{1}$, Comans $\mathrm{TA}^{2}$, Johnston $\mathrm{V}^{1}$

${ }^{1}$ Discipline of Physiotherapy, School of Health and Rehabilitation Sciences, The University of Queensland, Brisbane

Centre for Applied Health Economics, School of Medicine, Griffith University, Logan

Question: Do onsite workplace health-enhancing physical activity (HEPA) programs improve worker productivity?

Design: Two assessors used the Downs and Black checklist to determine the quality of eligible randomized controlled trials (RCTs)

Participants: Adult workers involved in onsite workplace, structured HEPA programs outside of work duties.

Outcome Measures: Any worker productivity measurement, including self-reported or actual sickness absences, quantitative measurements of job performance or qualitative questionnaires of work ability, whether these were primary or secondary outcomes.

Results: 8 studies were identified. There is consistent evidence that onsite workplace HEPA programs do not reduce sick leave. There appears to be inconsistent evidence of these programs impacting worker productivity. A high quality study of an onsite combination (aerobic, strengthening and flexibility) HEPA regime and a moderate quality study of a Tai Chi program improved self-reported worker productivity in female laundry workers and older female nurses respectively. Two high quality studies and four moderate quality studies did not show benefit. Studies that showed benefit were primarily those designed with productivity measures as primary outcomes, delivered to occupations involved with higher physical loads, had higher compliance and program intensity. The small number and inconsistency amongst studies limited further analyses.

Conclusion: There is inconsistent evidence that onsite workplace HEPA programs improve self-reported worker productivity.

\section{Key Practice Points:}

- Future high quality RCTs of onsite workplace HEPA programs should be designed around productivity outcomes, target at-risk groups, and investigate interventions of sufficient intensity.

- High attendance with improved recording is needed to achieve significant results in augmenting worker productivity.
VALIDATION OF AN IN VIVOTHREE

DIMENSIONAL METHOD FOR

INVESTIGATING THE KINEMATICS OF FEMOROACETABULAR IMPINGEMENT

Perriman DM1,2, Ward $\mathrm{T}^{1,2}$, Pickering $\mathrm{M}^{1,3}$, Scarvell JM ${ }^{1,4}$, Smith $\mathrm{PN}^{1,2}$

1 Trauma and Orthopaedic Research Unit, Canberra Hospital

${ }^{2}$ Australian National University

3University of New South Wales @ADFA

${ }^{4}$ University of Canberra

Question: How accurately does image registration measure in vivo hip kinematics?

Design: Cadaveric validation study.

Participants: One cadaveric specimen

Outcome Measures: A cadaveric hip and hemipelvis implanted with tantalum marker beads, underwent CT scanning to generate a 3D model. The stabilised hip/pelvis were flexed from approximately $0^{\circ}$ to $60^{\circ}$, simulating a clinical provocation test for hip impingement (flexion, adduction, internal rotation). In each position, fluoroscopic X-rays were taken at 5 different beam angles and the resultant angular measurements were compared to gold standard radiostereogramatic Analysis (RSA) data. Mean error with SD were calculated

Results: Mean angular error was -0.9o (SD 1.50). Mean in-plane displacement error was $-0.9 \mathrm{~mm}$ (SD $1.5 \mathrm{~mm}$ ), while mean out-of-plane error was $23.5 \mathrm{~mm}$ (SD $12.8 \mathrm{~mm})$.

Conclusion / Clinical significance: The errors measured for rotation and in-plane translations were comparable to results of Lin et al. 2012 who used used biplanar fluoroscopy with increased radiation exposure. Out-ofplane translation errors suggest that kinematic analysis is only optimized for two out of three of the orthogonal planes. Clinical applications will include assessment, categorization and measurement of treatment effect for FAl kinematics.

\section{Key Practice Points:}

- Image registration can accurately measure in vivo hip kinematics in two of of three planes.

- Image registration has potential to be a clinically relevant assessment and measurement tool.

- In vivo kinematic analysis will provide more accurate knowledge of FAI and the effect of interventions. 


\section{BACK PAIN ASSESSMENT CLINIC IN PRIMARY CARE: A FEASIBLE NEW MODEL OF HOSPITAL/COMMUNITY CARE}

Phan $\mathbf{U}^{1}$, de Gruchy $A^{1}$, Wicks I, 3, $^{2}$, Yuen $T^{5,6}$, Cunningham J7, MoijHY2,3

${ }^{1}$ Department of Physiotherapy, The Royal Melbourne Hospital, Melbourne 'Department of Rheumatology, The Royal Melbourne Hospital, Melbourne ${ }^{3}$ Department of Medicine, The University of Melbourne, Melbourne ${ }^{4}$ Walter and Eliza Hall Institute of Medical Research, Melbourne ${ }^{5}$ Department of Neurosurgery, The Royal Melbourne Hospital, Melbourne ${ }^{6}$ Department of Surgery, The University of Melbourne, Melbourne 7Orthopaedic Surgery, The Royal Melbourne Hospital, Melbourne

Question: Is a primary care-based physiotherapy-led back pain assessment clinic (BAC), supported by hospital-based rheumatology, neurosurgery and orthopaedics, a safe and effective model of care for patients with spinal pain?

Design: Mixed methods descriptive analysis.

Participants: Patients, rheumatologists, neurosurgeons, orthopaedic surgeons, advanced practice physiotherapists, hospital executives, administration staff, and community health service providers.

Outcome Measures: Waiting-times, health resource utilisation (BAC clinic activity, investigations, referral to specialist and allied health services), patient and staff satisfaction, and adverse incidents.

Results: The BAC model provides centralised triage of referrals, comprehensive assessment, optimisation of non-surgical management and streamlined access to community and specialist medical and surgical services. 402 patients have been triaged into BAC (244 accepted, 61 awaiting appointment, 91 not requiring appointment or non-contactable) BAC patients are seen within 12 weeks and commence community-based allied health interventions within 4 weeks of referral. Of the 184 patients that have attended, 61\% were referred for physiotherapy, $52 \%$ had medications adjusted, $28 \%$ had further investigations, $5 \%$ had spina injections and 6\% were referred to pain services. Two percent were referred to neurosurgery or orthopaedics. Patient and staff satisfaction has been high. There have been no adverse incidents.

Conclusion: Preliminary evaluation indicates that a collaborative, primary care-based, back pain clinic is a feasible, safe and effective model. Further evaluation is underway to determine cost-effectiveness and longterm impact.

\section{Key Practice Points:}

- BAC is a feasible model which improves patient access to care

- There is a high level of staff and patient satisfaction with the BAC model.

\section{HOW TO AVOID PARALYSIS BY ANALYSIS AND MAKE COMPLEX PATIENTS SIMPLE \\ Phillips $\mathrm{C}^{1}$ \\ ${ }^{1}$ DMA Clinical Pilates \& Physiotherapy}

Background: Chronic patients create unique challenges for clinicians, the presenting problem often being the tip of the iceberg. As a group they generally absorb $80 \%$ of funding and its common to see reductionist, single structure treatment, fail.

Aims / objectives: Behind most chronic presentations we find lists of co-morbidities which confuse decision making. We frequently overlook the patterns these co-morbidities form and the opportunity to use a big picture approach for rapid decision making.

Approach: Goldmans cardiac, decision tree, model has proven 4 key points are more effective for clinical decision making than 40 points. This model can be easily applied within musculoskeletal settings, using existing practice standards, to establish a directional treatment strategy. This nove approach allows a validated sub grouping

process that focuses treatment to a predominant side \& direction.

Conclusion: Directional models are common, well proven and still preferable to reductionist models. Directional filters also provide a tool for differentiation of radiological findings. The system is quick \& efficient with validation as a subgrouping / intervention prediction tool with excellent inter rater reliability This session will present the steps required to apply this model in the clinical setting and the supporting evidence being achieved in a variety of settings in every day practice on those problem patients.

\section{Key Practice Points}

- Identify patterns and significance of co-morbidities in chronic presentations

- Determine the 4 key points that will guide treatment

- Simplify management and moving away from a reductionist approach.

- Establish less is more as a valid clinical approach.

\section{IS A SLEEP INTERVENTION EFFECTIVE FOR LOW BACK PAIN WHEN GENETICS IS CONSIDERED? STUDY PROTOCOL OF A RANDOMISED CO-TWIN TRIAL}

Pinheiro $\mathrm{MB}^{1}$, Ferreira $\mathrm{ML}^{2,3}$, Refshauge $\mathrm{K}^{1}$, Grunstein $\mathrm{R}^{4}$, Hopper JL ${ }^{5}$, Maher $\mathrm{CG}^{2}$, Koes B ${ }^{6}$, Ordoñana JR ${ }^{7}$, Ho KK ${ }^{1}$, Ferreira PH ${ }^{1}$

${ }^{1}$ Faculty of Health Sciences, The University of Sydney, Sydney

${ }^{2}$ The George Institute for Global Health, Sydney Medical School, The University of Sydney, Sydney

3Institute of Bone and Joint Research, The Kolling Institute, Sydney Medical School, The University of Sydney, Sydney

${ }^{4}$ Woolcock Institute, The University of Sydney, Sydney

${ }^{5}$ Centre for Epidemiology \& Biostatistics, Melbourne School of Population and Global Health, The University of Melbourne, Melbourne

${ }^{6}$ Department of General Practice, Erasmus, Rotterdan, The Netherlands 7 Murcia Twin Registry, Department of Human Anatomy and Psychobiology, University of Murcia, and IMIB-Arrixaca, Spain

Question: What is the efficacy of a web-based sleep quality intervention in people with chronic low back pain and insomnia when the significant genetic influences that affect these conditions are controlled for? What is the feasibility of this study, specifically the recruitment and follow-up rate, adherence, diffusion, and twins' perceptions regarding the intervention?

Design: Randomised co-twin controlled trial.

Participants: Fifty monozygotic twins (25 complete pairs) with chronic low back pain and symptoms of insomnia will be enrolled.

Intervention: Participants will be randomly assigned to one of the two groups with each twin within a pair receiving either a web-based sleep intervention or a control intervention. The intervention will be an interactive online-based intervention tool based on cognitive behavioural therapy principles (intervention group) or web-based education program (control group) and will last for 6 weeks.

Outcome Measures: Outcomes will be measured at baseline, immediately post-treatment, and at a 3-month follow up post randomisation. Primary outcomes will be pain self-efficacy and patients' specific levels of function, while secondary outcomes will include pain intensity, disability, physical activity levels, sleep efficiency, and sleep quality. At the end of the study participants will answer a questionnaire about diffusion and a phone interview to investigate their opinion regarding the treatment. All data will be collected electronically

Conclusion: The results of this study will provide precise estimates into the efficacy of a sleep intervention for people with low back pain and insomnia because it will control for the significant contribution of genetics to both conditions.

\section{Key Practice Points:}

- This study will help to understand the effects of a sleep intervention for people with low back pain and insomnia using an innovative design that controls for genetics and web-based data collection methods.

- Results from this study will inform the implementation of policy in the treatment of low back pain. 


\section{SYMPTOMS OF DEPRESSION AS A PROGNOSTIC FACTOR FOR LOW BACK PAIN: A SYSTEMATIC REVIEW}

Pinheiro MB ${ }^{1}$, Ferreira $\mathrm{ML}^{2,3}$, Refshauge $\mathrm{K}^{1}$, Maher $\mathrm{CG}^{2}$, Ordoñana JR ${ }^{4}$, Andrade TB ${ }^{1}$, Tsathas $\mathrm{A}^{1}$, Ferreira $\mathrm{PH}^{1}$

${ }^{1}$ Faculty of Health Sciences, The University of Sydney, Sydney

${ }^{2}$ The George Institute for Global Health, Sydney Medical School, The

University of Sydney, Sydney

Institute of Bone and Joint Research, The Kolling Institute, Sydney Medical

School, The University of Sydney, Sydney

${ }^{4}$ Murcia Twin Registry, Department of Human Anatomy and Psychobiology,

University of Murcia, and IMIB-Arrixaca, Spain

Question: What is the effect of depression on the course of acute and subacute low back pain?

Design: Systematic review of cohort studies.

Participants: Participants with acute or sub-acute nonspecific low back pain (pain of less than 12 weeks duration).

Interventions: Not applicable. The prognostic factor of interest was depression or symptoms of depression assessed at baseline.

Outcome Measures: The outcomes of interest included: pain intensity, chronicity (non-recovery from low back pain), disability, return to work, health related quality of life, and patient satisfaction.

Results: Seventeen articles reporting 13 cohort studies were included in this review. There was considerable variability between studies in terms of method of assessment of depression and low back pain, statistical methods, and follow up length, which precluded the quantitative synthesis of the results. Definition of outcomes varied across studies, but overall they could be divided into work-related outcome measures, followed by disability, pain, self-perceived recovery, and mixed outcomes. Eleven out of 17 articles (or eight out of 13 cohorts) showed that symptoms of depression at baseline are related to worse low back pain outcomes (measured in various ways) at follow up.

Conclusions: The findings of this systematic review suggest that depression might have an adverse effect on the prognosis of low back pain. Future large studies enrolling an inception cohort and employing a standardized method for assessing depression and low back pain are needed.

\section{Key Practice Points:}

- There are few (heterogeneous) longitudinal studies that have investigated symptoms of depression as a prognostic factor for low back pain outcomes, which indicates the need for further research.

- Although not highly consistent, the evidence from the included studies points to a negative effect of symptoms of depression on low back pain outcomes.

\section{IS DEPRESSION A RISK FACTOR FOR LOW BACK PAIN? A PROSPECTIVE CO-TWIN STUDY}

Pinheiro MB ${ }^{1}$, Ferreira $\mathrm{ML}^{2,3}$, Refshauge $\mathrm{K}^{1}$, Colodro-Conde $\mathrm{L}^{4,5}$, González-Javier $\mathrm{F}^{4}$, Hopper J L ${ }^{6}$, Ordoñana JR ${ }^{4}$, Ferreira $\mathrm{PH}^{1}$

${ }^{1}$ Faculty of Health Sciences, The University of Sydney, Sydney

${ }^{2}$ The George Institute for Global Health, Sydney Medical School, The University of Sydney, Sydney

IInstitute of Bone and Joint Research, The Kolling Institute, Sydney Medical School, The University of Sydney, Sydney

${ }^{4}$ Murcia Twin Registry, Department of Human Anatomy and Psychobiology, University of Murcia, and IMIB-Arrixaca, Spain

${ }^{5}$ OIMR Berghofer Medical Research Institute, Brisbane

${ }^{6}$ Centre for Epidemiology \& Biostatistics, Melbourne School of Population and Global Health, The University of Melbourne, Melbourne

Question: Do symptoms of depression increase the risk of low back pain when genetics and early environmental influences are considered?

Design: Prospective observational co-twin study.

Participants: 1,607 twins from the Spanish Murcia Twin Registry who were free of low back pain at baseline.

Outcome Measures: Baseline data was collected in 2009-2011 and follow-up data in 2013. Twins answered questions on symptoms of depression (predictor) and low back pain (outcome). Firstly, the association was investigated using the complete sample (total sample analysis) Subsequent within pair case-control analyses were performed with all complete twin pairs discordant for low back pain regardless of zygosity, and then separately for dizygotic and monozygotic twins.

Results: In the total sample analysis, symptoms of depression at baseline did not significantly increase the risk of chronic low back pain (OR 1.40, $95 \%$ Cl 0.96 to 2.03). This risk was somewhat greater in the within-pair twin case-control analysis (OR 2.03,95\% Cl 0.95 to 4.36) and dizygotic only analysis (OR 2.15, 95\% Cl 0.97 to 4.77). No increase in risk was found in the monozygotic analysis (OR 1.00,95\% CI 0.06 to 15.99). A similar pattern was found for the other measures of depression and low back pain.
Conclusion: Symptoms of depression do not increase the risk of low back pain when high levels of adjustment for confounders were applied. This association appears to be confounded by genetic factors or even environmental factors and challenge the notion of depression as a causa factor for low back pain

\section{Key Practice Points:}

- People with symptoms of depression might not be at higher risk of developing low back pain.

- Genetic and environment factors need to be considered in the depression-low back pain relationship.

\section{PRACTICAL APPROACH TO UPPER LIMB REHABILITATION IN ATHLETES}

Pizzari T

La Trobe University Sports and Exercise Medicine Research Centre, Victoria, Australia

Upper body strength training is an essential component of an athlete's preparation for sport. In fact, for many athletes presenting to physiotherapy clinics with shoulder issues, strength training is their sport. The shoulder joint is potentially vulnerable to injury where high forces and repetitions are employed, particularly in the presence of incorrect technique. The sports physiotherapist needs to be skilled in the correct techniques of commonly used gym-based strength training exercises for athletes since high level strength training is an essential component of shoulder rehabilitation. This presentation will highlight frequent mistakes in strength training for shoulders and use video instruction to assist with increasing knowledge of correct technique and exercise parameters. A number of sports-specific exercises will be identified.

\section{Key Practice Points:}

- Correct techniques for commonly utilised gym-based shoulder strength exercises will be shown

- Exercises to avoid in the gym for particular shoulder conditions will be highlighted

- Sports-specific shoulder exercises will be identified

\section{PRINCIPLES OF SHOULDER REHABILITATION}

\section{Pizzari T}

La Trobe University Sports and Exercise Medicine Research Centre, Victoria, Australia

Assessment and management of shoulder conditions can present a challenge for the clinician since the shoulder is a complex joint requiring an intricate balance between mobility and stability. It can be difficult to know at what level to commence rehabilitation, what will work for each individua patient and what to do if things do not go as planned. This presentation will highlight an evidence and pathological-based approach to the selection of exercises for shoulder rehabilitation. Key principles that will benefit physiotherapists involved in the rehabilitation of shoulder conditions will be discussed, including the importance of assessment, scapula position and motion, humeral head position and motion, and pathological limitations. Emerging evidence of the value of external pacing of exercise to reduce intracortical inhibition will also be integrated since muscle inhibition is a consideration in ongoing shoulder problems.

\section{Key Practice Points:}

- The rehabilitation exercises will be clearly described and exercises parameters outlined, allowing immediate application in the clinical setting

- The suitability and limitation of rehabilitation exercises for particular presentations will be discussed and this knowledge will be useful for clinical practice 
UNRAVELLING THE GLUTEUS MINIMUS; IT'S NOT THE SIZE THAT COUNTS, BUT HOW YOU USE IT

\section{Pizzari T}

School of Allied Health, La Trobe University

The deep hip muscles, primarily the gluteus medius and minimus, are considered to be major stabilisers of the hip joint. This dynamic activity is essential for optimal joint reflexes, increasing joint stability and controlling joint forces. Atrophy or weakness of these periarticular muscles has been implicated in the development, progression, and severity of osteoarthritis of the hip and these deep hip muscles are commonly a focus of physiotherapy rehabilitation for a number of hip conditions. The gluteus minimus in particular has been theorised as being an important femoral head stabiliser due to the alignment of muscle fibres parallel to the femoral head, however there has been limited evaluation of the role of this muscle. The prescription of exercises for the gluteus minimus is based predominantly on its presumed roles and anecdotal evidence. This presentation will feature our electromyography (EMG) and magnetic resonance imaging (MRI) research into the gluteus medius and minimus in the normal population and pathological conditions such as osteoarthritis and gluteal tendinopathy. The normal functioning of deep gluteals will be revealed and exercises to rehabilitate these muscles will be discussed. The importance of the anterior gluteus minimus will be highlighted.

\section{HOW TO IMPROVE HOLISTIC MANAGEMENT OF INCONTINENCE POST-HOSPITALISATION WITH AGED CLIENTS. CAN A COMMUNITY PHYSIOTHERAPISTS HELP?}

\section{Fulford $\mathrm{A}^{1}$, Pendlebury $\mathrm{C}^{2}$, Podmore $\mathrm{V}^{3}$}

${ }^{1}$ Community Based Rehabilitation Services (CBRS), Royal Rehab, Sydney ¿Community Based Rehabilitation Services (CBRS), Royal Rehab, Sydney and Westmead Children's Hospital, Sydney

${ }^{3}$ Community Based Rehabilitation Services (CBRS), Royal Rehab, Sydney

Background: Within the community setting increasing numbers of clients are presenting with new continence issues post hospitalisation. Whilst on busy wards patients report having to use continence aids due to reduced mobility and needing assistance to access the toilet. Incontinence in the aged population is becoming normalised, due to product advertising and a general acceptance by staff, family and clients that it is part of the ageing process. For many elderly clients this is embarrassing and not discussed. This can lead to reluctance to leave their home and reduced quality of life. Could"hospital acquired" incontinence be better managed?

Aims / objectives: To gain practical suggestions for improving holistic management of these clients. Improve the knowledge and skills in recognising continence issues and appropriately managing these. Gain knowledge about sourcing and accessing continence resources and client education materials.

Approach: We will share our experiences in setting up a multidisciplinary working party to determine gaps in knowledge of team members in the community setting and how we worked towards becoming better advocates and educators for clients' continence management. Discussion will follow giving outlines of the continence awareness days that were run for allied heath staff, case managers and carers. Education focussed on identifying when new incontinence issues can be managed in the home setting, how and when to refer onto specialised physiotherapists and facilitate access to appropriate resources.

\section{Conclusion / Key Practice Points:}

- Assess continence issues

- Be skilled in client education and health promotion in continence management

- Facilitate access to client education and resources.

\section{REDUCING MANUAL TASKS RISKS IN UNCONTROLLED ENVIRONMENTS: HOW DO YOU KEEP PARAMEDICS SAFE?}

\section{Pollnitz P}

WorkFit Services, SA Health

Background: Paramedics perform heavy physical work in uncontrolled environments. Maguire et al. (2014) found that the paramedic profession is one of the most dangerous occupations in Australia, with a risk of serious injury more than seven times higher than the national average. The South Australian Ambulance Service (SAAS) engaged an Occupational Health Physiotherapist in May 2011 to review their manual tasks risk management system (including current equipment and work practices) in order to address the high rate of musculoskeletal injuries that paramedics suffer.

Aims: The presentation will outline the findings of that review, the system wide solutions being implemented and the results of this comprehensive program to protect emergency health services personnel. The findings translate to other areas and industries.

Methods: Analysing tasks performed and reviewing musculoskeletal incidents and claims revealed common problem areas and trends that could be targeted, some of which were surprising. The whole approach to manual tasks risk management at SAAS has been reviewed, with changes to ambulance environments and equipment. Removing high risk work practices and providing innovative alternatives to these is showing significant rewards. The use of scenario based training and assessment has improved the dynamic risk assessment and problem solving of paramedics, enabling them to make safer choices. The provision of equipment to reduce or eliminate hazardous tasks is a key part of this.

Results: SAAS has seen a marked reduction in the number and cost of lost time musculoskeletal injuries, demonstrated by graphs and data in the presentation.

\section{Key Practice Points:}

- The perception that the hierarchy of control can't be used in emergency services because of the unpredictable environments paramedics work within has been demonstrated to be false. There are many opportunities to influence the vehicles and equipment that paramedics work with and to improve their decision making to eliminate or reduce the hazardous characteristics of common manual tasks.

- Manual task risks can be controlled in ambulance services. Implementing innovative equipment solutions and different ways of moving patients creates markedly better results than the old manual handling paradigm of teaching them to 'lift right'.

\section{LEG POWER AS A PREDICTOR OF INJURY AND ILLNESS RISK IN POLICE RECRUITS}

Orr RM ${ }^{1}$, Pope $\mathbf{R}^{1}$, Peterson $\mathrm{S}^{1}$, Stierli $\mathrm{M}^{2}$, Hinton $\mathrm{B}^{2}$

${ }^{2}$ New South Wales Police, Sydney

Question: Does leg power, as measured by a vertical jump, predict injury and illness risk in a police population?

Design: Retrospective cohort study design.

Participants: New South Wales Police recruits $(n=1021)$ undergoing a basic police recruit training course.

Intervention A basic police recruit training course of 12 week duration.

Outcome Measures: Vertical Jump (VJ) height and formally reported illness and injuries.

Results: $15 \%$ of participants ( $n=158$, mean $\pm S D V J=42.03 \pm 7.35 \mathrm{~cm})$ reported an injury, 30\% ( $n=296, \mathrm{VJ}=41.88+7.48 \mathrm{~cm})$ an illness, and $38 \%$ $(n=390, V J=42.07 \pm 7.38 \mathrm{~cm})$ an illness and/or injury. The mean $V J$ heights for each of these groups was significantly lower $(p<.005)$ than those who did not suffer an injury (VJ $=44.00 \pm 7.56 \mathrm{~cm})$, illness $(\mathrm{VJ}=44.44 \pm 7.47 \mathrm{~cm})$ or a combination of either $(\mathrm{V}]=44.69 \pm 7.50 \mathrm{~cm})$. Correlations between $\mathrm{V}$ height and prevalence of illness and injury were low ( $r=-0.16$ and -0.09 , respectively) but significant ( $p<.005)$, with VJ height accounting for $2.6 \%$ and $0.8 \%$ of the variance in injury and illness rates, respectively.

Conclusion: Police recruits with lower VJ height are at a significantly greater risk of suffering an injury or illness during police basic recruit training.

\section{Key Practice Points:}

- Leg power is a useful predictor of injury and illness risk in recruits undergoing police training and potentially in other tactical populations.

- Increasing leg power in new recruits about to undergo police training my reduce their risk of injury or illness.

- Further research is required to assess the extent to which leg power is causal in these relationships. 


\section{ORTHOPAEDIC PHYSIOTHERAPY \\ SCREENING CLINIC (OPSC) - \\ RETROSPECTIVE COHORT STUDY OF LONG TERM PATIENT OUTCOMES}

Byrnes J, Murphy B, Pope $\mathbf{R}^{1}$

${ }^{1}$ Bond University, Gold Coast

Question: What are the long-term outcomes of patients who have received care under the Darling Downs Hospital and Health Services (DDHHS) OPSC clinic?

Design: Retrospective cohort study by manual patient chart audit.

Participants: Charts of the 214 patients who attended the OPSC between July 1, 2010 and June 30, 2011 were manually audited. Intervention: OPSC.

Outcome Measures: Clinical outcomes and documented care pathways recorded up to December 31, 2014, following discharge from the OPSC.

Procedure: Demographic and outcome data were manually extracted from patient charts by two of the researchers. Descriptive statistical analysis and inferential analyses were conducted to examine long term patient outcomes and differences between patient subgroups

Results: No patients who were removed from the orthopaedic waitlist following discharge from the OPSC re-presented. Significant differences in subsequent orthopaedic intervention status and rates of failure to attend orthopaedic appointments were observed depending on discharge status from the OPSC and on diagnostic group.

Conclusion: The OPSC model can be very effective, with good long term patient outcomes.

\section{Key Practice Points:}

- OPSC should continue to be implemented and supported.

- Orthopaedic outcomes vary with OPSC discharge status and diagnostic group, and these factors may constitute useful prognostic data to inform clinical decision-making/pathways.

\section{PEAK HEALTH FOR PEAK PERFORMANCE, LIVE LONGER, STRONGER, HEALTHIER AND HAPPIER}

\section{Popovic $\mathrm{H}$}

Be prepared to get a new lease on life, to re-ignite your spark, and to have more energy and vitality than you ever remember having. This fastpaced, information-packed session reveals the key steps to consistently performing at your peak, and how to thrive rather than merely survive. Everyone wants to be healthy and it's obvious that peak performance requires peak health. Yet the pace, pressures and demands of $21^{\text {st }}$ century living are actively working against us being healthy. Exhaustion has become the new normal, and $80 \%$ of visits to the doctor could be prevented if we made better lifestyle choices. But how do we make those choices in an already overstretched existence? And what exactly are those health-and-performance-enhancing choices?

\section{Key Practice Points:}

- The four simple daily choices that can prolong our lives by 14 YEARS

-Why energy management is more important than time management

- The four simple daily habits that sustain our energy and effectiveness all day long

- The four learnable skills that neuroscience has uncovered lead to optimal performance

- Why success is a choice and how to start choosing success in all areas of our lives

- How to create a dynamic workplace culture that actively supports everyone's optimal health and productivity
HEALTH PROFESSIONAL SELF CARE - DO YOU PRACTISE WHAT YOU PREACH?

\section{Popovic H}

We live in a culture that has confused self care with being selfish. Self care is something we tend to try and fit in only after we've fulfilled all our other duties, obligations and responsibilities. This presentation invites participants to think of self care differently: as something that needs to come first - at the top of our to-do list - because it enables us to fulfil all our other duties, obligations and responsibilities much more effectively and with more joy. How can self care be selfish when it allows us to give more? Is it selfish to service our car? We would think it irresponsible not to. Why don't we take the same approach with our bodies? This is easy to grasp intellectually yet self care is often tinged with guilt. Research has found that we feel guilty when we take time out for ourselves because there is always something else we 'should' be doing. At the same time, we fee guilty when we don't look after ourselves because we should be looking after ourselves! It seems that no matter what we do or don't do, we feel guilty. So we may as well look after ourselves!

\section{Key Practice Points:}

- What does self care mean to each of us as an individual?

- How can self care create time rather than encroach on our time?

- How to program the brain to accept self care as a natural and integral part of life

- The small things that make a big difference

- The big things that make a big differencel

- How to be an inspiring role model for everyone around us at work and at home

\section{PROMOTING PHYSICAL ACTIVITY AFTER STROKE VIA SELF-MANAGEMENT: A FEASIBILITY STUDY}

Preston E ${ }^{1,3}$, Ada L1 , Dean $\mathrm{C}^{2}$, Waddington $\mathrm{G}^{3}$, Stanton R ${ }^{1}$ School of Physiotherapy, Faculty of Health Sciences, University of Sydney, Australia

Discipline of Physiotherapy, Macquarie University, Australia ${ }^{3}$ Discipline of Physiotherapy, Faculty of Health, University of Canberra, Australia

Question: Is self-management feasible and effective in increasing physical activity after stroke? Can self-management improve health risk, self-efficacy, participation, quality of life and walking ability in mild stroke survivors?

Participants: Seventeen stroke survivors with mild disability who were discharged directly home were recruited from acute stroke units.

Intervention: A standardised self-management program was implemented in 5 sessions over 3 months, incorporating education, goal setting, identifying barriers, self-monitoring and feedback.

Outcomes measures: Feasibility was measured by examining compliance with study protocol. Clinical measures were: physical activity, health risk participation, quality of life and self-efficacy for exercise, and walking ability. Measures were collected at baseline, 3 months and 6 months.

Results: The study was feasible. $70 \%$ of participants completed the study and attended $98 \%$ of intervention sessions. At 3 months 33\% more participants completed 30 minutes of moderate activity/day $(95 \% \mathrm{Cl} 14$ to 61), and were completing 34 min/day more moderate activity (95\% Cl 10 to 58). Walking speed improved by $0.11 \mathrm{~m} / \mathrm{s}(95 \% \mathrm{Cl} 0.01$ to 0.21$)$, endurance by $38 \mathrm{~m}(95 \% \mathrm{Cl} 1-75)$, self-efficacy by 1.5 (95\% Cl 0.4 to 2.7$)$, quality of life by 17 ; ( $95 \%$ Cl 7 to 27$)$ and participation by 7 (95\% Cl 3 to 11$)$. These effects were maintained at follow-up.

Conclusion: Self-management appears to be feasible and has the potentia to increase physical activity in people after mild stroke.

\section{Key Practice Points:}

- Self-management is a promising intervention for increasing physical activity in mild stroke survivors

- A phase II randomized trial is warranted 
TEST-RETEST RELIABILITY OF THE NINE HOLE PEGTEST AND PURDUE PEGBOARD IN PEOPLE WITH PARKINSON'S DISEASE

Proud EL ${ }^{1}$, Bilney $\mathrm{B}^{1}$, Miller $\mathrm{KJ}^{1,2}$, , Morris $\mathrm{ME}^{3}$, McGinley $\mathrm{JL}^{1}$ ${ }^{1}$ Department of Physiotherapy, The University of Melbourne, Parkville Department of Physical Therapy, University of British Columbia, Canada ${ }^{3}$ Department of Physiotherapy, La Trobe University, Bundoora

Question: What is the test-retest reliability of the Nine Hole Pegtest and the Purdue Pegboard in people with Parkinson's disease?

Design: Prospective observational study.

Participants: Thirty volunteers with idiopathic Parkinson's disease (mean disease duration 6.4 years; median Hoehn and Yahr score 2).

Outcome Measures: Two manual dexterity measures, the Nine Hole Pegtest and Purdue Pegboard were administered i) in the 'on' phase of the medication cycle and ii) at the end of dose ('off'), on two days one week apart.

Results: Test-retest reliability ICCs $(2,1)$ for Nine Hole Pegtest dominant nondominant subtests were 0.79 / 0.70 'on' and 0.74 / 0.81 'off'. For Purdue Pegboard subtests (mean of three trials), ICCs $(2,3)$ ranged from 0.90 to 0.95 'on', and 0.93 to 0.97 'off'. Standard Errors of Measurement expressed as a percentage of the group mean for Nine Hole Pegtest subtests were $8.2 \%$ / 8.8\% 'on'; and 10.6\% / 7.6\% 'off'; and for Purdue Pegboard subtests $5.2 \%$ to $8.9 \%$ 'on'; $4.4 \%$ to $7.5 \%$ 'off'

Conclusion: Nine Hole Pegtest and Purdue Pegboard subtests showed moderate to good test-retest reliability and acceptable measurement error in people with mild to moderate Parkinson's disease, in both phases of the medication cycle,

\section{Key Practice Points}

- The Nine Hole Peg Test and Purdue Pegboard are reliable measures of manual dexterity in Parkinson's disease.

- Measurement error was generally greater in Nine Hole Pegtest subtests than Purdue Pegboard subtests.

- Knowledge of validity, responsiveness and clinically important change in this patient group would inform clinical practice.

\section{TENDINOPATHY: THEN, NOW AND WHERE TO FROM HERE?}

\section{Purdam C ${ }^{1}$, Spurrier $D^{2}$, Visentini $\mathrm{P}^{3}$, Laakso L ${ }^{4}$, Longbottom $]^{5}$,} Yelland $\mathrm{M}^{6}$, Gabel $\mathrm{P}^{7}$

${ }^{1}$ Australian Institute of Sport, Canberra

¿Mitcham Rehab Clinic, Adelaide

${ }^{3}$ Physiosport Brighton, Melbourne

${ }^{4}$ Griffith University School of Physiotherapy, OLD

${ }^{5}$ University of Hertfordshire, UK

${ }^{6}$ Griffith University School of Medicine and Primary Health Care, QLD

'Coolum Physiotherapy, QLD

This session aims to provide attendees with an up-to-date approach to the management of tendinopathy. A background of the current body of literature for tendon injuries will be presented and considered in a historical light, with expert opinion on recent and ongoing changes in the tendon injury paradigm and possible future directions. A wide-ranging discussion of the available treatment approaches will be provided. These will encompass an evidence-based perspective on the recommended treatment paradigms of loading and exercise rehabilitation, with particular emphasis on clinically applicable practice. The session will also consider the role of laser and needle-based therapies as adjunct treatments in the management of tendinopathy, with each perspective presented by experts in their own field. The session will be rounded off by a clinical case study utilizing a multi-modal approach in the daily management of achilles tendinopathy.

\section{Key practice points:}

- Historical understanding of tendinopathy management

- Evidence-based and clinically applicable exercise and loading approaches to tendinopathy rehabilitation

- Understanding of adjunct therapies available in the management of tendinopathy
USING THE SMART PHONE, MICROSOFT KINECT AND THE WII BOARD TO MEASURE CERVICAL RANGE OF MOTION, THORACIC KYPHOSIS AND BIG TOE STRENGTH

Quek J ${ }^{1,2}$, Brauer $\mathrm{S}^{1}$, Treleaven J1 ${ }^{1}$, Clark R ${ }^{3}$

${ }^{1}$ University of Queensland, Brisbane, Queensland

¿Singapore General Hospital, Singapore

${ }^{3}$ Australian Catholic University, Melbourne, Victoria

Background: In rehabilitation science, new technology such as smartphones, Nintendo Wii Board and the Microsoft Kinect are increasingly gaining global attention and popularity due to its tremendous potential to transform healthcare and clinical intervention in the community.

Aims/Objectives: The aims of the session are to 1) present the research findings of 3 studies investigating the use of the smart phone the Microsoft Kinect and the Nintendo Wii Board (NWB) to measure cervical range-of-motion (ROM), thoracic kyphosis and big toe strength respectively, 2) Demonstrate the use of these three devices and how they may be translated to clinical practice.

Approach: Results of the three studies will be briefly presented. (i) The smartphone application is valid and reliable to measure cervical ROM in flexion, extension and lateral flexion (ICC $=0.53-0.98$, Spearman $\rho=0.52$ 0.98 ) but not rotation (ICC $=0.05-0.33$ ). (ii) The Microsoft Kinect is valid and reliable to measure thoracic kyphosis when compared to the flexicurve (ICC=0.76-0.98). (iii) A new method using the NWB is reliable to measure big toe strength $(I C C=0.982)$. Next, a live demonstration of all three devices will be presented with opportunities for delegates to experiment using the devices. Delegates will be given access to download the application used for the smart phone.

Conclusion: This session will (i) provide clinically relevant insights to therapists and increase their confidence to use the smartphone, Microsoft Kinect and the NWB, (ii) demonstrate the use of these devices and (iii) broaden the repertoire of clinically relevant and accurate tools that are widely available that clinicians may use.

\section{MANAGING PAIN IN THE OLDER ADULT - A CLINICAL PERSPECTIVE}

\section{Rahmann $\mathrm{A}^{1,2}$}

${ }^{1}$ School of Physiotherapy, Australian Catholic University Banyo ${ }^{2}$ Brighton Rehabilitation Unit, Metro North Hospital and Health Service Pain is a common symptom in older adults, with $25-56 \%$ of communitydwelling adults reporting chronic pain and up to $75 \%$ of hospitalised older adults experiencing acute pain during admission. Pain in older adults is commonly persistent, multi-focal and multi-factorial, and is often underassessed and under-managed. Issues that can make the management of pain in older adults more challenging for the clinician are sensory and cognitive deficits, polypharmacy, co-morbidities and the incidence of undiagnosed depression. Added to these factors are the common attitudes and beliefs of older adults such as "I don't want to complain" or "I don't have any pain if I don't move around too much". Clinicians also report beliefs that pain is inevitable in older adults or that older adults are less sensitive to pain. Even more sobering statistics highlight the issues of older adults with a cognitive or communication deficit. Patients with advanced dementia hospitalised after hip fracture received a third the amount of opioid analgesia that older patients with intact cognition received. Similarly, patients with a cognitive impairment (MMSE <23) also received significantly less opioids than cognitively intact patients despite reporting similar pain intensities. This discussion will use a case-based approach to describe the assessment and clinical reasoning approach that I used to guide my physiotherapy management of a patient with complex pain issues admitted to our sub-acute inpatient rehabilitation unit. This gentleman was admitted with a recent below knee amputation following a long period with a poorly healing lower limb ulcer. To complicate matters he developed shingles and had an acute exacerbation of his chronic low back pain with sciatica.

\section{Key Practice Points:}

- Pain in older adults is often under-assessed and under-managed

- The appropriate management of pain in people with a cognitive or communication deficit is frequently sub-optimal

- Older adults require appropriate and often advanced clinical reasoning for optimal patient-focused management

- Multi-disciplinary involvement is a key ingredient to enable optimal management 


\section{ARE DIFFERENCES IN SELF-REPORTED PAIN, PERCEIVED EXERTION AND COPING STRATEGIES RELATED TO THE ADDITION OF AQUATIC EXERCISE EARLY AFTER TOTAL JOINT REPLACEMENT SURGERY?}

\section{Rahmann $\mathbf{A}^{1,2}$}

1School of Physiotherapy, Australian Catholic University, Brisbane ${ }^{2}$ Brighton Rehabilitation Unit, Metro North Hospital and Health Service Pain and psychological status can have an impact on functional recovery after joint replacement surgery. An initial pilot study examined differences in self-reported pain or perceived exertion amongst three different post-operative physiotherapy protocols. Eight participants recruited at Day 4 post-operatively $(67.6 \pm 5.7$ yrs) completed the protocols: water exercise (Day 4), intensive aquatic physiotherapy (Day 5) and ward based physiotherapy (Day 6). Self-rated pain was significantly less than baseline during both the intensive aquatic $(-1.6,95 \% \mathrm{Cl}-2.7$ to -0.5$)$ and water exercise programs $(-1.4,95 \% \mathrm{Cl}-2.5$ to -0.3$)$. There was a trend for pain to increase during ward exercise $(1.0,95 \% \mathrm{Cl}-0.1$ to 2 .1) compared to baseline. Perceived rate of exertion (PRE) was significantly different amongst the programs $(p=0.002)$ with post-hoc analysis revealing that the ward exercise program was perceived to be harder than either the water exercise $(p=0.010)$ or aquatic exercise programs ( $p=0.049)$, with no difference in PRE between the aquatic and water exercise programs ( $p$ $=0.420$ ). A second study examined the correlation between positive and negative coping strategies (Coping Strategies Questionnaire) and early postoperative functional recovery at Day 14 in the participants of a larger RCT (69.6+8.2years, 30 men). A moderate correlation was found between positive coping strategies and better self-reported function, pain and stiffness on the WOMAC arthritis scale. A higher positive coping strategies score also showed a trend for correlation with faster functional mobility (TUG and ten metre walk test). Although not significant, there was a trend for aquatic exercise to decrease catastrophising strategies more than ward-based physiotherapy in the early post-operative phase.

\section{Key Practice Points:}

- Self-rated pain was decreased during immersion, regardless of exercise type

- Clinicians need to be specific with aquatic exercise prescription in this early post-operative phase because the perception of less pain and less effort can lead to either under- or over-exercise

- Aquatic exercise early after surgery may help to decrease negative coping strategies

- Positive coping strategies are related to better self-rated abilities and increased functional mobility early after orthopaedic surgery

\section{TEAM BASED LEARNING FOR ENHANCING PHYSIOTHERAPY EDUCATION}

\section{Raigangar V}

Department of Physiotherapy, University of Sharjah

Purpose: To conduct and evaluate the effectiveness of a using team based learning to enhance physiotherapy education at the University of Sharjah (United Arab Emirates)

Relevance: Team-based learning (TBL) is a leaner centered, instructor directed small /large group instructional strategy that enables students to apply conceptual knowledge through a sequence of activities including individual work, team work and immediate feedback. This serves to make them more cohesive and encourages a positive approach towards working in multidisciplinary teams in practice.

Participants: 24 students enrolled in their final semester were engaged in a team based learning session. They were divided into four groups of six students each and asked to work collaboratively within their groups. Handouts for the session were provided prior to the class.

Methods: A three hour class was used to implement the Individual readiness assurance test (IRAT), the Group Readiness Assurance test (GRAT) followed by instructor led discussion and application to a case based scenario. This was then evaluated by pre/ post questionnaires and focus group interviews. Marks were allocated to both the IRAT and GRAT to be used in formative evaluation.

Analysis: This was done on the basis of the comments in the questionnaires and transcripts of the interviews which were classified into themes and reflected upon.

Results and Conclusion: There was initial apprehension by the entire class in engaging in such a session. Feedback obtained after the sessions demonstrated that it was in fact a very enjoyable and mutually beneficial session for all the students. Furthermore, the students expressed that they "learnt a lot" from each other and many more such future activities should be planned.

Implications: These collaborative activities add value to the assessment process for students. Guidelines for designing such sessions/modules in different aspects of physiotherapy education will be discussed. Recommendations will be provided on incorporating similar models of team based learning in hospitals as team work is crucial to the success of patient centered care to ensure high quality standards.
SOMATOSENSORY CHANGES

ASSOCIATED WITH CHRONIC NONSPECIFIC MUSCULOSKELETAL SHOULDER PAIN

Ramlu C ${ }^{1}$, Teys $\mathrm{P}^{1}$, Good D

${ }^{1}$ Australian Catholic University, Banyo, Brisbane

Question: Are somatosensory changes different in people with chronic shoulder pain compared with healthy controls?

Design: cross sectional pilot study.

Participants: 10 subjects with chronic shoulder pain and 10 age and gender matched healthy controls.

Outcome Measures: Quantitative sensory measures of pressure pain threshold, heat and cold pain threshold on affected shoulder or dominant shoulder, opposite shoulder, and tibialis anterior of the leg contralateral to the side of pain or dominance for the healthy subjects.

Results: There was a significant difference for pressure pain threshold between chronic shoulder pain subjects and healthy controls across all sites: $p=0.015$ affected shoulder versus dominant shoulder, $p=0.01$ unaffected shoulder and $p=0.003$ for tibialis anterior. There was no significant difference for heat pain threshold between the affected shoulder in shoulder pain subjects and the dominant shoulder of healthy controls ( $p=0.28$ ) but there were significant differences at both the unaffected side and non-dominant shoulders $(p=0.01)$ and the tibialis anterior $(p=0.00)$. There was a floor effect for the majority of the healthy controls for cold pain threshold. Pain was not felt before the cut-off temperature was reached so no further analysis was carried out for cold pain threshold.

Conclusion: Significantly lower pressure pain threshold and heat pain threshold values were found in subjects with chronic shoulder pain compared with healthy age and gender matched controls.

\section{Key Practice Points:}

- Somatosensory changes in subjects with chronic shoulder pain

- Lower PPT and HPT in subjects with chronic shoulder pain 


\section{IS IT MORE COST-EFFECTIVE TO EXPAND AN ADVANCED MUSCULOSKELETAL PHYSIOTHERAPY SERVICE, OR TRADITIONAL ORTHOPAEDIC PATHWAYS, TO ADDRESS UNMET DEMAND?}

Raymer $\mathrm{M}^{1}$, Standfield $\mathrm{L}^{2}$, O'Leary $\mathrm{S}^{1,3}$, Smith $\mathrm{D}^{4}$, Scuffham $\mathrm{P}^{2}$, Comans $T^{2,5}$

1Physiotherapy Department, Royal Brisbane and Women's Hospital, Metro North Hospital and Health Service, Brisbane

2Menzies Health Institute Queensland and Centre for Applied Health Economics, School of Medicine, Griffith University, Logan

${ }^{3}$ School of Health and Rehabilitation Sciences, University of Queensland, Brisbane

${ }^{4}$ Physiotherapy Department, Ipswich Hospital, West Moreton Hospital and Health Service, Ipswich

${ }^{5}$ Allied Health, Metro North Hospital and Health Service District

Question: Is it more cost-effective to expand an advanced musculoskeletal physiotherapy led service, or traditional pathways, to address unmet demand in Orthopaedic Outpatients?

Design: An economic model (Discrete Event Simulation, Simul8 ${ }^{\circ} 2014$ ) was used to synthesise data describing patient clinical pathways, outcomes and costs. The model compared options to address unmet demand on orthopaedic services over a five year timeframe and included an increase in any/ all of: (1) an advanced musculoskeletal physiotherapy led service, (2) traditional orthopaedic outpatient clinics, (3) orthopaedic surgery throughput.

Data was sourced from a prospective study of patients referred to orthopaedic services at two Queensland Hospitals, one with and one without an advanced musculoskeletal physiotherapy led service.

Outcome Measures: The primary outcome was the incremental costeffectiveness ratio (cost per additional quality-adjusted life-year-gained). Throughput (patients treated over five years) and waiting times were also calculated.

Results: Cost-effectiveness ratios ranged from $\$ 8301$ to $\$ 36218$ indicating all scenarios tested may be considered cost-effective in an Australian context. Doubling (ICER \$8301) or tripling (ICER \$8140) the scale of the advanced musculoskeletal physiotherapy led service was the most cost-effective alternative, resulting in the least cost per additional health unit gained.

Conclusion: Although any of the evaluated options may address unmet demands on orthopaedic outpatient services, the most cost-effective alternative is to increase the advanced musculoskeletal physiotherapy led assessment and treatment service. These findings can inform health service managers in decision making about resource allocation in orthopaedic settings.

\section{Key Practice Points:}

- Advanced musculoskeletal physiotherapy led services are increasingly adopted to improve access to services in Orthopaedic Outpatient settings

- Economic modeling can guide decision making about resource allocation where lengthy queues and unacceptable waiting times continue.

- This study indicates it is more cost-effective to increase the capacity of an advanced musculoskeletal physiotherapy led assessment and treatment service than traditional pathways to address unmet demand on Orthopaedic Outpatient services.

\section{PERFORMANCE SUCCESS OR FAILURE IS EXPLAINED BY WEEKS LOST TO INJURY AND ILLNESS IN ELITE AUSTRALIAN TRACK AND FIELD ATHLETES: A 5-YEAR PROSPECTIVE STUDY}

\section{Raysmith $\mathbf{B}^{1}$, Drew $\mathbf{M}^{1,2,3}$}

${ }^{1}$ Department of Physical Therapies, Australian Institute of Sport, Canberra, Australia

${ }^{2}$ Australian Centre for Research into Injury in Sport and its Prevention (ACRISP), Federation University, Australia

${ }^{3}$ Department of Physiotherapy, Faculty of Health, University of

Canberra, Australia

Objectives: To investigate the impact that training modification has on achieving performance goals. Previous research has shown an inverse relationship between injury burden and success in team sports. It is unknown whether this relationship exists within individual sport such as athletics.

Design: A prospective, cohort study ( $n=33$ International Track and Field Athletes; 76 athlete seasons) across five international competition seasons.
Methods: Athlete training status was recorded weekly over a 5-year period. Over the 6 -month preparation season, relationships between the volumes of planned training weeks completed, the number of illness/ injury events and the success or failure of a performance goal at major championships was investigated. Two-by-two table were constructed and attributable risks in the exposed (AFE) calculated. A mixed-model, logistic regression was used to determine the relationship between failure and burden per injury and/or illness.

Results: The likelihood of achieving a performance goal was increased by 7 -times in those that completed $>80 \%$ of planned training weeks ( $R R=7.19,95 \% C l, 1.84$ to 27.89 ). In this cohort $86 \%$ of successful seasons could be attributed to training availability (AFE=0.86, 95\%Cl, 0.46 to $0.96)$. The majority of new injuries occurred within the first month of the preparation season (30\%) and most illnesses occurred within 2-months of the event (50\%). For every week lost to full training the chance of success significantly reduced (OR=0.74, 95\% Cl 0.58 to 0.94$)$.

Conclusions: Injuries and illnesses, and their influence on training availability, during preparation are major determinants of an athlete's chance of performance goal success or failure at the international level.

\section{Key Practice Points:}

- Achieving consistent and uninterrupted training is imperative to improving the chances of the athlete reaching their key performance goal for the season.

- Successful athletes sustain lower accumulation of modified training weeks due to injury and illness.

- Successful athletes sustained less than two training modification episodes due to injuries and/or illness in the 6-months prior to a major international event.

\section{HOW TO ASSESS CONTRACTION OF THE CERVICAL DEEP MUSCLE EXTENSORS USING REAL-TIME ULTRASOUND}

\section{Rebbeck T ${ }^{1}$, Desa V, Shirley D, Clarke J, Leaver A}

${ }^{1}$ Discipline of Physiotherapy, Faculty of Health Sciences, University of Sydney

Background: Deep cervical extensor (DCE) muscles become impaired in cervical spine disorders and lead to key functional restrictions (eg inability to hold the head up). Whilst there are several methods to evaluate the impairment of the DCE'S, real time ultrasound (RTUS) is less invasive, less expensive and more clinically accessible, allowing for observation of the contraction of the DCE's in real time. Our work recently demonstrated the reliability of a clinical protocol, whereby impairment of the DCE is evaluated by observing the change in the muscle dimensions under load using video RTUS.

Aims / objectives: Participants will understand the role of the DCE's and how they become impaired in cervical spine disorders. Participants will have the clinical skills to assess the contraction of the DCE's using video RTUS as well as understanding how to interpret this information in context of a comprehensive cervical spine assessment.

Approach: A 10 minute presentation on the background research will be followed by a demonstration of the assessment protocol using video RTUS. Participants will then have the opportunity to practice. An interactive discussion will follow on how to interpret and integrate this information into clinical practise. Hand-outs on the assessment protocol will be provided.

\section{Conclusion / Key Practice Points:}

- Participants will have an additional clinical skill in the assessment of DCE's

- They will understand how to incorporate this into the routine assessment of cervical spine disorders

- Clinical reasoning on how to interpret and use RTUS in rehabilitation of the cervical extensors will enhance clinical practise 


\section{A NEW DIRECTION FOR WHIPLASH: DEVELOPMENT OF A RISK-BASED AND GUIDELINE -BASED CLINICAL PATHWAY OF CARE}

Rebbeck $T^{1}$, Leaver $A^{1}$, Ritchie $C^{2}$, Sterling $M^{2}$

IUniversity of Sydney

Griffith University

Question: Will a risk and guideline- based clinical pathway of care improve health outcomes for whiplash?

Design: Development of a clinical pathway of care

Participants: Participants were key stakeholders in the pathway of care. They included guideline developers and implementers, developers of a risk assessment tool, primary and secondary care clinicians, researchers and health policy makers.

Intervention: This paper describes the development of a novel clinical pathway of care. Firstly, a recently validated clinical prediction rule (CPR) to stratify patients into low, medium/ high risk of non-recovery was adapted for the pathway. Next, the interventions from a recently published guideline for acute whiplash were matched to the risk stratification. Finally a model of care utilizing primary care and specialist clinicians was developed and applied to the risk stratification. Key stakeholders were consulted to inform the process, content and outcome measures of the pathway

Outcome Measures: The outcome is a published clinical pathway of care encompassing risk stratification, matched guideline-based interventions and novel service delivery. The effect of the pathway on health care processes and outcomes (eg global perceived erect and self-efficacy) will be evaluated in a recently funded randomised controlled trial.

Results: This is the first pathway of care for whiplash to be developed and published. It has created a unique partnership amongst policy makers, researchers and service providers in multiple disciplines managing people with whiplash.

Conclusion: Successful development of the pathway occurred due to researchers, clinicians and policy makers collaborating with the ultimate aim of improving health outcomes for people with whiplash.

\section{Key Practice Points:}

- Involve key stakeholders from research, clinical practice and health care policy in the development of a pathway

- Ensure multi-disciplinary representation

- Qualitative research to inform the pathway was useful.

\section{HOW TO ASSESS FOR PAIN SENSITISATION IN THE CLINIC: NECK AND ARM PAIN FOCUS}

\section{Rebbeck T ${ }^{1}$, Moloney $\mathrm{N}^{2}$, Beales $\mathrm{D}^{3}$}

${ }^{1}$ Discipline of Physiotherapy, Faculty of Health Sciences, University of Sydney ${ }^{2}$ Dept. Health Professions, Macquarie University

${ }^{3}$ School of Physiotherapy and Exercise Science, Curtin University

Background: Increased pain sensitisation assessed by quantitative sensory testing (QST), such as cold hyperalgesia, is associated with poor outcomes in cervical pain states. Thus, more detailed pain assessments of pain are warranted in clinical practice. Our work has demonstrated a relationship between clinical tests of pain sensitivity (ice pain and pressure pain tests) and OST. Other clinical tests used to assess for pain sensitisation include nerve trunk palpation, conditioned pain modulation and 2-point discrimination. Identification of pain sensitisation in the clinic is important to not only sub-classify pain, but to inform appropriate management.

Aims / objectives: To improve participants' knowledge, skills and clinical reasoning in the clinical assessment of pain sensitisation. Participants will be competent in clinical application of the ice-pain test, pressure pain thresholds, 2-point discrimination, upper limb nerve trunk palpation and conditioned pain modulation. Participants will understand how to interpret these tests to sub-classify pain and direct treatment.

Approach: The presenters will provide a lecture style background introduction (10 mins) followed by a practical demonstration of the tests (10 mins). Participants will then have the opportunity to practise 1-2 of these tests (10 minutes). Learning materials provided include a manual on how to perform these tests.

Conclusion / Key Practice Points:

- Participants will be able to more accurately assess for pain sensitisation in their clinic.

- Participants will understand the added benefits and limitations of using these tests in clinical practice

- Participants will be able to use information gleaned from pain assessment to inform treatment
PHYSIOTHERAPY FOLLOWING TOTAL KNEE REPLACEMENT: AN AUDIT OF OUTPATIENT PRACTICE

Redmond $\mathrm{C}^{1}$, Alt $\mathrm{T}^{1}$, Crouch $\mathrm{D}^{1}$, Hewitt $\mathrm{T}^{2}$

${ }^{1}$ Physiotherapy, School of Health Sciences, Flinders University, Daw Park 2Physiotherapy, Repatriation General Hospital, Daw Park

Question: Did current outpatient physiotherapy practice reflect locally constructed standards of practice?

Design: Clinical practice at the Repatriation General Hospital (RGH) was compared with treatment standards, constructed from a literature review. Patient records were compared to these standards.

Participants: Of the 147 patients who had a total knee replacement during two quarterly periods in 2012, 79 attended outpatient physiotherapy appointments at the RGH, of which 74 medical and 76 physiotherapy records were available for review.

Outcome Measures: Treatment, outcomes and service delivery timeframes were recorded and analysed descriptively.

Results: Patients had a median of 2 outpatient physiotherapy appointments (range, 1-7 visits) prior to discharge. Most patients progressed from mobilising with a rollator frame $(66 / 74,89 \%)$ to mobilising without aids $(21 / 42,50 \%)$ or with a single point stick (13/42, $31 \%$ ) at discharge. The predominant treatment was an exercise program $(46 / 79,58 \%)$, consisting of range of movement (24/25, 96\%), strengthening $(22 / 25,88 \%)$ and balance exercises $(3 / 25,12 \%)$ or group therapy (27/79, 34\%).

Conclusion: Physiotherapists usually assessed mobility, prescribed home exercises and referred patients to group therapy. Our recommendations included using functional positions to progressively strengthening quadriceps and using a timed walking test to monitor recovery following total knee replacement.

\section{Key Practice Points:}

- Clinical audit assists in understanding if research is being translated into practice.

- Interpreting patient records relies on consistent terminology and clear descriptions.

- Using functional outcomes, such as walking speed, or stair ascending and descending, will assist in defining recovery in outpatient physiotherapy.

\section{THE PAST, THE PRESENT AND THE FUTURE OF CYSTIC FIBROSIS CARE}

\section{Reid DW}

Adult cystic fibrosis Centre, the Prince Charles Hospital, Brisbane Over the past 30 years, outcomes in CF have improved rapidly and the health care system is struggling to cope with the burgeoning numbers of adults, which requires new models of care to be considered. Dr Reid will review the Past, the Present and the Future of Cystic Fibrosis care, which will hopefully provide novel insights into the unique challenges ahead, and to some of the solutions. 
HEAD REPOSITIONING ACCURACY IN YOUNG PEOPLE WITH INSIDIOUS ONSET NECK PAIN ASSOCIATED WITH SUSTAINED FLEXION

Portelli $A^{1}$, Reid SA ${ }^{1}$

'School of Physiotherapy, Australian Catholic University, North Sydney

Question: Do young people with insidious onset neck pain who spend long periods in sustained flexion while using unsupported electronic mediums such as phones and ipads have deficits in head repositioning accuracy (HRA) compared to asymptomatic controls

Design: Two-group comparative observational study.

Participants: Forty four young (18-35 years) adults with $(n=22)$ and without $(n=22)$ neck pain

Outcome Measures: Proprioception measured with the HRA test during cervical flexion, extension left and right rotation. Intensity of neck pain was recorded with a visual analogue scale (VAS). T-tests determined differences in variables between neck pain and control groups and Pearson's bivariate analysis was used to assess correlations.

Results: Subjects with neck pain displayed significantly $(p=0.02)$ poorer HRA compared to asymptomatic controls during cervical flexion, but not with other movements. A moderate correlation ( $p<0.05)$ was found for time spent on unsupported electronic mediums and cervical spine pain intensity and for cervical spine pain intensity and deficits in head HRA during flexion.

Conclusion: The results indicate that the more time young people spend in prolonged flexion on unsupported electronic mediums, the greater their neck pain, and the poorer their proprioception is during flexion.

\section{Key Practice Points:}

- Advice should be given to patients not to spend long periods of time in sustained flexion on unsupported electronic mediums.

- Treatments that address cervical proprioceptive deficits such as head repositioning may be required.

- Specific treatment addressing neck posture and movement control into flexion for example using the deep neck flexors, may be required.

\section{SOCIAL MEDIA USE IN HEALTH PROFESSIONAL EDUCATION: PRELIMINARY FINDINGS FROM AN INTERNATIONAL AND INTERDISCIPLINARY STUDY}

\section{Remedios L}

The University of Melbourne

Questions: 1) How do students use social media (SoMe) in health professional education?

Design: An International, Interdisciplinary team of educators and students from eight Universities across the Univeritas 21 consortium, collaborated on the design and implementation of this study. Following ethics approval from the participating universities, a survey was hosted on fluid survey and opened to students and educators across the eight universities.

Participants: 1,757 students completed the survey. Students were from the University of British Columbia, University College Dublin, University of Birmingham, University of Nottingham, Fudan University, University of Hong Kong, University of Melbourne and Tecnologico de Monterrey and represented medicine, dentistry, pharmacy, nursing and physiotherapy

Outcome Measure: A 24 item questionnaire gathered demographic data, information on the formal and informal use of SoMe and knowledge and use of SoMe guidelines.

Results: The majority of students reported using SoMe often (37\%) or almost always (18\%) and used it both formally and informally to share learning resources (36\% \& 57\%) and exam preparation (32\%, 50.8\%) Close to half the students were not aware of professional SoMe guidelines or were aware of the implications of breaking rules.

Conclusion: Health professional students make use of social media for learning, although they have limited knowledge of SoMe policy guidelines. This has implications for their professional practice.

\section{Key Practice Points:}

- Educators can promote the use of social media for learning - Educators should include information on SoMe policy guidelines in curriculum.

- Effort should be made to ensure students understand the risks of unprofessional use of SoMe.
DOSE AND CONTENT OF STUDENT-LED

PHYSICAL THERAPY SESSIONS: AN OBSERVATIONAL STUDY OF PEOPLE WITH STROKE AND TRAUMATIC BRAIN INJURY

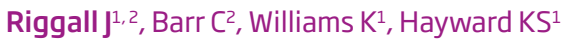

${ }^{1}$ Division of Physiotherapy, School of Health and Rehabilitation Sciences, The University of Queensland, Brisbane Australia

2School of Health Sciences, Flinders University, Adelaide Australia

Question: What is the dose and content of therapy provided by final year physiotherapy students working under supervision to people with stroke and traumatic brain injury (TBI) undergoing outpatient rehabilitation?

Design: Observational Study ( $n=16$ stroke sessions and $n=16$

TBI sessions)

Participants: Student physiotherapists under supervision provided 1-hour therapy sessions to client subjects with stroke and TBI. An independent observer documented the dose (number of repetitions) and content (passive, active, functional, sensory) of therapy; and level of function Intervention: Not applicable

Outcome Measures: An observation protocol to guide the classification of therapy content into categories (upper limb, lower limb, mobility) and subcategories (active, passive, functional, sensory) was used to guide data collection. Analysis of the dose and content of therapy was completed, using the Mann Whitney U Test, based on client diagnosis, level of function (modified Rankin Score and Disability Rating Scale) and student achievement (Assessment of Physiotherapy Practice form).

Results: Overall, gait was commonly addressed ( $94 \%$ of sessions; steps: stroke mean 323 SD 159, TBI mean 625 SD 642), but upper limb tasks were not (25\% of sessions; functional arm repetitions: stroke mean 72 SD 24, TB mean 84 SD 81). There were no significant differences in dose or content of therapy provided based on client diagnosis or level of function. Higher achieving students however, completed more upper limb repetitions per minute compared to lower achieving students $(p=0.03)$

Conclusion: Physiotherapy students can deliver a high dose of therapy that addresses a number of content categories.

\section{Key Practice Points:}

- Student physiotherapists can deliver a high dose of therapy with a predominant focus on functional tasks including gait.

- Investigation of other factors potentially contributing to the lower dose of upper limb therapy is warranted.

- Comparison of student to practicing therapist dose is warranted.

\section{KNOWLEDGE TRANSLATION IN \\ PHYSIOTHERAPY - FROM CLINIC TO RESEARCH AND BACK TO THE CLINIC}

\section{Risberg $\mathrm{MA}^{1,2}$}

${ }^{1}$ Department of Sport Medicine, Norwegian School Sport Sciences, Oslo Norway

2Department of Orthopedic Surgery, Oslo University Hospital, Oslo Norway

Only a small percentage of knowledge developed through research is translated into clinical practice. Hence, one of the most consistent findings is the failure to translate research into practice and policy. Globally, we spend millions of dollars on clinical research, but still health care professionals fail to provide evidence based level of care. Furthermore, translational research is needed to understand the mechanisms for why physical therapy interventions work. We need to better understand the continuum from "bench to bedside" in physical therapy. Hence, first, understanding the continuum from "bench to bedside", and secondly understanding the process of implementation of knowledge to clinic settings are at least two major aspects within knowledge translation in physical therapy. First line treatment for patients with mild to moderate knee and/or hip osteoarthritis is patient education and exercise

interventions. Still, many patients do not receive this evidence based leve of care. Implementation of research knowledge is lacking. Furthermore surgical interventions are used for patients with early knee OA despite the fact that we know they should receive other types of interventions as first line treatment. Some interventions are translated into clinical practice without high quality research, and others are not implemented despite high quality research knowledge. Femoroacetabular impingement (FAI) is also such and area. Exercise therapy for patients with tendinopathy has shown to be effective. The area of tendinopathy is one of few areas that we have undertaken translational research to better understand why and how exercise therapy may work for these patients. Mechanotherapy is a field of future development to improve our understanding of different musculoskeletal and sport physical therapy interventions. 


\section{EVIDENCE FOR ACL REHABILITATION - WHERE ARE WE AND WHERE ARE WE GOING}

\section{Risberg $M A^{1,2}$}

${ }^{1}$ Department of Sport Medicine, Norwegian School Sport Sciences, Oslo Norway

2Department of Orthopedic Surgery, Oslo University Hospital, Oslo Norway

Background: Treatment options after ACL injury is either 1) ACL reconstruction and rehabilitation, 2) prehab, ACL reconstruction, and rehabilitation or 3 ) rehabilitation alone. What is the evidence for surgery versus rehab alone, for prehab and for type of rehabilitation programs?

Objective: The objective of this presentation is to go through the most recent evidence for the $A C L$ rehabilitation and present our treatment algorithm for ACL injured individuals.

Methods: A literature review will be presented of the effect of surgery or rehabilitation only after $A C L$ injury, for the effect preoperative programs for $A C L$ injured individuals who are going through $A C L$ reconstruction (prehab), and to present the evidence for different interventions to optimize muscle function and functional outcomes after $\mathrm{ACL}$ reconstruction.

Results: The most recent literature continues to support that a subgroup of patients with ACL injury do well without surgery, although the majority still seem to prefer surgical treatment with postoperative rehabilitation. We must acknowledge that the ACL population is not a homogeneous group. Patients who undergo prehab prior to ACL reconstruction have significantly better outcome compared to those without prehab. We still need to optimize postoperative muscle function after ACL injury and reconstruction. Quadriceps inhibition must not be overlooked. Impairments and functional disabilities after ACL reconstruction need to be addressed and evaluated prior to return to sport. Patients who return early to pivoting sports seem to be at a higher risk of re-injury. Patients with additional meniscus injury have a significantly higher risk of knee OA and worse functional outcome long term. Risk factors for returning to sport should continue to be addressed in future large, high quality, prospective cohort studies.

Conclusion: There are at least three main treatment options for ACL injured individuals. The ACL population is a heterogeneous group and rehabilitation needs to target individual goals and preinjury level of functional performance, as well as individual impairments and functional disabilities. We need better screening tests to predict outcome for ACL injured individuals. Current evidence indicate that ACL injured individuals should receive non-operative interventions before surgical intervention is considered. The rehabilitation programs need to optimize interventions targeting muscle dysfunctions and improved functional performance. All patients should be tested for impairments and disabilities prior to return to sport. More research is needed on how exercises affect knee joint loading long term. Finally, significant risk factors for re-injuries should be studied in future high quality, prospective cohorts to develop better prevent strategies for meniscus injury and re-rupture of graft.

\section{EVIDENCE FOR REHABILITATION FOR KNEE OA PATIENTS - IMPLEMENTATION OF GUIDELINES}

\section{Risberg $M A^{1,2}$}

${ }^{1}$ Department of Sport Medicine, Norwegian School Sport Sciences, Oslo Norway

Department of Orthopedic Surgery, Oslo University Hospital, Oslo Norway

Background: First line treatment for patients with knee osteoarthritis $(\mathrm{OA})$ is exercises, patient education, and weight reduction (for those who are overweight or obese). However, we lack knowledge on type of exercises and physical activities (dose-response). Furthermore, there is lack of evidence on how exercises influence structural changes associated with knee OA.

Objective: The objective of this presentation is to go through the most recent evidence for the effect of exercises for patients with knee OA. Furthermore, the aim is to present current evidence for structural changes on knee articular cartilage following exercises.

Methods: A literature review will be presented of the efficacy of exercises on symptoms and dysfunctions for patients with knee $O A$, and current literature on how exercises may influence knee joint loading.

Results: The most recent literature continues to support the efficacy of exercises for symptoms and dysfunctions for patients with knee OA. A recent Cochrane review from 2015 (Fransen et al) concluded that high-quality evidence indicates that land-based therapeutic exercise is beneficial for knee OA. Many high quality guidelines for non-surgical treatment of knee OA have been published, hence, implementation of these guidelines is needed.

Furthermore, we need to implement interventions targeting modifiable factors associated with disabilities in OA patients. But we lack knowledge on what type of muscle impairments that causes disabilities as well as how exercises influence knee joint loading related to development and progression of OA. New magnetic resonance imaging studies have reported changes in articular cartilage after different types of exercises in health subjects as well as in those with early OA.

Conclusion: The literature continues to support that exercises should be the first-line treatment for patients with knee OA. There is no shortage of endorsement or guidance for the use of exercises and physical activity as an effective therapeutic modality for knee OA. However, a significant shortage appears in the implementation of exercises and physical activity in clinical settings. Furthermore, the mechanisms for why exercises have positive effects on symptoms and function is lacking. The muscles of the lower extremity, particular the quadriceps, have shown to play an important role in the management of patients with knee OA. But we still lack knowledge on what specific muscle impairments that causes disabilities in OA patients. Finally, we need a stronger focus towards prevention of onset and progression of OA rather than only palliative treatment of symptoms and dysfunction.

\section{GAIT - CONNECTING BRAIN AND BEHAVIOUR}

\section{Rochester L}

Institute of Neuroscience, Newcastle University, UK

For the past decade, the notion that safe and effective gait is due solely to an intact motor system has given way to a more complex model that reflects the cognitive control of gait. Evidence supporting this comes from diverse sources including: behavioural; imaging; neurophysiological; and neuropathological experiments. This presentation will identify how the connections between gait and the brain are helping to elucidate the mechanisms of gait impairment, cognitive impairment, and falls risk and how they are driving changes in physical rehabilitation. More specifically, behavioural, neurophysiological and imaging studies will illustrate the shared neural and pathophysiological correlates of gait and cognitive impairment in order to demonstrate the role of cognition and cognitive impairment in gait impairment. The intimate relationship between gait and cognition has also focused attention on gait measurement and what it can reveal about future cognitive states, and will be explored in relation to prediction of cognitive decline and falls risk. Finally, interventions that target attentional control of movement will be presented along with interventions aimed at improving age-related (cognitive) risk factors.

These will include pharmacological (e.g. cholinesterase inhibitors), and nonpharmacological (eg attention training, dual task and exercise) approaches, heralding a new era of therapeutic development. The significance of these developments will be discussed with respect to clinical practice. Throughout Parkinson's disease will be used as the central model, with reference to ageing and dementia where relevant and a detailed model of gait will be adopted to illustrate the discussion.

\section{Key Practice Points:}

- Cognition, particularly attention contributes to gait impairment and falls risk in ageing and neurodegenerative disease

- Assessment should target both motor and cognitive contributions to movement

- Rehabilitation therapies should focus on both the motor and non-motor attributes of gait impairment 


\section{AMBULATORY ACTIVITY - PUSHING THE BOUNDARIES OF MEASUREMENT}

\section{Rochester L}

Institute of Neuroscience, Newcastle University, UK

Quantitative measurement of ambulatory activity has an importan role to play in neurodegenerative disease to inform disease mechanism and progression, patient management and efficacy of interventions. Frameworks to characterise ambulatory activity are being developed which take a more comprehensive view and this approach is being driven by the application of body worn sensors (such as accelerometers). These frameworks expand the boundaries of measurement by conceptualising ambulatory activity at a macro level (volume, pattern and variability of ambulatory activity) as well as a micro level which includes quantitative measures of gait (spatiotemporal and gait dynamics). This expands clinic and home based assessment and monitoring of treatment efficacy. Moreover sensory-based quantification of gait provides a more complete picture that accounts for attentional fluctuations and environmental demands which are difficult to replicate in the laboratory or clinic. By applying this approach to a large cohort of people with Parkinson's, it is possible to capture the impact of very early disease informing enhanced disease management and self-management. Drawing from our own and others work in Parkinson's and healthy adults, the utility of this approach for disease detection, progression and prediction of cognitive decline and falls will be explored together with the challenges and opportunities for future assessment and intervention. Throughout the presentation, a broad measurement framework to capture the expanding range of micro and macro metrics using body worn sensors will be adopted. Recommendations for future work in this developing area will be discussed as we move to an era of measuring 'gait in the wild' and the potential it will provide for rehabilitation!

\section{Key Practice Points}

- Sensors provide opportunities for enhanced diagnostics and disease management expanding the role of the rehabilitation professional

- Sensors provide tools for patients for enhanced self-management to maintain and monitor activity to reduce the burden of disease

\section{CLINICAL, IMAGING AND OTHER FACTORS ASSOCIATED WITH PLANTAR HEEL PAIN: A SYSTEMATIC REVIEW WITH META- ANALYSIS}

van Leeuwen $\mathrm{KDB}^{1}$, Rogers $\mathrm{J}^{2}$, Winzenberg $\mathrm{T}^{2}$, Bierma-Zeinstra SMA ${ }^{1}$, van Middelkoop $\mathrm{M}^{1}$

${ }^{1}$ Department of General Practice, Erasmus MC Medical University Rotterdam, The Netherlands

zMenzies Institute for Medical Research, Hobart

Question: What factors are associated with plantar heel pain?

Design: Systematic review of observational studies with meta-analysis.

Participants: Adults with a clinical diagnosis of plantar heel pain and healthy controls.

Outcome Measures: Data were extracted on measures of exposures (for any clinical, imaging or other factor).

Results: A search of five databases to June 42014 yielded 51 eligible studies (2114 heel pain participants; 3091 controls), investigating 104 factors. There were one cohort, 46 case-control, and four cross-sectional studies. Pooling was possible for 12 factors. Compared to controls, planta heel pain participants demonstrated a thicker heel fat pad, a thicker, less echogenic, more hyperaemic plantar fascia, and increased perifascial fluid collection. In addition people with plantar heel pain were more likely to have a calcaneal spur and increased calcaneal uptake of Technetium-99. Pooled clinical data showed that heel pain participants were more likely to have a higher body mass index. Hallux extension mobility was not significantly different. Data for other exposures, including common lower limb clinical measures, were insufficient or too heterogeneous to pool.

Conclusion: There is strong evidence for imaging associations across multiple tissues in plantar heel pain. Aside from body mass index, the relationship between plantar heel pain and clinical factors remains poorly understood.

\section{Key Practice Points:}

- Structures other than the plantar fascia should also be considered in heel pain assessment

- Supporting evidence for commonly used foot and ankle measures remains limited

- The role of body mass in disease mechanisms and management requires further investigation.

\section{STROKE PATIENTS SHOW LOW PHYSICAL, SOCIAL AND COGNITIVE ACTIVITY LEVELS DURING USUAL CARE IN AN ACUTE STROKE UNIT}

Rosbergen ICM ${ }^{1,2}$, Grimley RS 3,4, Hayward KS2, Walker KC', Rowley $\mathrm{D}^{5}$, Campbell AM ${ }^{1}$, McGufficke $\mathrm{S}^{1}$, Robertson $\mathrm{ST}^{1}$, Trinder $\rfloor^{5}$, Janssen $\mathrm{H}^{6}$, Brauer $\mathrm{SG}^{2}$

${ }^{1}$ Allied Health Medical Services, Sunshine Coast Hospital and Health Service, Nambour

2Division of Physiotherapy, School of Health and Rehabilitation Sciences, The University of Queensland, Brisbane

${ }^{3}$ Sunshine Coast Clinical School, The University of Queensland, Nambour ${ }^{4}$ Department of Medicine, Sunshine Coast Hospital and Health Service, Nambour

${ }^{5}$ Nursing and Midwifery, Sunshine Coast Hospital and Health Service, Nambour ${ }^{6}$ Hunter Medical Research Institute, Newcastle

Question: What levels of physical, social and cognitive activity do people with a stroke undertake in an Acute Stroke Unit?

Design: Prospective observational study.

Participants: Thirty adults with stroke.

Intervention: Usual care.

Outcome Measures: Behavioral mapping was used to record body position, physical, social and cognitive activity every 10 minutes from $7.30 \mathrm{am}$ to 7.30pm across two weekdays and one weekend day within the first week of admission. Mapping variables were explored as a proportion of observed time.

Results: Mean age of participants was 76 (SD13) years, with 17 mild, 9 moderate and 4 severe stroke participants. Participants spent $65 \%$ in supine position. Physical, social and cognitive activity was noted in $21 \%$, $28 \%$ and $42 \%$ of observations. Physical activities observed included: eating/drinking 9\%, self-care 4\%, upper limb activity 3.5\% and standing/ walking activity $1.6 \%$. Main social and cognitive subcategories were talking $25 \%$, listening $28 \%$, reading $6 \%$ and watching TV $6 \%$. Less than $1 \%$ was spent on activities such as touching, laughing, writing, craft, mind games or computer use

Conclusion: Despite guidelines recommending early activity, stroke survivors spend most of the day supine and inactive early after stroke. An upcoming study will determine whether an enriched environment in an Acute Stroke Unit can increase all activity levels.

Trial registration:ANZCTR12614000679684

Key Practice Points:

- Patients show low levels of physical, social and cognitive activity in the acute setting.

- There is a need for service delivery models that can increase all activity levels.

- Emerging evidence shows that social and cognitive engagement is important for stroke recovery. 


\section{WHY HAVEN'T WE SOLVED THE MSD PROBLEM?}

\section{Rothmore P}

Faculty of Health Sciences, University of Adelaide

Our knowledge of MSDs has increased substantially in recent years. Clinical diagnostic skills have improved along with a greater understanding of tissue pathomechanics and pain mechanisms. The contribution of physical and psychosocial risk factors - and their interaction - in the development of MSDs is also now widely acknowledged and understood as is the importance of multi-factorial interventions. Why then have we not solved the MSD problem?

Even when workplace interventions designed to reduce the burden of MSDs are developed with "state of the art" knowledge they of little use if they are not properly implemented or are simply ignored. Injury prevention advice invariably proposes changes to the work system, the work environment and individual work practices. This involves a change in behaviour. Contemporary research suggests that an understanding of the behaviour change process, and more specifically its application in the development of targeted workplace interventions, may improve the implementation, and effectiveness, of advice at both an individual and organisational level. The most frequently applied of the behaviour change methods in workplaces settings has been the Stage of Change framework. In this framework readiness to change is assessed using a short series of closed questions after which participants are assigned to one of five stages. Once the stage of change is determined, advice is then tailored accordingly to improve its effectiveness. This presentation will outline the results of contemporary research incorporating this approach and present a model for its incorporation in the development of workplace interventions.

\section{Key Practice Points:}

- Injury prevention advice invariably involves a change in individual and organisational behaviour

- The implementation and effectiveness of injury prevention advice may be improved by the targeting of advice according to behaviour change principles

\section{HOW TO BUILD YOUR PROFESSIONAL BRAND WITH A PERSONAL WEBSITE}

\section{Ruscoe $\mathbf{G}^{1}$}

IManaging Director, dot.physio

Background: Physiotherapists operate in a competitive health environment where they themselves are often the brand. Subsequently self-promotion is the important for career success. As the world moves online the opportunities for significant self-promotion through digital strategies are great. Knowledge about building their professional brand with a personal website will help physiotherapists not only raise public awareness of their unique skills, interests and talents but will also facilitate their future self-development through cognitive consonance or the self-fulfilling prophecy effect. Furthermore the physiotherapy profession will benefit from the greater variety, expertise and awareness that is created by physiotherapists developing and promoting their unique points of difference.

Aims / objectives: The aim of the session is to raise awareness of the benefits of personal branding and to provide information on the online options available. The primary objectives are to increase confidence, decrease common errors and ultimately, facilitate personal professional promotion. By the end of the session participants will know: 1. the benefits of self-promotion and brand building online

2. why a personal website is the central online strategy

3. the best kind of domain names and where to access them

4. the best way to structure a personal website

5. the best kind of content to put on their personal website

6. the options available regarding website building and hosting.

Approach: The session will be delivered in lecture style drawing from real life examples to demonstrate good and bad strategies. Participants attending with their own smartphones, tablets or laptops will be able to further investigate sites exposed. A list of website addresses where high quality resources can be located will be provided.

Conclusion / Key Practice Points: A successful career requires selfpromotion. As the world moves online a personal professional website is a key strategy to future success. With the knowledge gained from the presentation:

- Participants will be able to build, high quality, personal websites to promote their professional brand and share their unique interests and services

- Participants will continually refine and develop their expertise through this informal method of self-appraisal and subsequently experience greater demand for their services and greater satisfaction with their career.

- The profile of the profession will be raised with the greater number and range of physiotherapy personal websites on line.
THE RELIABILITY OF THE MOVEMENT COMPETENCY SCREEN (MCS)

Russell ${ }^{1}{ }^{1}$, Hing $W^{1}, O^{\prime}$ Grady $L^{2}$, Reid $D^{2}$

${ }^{1}$ Bond University, Queensland

${ }^{2}$ Auckland University of Technology, Auckland

Question: What is the inter- and intra-rater reliability of the Movement Competency Screen (MCS)?

Design: Cross sectional study

Participants: 22 female New Zealand Water Polo athletes

Intervention: Participants were videoed performing three repetitions

of the seven MCS movements (squat, lunge, twist, bend, pull, push up and single leg squat) in both frontal and sagittal planes. Two novice raters (<2hrs experience) independently assigned a load level (1-3) to each movement for each subject to give an overall score out of 21 to determine the inter-rater reliability of the tool. The raters then independently rescored all movements 1 week later in random order to determine the intra-rater reliability.

Outcome measure: A two way mixed intraclass correlation coefficient (ICC) and $95 \% \mathrm{Cl}$

Results: The intra-rater reliability for both raters for the overall score was very high (ICC 0.94 and ICC 0.90). The inter-rater reliability for the overall score was low (ICC 0.48). The intra-rater reliability of the individual components of the MCS ranged from very low (Push Up ICC 0.27 and Lunge ICC 0.22 ) to very high (Pull ICC 0.93 and ICC 1.00 single leg squat) for both raters.

Conclusion: The MCS had high intra-rater reliability with novice raters but low inter-rater reliability. There was a large variation in the reliability of individual MCS movements. The poor inter-rater reliability of the MCS may be a practical limitation of the tool.

\section{Key Practice Points:}

- The MCS is an effective whole body movement screen when conducted by one rater to assess functional change

- If conducted by 2 or more raters, in its current form, it is not reliable

- Further research is needed into ways reduce the ambiguity in competency criteria, to improve MCS inter-rater reliability

\section{VENTILATOR HYPERINFLATION - SAFETY AND FEASIBILITY OF TRANSLATING EVIDENCE INTO PRACTICE IN ICU}

\section{Marzano $\mathrm{V}^{1}$, Bissett $\mathrm{B}^{1,2}$, Leditschke $\mathrm{A}^{3}$, Ryall $\mathrm{T}^{1}$}

${ }^{1}$ Physiotherapy Department, Canberra Hospital, ACT Health, Canberra ACT 2605

2Discipline of Physiotherapy, University of Canberra, Canberra ACT 2617 IIntensive Care Unit, Canberra Hospital, ACT Health, Canberra, ACT 2605

Question: Is it feasible and safe to implement ventilator hyperinflation (VHI) in a tertiary intensive care unit where standard practice is manual hyperinflation (MHI)?

Design: A prospective observational pre and post intervention study. Participants: Adult mechanically ventilated patients with evidence of sputum retention or alveolar collapse.

Intervention: An audit of MHI practice was conducted over three months Following implementation of an evidence-based $\mathrm{VHI}$ guideline with physiotherapy staff education and training, a subsequent audit of $\mathrm{VHI}$ practices was conducted over 3 months.

Outcome Measures: The frequency, prescription, contraindications, incidence of adverse outcomes and requirements for nursing assistance during physiotherapy treatment was collected for both MHI and VHI. All data was recorded by treating physiotherapists at the time of care and extracted to a purpose-built database.

Results: Following training of physiotherapists, VHI was feasible occurring 109 occasions in the 3 month period, an increase from 56 occasions of $\mathrm{MHI}$ in the previous period. VHI required less nursing assistance ( $49 \%$ with $\mathrm{MHI}$ compared to $0 \% \mathrm{VHI}$ ) and VHI was safe with an adverse event rate of $2.7 \%$ compared to $10.7 \%$ for MHI. All VHI treatments maintained peak airway pressures below $40 \mathrm{cmH} 20$ in accordance with safety guidelines.

Conclusion: Implementing VHI in a tertiary ICU is feasible and safe utilising a quality improvement process.

\section{Key Practice Points:}

- VHI can be safely implemented in ICU where standard practice is MHI

- There are fewer adverse events with VHI than MHI regardless of acuity

- $\mathrm{VHI}$ requires less nursing assistance to effectively implement physiotherapy treatment 


\section{BRAIN POWER}

\section{Sampson T}

Todd Sampson is the writer, host and human guinea pig of the awardwinning Discovery Science Series called REDESIGN MY BRAIN. The premise of the series was simple. For over 70 years, science has told us that our brains are fixed. You are born with the brain you have, it develops rapidly until age 7 and then it starts a steady decline from age 30 until you die. There was nothing you could do about it. But science has now proven that to be false. In fact, modern science has now shown that our brain is plastic and that we ALL have the ability to improve it at any age and at any time. This presentation will show you how to boost your Brain Power. Todd will not only take you behind the scenes of this extraordinary scientific journey, he will also provide you with the practical tools, strategies and techniques to improve your brain. We are all capable of more than we realise... and modern science is showing us how.

\section{LIVING AND LEARNING LONG-TERM - STUDENT'S PERSPECTIVES OF FULL YEAR PHYSIOTHERAPY PLACEMENTS}

\section{Sanderson M, McCarroll E, Kingston K, Neal M, Robinson T,} Wells H, Pilcher C

The University of Newcastle Department of Rural Health

Question: What are student perspectives of a long term clinical placement program in a rural area?

Design: Students will report on their experience on a long term clinical placement.

Participants: Seven physiotherapy students.

Intervention Student opted to undertake a full academic year in a rura area including their clinical and academic requirements. The placement provided the opportunity to participate in community engagement and interprofessional learning activities.

Outcome measures: Students will detail their experiences during this placement including how these experience have influenced learning and career plans.

Results: Long-term placements gave students opportunities to develop stronger links within the community, other health students and each other. Students engaged with the community through a range projects and volunteering. Knowing they would be living in one location for the year provided opportunity to commit to work and extra-curricular activities. Negative aspects were feeling distant from main campus and that some complex conditions were not managed at the rural hospitals.

Conclusion: Long-term clinical placements allowed students to develop greater understanding of the people and health issues within the community. It also provided stability of both their learning environments and the people they were learning with. The negative aspects of the placement are similar to those if completing placements in any unfamiliar environment.

\section{Key Practice Points:}

- Long-term placements provide an opportunity for students to gain an in-depth understanding of the community within which they are living and learning.

- Students were able to develop supportive relationships with staff and other students on order to facilitate learning.

\section{COMPARISON OF BUBBLE POSITIVE EXPIRATORY DEVICES: A BENCH- TOP STUDY}

Santos $M D^{1,2}$, Eisenhuth $]^{2}$, Milross $M A^{2}$, Alison JA2,3

1Physiotherapy Department, Prince of Wales Hospital, Randwick, NSW

${ }^{2}$ Clinical and Rehabilitation Sciences, The University of Sydney,

Lidcombe, NSW

3Physiotherapy Department, Royal Prince Alfred Hospital, Camperdown, NSW

Question: Does the bubble-positive expiratory pressure (bubble-PEP),

AguaPEP $^{\circledast}$, Hydrapep $^{\circledR}$ and Therabubble ${ }^{\circledR}$ devices, vary in regards to

pressures and oscillations produced, at different flow rates.

Design: Bench-top experimental study. PEP devices with a $10 \mathrm{~cm}$ water column height; flow rates of 5, 10, 15, 20 and $25 \mathrm{~L} / \mathrm{min}$

\section{Participants: Nil}

Outcome Measures: Positive expiratory pressure $(\mathrm{cmH2O})$ and oscillation frequency $(\mathrm{Hz})$.

Results: Bubble-PEP device constructed with a $10 \mathrm{~mm}$ internal diameter (ID) tubing and: (a) the tubing resting $3 \mathrm{~cm}$ from bottom of the bottle, produced mean (SD) PEP of $10.4(0.14) \mathrm{cmH} 20$ at $5 \mathrm{~L} / \mathrm{min}$ to $10.8(0.24)$ at $25 \mathrm{~L} / \mathrm{min}$ with oscillations between $13-17 \mathrm{~Hz}$; (b) the tubing resting at bottom of the bottle, produced PEP from $10.9(0.01) \mathrm{cmH} 2 \mathrm{O}$ at $5 \mathrm{~L} / \mathrm{min}$ to $12.9(0.08) \mathrm{cmH} 20$ at $25 \mathrm{~L} / \mathrm{min}$ with oscillations between $12-14 \mathrm{~Hz}$. AguaPEP ${ }^{\oplus}$ produced PEP from $9.7(0.02) \mathrm{cmH} 20$ at $5 \mathrm{~L} / \mathrm{min}$ to $11.5(0.02)$ at $25 \mathrm{~L} / \mathrm{min}$ with oscillations between $11-17 \mathrm{~Hz}$. Hydrapep produced PEP from $9.6(0.35) \mathrm{cmH} 20$ at $5 \mathrm{~L} / \mathrm{min}$ to $10.7(0.39)$ at $25 \mathrm{~L} / \mathrm{min}$ with oscillations between $14-17 \mathrm{~Hz}$. Therabubble ${ }^{\oplus}$ produced PEP from $8.6(0.01) \mathrm{cmH} 20$ at 5 $\mathrm{L} / \mathrm{min}$ to $12.8(0.03)$ at $25 \mathrm{~L} / \mathrm{min}$ with oscillations between $14-17 \mathrm{~Hz}$.

Conclusion: The bubble-PEP (a) device with a $10 \mathrm{~cm}$ water column height, $10 \mathrm{~mm}$ ID tubing and the tubing resting $3 \mathrm{~cm}$ from the bottom of the bottle, maintained stable PEP despite variable flow rates. All PEP devices had similar oscillations.

\section{Key Practice Points:}

- Design characteristics of the PEP device can influence the pressures generated.

- Although there was some variability in the pressures generated by bubble-PEP (b), AguaPEP ${ }^{\oplus}$, Hydrapep ${ }^{\circledR}$ and Therabubble ${ }^{\oplus}$ devices with increasing flow rates, this may not be clinically relevant.

- Further research is warranted to determine the effects of bubble PEP devices in people with respiratory impairments.

\section{TUBING INTERNAL DIAMETERS AND FLOW RATES AFFECT THE PRESSURES GENERATED BY THE BUBBLE-POSITIVE EXPIRATORY PRESSURE DEVICE}

Santos $M D^{1,2}$, Eisenhuth $]^{2}$, Milross $M A^{2}$, Alison JA2, 3

${ }^{1}$ Physiotherapy Department, Prince of Wales Hospital, Randwick, NSW ${ }^{2}$ Clinical and Rehabilitation Sciences, The University of Sydney,

Lidcombe, NSW

${ }^{3}$ Physiotherapy Department, Royal Prince Alfred Hospital, Camperdown, NSW

Question: Do the internal diameter (ID) of the tubing and flow rate through the tubing affect the pressures generated by the bubble-positive expiratory pressure (bubble-PEP) device?

Design: Bench-top experimental study. Bubble-PEP device with $10 \mathrm{~cm}$ water column height; tubing length $30 \mathrm{~cm}$; tubing ID (mm) 2, 4, 5, 7, 8, 10; tubing resting $3 \mathrm{~cm}$ from bottom of the bottle; flow rates of 5, 10, 15, 20, $25 \mathrm{~L} / \mathrm{min}$.

\section{Participants: Nil.}

Outcome Measures: Positive expiratory pressure $(\mathrm{cmH2O})$ and oscillation frequency $(\mathrm{Hz})$

Results: Bubble-PEP device constructed with: (a) $2 \mathrm{~mm}$ ID tubing produced mean (SD) PEP of $20.1(0.4) \mathrm{cmH} 20$ and $41.8(0.3) \mathrm{cmH} 20$ at 5 and $10 \mathrm{~L} /$ min respectively with oscillations of $15-19 \mathrm{~Hz}$ : (b) $4 \mathrm{~mm} \mathrm{ID} \mathrm{produced} \mathrm{PEP}$ from $12.5(0.19) \mathrm{cmH} 20$ at $5 \mathrm{~L} / \mathrm{min}$ to $41.5(0.28) \mathrm{cmH} 20$ at $25 \mathrm{~L} / \mathrm{min}$ with oscillations 14-18 Hz; (c) 5 mm ID produced PEP from $10.9(0.11) \mathrm{cmH} 20$ at 5 $\mathrm{L} / \mathrm{min}$ to $15.8(0.09) \mathrm{cmH} 20$ at $25 \mathrm{~L} / \mathrm{min}$ with oscillations $17-18 \mathrm{~Hz}$; (d) $7 \mathrm{~mm}$ ID produced PEP from $10.7(0.03) \mathrm{cmH} 20$ at $5 \mathrm{~L} / \mathrm{min}$ to $12.7(0.17) \mathrm{cmH} 20$ at $25 \mathrm{~L} / \mathrm{min}$ with oscillations $14-17 \mathrm{~Hz}$; (e) $8 \mathrm{~mm}$ ID produced PEP of $9.4(0.09)$ at $5 \mathrm{~L} / \mathrm{min}$ to 10.7 (0.12) $\mathrm{cmH} 20$ at $25 \mathrm{~L} / \mathrm{min}$ with oscillations $14-18 \mathrm{~Hz}$; (f) 10 $\mathrm{mm}$ ID produced PEP of $10.4(0.14) \mathrm{cmH} 20$ at $5 \mathrm{~L} / \mathrm{min}$ to $10.8(0.24)$ at $25 \mathrm{~L} /$ min with oscillations between $13-17 \mathrm{~Hz}$.

Conclusion: When tubing of $10 \mathrm{~mm}$ ID was used, stable PEP was generated despite variable flow rates.

\section{Key Practice Points:}

- Design characteristics of the bubble-PEP device can influence the pressures generated.

- Narrower tubing and higher flow rates can result in higher PEP being generated.

- In clinical practice, to ensure that the pressure generated by the bubble$\mathrm{PEP}$ is equal to the height of the water column, regardless of flow rates, tubing with $10 \mathrm{~mm}$ ID is recommended. 


\section{INTERACTIVE VISUALIZATION OF DEEP KNEE FLEXION, IN FOUR DIMENSIONS AND IN VIVO}

\author{
Scarvell JM1,2, Perriman DM2, 3, Smith $\mathrm{PN}^{2,3}$, Pickering $\mathrm{MR}^{4}$ \\ IUniversity of Canberra \\ Trauma and Orthopaedic Research Unit, Canberra Hospital \\ ${ }^{3}$ Australian National University \\ ${ }^{4}$ University of New South Wales, Canberra
}

Question: What can we learn about deep knee flexion by 4-dimensional visualisation of the knee?

Design: Observational study

Participants: 16 healthy participants

Intervention While participants knelt, fluoroscopy images were captured at 15 frames/second. Images were registered to CT and used to create dynamic 4D models. The 4D model can be viewed in any perspective to observe the motion.

Outcome Measures: Kinematic analysis in 6 degrees of freedom.

Results: Flexion from 90 to 140 degrees required 70 degrees of rotation, 2 degrees of adduction, and $6.5 \mathrm{~mm}$ of AP translation. A reversal in condylar translation occurred at 120 degrees. Viewing the knee model from a range of perspectives illuminates different features of the motion. In deep flexion it can be seen that the medial tibial condyle locks into the posterior femur. The medial meniscus is trapped within bony boundaries. Internal rotation of the tibia is essential to this deep flexion lock.

Conclusion: Painful deep flexion exposes pathology in the menisci, ligaments, and motion of the knee, yet it is important for a variety of functional and cultural reasons. This new tool enables to better understand joint behaviour in compression and extremes of motion.

\section{Key Practice Points:}

- Visualisation of the bony architecture in deep flexion demonstrates how much rotation is required.

- To fully flex the knee requires full rotation, and distraction of the anterior knee capsule

- Manual therapy distraction, rotation and an A-P to the tibia are likely to be most effective for deep flexion.

\section{TRACKING MOTOR CORTICAL CHANGES IN THE TRANSITION TO SUSTAINED MUSCLE PAIN}

Schabrun SM${ }^{1}$, Christensen SW², Mrachacz-Kersting N², Graven-Nielsen $\mathrm{T}^{2}$

${ }^{1}$ University of Western Sydney, School of Science and Health, Locked bag 1797 Penrith, NSW 2751, Australia

2Center for Neuroplasticity and Pain (CNAP), SMI, Department of Health

Science and Technology, Faculty of Medicine, Aalborg University,

Aalborg, Denmark

Question: How is the organisation and function of the primary motor cortex (M1) altered in the transition to sustained muscle pain?

Design: Observational

Participants: 12 healthy individuals

Intervention: Repeated intramuscular injection of nerve growth factor (NGF) induces deep tissue hyperalgesia reminiscent of musculoskeletal pain. NGF was injected into the dominant extensor carpi radialis brevis on Day 0 and Day 2

Outcome Measures: Quantitative sensory and motor testing, and assessment of M1 organisation (transcranial magnetic stimulation) and function (intracortical networks), were performed prior to injection on Days 0 and 2 and again on Day 4 and 14.

Results: Pain and pressure pain sensitivity were increased in paralle with reduced function at Day 2, and further increased at Day 4. M1 reorganisation was evident at Day 4 characterised by increased map volume and an increased number of map peaks. These changes were accompanied by reduced intracortical inhibition and increased intracortical facilitation. M1 adaptations returned towards baseline at Day 14.

Conclusion: These data provide the first evidence of the nature and timecourse of altered $\mathrm{M} 1$ organisation and function in response to sustained muscle pain

\section{Key Practice Points:}

- NGF is a novel pain model that can be used to examine cortical changes in the transition to sustained muscle pain.

- Reorganisation of M1 occurs after 4 days of sustained muscle pain and is charactered by an enlarged cortical representation with a greater number of discrete peaks.

- Altered M1 function is also present at Day 4 characterised by a loss of inhibition in local intracortical networks.

\section{TRANSCRANIAL DIRECT CURRENT STIMULATION DOES NOT ENHANCE DUAL-TASK GAIT TRAINING IN PARKINSON'S DISEASE}

Schabrun SM${ }^{1}$, Lamont RM², Brauer SG²

${ }^{1}$ University of Western Sydney, School of Science and Health, Locked bag 1797 Penrith, NSW 2751, Australia

2School of Health and Rehabilitation Sciences, The University of Queensland, St Lucia, Brisbane, Queensland 4062, Australia

Question: Does the addition of transcranial direct current stimulation (tDCS) improve gait and motor function beyond that of dual task gait training alone in Parkinson's disease (PD)?

Design: Pilot, randomized, double-blind, sham-controlled trial with 12 week follow-up

Participants: Sixteen individuals diagnosed with idiopathic PD

Intervention: Participants were randomized into a dual task gait training group with either i) active anodal tDCS to the primary motor cortex or ii sham tDCS. Training was applied in nine, 20 minute sessions, performed over 3 weeks.

Outcome Measures: The primary outcome was gait speed when walking and undertaking concurrent cognitive tasks. Secondary measures included step length and cadence, and the Timed Up and Go (TUG) test under single and dual task conditions.

Results: Gait speed, step length and cadence improved in both groups under all dual task conditions with training and improvements were maintained at follow-up. There was no difference between the active and sham tDCS groups. There was no change in TUG performance over time or between groups, but the active tDCS group improved their concurrent cognitive task correct response rate post training.

Conclusion: tDCS may not be an effective adjunct to dual task gait training in PD.

\section{Key Practice Points:}

- Three weeks of dual task gait training is effective at improving gait while concurrent tasks are performed in individuals with PD.

- Improvements are maintained at 12 weeks follow-up.

- The addition of tDCS did not result in greater improvements than training alone.

\section{THE BRAIN'S RESPONSE TO PLASTICITY- BASED TREATMENTS IS ALTERED IN PERSISTENT LOW BACK PAIN}

Schabrun SM ${ }^{1}$, Burns $\mathrm{E}^{1}$, Hodges PW ${ }^{2}$

IUniversity of Western Sydney, School of Science and Health, Locked bag 1797 Penrith, NSW 2751, Australia

2The University of Queensland, NHMRC Centre of Clinical Research Excellence in Spinal Pain, Injury and Health and School of Health and Rehabilitations Sciences, Brisbane, Queensland 4072, Australia

Question: Is the brain's response to plasticity-based treatments altered in individuals with persistent low back pain (LBP) compared with painfree individuals?

Design: Cross-over

Participants: 10 individuals with persistent LBP and 10 age-and gendermatched painfree individuals

Intervention: Two interventions known to induce plasticity in primary motor cortex (M1) were applied: i) transcranial direct current stimulation (tDCS), ii) peripheral electrical stimulation (PES) and iii) combined tDCS and PES. A sham control was also included. Each intervention was applied for 30 minutes (order randomised) with 1-week wash-out between treatments.

Outcome Measures: M1 excitability was assessed using transcranial magnetic stimulation before and after each intervention.

Results: The brain's response to PES (depression of excitability) and the sham control (no change) was similar between groups. However, when tDCS was applied alone (increased excitability in controls, no change in LBP) and when tDCS and PES were combined (no change in controls, increased excitability in LBP) the brain's response differed between individuals with persistent LBP and controls.

Conclusion: These data suggest individuals with persistent LBP have an altered response to some plasticity-based treatments that target the brain.

\section{Key Practice Points:}

- Individuals with persistent LBP do not display typical cortical responses to some plasticity-based treatments that target the brain.

- This finding may explain the high variability and lack of effect of plasticitybased treatments such as tDCS currently observed in the literature.

- The brain's response to treatment, and how this might be affected by the presence of pain, should be considered in the design of studies examining plasticity-based treatments. 


\section{THE PROBLEM AND COST OF HEALTH RELATED WORKLESSNESS}

\section{Schofield D}

The Boden Institute, The University of Sydney

Murdoch Children's Research Institute

Garvan Institute of Medical Research

Chronic pain and disability can have devastating health impacts on patients. However, the extent of the impacts of chronic pain and disability are often overlooked. These include loss of income for those who can no longer work, risk of poverty, reduced capacity to save and reduced income in retirement. For government the costs include reduced taxation revenue and additional welfare payments. Effective interventions may not only reduce the health impacts of chronic pain and disability, but also have significant economic benefits. Examples of the potential economic benefits of investing in chronic pain management will be presented.

\section{MEASURES OF GAS EXCHANGE DURING SIX MINUTE WALK TEST PREDICT CHANGES IN DISEASE SEVERITY IN PULMONARY ARTERY HYPERTENSION}

Seale $\mathrm{H}^{1}$, Harris $]^{1}$, Hall $\mathrm{K}^{1}$, Kermeen $\mathrm{F}^{1}$, Morris NR ${ }^{1,2}$

${ }^{1}$ Queensland Lung Transplant Service, The Prince Charles Hospital, Brisbane ¿School of Allied Health Sciences, Griffith University, Gold Coast

Introduction: The six minute walk distance (6MWD) is a validated measure of functional capacity and is related to mortality and morbidity in pulmonary arterial hypertension (PAH). It is unknown if changes in gas exchange measures are sensitive to changes in disease status.

Question: To determine relationship between changes in disease severity as measured by right ventricular systolic pressure (RVSP) and indices of gas exchange measured during a 6 MWT

Design: Prospective longitudinal observational study.

Method: 17 group 1 PAH patients $(12 F, 45.7 \pm 13.8$ yr, NYHA $2.3 \pm 0.5)$ completed two 6MWT - mean 623+203 days apart. Gas exchange was measured using a portable metabolic cart. End exercise measurements of oxygen uptake (VO2), VE/VCO2 and PETCO2 were determined from 60 s averages. Echocardiography and standard measurements of right ventricular function were measured on the same day.

Results: There was no statistical change in the 6MWD (pre: $556 \pm 76$; post: $541 \pm 115 \mathrm{~m} \mathrm{p}>0.5$ ), however a small significant reduction in RVSP (pre 71.2 \pm 21 ; post: $62.2 \pm 17 \mathrm{mmHg}, \mathrm{p}=0.02)$. Changes in RVSP correlated significantly with end exercise VE/VCO2 $(r=0.83, p<0.05)$ PETCO2 $(r=-0.74$, $p<0.05)$ and 6MWD ( $r=-0.57, p<0.05)$. No correlation with the change in VOz from baseline to follow up.

Conclusions: This study demonstrates that changes in gas exchange measures from a 6MWT relate to changes in disease severity in a small sample of patients with group $1 \mathrm{PAH}$.

\section{Key Practice Points:}

- Gas exchange measures are sensitive to disease progression

- Research needed in other larger patient cohorts

\section{EARLY MOBILIZATION OF A CONTINUOUS FLOW VENTRICULAR ASSIST DEVICE PATIENT COHORT: A TERTIARY CENTRE EXPERIENCE}

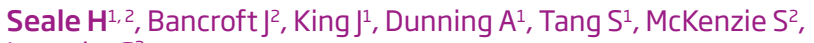
Javorsky $G^{2}$

${ }^{1}$ The Physiotherapy Department, The Prince Charles Hospital, Brisbane ${ }^{2}$ Advanced Heart Failure and cardiac transplant unit, The Prince Charles Hospital, Brisbane

Background: Ventricular assist devices (VAD) are a management strategy as a bridge to transplantation for selected patients with end stage heart failure. Durable mechanical support has increased due to improved safety, demand and progressive VAD miniaturization. Early mobilization and rehabilitation in ICU patients has been associated with decreased length of stay( LOS). No data exists on early mobilization of VAD patients.

Question: To report initial experience of early mobilization in VAD patient cohort

Design: prospective observational study

Method: 21 consecutive patients :age $35+13,14$ male, Ejection Fraction (EF) $13+5 \%, C I 2.1+0.6$, NYHA $3.6+0.6$, Intermacs score $1(9.5 \%) 2$ $(47.6 \%) 3(42.8 \%)$ with diagnosis of Dilated Cardiomyopathy 17 , Ischaemic Cardiomypathy 3, and Hypertrophic Obstructive Cardiomyopathy 1 undergoing insertion of continuous flow left VAD
Results: Median length of ICU stay: 14 days ( 7-20 25-75 quartile). 3 patients required ECMO pre VAD and 1 post. 90 day mortality: 3 (14.3\%). $16(76 \%)$ survived to transplant. 2 still awaiting transplant. Mean duration in days: first stand $3.4+2.6$, sit out of bed $4.2+3.1$ first walk $6.3+5.3$. ICU LOS correlated significantly with time to first mobilisation $(r=0.77 P<0.05)$ No correlation seen between ICU length of stay, EF, Cl, and Intermacs score.

Conclusion: Early mobilization was safe and well tolerated. Early mobilization correlated with reduced LOS in ICU in continuous flow VAD patient cohort. ICU length of stay didn't correlate with markers of disease severity.

Key Practice Points:

- Early mobilization associated with reduced LOS

\section{CRICKETERS - ARE THEY A PAIN IN THE BACK? CAN ELECTRO-DRY NEEDLING HELP THEM?}

\section{Selvaratnam $\mathrm{PJ}^{1,2}$}

${ }^{1}$ The University of Melbourne, Faculty of Medicine, Dentistry and Health Sciences, Melbourne Australia

¿The Melbourne Spinal and Sports Medicine Clinic

Cricketers at the international level and at grass roots level sustain injuries to the lumbar and cervical regions, the peripheral joints and muscles. The team approach by Musculoskeletal and Sports Physiotherapists in conjunction with Sports Physicians, coaches, high performance exercise physiologists and biomechanists provides optimal assistance to the cricketer's recovery. Injury management by health professionals may include appropriate medication, mobilization, active rehabilitation interventions, biomechanical analysis, fitness training and mind training to name a few. Wet needling with local anaesthetic or corticosteroids may assist the lumbar conditions of some cricketers. Since 1970's dry needling with acupuncture needles has been used in sports people including cricketers to assist recovery from trauma. This case presentation will discuss the spinal rehabilitation challenges of a state cricketer who represented the Australian under 19 team. The cricketer presented with chronic lumbar and referred pain. The CT scans revealed a unilateral L5 pars defect. The treatment approach with dry needling (DN) and electrical stimulation (electroacupuncture) is discussed as well as the possible neurophysiological effects at the injury site and at the brain. Discussion will also include the evidence for DN from research conducted in Australian Rules footballers with back related hamstring injuries. These neurophysiological effects of DN may benefit sports people in their return to sport in conjunction with physiotherapy rehabilitation, biomechanical analysis and mind training.

\section{Key Practice Points:}

- Dry Needling is beneficial in pain modulation locally and centrally in sports people

- Dry Needling can reduce muscle trigger point activity and enhance Physiotherapy rehabilitation. It is cost effective with minimal side-effects and should be considered early in the rehabilitation programme and not as a last resort

- Electroacupuncture may speed neural modulation and muscle relaxation 


\section{CAN TELEHEALTH SUBSTITUTE REVIEW APPOINTMENTS BY THE ADVANCED MUSCULOSKELETAL PHYSIOTHERAPISTS WORKING IN THE POST ARTHROPLASTY REVIEW CLINIC?}

Senserrick CA, Page C], Carroll S

St Vincent's Hospital

Design: Observational prospective cohort study

Participants: 100 consecutive patients attending the advanced musculoskeletal physiotherapy led post arthroplasty clinic

Intervention A survey was administered, explaining what telehealth involved, asking if patients would be interested in participating and if not, why?

Outcome Measures: Data was collected and analysed via a simple excel spreadsheet.

Results: $74 \%$ of the patients were willing to consider telehealth as an alternative to face to face contact for post arthroplasty reviews. Analysis of patients who declined has allowed clinicians to develop a target group within this population who are suitable to consider for telehealth. Technology has been set up to allow telehealth consultations in the clinic and a pilot trial of 25 patients has commenced.

Conclusion: Patients attending advanced musculoskeletal physiotherapy led post arthroplasty review clinics are willing to consider telehealth conference rather than face to face consultations.

\section{Key Practice Points:}

- Patients in post arthroplasty review clinics are willing to consider telehealth consultations as an alternative to face-to-face reviews

- The technology is available and can be used on personal computers, tablets or smartphones

- Consumer feedback from 100 consecutive patients has been utilised to determine a target group for telehealth consultations

- A pilot study has commenced to test the telehealth service and this will refine St Vincent's current model of care

\section{TRANSABDOMINAL ULTRASOUND TO ASSESS PELVIC FLOOR MUSCLE PERFORMANCE DURING ABDOMINAL CURL IN EXERCISING WOMEN}

\section{Serrao C, Barton A, Thompson J, Briffa KN}

${ }^{1}$ School of Physiotherapy and Exercise Science Curtin University, Perth

Question: Are abdominal curl-up activities safe for exercising women to perform?

Design: Cross sectional exploratory study

Participants: Convenience sample of ninety women participating in a variety of group exercise

Intervention: All participants completed a survey and transabdominal ultrasound assessment, performed by two qualified Continence and Women's Health Physiotherapists with experience in ultrasound scanning. The assessment comprised three attempts of a pelvic floor muscle contraction and an abdominal curl-up exercise in crook lying, with standardised instructions

Outcome Measures: Bladder base displacement (in millimetres) was measured to determine correct elevating pelvic floor muscle contractions and amount of movement during abdominal curl-up.

Results: Twenty-five percent ( $n=23)$ of women were unable to demonstrate an elevating pelvic floor muscle contraction and all women displayed bladder base depression on abdominal curl-up (range 0.33$31.2 \mathrm{~mm}$ ). Parous women displayed significantly more bladder base depression than nulliparous women $(15.5$ [7.3] mm vs 11.4 [5.8] mm, $p<0.009)$. Sixty percent $(n=54)$ reported stress urinary incontinence. There was no association between stress urinary incontinence and the ability to perform an elevating pelvic floor muscle contraction ( $p=0.278$ ) or the amount of depression with abdominal curl-up in women with an without stress urinary incontinence respectively $(14.1$ [7.6] mm versus 14.2 [6.7] mm)

Conclusion: Transabdominal ultrasound identified that $25 \%$ of women who participated in group exercise were unable to perform a correctly elevating pelvic floor muscle contraction and all depressed the bladder base on abdominal curl-up.

\section{Key Practice Point:}

- Exercising women may be at risk of pelvic floor muscle dysfunction when performing abdominal curl-up activities.

\section{PHYSICAL THERAPISTS' WAYS OF THINKING AND TALKING ABOUT OVERWEIGHT AND OBESITY: CLINICAL IMPLICATIONS}

Setchell $\mathrm{J}^{1}$, Watson $\mathrm{BM}^{1}$, Gard $\mathrm{M}^{2}$, Jones $\mathrm{L}^{3}$

${ }^{1}$ School of Psychology, Faculty of Health and Behavioural Sciences,

UQ, Brisbane

¿School of Human Movement and Nutrition Sciences, Faculty of Health and Behavioural Sciences, UQ, Brisbane

${ }^{3}$ School of Applied Psychology, Griffith University, Brisbane

Question: How do physiotherapists think and talk about people who are overweight? What are clinical implications of these weight discourses? How health professionals think and talk about weight is important because it can influence their behaviour towards patients who are overweight.

Design: An interpretive qualitative design was employed.

Participants: Twenty-seven qualified Australian physiotherapists from a range of sub-disciplines.

Methods: Data were collected from six focus groups of 4-6 physiotherapists in Queensland, Australia that discussed weight in a physiotherapy environment. Topics discussed included: physiotherapists' role in weight management, causes of weight, and what it is like to treat patients who are overweight. Group sessions were audio recorded and transcribed. Data collection and analysis were driven by qualitative methodology grounded in discourse analysis.

Results: Four main weight discourses (ways of thinking/speaking about weight) were identified in data analysis. Participants described patients who are overweight as 1) little affected by stigma, and 2) difficult to treat. Further, participants portrayed weight as 3) having simple causes, and 4) important in physiotherapy. Alternate weight discourses were less frequent in these data.

Conclusion: Results indicated some physiotherapists' understandings of weight might lead to negative interactions with patients who are overweight. Findings suggest physiotherapists require more nuanced understandings of: how patients who are overweight might feel in a physiotherapy setting; the complexity of causes of weight; and possible benefits and disadvantages of introducing weight management discussions with patients. Therefore, education should encourage complex understandings of working with patients of all sizes including knowledge of weight stigma.

\section{Key Practice Points:}

- Physiotherapists often lack a nuanced understanding of: weight stigma, the complexity of causes of weight and when to introduce weight management discussions.

- Physiotherapists often view working with patients who are overweight as 'difficult'.

- Education should encourage complex understandings of weight including weight stigma, and develop more positive ways of thinking about patients who are overweight.

\section{EFFECT OF NEURAL MOBILIZATION AND NERVE AND TENDON GLIDING EXERCISES IN CARPAL TUNNEL SYNDROME- RCT}

Shah $\mathrm{S}^{1}$, Purohit $\mathrm{M}^{1}$, Patel $\mathrm{K}^{1}$

${ }^{1}$ K.M.Patel Institute of Physiotherapy

Question: What is the effect of neural mobilization and nerve and tendon gliding exercises in mild to moderate carpal tunnel syndrome?

Design: Randomised clinical trial with concealed allocation and accessor blinding.

Participants: 60 hands diagnosed with CTS, clinically as well as electrophysiologically were selected and randomly assigned into 2 groups. Intervention: Group A was given ultrasound, neural mobilization and night splinting. Group B was given ultrasound, nerve and tendon gliding exercises and night splinting. This treatment was given for twelve consecutive sessions. Assessment was taken prior and at the end of twelve sessions.

Outcome Measures: The subjects were assessed in terms of visual analogue scale, symptom severity scale, functional severity scale, grip strength, pinch strength and two point discrimination.

Results: Intra-group analysis showed that both the groups had significant improvement in all outcome measures. Inter-group analysis proved that neural mobilization is more effective with respect to all outcome measures than nerve and tendon gliding exercises except 2 Point discrimination.

Conclusion: Neural mobilization and nerve and tendon gliding exercises are effective in treating mild to moderate CTS but neural mobilization is more effective than nerve and tendon gliding exercises.

\section{Key Practice Points:}

- Neural mobilization and nerve and tendon gliding exercises are effective conservative methods to manage mild to moderate carpal tunnel syndrome. 


\section{A FEASIBILITY STUDY INVESTIGATING A SUPERVISED EXERCISE PROGRAM FOR FULL THICKNESS TEARS OF THE SUPRASPINATUS MUSCLE}

Burley $\mathrm{S}^{1}$, Meek L'1, Shea M², Pope R $^{2}$

1Sport and Spinal Physiotherapy Practice, Sunshine Coast, Queensland ${ }^{2}$ Bond University, Gold Coast, Queensland

Question: What is the feasibility and acceptability of a standardised supervised exercise program for patients with full thickness tears of the supraspinatus muscle and what is the effectiveness of this program?

Design: Prospective feasibility study of a single cohort with a preliminary assessment of the program's effectiveness. Approved by Bond University Human Research Ethics Committee (R01897).

Participants: five adults with full thickness tears of the supraspinatus muscle

Intervention: A standardized exercise program already prescribed as part of the patient's treatment.

Outcome Measures: Exercise compliance and acceptability, assessed by an end of study questionnaire. Shoulder Pain and Disability Index (SPADI) questionnaire. Active shoulder flexion and abduction range of motion.

Results: Self-reported exercise compliance was $>90 \%$, and participants reported the exercise prescription was readily performed and acceptable. On average, pain and disability scores decreased by $32 \%$ and $36 \%$, respectively ( $p=.07$ for both, approaching significance and indicating harm is unlikely). The mean increases in shoulder flexion and abduction range of motion were 15 and 26 degrees, respectively.

Conclusion: Program feasibility was assessed as good and risk of harm from the program unlikely, with a larger effectiveness tria therefore warranted.

\section{Key Practice Points:}

- This standardised exercise program, for full-thickness tears of the supraspinatus muscle, was well tolerated and deemed feasible and acceptable by patients.

- Exercise program compliance levels were high and preliminary effectiveness analysis indicated harm from the program to be very unlikely.

- Based on these results, a larger effectiveness trial is now warranted.

\section{MATERNAL BIRTH TRAUMA}

\section{Shek C}

Liverpool Clinical School, Western Sydney University

Maternal birth trauma is an important cause of pelvic floor dysfunction. Levator trauma, both macroscopic and microscopic, is an important risk factor for pelvic organ prolapse; and obstetric anal sphincter injuries, a risk factor for anal incontinence. Both conditions are highly prevalent. The talk will give the audience an update on both forms of maternal birth injuries.

\section{THE EFFECT OF EXERCISE ON MUSCLE MASS IN OLDER PEOPLE: SYSTEMATIC REVIEW AND META-ANALYSIS OF RANDOMISED CONTROLLED TRIALS}

\section{Sherrington $\mathrm{C}^{1}$, Tavares $\mathrm{G}^{1,2}$, Fairhall $\mathrm{N}^{1}$, Tiedemann $\mathrm{A}^{1}$}

${ }^{1}$ The George Institute for Global Health, The University of Sydney, Sydney ¿Pontifícia Universidade Católica do Rio Grande do Sul, Porto Alegre, Brazil

Question: Does exercise increase muscle mass in older people?

Design: Systematic review with meta-analysis of randomised trials.

Participants: People aged 60 years and above.

Intervention: Exercise, delivered alone or as a component of a multifaceted intervention.

Outcome Measures: We extracted data on muscle mass and the secondary outcomes strength and mobility. Standardised mean differences and 95\% confidence intervals were calculated using a random effects model.

Results: Thirty-eight trials involving 2504 older adults tested the effect of exercise on muscle mass (48 comparisons, as 11 trials had more than one intervention group) and found a small beneficial effect of exercise (Hedges' g $=0.10,95 \% \mathrm{Cl} 0.01$ to 0.18). Following an average of 25 weeks of exercise, muscle mass improved by $0.72 \mathrm{~kg}$. The effect on muscle mass was bigger in programs that contained a strength training component, but this difference was not statistically significant (effect of strength in meta-regression, $p=0.47$ ). Exercise also had a moderate effect on strength (Hedges' $9.77,95 \% \mathrm{Cl} 0.51$ to 1.04 ) and mobility (Hedges' g $0.77,95 \% \mathrm{Cl}$ 0.51 to 1.04 ).
Conclusion: Exercise produced small improvements in muscle mass in older people, and moderate gains in strength and mobility. Further investigation is required to determine the optimal type to exercise to increase muscle mass and functioning in older people.

\section{Key Practice Points:}

- Exercise increases muscle mass in people aged over 60

- Exercise should be prescribed to prevent and reduce sarcopenia.

- Indications are that exercise should contain a strength component to increase muscle mass.

\section{VALIDITY OF THE FITBIT ACTIVITY TRACKER FOR MEASURING STEPS IN COMMUNITY-DWELLING OLDER ADULTS}

Paul S1, Tiedemann A ${ }^{1}$, Hassett L1, 2, Ramsay E ${ }^{1}$, Kirkham C1, Chagpar $\mathrm{S}^{1}$, Sherrington $\mathrm{C}^{1}$

${ }^{1}$ The George Institute for Global Health, The University of Sydney, Sydney 2Faculty of Health Sciences, The University of Sydney, Sydney, Australia

Question: What is the criterion validity of Fitbitstep counts compared to visual count and ActiGraph accelerometer step counts? What is the accuracy of ActiGraph step counts compared to visual count, in communitydwelling older people.

Design: Observational study

Participants: Thirty-two community-dwelling adults aged 60+

Outcome Measures: Step counts from Fitbit and ActiGraph devices simultaneously during a 2-minute walk test (2MWT) then during waking hours over a 7-day period. A physiotherapist also counted the steps taken during the 2MWT.

Results: There was excellent agreement between Fitbit and visually counted steps (intraclass correlation coefficient [ICC2,1] = 0.88, 95\% $\mathrm{Cl}$ 0.76-0.94) from the 2MWT, and good agreement between Fitbit and ActiGraph (ICC2,1 = 0.66, 95\% Cl 0.41-0.82) and between ActiGraph and visual counted steps (ICC2,1 = 0.60,95\% CI 0.33-0.79). There was excellent agreement between the Fitbit and ActiGraph in average steps/day over 7 days (ICC2.1 = 0.94,95\% Cl 0.88-0.97). Percentage agreement was closest for Fitbit steps compared to visual count (mean 0\%, SD 4\%) and least for Fitbit average steps/day compared to the ActiGraph (mean 13\%, SD 25\%)

Conclusion: The Fitbit accurately tracked steps during the 2MWT but the ActiGraph appeared to underestimate steps. There was strong agreement between Fitbitand ActiGraph counted steps.

\section{Key Practice Points:}

- The Fitbit tracker is sufficiently accurate to be used among communitydwelling older adults to monitor and give feedback on step counts.

\section{OPTIMISING OUTCOMES AFTER HIP FRACTURE}

\section{Sherrington C}

The George Institute for Global Health, The University of Sydney

Hip fracture is a common and increasing global public health problem. Unfortunately mobility outcomes after hip fracture in older people are generally poor. Carefully designed exercise interventions have the potential to improve outcomes after hip fracture. This presentation will overview research about outcomes after hip fracture in older people as well as the results of randomised trials evaluating exercise interventions to enhance outcomes after fracture.

\section{Key Practice Points:}

- This presentation aims to assist participants to apply an evidence-based approach to the physiotherapy care of older hip fracture survivors 


\section{THE RECURRENT HAMSTRING INJURY: WHY?}

\section{Shield A}

Queensland University of Technology

Hamstring injury recurrence rates are high and not completely explained. We have recently proposed that persistent neuromuscular inhibition occurs after moderate to severe hamstring strains and that this inhibition contributes to injury recurrence, partly via its negative effects on eccentric strength and muscle architecture (1). This presentation discusses a range of studies with varying methodologies that have been conducted by the QUT - ACU Hamstring Injury Group in recent years. We have evidence for persistent and previously unrecognised neuromuscular inhibition via a range of methods including surface EMG (sEMG) during isokinetic dynamometry (2,3), functional MRI (4) and twitch interpolation. Furthermore, we have observed shorter fascicles in previously injured BFIh muscles than in contralateral uninjured muscles (5) while our prospective studies have shown that low levels of eccentric strength $(6,7)$ and short biceps femoris long head (BFlh) fascicles (7) are risk factors for future hamstring strains in elite Australian football and soccer. While further work is required to conclusively establish the role of neuromuscular inhibition in hamstring strain injury recurrence, the evidence so far is consistent with this possibility. Future rehabilitation studies will be needed to establish the methods that will best correct the deficits known to occur after hamstring strain.

\section{Key Practice Points:}

Rehabilitation and return to play strategies should take into account restoration of...

\section{- eccentric strength}

- the capacity to activate the hamstrings during active lengthening - restoring or obtaining 'desirable' fascicle lengths. New methods for assessing eccentric strength and ultrasound examination of hamstring muscle architecture may become increasingly common in clinical practice in the future

\section{FIND TIME TO EXERCISE OR FIND TIME FOR ILLNESS}

\section{Shields $\mathbf{N}^{1,2}$}

${ }^{1}$ School of Allied Health, La Trobe University

2Northern Health

'Those who think they have not time for bodily exercise will sooner or later have to find time for illness.' Attributed to the Earl of Derby in 1873, this quotation challenges us to reconsider the importance of physical activity in our lives. Participation in physical activity is integral to every person's health and well-being, yet as a nation Australians are now less active and more obese than at any other time in history. Physical activity is particularly important for people with disability. They are at higher risk of developing chronic health conditions such as insulin resistance and of developing secondary conditions associated with their primary disability such as depression and social isolation. Increasing evidence indicates people with disability are less active than people without disability and are less likely to engage in recommended amounts of physical activity. The reasons for this are complex and multifactorial, and include personal, social, environmental, and economic barriers to their participation. Physiotherapists can play a pivotal role in connecting people with disability to their communities through engagement in physical activity, exercise and sport. Our expertise in exercise prescription, design and implementation can facilitate their participation. However, we need to be focused and proactive about promoting physical activity and work in partnership with disability and recreation agencies based in the community.

\section{HOW TWIN STUDIES WILL ADVANCE UNDERSTANDING OF AETIOLOGY OF MUSCULOSKELETAL DISORDERS AND GUIDE SELECTION OF OPTIMAL TREATMENT}

Shirley $\mathrm{D}^{1}$, Ferreira $\mathrm{P}^{1}$

${ }^{1}$ Arthritis and Musculoskeletal Research Group, University of Sydney

Background: Musculoskeletal disorders are common and a leading cause of disability. Despite the magnitude of the problem no definitive risk factors have been identified for musculoskeletal disorders, including low back pain. Twin research, with the support of twin registries, provides a new and exciting possibility to consider the effect of genetics and the early shared environment to answer questions on causation.

Aims / objectives: The aim of this session is to discuss a new and innovative approach to explore the aetiology of musculoskeletal disorders (including low back pain) using twin research design. At the conclusion of this session participants should understand how to explore the role of genetics and early shared environment in the aetiology of musculoskeletal disorders and consider the use of the twin approach.

Approach: This session provide a 10 minute presentation of the utility of twin registries, including the Australian Twin Registry, and provide examples of published studies that have explored the role of potential risk factors for musculoskeletal pain. This will be followed by a discussion on how the findings of these studies have the potential to optimise clinical management of musculoskeletal disorders.

\section{Conclusion / Key Practice Points:}

- Twin registries are a useful resource for researchers investigating the aetiology of musculoskeletal disorders including LBP.

- Twin studies are a new and innovative approach to exploring risk factors for musculoskeletal conditions such as LBP

- Consideration of genetics and the early shared environment provide an exciting opportunity to isolate risk factors and identify cleaner and more precise estimates of risks.

- Understanding the aetiology of musculoskeletal disorders such as LBP will help to identify characteristics of individuals that make them more likely to respond to particular management approaches, thus having the potential to optimise treatment choices to provide the most effective outcomes.

\section{HOW TO INCORPORATE PHYSICAL ACTIVITY ADVICE FOR PREVENTION OF NON COMMUNICABLE DISEASE INTO USUAL PHYSIOTHERAPY PRACTICE \\ Shirley $\mathbf{D}^{1}$ \\ ${ }^{1}$ Arthritis and Musculoskeletal Research Group, University of Sydney}

Background: Physical inactivity is the fourth highest risk factor for non communicable diseases (NCDs) such as cardiovascular disease, diabetes and some cancers. Physiotherapists are ideally placed to provide physical activity interventions into usual practice. Most physiotherapists believe it is part of their role and health promotion for NCD prevention is recognised as a key area of practice by the World Confederation of Physical Therapy and the Australian Physiotherapy Association. Despite this only about $50 \%$ of physiotherapists provide physical activity advice for prevention of NCD and only about 33\% can correctly describe the recommended physical activity guidelines.

Aims / objectives: The aim of this session is to discuss the physical activity guidelines and to provide practical, evidence based recommendations of how physical activity advice can be incorporated into usual physiotherapy practice. At the end of the session participants will understand where to access information about physical activity recommendations, screen patients for physical activity levels and devise and deliver physical activity interventions as a primary prevention for NCD.

Approach: This session will commence with a 10 minute presentation on the background to physical activity interventions for prevention of NCD in primary care (specifically in relations to physiotherapy practice), followed by an interactive discussion with participants on incorporating physical activity intervention into practice, identifying and overcoming barriers to the physiotherapist and the patient and identifying and compiling resources.

Conclusion / Key Practice Points:

- Participants will be able to identify patients who do not meet physical activity guidelines

- Knowledge about delivery of physical activity interventions will be updated

- Practical solutions for implementation of physical activity interventions in to practice will be highlighted including strategies for overcoming barriers for physiotherapists and patients

- Resources will be identified 


\section{EFFECTIVENESS OF GYROKINESIS ${ }^{-}$ BASED TRAINING ON PHYSICAL FUNCTION AND QUALITY OF LIFE IN BREAST CANCER SURVIVORS}

Siao MR, Wang T]

National Yang Ming University

Question: Is Gyrokinesis-based training effective on improving physical function and quality of life in breast cancer survivors?

Design: A center-stratified randomized controlled trial was conducted with two post-treatment assessments of breast cancer survivors assigned to either 12 weeks of 90 -minute twice per week Gyrokinesis-based training classes or a wait-list control.

Participants: 60 women diagnosed with breast cancer and were within 5 years after finishing adjuvant therapy were recruited.

Outcome measure: Outcome of physical function included shoulder mobility, resting kyphosis angle and thoracic mobility, quality of life (FACT-B traditional Chinese version 4, FACT-B), self-perceived movement freedom (Freedom of Movement Inventory, FOMI) and self-perceived improvements (Global rating of changed score, GRCS).

Result: At 12-week, the experimental group showed significant improvements in shoulder mobility in all directions of the cancer-side arm $(p \leqq 0.001)$, upright habitual sitting position $(p=0.008)$, self-perceived freedom of movement $(p=0.001)$ and self-perceived improvement $(p \leqq 0.001)$, while the control group did not shown any significant improvements except the quality of life outcome (FACT-B). Frequency of exercise was used as a covariate for analysis on the FACT-B total score and found that the experimental group demonstrated significant improvement both in the post-test and the follow-up test $(p=0.001 \sim 0.002)$.

Conclusion: Gyrokinesis-based training is effective on improving physical function and quality of life in breast cancer survivors.

\section{Key Practice Points:}

- Gyrokinesis can be used in treating restriction of shoulder and thoracic joint of breast cancer survivors.

- Gyrokinesis-based training is beneficial to quality of life of breast cancer survivors.

\section{PAIN AND NEUROPLASTICITY: WHAT DOES IT MEAN FOR PHYSIOTHERAPY?}

\section{Siddall $\mathbf{P}^{1,2}$}

${ }^{1}$ Department of Pain Management, Greenwich Hospital, HammondCare, Greenwich, NSW

2Sydney Medical School-Northern, University of Sydney, NSW

Over the last 25 years, there has been a huge shift in the way that we see pain. From a view that saw pain largely as a message that had to be eliminated or at least reduced, we now recognise that the periphery is much less important in our perception of pain than once thought. There has been a steadily growing recognition of the importance of central nervous system processes such as pain modulation and neuroplasticity in our experience of pain. Although the process of pain modulation has been recognised and studied for over a century, there are more recent findings that indicate that pain modulation is the defining element for many people with chronic pain. The development of brain imaging techniques has also opened up a new vista for understanding the complex and at times confusing world of the brain and pain. We now know that there is a vast array of brain changes that occur in association with chronic pain. These findings have thrown up interesting and important questions regarding the role of the physiotherapist in treating and managing pain. Is pain management still about finding and treating the source of pain? Or is treating pain now more about treating the central nervous system? Or yet again, should the role of the physiotherapist be less about treating pain and more about helping people achieve their functional goals? What do the findings from pain neuroscience tell us? What is their relevance and what do they mean for physiotherapists today?

\section{Key Practice Points:}

- Refresh knowledge regarding the relative contribution of peripheral and central processes contributing to pain

- Understanding of the implications of this knowledge for the role of physiotherapists in trying to identify and treat a source of pain

- Appreciation of the potential to influence other aspects of the pain experience through strategies directed at the central nervous system
THE LESSER KNOWN CONSEQUENCES OF SPINAL CORD INJURY

\section{Siddall $\mathrm{P}^{1,2}$}

${ }^{1}$ Department of Pain Management, Greenwich Hospital, HammondCare, Greenwich, NSW

¿Sydney Medical School-Northern, University of Sydney, NSW

Immersion results in profound physiological changes that may have other consequences in people with a spinal cord injury. For example changes in circulation during and after immersion commonly result in substantial changes in blood pressure that need to be monitored and managed. However, immersion can result in even more dramatic effects in people with spinal cord injury because of the major changes in autonomic function and cardiovascular autoregulation. These effects may not only be substantial and require forethought or attention but may also limit the ability of some people to engage in this type of treatment. Another effect of immersion on people with spinal cord injuries include changes in pain. Immersion may have positive effects on musculoskeletal pain by reducing pain associated with posture and weight bearing. In addition, people with neuropathic pain following spinal cord injury often report a reduction in pain during immersion. The reason for this reduction in neuropathic pain is not well known and may be frustrating for people who find this reduction can't be sustained. What are the possible reasons for the reduction in pain during immersion and can we use this information to help people with pain? Key Practice Points:

- Appreciate the potential effects of immersion on some of the consequences of spinal cord injury including autonomic dysregulation and pain

- Understanding of the implications of this knowledge for the use of immersion and potential adverse as well as positive effects

\section{DEVELOPMENT OF A CONSENSUS ON 'USUAL CARE' UPPER LIMB REHABILITATION EARLY POST-STROKE AMONGST WESTERN AUSTRALIAN THERAPISTS}

Garcia-Vega J $^{1,2}$, Gregory $\mathrm{G}^{1}$, Lind $\mathrm{C}^{2,3}$, Singer BJ ${ }^{2}$

${ }^{1}$ Physiotherapy Department, Sir Charles Gairdner Hospital, Perth 2Centre for Musculoskeletal Studies, Surgery, The University of Western Australia, Perth

${ }^{3}$ Department of Neurosurgery, Sir Charles Gairdner Hospital, Perth

Question: To obtain consensus amongst therapists on commonly reported therapy interventions for moderate to severe upper limb deficits post-stroke, to inform the development of a 'standard package of care' for a subsequent interventional trial, and a recording tool to facilitate an accurate description of the treatment given to trial participants as per extended CONSORT guidelines. An additional goal was to gain agreement from therapists to abstain from treating the upper limb during the interventional trial.

Design: Retrospective audit and qualitative study (modified Delphi method).

Participants: 21 physiotherapy and occupational therapists working in acute stroke care and two rehabilitation services participated in the qualitative study.

Outcome Measures: An audit of upper limb therapy documentation was conducted from notes of all first time ischaemic middle cerebral artery strokes admitted to an acute stroke service over a nine month period. A list of interventions was developed and distributed to therapists.

Results: Following discussions with each service to define the described interventions, an intervention template for patients with moderate to severe upper limb impairments post-acute stroke was developed. This treatment template was refined until agreement was reached that it represented 'usual care' as provided by their respective services, and was then used to guide and document the 'standard package of care' for a subsequent pilot study. All therapists complied with not treating the upper limb during this trial.

Conclusion: Therapists were able to reach a consensus regarding 'usual care' early upper limb management post-stroke. A template can facilitate data collection in an interventional study. 
LONG-TERM EFFICACY OF BOTULINUM TOXIN TYPE A INJECTION TO THE DISTAL VASTUS LATERALIS MUSCLE FOR REFRACTORY ANTERIOR KNEE PAIN

Singer $\mathrm{BJ}^{1}$, Silbert $\mathrm{B}^{2}$, Silbert $\mathrm{PL}^{1}$, Singer $\mathrm{KP}{ }^{1}$

${ }^{1}$ Centre for Musculoskeletal Studies, School of Surgery, The University of Western Australia

2Faculty of Medicine, Dentistry and Health Science, The University of Western Australia

Question: To examine long term outcomes of Botulinum toxin type A injection to vastus lateralis muscle for refractory anterior knee pain associated with quadriceps muscle imbalance.

Participants: Two cohorts (private clinic referrals $n=53$, previous research participants $n=23$ ) of patients with anterior knee pain were retrospectively surveyed. Intervention: Intramuscular injection of Botulinum toxin type A into the distal vastus lateralis muscle was administered by one neurologist.

Outcome measures: Self-reported benefit, duration of symptom relief and uptake of knee surgery post-injection.

Results: Average duration( \pm SD) of symptoms prior to injection was $76 \pm 98$ months. Thirty-eight of 46 contactable private patients reported benefit from injection (average duration $25 \pm 21$ months). Symptom relief was ongoing in 29 of those surveyed, while nine individuals reported symptom recurrence after an average of $14 \pm 21$ months. Twenty nine who benefitted from injection were originally considering surgical treatment, however only four ultimately underwent knee surgery. Six of eight non-responders went on to have surgery, with only one reporting symptom improvement. Nineteen of 23 previous research participants were contactable. Initially, all had responded favourably to injection. Fifteen subjects continued to report symptomatic benefit (average duration $44 \pm 20$ months). Two subjects had proceeded to surgical intervention, with one reporting relief of symptoms

Conclusions: A single Botulinum toxin type A injection into vastus lateralis muscle led to improved function and relief of knee-related symptoms in 57 of 65 individuals treated. Improvements were sustained at follow-up in 44 of 57 cases (average duration of benefit $34 \pm 25$ months). Knee surgery was avoided in the majority of individuals.

\section{AN INVESTIGATION OF FACTORS INFLUENCING PHYSICAL ACTIVITY LEVELS IN PEOPLE LIVING IN THE COMMUNITY AFTER STROKE}

\section{Jackson $\mathrm{SM}^{1,2}$, Mercer $\mathrm{C}^{2}$, Singer B] ${ }^{1}$}

${ }^{1}$ Centre for Musculoskeletal Studies, School of Surgery, The University of Western Australia, Perth

Physiotherapy Department, Western Sussex Hospitals NHS Foundation Trust, Worthing, United Kingdom (UK)

Question: This research explored levels of, and barriers to, physical activity in a cohort of people living in the UK post stroke.

Design: A questionnaire was used to elicit participants' self-reported physical activity levels, their beliefs and knowledge about, and barriers related to, physical activity. Spearman's rank correlation coefficient was used to explore associations between self-reported physical activity and: age, gender, level of mobility, fear of falling, beliefs, available supports and socioeconomic status.

Participants: 76 questionnaires were returned. Most participants identified as White British, average age was 75. Most were within two years post stroke. 59\% were male. $60 \%$ were coded as community ambulators.

Results: $54 \%$ of respondents did not undertake sufficient physical activity to meet current guidelines, while 63\% reported that they of ten sit for prolonged periods. Common barriers to undertaking physical activity included fear of falling, stroke-related disability, pain and fatigue. Low levels of physical activity were associated with fear of falling (Rho -0.402, $p=0.002$ ), poorer functional mobility (Rho $0.458 p=0.000$ ), prolonged sitting (Rho $-0.455, p=0.000$ ), and belief that physical activity would not improve health (Rho -.337, $\mathrm{p}=0.013$ ) or may cause another stroke (Rho -.305, $p=0.035$ ). Interpersonal and environmental barriers reported by participants included a lack of social support, difficulties with transport and the influence of inclement weather.

Conclusion: Participants reported a number of interlinking and overlapping personal, interpersonal and environmental barriers to undertaking physical activity. The majority reported levels of physical activity that are insufficient to meet current guidelines as well as prolonged periods of being sedentary.
PHYSIOTHERAPISTS' ASSESSMENT OF

PATIENTS' PSYCHOSOCIAL STATUS: ARE WE STANDING ON THIN ICE? A QUALITATIVE DESCRIPTIVE STUDY

Singla MM, Jones M, Edwards I, Kumar S

Therapia Physiotherapy and Pilates, International Centre for Allied Health Evidence, University of South Australia

Background: While physiotherapists are generally well educated to assess and manage the physical and many environmental dimensions of their patient's health conditions, assessment, evaluation and management of psychological and social factors is often less developed and less structured.

Objective: To explore private practice physiotherapists' assessment of patients' psychosocial status.

Methods: A qualitative descriptive research design was used in this study. Participants were recruited through purposeful sampling and potential informants were invited to participate through letters or phone calls and data was collected via semi-structured interviews. Nine interviews were conducted at the participants' workplaces. Data collection and analysis were conducted simultaneously and common concepts and themes were recognised, coded and grouped accordingly. Strategies to ensure research rigour and trustworthiness were implemented.

Findings: The physiotherapists' in this study demonstrated and acknowledged a poor understanding of the role of psychosocial factors in their patients' clinical presentations. Three themes were identified physiotherapists' capacity to conduct psychosocial assessment; the barriers while conducting psychosocial assessment; and the suggestions the therapists provided to overcome these barriers.

Conclusion: Physiotherapists in this study were unclear about the assessment of psychosocial factors. The barriers to assessment of psychosocial factors ranged from individual shortcomings to limitations in professional networks and time constraints. The most consistent barrier highlighted was participants' lack of formal education in psychosocial theory and assessment.

\section{Key Practice Points:}

- Ambiguity and uncertainty prevails in physiotherapy practice of assessment of psychosocial factors

- Multiple barriers to psychosocial assessment exist with lack of formal education being the main barrier. 


\section{EXTENDED SCOPE PHYSIOTHERAPY- LED MULTIDISCIPLINARY MANAGEMENT, USING THE INTERNATIONAL CLASSIFICATION OF FUNCTIONING, DISABILITY AND HEALTH (ICF) MODEL FOR CLIENTS WITH DISABILITIES}

Sloane J, Chau C, Blackburn C

Inner East Community Health

Background: The World Health Organisation (WHO) developed the ICF model in 2001 - a globally accepted framework, embracing a client-centred holistic approach to a person's health rather than disability. The model emphasises communication within and beyond the multidisciplinary team. It determines team roles, connects the team, clients and carers, with external clinicians and specialists.

Design: The physiotherapist-led Complex Disability Support Team (CDST) aimed to "Connect" the facets of client health and support services, improving the quality and effectiveness of client care, minimising impairments, improving function and participation, whilst reducing carer burden/stress and acute health costs.

Intervention: IECHS therapists identified significant health care gaps for people with neuro-developmental disabilities and employed a senio mentor physiotherapist with disability expertise, to improve the model of care.

A CDST was formed, allied health 'scopes of practice' defined and referral pathways developed to connect with key health and support services, GPs and specialists. IECHS instituted a patient-centered, evidence-based, multidisciplinary framework, to identify the health needs of CDST clients. This utilised new assessment templates and outcome-focused, goaldirected care plans, regularly reviewed via a standard format. One of many successful case studies will be presented.

Results: The ICF model implemented provided timely and appropriate delivery of therapeutic intervention in a cost effective manner. Clients reported improved levels of function and social inclusion through increased community activity and participation.

Conclusion: Utilising the ICF model, IECHS has successfully developed specialised client-centred teams, focused on improving client quality of life and health outcomes for people with a disability.

Key Practice Points:

- Using the ICF model provides a workable framework for community therapists.

- Connecting client centred teams can significantly improve client quality of life

- All therapist skills are improved by the collaborative communication of the multidisciplinary team

- Reduced therapy time impacts on overall health costs, while still achieving significant outcomes for the client.

\section{PREDICTING SUCCESS IN MEDICAL DIAGNOSIS AND TREATMENT OF WHIPLASH-MEDIATED CERVICAL FACET JOINT NOCICEPTION? AN EMERGING ROLE FOR PHYSIOTHERAPISTS}

Smith $A^{1}$, Schnieder $G^{3}$, Jull $G^{2}$, Sterling $M^{1}$

${ }^{1}$ CONROD Injury Research Centre, Griffith University, Queensland

2Division of Physiotherapy, University of Queensland, Queensland

${ }^{3}$ Faculty of Rehabilitation Medicine, University of Alberta, Canada

Question: Can physiotherapists predict which patients will respond to medical-based diagnosis and treatment of cervical facet joint nociception?

Design: Two prospective cohort studies

Participants: 178 individuals with whiplash-associateddisorder symptoms

Intervention: Controlled cervical medial branch blocks ( $n=125)$ and cervical radiofrequency neurotomy $(n=53)$

Outcome Measures: Multivariate regression analyses were performed in the derivation of clinical decision guides. Predictor variables measured at baseline (prior to cervical facet joint injections and radiofrequency neurotomy) included self-reported pain, disability, post-traumatic stress symptoms, pain catastrophizing, common clinical examination (palpation and provocation) tests and measures of sensory hypersensitivity (pressure and cold pain thresholds). The outcome measure for determination of facet joint 'success' was (pain relief $\geq 80 \%$ ), whilst post-neurotomy 'success' was perceived Global Rating of Change (scores $\geq 4$ ) 3 months post-neurotomy

Results: A clinical decision guide involving the findings of the manual spinal examination, palpation for segmental tenderness, and extensionrotation test demonstrated a positive likelihood ratio of $4.94(95 \% \mathrm{Cl}$
2.8 to 8.2). Multivariable logistic regression demonstrated 'success' of radiofrequency neurotomy was predicted by lower levels of disability and pain catastrophizing (both $p<0.05$ )

Discussion: Routine clinical examination tests may be useful for identifying patients suitable for diagnostic facet joint blocks. Simple questionnaires predicted success of radiofrequency neurotomy.

\section{Key Practice Points:}

- Physiotherapists can adequately determine which individuals may benefit from cervical facet joint injections.

- Individuals with chronic whiplash-associated-disorder symptoms respond to cervical radiofrequency, irrespective of physical or psychological features evident in their clinical manifestations.

Ethics ID: E-22082 (University of Calgary Conjoint Health Research Ethics Board)

\section{UNDERSTANDING THE RELATIONSHIPS} BETWEEN URINARY INCONTINENCE, RESPIRATORY PROBLEMS AND

\section{BACK PAIN}

\section{Smith MD}

School of Health and Rehabilitation Sciences, The University of Queensland St. Lucia, QLD

Question: This talk will highlight findings from a series of studies that aimed to investigate epidemiological and physiological associations between urinary incontinence $(\mathrm{UI})$, respiratory problems and back pain (BP).

Design: Epidemiological associations were investigated using crosssectional and longitudinal analyses with consideration of confounding factors. A case-control study designed was used for physiological experiments.

Participants: Women ( $\mathrm{n}=38,050)$ from the Australian Longitudinal Study on Women's Health participated in the epidemiological studies. Women with stress UI ( $n=16)$, individuals with respiratory disease $(n=15)$ and healthy controls $(n=29)$ participated in the physiological studies.

Outcome Measures: Self-report postal survey data were analysed to determine associations between the presence and development of UI, respiratory problems and BP. Electromyographic activity (EMG) of the pelvic floor (PF; Ul only), abdominal and erector spinae muscles were recorded during perturbation tasks. Amplitude and timing of EMG activity were calculated.

Results: There is a strong epidemiological relationship between the presence of UI, respiratory problems and BP among women. Longitudinal analyses indicate that the presence of one condition increases risk of the development of another. Women with stress Ul have delayed but increased PF muscle activity, and early and increased abdominal muscle activity associated with postural perturbations. Abdominal muscle activity is increased during perturbations in people with respiratory disease.

Conclusion: Physiological studies identify similar increases in superficial trunk muscle activity in individuals with stress UI, respiratory disease and BP. Altered abdominal muscle activity could negatively impact on the spine, continence and respiratory mechanisms, and may help to explain the association between these conditions.

\section{Key Practice Points:}

- Consider the interaction between abdominal and PF muscle function when managing women with stress UI

- Timing of PF muscle activation may be more important than amplitude of PF muscle activity in women with stress UI

- Management of co-morbidities is important when treating individuals with UI, respiratory disease and low BP 


\section{AQUATIC PHYSIOTHERAPY AND CANCER REHABILITATION: A LITERATURE REVIEW}

\section{Smith $\mathrm{SE}^{1,2}$}

${ }^{1}$ Bendigo Health

2Cobaw Community Health Kyneton

Question: What is known to reduce risk and inform effective practice in aquatic

physiotherapy with survivors of cancer?

Design: A narrative review of qualitative and quantitative studies following a systematic search of the CINAHL, MEDLINE, PEDro and Cochrane databases of all studies in aquatic exercise in survivors of cancer.

Participants: Cancer survivors.

Intervention: Any form of water based exercises.

Outcome Measures: Measures of lymphedema, cancer-related fatigue, reduced range of motion and reduced quality of life indicators were included.

Results: Nine studies were reviewed including five randomised controlled trials. Eight studies included females who had completed active treatment for breast cancer. One case description discussed lymphedema after gynaecological cancer. High adherence rates with aquatic exercise were achieved in all the studies with either transient or no adverse outcomes recorded in these participants. Hydrostatic pressure may be beneficial for managing lymphedema and the low impact aquatic environment may be helpful in addressing cancer related fatigue.

Conclusion: Initial research indicates that the aquatic environment may be safe and well tolerated for cancer survivors. Long term effectiveness has not been adequately studied.

\section{Key Practice Points:}

- Aquatic physiotherapy can be considered a safe and effective option for rehabilitation following treatment for breast cancer.

- The unique properties of water are advantageous for the management of common adverse outcomes such as lymphedema and cancer related fatigue.

-Whilst there is limited research currently available evaluating aquatic physiotherapy and its application to cancer rehabilitation, land based cancer rehabilitation quidelines can inform practice.

\section{THE EFFECTS OF SHOULDER TAPING ON SCAPULOTHORACIC MUSCLE CONTRACTION AND CORTICOMOTOR EXCITABILITY OF SCAPULAR AND SHOULDER MUSCLES}

Snodgrass $\mathrm{SJ}^{1}$, Farrell SF${ }^{1}$, Tsao $\mathrm{H}^{2}$, Osmotherly PG ${ }^{1}$, Rivett DA ${ }^{1}$, Chipchase $L^{3}$, Schabrun $S^{3}$

${ }^{1}$ Discipline of Physiotherapy, School of Health Sciences, The University of Newcastle, Newcastle

2The Royal Brisbane and Women's Hospital, Queensland Health, Brisbane 3University of Western Sydney, Brain Rehabilitation and Neuroplasticity Unit, Sydney

Question: Does shoulder taping affect the magnitude and timing of scapular muscle contraction and the corticomotor excitability of the scapular and shoulder muscles?

Design: Pre-post comparison.

Participants: 15 healthy individuals.

Intervention: Rigid strapping tape applied from the anterior glenohumeral joint to the inferior angle of the scapula on the dominant arm.

Outcome Measures: Surface electromyography (onset of upper trapezius, lower trapezius and serratus anterior activation relative to deltoid, and peak root mean square [RMS]) during active shoulder flexion and abduction, and amplitude of motor-evoked potential [MEP] to transcrania magnetic stimulation. Repeated measures analysis of variance with Bonferroni post-hoc tests determined differences in outcomes before, immediately after and 24 hours post-taping.

Results: During arm elevation after taping, there was earlier onset of upper and lower trapezius activation, but this was not maintained 24 hours post-taping. These results were most pronounced for upper trapezius during abduction post-taping (mean 34.2ms after deltoid onset, SD $118.2)$ compared to pre-taping (93.9ms, SD 106.3, $p=.021)$, and for lower trapezius during flexion post-taping (mean $110.0 \mathrm{~ms}$ before deltoid onset SD 109.8) compared to pre-taping (5.9ms, SD 92.3, $p=.056)$. Taping had no effect on MEPs or peak RMS during arm elevation.

Conclusion: Shoulder taping in healthy individuals changes the timing of scapular muscle contraction during arm elevation, but these changes are not maintained 24 hours post-tape and are not explained by altered corticomotor excitability.

\section{Key Practice Points:}

- Earlier onset of scapular muscles during arm elevation on the day of shoulder tape application suggests that this may be an ideal time to retrain these muscles to activate during shoulder elevation.

- The effects of shoulder taping on the timing of scapular muscle contraction are not maintained 24 hours after tape application, suggesting that additional interventions would be needed for longer term effects.

\section{PHYSICAL CHARACTERISTICS PREDISPOSING RUGBY UNION PLAYERS TO NECK INJURIES}

Snodgrass S] ${ }^{1}$, Osmotherly PG ${ }^{1}$, Reid SA ${ }^{2}$, Milburn PD ${ }^{3}$, Rivett DA ${ }^{1}$

${ }^{1}$ Discipline of Physiotherapy, School of Health Sciences, The University of Newcastle, Newcastle

¿School of Physiotherapy, Faculty of Health Sciences, Australian Catholic University, Sydney

${ }^{3}$ Griffith Health Institute, Griffith University, Gold Coast

Question: What are the physical characteristics associated with neck injury history and increased risk of sustaining a neck injury in rugby union players?

Design: Prospective cohort study

Participants: Semi-professional players $(n=142)$ from six NSW rugby union clubs.

Outcome Measures: Pre-season cervical spine range of motion (ROM), neck muscle strength, neck sensorimotor proprioception (joint position error), neck anthropometry, previous neck injury history, current neck symptoms, playing position, competition level, age, and years playing rugby. In-season neck pain and injury via team physiotherapist reports and player telephone interviews. Logistic regression determined factors associated with neck injury history and incidence.

Results: Sixty-five (46\%) players reported a previous neck injury, and 11 (8\%) sustained a neck injury during the competitive season. Age (OR 1.14, $95 \% \mathrm{Cl} 1.03-1.25, \mathrm{p}<.01)$ and years playing rugby $(1.12,0.84-3.19, \mathrm{p}<.01)$ were associated with neck injury history. Pre-season lateral flexion ROM was less in players sustaining a neck injury during the season (median left 23.6 $6^{\circ}$, IQR 21.8-26.2; right 27.9, $23.6-32.5$ ) than in other players (left $34.8^{\circ}$ 28.8-41.0, $p<.01$; right 39.1, 28.9-48.1, $\mathrm{p}=.03$ ). Decreased left lateral flexion ROM was associated with increased risk of sustaining a neck injury (OR 0.85, 95\% Cl 0.76-0.94, $\mathrm{p}<.01$ ).

Conclusion: Decreased cervical lateral flexion ROM may contribute to neck injury risk in rugby union players. However, few physical characteristics predicted neck injury incidence, suggesting additional factors should be explored in this cohort to determine injury risk. Acknowledgements: NSW Sporting Injuries Committee and NSW Rugby Union.

\section{Key Practice Points:}

- Older and longer playing rugby union players are more likely to report having had a neck injury over the course of their career.

- Injured players demonstrate less cervical lateral flexion ROM suggesting consideration of lateral flexion ROM in the prevention of injury is reasonable. A certain amount of lateral flexion may be required to minimise the risk of injury when rucking or tackling, for example.

- Neck injury incidence was low, suggesting possible under-reporting by players. A strong positive player relationship with the team physiotherapist appeared to improve injury reporting. 


\section{PHYSIOTHERAPISTS' PERSPECTIVES OF CURRENT MANAGEMENT OF ANTERIOR CRUCIATE LIGAMENT INJURIES}

Von Aesch $A^{1}$, Perry $M^{2}$, Sole $\mathbf{G}^{1}$

${ }^{1}$ Centre for Health, Activity and Rehabilitation Research, School of

Physiotherapy, University of Otago, Dunedin, New Zealand

2Centre for Health, Activity and Rehabilitation Research, School of

Physiotherapy, University of Otago, Wellington, New Zealand

Question: What are strengths and challenges of current management of patients with anterior cruciate ligament $(A C L)$ injuries, as perceived by physiotherapists?

Design: Qualitative study

Participants: Fifteen physiotherapists from private clinics on the South Island, New Zealand, were recruited via purposive sampling.

Outcome Measures: Semi-structured interviews were conducted using individual or small group, face-to-face, video or telephonic methods. The interviews were recorded, transcribed verbatim and the general inductive approach was used to develop themes.

Results: Participants strove to provide expert and evidence-informed care, applying principles of bio-psychosocial management. The importance of recognising and addressing psychological factors, such as patients' lack of confidence, was raised. Their main concerns were complex referral processes which extended the rehabilitation period, and sub-optimal interprofessional communication. Participants suggested translational research was needed for clear directions for exercise prescription and milestones for return to sports and activity following ACL injury.

Conclusion: Participants upheld high professional profiles by providing expert ACL injury management. Potential areas of improvement include simplifying the referral process and enhancing communication between physiotherapists and other health professionals. Continued education for addressing psychological factors related to rehabilitation of ACL injuries should be considered. Future research should focus on use of a milestoneapproach for rehabilitation and return to sport and activity.

\section{Key Practice Points:}

- Physiotherapists were confident employing a milestone approach to rehabilitation progression for patients with ACL injuries.

- Emphasis on a bio-psychosocial approach to rehabilitation is needed for continued education.

- The referral process for patients was often considered to be complex and delayed.

\section{GREATER REDUCTION IN WALKING SPEED AND AUTOMATICITY OBSERVED IN ADULTS AS SEVERITY OF HEARING IMPAIRMENT INCREASES}

Soles $\mathrm{K}^{1}$, Martin $\mathrm{F}^{1}$, Leung $\mathrm{A}^{1}$, McMahon $\mathrm{C}^{2}$, Scrivener $\mathrm{K}^{\mathrm{C}}$, Dean $\mathrm{CM}^{1}$ ${ }^{1}$ Department of Health Professions, Macquarie University

Department of Linguistics, Macquarie University

Question Does walking performance differ with reference to normative data and according to the severity of hearing impairment?

Design: Observational study

Participants: 43 adult clients (mean age 69 SD 14) seeking audiology services at a University Clinic. This total hearing group were divided into subgroups based on severity of hearing impairment of the better ear: normal ( $n=14)$, mild: $(n=15)$ and moderate/severe $(n=16)$.

Outcome Measures: Distance in 6MWT, 10 MWT speed under fast, comfortable, dual manual, dual cognitive and triple (cognitive and manual) conditions and automaticity index.

Results: The total hearing group walked at comparable speeds and cover comparable distances in six minutes to normative data. The total hearing group have a significant reduction (-6\% $95 \mathrm{Cl}-12$ to -0) in automaticity of walking when the walking involved an additional manual task compared with normative data. The reduction in automaticity was three times more $(-19 \% \mathrm{Cl}-35$ to -2$)$ pronounced in those with moderate/severe hearing impairment. Severity of hearing impairment also had a significant effect on most outcomes, with worse performance in the moderate/severe hearing impairment subgroup. Walking automaticity of the moderate/ severe subgroup on average was worse (MD - $14,95 \mathrm{Cl}-35$ to 2) than stroke data.

Conclusion: Adults with moderate/severe hearing impairment have a significant reduction in walking performance. Physiotherapy assessment and intervention services for those with hearing impairment warrant development and evaluation.

\section{Key Practice Points:}

- Hearing impairment negatively affects walking performance

- Training walking automaticity for hearing impaired warrants investigation

- Integrated physiotherapy services within hearing clinics is indicated.

\section{RELIABILITY OF THE COMBINED ELEVATION TEST IN SWIMMERS}

Sorrell-Saunders $\mathrm{M}^{1}$, Marshall $\mathrm{N}^{1,2}$, Carter j ${ }^{2}$, Pope R ${ }^{1}$

${ }^{1}$ Bond Institute of Health \& Sport, Faculty of Health Science \& Medicine, Bond University, Gold Coast

¿Surflife Physio, Gold Coast

Question: Can the combined elevation test be reliably performed in a healthy adult swimming population?

Design: Combined intra-observer and inter-observer reliability study

Participants: 49 swimmers with a mean $( \pm$ SD) age of 46.23 ( \pm 11.82 ) years recruited from a local swim squad on the Gold Coast, Australia.

Intervention: Two observers conducted the measurements, a

physiotherapist with a single year of clinical experience, and a second year post-graduate physiotherapy student. A single observer performed three measurements with 49 participants to assess intra-observer reliability. 22 of the participants were measured an additional three times by the second observer for assessment of inter-observer reliability.

Outcome Measures: Combined elevation test measurements.

Results: Inter-observer reliability ICC3.3 0.96 (95\% CI 0.90 to 0.98: SEM $2.74 \mathrm{~cm}$ ) and intra-observer reliability ICC1,30.99 (95\% CI 0.98 to 0.99; SEM $2.14 \mathrm{~cm})$. Both indicated high levels of agreement between measures.

Conclusion: The combined elevation test exhibits high levels of reliability in healthy adult swimmers when performed by both a single observer and differing observers of varying clinical experience levels.

\section{Key Practice Points:}

- The combined elevation test exhibits strong intra- and interobserver reliability

- The combined elevation test is a practical tool for functional assessment in swimming populations

- More research is required to validate the combined elevation test

\section{A RETROSPECTIVE COHORT STUDY OF OUICKDASH SCORES FOR COMMON ACUTE TRAUMA CONDITIONS PRESENTING FOR HAND THERAPY}

Southam $\mathrm{M}^{1}$, Driessens $\mathrm{S}^{2}$, Burton $\mathrm{C}^{2}$, Pope $\mathrm{R}^{1}$, Thurnwald $\mathrm{P}^{1}$

${ }^{1}$ Bond University, Gold Coast

¿Logan Hospital, Queensland Health, Logan City

Questions: What are the typical ranges and distributions of QuickDASH scores associated with specific upper limb pathologies? Do factors including age, gender, hand dominance, smoking history, diabetes status, occupation, and therapy attendance affect QuickDASH scores?

Design: A retrospective cohort study involving patient chart review and covering a five-year period from January 2009 - December 2014.

Participants: Four hundred and eighty-one patients who attended a hand elbow and wrist therapy service were identified, with one of five upper limb conditions: a flexor or extensor tendon injury/repair, a distal-radius fracture, a metacarpal fracture, or complex trauma.

Outcome Measures: Initial and final QuickDASH scores and QuickDASH change scores.

Results: The distributions of initial and final QuickDASH scores were determined and plotted, and will be presented for these upper limb conditions, together with $80 \%$ cut-points to aid clinical decision-making. The therapy period was consistently associated with a substantial shift in the distribution of QuickDASH scores from high to low. Age, initial QuickDASH result, time between injury and initial therapy session, and attendance all affected QuickDASH change and final scores.

Conclusion: The QuickDASH is a valuable outcome measure for clinicians to demonstrate progress and to identify higher-risk patients, by reference to typical score distributions.

\section{Key Practice Points:}

- The QuickDASH is widely used in clinical settings and these typical score distributions, based on empirical evidence, will increase the utility of QuickDASH scores for clinical decision-making. 


\section{THE EFFECTS OF PRETERM BIRTH ON INFANTS AND THEIR FAMILIES}

\section{Spittle $\mathbf{A}^{1,2,3}$}

${ }^{1}$ University of Melbourne, Australia

2Murdoch Childrens Research Institute, Australia

${ }^{3}$ The Royal Women's Hospital, Melbourne, Australia

Over 6,400 infants are born at <34 weeks' gestation in Australia annually, many of whom will experience substantial developmental challenges, including motor, cognitive, academic and mental health problems. Preterm birth can dramatically disrupt family functioning, and up to $63 \%$ of mothers of preterm infants report symptoms of depression in the first few months after the birth. Higher rates of parental psychological distress have also been reported across early childhood. Results of our prospective longitudinal study including 150 infants born <30 week's gestation and their parental well-being, from birth to two years will be presented. Intervention with at-risk children in the early years is one of the most cost-effective strategies to improve future outcomes and productivity for preterm children, and the evidence for this will be presented. Furthermore, the parent-child relationship provides the child's most immediate environment for development, and the parent-child relationship is proposed to be the mechanism for many of the positive effects of early intervention programs for preterm children. Novel and more established evidence-based intervention approaches for preterm children will be discussed, including long-term findings from our randomised controlled trial of a preventative care program in the first year of life for infants born preterm and their families.

\section{Key Practice Points:}

- Mothers and fathers have high rates of anxiety and depression following the birth of a preterm baby

- Early interventions involving parents have the best outcomes for preterm children

- Physiotherapists have an important role in enhancing the parentchild relationship

\section{THE EFFECT OF INFORMATION FEEDBACK ON TRAINING STANDING UP FOLLOWING STROKE: A FEASIBILITY STUDY}

Stanton $\mathbf{R}^{1}$, Ada $\mathrm{L}^{1}$, Dean $\mathrm{C}^{2}$, Preston $\mathrm{E}^{1}$

${ }^{1}$ School of Physiotherapy, Faculty of Health Sciences, University of Sydney, Australia

Discipline of Physiotherapy, Macquarie University, Australia

Question: Is information feedback a feasible and effective strategy to improve the ability to stand up in people following stroke?

Design: Single group feasibility study with pre-post measures.

Participants: Twenty people with hemiplegic stroke in inpatient rehabilitation.

Intervention: Ten sessions over two weeks of information feedback about foot placement during training of standing up from a chair. Progression involved increasing repetitions, increasing difficulty and fading feedback

Outcome Measures: Feasibility was determined by adherence, time taken, satisfaction, and safety. Clinical outcomes were the time taken to stand up and rating of performance by experienced therapists measured using the 5-Times-Sit-To-Stand-Test; and carryover into daily activities measured by covert observation.

Results: The study was feasible with $97 \%$ of sessions completed, taking 19 (6) to 25 (10) minutes. Participants understood (4.6/5), found useful (4.6/5), challenging (4.4/5), and would recommend (4.7/5) the training. 9 falls occurred during the study, none during training; of these, 2 were injurious. The time to stand up five times decreased by $24(95 \% \mathrm{Cl}-48$ to $-1) \mathrm{s}$, and the quality of standing improved by $1.0 / 10.0$ ( $95 \% \mathrm{Cl} 0.2$ to 1.8 ). Carryover of the correct foot placement occurred to real life, with the beginning foot position correct 2.1/3.0 (95\% Cl 1.6 to 2.6) and end foot position correct 1.8/3.0 (95\% Cl 1.2 to 2.4 ) occasions.

Conclusion: The intervention is feasible and has the potential to improve the ability to stand up.

Trial registration: ANZCTR12613000620729

\section{Key Practice Points:}

- This promising intervention warrants a randomised trial so its efficacy can be tested.
THE LOCATION OF NECK PAIN MATTERS TO IMPLICIT MOTOR IMAGERY DISRUPTION: IMPLICATIONS FOR CORTICAL INVOLVEMENT

Stanton $\mathrm{TR}^{1,2}$, Leake $\mathrm{HB}^{1}$, Wallwork $\mathrm{SB}^{1}$, Moseley $\mathrm{GL}^{1,2}$

${ }^{1}$ The Sansom Institute for Health Research, The University of South Australia, Adelaide, Australia

2Neuroscience Research Australia, Sydney, Australia

Question: Are disruptions in implicit motor imagery performance influenced by the site of neck pain?

Design: Randomised, repeated measures.

Participants: Participants that completed an online task ( $n=1608)$ were divided into groups based on neck pain location: none, left-sided, rightsided, bilateral.

Intervention: Implicit motor imagery tasks involving left/right judgements of images of head/neck rotation; participants identified the direction of neck rotation.

Outcome Measures: Accuracy and response time (RT). Repeated measures ANOVAs determined if the groups differed for images of left versus right neck rotation.

Results: Accuracy was higher for images of right neck rotation than for images of left neck rotation ( $p=0.001)$; no differences existed for RT $(p=0.09)$. A main effect of group occurred: those without pain were more accurate and quicker than those with bilateral pain $(p=0.009, p=0.031)$ and left-sided pain ( $p=0.045, p=0.037)$; those with right-sided pain were more accurate than those with bilateral pain $(p=0.0026)$ but were no quicker ( $p>0.05$ ). A significant image $x$ group interaction occurred for accuracy only: the accuracy advantage for right over left images was greatest in those with right-sided pain (versus other groups)

Conclusions: Neck pain location matters: having bilateral or left-sided neck pain consistently impairs implicit motor imagery performance. That decreased accuracy also occurred for left neck rotation images suggests that interactions between pain site and cortical spatial processing (known to be lateralised) may occur.

Key Practice Points:

- It may be relevant to consider the location of neck pain during assessment/treatment as this may identify those with increased disruption to cortical proprioceptive representation.

\section{WHIPLASH: LATEST EVIDENCE FOR REHABILITATION AND RECOVERY}

\section{Sterling $\mathrm{M}^{1}$}

${ }^{1}$ CRE Road Traffic Injury, CONROD research Injury Centre, Menzies Health Institute Queensland, Griffith University

Consistent international data indicate that up to $50 \%$ of people will not have recovered one year post whiplash injury. Whilst there is variability in recovery pathways, recent exploration using trajectory modelling analytical techniques has identified distinct recovery pathways (trajectories). Various physical and psychological factors have also been identified as being prognostic of poor health outcomes following whiplash injury, including initial pain intensity, initial disability, pain catastrophizing, posttraumatic stress symptoms, recovery expectations and signs of central sensitisation. Many trials have investigated relatively simple interventions such as exercise programs that may not be sufficient to address the complex clinical presentation seen in those with already chronic symptoms or those individuals with acute whiplash pain identified as being at high risk of poor-recovery. A recent high quality trial showed little benefit of an early multi-disciplinary intervention on the incidence of chronic pain development. Another high quality trial of an intensive physical rehabilitation program for people with chronic whiplash showed no greater effect than simple advice. In overall terms, currently available trials for whiplash could be described as showing mostly small effects in improving health outcomes. Whilst the question of how to prevent the transition from acute to chronic pain after whiplash injury cannot yet be answered, this presentation will outline and discuss possible ways forward for the management of this condition. 
HOW TO ASSESS AND MANAGE

PSYCHOLOGICAL FACTORS IN

PHYSIOTHERAPY OF MUSCULOSKELETAL PAIN CONDITIONS

\section{Sterling $\mathrm{M}^{1}$, McGregor $\mathrm{L}^{\mathrm{1}}$}

${ }^{1}$ CRE Road Traffic Injury, CONROD research Injury Centre, Menzies Health Institute Queensland, Griffith University

Background: Stress, anxiety and depression are common features of many musculoskeletal conditions. Physiotherapists usually don't consider these factors as a standard part of their patient assessment. They also report difficulty in communicating effectively with distressed patients and how to raise issues such as the impact of psychological factors on physical function and how and when to initiate psychological referral.

Aims / objectives: This interactive and practical workshop will:

- Develop knowledge and skills in identifying common psychological factors such as stress, anxiety and depression and how these may impact physical outcomes.

- Provide resources for managing psychological factors within physiotherapy treatment including: assessment measures and evidence based treatment techniques.

- Provide information and strategies for when and how to make a referral.

By the end of the session participants will understand how to screen for psychological factors in patients with musculoskeletal pain and develop skills in managing psychological distress.

Approach:The session with be delivered by a physiotherapist an psychologist. The session will include hands on practical experience including demonstrations, case studies and group work.

\section{Conclusion / Key Practice Points:}

The session will contribute to participants' clinical practice by:

- Providing knowledge of which psychological factors to assess for, tools to use for assessment and interpretation.

- Providing confidence in patient communication about the impact of psychological issues in order to optimise the effects of physiotherapy interventions

- Provide skills of when and how to refer patients to mental health practitioners as necessary.

\section{DOES THE VST DIFFERENTIATE}

CLINICAL PRESENTATIONS OF PATIENTS

REFERRED TO A PHYSIOTHERAPYLED VESTIBULAR SERVICE IN A TERTIARY HOSPITAL?

Stewart V ${ }^{1,2}$, Mendis $D^{3}$, Low Choy $\mathrm{N}^{1,2}$

${ }^{1}$ The Prince Charles Hospital, Brisbane

${ }^{2}$ Australian Catholic University (McAuley Campus), Brisbane

${ }^{3}$ Centre for Musculoskeletal Research, Mary Mackillop Institute of Health Research, Brisbane

Questions: Are there differences and associations between the Vestibular Screening Tool (VST), Dizziness Handicap Inventory (DHI), and mobility scores for clinical diagnostic groups referred for vestibular rehabilitation?

Design: Prospective observational study.

Participants: Patients referred to a tertiary hospital's physiotherapy-led vestibular service $(n=275)$ from acute hospital and community-based services. Clinical diagnostic groups included Benign Paroxysmal Positional Vertigo (BPPV), Vestibular Neuritis (VN), other vestibular and nonvestibular disorders.

Outcome Measures: The VST, DHI and mobility measures (Timed Up and Go, ten-metre walk-test, Functional Gait Assessment-FGA) were recorded.

Results: Patients diagnosed with BPPV, VN or other vestibular disorders had significantly higher scores on the VST $(p<0.001)$ and DHI $(p<0.001)$ than non-vestibular patients, but no significant differences between diagnostic groups for mobility measures ( $p>0.05)$. The VST was highly associated with the $\mathrm{DHI}(r=0.714 ; \mathrm{p}<0.001)$, particularly the physical sub-score $(r=0.768 ; p<0.001)$ and moderately associated with the FGA $(r=-0.428 ; p<0.001)$. No differences were found between patients in diagnostic categories referred from hospital or community-based services.

Conclusion: Higher VST scores were indicative of vestibular disorders. Mobility measures did not differentiate vestibular from non-vestibular patients. The VST demonstrates concurrent validity with DHI and only moderate association with mobility measures.

\section{Key Practice Points:}

- The VST can assist in differentiating vestibular from non-

vestibular disorders.

- The VST could be utilised to screen and refer patients with dizziness for vestibular assessment.

- Mobility measures do not appear to differentiate vestibular and nonvestibular disorders on initial assessment.
CONCURRENT VALIDITY OF THE VESTIBULAR SCREENING TOOL WITH THE DIZZINESS HANDICAP INVENTORY, COMPARING ACUTE AND COMMUNITY PRESENTATIONS ACROSS THREE TIME-POINTS

Stewart $\mathbf{V}^{1,2}$, Mendis $\mathrm{D}^{3}$, Low Choy $\mathrm{N}^{12}$

${ }^{1}$ The Prince Charles Hospital, Brisbane

${ }^{2}$ Australian Catholic University (McAuley Campus), Brisbane

${ }^{3}$ Centre for Musculoskeletal Research, Mary Mackillop Institute of Health Research, Brisbane

Question: Does the Vestibular Screening tool (VST) demonstrate concurrent validity with the Dizziness Handicap Inventory (DHI) at initial, discharge and follow-up whether referred from hospital or via community?

Design: Prospective observational study

Participants: Patients referred to a tertiary hospital's physiotherapyled vestibular service ( $n=271)$ from acute hospital or community-based services.

Outcome Measures: VST and DHI completed concurrently on initial, discharge, and 3-month follow-up assessments.

Results: VST scores were highly correlated with $\mathrm{DHI}$ total scores on initial $(r=0.714: p<0.001)$ discharge $(r=0.996$ : $p<0.001)$ and 3-month follow-up $(r=0.755 ; p<0.001)$. Correlations between VST and DHI physical sub-scores at each time-point $(r>0.768$; $p<0.001)$ were higher than with $\mathrm{DHI}$ total scores. The acute group's VST scores were more highly associated with DHI total scores on initial $(r=0.763$; $p<0.001)$ and discharge $(r=$ $0.676 ; p<0.001$ ) than the community-referred group on initial $(r=0.584$ $p<0.001)$ and discharge $(r=0.644 ; p<0.001)$. Both groups were highly correlated on follow-up $(r \geq 0.727 ; p<0.001)$.

Conclusion: The VST is highly correlated with DHI scores, across three time-points when tested with dizzy hospital patients and communityreferred patients presenting to a vestibular service.

\section{Key Practice Points:}

- The VST could be used in acute hospital or community-based services.

- The VST could be a screening tool to refer patients to physiotherapy-led vestibular services.

- Research is required to determine if VST scores reflect changes in physical/ functional abilities.

\section{WHAT IS EXCESSIVE OCCUPATIONAL SITTING?}

\section{Straker L}

School of Physiotherapy and Exercise Science, Curtin University Perth, Australia

Recently there have been frequent media stories about the hazards of too much sitting, however what is the evidence base for considering sitting a health hazard? Further, to what extent is occupational exposure common and substantial? Also, is there any evidence to determine a threshold for an excessive amount of sitting, both in total life exposure and in occupational exposure? Should any threshold consider both total accumulation and pattern of accumulation? If occupational sitting is a health hazard, is it possible to design a safe system of work for currently sedentary occupations? Is standing a suitable work posture alternative to sitting? Finally, what control options are practicable and have demonstrated efficacy?

Key Practice Points:

- This presentation will inform practice through a better understanding of the consequences associated with excessive sitting, the role of occupation in sitting exposure and evidence to support contemporary control options 


\section{ABSENTEEISM, PRESENTEEISM AND MUSCULOSKELETAL DISCORDERS IN YOUNG WORKERS}

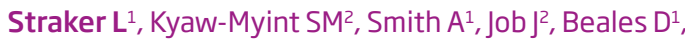
O'Sullivan $\mathrm{P}^{1}$

${ }^{1}$ School of Physiotherapy and Exercise Science, Curtin University, Perth, Australia

¿Safe Work Australia, Canberra, Australia

Question: Do young workers report substantial absenteeism and presenteeism? Are absenteeism and presenteeism related to back and neck pain in young workers?

Design: Cross-sectional survey of ongoing longitudinal community cohort.

Participants: 1146 young (mean age 22 years) adults from the Western Australian Pregnancy Cohort (Raine) Study provided data.

Outcome Measures: Absenteeism and presenteeism data were collected using the Health Performance Questionnaire, enabling estimates of time lost per year. Back pain and neck pain data included self report of health professional diagnosed disorders.

Results: Young workers reported mean loss of 53 hours/year due to health-related absenteeism and 302 hours/year due to presenteeism. 17\% reported current diagnosed back pain and $9 \%$ reported current diagnosed neck pain. Young workers with back pain had more health-related absenteeism than those without back pain (81 hours/year vs 41 hours/ year) but those with neck pain did not have significantly greater health related absenteeism than those without neck pain (69 hours/year vs 51 hours/year). There were no differences in presenteeism between those with and without back or neck pain.

Conclusion: Young worker absenteeism and presenteeism are important issues, with musculoskeletal disorders related to greater productivity loss.

\section{Key Practice Points:}

- Health-related productivity is an important aspect to consider in occupational physiotherapy practice.

- Back pain is common in young workers.

- Back pain is related to greater work productivity loss in the form of health-related absenteeism

\section{QUALITY INDICATORS FOR MUSCULOSKELETAL INJURY MANAGEMENT IN THE EMERGENCY DEPARTMENT: A SYSTEMATIC REVIEW}

Strudwick $\mathbf{K}^{1,2,3}$, Nelson $M^{1,3}$, Martin-Khan $M^{4,5}$, Bourke $M^{1,3}$, Bell $A^{2,6}$, Russell $\mathrm{T}^{3}$

${ }^{1}$ Physiotherapy Department, QEll Jubilee Hospital, Metro South Health, Brisbane

¿Emergency Department, QEll Jubilee Hospital, Metro South Health, Brisbane. ${ }^{3}$ School of Health and Rehabilitation Sciences, The University of Queensland, Brisbane

${ }^{4}$ Centre for Research in Geriatric Medicine, The University of Queensland,

Brisbane

${ }^{5}$ Centre for Online Health, The University of Queensland, Brisbane

"School of Medicine, The University of Queensland, Brisbane

Question: What quality indicators used in Emergency Departments are relevant to musculoskeletal injuries? What is their methodological quality?

Design: A systematic review of articles containing at least one quality indicator measuring the care of musculoskeletal injuries in Emergency Departments.

Participants: Emergency Departments.

Intervention: MEDLINE, EMBASE, CINAHL and grey literature were searched. Articles were included that described a quality indicator and its development process, related to the care of musculoskeletal injuries in Emergency Departments. Critical appraisal of relevant quality indicators was conducted.

Outcome Measures: Quality indicators were rated against the Appraisal of Indicators through Research and Evaluation (AIRE) Instrument, and categorised using quality frameworks of Donabedian and the Institute of Medicine.

Results: Fifteen articles were included, containing 71 relevant indicators Pain ( $n=17)$ and fracture management $(n=13)$ quality indicators were predominant. Only ten indicators scored $\geq 50 \%$ across all AIRE Instrument domains and these were related to pain management and appropriate imaging of the spine.

Conclusion: The methodological quality for the development of most musculoskeletal quality indicators used in Emergency Departments is poor Recommendations for a core set of quality indicators that address the complete spectrum of musculoskeletal injury management in emergency medicine is not possible.

\section{Key Practice Points:}

- There is a lack of high-level evidence surrounding the type of quality indicators used in Emergency Departments to measure quality of care for musculoskeletal injuries.

- Development of quality indicators in this area is needed given time pressures and evolving models of care in Emergency Departments, including primary contact physiotherapy.

\section{DEVELOPMENT OF A COMPETENCY FRAMEWORK: PHYSIOTHERAPISTS PERCEPTIONS OF WORKPLACE KNOWLEDGE AND SKILLS}

Sturt $\mathbf{R}^{1}$, Harding $\mathrm{P}^{1}$, Sayer $]^{1}$, Burge $A T^{1}$

${ }^{1}$ Department of Physiotherapy, Alfred Health, Melbourne

Question: What are acute hospital-based physiotherapists' perceptions around learning, professional development and workplace competency?

Design: Qualitative study utilising survey questions, semi-structured interviews and focus groups.

Participants: A convenience sample of 46 qualified physiotherapists in a metropolitan hospital network.

Outcome Measures: Survey results were quantitatively analysed and thematic analysis of interviews and focus groups was used to provide a collective insight into physiotherapists' perceptions.

Results: Five main themes emerged: confidence in clinical knowledge and skills is mostly high but affected negatively by perception of isolation in less experienced staff; assessment and feedback around competency is limited, predominantly informal and unstructured; staff openly acknowledge their clinical and non-clinical knowledge and skill limitations, but readily identify clinical risk if more experienced; staff draw on a range of strategies to support their learning, but rely mainly on informal workplace discussion; while staff feel supported to learn outside of work hours, dedicated time and resources within work time would be welcomed.

Conclusion: Physiotherapists report limited feedback regarding their workplace knowledge and skills. Physiotherapists rely on informal discussion to support their competency development. Physiotherapist's knowledge and skills may be enhanced through dedicated resources within work time.

Key Practice Points:

- Less experienced physiotherapists may benefit from structured feedback regarding knowledge, skills and identifying clinical risk.

- Physiotherapists may benefit from more frequent and structured assessment around clinical and non-clinical knowledge and skills

- Physiotherapists could consider utilizing a broader range of workplace assessment strategies 
PROLONGED LAPAROSCOPIC AND LOWER ABDOMINAL SURGERY: THE RATE AND COST OF POSTOPERATIVE PULMONARY COMPLICATIONS AND IMPLICATIONS FOR PHYSIOTHERAPY MANAGEMENT

\section{Boden IJ, ${ }^{1,2}$, Sullivan KE $\mathrm{K}^{1,3}$}

IPhysiotherapy Department, Launceston General Hospital, Launceston Department of Physiotherapy, The University of Melbourne, Melbourne ${ }^{3}$ Department of Physiotherapy, Monash University, Melbourne

Question: What is the incidence of respiratory complications and hospital costs following prolonged laparoscopic and lower abdominal surgery?

Design: Prospective multi-centre observational study.

Participants: One-hundred and sixty-five patients having laparoscopic colorectal surgery, advanced laparoscopic upper gastrointestinal surgery, open abdominal hernia repair or open lower abdominal surgery longer than three hours. Patients received no pre- or post-operative physiotherapy.

Outcome Measures: Post-operative respiratory complications were assessed daily using standardised diagnostic criteria. Hospital costs were collected from standardised government clinical activity coding and costing data for each participant.

Results: Respiratory complication rate was 10\% (95\% Cl, 6\% to 15\%). Length of hospital stay for patients with a respiratory complication was significantly higher than those without (mean difference 6.07 days, $95 \% \mathrm{Cl}$ 4 to 8 days, $p=0.0023$ ). Hospital costs were also higher (mean difference $\$ 13,639,95 \%$ CI \$6, 319 to $\$ 20,959)$

Conclusion: Compared to patients after open upper abdominal surgery, patients having prolonged laparoscopic or lower abdominal surgeries are only slightly less likely to get a respiratory complication postoperatively. Prophylactic physiotherapy intervention may be warranted in this patient group given the increasing complexity and length of procedures previously considered low risk.

\section{Key Practice Points:}

- Respiratory complications following prolonged laparoscopic, open lower abdominal or open hernia surgery are comparatively high, have large cost implications, and increase length of stay.

- Provision of physiotherapy to prevent respiratory complications in this population needs to be considered.

- A risk prediction model for this population model would assist in allocating physiotherapy resources appropriately.

\section{A TMS-GUIDED INTERVENTION INDUCES PLASTICITY IN THE MOTOR CORTEX AND REDUCES PAIN IN CHRONIC KNEE OSTEOARTHRITIS}

Chipchase $L^{1}$, Summers $S^{1}$, Baptista AF², Te $M^{1}$, Schabrun SM ${ }^{1}$

${ }^{1}$ School of Science and Health, University of Western Sydney, Sydney

${ }^{2}$ Biomorphology Department, Health Sciences Institute, Federal University of Bahia, Salvador

Question: Does a TMS-guided exercise and electrical stimulation intervention reduce pain and improve function in OA knee?

Design: Single case study (ABA design).

Participants: A 66-year-old woman diagnosed with knee OA.

Intervention: Transcranial magnetic stimulation was used to compare the corticomotor maps of rectus femoris, vastus lateralis and vastus medialis between the participant and an age and gender matched control. A home exercise program with electrical stimulation was designed to target specific alterations in motor cortical organisation observed in the participant compared to the control. Data were collected over three phases: baseline for 4 weeks (A), 2 week intervention phase (B), and a 4 week final phase where the intervention was withdrawn $(A)$.

Outcome Measures: The Intermittent and Constant Osteoarthritis Pain (ICOAP) questionnaire and the Western Ontario and McMasters Universities OA index (WOMAC) were completed every week. The two standard deviation band method was used for statistical analysis.

Results: Significant improvements in the three scales of the ICOAP were seen following the intervention $(p<0.05)$. However, there was no statistically significant change in WOMAC scores following the intervention.

Conclusion: This is the first study to assess the effect of a TMS-guided intervention designed to target neurophysiological changes occurring at the level of the cortex in a knee OA individual. Results demonstrate significant pain reductions in knee $\mathrm{OA}$.

\section{Key Practice Points:}

- A TMS-guided intervention to target changes in motor cortical organisation improved the severity of pain in a knee OA individual.

- Further research with larger samples is required to confirm these findings.
RISING TO THE PARADOX: THE BURDEN OF CHRONIC DISEASE AND THE ROLE OF PHYSIOTHERAPY WITHIN THE HEALTH SYSTEM

Swan N, Dennis S, Coombes J, Martin J, Barnett C, Jones T

As life expectancy increases so does disability free life expectancy. But what isn't changing much is the gap created by socio-economic inequality. People in better off postcodes are fit and active and live long lives whilst people with fewer years of education and with less disposable income are living shorter lives with more disability. Meantime the burden of chronic illness increases. These are the major challenges facing Australia's healthcare system. Is the physiotherapy profession willing and able to tackle this challenge of access, with high quality, efficient and safe interventions, that evaluates outcomes? And if so, how and who pays?

INTERNATIONAL CLINICAL PLACEMENTS FOR UNDERGRADUATE ALLIED HEALTH STUDENTS: ARE THEY WORTH THE RESOURCES INVESTED?

\section{Tan B-K}

Faculty of Health Sciences, Curtin University; Armadale Health Service, Perth, Western Australia

Fieldwork or clinical placement has been an integral part of clinical training for Physiotherapy and other allied health students, not only to develop their clinical skills but to develop important attributes such as team work communication and problem solving skills. With the current emphasis on Global Citizenship, there is a growing trend to send students on overseas clinical placements. International fieldwork learning can be particularly rich as it facilitates the development of students' cultural competence, key discipline skills in real-world contexts and employability skills. However, these international placements are often expensive (for the students) and resource intensive (for the university). Many students who have participated in international placements often claimed that the experience was 'life-changing'. Their benefits, however, have not been systematically investigated. This presentation describes Curtin University Faculty of Health Sciences' international and interprofessional 'Go Global' program and three cross-sectional studies using mixed methods design conducted between 2011-2014 to evaluate if the program achieved its intended aims and learning outcomes. Participants included students (recent Go Global participants); Health Sciences graduates (in the workforce for at least 6 months and including those who did not have an international placement) and graduates of the Go Global program for more than two years.

Key Findings: The Go Global program facilitated the development of interprofessional capabilities (teamwork, collaboration, increased professional role understanding), communication skills (cross-cultural and non-verbal communication), resilience (including independence, confidence and developing their problem solving and negotiation skills), and cultural sensitivity (empathy and understanding cultural influence on health beliefs).

\section{Key Practice Points:}

- International placements, whilst resource intensive, are valuable opportunities to build the capacity of the future global health workforce, in particular intercultural understanding, interprofessional practice capabilities and capabilities to navigate varying social contexts 


\section{REORGANISATION OF THE PRIMARY MOTOR CORTEX IN PERSISTENT ANTERIOR KNEE PAIN}

Te $M^{1}$, Baptista $\mathrm{AF}^{2}$, Schabrun SM1, Chipchase LS1

${ }^{1}$ School of Science and Health, University of Western Sydney, Sydney Biomorphology Department, Health Sciences Institute, Federal University of Bahia, Salvador

Question: Is corticomotor excitability and organisation of the quadriceps muscles altered in persistent anterior knee pain (AKP) compared with painfree controls?

Design: Observational, cross-sectional study.

Participants: Eleven participants with persistent AKP, and 11 healthy ageand gender-matched control participants.

Outcome Measures: Transcranial magnetic stimulation was used to measure corticomotor excitability and organisation of three quadriceps muscles: rectus femoris (RF), vastus lateralis (VL) and vastus medialis (VM). Outcome measures included map volume (excitability), distance between maps and number of discrete peaks (organisation).

Results: Corticomotor excitability was less in AKP participants compared to pain-free controls ( $p<0.001)$. The distance between the motor maps of RF and VL was less in AKP compared to controls ( $p=0.006)$, but did not differ between other muscles ( $p>0.05$ ). Additionally, the number of discrete peaks in VL was significantly less in AKP compared to controls ( $p=$ $0.005)$, but not for other muscles ( $p>0.05$ ).

Conclusion: These data provide evidence of reduced corticomotor excitability and altered organisation (characterised by increased overlap in the corticomotor representations of RF and VL, and reduced number of discrete peaks for VL), in persistent AKP compared with pain-free controls. These cortical changes could contribute to a loss of motor control of the quadriceps in people with AKP.

\section{Key Practice Points:}

- Reduced cortical excitability and altered motor cortex organisation is present in people with AKP.

- Cortical reorganisation is present in VL and RF.

- Longitudinal studies with larger sample sizes are required.

\section{CHOOSING THE RIGHT TOOL}

\section{Thomas DT}

There are a multitude of tools available to help identify and assess the work-related musculoskeletal disorder risk. These range from measuring devices, checklists, surveys and analysis tools. These tools can be subjective or objective in nature. Many analysis tools attempt to quantify physical work load and psychosocial work factors placed on the employee. The choice of tool or tools to be used at the workplace will be determined by the task being assessed, the target audience of any findings, the assessors' understanding of the tool and their competency with the use of the tool. Every tool is designed with a specific use in mind. Researchers often made assumptions when developing tools. No one tool is perfect or better than another tool in every situation and every tool has its limitations. It is only through the thorough understanding of a tool's limitations, underlying assumptions and uses, can the most appropriate tools be chosen for the worksite assessment and then used effectively. This presentation will review some commonly used tools (Revised NIOSH lifting equation, RULA etc.) in terms of their use, limitations and underlying assumptions.

\section{THE EFFECT OF END-RANGE CERVICAL ROTATION ON CERVICAL ARTERIAL BLOOD FLOW AND CEREBRAL INFLOW: SUB ANALYSIS OF AN MRI STUDY}

\section{Thomas LC 1,2 , MacLeod L1,2, Osmotherly $\mathrm{P}^{1,2}$}

\section{University of Queensland, The University of Newcastle}

Question: What is the mean effect of end-range cervical rotation on blood flow in the cervical arteries and overall cerebral inflow and are there individual variations?

Design: Experimental observational study.

Participants: 20 healthy adults

Intervention: Participants were imaged with magnetic resonance angiography in the neutral head position and at end-range rotation to the left and right.

Outcome Measures: Average blood flow volume ( $\mathrm{ml} / \mathrm{s})$ in the cervical arteries was measured in 3 neck positions: neutral, end range rotation (left and right) and compared between the neutral and end-range rotation positions.Total cerebral inflow was determined from the sum of average flow volume in the cervical arteries and compared between positions.
Results: Average blood flow volume decreased in the vertebral and internal carotid arteries on contralateral rotation, compared to neutral, statistically significant on left rotation only. Ipsilateral rotation had no effect. Total cerebral inflow was not significantly affected by rotation in either direction.

Conclusion: In healthy adults the cerebral vasculature appears to compensate for decreased flow in one or more arteries by increasing flow in other arteries, to maintain cerebral perfusion. Sustained end-range rotation may therefore reflect the compensatory capacity of the system as a whole rather than isolated vertebrobasilar function.

\section{Key Practice Points:}

- In healthy individuals blood flow volume in the cervical arteries generally decreases on end-range neck contralateral rotation

- Blood flow reduction in one cervical artery appears to be adequately compensated by the other vessels in participants with normal vascular anatomy.

- Pre-manipulative testing involving sustained rotation may still be warranted to detect those with inadequate collateral flow

- Clinicians should monitor patients for vertebrobasilar insufficiency in case of abnormal vascular anatomy.

\section{HOW TO CONDUCT A SCREENING PROTOCOL PRIOR TO MANUAL TREATMENT OF THE CERVICAL SPINE}

Thomas LC, Der A, Field E, Ho K, Seth T, Treleaven J The University of Queensland

Background: Manual therapy of the cervical spine has in rare cases been associated with adverse events. Current screening practices are under review in light of suggestions that they may not adequately identify those at risk of neurovascular compromise or with an ischaemic event already in progress and some frameworks have been suggested which may lack clinical utility. There is a need for a succinct protocol which can be conducted quickly within a normal clinical encounter on patients of concern.

Aims / objectives: To demonstrate a quick screening examination covering domains of balance, co-ordination, cranial nerve function and VBI which might be undertaken prior to treatment of the cervica spine. Participants will also have the opportunity to participate in some discussion about the application and clinical utility of these tests.

Approach: The test protocol will be demonstrated by the presenter and participants given the opportunity to practice with the assistance of 4 facilitators who have used the protocol extensively and are familiar with expected findings. Participants will be supplied with material (booklet) outlining the history component of the assessment, pictures and descriptions of the tests, and 'normal' values.

\section{Conclusion / Key Practice Points:}

- Participants will have practical experience of performing basic tests of balance, co-ordination, cranial nerve function, VBI as quick screening tests

- This session will enable musculoskeletal practitioners to incorporate basic neurological functional type screening into their practice

- Familiarity with the screening test protocol should help improve safety in musculoskeletal practice and help identify individuals with neurovascular compromise or ischaemic event in progress.

\section{EVOLVING CONCEPTS IN SIMULATION EDUCATION}

\section{Thomas P}

The Royal Brisbane and Women's Hospital, Brisbane Australia

Over the past decade, there has been a surge in interest and utilisation of simulation-based healthcare education in university and workplace settings. Cardiorespiratory physiotherapy has emerged as an effective area for its successful implementation. This presentation will provide an overview of current applications of simulation training in university and hospital settings and explore novel ways of applying simulation to make it accessible and tailored towards achieving desired learning outcomes. Evolving concepts in simulation education include directions for educator training, delivering education at the right time - in the right place, the integration of computer based technology, and the ongoing development of simulation modalities and their matching to the desired learning objectives. A variety of examples will be presented including "insitu" simulation and variations of hybrid standardised patients. 
WHEELCHAIR SPORTS PARTICIPATION; INJURIES, BENEFITS AND BARRIERS

Thompson ER ${ }^{1-3}$, Snodgrass SJ' $]^{1}$ Osmotherly PG ${ }^{1}$

${ }^{1}$ Discipline of Physiotherapy, School of Health Sciences, The University of Newcastle, Newcastle

2Sapphire Coast Physiotherapy, Bega

${ }^{3}$ Bega District Hospital, New South Wales Health, Bega

Question: What is the nature and management of injuries sustained by wheelchair sports participants? What are the perceived benefits and barriers to wheelchair sports participation?

Design: Cross-sectional descriptive study.

Participants: 278 members of Wheelchair Sports New South Wales.

Outcome measure: Questionnaire items collecting information on demographics and sports played, injuries sustained, prevention and management practices, and benefits and barriers to participation.

Results: Seventy-one questionnaires were returned (response rate 26\%). Injuries most frequently affected the shoulder $(25 \%, n=11)$, were muscle strains $(32 \%, n=14)$, and caused by contact $(48 \%, n=21)$. Injury prevention strategies were reported by $75 \%$ of the sample $(n=53)$. The main participation benefits were improvements in fitness $(85 \%, n=59)$, opportunities for friendship ( $83 \%, n=59)$, and setting goals ( $91 \%, n=63$ ). Barriers were the cost of sports wheelchairs $(68 \%, n=45)$, availability of competitions $(62 \%, n=43)$, and distance required to travel for training/ competition $(71 \%, n=49)$.

Conclusion: There are many perceived benefits to participation in wheelchair sport which should be promoted in improving the health of individuals with disabilities.

\section{Key Practice Points:}

- Injuries in wheelchair sport are common so physiotherapists should endeavour to assist athletes with disabilities with prevention and management strategies.

- As the majority of wheelchair athletes report fitness, social and personal benefits from participating in sport, physiotherapists can confidently promote these benefits to persons with disabilities to encourage greater participation.

- Physiotherapists should work to address the reported barriers to wheelchair sport participation

\section{HOW RELIABLE IS AN INSTRUMENTED FOOTPLATE IN MEASURING APPLIED TORQUE AND ANGULAR DISPLACEMENT OF THE ANKLE?}

Thomson $D^{1,2}$, Gupta $A^{1}$, Crosbie ${ }^{1}$

${ }^{1}$ School of Science and Health, University of Western Sydney

2Physiotherapy Department, Liverpool Hospital

Question: Are the relationships between applied torque and angular displacement at the ankle reliable within and between test sessions? Design: An intra- and inter-session repeated measures design.

Participants: Twelve healthy young volunteers.

Outcome Measures: An instrumented footplate was rhythmically oscillated to obtain measures of applied torque and angular displacement at the ankle. Intra-class correlation coefficients (ICC) were sought to determine the reliability for values of angular displacement (degrees) at given torques of $0.1,0.15$ and $0.2 \mathrm{Nm} . \mathrm{kg}^{-1}$. The magnitude of applied torque required to achieve a given angle of 0,5 and 10 degrees was also collected. Data regarding intra-session reliability were collected at intervals of 0,15 and 45 minutes and were analysed using a one-way repeated measures ANOVA. Inter-session reliability was determined using a paired t-test and compared the initial measure to a subsequent measure taken two weeks later. Measures were collected with the knee extended and flexed. Surface EMG was collected for soleus and tibialis anterior to ensure absence of muscle activity.

Results: No significant intra- or inter-session difference was noted between measurements ( $p>0.07$ ). ICC's for both intra-session ( $r>0.94$ ) and inter-session ( $r>0.85$ ) reliability were consistently 'excellent' for all investigated variables.

Conclusion: The instrument was consistent and reliable in collecting torque/angle relationships.

\section{Key Practice Points:}

- The use of an instrumented footplate provides a consistent and reliable method of obtaining angular torque and displacement values

- This method provides a simple and accurate way to determine stiffness and extensibility about the ankle joint
THE EFFECT OF MASSAGE AND HEAT ON THE PASSIVE MECHANICAL PROPERTIES OF THE CALF MUSCLE COMPLEX

Thomson $D^{1,2}$, Gupta $A^{1}$, Crosbie $\rfloor^{1}$

${ }^{1}$ School of Science and Health, University of Western Sydney

2Physiotherapy Department, Liverpool Hospital

Question: Does deep soft-tissue massage or superficial heat have an effect on the passive mechanical properties of the healthy calf muscle complex?

Design: A randomised, repeated measures cross-over study with assessor blinding.

Participants: Twenty-nine healthy young volunteers.

Intervention: Participants received a ten minute application of massage or superficial heat to a randomised limb, whilst the contralateral limb was a resting control. Intervention and control legs were then switched following a 2 week washout period.

Outcome Measures: An instrumented footplate was rhythmically oscillated to obtain measures of passive stiffness (quotient of torque and displacement curve between 0.1 and $0.2 \mathrm{Nm} \cdot \mathrm{kg}^{-1}$ ) and extensibility (angular displacement at torque of $0.2 \mathrm{Nm}^{\mathrm{kg}} \mathrm{g}^{-1}$ ) before, immediately after, and 30 minutes after the intervention. Measures were collected in positions of knee extension, and 30 degrees of knee flexion. Surface EMG was collected for soleus and tibialis anterior to ensure absence of muscle activity. Analysis was performed with a $4 \times 3$ repeated measures ANOVA with respect to intervention and time.

Results: There were no statistically significant differences in the passive stiffness ( $p>0.8)$ or extensibility ( $p>0.35)$ of the calf muscle complex due to intervention over the time periods analysed. No significant differences were observed in either knee position.

Conclusion: Massage and heat provided no mechanical changes to healthy calf tissue.

Key Practice Points:

- Neither massage nor heat appear to provide a significant passive mechanical effect in the healthy calf muscle complex

- Future studies should investigate the efficacy of massage and surface heating in clinical populations

\section{A MULTI-DISCIPLINARY, COLLABORATIVE APPROACH TO LOAD MANAGEMENT IN A HIGH PERFORMANCE TRAINING ENVIRONMENT}

\section{Toomey M}

QueenslandAcademy of Sport

Understanding the optimal application and progression of load is critical in ensuring that athletes achieve the desired benefits of undertaking a high performance training program, such that they develop the physicality, resilience and competitive mindset required to achieve their performance goals. Equally, monitoring and understanding the impact of unplanned, significant or habitual variations in load application is critical to understanding and managing an athlete's risk of injury and illness during the training cycle. This presentation highlights the benefit to athletes and sporting organisations when load management is optimised in a multidisciplinary, collaborative environment. Conversations which bring the expertise of the sports scientist, strength and conditioning coach, health service provider, technical coach and the athlete to the table to formulate loading strategies; monitor and measure the effect of load and where necessary, make adjustments to the application of load at particular times in the athlete training cycle or in response to particular events create the greatest likelihood that the elite athlete's pathway to achieving their performance goals.

\section{Key Practice Points:}

- The learning outcomes from this session will contribute to participants' practice by increasing their understanding of the need to engage collaboratively with the team working around an athlete to develop and deliver effective load management and injury risk reduction strategies 
HOW TO SET UP COLLABORATIVE

PARTNERSHIPS BETWEEN FITNESS

FACILITIES AND PHYSIOTHERAPY

SERVICES TO ENABLE SUCCESSFUL CLIENT SELF-HEALTH MANAGEMENT

Tran $\mathrm{M}^{1}$, Farrugia $\mathrm{L}^{1}$, Brophy $]^{2}$, Bailey $\mathrm{K}^{1}$

ISt Vincent's Hospital Melbourne, Vic

¿Department of Health and Human Services, Vic

Background: With the increasing burden of chronic disease on healthcare systems, physiotherapists should consider innovative approaches to facilitate ongoing client adherence to physical activity guidelines. Community gyms and fitness centres are currently diversifying to serve a wider population with medical conditions and can provide an appropriate and safe environment for this cohort

Aims/objectives: Participants will gain an appreciation of the stages involved when engaging with an external gym and leisure centre facility to ensure a successful partnership including:

- Addressing client barriers of hesitation, unfamiliarity and

membership cost

- The types of health and well-being programs currently running in community fitness and leisure facilities and how they can benefit clients

- Typical client outcomes (objective, clinical and subjective client focus group feedback) when they transition to gym settings

- Boundaries of care between health professionals and gym staff and how to conduct a training needs analysis

- Understanding who your stakeholders may be and how to manage these relationships

- A clinical and workforce evaluation framework to measure level of success

- Considerations for legal and clinical risk management

Approach: Sample materials including assessment tools, survey

questionnaires, gym training packages and evaluation frameworks will be distributed. There will also be interactive discussion with the audience to share experience and knowledge.

\section{Conclusion/Key Practice Points:}

- Community gym participation ensures clients reach physical activity targets for long-term optimal health outcomes.

- A carefully planned approach when engaging with gyms ensures better client adherence and outcomes leading to a successful partnership.

\section{TRANSITION OF PHASE-TWO}

\section{CARDIOPULMONARY REHAB CLIENTS TO INDEPENDENT EXERCISE IN COMMUNITY FITNESS CENTRES}

Tran $\mathrm{M}^{1}$, Farrugia $\mathrm{L}^{1}$, Brophy $\rfloor^{2}$, Bailey $\mathrm{K}^{1}$

${ }^{1}$ St Vincent's Hospital Melbourne, Vic

2Department of Health and Human Services, Vic

Question: Is exercise in community gyms safe and effective for people with chronic cardiopulmonary conditions post rehab programs?

Design: Prospective observational cohort study

Participants: Hospital phase-two cardiopulmonary rehab clients ( $n=63$ ) who volunteered to continue their exercise regime independently

Intervention: Guided transition to independent self-directed exercise in a community gym by hospital-trained staff and financial assistance of gym membership

Outcome Measures: Physical and psychosocial data before and after phase 2 hospital rehab, at commencement at the gym and at 3 months post participation.

Results: Clients' mean age was 65.9 (62\% male). At 3 months post gym commencement, 6MWT distance improved from $501 \mathrm{~m}$ to $549 \mathrm{~m}(+48 \mathrm{~m}$ or $+10 \%)(p=0.006), 1$ RM seated row improved from $40.0 \mathrm{~kg}$ to $43.6 \mathrm{~kg}$ $(p=0.055), 1$ RM leg press improved from $245 \mathrm{~kg}$ to $286 \mathrm{~kg}(p=0.001)$. All SF-36 domains and the physical and mental component scores remained unchanged in the gym setting, while PHQ-9 score (depression screening tool) improved $(p=0.001)$. There were zero safety incidents during this trial.

Conclusion: Guided gym transition is safe and beneficial for people with chronic cardiopulmonary conditions. Physical and psychological improvements are realised beyond the initial hospital rehabilitation program.

Key Practice Points:

- People with chronic cardiorespiratory conditions show a marked improvement in physical and psychosocial outcomes beyond the gains made in rehab when transitioned to the gym setting

- Local community gyms can have a positive, therapeutic influence on clients' exercise capacity and mood

- Barriers to community gym attendance, such as unfamiliarity and cost need to be addressed
ADDITIONAL STANDING BALANCE CIRCUIT CLASSES DURING INPATIENT REHABILITATION IMPROVED BALANCE OUTCOMES: AN ASSESSOR BLINDED RANDOMISED CONTROLLED TRIAL

Treacy $\mathrm{D}^{1}$, Schurr $\mathrm{K}^{2}$, Lloyd B ${ }^{3}$, Sherrington $\mathrm{C}^{1}$

${ }^{1}$ The George Institute for Global Health, University of Sydney, Australia 2Bankstown-Lidcombe Hospital, Sydney, Australia

${ }^{3}$ Centre for education and workforce development, Sydney Local Health District, Australia

Question: To evaluate the impact on balance of six 1-hour circuit classes which targeted balance in addition to usual therapy for rehabilitation inpatients.

Design: A randomised controlled trial with concealed allocation, assessor blinding and intention to treat analysis with two week and three month follow up

Participants: 162 general rehabilitation inpatients from BankstownLidcombe hospital

Intervention: Intervention group participants received six 1-hour standing balance circuit classes over a two-week period in addition to usual therapy. Control group participants received usual therapy alone

Outcome Measures: The primary outcome measures were balance at two weeks and at three months after randomisation. The secondary outcome measures were mobility; self reported physical functioning; fall incidence; and hospital readmissions.

Results: Standing balance performance was better in the intervention group than the control group at two weeks (between group difference after adjusting for baseline values 3.3 seconds; $95 \%$ Cl 0.84 to $5.7, \mathrm{p}=$ 0.009 ) and three months ( 3.4 seconds $95 \% \mathrm{Cl}-0.56$ to $7.38, p=0.092)$.

Conclusion: Two weeks of standing balance circuit classes in addition to usual therapy improved balance in general rehabilitation inpatients at two weeks and the effects may have been maintained at three months.

\section{Key Practice Points:}

- A high intensity challenging balance exercise program can be provided safely and effectively in a group environment to older rehabilitation inpatient

- Elderly individuals with a high number of co-morbidities rea able to perform high amounts of practice

\section{HOW TO PRESCRIBE EXERCISES FOR CERVICAL KINEMATIC IMPAIRMENT IN NECK PAIN}

Treleaven J1', Sarig-Bahat ${ }^{2}$

${ }^{1}$ Cervical spine and whiplash unit CCRE spine, Division of Physiotherapy, SHRS, University of QLD, Queensland

¿Department of Physical Therapy, Faculty of Social Welfare \& Health Sciences, University of Haifa, Israel

Background: Cervical kinematic impairments such as altered range, velocity, smoothness and accuracy of head motion are commonly seen in people with neck pain. This has particular functional relevance given the importance of the neck to respond fully, quickly and accurately to surrounding stimuli. A recent pilot RCT demonstrated the benefits of kinematic exercises in those with neck pain. (Sarig-Bahat et al 2014).

Aims / objectives: The aim of the session is to provide exercise based examples of ways to improve cervical kinematics for patients with neck pain using either a virtual reality device or a simple laser method.

Approach: Participants will have an opportunity to observe and then practice the laser method exercises in a small group environment with feedback from the instructor. Written handouts will be provided.

Conclusion / Key Practice Points:

- Therapists will have observed exercise methods to address several kinematic deficits associated with neck pain.

- Therapists will have had an opportunity to practice these and be given some feedback on these tasks.

- Therapists will have exposure to new virtual reality technology in the provision of these exercises.

- Written handouts and supplementary material to assist exercise set up will be provided.

- This workshop should give therapists confidence to apply this exercise approach when treating patients with neck pain. 


\section{HOW TO PRESCRIBE EXERCISES FOR SENSORIMOTOR CONTROL DISTURBANCES IN NECK PAIN}

Treleaven $]^{1}$

${ }^{1}$ Cervical spine and whiplash unit CCRE spine, Division of Physiotherapy, SHRS, University of QLD, Queensland

Background: Sensorimotor control deficits are commonly seen in people with neck pain and can be associated with symptoms of dizziness, visual disturbances and impairments in head, trunk and eye movement control and postural stability. Specific exercises directed towards these impairments can be a useful adjunct to musculoskeletal physiotherapy in the treatment of neck pain.

Aims / objectives: The aim of the session is to provide exercise based examples of ways to improve head movement and position sense, eyehead coordination, gaze stability and trunk head co-ordination relevant for patients with neck pain

Approach: Participants will have an opportunity to observe and then practice some of these in a small group environment with feedback from the instructor. Written handouts will be provided.

\section{Conclusion / Key Practice Points:}

- Therapists will have observed exercise methods to address several sensorimotor deficits associated with neck pain.

- Therapists will have had an opportunity to practice some of these and be given some feedback on these tasks.

- Written handouts and supplementary material to assist exercise set up will be provided.

- This workshop should give therapists confidence to apply this exercise approach when treating patients with neck pain.

\section{PHYSICAL AND PSYCHOLOGICAL FACTORS ASSOCIATED WITH ONGOING SIGNS AND SYMPTOMS OF DIZZINESS IN PATIENTS WITH PERSISTENT WHIPLASH}

Treleaven $\mathrm{J}^{1}$, Ludvigsson $\mathrm{L}^{2,3}$, Peterson $\mathrm{G}^{2,4}$, Kammerlind A-S ${ }^{2,5}$ Peolsson $A^{2}$

${ }^{1}$ The Division of Physiotherapy, CCRE-Spine, School of Health and Rehabilitation Sciences, The University of Queensland, Brisbane, Australia 'Department of Medical and Health Sciences, Physiotherapy, Faculty of Health Sciences, Linköping University, Sweden

${ }^{3}$ Rehab Väst, County Council of Östergötland, Sweden

${ }^{4}$ Centre for Clinical Research Sörmland, Uppsala University, Sweden

${ }^{5}$ The Academy for Healthcare, County Council Jönköping, Sweden

Question: The aim of this study was to compare physical and psychological features in patients with whiplash with and without dizziness at a 12 month follow-up following neck specific or general exercise to determine factors associated with ongoing signs and symptoms of dizziness.

Design: Observational study within the framework of an RCT.

Participants: One hundred and sixty participants with persistent whiplash.

Outcome Measures: Dizziness and neck disability, static and dynamic clinical balance tests, neck muscle endurance, cervical range of motion, and several psychological questionnaires were assessed at the 12 month follow-up

Results: Fifty-eight\% of the subjects reported dizziness at 12 months They were significantly older, had higher levels of neck pain, poore endurance of the neck flexors and less movement and poorer psychological states compared to those not reporting these symptoms.

Conclusion: Many patients still complain of symptoms of dizziness following neck specific or general exercise. Dizziness may be inhibiting these patients from improving in neck pain, psychological and physical neck function or alternatively could be as a result of these factors.

\section{Key Practice Points:}

- Dizziness and balance deficit is moderate and common in those with persistent whiplash

- Ongoing balance deficits could have implications for future falls prevention.

- Subjects with dizziness, overall have significantly higher levels of pain and poorer neck physical and psychological function as compared to those not reporting these symptoms.

- The results point to a need for physiotherapy intervention and rehabilitation specifically addressing these impairments.
IMPROVEMENTS IN PAIN DURING MANUAL THERAPY TREATMENT ARE ASSOCIATED WITH RATE AND EXTENT OF RECOVERY

Trott CA ${ }^{1}$, Aguila $\mathrm{MR}^{1}$, Leaver AM ${ }^{1}$

${ }^{1}$ Faculty of Health Sciences. The University of Sydney

Question: Do changes in pain during a treatment session and over the course of several sessions of manual therapy care relate to longer term outcomes

Design: Observational study

Participants: 181 people with a new episode of non-specific neck pain.

Intervention: A course of mobilisation or manipulation on four occasions over 2 weeks.

Outcome Measures: The clinical course of the episode of neck pain was described by plotting the average pain scores at the beginning and end of each treatment session over the course of treatment. The relationship between the within session changes in pain and recovery were explored using multivariate regression analysis.

Results: There was statistically significant improvement during each treatment session with slight relapse between sessions. The change in pain scores after the first treatment session was independently associated with high GPE scores at three months ( $B=0.2,95 \% \mathrm{Cl} 0.01$ to 0.4). Minor adverse events such as increased pain or headache were not associated with long term outcome.

Conclusion: Improvements in symptoms within a treatment session are associated with a faster rate of recovery from an episode of neck pain and with patient's overall perception of the benefits of treatment. Minor adverse effect are frequently reported but do not impact on the rate or extent of recovery.

\section{Key Practice Points:}

- This study can assist a patients' understanding of the trajectory of their recovery

- Patients should expect improvement in symptoms with manual with slight relapses between sessions

- Early favorable results help to predict longer term outcomes.

\section{EXAMINING THE PERSPECTIVES OF STAKEHOLDERS REGARDING THE BARRIERS AND STRATEGIES TO RETURN TO WORK: A QUALITATIVE STUDY}

Truong AP ${ }^{1}$, Peters $\mathrm{SE}^{1,2}$, Johnston $\mathrm{V}^{1}$

${ }^{1}$ School of Health and Rehabilitation Sciences, Faculty of Health and Behavioural Sciences, The University of Queensland

¿Brisbane Hand and Upper Limb Research Institute

Question: What do stakeholders identify as the return to work barriers and strategies for a worker with a complex workers compensation claim for a non-traumatic upper extremity musculoskeletal disorder?

Design: Survey responses to open-ended questions about a case scenario. Participants: 621 respondents consisting of 488 healthcare providers, 62 employers, 55 insurers and 16 lawyers.

Outcome Measures: Responses to questions on the return to work barriers and strategies were mapped to the biopsychosocial model. Pearson's Chi Square and Fisher's Exact Test were performed to compare the proportion of identified barriers and strategies between stakeholder groups.

Results: 35 barriers for return to work (11 biological, 15 psychological and 9 social barriers) and 16 types of return to work strategies (4 biological 6 psychological and 6 social interventions) were identified. Healthcare providers, employers, insurers and lawyers alike identified more psychological than biological or social barriers. However, stakeholders identified strategies to address these barriers differently, which may be related to their professional expertise. Employers and insurers nominated more psychosocial strategies while healthcare providers reported more biological strategies. Stakeholders did not consistently recommend strategies for some of the modifiable barriers identified.

Conclusion: Healthcare professionals, employers, insurers and lawyers tend to perceive similar barriers for return to work but developed different strategies according to their area of expertise.

Key Practice Points:

- Stakeholders identified similar barriers to return to work

- Stakeholders nominated quite diverse return to work strategies

- Stakeholders were able to identify more barriers than they were able to nominate strategies 
HOME-BASED TELEREHABILITATION IN PEOPLE WITH CHRONIC OBSTRUCTIVE PULMONARY DISEASE: SATISFACTION LEVELS OF PARTICIPANTS AND THEIR PARTNERS

Tsai LL ${ }^{1,2}$, McNamara RJ ${ }^{1,3}$, Moddel C ${ }^{4}$, Alison JA ${ }^{2,5}$, McKenzie DK ${ }^{3}$, McKeough Z] $]^{2}$

IPhysiotherapy Department, Prince of Wales Hospital, Randwick, NSW 2Clinical and Rehabilitation Sciences, The University of Sydney, Lidcombe, NSW

${ }^{3}$ Respiratory and Sleep Medicine Department, Prince of Wales Hospital, Randwick, NSW

${ }^{4}$ Agency for Clinical Innovation, Chatswood, NSW

SPhysiotherapy Department, Royal Prince Alfred Hospital, Camperdown, NSW Question: What is the level of satisfaction reported by both participants and their partners after a home-based telerehabilitation program (TR) in people with chronic obstructive pulmonary disease (COPD)?

Design: Participants with COPD completed an eight week TR exercise program at home. At the end of TR, participants completed the Client Satisfaction Questionnaire (CSQ8) and a purpose-designed survey regarding satisfaction with the program. Partners of the participants also completed a purpose-designed survey regarding their satisfaction with the program.

Participants: Fifteen participants with COPD and six partners were recruited to complete the surveys.

Intervention: The TR program consisted of lower limb cycling, walking and functional leg exercises three times a week for eight weeks with supervision by a physiotherapist using videoconferencing technology.

Outcome Measures: CSQ8 (a maximum score of 32 indicates 100\% satisfaction) and two purpose-designed surveys.

Results: Fourteen participants with COPD (mean (SD) age 74 (8) years, FEV1 \% pred. 58 (22)) and five participants' partners completed the surveys. Satisfaction with the program amongst patients was high (CSQ8 mean (SD) score 31 (2)). Based on the survey $93 \%$ of participants were highly satisfied with the quality of the exercise sessions, the information provided about COPD, and indicated they would definitely continue the program if it was offered. One-hundred percent of participants' partners reported it helped participants stay motivated to exercise at home.

Conclusion: High satisfaction from both participants and their partners was reported at the end of an eight week home-based TR program in people with COPD

\section{Key Practice Points:}

- People with COPD were highly satisfied with home based telerehabilitation

- People with COPD are highly likely to continue a home-based telerehabilitation program

- Participants' partners reported that a home-based telerehabilitation program helped the participants to stay motivated to exercise at home

\section{DOES BENDING SPEED INFLUENCE THE LUMBO-PELVIC KINEMATICS AND COORDINATION IN PEOPLE WITH LOW BACK PAIN?}

Tsang $\mathrm{S}^{1}$, Szeto $\mathrm{G}^{1}$, Chan $\mathrm{I}^{1}$, Ho $\rfloor^{1}$, Leung $\mathrm{L}^{1}$, Li R ${ }^{1}$, Tang $\mathrm{WH}^{1}$ ${ }^{1}$ Department of Rehabilitation Sciences, The Hong Kong Polytechnic University, Hong Kong

Design: Cross sectional, between-participants study

Participants: Seventeen (3 F) adults with low back pain [LBP] and seventeen age and gender matched healthy controls [HC]

Intervention: Participants performed forward bending in standing at a wide range of movement speeds. Five speed conditions were: very slow, slow, normal, fast and very fast paced at 20, 30, 40, 50 and 60 beats per minutes respectively.

Outcome measures: Angular displacement, velocity and acceleration, and movement coordination (cross correlation analysis) of the lumbo pelvic region.

Results: Angular velocity and acceleration of the lumbar spine and hip joint increased with the bending speed escalated. These variables were significantly decreased in LBP in almost all speed conditions ( $p<001)$. Participants with LBP displayed a consistent pattern of lumbo-pelvic coordination (table shows the cross correlation coefficient values) across all speed conditions which were significantly different compared to $\mathrm{HC}\left({ }^{*} p<0.01\right)$.

Conclusion: Bending speed influenced the differential kinematics and movement coordination of the lumbo-pelvic region. People with LBP displayed a significantly altered coordination of the lumbo-pelvic region during forward bending.

\section{Key Practice Points:}

- Differential kinematics are more sensitive outcome indicators for movement analysis

- People with LBP adapted an altered lumbo-pelvic coordination pattern that is independent to movement speed.

\section{CHILDHOOD OBESITY: A CALL TO ACTION FOR PHYSIOTHERAPISTS}

\section{Tsiros MD}

Alliance for Research in Exercise, Nutrition and Activity, Sansom Institute for Health Research, School of Health Sciences, University of South Australia Increasing evidence suggests that obesity may be linked with reduced physical functioning and disability in children. Common obesity-related impairments may include limitations in cardiorespiratory fitness and muscle strength, increased pain, reduced balance and motor skill proficiency and other musculoskeletal issues. Limitations in daily functioning are also seen, with some obese children having difficulty with simple daily tasks like walking, climbing stairs and rising from a chair. Hence, it is not surprising that children with obesity tend to have poor physical health-related quality of life and lower participation in physical activity. This presentation will be a'call to action' for physiotherapists, discussing a number of important questions. What role do physiotherapists have to play in childhood obesity management? How do we 'find the right fit' when prescribing physical activity for children? Furthermore, what factors should physiotherapists be mindful of when working with children who may be overweight or obese? Lastly, how can we develop strategies to limit obesity-related disability in childhood and beyond? If we can get children moving and functioning better, then we may help them to be more active which will assist their weight management in the long-term.

\section{Key Practice Points:}

This session will assist physiotherapists working with children to: - Identify overweight/obesity in children

- Understand how obesity impacts children's movement and function

- Identify considerations for interventions to promote function and physical activity

\section{HOW TO USE AN ADVOCACY ENOUIRY APPROACH IN STUDENT FEEDBACK TO IMPROVE STUDENT ENGAGEMENT AND THE EFFECTIVENESS OF THE CONVERSATION}

\section{Tuttle $\mathbf{N}^{1,2}$}

${ }^{1}$ School of Allied Health Sciences, Griffith University

2Menzies Health Institute Queensland

Background: Feedback is essential to maximise student learning but student engagement in the process is often less than ideal. In addition, when feedback is focussed on specific observed behaviours its generalisability can be limited. The advocacy enquiry approach originates from the debriefing process in simulated learning environments and has the potential to address both student engagement and generalisability of learning. The elements of the approach are a pre-judgemental juxtaposition of observations and a preferred standard. The educator's curiosity then opens the conversation to consider not just the specific performance, but also the understanding and attitudes that underpin the student's performance.

Aims / objectives: Introduce participants to the advocacy enquiry approach to feedback. Specifically by the end of the session participants will 1) identify components of the approach and 2) have experience through role-play of providing and responding to feedback using this approach.

Approach: A brief presentation of the principles will be followed by a 'fishbowl' session where one participant role-plays providing feedback. Participants in small groups will each experience both sides of providing and responding to feedback using an advocacy enquiry model.

\section{Conclusion / Key Practice Points:}

- The advocacy enquiry approach provides one structure that can be useful in the feedback process

- The approach is more likely to be useful when sufficient time is available and the student has reasonable insight and ability to reflect on their performance.

- The approach can be useful to increase student engagement and have an impact on practice beyond the specific situation being discussed. 
HOW TO IMPROVE REASONING

PROCESSES USED IN DAY-TO-DAY

CLINICAL PRACTICE BY APPLYING PRINCIPLES FROM N-OF-ONE TRIALS

\section{Tuttle $\mathbf{N}^{1,2}$, Evans $\mathrm{K}^{1,2}$}

${ }^{1}$ School of Allied Health Sciences, Griffith University

2Menzies Health Institute of Queensland

Background: Discussion of clinical reasoning often focusses on determining the initial diagnosis and/or management plan. The majority of reasoning in day-to-day practice however is based on patient response and guides the progression and modification of ongoing treatment. As commonly applied, this method relies on a assumptions that may not be valid and, in some cases, may actually limit the effectiveness of patient management

Aims / objectives: The aim of this session is to enable participants to improve the rigor and extend their repertoire of their reasoning processes related to ongoing patient management in day-to-day practice. By the end of the session, participants will 1) identify some of the assumptions underlying their current reasoning processes and 2) construct additional patterns of intervention and reassessement that are more rigorous and potentially more effective and efficient.

Approach: The common method of assessment and reassessment used in clinical practice will be deconstructed. Alternative structures that can be used in day-to-day practice based on n-of-one study designs including the concepts of super-responders will be explored. Participants in small groups will explore structures of treatment and reassessment (n-of-one study designs) that can be used for individual patients in day-to-day practice in their own clinical practice.

\section{Conclusion / Key Practice Points:}

- An understanding assumptions that underly clinical reasoning will result in more effective patterns of treatment and reassessment

- The use of more rigorous reasoning methods can enable therapists to learn more from their patients and become more aware of how their own biases influence decision making.

\section{THE EFFECT OF ANTERIOR CRUCIATE LIGAMENT INJURY ON THE MAGNITUDE OF ACTIVATION OF THE QUADRICEPS AND HAMSTRINGS}

Tyson KP1 , Keays SL 1,2 , Bullock-Saxton JE ${ }^{1,3}$, Newcombe PA ${ }^{4}$ ${ }^{1}$ Private Physiotherapy Practice

2Faculty of Health and Sport Science, University of the Sunshine Coast, Sippy Downs

${ }^{3}$ School of Physiotherapy, Australian Catholic University, Banyo

${ }^{4}$ School of Psychology, The University of Queensland, St Lucia

Question: Does anterior cruciate ligament injury affect the magnitude of activation of the quadriceps and hamstrings?

Design: Controlled cohort study

Participants: 15 subjects with chronic unilateral anterior cruciate ligament-deficiency and 7 uninjured control subjects.

Intervention: Surface electromyography of Vastus lateralis, Vastus medialis and Biceps femoris was recorded on each leg during a maximum voluntary isometric contraction, single-leg hop and a kick forward. Electromyography data was collected and analysed by an assessor blind to side of injury and subject condition using a customised program.

Outcome Measures: Root mean square, calculated over 100ms epochs was compared between injured and control limbs. The epochs reflected the highest root mean square value for each muscle during hop take-off and landing phases, and the entire kick phase. A series of paired t-tests compared the left and right control legs, and uninjured and injured legs; while an independent-samples t-test compared the injured and control legs.

Results: There were no significant differences in magnitude of activation between the control legs. Biceps femoris exhibited increased magnitude of activity throughout all tested phases of the hop and kick in the injured limb compared to uninjured $(p \leq 0.06)$ and control limbs $(p \leq 0.02)$. Vastus lateralis exhibited a trend towards significance for the kick $(p=0.08)$ only

Conclusion: Hamstrings show significant increases in the magnitude of activation following anterior cruciate ligament injury during both activities tested while quadriceps showed no significant increase. Increased hamstring activity may result to compensate for loss of joint stability.

\section{Key Practice Points:}

- Physiotherapist guided rehabilitation following anterior cruciate ligament injury must include emphasis on improvement of the magnitude of activation of the supporting musculature.

- Hamstring emphasis throughout rehabilitation of anterior cruciate ligament injured patients should not be at the expense of quadriceps exercise

\section{CLASSIFICATION IN SPORT \\ FOR ATHLETES WITH PHYSICAL IMPAIRMENTS}

\section{Ungerer G}

Queensland Spinal Cord Injuries Service, International Wheelchair Rugby Federation

Physical impairments lead to a competitive disadvantage in sport, by limiting the performance of sports-specific activities. Classification aims to minimise the impacts of physical impairment on sports performance, and helps to ensure that the success of the athlete is determined by skill, fitness, power, endurance, body dimensions, psychology and other attributes relevant to the sport. Classification systems have existed since the mid-1940s, and were initially based on medical diagnosis alone, with athletes who had the same impairment type competing against each other during competition. Modern classification systems are increasingly evidence based, and focus on the effect the physical impairment has on activities specific to each sport. As such, modern classification systems incorporate the assessment of performance of key sports-specific activities in addition to traditional assessments of impairment, such as muscle power, co-ordination, limb length and range of movement. For some sports, these classification systems allow athletes with different impairment types to compete alongside or against each other, breaking down barriers that existed in more medical models. Wheelchair Rugby classification was first developed in 1990 and has evolved significantly into a classification system that allows the inclusion of athletes with many different impairments types. Developed initially as a system that catered exclusively for athletes with spinal cord injury, the system has evolved, based on available evidence and input from athletes, to allow the inclusion of athletes with tetraplegia-equivalent impairment types, such as congenital limb loss. As an inclusive sport, challenges remain in the development of assessment tools for a variety of impairment types within the one classification system.

\section{A NATIONAL SURVEY OF INTENSIVE CARE SENIOR PHYSIOTHERAPISTS REGARDING RESPIRATORY MANAGEMENT OF INTUBATED ADULTS WITH COMMUNITY ACQUIRED PNEUMONIA}

\section{Van der Lee $\mathrm{LM}^{1,2}$, Hill AM ${ }^{1}$, Patman S}

'School of Physiotherapy, The University of Notre Dame Australia, Fremantle 2Fiona Stanley Hospital, Department of Health Western Australia, Perth

Question: What is current physiotherapy practice for intubated adult patients with community acquired pneumonia in intensive care units within Australia?

Design: Cross-sectional, mixed methods survey

Participants: Senior intensive care physiotherapists, with a minimum of one year experience in the position, were recruited from 72 Australian public and private hospitals.

Outcome Measures: An online questionnaire was developed and piloted to explore current clinical practice and clinical reasoning. The domains were structured based on common aspects of physiotherapy assessment, rationale and intervention options for intubated and mechanically ventilated patients. Quantitative data were collected using categorical and Likert scales and qualitative data were collected using open ended responses.

Results: There were 67 respondents (65\% response rate). Respondents main rationale for intervention were improved airway clearance (98.3\%), alveolar recruitment (74.6\%) and gas exchange (32.2\%). Intervention included positioning alternate side lying (80.6\%) or affected lung uppermost (82.3\%), vibrations (53.3\%), hyperinflation techniques (34.6\%), and suction (92\%). Vibrations were used when sputum is excessive (20.4\%). Manual hyperinflation was chosen for high sputum load $(29.8 \%)$ due to specific aspects of the technique (21.3\%). Ventilator hyperinflation was chosen if there were concerns about ventilator disconnection (29.5\%), such as loss of high positive end expiratory pressure (63.6\%) or risk of airborne pathogen transmission (18.2\%)

Conclusion: Senior physiotherapists rationalise that a significant physiotherapy role exists during the intubated period for community acquired pneumonia.

\section{Key Practice Points:}

- Airway clearance is the greatest reason for intervention.

- Positioning, vibrations and hyperinflation techniques are used.

- Further research is required to establish impact on patient outcomes. 
WHICH FACTORS AFFECT THE DURATION AND FREQUENCY OF RESPIRATORY PHYSIOTHERAPY FOR INTUBATED ADULTS WITH COMMUNITY ACQUIRED PNEUMONIA? A NATIONAL SURVEY

\section{Van der Lee $\mathrm{LM}^{1,2}$, Hill AM1, Patman $\mathrm{S}^{1}$}

${ }^{1}$ School of Physiotherapy, The University of Notre Dame Australia, Fremantle 2Fiona Stanley Hospital, Department of Health Western Australia, Perth

Question: Which factors influence duration and frequency of respiratory physiotherapy intervention for intubated patients with community acquired pneumonia?

Design: Cross-sectional, mixed methods survey.

Participants: Senior intensive care physiotherapists were recruited from 72 Australian public and private hospitals.

Outcome Measures: Respondents were nested within a larger online survey, developed and piloted to explore their current clinical practice and clinical reasoning for intubated patients with community acquired pneumonia. Quantitative and qualitative data were collected using categorical and Likert scales, and open-ended responses to explore factors which influenced the delivery of respiratory physiotherapy intervention during the acute intubated period.

Results: There were 67 respondents (response rate 65\%). The average intervention lasted between 16 and 30 minutes (70\% of respondents) and was delivered once (44\%) or twice (44\%) daily. Duration of intervention was most affected by sputum volume (95\%), viscosity (93\%) and purulence (88\%), cough effectiveness (95\%), chest-xray (87\%) and auscultation (84\%). Sixty percent of respondents reported that workload and staffing issues affected intervention duration and frequency. Intervention time was more likely to be increased when there were greater staffing levels $(p=0.013)$.

Conclusions: Duration and frequency of intervention for severe community acquired pneumonia requiring intubation and mechanical ventilation is determined by ongoing assessment of patient-centred variables, but tempered by workforce issues.

\section{Key Practice Points:}

- Intubated patients with community acquired pneumonia are commonly treated once or twice a day.

- Length of treatment is most influenced by quality and quantity of sputum.

- Length of treatment is likely to be longer in units with greater staffing capacity.

\section{A NATIONAL YOUTH SPORTS INJURY PREVENTION PROGRAM: FANTASY OR POSSIBILITY?}

\section{Vertullo C}

Knee Research Australia \& Centre for Musculoskeletal Research, Menzies Health Institute Griffith University

Rupture of the ACL is a devastating event often resulting in life long disability and resultant osteoarthritis, particularly if meniscal injury occurs. Fortunately it is mostly preventable and universal neuromuscular training programs are both efficient and cost effective, reducing ACL injury rates $50-80 \%$ of the time. Despite the demonstrable public health impact of joint injury and the known efficacy of prevention of these trials, program dissemination and implementation has been limited in Australia. The implementation of a universal Federally funded $A C L$ sports injury program in Australia is discussed.

\section{HOW TO INCORPORATE AQUATIC THERAPY INTO REHABILITATION OF PATIENTS AFTER COMPLEX ORTHOPAEDIC SURGERY IN THE RESTRICTED WEIGHT BEARING PERIOD}

\section{Villalta $\mathbf{E}^{1}$, Senserrick $\mathrm{C}^{1}$}

\section{'St Vincent's Hospital, Melbourne}

Background: Surgical management of conditions such as musculoskeletal tumours, complex fractures and revision arthroplasty often involves resection of muscles, bone and nerves and insertion of metalware. Physiotherapists can find themselves challenged by treatment options in this unique population during the rehabilitation phase, particularly during lengthy weight- bearing restrictions. Aquatic therapy can be a helpful adjunct to land based therapy, however therapists are often unsure which techniques to use, what exercises are most effective and what assessment methods are suitable, particularly in this environment.

\section{Aims / objectives:}

Understanding of what structures are affected during complex lower limb orthopaedic surgery and how this will affect recovery

Ability to prescribe a variety of lower limb strengthening and contro exercises during the restricted (non-, touch and partial weightbearing) period

Increased understanding of safety considerations and training effects in water

Approach: Two experienced presenters will use medical imaging to facilitate discussion on affected structures and impact on function using of a variety of case studies (eg: revision hip replacement, hemipelvectomy, periprosthetic fracture)

Video playback of patients at different stages of rehabilitation, as well as demonstration of exercises using video (for aquatic exercises) and simple equipment (mats, fitball, theraband) for land based examples.

Conclusion / Key Practice Points:

- Improved confidence with managing patients post complex orthopaedic surgery

- Exercise progression ideas in the aquatic environment for patients in the restricted weight bearing phase

- Exercise progression ideas on land

- Outcome measures that can be used in pool and land

\section{HOW TO ASSESS FOR PELVIS POSITION IN A BIKEFIT: EVIDENCE AND CLINICAL PRACTICE}

Visentini $\mathrm{P}^{1}$, Wisbey-Roth $\mathrm{T}^{2}$

${ }^{1}$ Physiosports Brighton and Latrobe University

2"Take Control" Active Rehab; Bounce back active rehabilitation; Aust. Catholic University

Background: Sports physiotherapists are heavily involved in the management of pain and injury in cycling. As with all sports a knowledge of the biomechanical aspects of the sport is essential, and an extremely important component of management is to clarify that the BikeFit is appropriate. Hence, it is advantageous for Sports Physiotherapists with a special interest in BikeFit to understand the evidence, history and practice of BikeFit. The position of the pelvis is of the utmost importance.

\section{Aims / objectives:}

1. To present the theoretical evidence behind knee angle measures, and measures of fore-aft positioning of the saddle in relation to optimal performance and injury prevention.

2. To demonstrate and practice knee angle and fore-aft positioning measures and their interpretation.

\section{Approach:}

1. Initiate with 5 mins of slides outlining theory including validity and reliability of knee angle and fore-aft measures

2. Demonstration of knee angle and fore-aft positioning rmeasures using a bike and rider, including App based technology, goniometer use, plumb line and observation.

3. Group exercise involving problem solving and clinically reasoning different presentations of pelvis position on the bike.

\section{Conclusion / Key Practice Points:}

- Awareness that components of BikeFit are extremely important in the assessment of cycling performance and injury management.

- Ability to clinically measure the appropriateness and accuracy of pelvic position on the bike

- Ability to discuss sports specific technique more readily with cyclists.

- Ability to clinically reason through the issue of cycling pelvic position and its relationship to injury and performance.

\section{GETTING STARTED ON A RESEARCH}

\section{STUDY. CAN YOU HAVE AN ACADEMIC CAREER IN THE SPORTING FIELD?}

\section{Waddington $\mathrm{G}^{1,2}$}

${ }^{1}$ Australian Institute of Sport, Bruce, ACT

2University of Canberra, Bruce, ACT

In a world demanding ever more evidence based support for interventions the domain of sport and exercise is no different with increasing demands on clinicians to, at a minimum, have a high level capacity for evaluating research that might affect their practice. At the other end of the spectrum is the clinician researcher who is actively driving research into new aspects of their practice domains. This session will explore the first steps to getting started across the range from an effective research consumer to that of a clinician researcher. 


\section{VOCATIVE ANECDOTE WRITING AS A TOOL FOR PHYSIOTHERAPY STUDENT REFLECTION}

Wakely L1 ${ }^{1}$ Croker $C^{2}$, Tinlin L ${ }^{2}$, Croker $A^{1}$

${ }^{1}$ The University of Newcastle Department of Rural Health

${ }^{2}$ The University of Newcastle School of Health Sciences

Question: Does vocative anecdote writing assist physiotherapy student's reflection on complex areas of professional practice?

Design: This descriptive paper details a process of facilitating physiotherapy student reflection on how they learn about other health disciplines.

Participants: Two second-year physiotherapy students.

Intervention The students were guided through a process of vocative anecdote writing to facilitate when and how they had learnt about other roles in health. They were asked to write accounts that would allow the reader to imagine their experience.

Outcome Measures: Anecdotes and reflections published, teaching practices changed.

Results: Student-generated anecdotes facilitated or resulted from discussion of complex issues such as the socialisation of our learning, the difficulty understanding the similarities and difference within professional roles and the difficulty of trying to understand other professional roles while trying to establish their own professional identity. Findings have been incorporated into teaching of other students.

Conclusion: This method of refection was excellent for facilitating deep thinking around complex issues. The student's anecdotes demonstrated that early in their learning they struggle with concepts such as professiona identity and fitting into the health system. Vocative anecdote writing allowed them to reflect on their own experiences in a way that was safe and non-judgemental.

\section{Key Practice Points}

- Vocative anecdote writing is a useful tool for facilitating reflective practice for physiotherapy students.

- Physiotherapy students begin to grapple with complex practice areas such as professional identity early in their learning.

- Educators need to recognise these issues that students are grappling with in order to facilitate learning.

\section{COPING WITH DEMAND WHILE CARING FOR THE CLINICIANS: STRATEGIES TO ENHANCE PROFESSIONAL PLACEMENT OPPORTUNITIES FOR PHYSIOTHERAPY STUDENTS}

Wakely L ${ }^{1}$, Leys $]^{1}$

${ }^{1}$ The University of Newcastle Department of Rural Health

Question: How do we increase clinical placement capacity to meet demand, yet still consider the needs of clinicians?

Design: This paper details strategies implemented to improve the robustness of a clinical placement model.

Participants: Physiotherapy students and clinical educators. Intervention: A range of strategies including individual consultation, education programs and targeting junior physiotherapists were implemented to increase the number of placements offered. Communication between students of different year groups was facilitated to encourage their peers to request these placement opportunities.

Outcome Measures: Numbers of student placement and student placement evaluations.

Results: From 2011 to 2014 placement numbers increased from 11 to 84 student placements. Placement weeks in this time have increased from 64 in 2011 to 420 in 2014 . Fifteen new placement sites continue to take students placements. In post-placement evaluations students reported high levels of satisfaction with these placements.

Conclusion: These strategies aimed to improve the culture of student supervision across a range of sites. The focus on the development of new placements sites has resulted in a greater number of supervisors sharing the load and therefore a more robust placement structure as it is less reliant on a small number of individual clinicians. Despite these changes students reported being satisfied with the supervision and clinical opportunities while on placement.

\section{Key Practice Points:}

- Individual consultation and intervention was key to addressing barriers to clinical supervision.

- Strategies to encourage students to discuss placements with peers has resulted in greater uptake of placement opportunities.
MAXIMUM TOLERATED DOSE OF WALKING FOR PEOPLE WITH SEVERE OSTEOARTHRITIS OF THE KNEE: A PHASE I TRIAL

Wallis $\mathrm{JA}^{1,2}$, Webster $K E^{1}$, Levinger $\mathrm{P}^{3}$, Singh $\mathrm{PJ} \mathrm{J}^{2}$, Fong $\mathrm{C}^{2}$, Taylor $\mathrm{NF}^{1,2}$

${ }^{1}$ La Trobe University, Melbourne

Eastern Health, Melbourne

${ }^{3}$ Victoria University, Melbourne

Question: How much walking can be safely tolerated for people with severe knee osteoarthritis?

Design: Phase I dose response trial with escalating walking doses of 10 $20,35,50,70$, and 95 minutes, prescribed over one week, at moderate intensity. The primary stopping rule was a substantial increase in knee pain

Participants: Twenty-four participants with severe knee osteoarthritis. Outcome Measures: The primary outcome was an estimation of the maximum tolerated dose of walking. The secondary outcomes were pain, stiffness and activity limitation (WOMAC).

Results: Three participants were assigned to each dose between 10 and 70 minutes, and nine participants assigned to the 95-minute dose. The trial was stopped at 95 minutes due to the increase in knee pain occurring at this dose. Therefore, the maximum tolerated dose was 70 minutes.

There was a moderate association between dose and increased activity (linear $R^{2}=0.31$, cubic $R^{2}=0.69$ ) and reduced stiffness (linear $R^{2}=0.20$, cubic $\left.\mathrm{R}^{2}=0.52\right)$, with increased benefits at moderate to higher doses.

Conclusion: There is preliminary evidence that 70 minutes per week of moderate intensity supervised walking was safe for people with severe knee osteoarthritis; for higher doses there was a risk of exacerbating knee pain

Key Practice Points:

- Encouraging people with severe knee osteoarthritis to be active by prescribing 70 minutes per week of walking may be done without increasing short-term knee pain.

- It is not known if sustained levels of physical activity can lead to health benefits without exacerbating knee pain in this population.

\section{PASSIVE RANGE OF MOVEMENT OF THE SHOULDER: A STANDARDISED METHOD FOR MEASUREMENT AND ASSESSMENT OF INTRARATER RELIABILITY}

\section{Dougherty $]^{1}$, Walmsley S ${ }^{1}$, Osmotherly PG1}

${ }^{1}$ School of Health Sciences, The University of Newcastle, Newcastle, NSW

Question: Using digital inclinometry is a standardised method for measuring the passive range of movement of seven shoulder movements reliable and reproducible in a large asymptomatic population?

Design: Repeated measures.

Participants: Ninety adults with pain-free shoulder movement and no history of shoulder pain in the preceding 12 months.

Outcome Measures: Measurement of seven passive shoulder movements including; total shoulder flexion and abduction, gleno-humeral flexion and abduction, external rotation in neutral abduction and internal and external rotation in 90 degrees of abduction was performed with a digital inclinometer by a single assessor on two occasions, then repeated one week later. Reliability of measurement expressed in terms of interclass correlation coefficients (ICCS) and standard error of measurement (SEM) was determined for each shoulder range of movement.

Results: The intrarater reliablitiy of this method was substantial for total shoulder flexion (ICC $=0.82$, SEM $=12.3$ degrees), moderate for all other movements $($ ICC range $=0.64-0.75)$ with the exception of external rotation in neutral which was slight (ICC 0.28, SEM 31 degrees). There was moderate reliability at one week follow up measurement for all movements (ICC range $=0.60-0.77$ ).

Conclusion: The method of measurement described in this study has moderate to substantial reliability for the majority of the seven tested passive shoulder movements. At one week follow-up moderate reliability was maintained for all movements. This study provides a clinically reproducible method of measurement of passive shoulder range of movement as well providing a basis for future research in a variety of populations.

\section{Key Practice Points:}

- Passive shoulder range of movement can be reliably and reproducibly measured using a standardised method in the majority of movements with the exception of external rotation in neutral abduction

- Digital inclinometry should be considered when reliable measurement of passive shoulder range of movement is required

- The standardised set of shoulder movements described may be valuable to use in future research as well as in the clinical setting 


\section{HYDROTHERAPY WITH DUAL SENSORY IMPAIRMENT}

\section{Warburton M}

Gateway Physiotherapy, Brisbane, Australia

Outline of presentation: Deaf-blindness is a combination of vision and hearing loss that can be congenital or acquired. There are several causes of deaf-blindness and congenital Rubella syndrome is one of them. Mobility and communication are significantly affected areas for the deaf-blind person, and touch becomes the primary information gathering sense. Buoyancy, warmth, hydrostatic pressure, viscosity and turbulence are elements experienced during a hydrotherapy session, and may provide a unique hydro-therapeutic environment for the person with DSI.

Purpose: To establish what measurable benefits could be achieved from hydrotherapy with a DSI person.

Methods: Hydrotherapy was provided for 8-weeks, 2 x week, 35-minute session duration.

Each session consisted of mobility encouragement and supervision, and activities to stimulate the somato-sensory system. Somato-sensory activities focused on tactile exploration including objects of various shape, size, weight, contour, texture, elasticity, pliability, softness and hardness. Outcomes were measured using the Goal Attainment Scale (GAS) and included mobility distance, attendance, and timed tactile responsiveness to varying objects.

Results: Mobility distance and attendance exceeded baseline expectations. Timed tactile responsiveness changed positively from baseline. Average scale scores were 1.00 with an overall GAS t-score of 63.69.

\section{Key Practice Points:}

- Hydrotherapy can be a quantifiable physio-therapeutic option for persons with DSI

- It provides a relatively safe environment for mobility and allows the somato-sensory system to be fully engaged - important for the DSI population

- Physiotherapists should consider hydrotherapy for DSI people. It offers unique properties not available on land.

\section{HYDROTHERAPY INCIDENTS: OCCURRENCES, PREVENTION AND MANAGEMENT}

\section{Warburton M}

\section{Gateway Physiotherapy, Brisbane, Australia}

Incidents in and around the hydrotherapy setting can and do occur. Most are minor incidents and are easily managed. Some are major with sometimes upsetting and traumatic consequences. This presentation will discuss some of the more common incidents that can occur in the hydrotherapy arena, together with some unexpected events and examples that have occurred. It will discuss how to prevent and prepare for incidents, and how best to manage them.

\section{Key Practice Points:}

- Incidents can and do occur

- Physiotherapists should be alert to the potential for an incident

- Incident preparation and training can assist in the management of hydrotherapy incidents extensor strength (BES) was measured with a hand-held dynamometer. Isometric lower extremity strength (LES) was examined with a leg extension dynamometer. Functional performance (Timed up-and-go [TUGT], functional reach [FRT], and five-times sit-to-stand [FTSTS]) was examined. Multiple regression analysis was used to determine the degree to which lean mass and physical performance predicted variance in LS and FN BMD.

Results: Lean mass explained the majority of the variance in LS BMD $\left(R^{2}=24.4 \%, p=0.001\right)$. BES contributed a further $6.7 \%$. Lean mass also explained the majority of the variance in FN BMD $\left(R^{2}=11.0 \%, p=0.001\right)$. FTSTS contributed a further $4.6 \%$

Conclusion: Lean mass predicted a greater amount of the variance in LS and FN BMD than physical performance.

\section{Key Practice Points:}

- The weaker relationship of physical performance than lean mass to BMD suggests genes play a dominant role in the determination of bone mass

- The observed contribution of physical performance to BMD indicates strength and function are likely to be crucial to the maintenance of bone heal

\section{A SIMPLE TEST OF LOWER EXTREMITY STRENGTH: VALIDITY AND RELIABILITY}

Weeks BK ${ }^{1,2}$, Little $A^{2}$, Harding $A^{2}$, Watson $\mathrm{SL}^{1,2}$, Beck BR ${ }^{1,2}$, Horan $\mathrm{SA}^{1,2}$

${ }^{1}$ Centre for Musculoskeletal Research, Menzies Health Institute Queensland, Griffith University, Gold Coast, Australia

¿School of Allied Health Sciences, Griffith University, Gold Coast, Australia

${ }^{3}$ School of Medicine, Griffith University, Gold Coast, Australia

Question: Is a simple, isometric lower extremity strength

(LES) test reliable and valid with reference to laboratory-based isokinetic dynamometry?

Design: Cross-sectional validation study with short- and long-term repeated measures reliability analysis.

Participants: Fifty-one adults (26M, 25F) aged $46.7 \pm 20.8$ years with no recent surgery or medical conditions that affect muscle mass or strength. Outcome Measures: Participants attended two testing sessions separated by one week. Each session involved an isometric LES test (three trials) with a simple lower limb dynamometer (TTM Muscular Meter, Japan), and three isometric knee flexion-extension trials using an isokinetic dynamometer (Biodex Medical Systems, USA). Test-retest reliability and validation of the LES test was examined using Pearson's correlations.

Results: A strong positive relationship was observed between LES measured with the leg dynamometer and LES measured with Biodex ( $r$ = $0.83, p<0.001)$. Repeated measures of LES with the leg dynamometer were strongly associated $(r=0.96-0.98, p<0.001)$. Test-retest reliability for the leg dynamometer was excellent $(r=0.97, p<0.001)$, which compared favourably to that for the Biodex $(r=0.94, p<0.001)$.

Conclusion: Our findings indicate excellent validity and reliability of a simple LES test against the gold standard. The test would be easily incorporated into clinical settings.

Key Practice Points:

A simple isometric test of lower extremity strength:

- derives comparable measures to that of isokinetic dynamometry;

- exhibits high short and long term test-retest reliability; and

- is suitable for clinical use where isokinetic testing may be impractical

\section{LEAN MASS OR PHYSICAL FUNCTION: WHAT IS THE STRONGEST PREDICTOR OF BONE MASS IN POSTMENOPAUSAL WOMEN?}

Watson $\mathrm{SL}^{1,2}$, Weeks $B K^{1,2}$, Horan $\mathrm{SA}^{1,2}$, Harding $\mathrm{A}^{1,2}$, Little A, Beck BR ${ }^{1,2}$

${ }^{1}$ Centre for Musculoskeletal Research, Menzies Health Institute Queensland, Griffith University, Gold Coast, Australia

2School of Allied Health Sciences, Griffith University, Gold Coast, Australia

Question: Does muscle mass or function best predict bone mass in postmenopausal women?

Design: Observational cohort study

Participants: Eighty-five postmenopausal women (64.7 + 6.1 yrs; 64.4 $\pm 11.3 \mathrm{~kg} ; 161.5 \pm 6.5 \mathrm{~cm}$, bone mineral density [BMD] lumbar spine [LS] T-score $-1.88 \pm 1.11$ ), free from conditions and medications that influence bone and physical function.

Outcome measure: Whole body lean mass and BMD at the femoral neck (FN) and LS were determined from densitometry (XR-800, Norland). Back 


\section{TREATING CHRONIC LOW BACK PAIN WITH PILATES EXERCISE: A CLINICAL PRACTICE GUIDELINE FOR PHYSIOTHERAPISTS}

Wells C1,2, Kolt GS², Marshall P2, Hill B³ , Bialocerkowski A $^{3}$

${ }^{1}$ University of Canberra

¿University of Western Sydney

${ }^{3}$ Griffith University

Question: Is Pilates exercise effective in treating people with chronic low back pain? If so, how is it best applied?

Design: A clinical practice guideline was developed from a systematic review of research evidence. Recommendations were generated by the synthesis of findings from papers representing the highest level and quality of evidence available. Recommendations were graded using a modified Grading of Recommendations Assessment, Development, and Evaluation system. The guideline was externally reviewed by several guideline experts and physiotherapists, and then adjusted prior to completion.

Results: Recommendations were developed from 33 research papers. Several high quality trials explored the effectiveness of Pilates exercise. Only expert opinion evidence described Pilates exercise and how to select suitable candidates.

Conclusion: This guideline provides a weak recommendation for the use of Pilates exercise to treat people with chronic low back pain. Recommendations should be cautiously applied due to underlying research being limited in amount and quality.

\section{Key Practice Points:}

- Pilates exercise may offer superior benefits to usual care and physical activity in the short term (Grade C), and at least equivalent benefits when compared to other treatments (Grade B).

- Supervised and individualised exercise sessions of 30 to 60 minutes duration, twice per week for 3 to 6 months are recommended, along with use of specialised equipment and home exercises (Grade F).

- People may benefit if they have poor body awareness and maladaptive motor patterns. People may not benefit if they have eclampsia, significant radiculopathy, an unstable fracture or spondylolisthesis (Grade F)

\section{A CARER-FOCUSED INTERVENTION TO IMPROVE INCIDENTAL PHYSICAL ACTIVITY LEVELS IN PEOPLE WITH AN INTELLECTUAL DISABILITY: A PILOT STUDY}

Weston $\mathrm{D}^{1}$, Milne $\mathrm{N}^{1}$, Crompton $\mathrm{A}^{2}$, Chesney $]^{2}$, Keogh $\mathrm{H}^{2}$

${ }^{1}$ Faculty of Health Science and Medicine, Bond University

2Department of Family and Community Services Aging, Disability \& Home Care

Question: Can a carer-centred coaching program with weekly support by a physiotherapist, increase incidental physical activity levels and energy expenditure for individuals with intellectual disability?

Design: Quasi-experimental pre-post pilot study.

Participants: Ten individuals (males: $n=3$; females: $n=7$, mean age: 37.14yr) with intellectual disability and 24 of their carers recruited from group homes and day programs.

Intervention: The study protocol included informal information sessions to carers in day programs, initial home visit with participants/carers, pre-data collection using SenseWear accelerometry to measure physical activity and energy expenditure, a follow up visit discussing the SenseWear report with carers, six consecutive weekly coaching sessions with carers, post-data collection using SenseWear.

Outcomes Measures: Physical Activity Levels, Total Energy Expenditure (kJ) and Metabolic Equivalents (METs) were determined using SenseWear accelerometry pre- and post-intervention.

Results: Increased physical activity levels were achieved from this six-week carer-focused intervention (PAL: $t=-2.46, D F=6, p=.053$ ) which resulted in significant increases in mean Total Energy Expenditure as measured in $\mathrm{kJ}(\mathrm{t}=2.554, \mathrm{DF}=6, \mathrm{p}=.043)$ and METs $(\mathrm{t}=-2.640, \mathrm{DF}=6, \mathrm{p}=.039)$

Conclusion: A six-week carer focused intervention using a weekly coaching method by physiotherapists can increase physical activity and energy expenditure of individuals with intellectual disability in the shortterm.

\section{Key Practice Points:}

- Australians as a whole are inactive, with only $34 \%$ of individuals with intellectual disability meeting National Health Guidelines for physical activity

- The carer-focused coaching method is simple and effective, and should be considered when trying to effect behaviour change regarding increasing physical activity levels in individuals with intellectual disability who require carer support
THE DISTRIBUTION OF POSITIVE WORK AND POWER GENERATION AMONGST THE LOWER-LIMB JOINTS DURING WALKING NORMALISES FOLLOWING RECOVERY FROM TRAUMATIC BRAIN INJURY

Williams $\mathrm{G}^{1,2}$, Schache $\mathrm{A}^{2}$

${ }^{1}$ Epworth Hospital, Melbourne

¿University of Melbourne, Melbourne

Objective: To determine whether better walking performance following recovery from traumatic brain injury (TBI) is attributable to an accentuation of compensatory strategies or an improvement in the way the lower-limb joints perform positive work and generate power.

Setting: A large metropolitan rehabilitation hospital

Participants: Thirty-five ambulant people with extremely-severe TBI who were attending physiotherapy for mobility limitations, and a comparative sample of 25 healthy controls (HC)

Design: Cross-sectional cohort study with six month follow-up.

Main Measures: Positive work and average power generated by the hip, knee and ankle during stance as well as self-selected gait velocity.

Results: In comparison to HCs, TBI participants walked at baseline with a significantly $(p<01)$ reduced contribution from the ankle to total lowerlimb positive work and average power generation during stance, and a significantly $(p=.03)$ greater contribution from the hip. However, this compensatory strategy resolved over time such that at six month followup no significant differences in the relative contributions from the ankle and hip were identified for the TBI participants when compared to HCs.

Conclusion: Better walking performance following recovery from TBI is attributable to an improvement in the way the lower-limb joints perform positive work and generate power rather than an accentuation of compensatory strategies. 


\section{AN ACUPUNCTURE INTERVENTION TO PROMOTE BRAIN PLASTICITY FOR STROKE RECOVERY BASED ON FINDINGS FROM HUMAN NEUROSCIENCE: A SYSTEMATIC REVIEW}

Bradnam L1,2, Xydias S², Paparella J2, Cheary S³, Williams J ${ }^{2}$, Lennon $\mathrm{S}^{2}$

${ }^{1}$ Discipline of Physiotherapy, Graduate School of Health, University of Technology Sydney, Sydney, Australia

'Discipline of Physiotherapy, School of Health Sciences, Faculty of Medicine, Nursing and Health Sciences, Flinders University, South Australia

${ }^{3}$ Department of Physiotherapy, Repatriation General Hospital, Adelaide, South Australia

Question: Acupuncture intervention in stroke rehabilitation is not recommended in current stroke guidelines and recent systematic reviews do not support its use. This review aimed to identify elements of sensory stimulation interventions including acupuncture, peripheral nerve stimulation, vibration and muscle stimulation which promote neuroplastic change in the motor brain. From these, an acupuncture treatment protocol developed based on common elements across studies and compared to reported interventions in clinical trials.

Design: Two systematic literature searches were undertaken to identify studies investigating effects of sensory stimulation on brain motor function, demonstrated with TMS or fMRI and clinical trials investigating the efficacy of acupuncture for stroke recovery. The databases searched included: Proquest, CINHAL, Scopus and the Cochrane Library using combinations of key words: stroke, acupuncture, dry needling, recovery, neural plasticity, efficacy, functional gains, motor outcomes and rehabilitation.

Participants: Neurophysiological studies included adults post stroke and healthy adults. Clinical trials included adult human participants in the acute to subacute phase post-stroke.

Outcome Measures: Quality of included studies was critically appraised using the PEDRO scale.

Results: Key characteristics / parameters of interventions were recorded and synthesised from identified neurophysiological studies. An evidenceinformed 'best practice' intervention was developed and compared to interventions used in stroke clinical trials. There was a limited presence of key elements found to promote brain neuroplasticity, which may help to explain the lack of positive effect.

Conclusion: The effect of the proposed acupuncture intervention on cortical neurophysiology remains to be evaluated in a clinical trial of stroke rehabilitation.

\section{Key Practice Points:}

- Acupuncture is not recommended for stroke rehabilitation by current guidelines

- An acupuncture intervention aimed to promote brain plasticity may improve outcomes in clinical trials of stroke rehabilitation

- Guidelines for an acupuncture intervention to promote plasticity are proposed but remain to be tested for both neurophysiologic and clinical effects

- Tested for both neurophysiologic and clinical effects

\section{Key Practice Points:}

- A case based summary of 3 of the musculoskeletal issues seen in the equine athlete and the management strategies used in their rehabilitation.

- There will be different experiences in managing these and hopefully will promote discussion of others opinions and approach.

\section{MEASUREMENTS AND RANKING OF MUSCLE SIZE OF ABDOMINAL AND LUMBAR MULTIFIDUS MUSCLES AMONG OLDER ADULTS: A CROSS-SECTIONAL ULTRASOUND STUDY}

Wilson $\mathbf{A}^{1}$, Hides $]^{2}$, Blizzard L1, Callisaya $\mathrm{M}^{1}{ }^{1}$, Srikanth $\mathrm{V}^{3}$, Winzenberg $T^{1}$

${ }^{1}$ Menzies Institute for Medical Research, University of Tasmania, Hobart ${ }^{2}$ Centre for Musculoskeletal Research, Mary MacKillop Institute for Health Research, Australian Catholic University, Brisbane

${ }^{3}$ Department of Medicine, Monash University, Melbourne

Questions: Does abdominal and lumbar multifidus muscle size, and ranking of size, vary with age and sex in older adults?

Design: Cross-sectional study.

Participants: Community-dwelling adults aged $65-89$ years $(n=241)$ were randomly selected from the Tasmanian electoral roll.

Outcome Measures: Ultrasound images of resting thickness of rectus abdominis: resting and contracted thickness of external oblique interna oblique, transversus abdominis and lumbar multifidus; and resting crosssectional area of multifidus.

Results: Age was inversely associated with size of all muscles. Other than external oblique, males had larger muscles than females in all age groups. For males the most common ranking (32\%) of muscle thickness was rectus $>$ internal $>$ external $>$ transversus. This differed for females, with the most common ranking (30\%) being external > internal > rectus $>$ transversus. For both sexes the most common ranking for lumbar multifidus thickness was lumbar $4>5>2>3$. Cross-sectional area ranking was lumbar $5>4>3>2$.

Conclusion: With advancing age, females in particular, have smaller abdominal and lumbar multifidus muscles. The rankings of multifidus and abdominal muscles do not follow the patterns established for younger adults.

\section{Key Practice Points:}

- We provide age-related data for these muscles which will assist practitioners working with older adults.

- Differences between males and females, and rankings of muscle size, require further investigation to ascertain their effect on disability and function.

- The above findings may have implications in the future for the design of rehabilitation programmes in this age group.

\section{A MULTIDISCIPLINARY APPROACH TO MUSCULOSKELETAL ISSUES IN THE EQUINE ATHLETE}

\section{Williams P}

Samford Valley Veterinary Hospital, Samford, Queensland

Musculoskeletal issues in the equine athlete are one of the main causes of performance problems. This is true of all the equine disciplines ranging from the TB racehorses, Eventers, Showjumpers, Dressage horses, Western Performance horses and also including general leisure and trail riding horses. Horse owners are renowned for seeking multiple opinions from many different sources in such cases. They often seek opinions prior to veterinary involvement, from chiropractors, massage therapists and body workers. As an equine veterinary surgeon with an interest in the diagnosis and rehabilitation of musculoskeletal issues, I find that the best results in managing musculoskeletal problems are achieved by the integration of veterinary surgeon, trainer, rider, physiotherapist and farrier. This presentation aims to examine that approach with case based summaries including the initial diagnostic investigation (clinica examination, regional anaesthesia, radiography and ultrasonograhy) and then to consider the multidisciplinary strategies used in the rehabilitation. Cases to be looked at are proximal suspensory ligament desmitis, navicular disease and impingement of the dorsal spinous processes. The management of these cases with veterinary methods, farriery and physiotherapy will be discussed. 


\section{BIG NEEDS, BIG STATE, BIG CHALLENGE: RESPIRATORY PHYSIOTHERAPY \\ FOR CHILDREN DELIVERED ACROSS QUEENSLAND}

\section{Wilson C]}

Lady Cilento Children's Hospital, Brisbane

The physiotherapy management of children with respiratory conditions is resource-intensive, and the geography of Queensland creates challenges around appropriate and equitable access to healthcare. During the 1980's Royal Children's Hospital (Brisbane) commenced outreach clinics for young people with a wide range of chronic and complex lung conditions, in recognition of the needs of ongoing management beyond southeast Queensland. The Lady Cilento Children's Hospital (LCCH) opened on $29^{\text {t }}$ November 2014 with the merger of Queensland's two paediatric hospitals: Royal Children's and Mater Children's Hospital. LCCH now manages over 400 children with Cystic Fibrosis, and many more with non-CF bronchiectasis. The need for high quality physiotherapy services based on available evidence, agreed standards of care, and contemporary best practice forms an ongoing challenge for these and less common diagnoses. Physiotherapy clinical reasoning across the spectrum of paediatric respiratory illness, from early and potentially reversible conditions, through to complexities of irreversible suppurative and structural illnesses is described. The context of statewide outreach services is used to define a model of service delivery which aims to involve all stakeholders via local and remote clinical assessment, collaborative problem-solving, interpretation and service planning, particularly for physiotherapists whose clinical experience and primary roles are not in paediatric respiratory conditions. Clinical cases from Indigenous Respiratory Outreach Care, Cystic Fibrosis, and Respiratory clinics will be presented, outlining principles of physiotherapy management, education and research as foundations for sustainable and effective services across this large state.

\section{Key Practice Points:}

- Outreach visits and increased access to Telehealth services allow greater ongoing collaboration with local healthcare providers including clients, Indigenous Health Workers, Nurses, and Physiotherapists to effectively support ongoing and sustainable physiotherapy management. (Cox N 2012, Dinesen B 2012)

- Improving quality of care requires a good match between the conditions or context for care delivery, and the strategies used to achieve improvement (Kaplan et al 2012)

- Prescribed exercise and airway clearance strategies are among modifiable factors which can improve the clinical outcome and consequences of illness in chronic suppurative lung disease, cystic fibrosis and bronchiectasis (Chang 2012, Dwyer 2011, Lee 2014, Reix 2012, van der Schans 2003)

\section{MECHANICAL NOCICEPTIVE THRESHOLDS FOLLOWING PHYSIOTHERAPY IN UNRIDDEN HORSES}

Winchester $\mathrm{FI}^{1}$, Varcoe-Cocks $\mathrm{K}^{2}$, Jackson $\mathrm{MA}^{3}$, Walmsley $\mathrm{ME}^{4}$

${ }^{1}$ All Creatures Physiotherapy, Donvale

2Topline Equine Physiotherapy, Upwey

3Higher Education Primary Industries, Melbourne Polytechnic, Epping

${ }^{4}$ Faculty of Veterinary Science, The University of Melbourne, Werribee

Question: Does physiotherapy reduce mechanical nociceptive thresholds in unridden horses over a four week period?

Design: Blinded case controlled study.

Participants: Fifty unridden horses with no known history of back pain, all horses had been unridden for at least the preceding 4 months.

Intervention: Case horses received physiotherapy aiming to reduce muscle hypertonicity on day one.

Outcome Measures: Muscle sensitivity was determined by pressure algometry at 9 anatomical landmarks on both sides of each horse 30 minutes post treatment, 1- and 4 weeks post intervention.

Results: There was a significant reduction in mechanical nociceptive thresholds at the lateral triceps from pre- to post treatment in case horses (mean dif $=0.9 ; p=0.047$ ) and a significant reduction in control mechanical nociceptive thresholds at L3 from time points pre- post (mean dif =1.7; $p=0.001$ ), pre- 1 week (mean dif $=1.3 ; p=0.002$ ) and pre- 4 weeks (mean dif $=1.7 ; p<0.0001$ ) however these differences were within the MNT measurement variability observed.

Conclusions: This study does not support a reduction in muscle sensitivity following physiotherapy in unridden horses. Mechanical nociceptive thresholds were generally consistent over time. Statistically significant findings fell within the parameters of pressure algometry measurement error, making their biological relevance difficult to interpret.

\section{Key Practice Points:}

- Physiotherapy in unridden horses does not increase mechanical nociceptive thresholds.

- This study provides further information on the variation in mechanical nociceptive thresholds in unridden horses over time.

- Further research is required to determine the effects of manual therapy in horses.

\section{ARE CLINICAL TESTS USED TO ASSESS GLUTEAL MUSCLE FUNCTION VALID AND RELIABLE? A SYSTEMATIC REVIEW}

Window PJ', 2, Michaleff $\mathrm{ZA}^{2}$, Culvenor $\mathrm{AG}^{1,3}$, Tucker $\mathrm{KJ}^{4}$, O'Leary SP1,2

${ }^{1}$ School of Health and Rehabilitation Sciences, The University of Queensland, Brisbane

¿Physiotherapy Department, Royal Brisbane and Women's Hospital, Brisbane ${ }^{3}$ School of Allied Health, La Trobe University, Melbourne

${ }^{4}$ School of Biomedical Sciences, The University of Queensland, Brisbane

Question: Are commonly described clinical tests of gluteal muscle function (single-leg stance, single-leg squat, single-leg drop, prone hip extension, active hip abduction) valid and reliable?

Design: Systematic review.

Participants: Articles evaluating the reliability of the clinical tests, or their validity in terms of assessing gluteal function in adults (18-65 years), with and without lumbo-pelvic or lower extremity musculoskeletal disorders.

Outcome Measures: Test-retest reliability, and validity (content, construct, criterion and/or diagnostic validity). Methodological quality was assessed using the Qualsyst appraisal tool.

Results: Thirty-six studies across the five tests were included in the final analysis. Reliability data was available for all tests (excluding singleleg drop), and this varied widely (within and between tests), from poor (prevalence-adjusted-bias-adjusted kappa $=0.06$ ) to excellent (kappa $=1.00$ ). Single-leg stance was sensitive $(72.7 \%)$ and specific $(76.9 \%)$ in identifying a gluteus medius tear (partial/complete). Studies reported inconsistent and often conflicting correlations between test performance and objective measures of gluteal function.

Conclusion: Despite these clinical tests being widely accepted and used in clinical practice to assess gluteal muscle function there is only a modest amount of evidence supporting their use. Future studies are needed to standardise testing procedures and outcome measures in order to strengthen the conclusions that can be made.

\section{Key Practice Points:}

- Reliability of tests of gluteal function are inconsistent

- Only single-leg stance test has demonstrated diagnostic accuracy in determining specific gluteal pathology (gluteus medius tear).

- Clinicians need to recognise potential limitations in these tests as measures of gluteal function. 


\section{CLINICAL MEASURES OF BALANCE FOR PEOPLE WITH MULTIPLE SCLEROSIS AND CEREBELLAR ATAXIA}

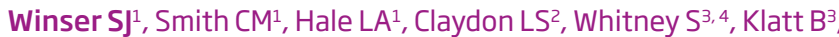
Mottershead $]^{5}$, Zaydan ${ }^{6}$, Heyman $\mathrm{R}^{6}$

${ }^{1}$ Centre for Health, Activity and Rehabilitation Research, School of Physiotherapy.University of Otago, New Zealand

2Department of Allied Health and Medicine, Anglia Ruskin University, UK ${ }^{3}$ School of Health and Rehabilitation Sciences, University of Pittsburgh, USA ${ }^{4}$ Rehabilitation Research Chair at King Saud University, Saudi Arabia ${ }^{5}$ Dunedin School of Medicine, Neurology, University of Otago, New Zealand ${ }^{6}$ School of Medicine, Neurology, University of Pittsburgh, USA

Question: How reliable and valid are the Berg Balance Scale, Timed Up and Go test, balance sub-components of the International Co-operative Ataxia Rating Scale and the Scale for the Assessment and Rating of Ataxia for assessing balance in people with multiple sclerosis related cerebellar ataxia?

Design: Cross-sectional observational multi-centre study.

Participants: Fifty participants with multiple sclerosis and secondary cerebellar ataxia, aged between 18 and 65 and were able to walk with or without support.

Outcome Measures: Barthel Index, Expanded Disability Status Scale two ataxia rating scales were assessed and disease duration recorded to estimate validity. Assessments were video-recorded and repeat assessments to estimate reliability were completed through observation of video recordings.

Results: Reliability was strong for all four measures (ICC range 0.95-0.99). Internal consistency was moderate to strong ( $\alpha$ range 0.72-0.94) Moderate to strong correlation found between the measures of balance ( $\rho S$ range $0.72-0.85)$. Poor to moderate correlation with disease severity, functional independence (Barthel Index) and disease duration ( $\rho$ S range - 0.37 to 0.76). Minimal detectable change was derived for the measures.

Conclusion: Tested measures had strong inter-rater and intra-rater reliability, internal consistency, convergent validity, discriminant validity and acceptable external validity. These measures are reliable and valid for assessing balance in people with multiple sclerosis and cerebellar ataxia.

\section{Key Practice Points:}

- Tested measures are recommended for clinical use.

- They are quick, easy and available at no cost

- Results can be generalized to other health conditions where cerebellar ataxia occurs.

\section{KNEE PAIN, INJURY AND MAGNETIC RESONANCE IMAGING ABNORMALITIES IN AUSTRALIAN RULES FOOTBALL PLAYERS: A COHORT STUDY}

Winzenberg $\mathrm{T}^{1,2}$, Humphries $\mathrm{D}^{3}$, Laslett $\mathrm{L}^{1}$, Pitchford $\mathrm{N}^{4,5}$, Khan $\mathrm{H}^{1}$, Abram F6, Pelletier J-P6, Martel-Pelletier J7, Jones G ${ }^{1}$, Aitken D

${ }^{1}$ Menzies Institute for Medical Research, University of Tasmania, Hobart, Australia

${ }^{2}$ Faculty of Health, University of Tasmania, Hobart, Australia

${ }^{3}$ The Sports Medicine Practice, Hobart, Hobart, Australia

4 Institute of Sport, Exercise and Active Living, Victoria University,

Melbourne, Australia

${ }^{5}$ AFL Tasmania, Hobart, Australia

6Medical Imaging Research \& Development, ArthroLab Inc. Montreal, Canada

'Osteoarthritis Research Unit, University of Montreal Hospital Research

Centre (CRCHUM), Montreal, Canada

Question: How common are MRI knee abnormalities in AFL players and what is their relationship with knee pain, function and injury?

Design: Observational longitudinal study over one season.

Participants: 75 male Tasmanian State Football League players (aged 16-30 years).

Outcome Measures: Knee pain (VAS), function (KOOS), MRI of both knees (assessing cartilage defects, bone marrow lesions, meniscal tears/extrusion, and effusion), past knee injury and surgery, and incident knee injuries.

Results: MRI abnormalities were common at baseline (67\% bone marrow lesions, 16\% meniscal tear/extrusion, $43 \%$ cartilage defects, $67 \%$ effusion). Bone marrow lesions were associated with higher pain and dysfunction (VAS 3 vs 17, $P<0.01$; KOOS 6 vs 18, $P=0.03$ ), previous injury ( $21 \%$ vs $53 \%, P=0.01)$ and previous surgery ( $0 \%$ vs $18 \%, P=0.03)$. Previous injury was more common in those with an effusion ( $30 \%$ vs $47 \%, P=0.07$ ) Previous surgery was more common in those with cartilage defects $(1 \%$ vs 21\%, $P<0.01$ ). Incident knee injuries were associated with worsening knee pain and function (VAS - 6 vs 27, $P<0.01$; KOOS -5 vs 21, $P<0.01$ ), new or enlarging bone marrow lesions ( $22 \%$ vs $67 \%, P<0.01)$ and incident cartilage defects ( $3 \%$ vs $17 \%, P=0.03$ )
Conclusion: MRI abnormalities commonly seen in osteoarthritis are common in AFL players. Their associations with pain, function, injury and surgery suggest they are clinically important.

\section{Key Practice Points:}

- Maintaining knee health is a challenge in AFL players.

- Knee structural abnormalities impact negatively on knee health.

- A major research priority should be to determine how physiotherapy can minimise the impacts of knee injury.

\section{HOW ARE HIGH RISK CHEST TRAUMA PATIENTS MANAGED AT A REGIONAL HEALTH SERVICE?}

\section{Winzer $\mathrm{B}^{1}$}

${ }^{1}$ Northeast Health Wangaratta

Question: What is the acuity of chest trauma patients admitted to Northeast Health Wangaratta? How are these patients managed? What is the complication rate?

Design: A retrospective audit.

Participants: Patients admitted to the critical care unit with three or more rib fractures, flail segment, bilateral rib fractures or a fractured sternum, 29/4/12 - 30/6/13

Outcomes measures: Patient acuity, time from emergency department (ED) presentation to initial physiotherapy contact, respiratory and pain management, complication rate.

Results: Twenty-three participants were included. The mean number of rib fractures per patient was four (range 1-10). Six patients had seven or more rib fractures. Additional injuries: pneumothorax $(n=7)$, sternal fracture $(n=5)$, lung contusion ( $n=5)$, flail $(n=3)$ and bilateral rib fractures $(n=2)$. Ninety-six per-cent of patients were reviewed by a physiotherapist during their admission: $48 \%$ within 24 hours of ED presentation Interventions included: standard-flow humidified oxygen $(n=7)$, high-flow oxygen $(n=1)$, non-invasive ventilation $(n=5)$, nasopharyngeal suctioning $(n=1)$ and postural drainage $(n=3)$. All patients received mobilisation and deep breathing exercises. Seventy-eight per-cent of patients were reviewed by the Acute Pain Service (APS) during their admission; 43\% within 24 hours of ED presentation. The complication rate was 35\%: pneumonia $(n=4)$, respiratory failure requiring intubation $(n=3)$ and ARDS $(n=1)$

Conclusion: The acuity of chest trauma patients at Northeast Health Wangaratta is high. Earlier referral to physiotherapy and APS would likely benefit this patient group.

\section{Key Practice Points:}

- Rural physiotherapists need to be adequately skilled to manage chest trauma patients.

- Ongoing training in cardiothoracic physiotherapy skills is warranted to assist generalist physiotherapists working rurally. 


\section{HOW TO ASSESS AND MANAGE FOR GLUTEAL FUNCTION IN THE CYCLING POPULATION}

Wisbey-Roth $\mathrm{T}^{1}$, Visentini $\mathrm{P}^{2}$

I"Take Control" Active Rehab; Bounce back active rehabilitation; Aust. Catholic University

2Physiosports Brighton, Latrobe University

Background: Sports physiotherapists are heavily involved in the management of pain and injury in cycling. As with all sports, knowledge of biomechanical aspects of the sport is essential, and in cycling the gluteal group is highly significant. An awareness of lower limb closed kinetic chain function is assumed.

Aims / objectives: The session will outline:

1. The theoretical significance and involvement of the gluteal bulk in the cycling pedal stroke

2. Assessment of the gluteal muscle group in the context of optimising cyclist performance.

3. Clinical Management and exercise intervention examples to optimise Gluteal Function

\section{Approach:}

4. Introduction 5 mins of slides outlining Gluteal activation in cycling theory and diagrammatic representations of gluteal activation in the pedal stroke.

5. Demonstration and practice of cycle specific gluteal activation assessment techniques.

6. Demonstration and practice of cycle specific gluteal retraining techniques.

7. Finish with Group Activity - group discussion and decide upon exercise type and entry point into rehabilitation program.

\section{Conclusion / Key Practice Points:}

- Awareness that Cycling muscle recruitment technique has specific effects on both performance and injuries sustained by the athlete.

- Cycle specific assessment tests to gauge gluteal activation and strength in cycle specific joint angles.

- Specific knowledge of the involvement of the gluteal bulk in cycle pedalling technique, and an ability to undertake a targeted exercise intervention program to address deficiencies in this component of the cycling closed kinetic chain.

- Ability to discuss sports specific technique more readily with cyclists.

- Ability to clinically reason through the creation of gluteal rehab exercises for cyclists.

\section{EFFECT OF A TWELVE MONTH HOME- BASED WALKING TRAINING PROGRAM IN MAINTAINING HEALTH RELATED QUALITY OF LIFE AND EXERCISE CAPACITY IN PEOPLE WITH COPD: A RANDOMISED CONTROLLED TRIAL}

Wootton SL ${ }^{1,2}, \mathrm{Ng} \mathrm{C}^{3}$, McKeough Z ${ }^{1}$, Jenkins $\mathrm{S}^{3,4}$, Hill K ${ }^{3}$, Eastwood P3,4, Hillman $D^{4}$, Jenkins $C^{5}$, Cecins $N^{4}$, Spencer $L^{1,6}$, Alison $\rfloor^{1,6}$

IPhysiotherapy, University of Sydney, NSW

Chronic Disease Community Rehabilitation Service, Northern Sydney Local Health District, NSW

${ }^{3}$ Physiotherapy, Curtin University, WA

${ }^{4}$ Pulmonary Physiology \& Physiotherapy, SCGH, WA

${ }^{5}$ Respiratory Medicine, Concord Hospital, NSW

${ }^{6}$ Physiotherapy, Royal Prince Alfred Hospital, Camperdown, NSW

Question: Following completion of an 8-week supervised walking training program, does a 12-month maintenance program comprising unsupervised home-based walking training, biofeedback, progressive goal setting and participant support improve the ability of people with COPD to maintain health-related quality of life (HRQoL) and/or exercise capacity, compared to simple instructions?

Design: Multi-centred, randomised controlled trial with concealed allocation and blinded assessment.

Participants: 95 participants with COPD.

Outcome Measures: HRQoL measured by the St George's Respiratory Questionnaire (SGRQ) and the Chronic Respiratory Disease Questionnaire (CRQ). Exercise capacity measured by the endurance shuttle walk test (ESWT) and six-minute walk test (6MWT).

Results: Seventy-five (79\%) participants (mean (SD) age 69 (8) years, forced expiratory volume in one second (FEV1) 43 (15) \% predicted) completed the study. There were no between-group differences in magnitude of change over the 12 month study period (mean difference between groups, 95\% CI) in SGRO total score (3.9 points, 95\% Cl -1.5 to 9.3), CRQ total score (1.8 points, $95 \% \mathrm{Cl}-4.6$ to 8.1) ESWT time (-54 sec, $95 \% \mathrm{Cl}-245$ to 137$)$ or $6 \mathrm{MWT}$ distance (-16m, $95 \% \mathrm{Cl}-46$ to 15$)$.

Conclusion: Unsupervised home-based walking training together with biofeedback, progressive goal setting and participant support was no better than simple instructions to do home-based walking training in maintaining the benefits of a short-term walking program.

Support: NHMRC project grant 570814

\section{Key Practice Point:}

- Following completion of an 8-week supervised walking training program, unsupervised home-based walking training, biofeedback using pedometers, progressive goal setting and participant support via telephone calls did not improve the ability of people with COPD to maintain HRQoL or exercise capacity over a 12 month period.

\section{SIMULATED LEARNING IN PAEDIATRIC ALLIED HEALTH - PAEDIATRIC WORKSHOP (SLIPAH)}

\section{Wright S, Kelly K, Thomas R}

Lady Cilento Children's Hospital

The aim of the workshop will be to expand participants' concepts of simulation and how it can be used to provide authentic, realistic paediatric experiences to physiotherapists in a safe environment across the spectrum of paediatrics. Simulation is not just about crisis management of the critically ill child, it is not just procedural assessment and technical skills. The skills taxonomy of physio is based around effective clinical decision making and the ability to engage and empower families. This one hour interactive workshop will give a taste of the possibilities that simulation provides in physiotherapy. This workshop will focus on how simulation can be used to translate the unique skills of the physio including:

1. Creating scenarios that are targeted and responsive to learning objectives including the NSQHS Standards

2. Adapting the delivery model according to participant capability (e.g. pause/discuss, immersive)

3. Matching manikins and equipment, from low to high technology, to the needs of the scenario

4. The role of confederates to promote the realism of a scenario

5. Debriefing techniques to assist in evaluation and feedback 


\section{A NATIONAL PROJECT TO EMBED ROLE- PLAY SIMULATION INTO AUSTRALIAN ENTRY-LEVEL PHYSIOTHERAPY CLINICAL EDUCATION PROGRAMS}

\section{Wright A}

School of Physiotherapy and Exercise Science, Curtin University, Perth, WA

Background: Simulation training provides a unique learning experience to enhance clinical education for students. Scenarios can be scripted to focus on specific conditions for students commencing their clinical training or they can be extended to provide advanced students with the opportunity to deal with challenging communications, interpersonal or safety issues. A consortium involving 7 Universities under the leadership of Prof Gwen Jull previously completed a major controlled trial which demonstrated that clinical placements incorporating simulation training could achieve equivalent educational outcomes to traditional clinical placements.

Purpose: Building on this work, Health Workforce Australia funded a consortium of 16 Universities to implement simulation training during the 2014/15 academic years. The project embedded a sustainable 5-day model of simulation (using professional role play actors as simulated patients) into entry-level physiotherapy clinical education programs as a substitute for traditional clinical placement time. A collaborative approach was taken, with the aim of encouraging profession-wide support. The project provided each physiotherapy program with the physical resources, simulation scenarios, staffing and training to enable simulation training to be embedded as a component of their clinical education programs. Each University had flexibility in relation to how they incorporated simulation training into their existing programs.

Outcomes: The project was successfully implemented across all of the participating Universities with 1789 students undertaking a period of simulation training across the three core areas of practice. The project was comprehensively evaluated and the presentation will provide data from the evaluations with a particular emphasis on findings related to cardiopulmonary clinical education.

\section{Key Practice Points:}

Participants attending this session will have an enhanced understanding of:

- the nature of simulation training

- the evidence base related to this form of education

- the potential value of utilizing simulation training in cardiopulmonary clinical education.

\section{PROFESSIONAL SEXUAL BOUNDARIES - SINGAPORE PHYSIOTHERAPISTS PERCEPTION: AN OBSERVATIONAL STUDY}

Yeung $\mathbf{M}^{1}$, Cooper ${ }^{2,3}$, Jones $A^{4}$, Jenkins $S^{2,3,5}$

${ }^{1}$ Singapore Institute of Technology, Academic Programmes, Singapore, Singapore 2Curtin University of Technology, Curtin Health Innovation Research Institute, Perth, Australia

${ }^{3}$ Sir Charles Gairdner Hospital, Physiotherapy Department, Perth, Australia ${ }^{4}$ Griffiths University, School of Allied Health Sciences, Brisbane, Australia ${ }^{5}$ Lung Institute of Western Australia, Perth, Australia

Background: Maintaining appropriate professional boundaries is an important ethical requirement of physiotherapists. Failure to operate within professional boundaries may lead to irreversible damages to the patient, profession and the public. Different cultures may have different views about appropriate sexual behaviour in general terms, but inadequately shown if these extend to professional setting.

Purpose: This study collected data from physiotherapists registered with the Allied Health Professions Council (AHPC) Singapore to the issues of sexual professional boundaries.

Methods: A self-complete questionnaire with was administered to registered physiotherapist in Singapore. Respondents were asked (1) their opinions about the actions of a physiotherapist in six vignettes highlighting professional sexual boundaries; (2) the incidence of sexual attraction and dating of their patients; and (3) their course(s) of action when confronted by an allegation of sexual misconduct against a colleague.

Results: Seventy-three responses were received (100\%). The Singapore results were similar to Hong Kong (HK) and Western Australia (WA), finding 4 of the 6 vignettes unacceptable. Singaporean physiotherapists were significantly $(P<0.01)$ less conservative than their WA peers. $84.7 \%$ of respondents opted acceptable for a physiotherapist working for a sports team to date a player, $49.3 \%$ opted acceptable to date a work colleague to whom they had provided professional ergonomics advice. 5.5\% reported feeling sexually attracted to a patient, and none (0\%) had ever dated a patient who at the time was receiving physiotherapy or dated their ex-patients.

Conclusions: Similarities and differences exist between Singapore, HK and WA physiotherapist with regard to professional sexual boundaries.

\section{Key Practice Points}

- The understanding of professional sexual boundaries is common across Eastern and Western culture.

\section{A SYSTEMATIC REVIEW OF AXILLARY WEB SYNDROME}

Yeung $\mathrm{WM}^{1}$, McPhail SM ${ }^{2,3}$,Kuys SS ${ }^{4,5}$

${ }^{1}$ Princess Alexandra Hospital, Brisbane

2School of Public Health and Social Work, Queensland University of Technology

${ }^{3}$ Centre for Functioning and Health Research, Metro South Hospital and Health Service

${ }^{4}$ School of Physiotherapy, Australian Catholic University, Brisbane

${ }^{5}$ School of Allied Health Sciences, Griffith Health Institute, Griffith University, Gold Coast

Question: What are the clinical presentation, diagnosis, frequency, natural progression, grading, pathoaetiology, risk factors, symptoms, interventions and outcomes of Axillary Web Syndrome (AWS)?

Design: Systematic review with narrative synthesis

Participants: Cancer patients following axillary lymphadenectomies Interventions: observation, physiotherapy, pharmacology, surgery Outcome Measures: Study quality was rated using NHMRC levels of evidence and Downs and Black tool.

Results: 37 studies of NHMRC level II to IV methodological quality were identified. Mean Downs and Black score for prospective and retrospective studies was 21 on a 28 -point scale (range 11 - 26). AWS diagnosis relies on inspection and palpation and grading has not been validated. AWS frequency has been reported to be as high as $85.4 \%$. Biopsies indicated venous and lymphatic pathoaetiology with five studies suggesting lymphatic involvement. Approximately 57\% studies reported AWS occurrence within eighth postoperative weeks however delayed presentation of greater than three months is possible. AWS commonly causes pain and shoulder abduction restriction, with timeframe of resolution usually within three months. Few studies (24.3\%) reported chronic symptoms beyond three months. Predisposing factors included extensiveness of surgery, younger age, lower body mass index, early arm oedema, numbness after intercostobrachial nerve injury, hematoma and ethnicity. Level IV studies indicated that pharmacology and/or physiotherapy may be safe and effective in promoting early recovery.

Conclusion: No gold standard exists for diagnosing, grading or treating AWS. Directions for future research include investigating possible relationship between AWS and lymphoedema; differentiating lymphatic versus venous involvement in AWS to understand implications on treatment; and determining treatment effectiveness.

\section{Key Practice Points:}

- AWS appears common following axillary lymphadenectomy for cancer Clinicians may consider pre-operative education or post-operative assessment to look for AWS

- AWS contributes to arm pain and disability. Diagnosis relies on subjective and objective examination (e.g. observation, range of movement (ROM) palpation) and differential diagnosis to rule out other pathologies.

- Although physiotherapy seems able to reduce symptoms, allow faster restoration of pre-morbid ROM and return to function; the strength of these finding is weak based on poorer quality studies and a lack of consistency between studies. Further research into physiotherapy management of AWS is worthwhile. 


\section{YOGA-BASED EXERCISE IMPROVES BALANCE AND MOBILITY IN OLDER PEOPLE: A SYSTEMATIC REVIEW}

Youkhana $\mathrm{S}^{1}$, Dean $\mathrm{CM}^{1}$, Wolff $\mathrm{M}^{2}$, Sherrington $\mathrm{C}^{3}$, Tiedemann $\mathrm{A}^{3}$

${ }^{1}$ Department of Health Professions, Macquarie University, Australia ¿Department of Clinical Sciences in Malmö, Lund University, Malmö, Sweden 3Musculoskeletal Division, The George Institute for Global Health, The University of Sydney, Australia

Question: What is the effect of yoga-based exercise on balance and physical mobility in people aged 60 years and older?

Design: Systematic review with meta-analysis of randomised controlled trials.

Participants: Trials were included if they involved participants aged 60 years and over.

Intervention: Trials were included if they involved physical yoga programs (excluding meditation and breathing exercises alone). No limitation was placed on the type, duration and frequency of yoga intervention.

Outcome Measures: Any standardised clinical or laboratory patient-based measure of balance was included such as the Short Physical Performance Battery, Berg Balance Scale and timed one leg stand. The physical mobility measures included the sit to stand test and gait speed tests.

Results: Six trials were identified that had data that could be included in a meta-analysis. Effect sizes were calculated as standardised mean differences (SMD) since different outcome measures were used. Across all participants, yoga interventions had a positive effect on both balance (SMD 0.34, 95\% CI 0.09 to 0.59 ) and physical mobility (SMD 0.50, 95\% CI 0.20 to 0.80).

Conclusion: Yoga interventions improve balance and physical mobility in people aged 60 years and over.

\section{Key Practice Points}

- Physiotherapists can confidently recommend yoga interventions for those aged 60 years and over to improve balance and physical mobility.

- Yoga-based exercise shows promise as a fall prevention intervention.

- A large randomised controlled trial investigating the effects of yoga on falls is warranted.

\section{DOES EDUCATION IMPACT THE RISK OF DEVELOPING LOW BACK PAIN WHEN GENETICS IS CONSIDERED? A POPULATION-BASED STUDY OF SPANISH TWINS}

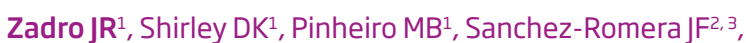
Ordoñana J $\mathrm{R}^{2,3}$, Ferreira $\mathrm{PH}^{1}$

${ }^{1}$ Discipline of Physiotherapy, Faculty of Health Sciences, The University of Sydney, Sydney, Australia

2Murcia Twin Registry, Department of Human Anatomy and Psychobiology, University of Murcia, Murcia, Spain

${ }_{3}$ Murcia Institute for Biomedical Research (IMIB-Arrixaca), Murcia, Spain

Question: Does gender influence the relationship between education and low back pain? What happens to this relationship when genetics is considered?

Design: Longitudinal twin case-control design.

Participants: 1,077 Spanish twins who reported the absence of low back pain at baseline were included in the analysis and followed over four years.

Outcome Measures: Low back pain was assessed by the following question: "Was your back pain bad enough to limit your usual activities or change your daily routine for more than one day?"

Results: Females with general secondary education have a significantly reduced risk of developing low back pain $(\mathrm{OR}=0.5,95 \% \mathrm{Cl} 0.3$ to $1.0, \mathrm{p}=$ 0.04 ) while males with the same education had an increased risk (OR = $1.5,95 \% \mathrm{Cl} 0.9$ to $2.3, \mathrm{p}=0.09$ ). In twins with university education the risk of developing low back pain decreased when controlling for genetics (OR decreased from 0.9 to 0.5 ), however, this was not statistically significant.

Conclusion: Educational status affects low back pain differently in males and females. After adjusting for genetics the relationship between education and the incidence of low back pain appears to strengthen, suggesting a direct causal link, although this needs to be further tested with greater sample sizes.

\section{Key Practice Points:}

- The risk for developing low back pain in males and females with basic vocational education is different.

- Results from previous studies investigating the relationship between education and low back pain might have been biased by not controlling for genetics.

\section{Ethics Approval}

All registry and data collection procedures involved in this study were approved by the University of Murcia Research Ethics Committee. 


\section{AUTHORS' INDEX}

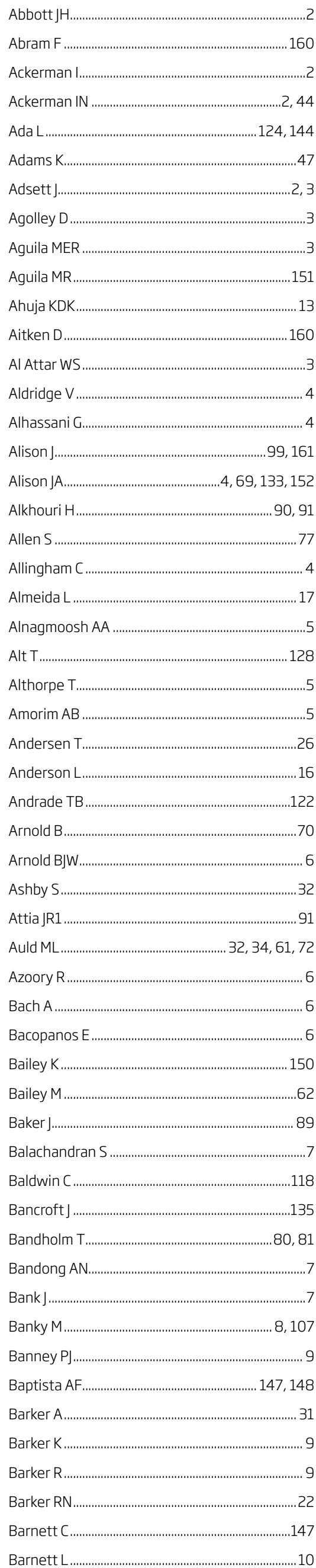

\begin{tabular}{|c|c|}
\hline Barr C 129 & Bowles K \\
\hline Barradell S & Box RC \\
\hline Barry CM & Boyd R \\
\hline Barry RK & Bradley S \\
\hline Barton A & Bradley T \\
\hline Basteris A & Bradnam L . . . . . . \\
\hline 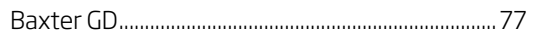 & Bradnam LV . \\
\hline Beach LJ & 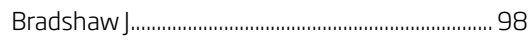 \\
\hline Beales D $\ldots \ldots \ldots \ldots \ldots \ldots \ldots \ldots \ldots \ldots \ldots \ldots \ldots \ldots \ldots \ldots \ldots \ldots$ & Braithwaite FA \\
\hline Beall P & Brand CA \\
\hline Beck BR & 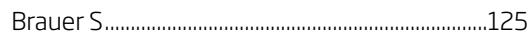 \\
\hline Beeson E & Brauer SG $\ldots \ldots \ldots \ldots \ldots \ldots . . .22,28,82,89,90,131,134$ \\
\hline Bell A & Breckenridge J \\
\hline Bellomo R & Brennan F ......................................... 44 \\
\hline Benjamin D & Brennan PC \\
\hline 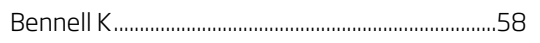 & Briffa KN \\
\hline Bennett L & Briffa N \\
\hline 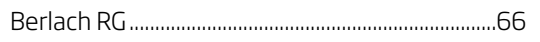 & 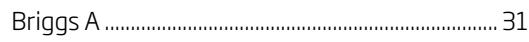 \\
\hline Berney S................................................11, 62, 118 & 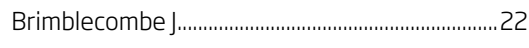 \\
\hline 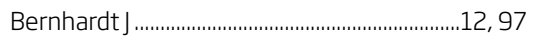 & 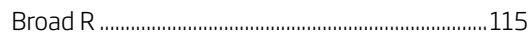 \\
\hline Bew P & Brock K \\
\hline Bialocerkowski A & Broecker M \\
\hline Bierma-Zeinstra SMA _.........................................131 & Brooksbank J \\
\hline Bilney B .............................................98, 125 & Brophy J.............................................. 150 \\
\hline Bilney BE & Brophy-Williams N ……...................................... 23 \\
\hline Bird ML & Broughton N \\
\hline 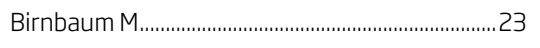 & 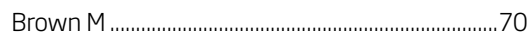 \\
\hline Birnbaum MA & Brown T \\
\hline Bisset L & Browning L . \\
\hline Bissett B & Bruder A \\
\hline Black S & Bryant AL \\
\hline Blackburn C ........................................................ & Bucknill A \\
\hline Blackstock FB & Buhagiar M........................................... 111 \\
\hline 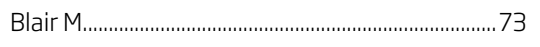 & Buhagiar MA \\
\hline Blair SN & Buhr $\mathrm{H}$ \\
\hline Blake EL & Bullock-Saxton ] ............................................. \\
\hline 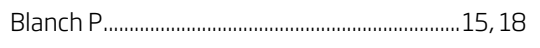 & Bullock-Saxton JE \\
\hline Blizzard L ................................................... & Bulsara C \\
\hline 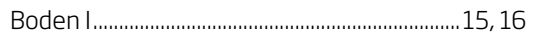 & Bunzli S \\
\hline Boden IJ & Burge AT \\
\hline Boettcher C & BurgesS C \\
\hline 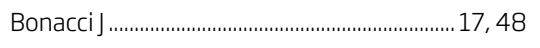 & Burkett B \\
\hline Bongers B & 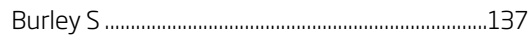 \\
\hline Boote C & Burn DE \\
\hline Borges Dario A ......................................... 18 & 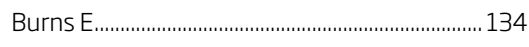 \\
\hline 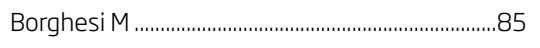 & Burton C \\
\hline Bott A & Buscemi V \\
\hline 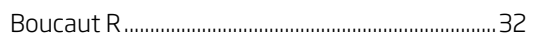 & Butler J \\
\hline 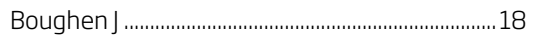 & 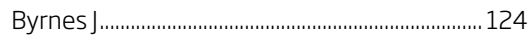 \\
\hline Bourke M........................................................... 146 & Cacciotti K \\
\hline Bousie JA & 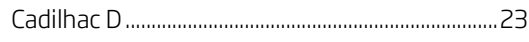 \\
\hline 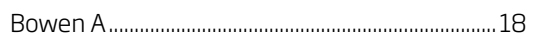 & Callisaya M...............................................13, 35, 158 \\
\hline 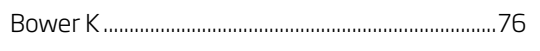 & Callister R ..........................................106, 114 \\
\hline Bower W & 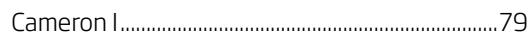 \\
\hline
\end{tabular}




\begin{tabular}{|c|c|c|}
\hline 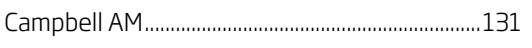 & 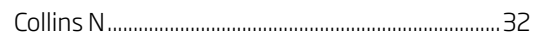 & 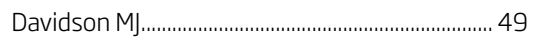 \\
\hline Campbell L ............ & 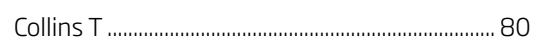 & Davies JM.................... \\
\hline 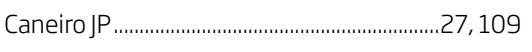 & 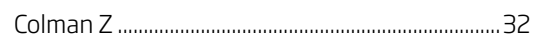 & Davies K...................................................... \\
\hline 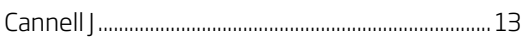 & Colodro-Conde L ….............................................. & Davies KN......................................................... \\
\hline Caple D & Coltman KA & 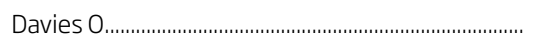 \\
\hline 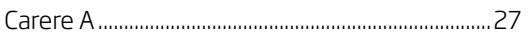 & 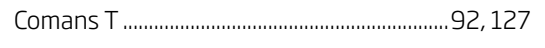 & 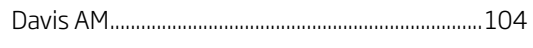 \\
\hline Carroll HG & 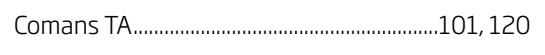 & 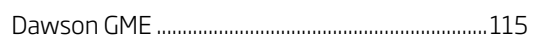 \\
\hline 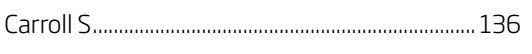 & 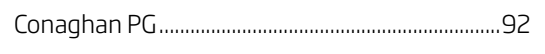 & 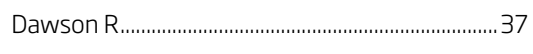 \\
\hline 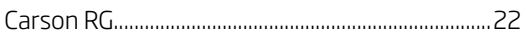 & Connelly L .............................................................. & 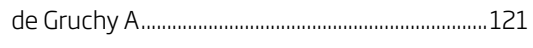 \\
\hline 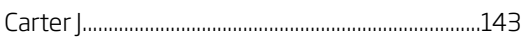 & 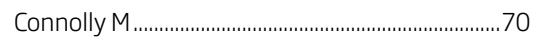 & 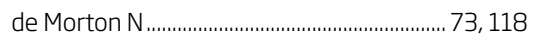 \\
\hline 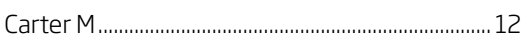 & 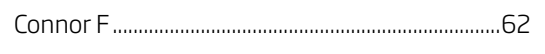 & 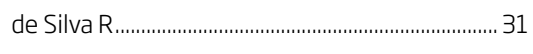 \\
\hline$\ldots$ & Conroy A ............................................ & 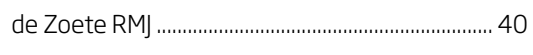 \\
\hline .......................... 80 & $\ldots$ & Dean B..............................................................38 \\
\hline Cassidy E .............. & Coombes BK.......................................... 33, 120 & Dean C .....................................................124,144 \\
\hline 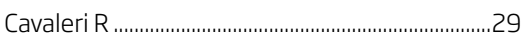 & 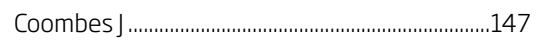 & Dean CM .....................................38, 74, 143, 163 \\
\hline Cavka B......................................................... & 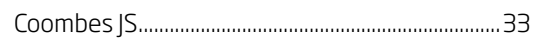 & Dear B..................................................................39 \\
\hline Cecins N ...................................................99, 161 & 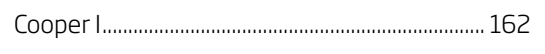 & 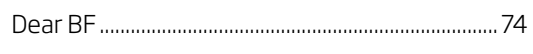 \\
\hline$\ldots$ & $\ldots$ & 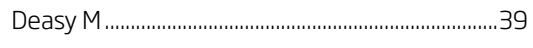 \\
\hline$\ldots 3,137$ & 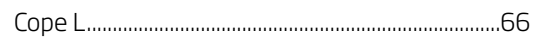 & Debenham J........................................................... \\
\hline$\ldots \ldots \ldots \ldots \ldots \ldots \ldots . .20$ & Corben LA ............................. & 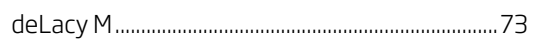 \\
\hline 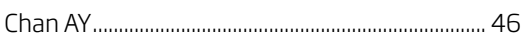 & Cornwall J........................................................... 42 & Delatycki MB.........................................104, 105 \\
\hline Chan AYP....................................................46, 59, 60 & Corrigan R .......................................................... & Demase B]..............................................................39 \\
\hline 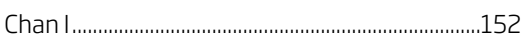 & 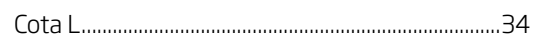 & Denehy L............... $7,11,15,16,45,56,62,85,118$ \\
\hline 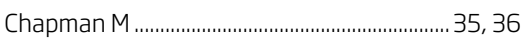 & 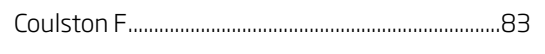 & 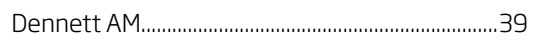 \\
\hline$\ldots 77$ & Coulter $\mathrm{C}$ & 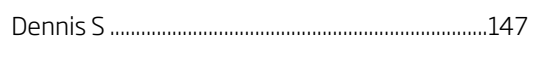 \\
\hline 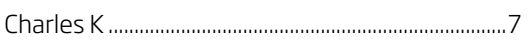 & 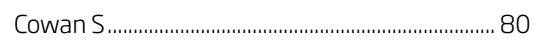 & Dennis SM............................................ \\
\hline$\ldots$ & 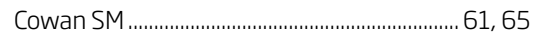 & 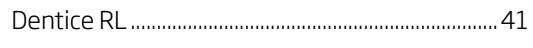 \\
\hline$\ldots \ldots \ldots \ldots \ldots \ldots \ldots \ldots . .28$ & Cox L $\ldots \ldots \ldots \ldots \ldots \ldots \ldots \ldots$ & Der A . \\
\hline 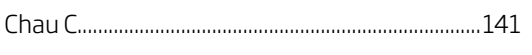 & Cox LE & 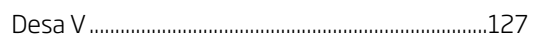 \\
\hline 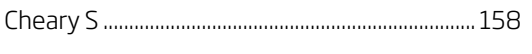 & $\ldots$ & Desa VG ................................................................. 40 \\
\hline$\ldots 79$ & 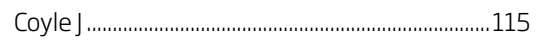 & Diggles A ............................................................ 45 \\
\hline$\ldots$ & 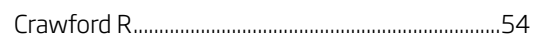 & 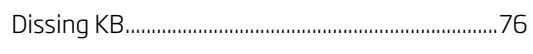 \\
\hline 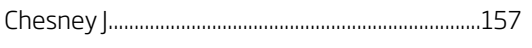 & 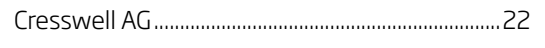 & 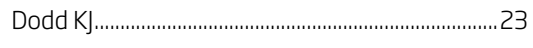 \\
\hline Chiarelli P ............................................................ 41 & Croker A & Donovan J........................................................... 94 \\
\hline Chiarelli PE....................................................53, 117 & Croker C & Donovan M ............................................................. 40 \\
\hline Chipchase L........................................... & Crompton A & 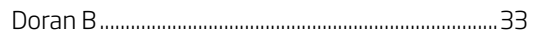 \\
\hline Chipchase LS..................................................... 148 & Crook TC & Dougherty J............................................................. \\
\hline 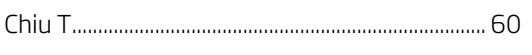 & Crosbie J............................................. 111, 149 & 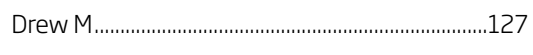 \\
\hline Chivers A ...................................................... 57 & Crossley KM............................. 44, 49, 79, 108 & 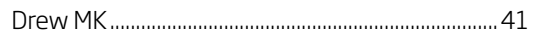 \\
\hline Chow R........................................................29, 30 & 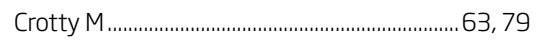 & Driessens S ......................................................143 \\
\hline 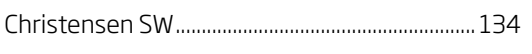 & 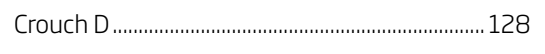 & Duguid L ............................................... \\
\hline Churilov L .................................................................97 & Cruickshank SE ..............................................34, 35 & Dunning A ...............................................................135 \\
\hline 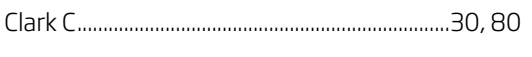 & Cuellar WA & 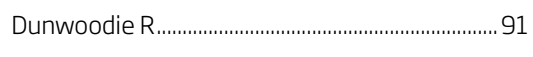 \\
\hline Clark R........................................... 30, 76, 125 & Culvenor AG ............................................. 44, 159 & 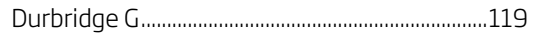 \\
\hline 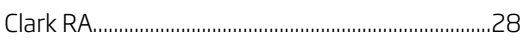 & Cunningham J ..................................................... & 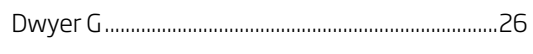 \\
\hline Clarke J...................................................28, 40,127 & Curtis R................................................................ & Eastwood P........................................................161 \\
\hline Claus A .................................................... 93 & Cynn HS ..................................................... & Edbrooke L......................................................11, 56 \\
\hline Clavisi O............................................................ 31 & Dafoe S & 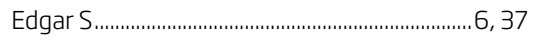 \\
\hline 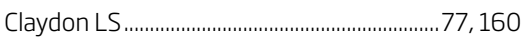 & Dallimore JT & 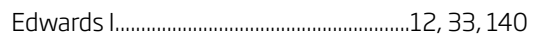 \\
\hline 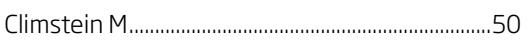 & 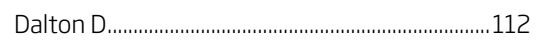 & 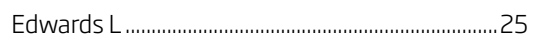 \\
\hline Coburn P........................................................... 31 & Dalton M & 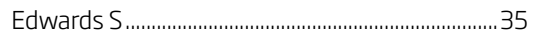 \\
\hline Coburn PT ...................................................... & 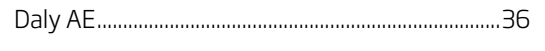 & Eisenhuth J ........................................................133 \\
\hline 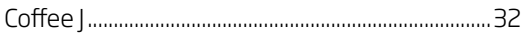 & 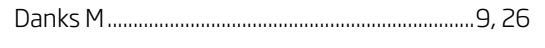 & El-Ansary D ............................................... 45 \\
\hline 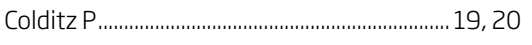 & 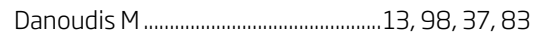 & 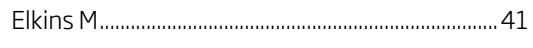 \\
\hline
\end{tabular}




\section{AUTHORS' INDEX}

Elkins MR ..................................................................101

Elliott S......................................................................... 41

Ellis R......................................................................... 77

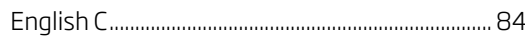

Enticott P ................................................................. 98

Etherton-Beer C...........................................................4

Evans D .................................................................99

Evans K .............................................................42, 153

Fagan A...................................................................... 91

Fairhall N …………………………...................................137

Farrell SF .................................................40, 42, 142

Farrugia L.................................................................... 150

Fearon AM …………………....................................43

Feil D ........................................................................85

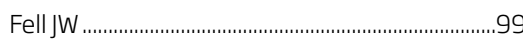

Ferrar K ………………………………………………4 43

Ferreira M....................................................................... 17

Ferreira ML ........... 5, 18, 43, 87, 88, 101, 121, 122

Ferreira P.

5, 138

Ferreira PH ......... 17, 18, 43, 87, 87, 121, 122, 163

Fetterplace K...........................................................11

Field E......................................................................148

Filbay SR.............................................................. 44

Finlay N ...................................................................... 44

Fiori S................................................................. 19, 20

Fisher BJ................................................................45

Fitzgerald J.............................................................4

Fitzgerald S................................................................4 45

Fitzhenry SK...........................................................45

Flicker L.................................................................... 47

Folbigg SL ..........................................................116

Fong C......................................................................

Ford J] .......................................................46, 59, 60

Ford KRMyer GD .......................................................117

Formosa D.................................................................103

Fortunato R ...................................................24, 111

Francis-Coad J..........................................................4 47

Francis-Cracknell A ...................................................47

Franettovich Smith MM................................. 48, 105

Fransen M...................................................... 5, 108

Fraser RM............................................................. 48

Frawley H............................................................... 48

Frawley HC .........................................................49, 85

Freke MD ……………………………………………… 49

Fripp J............................................................. 19, 20

Fritsch C.................................................................... 87

Fu S.......................................................................... 37

Fulford A ................................................................123

Fuller A ………............................................................ 111

Fuller LM................................................................... 64

Furness J..................................................................50

Gabbe B ................................................................62

Gabel CP.....................................................50, 51, 52

Gabel P ..................................................................125
Galea MP.................................................................52

Gallagher R.......................................................... 53

Gallagher S..............................................................38

Galloway G .................................................................119

Ganderton C................................................................4 43

Gane EM .......................................................................5

Garcia-Vega J......................................................... 139

Gard M .............................................................. 136

Gardiner $P$.................................................................. 84

Garth B...................................................................... 87

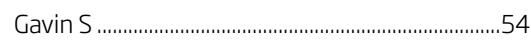

Gaylor $\mathrm{H}$................................................................

Gelderman D................................................................54

George J..........................................................19, 20

Georgiou-Karistianis N..............................104, 105

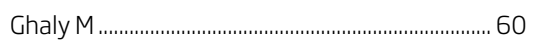

Gibbins IL.................................................................. 10

Gibbons P................................................................. 57

Gibbons T................................................................ 13

Gibbs A .................................................................115

Gibson A _.............................................................. 55

Gibson SJ.................................................................. 55

Gilby R....................................................................4

Giles M....................................................................53

Gill S........................................................................ 44

Ginn KA ...........................................................69, 99

Goff L .......................................................................5

González-Javier F.............................................122

Goo MR .............................................................. 55, 56

Good D................................................................

Gower A .....................................................................4

GraetzJ................................................................... 43

Graham PL .................................................................. 74

Granger CL.............................................56, 85, 118

Graven C ..................................................................23

Graven-Nielsen T..................................................... 134

Gray K ......................................................................... 57

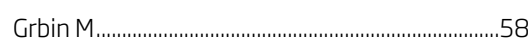

Greenstein C .........................................................57

Gregory G ........................................................... 139

Griffiths A.............................................................58

Grimaldi A................................................... 3, 23, 58

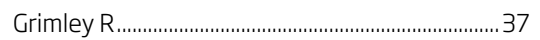

Grimley RS .......................................................131

Grudzinskas K...........................................................58

Grunstein R ....................................................... 121

Guirguis R.............................................................4 43

Gupta A ................................................................... 149

Guyatt SL .................................................................59

Guzzetta A.................................................................19

Haberberger RV ...........................................................10

Hahne AJ....................................................46, 59, 60

Haines T .................................................................... 60

Haines TP................................................... 9, 47, 101
Hakkennes S.............................................................. 44

Halaki M...................................................................69

Hale LA.......................................................................... 160

Haley C .............................................................................

Hall K .............................................................................135

Hallbach M.....................................................................43

Halloran T ............................................................... 73

Han HR................................................................ 61, 85

Hankin N............................................................... 32, 72

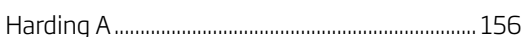

Harding AL..................................................................... 61

Harding $P$....................................................... 11, 61, 146

Hardy S.................................................................62

Harmer AR .....................................................................

Harris E ....................................................................72

Harris EC............................................................ 34, 61

Harris IA .....................................................................

Harris J ............................................................................135

Harris L.......................................................................4

Harrison L ...............................................................62

Harrold M ...................................................................62

Hart S..........................................................................62

Harvie DS .................................................................63

Hassett L..........................................................63, 137

Hatton AL ..................................................................54

Hau R ........................................................................23

Hay-Smith EJC ......................................................... 64

Hayes K .................................................................... 64

Hayward KS............................................ 22, 129, 131

Heerey J .............................................................................65

Henderson J ........................................................53

Hensman-Crook AS ...................................................65

Herbert RD ................................................................101

Hermans D.....................................................................65

Hestbaek L .................................................................76

Hewett TE ............................................................. 117

Hewitt B ........................................................................65

Hewitt T ...................................................................128

Heyman R ................................................................ 160

Heywood SE ........................................................65, 66

Hickey A .....................................................................2

Hickey L .......................................................................59

Hides J....................................................35, 105, 158

Hides JA................................................................ 48

Higgins A ...............................................................62

Higgins R................................................................. 98

Higgins RO ............................................................ 13

Hill A................................................................................. 91

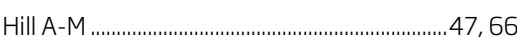

Hill AM .....................................................47, 153, 154

Hill B ............................................................. 30, 67, 157

Hill C .........................................................16, 99

Hill C] ...........................................................................25

Hill K …….............................................14, 97, 99, 161 


\begin{tabular}{|c|c|c|}
\hline Hiller A & Johnston C & 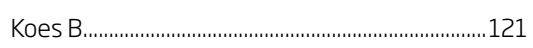 \\
\hline 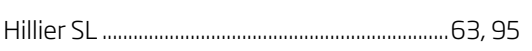 & Johnston CL..............................................10, 72 & 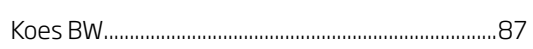 \\
\hline 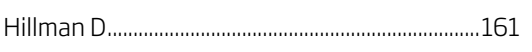 & Johnston LM.........................32, 34, 61, 72, 73, 83 & Kohler F.......................... \\
\hline Hing W...................................... 50, 67, 77, 104,132 & Johnston $\vee \ldots \ldots . . . . .18,29,40,73,75,115,120,151$ & Kolt GS \\
\hline Hing WA & 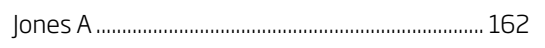 & König von Borstel U ..................................99 \\
\hline 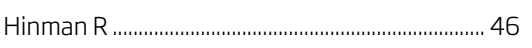 & 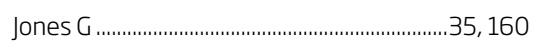 & 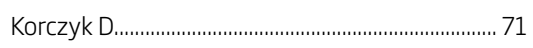 \\
\hline 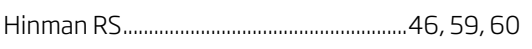 & Jones L.................................................... & 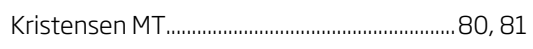 \\
\hline 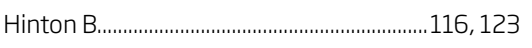 & 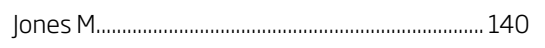 & 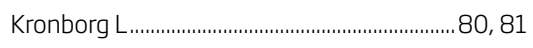 \\
\hline 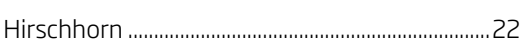 & 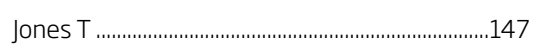 & 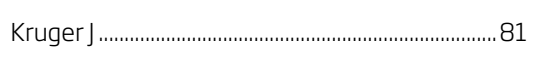 \\
\hline 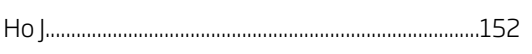 & 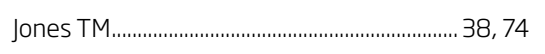 & 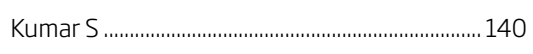 \\
\hline 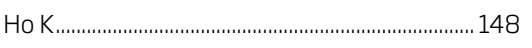 & 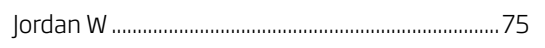 & 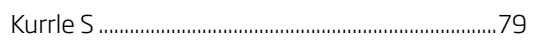 \\
\hline 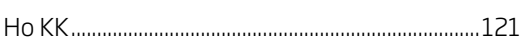 & 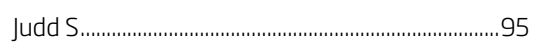 & 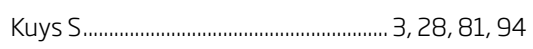 \\
\hline 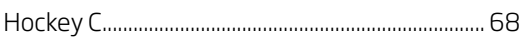 & 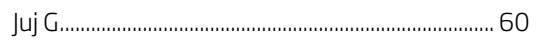 & 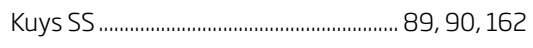 \\
\hline Hodges P...................................................58, 68 & Jull G............................................................... 29, 141 & Kwiatkowski SL ................................................. 81 \\
\hline Hodges PW ....................................... 134 & 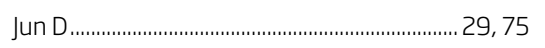 & 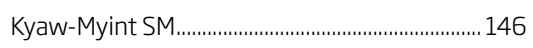 \\
\hline 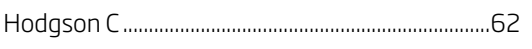 & 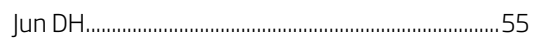 & Laakso E-L.................... \\
\hline Hodgson CL........................................................ & 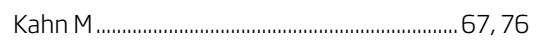 & Laakso L...................... \\
\hline 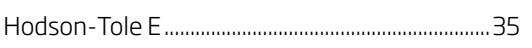 & 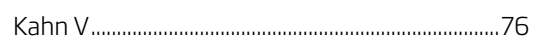 & Lacasse N.................. \\
\hline 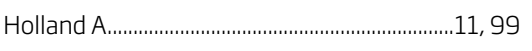 & Kammerlind A-S ................................................. & 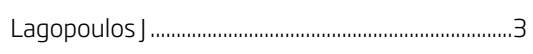 \\
\hline$\ldots$ & Kamper SJ..................... & Lahham A.................... \\
\hline$\ldots$ & Kannan P.................... & Lalor A ........................ \\
\hline 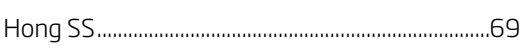 & 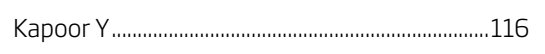 & Lambridis T ............................ \\
\hline 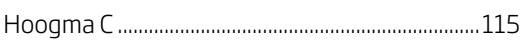 & 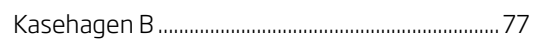 & Lamont RM......................... \\
\hline Hopper JL ............................................121, 122 & 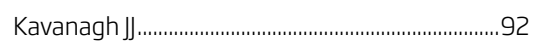 & 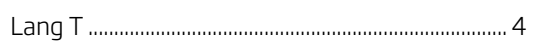 \\
\hline Horan S................................................................70 & 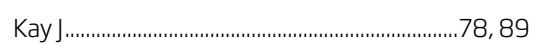 & Langford J....................... \\
\hline$\ldots, \ldots \ldots \ldots$ & $\ldots \ldots \ldots \ldots \ldots \ldots . . . .47$ & Laslett L........... \\
\hline Hordacre B.............. & $\ldots$ & Latimer [ ............... \\
\hline Hough J .................................................................26 & Keays SL ................................................ 93, & Lau P ............................. \\
\hline Howard VJ...............................................................99 & Kehlet H...................................................... & Law M.......................................................... \\
\hline 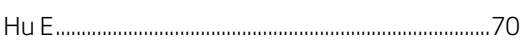 & 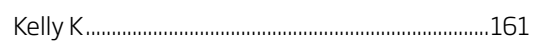 & Lazzarin T ...................................................... \\
\hline 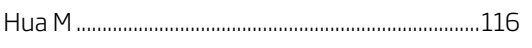 & Kelly RL & $\ldots$ \\
\hline Hughes D .................. & $\ldots . . . . . . . . . . . .19,108$ & 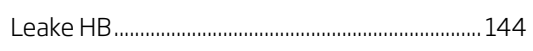 \\
\hline Humphries D........................................................ 160 & 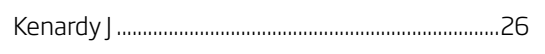 & Leaver A.......................................... 7, 40, 127, 128 \\
\hline Hunt $P$. . . . . . . . & Kennedy B & 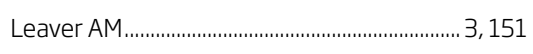 \\
\hline Hunter SW .................................................... 71 & Kent J........................ & Leditschke A ..................... \\
\hline 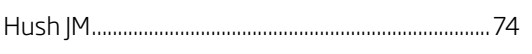 & 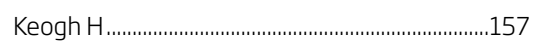 & Lee A .......................................... \\
\hline 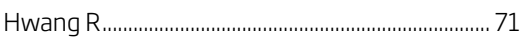 & 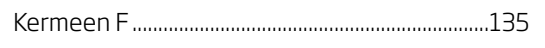 & Lee AL .............................. \\
\hline Ingram K .............. & Kersten P.................... & Lee D................. \\
\hline Irving $L$...................... & Kestell GR & Lee DCA ............................. \\
\hline Iwashyna T]............. & 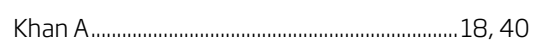 & Lees J.................... \\
\hline 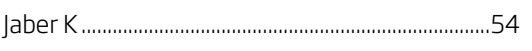 & 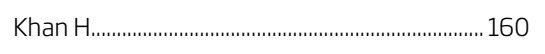 & Leet AS............... \\
\hline 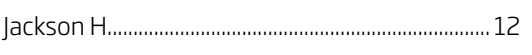 & 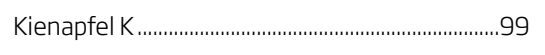 & Lennon S........................... \\
\hline 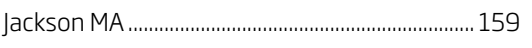 & Killington M & 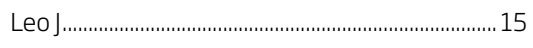 \\
\hline 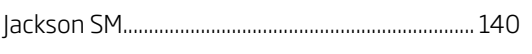 & 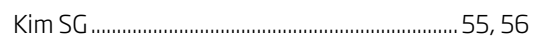 & 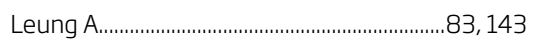 \\
\hline$\ldots$ & $\ldots$ & Leung B................. \\
\hline$\ldots$ & 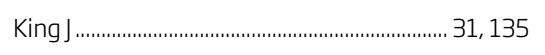 & Leung L.......... \\
\hline 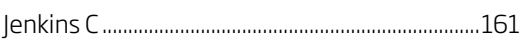 & Kingston K & Leung R ................ \\
\hline Jenkins S.....................................99, 161, 162 & 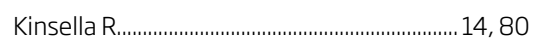 & Levi CR.................. \\
\hline 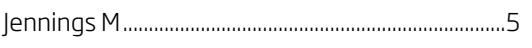 & Kirkham C & 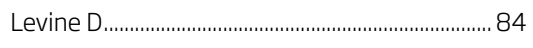 \\
\hline 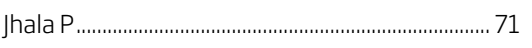 & 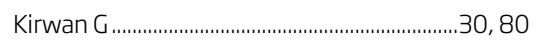 & Levinger $P_{\ldots} \ldots \ldots \ldots \ldots \ldots \ldots$ \\
\hline Jo Nijs .....................................................112, 113 & 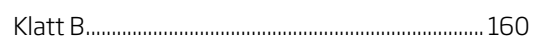 & 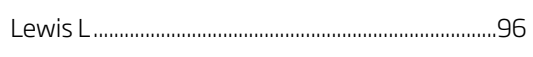 \\
\hline Job J .................................................................. 146 & 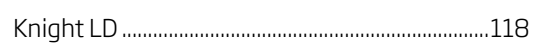 & 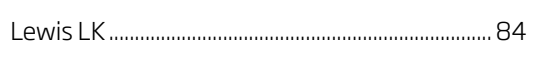 \\
\hline 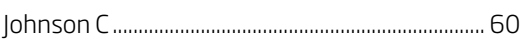 & Ko V & 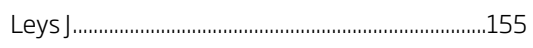 \\
\hline 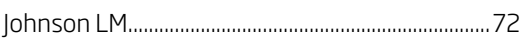 & 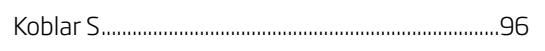 & Li Q........................................................... \\
\hline
\end{tabular}




\section{AUTHORS' INDEX}

\begin{tabular}{|c|c|}
\hline Li R & 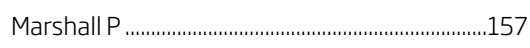 \\
\hline 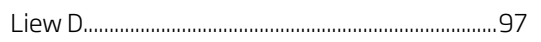 & 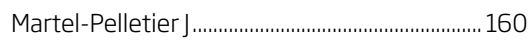 \\
\hline Lim M & Martin F \\
\hline Lim OB & Martin J \\
\hline Lin K-Y & 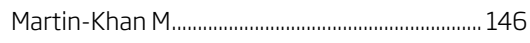 \\
\hline Lind C & Marzano V \\
\hline 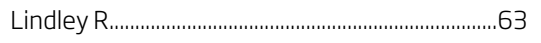 & Mason M \\
\hline Lion P & Mastwyk S \\
\hline Lisle K & Matthews M \\
\hline 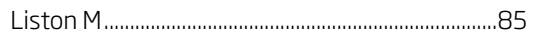 & Mattin S \\
\hline Little A & 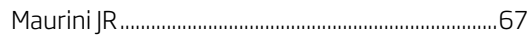 \\
\hline Lloyd B...................................................... 150 & Mawston G ...................................................... 77 \\
\hline Lloyd D & May K \\
\hline 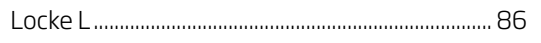 & May S \\
\hline Locke M & 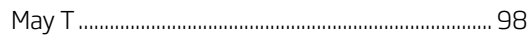 \\
\hline Longbottom J............................................. & McArdle A \\
\hline Louca C & McAtamney L ............................................ 27 \\
\hline Lovell G .................................................. 41 & McCarroll E \\
\hline Low A & McCarthy EA \\
\hline Low Choy N .........................................9, 94, 145 & McCarthy S .............................................. 90, 91 \\
\hline Lowe $\mathrm{H}$ & 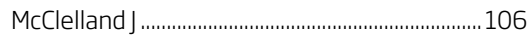 \\
\hline 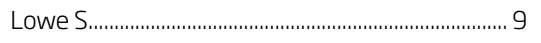 & McCluskey MA \\
\hline Lowell A & 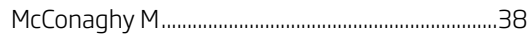 \\
\hline Ludvigsson L ................................ & McConville J.................................................... 15 \\
\hline Luker ] ........................................ & McCormick M.............................................. 4 \\
\hline MacDonald-Wicks L ........................... & McCutcheon L ............................................... 94 \\
\hline 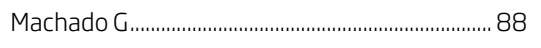 & McDermott F \\
\hline Machado GC ............................ & McDonald CF \\
\hline 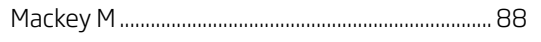 & McDonnell MN \\
\hline 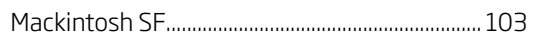 & McEvoy M.......................................... 21, 96 \\
\hline Mackney J & McGhee DE \\
\hline MacLeod L .................................. & McGhie JA \\
\hline 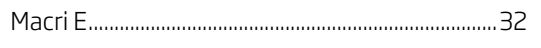 & 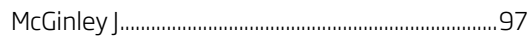 \\
\hline Madden VJ.............................................................63 & McGinley JL _.......................................... 13, 98, 125 \\
\hline Magarey ME …....................................... 88, 89 & McGough A \\
\hline 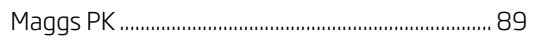 & 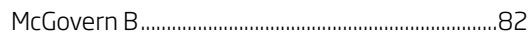 \\
\hline 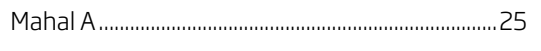 & McGowan CM \\
\hline Mahendran N......................................... 89, 90 & McGreevy P.................................................. 98, 99 \\
\hline Maher C & McGregor L \\
\hline Maher CG ............................................ 87, 121, 122 & 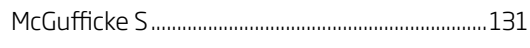 \\
\hline Maka K..................................................... 90, 91 & McKenzie DK \\
\hline 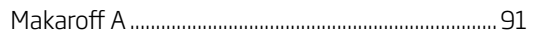 & McKenzie S........................................... 78, 135 \\
\hline 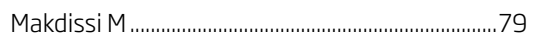 & McKeough Z \\
\hline Maloney S & McKeough Z]......................................... 100, 152 \\
\hline Mandrusiak A.............................................. 71, 83, 91 & 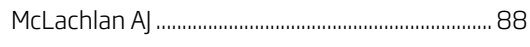 \\
\hline Mangharam J..................................................... 91 & 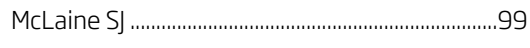 \\
\hline Manickaraj N ..............................................................92 & McLean A \\
\hline 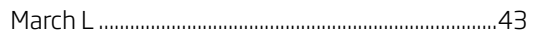 & McLean R \\
\hline 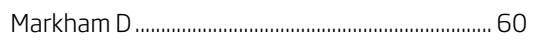 & McLellan C \\
\hline 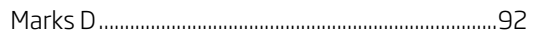 & McLoughlin E \\
\hline Marquez EL …......................................... 57 & McMahon C \\
\hline 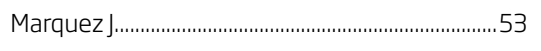 & 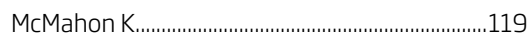 \\
\hline 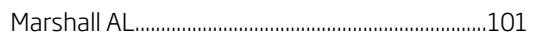 & McNamara RJ .....................................100, 101, 152 \\
\hline Marshall N .................................... & McPhail SM ..........................47, 54, 101, 102, 162 \\
\hline
\end{tabular}

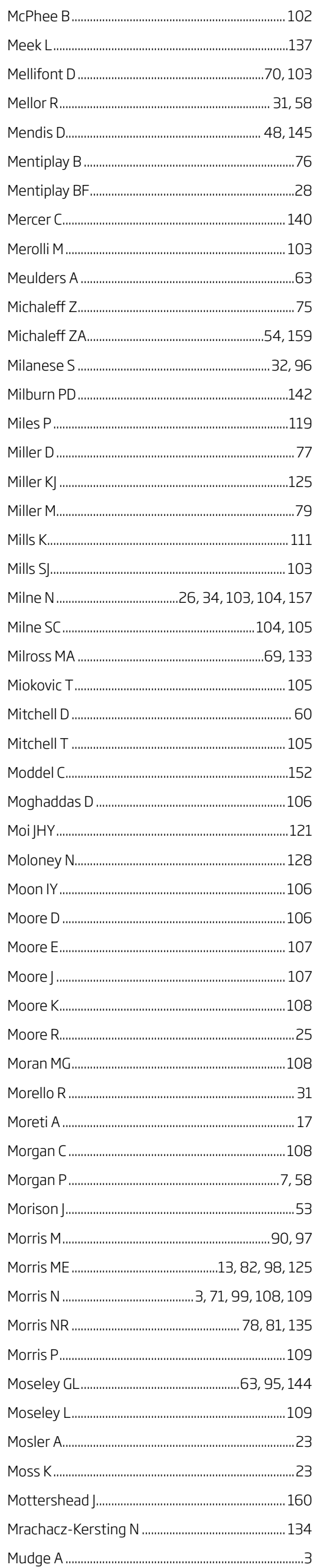




\begin{tabular}{|c|c|c|}
\hline 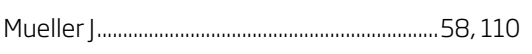 & Page C & 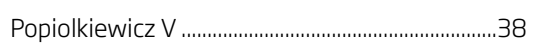 \\
\hline Mulcahy A .........................................................110 & Page C]......................................................... 136 & 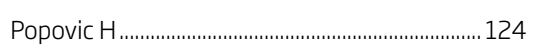 \\
\hline 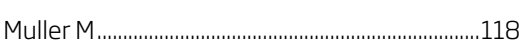 & 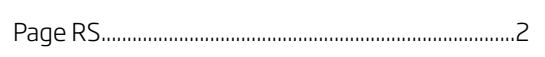 & 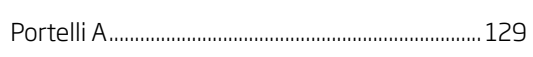 \\
\hline Mullins RM.............................................................110 & 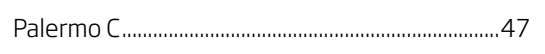 & 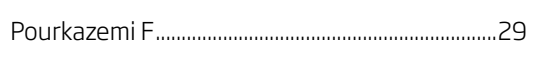 \\
\hline Murphy A................................................ 98, 105 & Pallant J ................................................................. 67 & Presneill J..............................................................62 \\
\hline 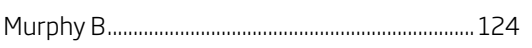 & Palm H & 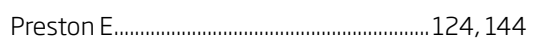 \\
\hline Murray M............................................ 47, 90, 91 & 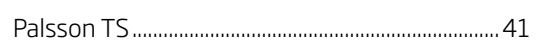 & Pritchard MG ..................................................79 \\
\hline 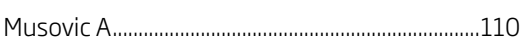 & Panizza B.......................................................... & Pritchard SA .................... \\
\hline 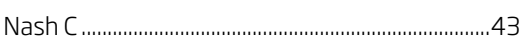 & 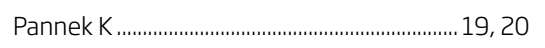 & Proud EL ............................. \\
\hline 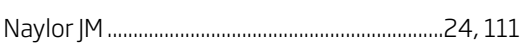 & 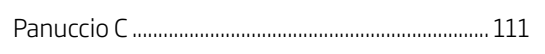 & 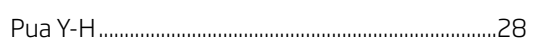 \\
\hline 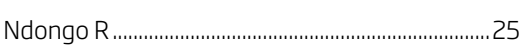 & 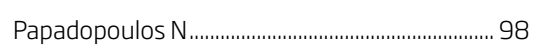 & 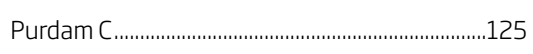 \\
\hline 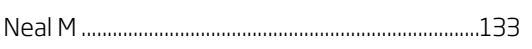 & 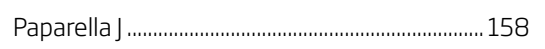 & Purohit M............................. \\
\hline 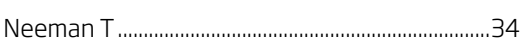 & 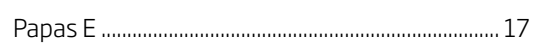 & Purvis T............................. \\
\hline 146 & Pappas E............................................ 3, 5, 117 & Quek J............. \\
\hline Neumann P .......................................... 86, 111 & 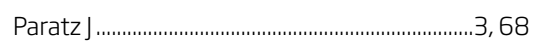 & Quinlan T......................... \\
\hline Newcombe PA.................................78, 93, 153 & 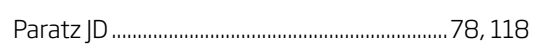 & Rabey M........................... \\
\hline 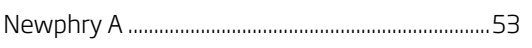 & Park JH & Rahmann A .......................... \\
\hline Newstead C]................................................... 10, 72 & 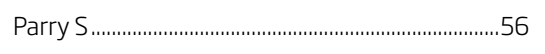 & Raigangar V .................................................... \\
\hline $\mathrm{Ng} C \ldots \ldots \ldots$ & Parry SM & 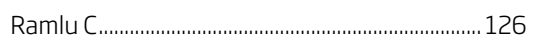 \\
\hline$\ldots$ & $\ldots$ & Ramsay E.......... \\
\hline 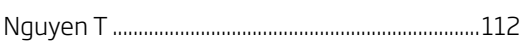 & Patman S....................................... 153, 154 & Raper D ................. \\
\hline 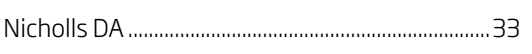 & 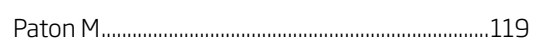 & Raymer M....................... \\
\hline 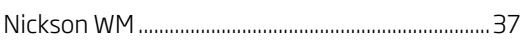 & 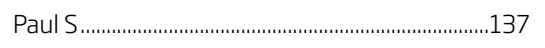 & 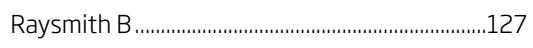 \\
\hline 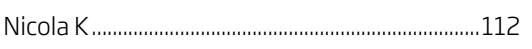 & 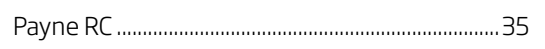 & Rebbeck T........................ 3, 7, 40, 127, 128 \\
\hline 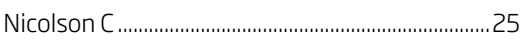 & 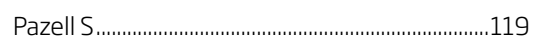 & 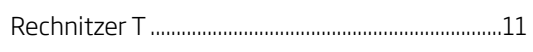 \\
\hline$\ldots \ldots \ldots \ldots \ldots \ldots . . .18,90$ & $\ldots$ & Redmond $\mathrm{C} \ldots \ldots . . .$. \\
\hline Nobre D................................. & $\ldots \ldots \ldots$ & Redpath A ....................... \\
\hline 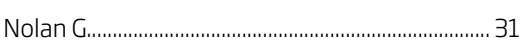 & 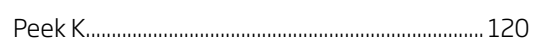 & Reeve J............................ \\
\hline Norton U................................................................113 & Pellegrino VA..................................................... 64 & Reeve JC ................................... \\
\hline 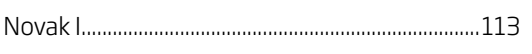 & 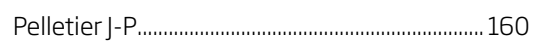 & Refshauge K....................................17, 18, 121, 122 \\
\hline Ntoumenopoulos G ..................................113, 114 & Pendlebury C . & 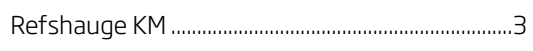 \\
\hline$\ldots \ldots \ldots \ldots \ldots \ldots \ldots \ldots \ldots \ldots \ldots \ldots . .114$ & Peolsson A.............. & ........................ \\
\hline O'Brien L.............................................................61 & 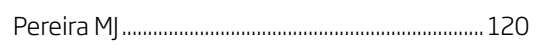 & Reid DW ............................ \\
\hline O'Connor D............................................................. 60 & 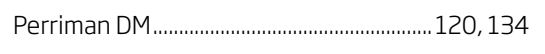 & Reid SA.................................................48, 129, 142 \\
\hline O'Connor LR ................................................. & 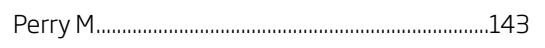 & Remedios L.............................. 56, 83, 118, 129 \\
\hline O'Dwyer M...................................................... & 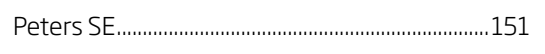 & Richards MC .....................................46, 59, 60 \\
\hline 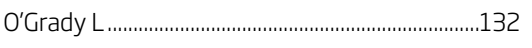 & Peterson G & 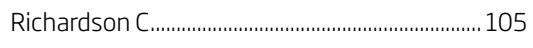 \\
\hline O'Keeffe D ............................................................115 & Peterson S............ & Riddiford-Harland DL.............................................97 \\
\hline O'Leary S..............................29, 54, 75, 92, 115, 127 & 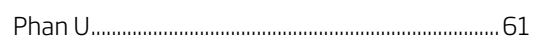 & Ridding BC ............................ \\
\hline 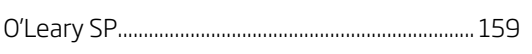 & 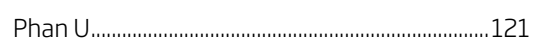 & Ridding MC............. \\
\hline O'Sullivan P........................................ 24, 27, 146 & 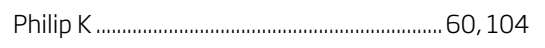 & Riggall ]....................... \\
\hline O'Sullivan-Pippia L .................................................70 & Phillips C ..........................................100, 121 & Rinehart N ......................... \\
\hline 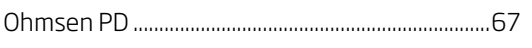 & 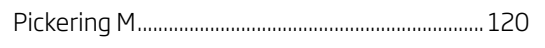 & 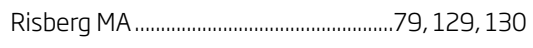 \\
\hline 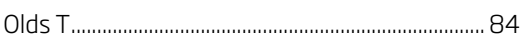 & 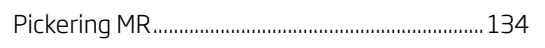 & $\ldots \ldots \ldots \ldots \ldots \ldots . .128$ \\
\hline Oliver J........................................................ 8, 67, 107 & Pilcher C & 9 \\
\hline 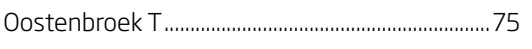 & Pinheiro MB........................................ 122, 163 & $\ldots \ldots \ldots \ldots . . .40,42,142$ \\
\hline Ordoñana JR .......................... 5, 18, 121, 122, 163 & 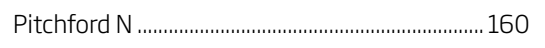 & Roberts $\mathrm{C}$ \\
\hline Orr R................................................ & Pizzari T ................................... 31, 106, 122, 123 & Robertson IK ..................... \\
\hline 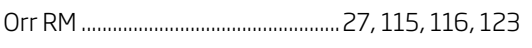 & 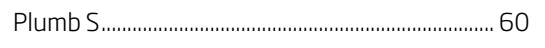 & 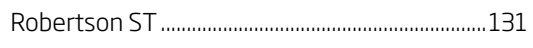 \\
\hline 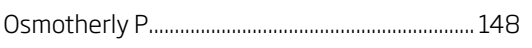 & Podmore V & 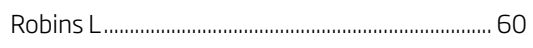 \\
\hline Osmotherly PG ............................... 10, 40, 41 & Pole N & 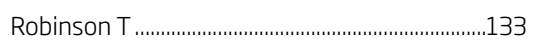 \\
\hline Osmotherly PG...... 42, 53, 116, 117, 142, 149, 155 & Pollnitz P & 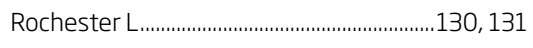 \\
\hline 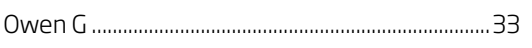 & Pope R...........34, 50, 115, 116, 123, 124, 137, 143 & 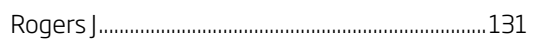 \\
\hline Pacey V ................................................4, 57, 117 & 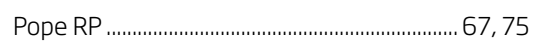 & Rollinson TC ....................... \\
\hline
\end{tabular}




\section{AUTHORS' INDEX}

Rosbergen ICM..................................................... 131

Rose S............................................................ 19, 20

Rothmore P ....................................................................132

Rothwell JC .............................................................96

Rowlands A ...................................................... 84

Rowley D ............................................................... 131

Royse A ...................................................................

Ruscoe G.............................................................132

Russell G..................................................................... 60

Russell N................................................................132

Russell T ........................................... 44, 49, 71, 146

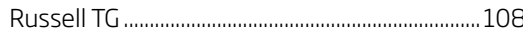

Rutkowski S ........................................................ 73

Ryall T .................................................................132

Ryan M............................................................ 90, 92

Rzewuska M............................................................... 88

Sackville G .................................................................... 31

Said C ..................................................................97

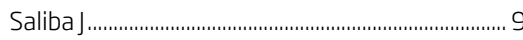

Salter K ..................................................................110

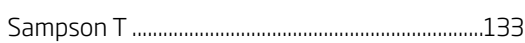

Samuels A .............................................................. 45

Sanchez-Romera JF ................................................... 163

Sanders RH ............................................................

Sanderson M ...................................................133

Santos MD …..................................................133

Sarig-Bahat …......................................................... 150

Sarkies M................................................................... 60

Sartori S...................................................................... 70

Saunders N ........................................................ 17

Savage J …............................................................. 78

Saxena M................................................................62 62

Sayer J............................................................ 80, 146

Scarvell ] ........................................................ 34, 43

Scarvell JM....................................................120, 134

Schabrun S..................................................................142

Schabrun SM ................................... 4, 134, 147, 148

Schache A ...............................................................157

Schache AG ....................................................... 79

Schippers M .............................................................101

Schnieder G................................................................. 141

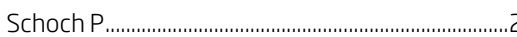

Schofield D ….................................................135

Schurr K ................................................. 63, 74, 150

Schütze R ..............................................................24

Scobie E ................................................................. 38

Scrivener K ............................................. 38, 74, 143

Scuffham P .............................................................127

Scuffham PA .........................................................92

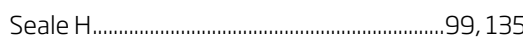

Selvaratnam PJ.........................................................135

Semciw A ....................................................... 39, 106

Senserrick C ........................................................... 154

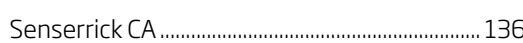

Serrao C .............................................................. 136

Setchell ] ....................................................33, 136

Seth T ..................................................................... 148

Shah S .................................................................... 136

Shaw B ................................................................. 80

Shea M ............................................................137

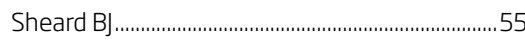

Shek C ....................................................................137

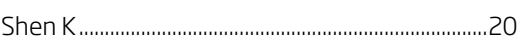

Sherrington C................................63, 137, 150, 163

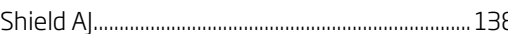

Shields N …................................................23, 55, 138

Shing CM ..............................................................99

Shinkfield P .........................................................58

Shirley D ............................................... 40, 127, 138

Shirley DK …………................................................. 163

Shiyko MP ......................................................... 117

Siao MR ………………………………………… 139

Siddall P ................................................................... 139

Silbert PL ............................................................ 140

Simic M............................................................ 5,17

Simmonds M] .......................................................... 104

Sims K …………………………………………………

Sims KJ.............................................................. 49

Sinclair M.................................................................... 117

Sinclair PJ...........................................................

Singer BJ ..................................................... 139, 140

Singer KP.............................................................. 140

Singer L........................................................................ 73

Singh PJ ..............................................................

Singla MM …............................................................ 140

Sjøgaard G .............................................................29

Skinner E _........................................................60, 62

Skinner EH.......................................... 9, 15, 16, 119

Slater SL .....................................................46, 59, 60

Sloane J ............................................................................ 141

Smart A ............................................................................. 83

Smith A …...........................................24, 27, 141, 146

Smith CM.......................................................... 160

Smith D .....................................................................127

Smith M............................................................. 54, 114

Smith MD

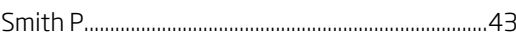

Smith PN ….......................................... 34, 120, 134

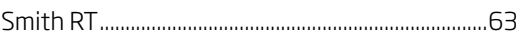

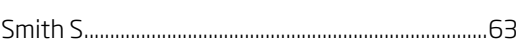

Smith SE .................................................................142

Smith ST ..................................................................... 13

Snodgrass S] ...........................40, 106, 114, 142, 149

Solberg OM....................................................................115

Sole G .........................................................................143

Soles K..............................................................143

Somerville L .............................................................. 104

Son Jl ................................................................... 61
Soomro N ................................................................... 3

Sorrell-Saunders M..............................................143

Southam M...............................................................143

Spencer L _................................................... 99, 161

Spencer LM ................................................................101

Spittle A .......................................................5

Spittle AJ.............................................................. 144

Spurrier D.................................................................125

Srikanth V ................................................................... 158

Stafford R ....................................................................... 68

Standage M......................................................... 84

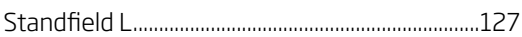

Stanton R ….............................................124, 144

Stanton TR ............................................................ 144

Stanton W............................................... 48, 94, 105

Staples K ............................................................. 70, 76

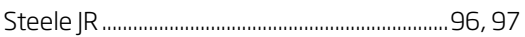

Steffens D ................................................................... 87

Steinfort D .............................................................56

Sterling M..................... 26, 119, 128, 141, 144, 145

Stewart V ..................................................................145

Stierli M.............................................................116, 123

Stiller K ................................................................ 35, 36

Stockton K ...........................................................118

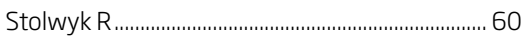

Straker L ................................................... 145, 146

Strudwick K .......................................................... 146

Stubbs N.................................................................. 35

Sturt R .................................................................. 146

Sullivan KE ....................................................147

Summers S ............................................................... 147

Sundberg S............................................................ 115

Surkitt LD ...............................................46, 59, 60

Sutherland P............................................................ 111

Swan N ..............................................................147

Symonds JT ..........................................................116

Szeto G ...................................................................152

Szoeke C ................................................................97

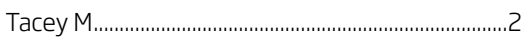

Tai G..................................................................... 104

Talevski J..................................................................... 31

Tan B-K ........................................................................147

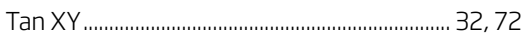

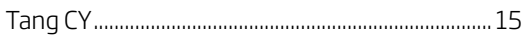

Tang S.................................................................135

Tang WH..............................................................152

Tavares G ...........................................................137

Taylor N.....................................................................110

Taylor NF ..............................23, 39, 46, 59, 60, 155

Te M................................................................. 147, 148

Terrill D............................................................................. 61

Teys $P$...................................................................... 126

Thewlis D ................................................................... 103

Thomas D ................................................................. 57 


\begin{tabular}{|c|c|}
\hline 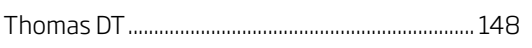 & 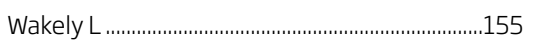 \\
\hline Thomas L & Waldron N \\
\hline 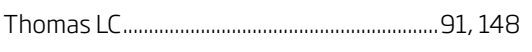 & Walker KC \\
\hline 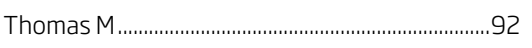 & Walker R............. \\
\hline 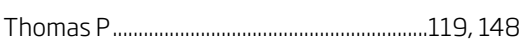 & 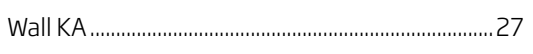 \\
\hline Thomas R & Wallis JA .................... \\
\hline 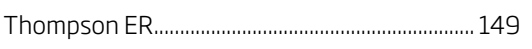 & 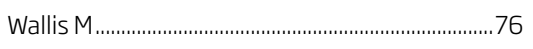 \\
\hline 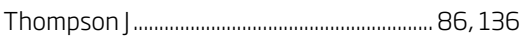 & Wallwork SB............... \\
\hline Thomson A .................. & Walmsley ME ............ \\
\hline 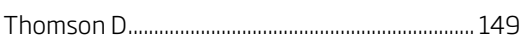 & Walmsley S............................ \\
\hline Thurnwald P ................................................ & 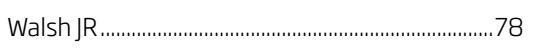 \\
\hline Tiedemann A ....................................... 5, 137, 163 & Walter K ..................... \\
\hline$\ldots$ & Walters J.................. \\
\hline Tipping C .................. & Wang TJ........................ \\
\hline Titov N..................... & 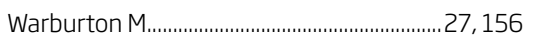 \\
\hline 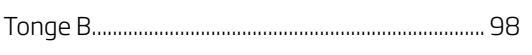 & 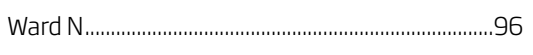 \\
\hline 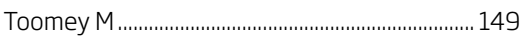 & 120 \\
\hline 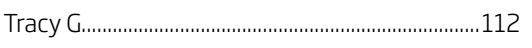 & .......................... 20 \\
\hline$\ldots$ & Watson BM.............. \\
\hline$\ldots$ & Watson SL............. \\
\hline$\ldots$ & Watter P............... \\
\hline Treleaven J............................63, 125, 148, 150, 151 & Webb G..................... \\
\hline 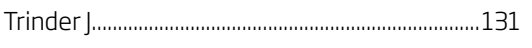 & 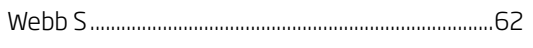 \\
\hline Trott CA & Weber $\mathrm{H}$ \\
\hline Truong AP............ & Webster KE......... \\
\hline$\ldots \ldots \ldots \ldots . .152$ & Weeks B............ \\
\hline Tsang S ................. & Weeks BK ................. \\
\hline 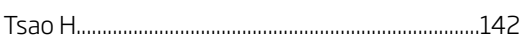 & 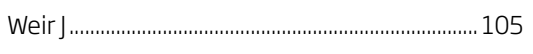 \\
\hline$\ldots \ldots \ldots \ldots \ldots \ldots \ldots . .122$ & Wells C ...................... \\
\hline 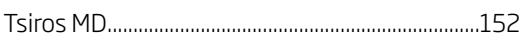 & Wells $\mathrm{H} \ldots \ldots \ldots \ldots \ldots \ldots \ldots$ \\
\hline 159 & Weston D................. \\
\hline Turczyniak M.......... & Whitney S........ \\
\hline 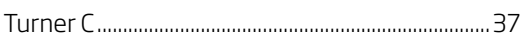 & 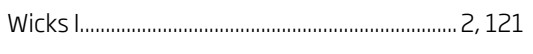 \\
\hline$\ldots \ldots \ldots \ldots \ldots . .42,152,153$ & $\ldots$ \\
\hline Tyson KP ................................. & $\ldots$ \\
\hline 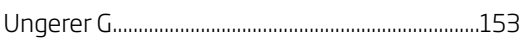 & 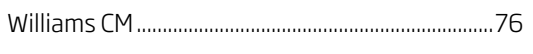 \\
\hline van den Berg MEL............. & $\ldots \ldots \ldots . . . .6,67,76,107,157$ \\
\hline Van der Lee LM.............................................153, 154 & $\ldots$ \\
\hline van Leeuwen KDB........... & Williams K............... \\
\hline van Middelkoop M............ & Williams L............... \\
\hline Van Zundert ........................ & Williams P................ \\
\hline 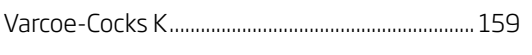 & Wilson A..................... \\
\hline Vargas JP & Wilson AM................ \\
\hline$\ldots$ & 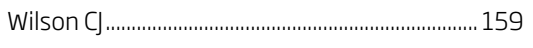 \\
\hline 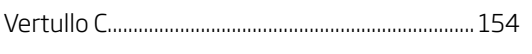 & 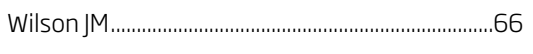 \\
\hline Vicenzino B ....................................17, 18, 32, 33, 58 & Winchester FI............. \\
\hline 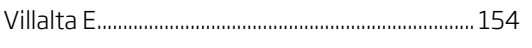 & 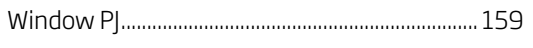 \\
\hline Visentini P ..........................................125, 154, 161 & 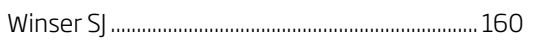 \\
\hline 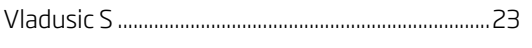 & Winzenberg T ..................................35, 131, 158, 160 \\
\hline 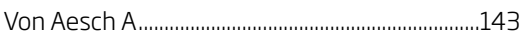 & Winzer B \\
\hline Waddington G ............................................... 124, 154 & Wisbey-Roth T.......................................3, 154, 161 \\
\hline 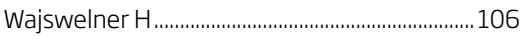 & 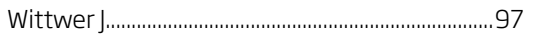 \\
\hline Wajswelner W & 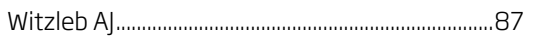 \\
\hline 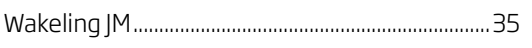 & Wolff M....................... \\
\hline
\end{tabular}

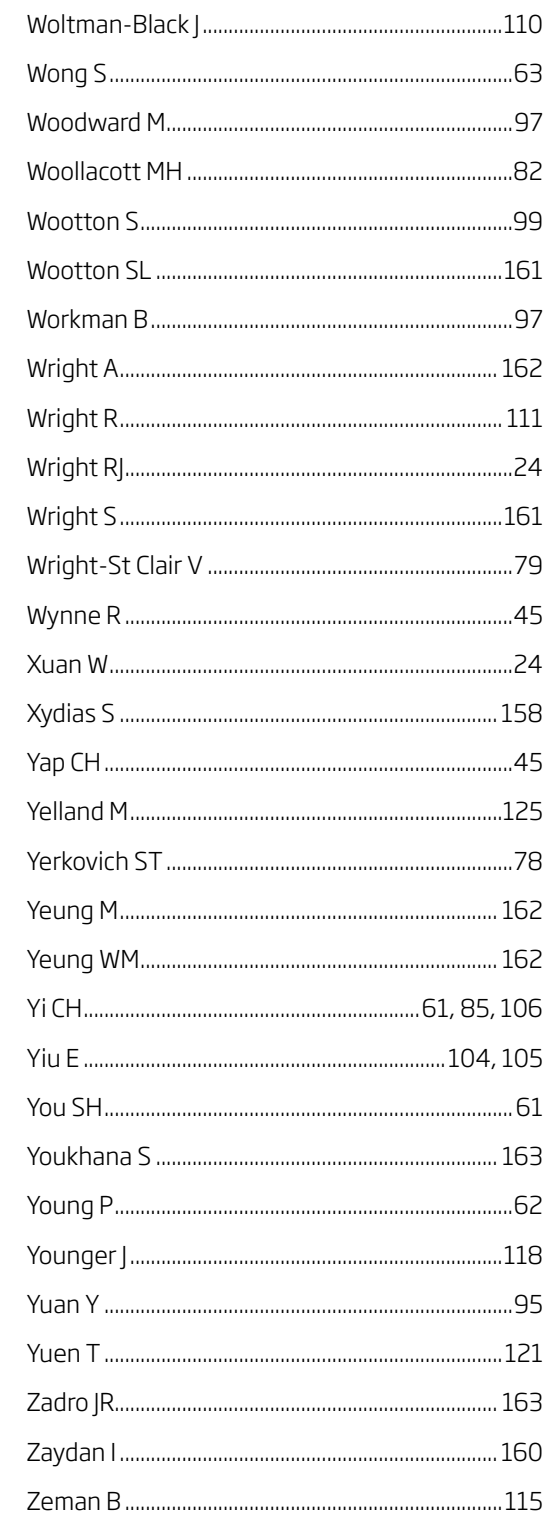



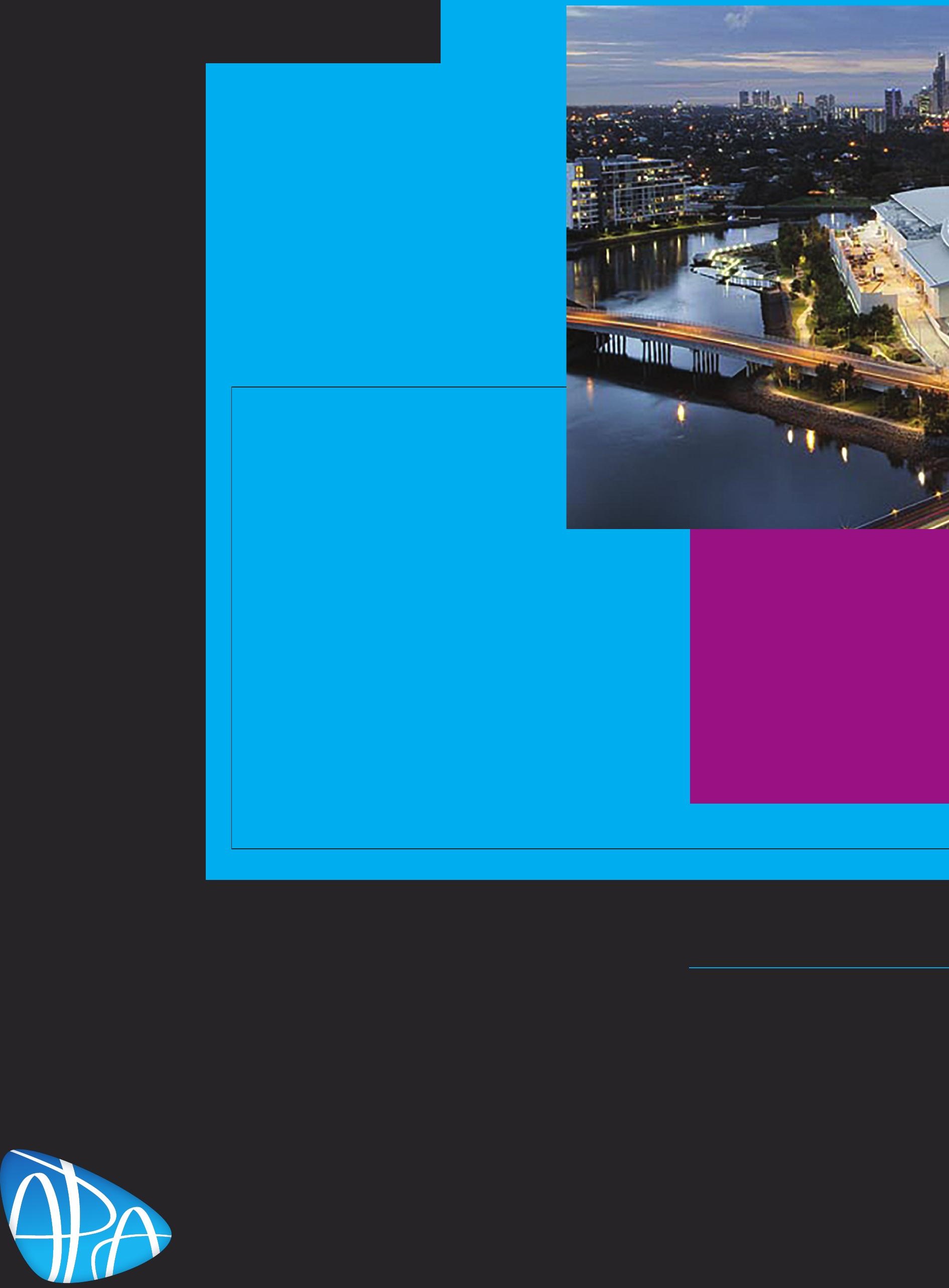

AUSTRALIAN PHYSIOTHERAPY ASSOCIATION 